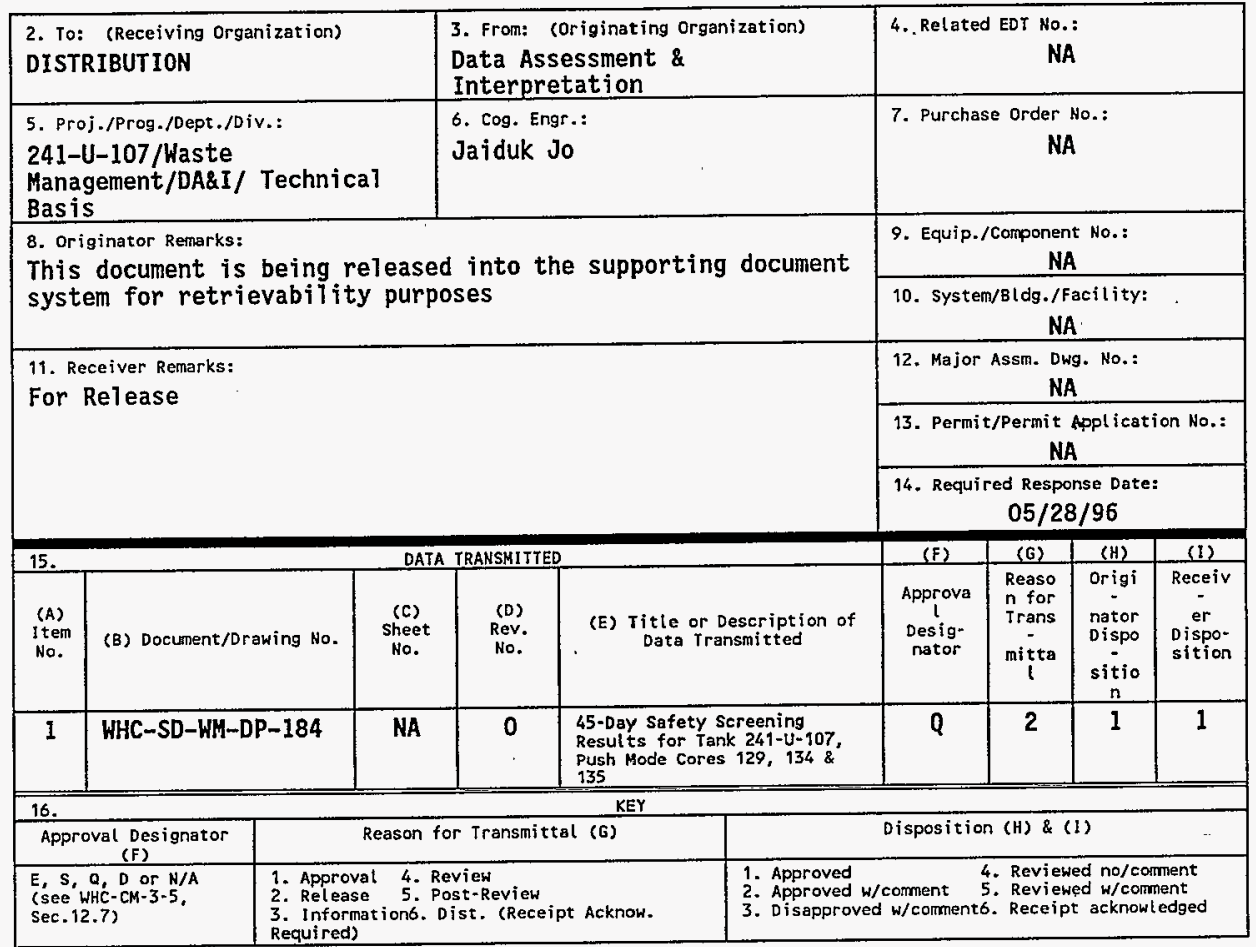

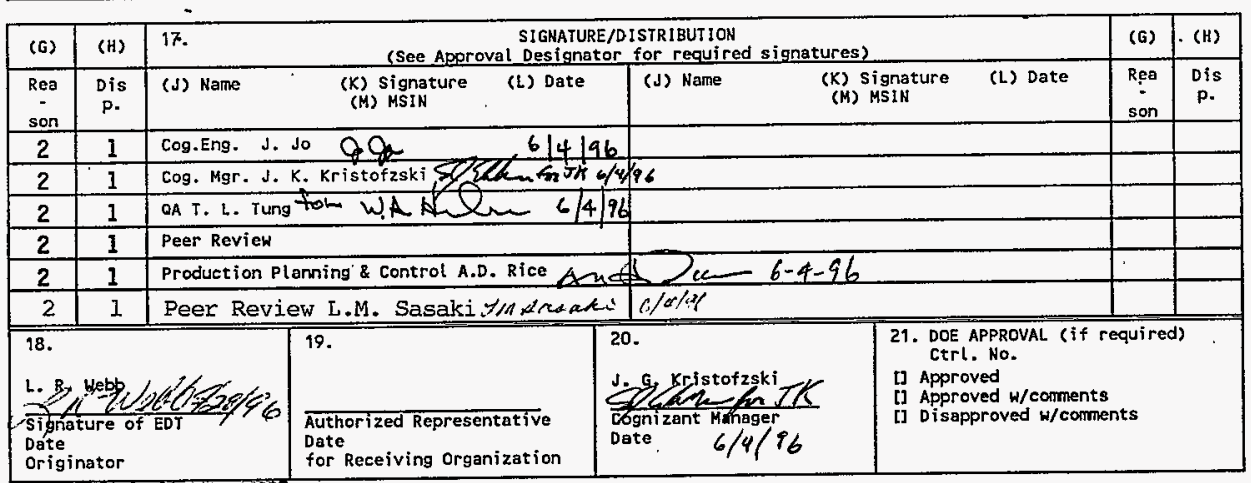

BD-7400-772-2 (04/94) GEFOS7 


\section{"45-DAY SAFETY SCREENING RESULTS FOR TANK 241-U-107, PUSH MODE CORES 129, 134 AND 135."}

Jaiduk Jo

Westinghouse Hanford Company, Richland, WA 99352

U.S. Department of Energy Contract DE-AC06-87RL10930

$\begin{array}{lll}\text { EDT/ECN: } & \text { EDT-614792 } & \text { UC: } 2070 \\ \text { Org Code: } & 79400 & \text { Charge Code: } \\ \text { B\&R Code: } & \text { EW } 3120074 & \text { Total Pages: } 310\end{array}$

Key Words: 45-Day Safety Screening Results for Tank 241-U-107, Push Mode Cores 129, 134 and 135, Tank 241-U-107

Abstract: N/A

TRADEMARK D1SCLAIMER. Reference hereín to any specific commercial product, process, or service by trade name, tradenark, manufacturer, or otherwise, does not necessarily constitute or imply its endorsement, recommendation, or favoring by the United States Government or any agency thereof or its contractors or subcontractors.

Printed in the United States of America. To obtain copies of this document, contact: WHC/BCS Document Control Services, P.O. Box 1970, Hailstop H6-08, Rjchlond HA. 09352 , Rhope (509) 372-2420. Fax (509) 376-4989.
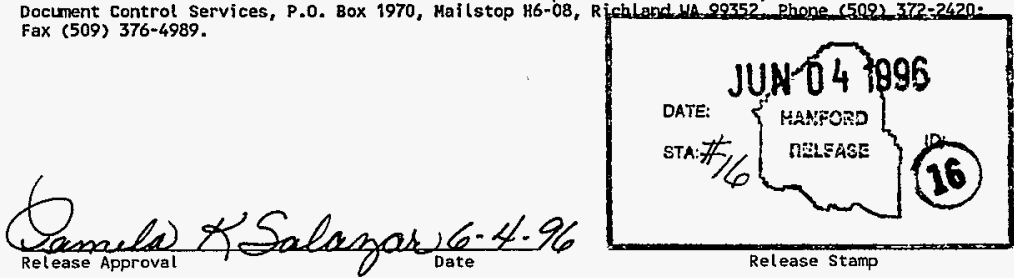

\section{Approved for Public Release}




\section{ANALYTICAL SERVICES}

\section{5-DAY SAFETY SCREENING RESULTS FOR TANK 241-U-107, PUSH MODE CORES 129, 134 AND 135}

Project Coordinator: JAIDUK JO

Prepared for the U.S. Department of Energy Office of Environmental Restoration and Waste Management

by

Westinghouse Hanford Company Box 1970

Richland, Washington 
WHC-SD-WM-DP-184, REV. 0

THIS PAGE WAS INTENTIONALLY LEFT BLANK 
WHC-SD-WM-DP-184, REV. 0

TABLE OF CONTENTS

Narrative ........................ . . . . . . .

Sample Data Summary . . . . . . . . . . . . . . 12

Dome Space Vapor Flanmability Data Sheets . . . . . . . . . . 30

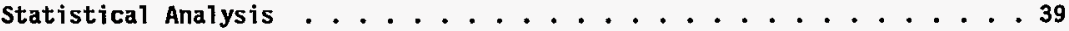

Inorganic Analyses . . . . . . . . . . . . . . . 44

Differential Scanning Calorimetry (DSC)

DSC Work1 ist \#5892 $(644,647) \ldots \ldots . . . . . . . . .446$

DSC Workl ist \# $5893(650,653) \ldots \ldots . . . . . . . .52$

DSC Workl ist \#6035 (683) . . . . . . . . . . . . . 58

DSC Worklist \# $6511(1055,1056) \ldots \ldots$. . . . . . . . . 62

DSC Workl ist \# $6512(1057,1058) \ldots \ldots 9$

DSC Work7 ist \# $6515(1064,1065) \ldots \ldots . \ldots . . \ldots 77$

DSC Workl ist \# $6516(1068,1143) \ldots \ldots . \ldots . . \ldots 83$

DSC Horkl ist \# $6624(1128)$. . . . . . . . . . . . . . 92

DSC Worklist \#6627 $(1059,1070) \ldots . . . . . . . . . . .997$

DSC Workl ist \# $6655(665,682) \ldots \ldots \ldots \ldots$

DSC Worklist \# $6753(1159)$. . . . . . . . . . . . . . . 112

DSC Workl ist \# $7587(1119,1120,1121) \ldots \ldots . . . \ldots . . . .116$

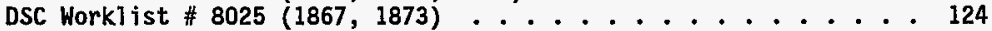

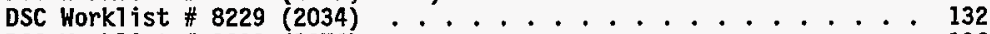

DSC Horkl ist \# $8230(1874) \ldots \ldots \ldots$

DSC Workl ist \# 8245 (2132) ................. 141

DSC Workl ist \# $8437(2140,2141) \ldots \ldots . . . . . . . . . .146$

DSC Workl ist \# 8438 (2142) . . . . . . . . . . . 152

DSC Workl ist \# 8439 (2133) . . . . . . . . . . . . 157

DSC Work list \# 8949 (1866) $\ldots \ldots \ldots 161$

DSC Workl ist \# $9021(665,682,2140,1119,1120,1121$, $2034,2141,2142) \ldots \ldots . . \ldots 165$

DSC Worklist \# $9022(650,653,644,647,683,1055$, $1128,1143,1159) \ldots \ldots . \ldots 168$

DSC Workl ist \# 9023 (2131, 1056, 1057, 1058, 1059, $1064,1065,1068,1070) \ldots \ldots . . . . . .171$

DSC Work list \# $9024(1867,1873,1874,2132,2133) \ldots \ldots . . . .174$

DSC Worklist \# $9028(2131) \ldots \ldots \ldots \ldots . . \ldots 176$

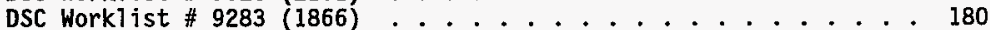

Thermogravimetric Analysis (TGA)

TGA Workl ist \#5890 $(650,653) \ldots \ldots \ldots \ldots . \ldots . \ldots 181$

TGA Workl ist \# 5891 (644, 647) . . . . . . . . . . 187

TGA Workl ist \# 6027 (683) .................. 193

TGA Workl ist \# $6499(1055,1056) \ldots \ldots . \ldots . \ldots 196$ 
WHC-SD-WM-DP-184, REV. 0

TABLE OF CONTENTS (Continued)

TGA Workl ist \# $6500(1057,1058) \ldots \ldots . \ldots . \ldots . . . . .203$

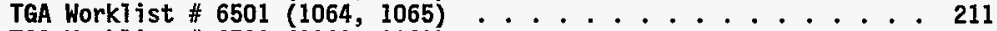

TGA Work1 ist \#6502 $(1068,1143) \ldots \ldots \ldots \ldots . \ldots . \ldots 216$

TGA Work7 ist \#6633 $(665,682) \ldots \ldots . . . . . . . . .225$

TGA Work 1 ist \# $6648(1059,1070)$. . . . . . . . . . . . . . 231

TGA Workl ist \#6656 (1128) . . . . . . . . . . 240

TGA Work ist \#6758 (1159) .............. 245

TGA Work $l$ ist \# $7585(1119,1120,1121) \ldots \ldots . \ldots . \ldots 251$

TGA Worklist \# 7908 (1867) $\ldots \ldots \ldots \ldots \ldots \ldots$

TGA Workl ist \# 8037 (1873) ................. 264

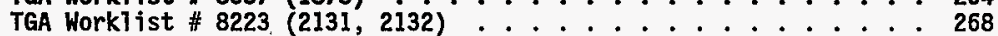

TGA Work list \#8224 (1874) $\ldots . . . . . . . . . . . . . .274$

TGA Work list \# 8226 (2034) . . . . . . . . . . . . . 279

TGA Work l ist \#8434 $(2140,2141) \ldots \ldots . \ldots . \ldots . . \ldots 283$

TGA Workl ist \# $8435(2142) \ldots \ldots . . . . . . . .289$

TGA Workl ist \#8436 (2133) . . . . . . . . . . . . 293

TGA Workl ist \# $8947(1866) \ldots \ldots . \ldots . . . \ldots 299$

This Document consists of pages 1 through 302, plus page 123.1, 194.1, 214.1. Also pages $i i, 2,13,31,40$ and 45 were intentionally left blank.

\section{TRADEMARKS:}

Perkin-Elmer is a Registered Trademark of Research and Manufacturing Company, Inc. Mettler is a Registered Trademark of Mettier Electronics 
WHC-SD-HM-DP-184, REV. 0

NARRATIVE

1 
WHC-SD-WM-DP-184, REV. 0

THIS PAGE WAS INTENTIONALLY LEFT BLANK 


\section{WHC-SD-WM-DP-184, REV. 0 \\ 45-Day Safety Screening Results for Tank 241-U-107, Push Mode \\ Cores 129,134 , and 135}

\section{SUMMARY}

This is the 45-day safety screening report for rotary mode core samples taken from risers 9, 7, and 2 of tank 241-U-107 (U-107) from February 6, 1996 through March 28, 1996. The samples were received at the 222-5 Laboratories from February 9, 1995 through April 2, 1996, and are undergoing analyses consistent with the safety screening data quality objective (DQO) (Dukelow et a1. 1995), the organic safety program DQO (Turner et a1. 1995), the historical DQO (Simpson and MCCain 1995), and the compatibility DQO (Fowler 1995). ATl subsegments from the three cores obtained are being analyzed for those tests identified in the sampling and analysis plan (Raphael 1996), except in circumstances where insufficient material prevented the analysis. This interim report, however, only summarizes the results of the primary safety screening analyses: differential scanning calorimetry (DSC), thermogravimetric analysis (TGA), total alpha analysis, and density/specific gravity anaiyses. The remaining analyses shall be discussed in the final report for this project. In addition to the analytical requirements of above references, a test for flammable gas concentration was requested; the data from these measurements obtained by the field operations crews are also provided.

None of the data indicate that the tank is "unsafe" when compared to the criteria in the Safety Screening Data Quality Objective (Dukelow et a]. 1995) with the exception of the energetic results for two samples (1 iquid samples). However, there is a high moisture content of greater than $45 \%$ in the 7 iquid portion of the tank. The one-sided 95-percent confidence interval for total alpha results are well below the notification 1 imit. Furthermore, the vapor in the tank U-107 dome space is far below the 25\% Tower flammability limit (LFL) stated in the SAP. Therefore, the results show that this tank may be considered "conditionaliy" safe.

Water solution with a lithium bromide tracer was used to soften the sample when the sampling crew encountered hard surfaces. However, no water solution blank was taken. If needed, the water solution blank data from the tank 241U-109 sampling event may be used. Bromide analys is by ion chromatography (IC) and Tithium analysis by inductively coupled plasma atomic emission spectroscopy (ICP) are being performed to determine the extent of water contamination in the samples. These results will be summarized in the final report for this analysis event.

\section{SCOPE}

This document serves as the 45-day report deliverable for the tank 241-U-107 core samples collected between February 6 and March 28, 1996. Each sample was received, extruded, and analyzed by the 222-S Laboratory in accordance with the SAP (Raphael 1995). 
WHC-SD-WM-DP-184, REV. 0

This report includes the primary safety screening results (tank headspace flammability, DSC, TGA, total alpha, and bulk density or specific gravity) and copies of a 11 DSC and TGA raw data scans as requested per the SAP.

Photographs of the extruded segments were taken and, although not included in this report, are available.

\section{SAMPLE RECEIPT AND EXTRUSION}

Eight segments per core were expected to be taken from this tank. Segment 1 was expected to be $26 \mathrm{~cm}$ (10 inches) in depth and 2 through 8 segments expected to be $48 \mathrm{~cm}$ (19 inches) in depth. However, the sampling crew encountered very hard surface at different levels for each core and the waste could not be further penetrated. Therefore, the three cores were only 3,6 , and 2 segments in legth.

Information on the receipt and extrusion of the core samples, including sampling date, sample receipt date, extrusion date, amount of sample, and sample description, is provided in Table 1 . No separabie organic liquid was observed in any of the segments, and no fjeld blank was delivered to the $222-5$ Laboratory with this sampling event. 


\begin{tabular}{|c|c|c|c|c|c|c|}
\hline 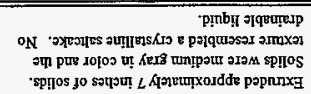 & s 乙゙દ9I & :p!os $\pi^{\circ} \mathrm{H}$ Iadd & $96 / 0 z / 20$ & $96 /$ St/R0 & $96 / 60 / 20$ & 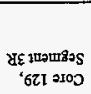 \\
\hline 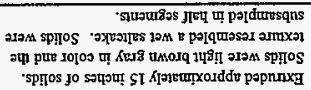 & $\begin{array}{l}31.6 \mathrm{LI} \\
86.6 \mathrm{I}\end{array}$ & 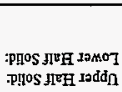 & 96/EI/Z0 & $96 / 60 / 20$ & $96 / L 0 / 20$ & $\begin{array}{l}\text { \& turuses } \\
\text { '6ZI 2105 }\end{array}$ \\
\hline $\mathrm{V} / \mathrm{N}$ & & 2tron & 96/EU/Z0 & $96 / 60 / 20$ & $96 / 60 / 20$ & 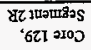 \\
\hline 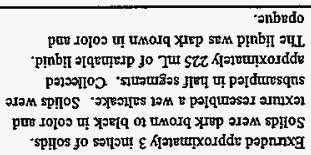 & $\begin{array}{l}\text { \& } \tau L E \\
8 \pi S L \\
\text { \& } \$ \varepsilon L Z\end{array}$ & 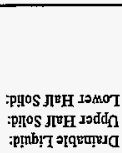 & 96/EI/20 & $96 / 60 / 20$ & $96 / 90 / 20$ & 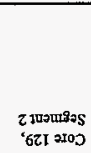 \\
\hline$V / N$ & & =ய0N & 96/Et/Z0 & $96 / 60 / 20$ & $96 / 60 / 20$ & 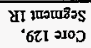 \\
\hline 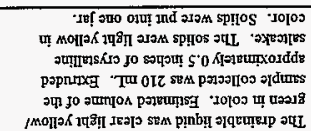 & $\begin{array}{c}85 \% \\
88^{*} 99 \tau\end{array}$ & 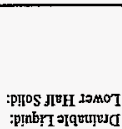 & $96 / \varepsilon t / 20$ & $96 / 60 / 20$ & $96 / 90 / 20$ & $\begin{array}{l}\text { ! 1t6ett8as } \\
\text { '6t! } 2100\end{array}$ \\
\hline 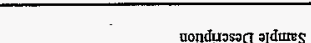 & & 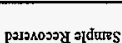 & papnnxस गеत & 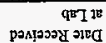 & pगdures गाष & गุdutrs \\
\hline
\end{tabular}

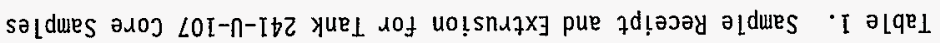

\section{0 ค}


WHC-SD-WM-DP-184, REV. 0

\begin{tabular}{|c|c|c|c|c|c|c|}
\hline Sample & Date Sampled & $\begin{array}{l}\text { Date Received } \\
\text { at Lab }\end{array}$ & Date Extruded & Ssmple Recovered & & Sample Desctiption \\
\hline $\begin{array}{l}\text { Core 134, } \\
\text { Segment } 1\end{array}$ & $02 / 16 / 96$ & $02 / 20 / 96$ & $02 / 23 / 96$ & None & & $N / A$ \\
\hline $\begin{array}{l}\text { Core 134, } \\
\text { Segment } 2\end{array}$ & $02 / 16 / 96$ & $02 / 20 / 96$ & $02 / 23 / 96$ & Upper Half Solid: & $249.2 \mathrm{~g}$ & $\begin{array}{l}\text { Extruded approximately } 12 \text { inches of solids. } \\
\text { Solids were white/grcy fin color and the texaine } \\
\text { resembled a dry saltcake. Solids were } \\
\text { subsampled in one jar. }\end{array}$ \\
\hline $\begin{array}{l}\text { Core 134, } \\
\text { Segment } 3\end{array}$ & $02 / 16 / 96$ & $02 / 20 / 96$ & $02 / 27 / 96$ & Upper Half Solid: & $143.9 \mathrm{~g}$ & $\begin{array}{l}\text { Extruded approximately } 6.5 \text { frches of solits. } \\
\text { Solids were medrum gray in color and the } \\
\text { texture resembled a salteake. }\end{array}$ \\
\hline $\begin{array}{l}\text { Core 134, } \\
\text { Segment } 4\end{array}$ & $02 / 16 / 96$ & $02 / 20 / 96$ & $02 / 27 / 96$ & $\begin{array}{l}\text { Lower Half Soltd: } \\
\text { Quarter Segment B: } \\
\text { Quaster Segment A: }\end{array}$ & $\begin{array}{r}184.4 \mathrm{~g} \\
92.8 \mathrm{~g} \\
81.8 \mathrm{~g}\end{array}$ & $\begin{array}{l}\text { Extruded approximately } 17.5 \text { inches of solids. } \\
\text { Facies was present, the lower section was } \\
\text { bleck sludge and the topper section was dark } \\
\text { gray salteake. The solids were subsampled in } \\
\text { quarter segments. }\end{array}$ \\
\hline $\begin{array}{l}\text { Core 134, } \\
\text { Segment } 5\end{array}$ & $02 / 16 / 96$ & $02 / 20 / 96$ & $02 / 27 / 96$ & $\begin{array}{l}\text { Quarter Segment B: } \\
\text { Quarter Segment A: }\end{array}$ & $\begin{array}{r}49.7 \mathrm{~g} \\
59.8 \mathrm{~B}\end{array}$ & $\begin{array}{l}\text { Exuruded approximately } 5 \text { inches of solids. } \\
\text { Solids were gray salicake (lower section) and } \\
\text { black sludge (upper section). Solids contained } \\
\text { facies. }\end{array}$ \\
\hline $\begin{array}{l}\text { Core 134, } \\
\text { Segment 5A }\end{array}$ & $02 / 20 / 96$ & $02 / 20 / 96$ & $02 / 27 / 96$ & $\begin{array}{l}\text { Quarter Segmenl A: } \\
\text { Liner Liquid: }\end{array}$ & $\begin{array}{l}19.4 \mathrm{~g} \\
32.8 \mathrm{~g}\end{array}$ & $\begin{array}{l}\text { Extruded approximately } 1 \text { inch of solids. } \\
\text { Sotids were gray in color with some brown } \\
\text { tint. The texture resembled a salcake. }\end{array}$ \\
\hline $\begin{array}{l}\text { Core 134, } \\
\text { Segment 5B }\end{array}$ & $02 / 21 / 96$ & $02 / 23 / 96$ & $02 / 27 / 96$ & Drainable Liquid: & $106.3 \mathrm{~g}$ & $\begin{array}{l}\text { Collected approxinately } 70 \mathrm{mI} \text { of drainable } \\
\text { liquid. The liquid was dark brown in color } \\
\text { and opaque. No solids exruded other than } \\
\text { those suspended in drainable liquid. }\end{array}$ \\
\hline $\begin{array}{l}\text { Core 134, } \\
\text { Segment } 6 \\
\end{array}$ & $02 / 21 / 96$ & $02 / 23 / 96$ & $02 / 27 / 96$ & None & & $\mathrm{N} / \mathrm{A}$ \\
\hline $\begin{array}{l}\text { Core 134, } \\
\text { Segment } 6 \mathrm{~A}\end{array}$ & $02 / 26 / 96$ & $02 / 23 / 96$ & $03 / 01 / 96$ & $\begin{array}{l}\text { Whole Segnent: } \\
\text { Drainatle liquid: } \\
\text { Liner Liquid: }\end{array}$ & $\begin{array}{l}29.3 \mathrm{~g} \\
89.6 \mathrm{~g} \\
16.8 \mathrm{~g}\end{array}$ & $\begin{array}{l}\text { Extruded approximately } 6 \text { inches of solids. } \\
\text { Solids were light browin in color and texture } \\
\text { resembled a wet salteake. Collected } \\
\text { approximately } 70 \mathrm{~mL} \text { of drainable liquid. The } \\
\text { liquid was light brown in color and opaque. }\end{array}$ \\
\hline
\end{tabular}




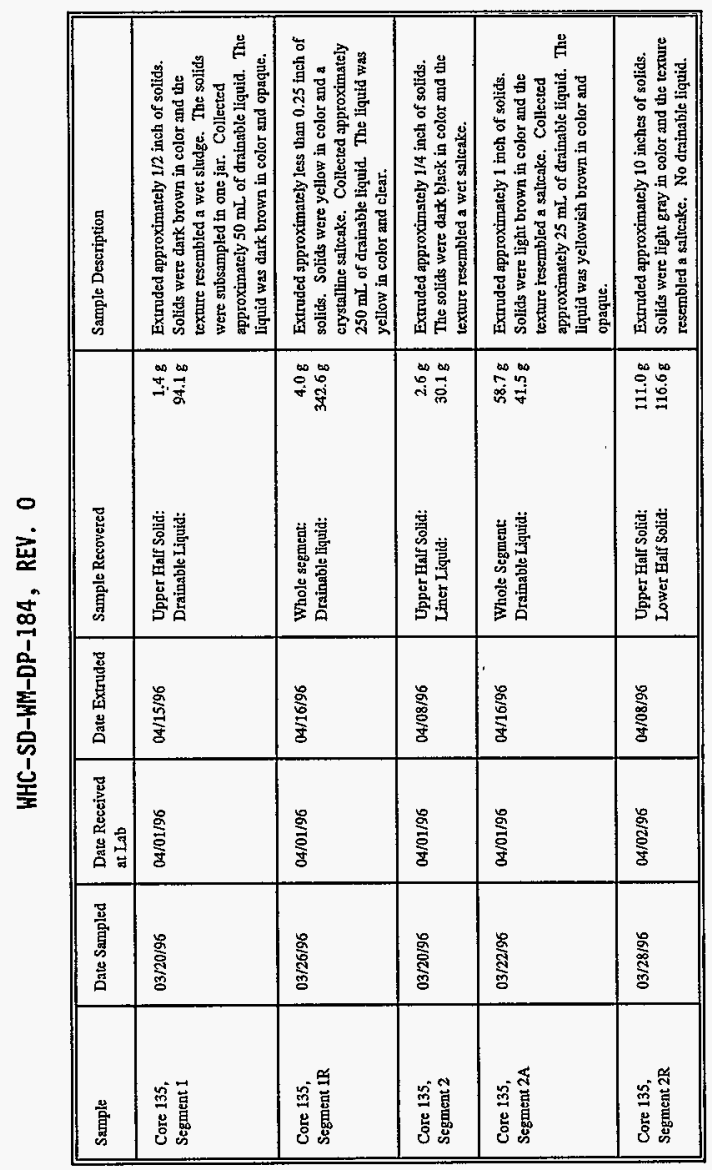




\section{TANK HEADSPACE FLAMMABILITY}

Prior to core sampling, the flammability of the tank headspace was measured to address the vapor flammability issue of the safety screening data quality objective (Dukelow et a7. 1995). The tank vapor space was sampled periodically from February 6 through February 21, 1996 to determine the flammability of the vapor space gasses. Sampling was done through risers 7 and 9 in the dome space. The average combustible gas meter readings were between 0 to 4 percent of the LFL (attachment), indicating no flammability concerns with this tank.

\section{ANALYTICAL RESULTS}

Analytical results appear in the Sample Data Summary and a discussion of the results is presented below. Any deviations from the requirements of the SAP are noted in the following sections.

\section{Thermogravimetric Analys is (TGA) Moisture}

The weight percent water by TGA was performed under a nitrogen purge using either procedure LA-560-112, Rev B-1 on a Mettler TG 50 instrument or procedure LA-514-114, Rev. C-1 on a Perkin Elmer TGA 7 instrument.

Results ranged from 0.73 to $49.9^{\circ}$ weight percent water for the solids. Drainable liquids ranged from 47.0 to 92.2 weight percent water. Three 1 iner liquid sample were analyzed and the results ranged from 89.1 to 98.2 weight percent water. The weight percent water for sample S96T001070 (Core 134, Segment 6A) was $92 \%$. The probable cause for the high water content in this sample is due to the contamination by the water solution.

Ali standards run with the sampies had recoveries which fell within the required range of 90 to 110 percent. Ten subsegments had RPD values that exceeded the desired 10 percent criterion. No reruns were requested due to the heterogeneity of the samples.

\section{Differential Scanning Calorimetry (DSC)}

Differential scanning calorimetry analyses under a nitrogen purge were performed using either procedure LA-514-113, Rev. $\mathrm{C}-1$ on a Mett1er DSC 20 instrument or procedure LA-514-114, Rev. C-1 on a Perkin Elmer DSC 7 instrument. It should be noted that the DSC scans from these two instruments show exotherms different1y; exotherms on the Perkin Eimer are shown as downward peaks while the Mettler shows them as upward peaks. Using the average percent water by TGA for each sample, dry weight basis results were calculated from the wet weight basis results. Both wet and dry results are given in the Sample Data Summary. 
WHC-SD-WM-DP-184, REV. 0

Sixteen out of twenty-eight of the waste samples showed no exotherm. Two of the twelve samples which had observable energetics had DSC exotherms that exceeded the action limit of 480 joules/g (dry weight basis) stated in the SAP (Raphael 1996) and the Safety Screening Data Quality objective (Dukelow et al. 1995). Appropriate notifications of a limit violation were made.

For the two samples with DSC results above 480 joules $/ g$, the upper limit of a one-sided $95 \%$ Confidence Interval were calculated (see attachment). The upper 7 imit of the $95 \%$ Confidence Intervals were 674.0 to 678.0 joules/g (dry weight basis). For the other ten samples with DSC results above 0 joules $/ g$, the upper limit of a one-sided $95 \%$ Confidence Intervals ranged from 16.6 to 467.8 joules/g (dry weight basis).

Standards run with the samples had recoveries which fell within the required range of 90 to 110 percent for all but two of the samples ( 112.5 and 113.9 percent). RPDs between the sample and duplicate sample analyses were below 10 percent for all but five of the samples. The RPDs for these five samples were ranged from 15.7 to 150 percent. No reruns were requested due to the heterogeneity of the samples.

\section{Total Alpha}

The total alpha analyses were performed using procedure LA-508-101, Rev. D-2. Solid samples were prepared by fusion prior to the total alpha analysis; the fusion was performed using procedure LA-549-141, Rev. D-0.

Individual sample and duplicate results ranged from $0.00243 \mu \mathrm{Ci} / \mathrm{g}$ to 0.91 $\mu \mathrm{Cj} / \mathrm{g}$ in the solids. In the drainable liquid, the results ranged from less than values from $<2.25 \mathrm{E}-03$ to $<1.72 \mathrm{E}-03 \mu \mathrm{Ci} / \mathrm{mL}$ with on $7 y$ one value reported that was not a less than value $(5.36 \mathrm{E}-03 \mu \mathrm{Ci} / \mathrm{mL})$. The low sample activities and high dissolved solids in the samples resulted in high RPDs and low spike recoveries for the tank U-107 samples. Six samples have spike recoveries out of range (from 66.0 to 87.2 percent) and 9 RPDs were above the 10 percent criterion with RPDs ranging from 11.3 to 56.1 percent. Thirteen standard recoveries were out of limits with values ranging from 110.9 to 119.5 percent.

The one-sided 95-percent upper confidence interval limit was calculated for samples where the total alpha results were above the detection limit. Results are presented in the attachment. The upper confidence intervals ranged from $2.40 \mathrm{E}-03 \mu \mathrm{Ci} / \mathrm{mL}$ to $1.65 \mathrm{E}-02 \mu \mathrm{Ci} / \mathrm{mL}$ for $7 \mathrm{iquid}$ samples and $3.20 \mathrm{E}-03 \mu \mathrm{Ci} / \mathrm{g}$ to $1.71 \mu \mathrm{Cj} / \mathrm{g}$ for solids. All of these results are well below the notification $1 \mathrm{imit}$ of $34.9 \mu \mathrm{Ci} / \mathrm{g}$; therefore, no reruns were requested. 
WHC-SD-WM-DP-184, REV. 0

\section{Bulk Density and Specific Gravity}

Bulk density measurements were performed on the solid samples using procedure 10-160-103, Rev. A-7, and specific gravity measurements were performed on the Tiquid samples using procedure LA-510-112, Rev. C-3.

Bulk densities of the soljds ranged from 1.30 to $1.76 \mathrm{~g} / \mathrm{mL}$. Bulk density for sample S96T001046 (Core 134, Segment 6A) was $1.1 \mathrm{~g} / \mathrm{mL}$. The probable cause for low density is due to the water solution contamination. A density of $1.5 \mathrm{~g} / \mathrm{mL}$ is assumed in converting the total alpha notification limit from $\mathrm{g} / \mathrm{L}$ of plutonium to $\mu \mathrm{Ci} / \mathrm{g}$. A value below 1.5 is conservative; therefore, the total aTpha notification should be adjusted to $34.9 \mu \mathrm{Ci} / \mathrm{g}$ (from density of 1.76 $\mathrm{g} / \mathrm{mL})$. The specifjc gravities of the liquids ranged from 1.38 to $1.67 \mathrm{~g} / \mathrm{mL}$. The standard recoveries for the specific gravity and density measurements were between 97.7 and 99.6 percent.

Project Coordinator: Jaiduk Jo 


\section{REFERENCES}

Dukelow, G. T., J. W. Hunt, H. Babad, and J. E. Meacham, 1995, Tank Safety Screening Data Quality Objective, WHC-SD-WM-SP-004, Rev. 2, Westinghouse Hanford Company, Richland, Washington.

Fowler, K. D., 1995, Data Quality Objectives for Tank Farms Waste Compatibi7ity Program, WHC-SD-WM-DQ0-001, Rev. 1, Westinghouse Hanford Company, Richland, Washington.

Raphae1, G. F., 1996, Tank 241-U-107 Push Mode Core Sampling and Analysis Plan, WHC-SD-WM-TSAP-076, Rev. 0, Westinghouse Hanford Company, Richland, Washington.

Simpson, B. C., and D. J. McCain, 1995, Historical Model Evaluation Data Requirements, WHC-SD-WM-DQO-018, Rev. OA, Westinghouse Hanford Company, Richland, Washington.

Turner, D. A., H. Babad, L. L. Buckley, and J. E. Meacham, 1995, Data Qua7ity Objective to Support Resolution of the Organic Complexant Safety Issue, WHC-SD-WM-DQ0-006, Rev. 2, Westinghouse Hanford Company, Richland, Washington. 
WHC-SD-WM-DP-184, REV. 0

SAMPLE DATA SUMHARY 
WHC-SD-WH-DP-184, REV, 0

THIS PAGE WAS INTENTIONALLY LEFT BLANK 
CORE MUMBER: 129

SEGMENT \#: $3 R$

SEGMENT PORTION: U Upper Half of Segment

\begin{tabular}{|c|c|c|c|c|c|c|c|c|c|c|c|c|c|c|}
\hline & & & & Action & Limits & & & & & & & & & \\
\hline Sample\# & A\# & Analyte & Unit & Lower & Upper & Standard \% & Blank & Result & Dupl icate & Average & RPD \% & Spk Rec \% & Det Limit & Count Err\% \\
\hline 5967001140 & & Bulk Density of Sample & $\mathrm{g} / \mathrm{mL}$ & None & None & $\mathrm{n} / \mathrm{a}$ & $n / a$ & 1.360 & $\mathrm{n} / \mathrm{a}$ & $n / a$ & $\mathrm{n} / \mathrm{a}$ & $n / a$ & $5.000 e^{-1}$ & $n / a$ \\
\hline 5967001143 & & \% Water by TGA on Perkin Elmer & $\%$ & None & None & 99,41 & $\mathrm{n} / \mathrm{a}$ & 4.960 & $6.360 \mathrm{e} 0$ & $5.650 \mathrm{e} 0$ & 24.7 & $n / a$ & $\mathrm{n} / \mathrm{a}$ & $n / a$ \\
\hline 01143 & & DSC Exotherm on Perkin Elmer & Joules $/ \mathrm{g}$ & None & None & 92.86 & $\mathrm{n} / \mathrm{a}$ & $0.00 \mathrm{e}+00$ & $0.000 \mathrm{e} 0$ & $0,000 \mathrm{e} 0$ & 0.00 & $\mathrm{n} / \mathrm{a}$ & $\mathrm{n} / \mathrm{a}$ & $n / a$ \\
\hline S96r001153 & & Alpha of Digested Solid & $u[i / g$ & $-7.0 \mathrm{e}+00$ & 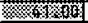 & 98.44 & $<1.450 \mathrm{e}-3$ & $4.64 \mathrm{e}_{02}$ & $3.220 \mathrm{e}-2$ & $3.930 \mathrm{e}-2$ & 36.1 & 73.82 & $4.000 e-3$ & $1.75 \mathrm{E}+01$ \\
\hline
\end{tabular}

5\%6r001153 Alpha of Digested Solid 
CORE NUMBER: 129

SEGMENT H: 1

SEGMENT PORTION: L Lower Half of Segment

\begin{tabular}{|c|c|c|c|c|c|c|c|c|c|c|c|c|c|c|}
\hline & & & & Action & Limits & & & & & & & & & \\
\hline Sampl e\# & $\mathrm{R}$ AA & Analyte & Unit & Lower & Upper & Standard \% & Blank & Result & Duplicate & Average & RPD \% & Spk Rec \% & Det Limit & Count Err\% \\
\hline 5967000683 & & $\%$ Water by TGA using Mettler & $\%$ & Mone & None & 102.3 & $n / a$ & 18.52 & $1.937 \mathrm{e} 1$ & $1.895 \mathrm{e} 1$ & 4.49 & $\mathrm{n} / \mathrm{a}$ & $n / a$ & $n / a$ \\
\hline S96T & & DSC Exotherm Dry Calculated & Joules/g Dry & $-7.0 \mathrm{e}+00$ & 4 (1) & $n / a$ & $\mathrm{n} / \mathrm{a}$ & & & $1.469 \mathrm{e} 1$ & 8.38 & $\mathrm{n} / \mathrm{a}$ & $\mathrm{n} / \mathrm{a}$ & n/a \\
\hline S96T000683 & & DSC Exotherm using Hettler & Joul es/g & Hone & None & 108.6 & $\mathrm{n} / \mathrm{a}$ & 12.40 & $1.140 \mathrm{e} 1$ & $1.190 \mathrm{e} 1$ & 8.40 & $n / a$ & $n / a$ & $n / a$ \\
\hline S96T000684 & $\mathbf{F}$ & Alpha of Digested Solid & LCi/g & $-7.0 \mathrm{e}+00$ & 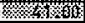 & 104.6 & $2.00 e-03$ & $2.43 \mathrm{e}: 03$ & $<5.12 E-3$ & $n / a$ & $\mathrm{n} / \mathrm{a}$ & 92.60 & $4.000 e-3$ & $1.06 E+02$ \\
\hline
\end{tabular}

Drainable Liquid: Drainable Liquid

\begin{tabular}{|c|c|c|c|c|c|c|c|c|c|c|c|c|c|c|}
\hline & & & & Action & Limits & & & & & & & & & \\
\hline Sampl e\# & A) & Analyte & Unit & Lower & Upper & Standard \% & BL角望 & Result & Dupureate & Average & RPD \% & Spk ReC \% & Det Limit & Count Err\% \\
\hline \$967000637 & & Specific Gravity & Sp.G. & Mone & None & 98.24 & n/a & 1884 & 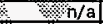 & $n / a$ & $n / a$ & $n / a$ & $1.000 \mathrm{e}-3$ & $n / a$ \\
\hline S967000665 & & $\%$ Water by TGA using Hettler & $\%$ & None & None & 99.61 & 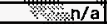 & 78594 & $5.451 \mathrm{e} 1$ & $5.322 \mathrm{e} 1$ & 4.83 & $n / a$ & $n / a$ & n/a \\
\hline$\$ 967000665$ & & DSC Exotherm Dry calculated & Joules/g Dry & $-7.0 \mathrm{e}+00$ & 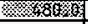 & $n / g:$ & od/a & W1.7e+QE & $1.148 \mathrm{e} 2$ & $1.160 \mathrm{e} 2$ & 2.07 & $n / a$ & $\mathrm{n} / \mathrm{a}$ & $n / a$ \\
\hline 5967000665 & & DSC Exotherm using Hettler & Joules/g & Mone & Hone & $105 \times 8$ & sola & 54.80 & $5.370 \mathrm{e} 1$ & $5.425 \mathrm{e}$ & 2.03 & $\mathrm{n} / \mathrm{a}$ & $\mathrm{n} / \mathrm{a}$ & $n / a$ \\
\hline 5967000665 & & Qlpha in Liquid Samples & $\mathrm{UCi} / \mathrm{mL}$ & $-7.0 \mathrm{e}+00$ & 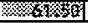 & . 98.04 & 3.2100 .2 & $s<1.728-02$ & $<1.72 E-2$ & $n / a$ & $n / a$ & 95.41 & $2.500 \mathrm{e}-2$ & $5.00 E+02$ \\
\hline
\end{tabular}

$\Rightarrow$ Limit violated

$\Rightarrow$ Selected Limit

约 
CORE NUPBER: 129

SEGHENT \#: 2

SEGMENT PORTION: U Upper HaLf of Segment

\begin{tabular}{|c|c|c|c|c|c|c|c|c|c|c|c|c|c|}
\hline & & & Action & Limits & & & & & & & & & \\
\hline Sample\# & A\# Analyte & Unit & LoHer & Upper & Standard \% & Blank & Result $t$ & Duplicate & Average & RPD \% & Spk Rec \% & Det Limit & Count Err\% \\
\hline \$9670 & Bulk Density of Sample & $\mathrm{g} / \mathrm{mL}$ & None & None & $\mathrm{n} / \mathrm{a}$ & $n / \mathbf{a}$ & 1.520 & $\mathrm{n} / \mathrm{a}$ & $\mathrm{n} / \mathrm{a}$ & $\mathrm{n} / \mathrm{a}$ & $n / a$ & $5.000 e^{-1}$ & $n \bar{a}$ \\
\hline S961 & $\%$ Water by IGA using Mettler & $\%$ & None & None & 100.2 & $n / a$ & 48.57 & $4.734 \mathrm{e} 1$ & 4.79591 & 2.56 & $n / a$ & $\mathrm{n} / \mathrm{a}$ & $n / a$ \\
\hline S96T0 & DSC Exotherm Dry calculated & Joules/g Dry & $-7.0 \mathrm{e}+00$ & 43 & $n / a$ & $\mathrm{n} / \mathbf{a}$ & $2.71 \mathrm{e}+02$ & $2.538 \mathrm{e} 2$ & $2.626 \mathrm{e} 2$ & 6.67 & $n / a$ & $n / a$ & n/a \\
\hline
\end{tabular}

S96r000644

$L$ Lower Half of Segment: $L$ Lower Half of Segment

\begin{tabular}{|c|c|c|c|c|c|c|c|c|c|c|c|c|c|c|}
\hline & & & & Action & Limits & & & & & & & & & \\
\hline Samoleff & A A\# $\mid$ & Analyte & Unit & Lower & Upper & Standard $\%$ & Blatu & Sesult & Dügriveate & Average & $\mathrm{RPD} \%$ & Spk Rec \% & Det Limit & Count Err\% \\
\hline $596 \mathrm{~T} 000645$ & & Bulk Density of Samole & $\mathrm{g} / \mathrm{mL}$ & None & None & $n / a$ & $n / a$ & 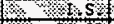 & 等 & $n / a$ & $n / a$ & $n / a$ & $5.000 e^{-1}$ & $n / a$ \\
\hline 5967000647 & & $\%$ Water by IGA using Mettler & $\%$ & None & None & 100.2 & $\mathrm{n} / \mathrm{a}$ & 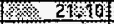 & $1.826 \mathrm{e} 1$ & $1.968 \mathrm{e} 1$ & 14.4 & $n / a$ & $\mathrm{n} / \mathbf{a}$ & $n / a$ \\
\hline 5967000647 & & DSC Exotherm Dry Calculated & Joules/g Dry & $-7.0 \mathrm{e}+00$ & 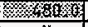 & 048 & nya & 10.95 & $1.021 \mathrm{e} 1$ & $1.108 \mathrm{e} 1$ & 15.7 & n/a & $\mathrm{n} / \mathrm{a}$ & $n / a$ \\
\hline 5967000647 & & DSC Exotherm using Mettler & Joules/g & None & None & $405 \%$ & $50 / a$ & 9.600 & 8.2000 & $8.900 \mathrm{e} 0$ & 15.7 & n/a & $\mathrm{n} / \mathrm{a}$ & $\mathrm{n} / \mathrm{a}$ \\
\hline 5967000687 & $F$ & Alpha of Digested Solid & $\mathrm{uCi} / \mathrm{g}$ & $-7.0 \mathrm{e}+00$ & 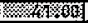 & 04.6 & $2.00 e^{2} \mathrm{3} 3$ & $1.54=01$ & $7.700 \mathrm{e}-1$ & $1.620 \mathrm{e}-1$ & 9.88 & $\mathrm{n} / \mathrm{a}$ & $4.000 \mathrm{e}-3$ & $1.01 \mathrm{E}+01$ \\
\hline
\end{tabular}
\begin{tabular}{l|l|l}
$\$ \$ 967000687$ & F & Alpha of Digested Solid \\
\hline
\end{tabular} uCi/g $\quad-7.0 \mathrm{e}+00 \mid$

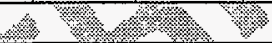

Drainable Liquid: Drainable Liquid

\begin{tabular}{|c|c|c|c|c|c|c|c|c|c|c|c|c|c|}
\hline Sample\# & A\#|Analyte & Unit & $\frac{\text { Action }}{\text { Lower }}$ & $\frac{\text { Limfos }}{\text { Wifipper }}$ & Ständara & Blank & Result & Duplicate & Average & RPD \% & Spk Rec \% & Det Limit & Count Err\% \\
\hline$\$ 961000641$ & Specific Gravity & Sp.G. & Note & Hone & 98.28 & $n / a$ & 1.435 & $n / a$ & $n / a$ & $\mathrm{n} / \mathrm{a}$ & $n / a$ & $1.000 \mathrm{e}-3$ & $\mathrm{n} / \mathrm{a}$ \\
\hline 5967000682 & $\%$ Water by IGA using Hettler & $\%$ & sitarie & Xote & 99.61 & $\mathrm{n} / \mathrm{a}$ & 49.79 & $4.955 \mathrm{el}$ & $4.967 \mathrm{e} 1$ & 0.48 & $\mathrm{n} / \mathrm{a}$ & $\mathrm{n} / \mathrm{a}$ & $n / a$ \\
\hline 5961000682 & DSC Exotherm Dry Calculated & soules/g Dry & Trige+00 & 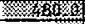 & 楑/a & $\mathrm{n} / \mathrm{a}$ & $1.30 \mathrm{e}+02$ & $1.266 \mathrm{e} 2$ & $1.281 \mathrm{e} 2$ & 2.42 & $\mathrm{n} / \mathrm{a}$ & $\mathrm{n} / \mathrm{a}$ & $\mathrm{n} / \mathrm{a}$ \\
\hline 5967000682 & DSC Exotherm using Hettler & Joules/g & None & None & 105.8 & $\mathrm{n} / \mathrm{a}$ & 65.30 & $6.370 \mathrm{e} 1$ & $6.450 \mathrm{e} 1$ & 2.48 & $\mathrm{n} / \mathrm{a}$ & $n / a$ & $\mathrm{n} / \mathrm{a}$ \\
\hline \$96T000682 & Alpha in Liquid Samples & $\mathrm{LCi} / \mathrm{mL}$ & $-7.0 \mathrm{e} 20$ & ystion & 98.04 & $\leq 1.210 \mathrm{e}-2$ & $<1.09 \mathrm{e}-02$ & $\leq 1.09 \mathrm{E}-2$ & $\mathrm{n} / \mathrm{a}$ & $\mathrm{n} / \mathrm{a}$ & $\mathrm{n} / \mathrm{a}$ & $2.500 e^{-2}$ & $5.00 E+02$ \\
\hline 5967000685 & Bulk Density of Sample & $\mathrm{g} / \mathrm{mL}$ & None & None & $\mathrm{n} / \mathrm{a}$ & $n / a$ & 1.400 & $\mathrm{n} / \mathrm{a}$ & $\mathrm{n} / \mathrm{a}$ & $\mathrm{n} / \mathrm{a}$ & $n / a$ & $5.000 \mathrm{e}-1$ & $n / a$ \\
\hline
\end{tabular}

$\Rightarrow$ Limit violated

$\Rightarrow$ Selected Limit

$\vec{\sigma}$ 
CORE NUMBER: 129

SEGMENT \#: 3

SEGMENT PORTION: U Upper Half of Segment

\begin{tabular}{|c|c|c|c|c|c|c|c|c|c|c|c|c|c|}
\hline & & & Action & Limits & & & & & & & & & \\
\hline Sample\# & A\# Analyte & Unit & Lower & Upper & Standard \% & Blank & Result & Duplicate & Average & RPD \% & Spk Rec \% & Det Limit & Count Err\% \\
\hline S967000648 & Bulk Density of Sample & $\mathrm{g} / \mathrm{mL}$ & None & None & $\mathrm{n} / \mathrm{a}$ & $n / a$ & 1.480 & $\mathrm{n} / \mathrm{a}$ & $n / a$ & $n / a$ & $\mathrm{n} / \mathrm{a}$ & $5.000 \mathrm{e}-1$ & $n / a$ \\
\hline 5967000650 & $\%$ Hater by TGA using Mettler & $\%$ & None & None & 102.1 & $\mathrm{n} / \mathrm{a}$ & 22.19 & $2.684 \mathrm{e}$ & $2.452 \mathrm{e} 1$ & 19.0 & $\mathrm{n} / \mathrm{a}$ & $n / a$ & $n / a$ \\
\hline & DSC Exotherm Dry Calculated & Joules/g DFY & $-7.0 \mathrm{e}+00$ & 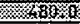 & $n / a$ & $n / a$ & 33.78 & $1.418 \mathrm{e} 1$ & $2.398 \mathrm{e} 1$ & 89.7 & $\mathrm{n} / \mathrm{a}$ & $\mathrm{n} / \mathrm{a}$ & $n / a$ \\
\hline S96T0 & DSC Exotherm using Mettler & Joules $/ 9$ & Hone & None & 106.2 & $n / a$ & $25 \times 50$ & 1.07001 & $1.810 \mathrm{e} 1$ & 81.8 & $\mathrm{n} / \mathrm{a}$ & $n / a$ & $\mathrm{n} / \mathrm{a}$ \\
\hline
\end{tabular}

\begin{tabular}{l|l} 
S967000650 & DSC Exotherm Dry Calculated \\
\hline S96T000650 & DSC Exotherm using Mettler
\end{tabular}

Joules/g

Hone

None 106.2

\begin{tabular}{|c|c|c|c|c|c|c|c|c|c|c|c|c|c|c|}
\hline & & & & Action & Limits & & & & & & & & & \\
\hline Samole\# & $\left.R\right|_{A \neq}$ & Analyte & Unit & Lower & Upper & Standard \% & 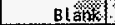 & Fresutt & Dupif cate & Average & RPD \% & Spk Rec \% & Det Limit & Count Err\% \\
\hline S96T000651 & & Bulk Density of Sample & $\mathrm{g} / \mathrm{mL}$ & None & None & $n / a$ & $\mathrm{n} / \mathrm{a}$ & 34460 & a n/a & $\mathrm{n} / \mathrm{a}$ & n/a & $n / a$ & $5.000 \mathrm{e}-1$ & $n / a$ \\
\hline 5967000653 & & $\%$ Water by TGA using Mettler & $\%$ & None & None & 102.1 & n/a & 带得 7678 & $1.743 \mathrm{e} 1$ & $1.711 \mathrm{e} 1$ & 3.80 & $n / a$ & $\mathrm{n} / \mathrm{a}$ & $n / a$ \\
\hline S961000653 & & DSC Exotherm Dry calculated & Joules/g Dry & $-7.0 \mathrm{e}+00$ & F & $n / 6$ & Sis/a & 16.7 .7 & $3.619 \mathrm{e}$ & $2.648 \mathrm{e} 1$ & 73.3 & n/a & $\mathrm{n} / \mathrm{a}$ & $n / a$ \\
\hline S967000653 & & DSC Exotherm using Mettler & Joules/g & Hone & None & $\sin 2$ & H yoj & 33.90 & $3.000 \mathrm{e}$ & $2.195 \mathrm{e} 1$ & 73.3 & $n / a$ & $n / a$ & $n / a$ \\
\hline \$967000689 & F & Alpha of Digested Solid & LCC $1 / 9$ & $-7.0 \mathrm{e}+00$ & 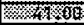 & 35.42 & 35.23003 & $3.36 \mathrm{z}-02$ & $3.710 \mathrm{e}-2$ & $3.530 e-2$ & 9.90 & 85.46 & $6.000 \mathrm{e}-3$ & $2.36 \mathrm{E}+01$ \\
\hline
\end{tabular}

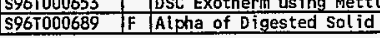

$\Rightarrow$ Limit violated

$\Rightarrow$ Selected Limit

5 
CORE NUMBER: 134

SEGHENT \#: SA

SEGHENT PORTION: Liner Liquid

\begin{tabular}{|c|c|c|c|c|c|c|c|c|c|c|c|c|c|}
\hline & & & Action & imits & & & & & & & & & \\
\hline Sample\# & Afl|Analyte & Unit & Lower & Upper & Standard \% & Blank & Result & Duplicate & Average & RPD \% & Spk Rec \% & Det Limit & Count Err\% \\
\hline S96T001110 & Alpha in Liquid Samples & $\mathrm{uCi} / \mathrm{mL}$ & $-7.0 \mathrm{e}+00$ & 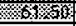 & 112.5 & $<4.4500-5$ & $<6.30 \mathrm{e}-05$ & $<4.54 \mathrm{E}-5$ & $n / a$ & $\mathrm{n} / \mathrm{a}$ & 98.05 & $90 e-4$ & $\mathrm{DE}+\overline{\mathrm{O} 2}$ \\
\hline S96T002034 & $\%$ Water by TGA using Mettler & $\%$ & None & None & 100.1 & $n / a$ & 98.17 & $9.814 \mathrm{e} 1$ & $9.816 \mathrm{e} 1$ & 0.03 & $n / a$ & $n / a$ & $n<a$ \\
\hline S967002034 & DSC Exotherm Dry Calculated & Joules/g Dry & $-7.0 \mathrm{e}+00$ & 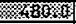 & $\mathrm{n} / \mathrm{a}$ & $\mathrm{n} / \mathrm{a}$ & $0.00 \mathrm{e}+00$ & & $0.000 \mathrm{e} 0$ & 0.00 & $n / a$ & $n / a$ & nLa \\
\hline 5967002034 & DSC Exotherm using Mettler & Joules/g & None & Mone & 110.7 & $n / a$ & $0.00 \mathrm{e}+00 \mathrm{0}$ & $0.000 \mathrm{e} 0$ & $0.000 \mathrm{e} 0$ & 0.00 & $\mathrm{n} / \mathrm{a}$ & $n / a$ & $n / a$ \\
\hline
\end{tabular}

\$967002034

Joules/g

W 3

\begin{tabular}{|c|c|c|c|c|c|c|c|c|c|c|c|c|c|c|}
\hline \multirow[b]{2}{*}{ Sample\# $\mathrm{R}$} & \multirow[b]{2}{*}{$\mathbf{R} \mid$ A\# } & \multirow{2}{*}{ Analyte } & \multirow[b]{2}{*}{ Unit } & \multicolumn{2}{|c|}{ Action Limits } & \multirow[b]{2}{*}{ Standard \% } & \multirow{2}{*}{ Bl䋞 } & \multirow{2}{*}{ 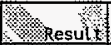 } & \multirow{2}{*}{ Dupplicate } & \multirow[b]{2}{*}{ Average } & \multirow[b]{2}{*}{ RPD \% } & \multirow[b]{2}{*}{ Spk Rec \% } & \multirow[b]{2}{*}{ Det Limit } & \multirow[b]{2}{*}{ Count Err\% } \\
\hline & & & & Lower & Upper & & & & & & & & & \\
\hline 5967001045 & & Bulk Density of Sample & $\mathrm{g} / \mathrm{mL}$ & None & None & $n / a$ & n/a & 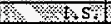 & $\mathrm{n} / \mathrm{a}$ & $n / a$ & $n / a$ & $\mathrm{n} / \mathrm{a}$ & $5.000 \mathrm{e}-1$ & $\mathrm{n} / \mathrm{a}$ \\
\hline 5967001059 & & $\%$ Water by IGA using Mettler & $\%$ & None & None & 100.1 & \$ & $3 \times 27 \times 5$ & $2.126 \mathrm{e} 1$ & $2.126 \mathrm{e} 1$ & 0.05 & $\mathrm{n} / \mathrm{a}$ & $\mathrm{n} / \mathrm{a}$ & $\mathrm{n} / \mathrm{a}$ \\
\hline S967001059 & & DSC Exotherm Dry Calculated & Joules/g_Dry & $-7.0 e+00$ & 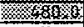 & $\mathrm{n} / \mathrm{R}$ & \&. $\quad \mathrm{s} / \mathrm{a}$ & $80.00+00$ & $0.000 \mathrm{e} 0$ & $0.000 \mathrm{e} 0$ & 0.00 & $\mathrm{n} / \mathrm{a}$ & $\mathrm{n} / \mathrm{a}$ & $\mathrm{n} / \mathrm{a}$ \\
\hline S967001059 & & DSC Exotherm using Mettler & Joules/g & None & Hone & 423 & 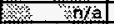 & $0.00 \mathrm{e}+00$ & $0.000 \mathrm{e} 0$ & $0.000 \mathrm{e} 0$ & 0.00 & $n / a$ & $n / a$ & $n / a$ \\
\hline 5967001096 & $\mathbf{F}$ & Alpha of Digested solid & uCi/g & $-7.0 \mathrm{e}+00$ & 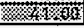 & $2 \times 19.5$ & $4.050 \mathrm{e}^{3} 3$ & $=1,830-01$ & $1.830 \mathrm{e}-1$ & $1.830 \mathrm{e}-1$ & 0.00 & $\mathrm{n} / \mathrm{a}$ & $9.000 \mathrm{e}-3$ & $1.41 E+01$ \\
\hline
\end{tabular}

$\Rightarrow$ Limit violated

$\Rightarrow$ Sel ected Limit

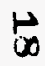


CORE NUMBER: 134

SEGMENT \#: $5 B$

SEGMENT PORTION: Drainable Liquid

\begin{tabular}{|c|c|c|c|c|c|c|c|c|c|c|c|c|c|c|}
\hline & & & & Action & Limits & & & & & & & & & \\
\hline Sample\# & A\# & Analyte & Unit & Lower & Upper & Standard \% & Blank & Result & Dupl icate & Average & RPD \% & Spk ReC \% & Det Limit & Count Err\% \\
\hline S96T001107 & & Specific Gravity & Sp.G. & None & None & 97.74 & $n / a$ & 1.437 & $1.419 \mathrm{e} 0$ & $1.428 \mathrm{eO}$ & 1.26 & $\mathrm{n} / \mathrm{a}$ & $1.000 \mathrm{e}-3$ & $n / a$ \\
\hline S96T001119 & & $\%$ Water by TGA on Perkin Elmer & $\%$ & None & Hone & 99.611 & $\mathrm{n} / \mathrm{a}$ & 51.01 & $5.114 \mathrm{e} 1$ & $5.108 \mathrm{e} 1$ & 0.25 & $n / a$ & $n / a$ & $n / a$ \\
\hline S96T001119 & & DSC Exotherm on Perkin Elmer & Joules/g & None & Mone & 97.64 & $\mathrm{n} / \mathrm{a}$ & $0.00 \mathrm{e}+00$ & 0.00000 & $0.000 \mathrm{e}$ & 0.00 & $\mathrm{n} / \mathrm{a}$ & $\mathrm{n} / \mathrm{a}$ & $n / a$ \\
\hline S967001119 & & ALpha in Liquid Samples & $\mathrm{UCI} / \mathrm{mL}$ & $-7.0 e+00$ & \% 65 & 112.5 & $\angle 4.450 e-5$ & $5.36 \mathrm{e} 203$ & $<<4.94 \mathrm{E}-3$ & $n / a$ & $\mathrm{n} / \mathrm{a}$ & $n / a$ & $5.000 \mathrm{e}-3$ & $7.34 \mathrm{E}+01$ \\
\hline
\end{tabular}

w Limit violated $\Rightarrow$ Selected Linit

$\frac{\omega}{6}$ 
CORE NUMBER: 134

SEGMENT \#: 6 A

SEGMENT PORTION: Liner Liquid

\begin{tabular}{|c|c|c|c|c|c|c|c|c|c|c|c|c|c|c|}
\hline & & & & Action & Limits & & & & & & & & & \\
\hline Sample\# & $\mathrm{R} \mid \mathrm{A} \neq \mathbf{1}$ & Analyte & Unit & Loher & Upper & Standard \% & B Lank & Result & Duplicate & Average & RPD \% & Spk Rec \% & Det Limit & Count Err\% \\
\hline $96700 \div 120$ & & $\%$ water by TGA on Perkin Elmer & $\%$ & None & Mone & 99.61 & $n / a$ & 89.27 & $8.910 \mathrm{e} 1$ & $8.919 \mathrm{e} 1$ & 0.19 & $\mathrm{n} / \mathrm{a}$ & $n / a$ & $n / a$ \\
\hline & & DSC Exotherm on Perkin Elmer & Joules/g & Mone & None & 97.64 & $n / a$ & $0.00 \mathrm{e}+00$ & $0.000 \mathrm{e}$ & $0.000 \mathrm{e} 0$ & 0.00 & n/a & $n / a$ & $n / a$ \\
\hline 9967001120 & & DSC Exotherm Dry Calculated & Joules/g Dry & $-7.0 \mathrm{e}+00$ & 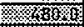 & $\mathrm{n} / \mathrm{a}$ & $\mathrm{n} / \mathrm{a}$ & $0.00 e+00$ & $0.000 \mathrm{e}$ & $0.000 e 0$ & 0.00 & $\mathrm{n} / \mathrm{a}$ & $\mathrm{n} / \mathrm{a}$ & $\mathrm{n} / \mathrm{a}$ \\
\hline
\end{tabular}

W Whole Segment: Whole Segment

\begin{tabular}{|c|c|c|c|c|c|c|c|c|c|c|c|c|c|c|}
\hline \multirow{2}{*}{ Sample\# $\mathrm{R}$} & \multirow{2}{*}{ A\# } & \multirow{2}{*}{ Analyte } & \multirow[b]{2}{*}{ Unit } & \multicolumn{2}{|c|}{ Action Limits } & \multirow[b]{2}{*}{ standard \% } & \multirow{2}{*}{\multicolumn{2}{|c|}{ 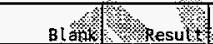 }} & \multirow{2}{*}{ Diphatate } & \multirow[b]{2}{*}{ Average } & \multirow[b]{2}{*}{ RPD \% } & \multirow[b]{2}{*}{ Spk Rec \% } & \multirow[b]{2}{*}{ Det Limit } & \multirow[b]{2}{*}{ Count Err\% } \\
\hline & & & & Laherl & Upper & & & & & & & & & \\
\hline S96T001046 & & Bulk Density of Sample & $\mathrm{g} / \mathrm{mL}$ & None & Mone & n/a & $\%$ n/a & What & n/a & $n / a$ & $n / a$ & $n / a$ & $5.000 e^{-1}$ & $n / a$ \\
\hline 5967001070 & & $\%$ Water by TGA using Mettler & $\%$ & Mone & Kone & 102.9 & \% & 到 $97 \times 4$ & $9.102 \mathrm{e} 1$ & $9.123 \mathrm{e} 1$ & 0.46 & $\mathrm{n} / \mathrm{a}$ & $\mathrm{n} / \mathrm{a}$ & $n / a$ \\
\hline 5967001070 & & DSC Exotherm Dry Calculated & Joules/g Dry & $-7.0 \mathrm{e}+00$ & 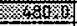 & $n / 20$ & $\mathrm{~s} / \mathrm{a}$ & $9600+0$, & $0.000 \mathrm{e}$ & $0.000 \mathrm{e} 0$ & 0.00 & $\mathrm{n} / \mathrm{a}$ & $\mathrm{n} / \mathrm{a}$ & $n / a$ \\
\hline S96T001070 & & DSC Exotherm using Mettler & Joules/g & None & Hone & 4630 & \% na & $0.000+00$ & 0.00000 & $0.000 \mathrm{e}$ & 0.00 & $\mathrm{n} / \mathrm{a}$ & $\mathrm{n} / \mathrm{a}$ & $n / a$ \\
\hline S967001097 & $F$ & Alpha of Digested solid & $\mathrm{uCi} / \mathrm{g}$ & $-7.0 e+00$ & 经地 & 9.5 & $4.050 e^{3}$ & $1.765-02$ & $1.270 \mathrm{e}-2$ & $1.520 \mathrm{e}-2$ & 32.3 & $n / a$ & $4.000 e-3$ & $3.46 E+01$ \\
\hline
\end{tabular}

\begin{tabular}{|l|l|l}
\hline S96T001097 & F & Alpha of Digested Solid \\
\hline
\end{tabular}

\section{\%}

\begin{tabular}{|c|c|c|c|c|c|c|c|c|c|c|c|c|c|c|}
\hline Samole:H & $|A|$ & Analyte & Unit & Action & Limitas & stindara & B Lank & Result & Duplicate & Average & RPD \% & Spk Rec \% & Det Limit & Count Err\%. \\
\hline 5001121 & & \% Water by IGA on Perkin Elmer & $\%$ & Norot & Honet & 99.6 & $n / a$ & 91.70 & $9.222 \mathrm{e} 1$ & $9.196 \mathrm{e} 1$ & $\frac{101}{101}$ & Spe kec/o & $\frac{n / a}{n / a}$ & $\frac{n / a}{n / a}$ \\
\hline$\frac{1121}{1121}$ & & Specific Gravity & Sp.G. & Fritive & allowe & 97.46 & $\mathrm{n} / \mathrm{a}$ & 1.026 & $1.009 \mathrm{e} 0$ & $1.018 \mathrm{e} 0$ & 1.67 & $n / a$ & $1.000 \mathrm{e}-3$ & $n / a$ \\
\hline 121 & & DSC Exotherm on Perkin Elmer & Joules/g & sone & Hone & 89764 & $n / a$ & $0.00 \mathrm{e}+00$ & $0.000 \mathrm{e}$ & $0.000 \mathrm{e} 0$ & 0.00 & $n / a$ & $n / a$ & $n / a$ \\
\hline & & DSC Exotherm Dry Calculated & Joules/g Dry & 7,00000 & 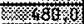 & $n / a$ & n/a & & & $0.000 \mathrm{e} 0$ & 0.00 & n/a & $n / a$ & $\mathrm{n} / \mathrm{a}$ \\
\hline$\frac{570001121}{596001121}$ & & Alpha in Liquid Samples & $\mathrm{uCi} / \mathrm{mL} / \mathrm{y}$ & $-7.0 \mathrm{~m}+000$ & 6h & 112.5 & $<4.450 \mathrm{e}-5$ & $<2.25 \mathrm{e}-03$ & $<2.29 E-3$ & $\mathrm{n} / \mathrm{a}$ & $\mathrm{n} / \mathbf{a}$ & $n / a$ & $5.000 e-3$ & $.00 E+02$ \\
\hline
\end{tabular}

$\Rightarrow$ Limit violated

$\Rightarrow$ Selected Limit

$\mathscr{8}$

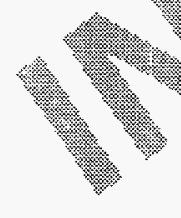


CORE MUMBER: 134

SEGMENT \#: 2

SEGMENT PORTION: U Upper Half of Segment

\begin{tabular}{|c|c|c|c|c|c|c|c|c|c|c|c|c|c|}
\hline & & & Action & Limits & & & & & & & & & \\
\hline Sample\# & A\#f Analyte & Unit & Lower & Upper & Standard \% & Blank & Result & Duplicate & Average & RPD \% & Spk Rec \% & Det Limit & Count Err\% \\
\hline 5967001043 & \begin{tabular}{|l|} 
Bulk Density of Sample \\
\end{tabular} & $g / m L$ & None & None & $\mathrm{n} / \mathrm{a}$ & $n / a$ & 1.410 & $\mathrm{n} / \mathrm{a}$ & $\mathrm{n} / \mathrm{a}$ & $n / a$ & n/a & $5.000 e^{-1}$ & n/a \\
\hline S96T001055 & $\%$ Water by TGA on Perkin Elmer & $\%$ & None & None & 100.3 & $\mathrm{n} / \mathrm{a}$ & 6.940 & & $7.270 \mathrm{e} 0$ & 9.08 & $\mathrm{n} / \mathrm{a}$ & $n / a$ & nja \\
\hline S96T0 & DSC Exotherm on Perkin Elmer & Joules/g & None & Mone & 96.63 & $n / a$ & $0.00 \mathrm{e}+\mathrm{QD}$ & & $0.000 \mathrm{e} 0$ & 0.00 & $n / a$ & $\mathrm{n} / \mathrm{a}$ & $n / \mathbf{a}$ \\
\hline S9610 & DSC Exotherm Dry Calculated & Joules/gDry & $-7.0 \mathrm{e}+00$ & 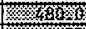 & $\mathrm{n} / \mathrm{a}$ & $n / a$ & 0.00 e*0:09: & $0,000 \mathrm{e} 0$ & $0.000 \mathrm{e} 0$ & 0.00 & $\mathrm{n} / \mathrm{a}$ & $n / a$ & $n / a$ \\
\hline 1089 & Alpha of Digested Sol id & uCi/g & $-7.0 \mathrm{e}+00$ & 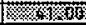 & 116.4 & $<3.220 \mathrm{e}-3$ & 1.530002 & $2.030 \mathrm{e}^{-2}$ & $1.780 \mathrm{e}-2$ & 28.1 & 71.31 & $6.000 \mathrm{e}-3$ & $3.77 \mathrm{E}+01$ \\
\hline
\end{tabular}

S961001089 F Alpha of Digested Sol id 
CORE NUMBER: 134

SEGMENT \#: 3

SEGMENT PORTION: U Upper Half of Segment

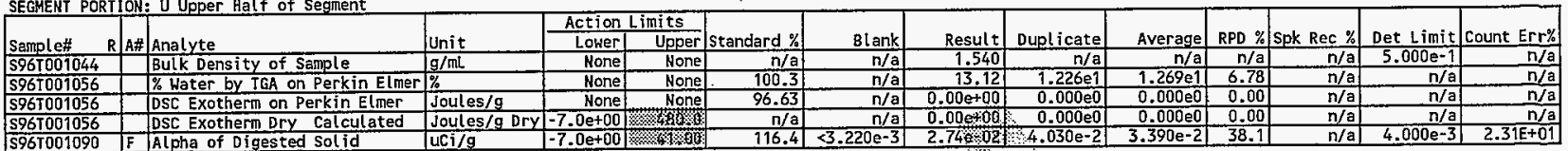

\section{$\Rightarrow$ Limit violated}

$\Rightarrow$ Selected Limit

N 
CORE NUMBER: 134

SEGMENT \#: 4

\begin{tabular}{|c|c|c|c|c|c|c|c|c|c|c|c|c|c|c|}
\hline & & & & Action & Limits & & & & & & & & & \\
\hline Sample\# & A A & Analyte & Unit & Lower & Upper & Standard \% & Btank & Result & Duplicate & Average & RPD \% & Spk Rec \% & Det Limit & Count Err\% \\
\hline S96T001061 & & Bulk Density of Sample & $\mathrm{g} / \mathrm{mL}$ & Mone & None & $n / a$ & $n / a$ & 1.740 & $\mathrm{n} / \mathrm{a}$ & $n / a$ & $n / a$ & $n / a$ & $5.000 e-1$ & $n / a$ \\
\hline S96T001064 & & $\%$ Water by TGR using Mettler & $\%$ & None & None & 102.4 & $\mathrm{n} / \mathrm{a}$ & 23.61 & $2.276 \mathrm{e} 1$ & $2.319 \mathrm{e} 1$ & 3.67 & $\mathrm{n} / \mathrm{a}$ & $\mathrm{n} / \mathrm{a}$ & $n / a$ \\
\hline 5967001064 & & DSC Exotherm Dry Calculated & Joules/g Dry & $-7.0 \mathrm{e}+00$ & 4BO & $n / a$ & n/a & $0.00 \mathrm{e}+00$ & $0.000 e^{0}$ & $0.000 \mathrm{e} 0$ & 0.00 & $n / a$ & $\mathrm{n} / \mathrm{a}$ & $n / a$ \\
\hline S96T001064 & & IDSC Exotherm using Rettler & Jaules/g & Hone & None & 106.2 & $n / a$ & $0.00 e+30$ & $0.000 \mathrm{e} 0$ & $0.000 \mathrm{e} 0$ & 0.00 & $n / a$ & $n / a$ & $n / a$ \\
\hline S96T001092 & $F$ & Alpha of Digested Solid & $\mathrm{IuCi} / \mathrm{g}$ & $-7.0 \mathrm{e}+00$ & W. & 110.9 & $<8.720 e-3$ & 7.61402 & $7.510 \mathrm{e}-2$ & $7.560 \mathrm{e}-2$ & 1.32 & 94.71 & $1.400 e^{-2}$ & $2.21 E+01$ \\
\hline
\end{tabular}

\begin{tabular}{|c|c|c|c|c|c|c|c|c|c|c|c|c|c|c|}
\hline & & & & Action & imits & & & & & & & & & \\
\hline Sample\# & \begin{tabular}{l|l}
$\mathbf{R}$ & AH \\
\end{tabular} & \# Analyte & Unit & Lower & Upper & Standard \% & \&larik & Resultis & Duptyeate & Average & RPD \% & Spk Rec \% & Det Limit & Count Err\% \\
\hline S96T001062 & & Bulk Density of Sample & $\mathrm{g} / \mathrm{mL}$ & None & Mone & $n / a$ & \%n/a & 1.830 & $\mathrm{n} / \mathrm{a}$ & $n / a$ & $n / a$ & $n / a$ & $5.000 \mathrm{e}^{-1}$ & $n / a$ \\
\hline S96T001065 & & $\%$ Water by TGA using Mettler & $\%$ & None & Nane & $102 \times 4$ & 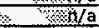 & 3.28 .65 & $2.764 \mathrm{e} 1$ & $2.814 \mathrm{e} 1$ & 1.47 & $\mathrm{n} / \mathrm{a}$ & $\mathrm{n} / \mathrm{a}$ & $\mathrm{n} / \mathrm{a}$ \\
\hline 5967001065 & & DSC Exotherm Dry Calculated & Joules/g Dry & $-7.0 \mathrm{e}+00$ & 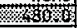 & सxial & 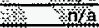 & $0 \% 09 e+00$ & 0.00000 & $0.000 \mathrm{e} 0$ & 0.00 & $\mathrm{n} / \mathrm{a}$ & $\mathrm{n} / \mathrm{a}$ & $n / a$ \\
\hline 5967001065 & & DSC Exotherm using Mettler & Joules/g & Hone & Mone & 106.2 & h & $0.00 \mathrm{e}+00$ & $0.000 \mathrm{e} 0$ & $0.000 \mathrm{e} 0$ & 0.00 & n/a & $\mathrm{n} / \mathrm{a}$ & $n / a$ \\
\hline S967001093 & $\mathbf{F}$ & Alpha of Digested Solid & $u C i / g$ & $-7.0 e+00$ & 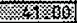 & 10.9 & $8 \times 8720 e^{35}$ & $9.65 \mathrm{e}-02$ & $9.800 \mathrm{e}-2$ & $9.730 \mathrm{e}-2$ & 0.54 & $\mathrm{n} / \mathrm{a}$ & $3.000 e-2$ & $3.03 E+01$ \\
\hline
\end{tabular}
\begin{tabular}{|l|l|l|l|l}
\hline S967001093 & F & Alpha of Digested Solid \\
\hline
\end{tabular}

\begin{tabular}{|c|c|c|c|c|c|c|c|c|c|c|c|c|c|c|}
\hline & & & & Action & Einfits & & & & & & & & & \\
\hline Sample\# & $\mathrm{R}$ A\# & Analyte & Unit & Lokise & tupers & Standard\% & Blank & Result & Dupl icate & Average & RPD \% & Spk Rec \% & Det Limit & Count Err\% \\
\hline S96T001047 & & Bulk Density of Sample & $g / m L$ & aftine & Wole & n/át & $\mathrm{n} / \mathrm{a}$ & 1.660 & $\mathrm{n} / \mathrm{a}$ & $n / a$ & $\mathrm{n} / \mathrm{a}$ & $\mathrm{n} / \mathrm{a}$ & $5.000 e-1$ & $n / a$ \\
\hline S96T001057 & & $\%$ Water by TGA using Mettler & $\%$ & Wyone & Hoñe & \%9997 & $\mathrm{n} / \mathrm{a}$ & 34.53 & $3.923 \mathrm{e} 1$ & $3.688 \mathrm{e} 1$ & 12.7 & $\mathrm{n} / \mathrm{a}$ & $n / a$ & $\mathrm{n} / \mathrm{a}$ \\
\hline 5967001057 & & DSC Exotherm Dry Calculated & Joules/g Dry & $7 \times 0+00$ & 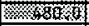 & $n / a$ & $\mathrm{n} / \mathrm{a}$ & $2.33 \mathrm{e}+02$ & $2.345 \mathrm{e} 2$ & $2.337 \mathrm{e} 2$ & 0.68 & $n / a$ & $n / a$ & $n / a$ \\
\hline 5967001057 & & DSC Exotherm using Mettler & Joules $k$ : & Noine & None; & 94.90 & $\mathrm{n} / \mathrm{a}$ & $1.47 e+02$ & $1.480 \mathrm{e} 2$ & $1.475 \mathrm{e} 2$ & 0.68 & $\mathrm{n} / \mathrm{a}$ & $n / a$ & $\mathrm{n} / \mathrm{a}$ \\
\hline S967001091 & $F$ & Alpha of Digested Solid & $\mathrm{LCi} / \mathrm{g}$ & $-7.0 e+039$ & 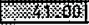 & 108.6 & $<1.180 \mathrm{e}-2$ & $2.85 e^{-01}$ & $3.190 \mathrm{e}-1$ & $3.020 e-1$ & 11.3 & $\mathrm{n} / \mathrm{a}$ & $2.000 e-2$ & $1.08 \mathrm{E}+01$ \\
\hline
\end{tabular}

\begin{tabular}{l|l|l|l}
5967001057 & & DSC Exotherm using Mett L \\
\hline 5967001097 & F & Al pha of Digested Solid \\
\hline
\end{tabular}

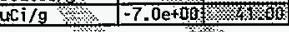

\section{$\Rightarrow$ Limit violated}

a Selected Limit 
CORE NUMBER: 134

SEGNENT \#: 5

SEGMENT PORTION: A TOR Quarter of Segment

\begin{tabular}{|c|c|c|c|c|c|c|c|c|c|c|c|c|c|c|}
\hline & & & & Action & Limfts & & & & & & & & & \\
\hline Sample\#̈ & A\# & Analyte & Unit & Lower & Upper & Standard $\%$ & Blank & Result & Duplicate & Average & RPO \% & Spk Rec \% & Det Limit & Count Err\% \\
\hline$\$ 967001067$ & & Bulk Density of Sample & $g / m L$ & None & None & $\mathrm{n} / \mathbf{a}$ & $\mathrm{n} / \mathrm{a}$ & 1.680 & $n / a$ & $\mathrm{n} / \mathrm{a}$ & $n / a$ & $n / a$ & $5.000 \mathrm{e}^{-1}$ & $n / a$ \\
\hline S967001068 & & \% Water by TGA on Perkin Elmer & $\%$ & None & None & 99.93 & $n / a$ & 38.28 & $4.335 \mathrm{e} 1$ & $4.081 \mathrm{e} 1$ & 12.4 & $n / a$ & $\mathrm{n} / \mathrm{a}$ & $n / a$ \\
\hline S96T001068 & & DSC Exotherm on Perkin Elmer & Joules/g & Kone & None & 93.78 & $\mathrm{n} / \mathrm{a}$ & $0.00 \mathrm{e}+00$ & $0.000 \mathrm{e} 0$ & $0.000 \mathrm{e} 0$ & 0.00 & $\mathrm{n} / \mathrm{a}$ & $n / a$ & $n / a$ \\
\hline 5967001068 & & OSC Exotherm Dry Calculated & Joules/g Dry & $-7.0 \mathrm{e}+00$ & 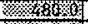 & $\mathrm{n} / \mathrm{a}$ & $\mathrm{n} / \mathrm{a}$ & $0.00 \mathrm{e}+0 \mathrm{~g}$ & $0.000 \mathrm{e} 0$ & $0.000 \mathrm{e} 0$ & 0.00 & $n / a$ & $n / a$ & $\mathrm{n} / \mathrm{a}$ \\
\hline S96T001095 & $\mathbf{F}$ & Alpha of Digested Solid & $\mathrm{uCi} / \mathrm{g}$ & $-7.0 \mathrm{e}+00$ & 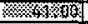 & 119.5 & $<7.050 \mathrm{e}-3$ & $9.10 \mathrm{ex0}$ & $6.090 \mathrm{e}-1$ & $7.600 e^{-1}$ & 39.6 & 87.19 & $1.500 \mathrm{e}-2$ & $B E+00$ \\
\hline
\end{tabular}

B Second Quarter of Segment: B Second Quarter of Segment

\begin{tabular}{|c|c|c|c|c|c|c|c|c|c|c|c|c|c|c|}
\hline & & & & Action & Limits & & & & & & & & & \\
\hline Sample\# & R A\# & Analyte & Unit & Lower & Upper & Standard \% & Blank & Resultz & Suptecte & Average & RPD \% & Spk Rec \% & Det Limit & Count Erri \\
\hline 5967001058 & & \% Water by TGA using Hettler & $\%$ & None & None & 99.97 & $p / a$ & 落 4427 & $2.819 e 1$ & $3.620 \mathrm{e} 1$ & 44.3 & $\mathrm{n} / \mathrm{a}$ & $\mathrm{n} / \mathrm{a}$ & $\underline{n}$ \\
\hline 5967001058 & & DSC Exotherm Dry Calculated & Joules/g Dry & $-7.0 e+00$ & Schof & nas & i/a & 18.13 & $1.255 \mathrm{e} 2$ & $7.181 \mathrm{e} 1$ & 150 & $\mathrm{n} / \mathrm{a}$ & $\mathrm{n} / \mathrm{a}$ & n \\
\hline S96T001058 & & DSC Exotherm using Mettler & Joules/g & Hone & None & 96,90 & $x$ & 11.50 & $7.970 \mathrm{e} 1$ & $4.560 \mathrm{e} 1$ & 150 & $n / a$ & $\mathrm{n} / \mathrm{a}$ & $\mathrm{n}$ \\
\hline$\$ 967001066$ & & Bulk Density of Sample & $\mathrm{g} / \mathrm{mL}$ & Hone & None & h/a & 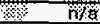 & 8760 & $\mathrm{n} / \mathrm{a}$ & $\mathrm{n} / \mathrm{a}$ & $\mathrm{n} / \mathrm{a}$ & $n / a$ & $5.000 e^{-1}$ & \\
\hline S967001094 & $\mathbf{F}$ & Alpha of Digested Solid & UCi/g & $-7.0 e+00$ & (x) & 910.9 & $820 e^{2}-3$ & $4.30 \mathrm{e}-01$ & $3.820 \mathrm{e}-1$ & $4.060 e^{-1}$ & 11.8 & $n / a$ & $2.900 \mathrm{e}-2$ & $1.28 E+0$ \\
\hline
\end{tabular}

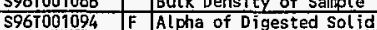

g/mL

$\Rightarrow$ Limit violated

\% Selected Limit

N 
CORE NUMBER: 135

SEGMENT \#: $1 R$

\begin{tabular}{|c|c|c|c|c|c|c|c|c|c|c|c|c|c|c|}
\hline & & & & Action & Limits & & & & & & & & & \\
\hline Sample\# & $\mathrm{R}$ A\# & Anglyte & Unit & Lower & Upper & standard \% & Blank & Result & Duplicate & Average & RPD \% & Spk Rec \% & Det Limit & Count Err\% \\
\hline S96T002132 & & $\%$ Water by TGA using Mettler & $\%$ & None & None & 99.70 & $n / a$ & 18.55 & $1.645 \mathrm{e} 1$ & $1.750 \mathrm{e} 1$ & 12.0 & $\mathrm{n} / \mathrm{a}$ & $n / a$ & $n / a$ \\
\hline 5967002132 & & DSC Exotherm Dry Calculated & Joules/g Dry & $-7.0 e+00$ & 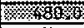 & $\mathrm{n} / \mathrm{a}$ & $n / a$ & $0.00 \mathrm{e}+00$ & 0.00000 & $0.000 \mathrm{eO}$ & 0.00 & $n / a$ & $\mathrm{n} / \mathrm{a}$ & $n / a$ \\
\hline 5967002132 & & DSC Exotherm using Mettler & Joules/g & None & None & 104.7 & $n / a$ & $0.00 \mathrm{e}+00$ & $0.000 \mathrm{e}$ & $0.000 \mathrm{e}$ & 0,00 & $\mathrm{n} / \mathrm{a}$ & $n / a$ & $n / a$ \\
\hline & & & & $-7.0 \mathrm{e}+00$ & 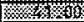 & 98.44 & $<1.750 \mathrm{e}-\overline{3}$ & $<2.88 \mathrm{e}+33$ & $<2.76 E-3$ & $\mathrm{n} / \mathrm{a}$ & $\mathrm{n} / \mathrm{a}$ & $n / a$ & $4.000 \mathrm{e}-3$ & $2.53 E+02$ \\
\hline
\end{tabular}

S96T002135 F Alpha of Digested Solid

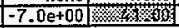

\begin{tabular}{l|l|l|l|}
98.44 & $<1.750 \mathrm{e}-3$ & $<2.88 \mathrm{e}+03$ & $<2.76 \mathrm{E}-3$
\end{tabular}

\section{$\checkmark$ v}

\begin{tabular}{|c|c|c|c|c|c|c|c|c|c|c|c|c|c|}
\hline & & & & Action & Limits & & & & & & & & \\
\hline Sample\# & R A\# & Analyte & Unit & Lower & Upper & Standard \% & BLâk & Duplicate & Average & $\mathrm{RPD} \%$ & Spk Rec \% & Det Limit & Count Err\% \\
\hline 5967002141 & & \% Water by TGA using Mettler & $\%$ & None & None & 99.53 & $A, n / a$, $/ 7,03$ & 4.7021 & $4.703 \mathrm{e} 1$ & 0.02 & $n / a$ & $n / a$ & $\mathrm{n} / \mathrm{a}$ \\
\hline 2141 & & Specific Gravity & SP.G. & None & None & 97.93 & $3 \times 2, n / a$ & 1.41200 & $1.443 \mathrm{e} 0$ & 4.30 & $\mathrm{n} / \mathrm{a}$ & $1.000 e^{-3}$ & $n / a$ \\
\hline 141 & & IDSC Exotherm Dry Calculated & Joules/g Dry & $-7.0 \mathrm{e}+00$ & 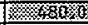 & n/a & WV/a s $259 e+02$ & $5.160 \mathrm{e} 2$ & $5.376 \mathrm{e}^{2}$ & 8.04 & $\mathrm{n} / \mathrm{a}$ & $n / a$ & $n / a$ \\
\hline 141 & & DSC Exotherm using Mettler & Joules $/ 9$ & None & None & 106.5 & * $\quad 81 \mathrm{a} \quad 2806 \mathrm{e}+02$ & $2.733 e^{2}$ & $2.848 \mathrm{e}^{2}$ & 8.04 & $\mathrm{n} / \mathrm{a}$ & $n / a$ & $n / a$ \\
\hline 5967002141 & & Alpha in Liquid Samples & $\mathrm{uCi} / \mathrm{mL}$ & $-7.0 \mathrm{e}+00$ & 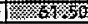 & 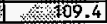 & $01.330 \mathrm{e} / 2<<1.05 \mathrm{e}-02$ & $<1.65 \mathrm{E}-2$ & $\mathrm{n} / \mathrm{a}$ & $\mathrm{n} / \mathrm{a}$ & $\mathrm{n} / \mathrm{a}$ & $2.000 e-2$ & $1.67 \mathrm{E}+02$ \\
\hline
\end{tabular}

\section{$\Rightarrow$ Limit violated}

$\Rightarrow$ Selected Limit

败 
CORE NUMBER: 135

SEGMENT \#: $2 \mathrm{~A}$

SEGMENT PORTIOH: Wh whole Segment

\begin{tabular}{|c|c|c|c|c|c|c|c|c|c|c|c|c|c|c|}
\hline & & & & Action & Limits & & & & & & & & & \\
\hline Sample\# & A Al th & Analyte & Unit & Lower & Upper & Standard \% & BLank & Result & Duplicate & Average & RPD \% & Spk Rec \% & Det Limit & Count Err\% \\
\hline 5961002130 & & Bulk Density of Sample & $\mathrm{g} / \mathrm{mL}$ & None & Mone & $n / a$ & $\mathrm{n} / \mathrm{a}$ & & $n / a$ & $\mathrm{n} / \mathrm{a}$ & $\mathrm{n} / \mathrm{a}$ & $\mathrm{n} / \mathrm{a}$ & $5.000 \mathrm{e}-1$ & $n / a$ \\
\hline 5967002133 & & \% Water by TGA using Hettler & $\%$ & None & Mone & 99.53 & $\mathrm{n} / \mathrm{a}$ & 16.38 & $1.546 \mathrm{e} 1$ & $1.592 \mathrm{e} 1$ & 5.78 & $\mathrm{n} / \mathrm{a}$ & n/a & $n / a$ \\
\hline S967002133 & & DSC Exotherm Dry Calculated & Joules/g Dry & $-7.0 \mathrm{e}+00$ & 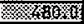 & $n / a$ & $\mathrm{n} / \mathrm{a}$ & $0.00 \mathrm{e}+00$ & 0.00000 & $0.000 \mathrm{e} 0$ & 0.00 & $n / a$ & $\mathrm{n} / \mathrm{a}$ & $\mathrm{n} / \mathrm{a}$ \\
\hline 5967002133 & & DSSC Exotherm using Mettler & Joules/g & None & None & 102.6 & $n / a$ & $0.000+00$ & $0.000 \mathrm{e}$ & $0.000 \mathrm{e}$ & 0.00 & $\mathrm{n} / \mathrm{a}$ & $n / a$ & $n / a$ \\
\hline \$96T002136 & $\bar{F}$ & Alpha of Digested Solid & uCi/g & $-7.0 \mathrm{e}+00$ & 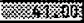 & 115.6 & $<1.730 e^{-3}$ & 1.09 ब. & $1.940 \mathrm{e}-2$ & $1.520 \mathrm{e}-2$ & 56.1 & $\mathrm{n} / \mathrm{a}$ & $4.000 \mathrm{e}-3$ & $4.07 E+01$ \\
\hline
\end{tabular}
Drainable Liquid: Drainable Liquid

\begin{tabular}{|c|c|c|c|c|c|c|c|c|c|c|c|c|c|c|}
\hline & & & & Action & Limits & & & & & & & & & \\
\hline Sample\# & R|AH & Analyte & Unit & Lower & Upper & Standard \% & 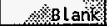 & resulta & Dupticate & Average & RPD $\%$ & Spk Rec \% & Det Limit & Count Err\% \\
\hline \begin{tabular}{|l|}
5961002142 \\
\end{tabular} & & $\%$ Water by TGA using Mettler & $\%$ & None & None & 99.88 & \% $\mathrm{n} / \mathrm{a}$ & 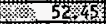 & $5.218 \mathrm{e} 1$ & $5.237 \mathrm{e}$ & 0.52 & $\mathrm{n} / \mathrm{a}$ & $\mathrm{n} / \mathrm{a}$ & $n / a$ \\
\hline \$961002142 & & Specific Gravity & Sp.G. & Hone & None & 97.93 & 40 & 3.665 & $1.373 \mathrm{e} 0$ & $1.519 \mathrm{e} 0$ & 19.2 & $\mathrm{n} / \mathrm{a}$ & $1.000 \mathrm{e}-3$ & $n / a$ \\
\hline S961002142 & & DSC Exotherm on Perkin Elmer & Joules/g & None & None & 4010 & 10 & $0.0 \mathrm{pe}+00$ & 0.0000 & $0.000 \mathrm{e} 0$ & 0.00 & $\mathrm{n} / \mathrm{a}$ & $n / a$ & $n / a$ \\
\hline$\$ 961002142$ & & DSC Exotherm Dry calculated & Joules/g Dry & $-7.0 \mathrm{e}+00$ & 1) & \% n/a & W & $0.000+00$ & $0.000 \mathrm{e} 0$ & $0.000 \mathrm{e} 0$ & 0.00 & $n / a$ & $\mathrm{n} / \mathrm{a}$ & $\mathrm{n} / \mathrm{a}$ \\
\hline$\$ 967002142$ & & Alpha in Liguid Samples & $\mathrm{UCi} / \mathrm{mL}$ & $-7.0 \mathrm{e}+00$ & 1. & 309.4 & $330 e^{-2}$ & $<1.17 e^{-02}$ & $<1.17 \mathrm{E}-2$ & $\mathrm{n} / \mathrm{a}$ & $\mathrm{n} / \mathrm{a}$ & $\mathrm{n} / \mathrm{a}$ & $2.000 \mathrm{e}-2$ & $5.00 E+02$ \\
\hline
\end{tabular}

$\Rightarrow$ Limit violated

要 $\quad$ Selected Limit

มู 
CORE NUMBER: 135

SEGWENT \#: 2R

SEGMENI PORTION: U Upper Half of Segment

\begin{tabular}{|c|c|c|c|c|c|c|c|c|c|c|c|c|c|c|}
\hline & & & & Action & Limits & & & & & & & & & \\
\hline Sample贲 & A\# & Analyte & Unit & Lower & Upper & Standard \% & Blank & Result & Dupl icate & Average & RPD \% & Spk Rec \% & Det Limit & Count Er $\mathrm{r} \%$ \\
\hline S967001869 & & Bulk Density of Samole & $\mathrm{g} / \mathrm{mL}$ & None & None & $\mathrm{n} / \mathrm{a}$ & $\mathrm{n} / \mathrm{a}$ & 1.300 & $\mathrm{n} / \mathrm{a}$ & $\mathrm{n} / \mathrm{a}$ & $n / a$ & $\mathrm{n} / \mathrm{a}$ & $5.000 \mathrm{e}-1$ & $\mathrm{n} / \mathrm{a}$ \\
\hline 5967001873 & & $\%$ Hater by TGA using Mettler & $\%$ & Hone & Mone & 99.22 & $\mathrm{n} / \mathrm{a}$ & 4.670 & $4.150 \mathrm{e} 0$ & $4.410 \mathrm{e} 0$ & 11.8 & $n / a$ & $n / a$ & $n / a$ \\
\hline S96T001873 & & DSC Exotherm Dry Calculated & Joules/g Dry & $-7.0 e+00$ & 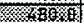 & $\mathrm{n} / \mathrm{a}$ & $n / a$ & $0.00 \mathrm{e}+00$ & $0.000 \mathrm{e} 0$ & $0.000 \mathrm{e}$ & 0.00 & $n / a$ & $\mathrm{n} / \mathrm{a}$ & n/a \\
\hline \$96T001873 & & DSC Exotherm using Hettler & Joules $/ g$ & None & None & 96.31 & $n / a$ & $0.000 \% 00$ & 0.00000 & $0.000 \mathrm{e} 0$ & 0.00 & $n / a$ & $\mathrm{n} / \mathrm{a}$ & $n / a$ \\
\hline \$967001879 & $\overline{\mathbf{F}}$ & Alpha of Digested Solid & $\mathrm{uCi} / \mathrm{g}$ & $-7.0 \mathrm{e}+00$ & 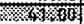 & 115.6 & $<1.730 \mathrm{e}-3$ & $1.47 \mathrm{es}$ & $1.510 \mathrm{e}-2$ & $1.490 \mathrm{e}-2$ & 2.68 & 66.02 & $4.000 \mathrm{e}-3$ & $3.33 E+01$ \\
\hline
\end{tabular}

L. Lower Half of Segment: L Lower Half of Segment

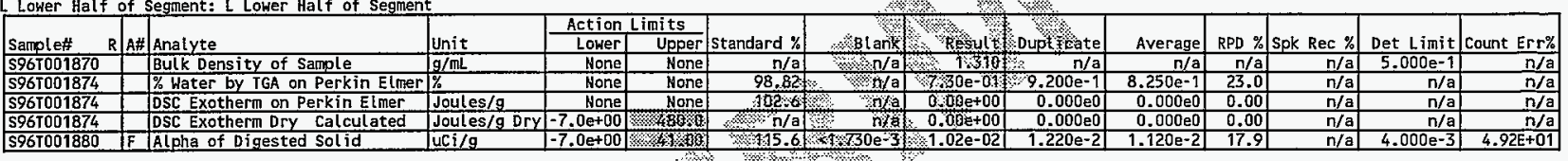

\section{$\Rightarrow$ Limit violated}

S Selected Limit

N 
CORE NUMBER: 135

SEGMENT \#: 1

SEGMENT PORTION: U Upper Half of Segment

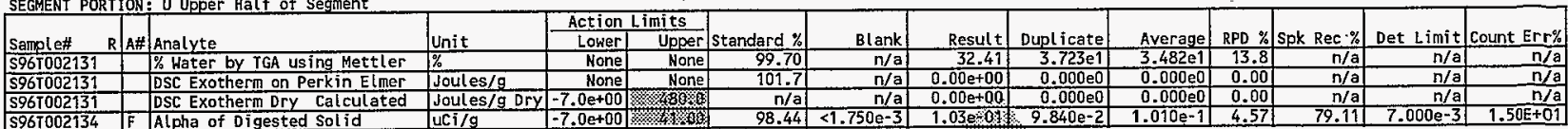
\begin{tabular}{|l|l|l|} 
S967002131 & & DSC Exotherm Dry Calculated \\
\hline S967002134 & F & Alpha of Digested Sol id \\
\hline
\end{tabular} uCi/g

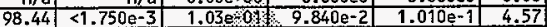

\begin{tabular}{r|r|r}
\hline 79.11 & $7.000 \mathrm{e}-3$ & $1.50 \mathrm{E}+01$ \\
\hline
\end{tabular}

Drainable Liquid: Drainable Liquid

\begin{tabular}{|c|c|c|c|c|c|c|c|c|c|c|c|c|c|c|}
\hline & & & & Action & Limits & & & 业 & W & & & & & \\
\hline Sample\# & R|A\# & Analyte & Unit & Lower & Upper & Standard \% & Blakta & Resul $\mathrm{t}$ & Dusficate & Average & RPD \% & Spk Rec \% & Det Limit & Count Er r \% \\
\hline 02140 & & \% water by TGA using Mettler & $\%$ & Hone & None & 99.53 & $\infty, n / 2$ & 552.73 & $5228 \mathrm{e} 1$ & $5.250 \mathrm{e} 1$ & 0.86 & $n / a$ & $\mathrm{n} / \mathrm{a}$ & $n / a$ \\
\hline & & fic Gravity & Sp.G. & None & Mone & 97.93 & $n / a$ & 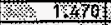 & $1.392 \mathrm{e} 0$ & $1.431 \mathrm{e} 0$ & 5.45 & $n / a$ & $1.000 \mathrm{e}-3$ & $n / a$ \\
\hline 596 & & DSC Exotherm Dry Calculated & Joules/g Dry & $-7.0 \mathrm{e}+00$ & 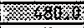 & aka & \%ua & $-40 \mathrm{e}+02$ & $5.776 \mathrm{e} 2$ & $5.588 \mathrm{e} 2$ & 6.75 & $n / a$ & $\mathrm{n} / \mathrm{a}$ & $n / a$ \\
\hline & & DSC Exotherin using Hettler & Joules/g & Hone & None & $406: 5$ & No & $2.56 \mathrm{e}+02$ & $2.743 \mathrm{e} 2$ & $2.654 \mathrm{e} 2$ & 6.75 & $n / a$ & $n / a$ & $n / a$ \\
\hline S967002140 & & Alpha in Liquid samples & $\mathrm{uCi} / \mathrm{mL}$ & $-7.0 \mathrm{e}+00$ & 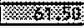 & $\$ 09.4$ & $3.3300 \times 2$ & $<1.336-02$ & $<1.33 E-2$ & $\mathrm{n} / \mathrm{a}$ & $n / a$ & 99.44 & $2.000 \mathrm{e}-2$ & $5.00 E+02$ \\
\hline
\end{tabular}

$\Rightarrow$ Limit violated

$\Rightarrow$ Selected Limit

番

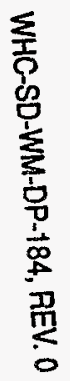


CORE NUMBER: 135

SEGMENT \#: 2

SEGHENT PORTION: Liner Liquid

\begin{tabular}{|c|c|c|c|c|c|c|c|c|c|c|c|c|c|c|}
\hline \multirow[b]{2}{*}{ Sample\# R } & \multirow[b]{2}{*}{ Aff } & \multirow[b]{2}{*}{ Analyte } & \multirow[b]{2}{*}{ Unit } & \multicolumn{2}{|c|}{ Action Limits } & \multirow[b]{2}{*}{ Standard \% } & \multirow[b]{2}{*}{$B$ Lank } & \multirow[b]{2}{*}{ Result } & \multirow[b]{2}{*}{ Duplicate } & \multirow[b]{2}{*}{ Average } & \multirow[b]{2}{*}{ RPD \% } & \multirow[b]{2}{*}{ Spk Rec \% } & \multirow[b]{2}{*}{ Det Limit } & \multirow[b]{2}{*}{ Count Err\% } \\
\hline & & & & Lower & Upper & & & & & & & & & \\
\hline \$961001865 & & Specific Gravity & Sp.G. & None & Hone & 98.38 & $n / a$ & 1.030 & $1.030 \mathrm{e} 0$ & $1.030 \mathrm{e}$ & 0.00 & $\mathrm{n} / \mathrm{a}$ & $1.000 \mathrm{e}-3$ & $n / a$ \\
\hline 5967001866 & & \% Water by TGA using Mettler & $\%$ & None & None & 100.3 & $\mathrm{n} / \mathrm{a}$ & 94.44 & $9.393 \mathrm{e} 1$ & $9.419 \mathrm{e} 1$ & 0.54 & $n / a$ & $n / a$ & $n / a$ \\
\hline$\$ 967001866$ & & DSC Exotherm Dry Calculated & Joules/g Dry & $-7.0 \mathrm{e}+00$ & 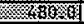 & $\mathrm{n} / \mathrm{a}$ & $\mathrm{n} / \mathrm{a}$ & $0.00 \mathrm{e}+00$ & $0.000 \mathrm{e} 0$ & $0.000 \mathrm{e} 0$ & 0.00 & $\mathrm{n} / \mathrm{a}$ & $n / a$ & $\mathrm{n} / \mathrm{a}$ \\
\hline \$96T001866 & & DSC Exothem using Mettler & Joules/g & None & Mone & 113.5 & $n / a$ & $0.00 \mathrm{e}+0 \mathrm{0}$ & $0.000 \mathrm{e} 0$ & $0.000 \mathrm{e} 0$ & 0.00 & $\mathrm{n} / \mathrm{a}$ & $n / a$ & $n / a$ \\
\hline S96T001866 & & Alpha in Liquid Samples & $\mathrm{uC} \mathbf{i} / \mathrm{mL}$ & $-7.0 \mathrm{e}+00$ & 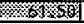 & 112.5 & $<7.280 \mathrm{e}-4$ & $<5.510 .04$ & $2 \times 6.41 E-4$ & $n / a$ & $\mathrm{n} / \mathrm{a}$ & 114.8 & $1.000 \mathrm{e}-3$ & $5.00 E+02$ \\
\hline
\end{tabular}

U Upper Half of Segment: U Upper Half of Segment

\begin{tabular}{|c|c|c|c|c|c|c|c|c|c|c|c|c|c|c|}
\hline & & & & Action & Limits & & ) & & \% & & & & & \\
\hline Sample\# $\quad \mathrm{B}$ & 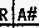 & Analyte & Unit & Lower & Upper & Standard \% & \% Blank & kesultit & Duptictate & Average & RPD \% & Spk Rec \% & Det Limit & Count Err\% \\
\hline 596T001867 & & $\%$ Water by TGA using Mettler & $\%$ & Hone & None & 99.19 & $n / a$ & $49 \%$ & $4.974 \mathrm{e} 1$ & $4.982 \mathrm{el}$ & 0.32 & n/a & $n / a$ & $n / a$ \\
\hline 5967001867 & & DSC Exotherm Dry Calculated & Joules/g Dry & $-7.0 e+00$ & 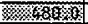 & $\mathrm{n} / \mathrm{s}$ & o/a & $280 e+02$ & $2.096 \mathrm{e} 2$ & $2.449 \mathrm{e} 2$ & 28.8 & n/a & $n / a$ & $n / a$ \\
\hline 5967001867 & & DSC Exotherm using Mettler & Joules/g & Hone & Hone & $96 \% 3 \div$ & nica & Tyte+02 & $1.052 \mathrm{e} 2$ & $1.229 \mathrm{e} 2$ & 28.8 & $\mathrm{n} / \mathrm{a}$ & $n / a$ & $n / a$ \\
\hline 5967001868 & $\bar{F}$ & Alpha of Digested Solid & uCi $/ g$ & $-7.0 \mathrm{e}+00$ & 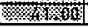 & 98.44 & $\times 1.75003$ & $3.29-01$ & $3.480 \mathrm{e}-1$ & $3.390 e-1$ & 5.61 & $\mathrm{n} / \mathrm{a}$ & $4.000 \mathrm{e}-3$ & $6.07 \mathrm{E}+00$ \\
\hline
\end{tabular}

S967001867 \% Water by TLA using Mettler S960001867 $\quad$ DSC Exotherm using Mettler uci $/ \mathrm{g}$

$-7.0 \mathrm{e}+00$ None

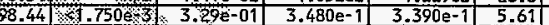

\section{$\Rightarrow$ Linit violated}

$\Rightarrow$ Selected Limit

청 
WHC-SD-WM-DP-184, REV. 0

DOME SPACE VAPOR FLAMMABILITY DATA SHEETS 
WHC-SD-WM-DP-184, REV, 0

THIS PAGE WAS INTENTIONALLY LEFT BLANK

31 
$05 / 23 / 96 \quad 13: 23 \quad \mathscr{B} 509 \quad 3737226$

ENG. $2750 \mathrm{E}$ K109

G012

WHC-SD-WM-DP-184, REV . 0

CONTROLLER COPY

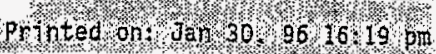

DATA SHEET 7 - IH\&S VAPOR SURVEY

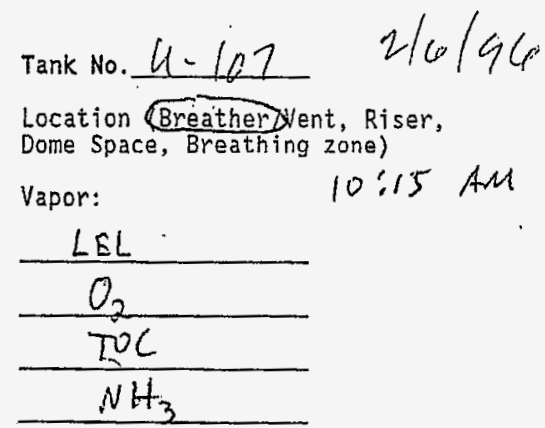

Tank No. U-10

Location (Breather/Vent, Riser, (Dome Space, Breathing zone) 10:0040M Vapor:

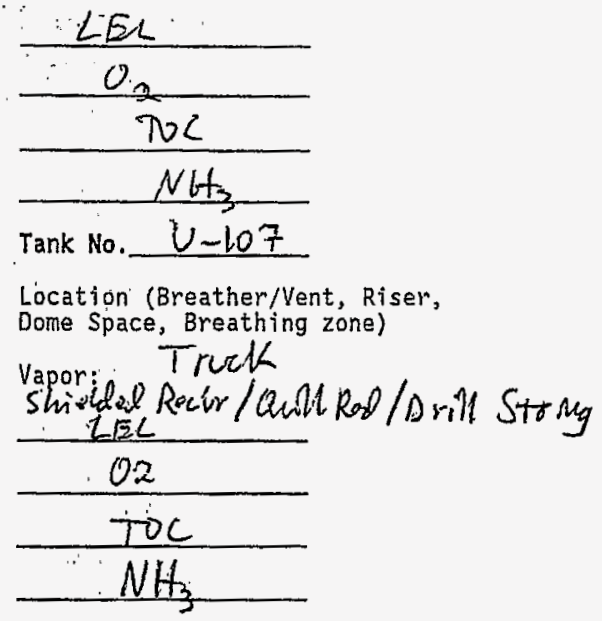

Riser No. 9

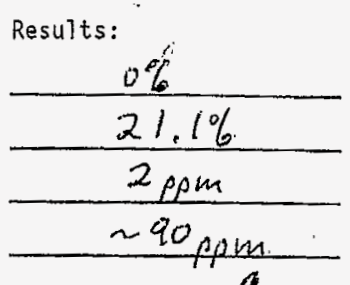

Riser No. $\frac{9}{\square}$

\begin{tabular}{ll}
\hline Results: & $1 \%$ \\
\hline$\frac{20.84:}{14}$ \\
\hline$\frac{400 \text { pion }}{9}$ \\
\hline Riser No. $\frac{9}{}$
\end{tabular}

Results:

$\frac{0 \% / 0 \% / 0 \%}{20.9 \% / 14 \% / 12 \%}$
0 ppm $/ \angle 2 p$ pm $/ \leq 2 p p m$
$-1<5 p p m / \leq 5 p p m$

32

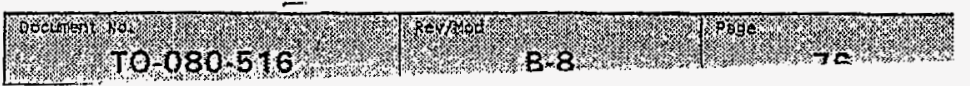




\section{DATA SHEET. 9 - IH\&S FLAMMABLE GAS MONITORING}

Tank No. U./ 107 Riser No. 9

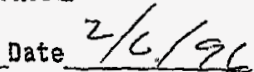

NOTE- Vapor survey for single Shell Tanks with hand held combustible gas meter and probe per HASP or SHMS sha11 be recorded every 15 minutes.

NOTE- Vapor survey for Double Shel1 Tank in vent header or SHMS, if operable, recorded every 15 minutes with hand held combustible gas meter per HASP.

Location (Vent Header on Dome Space)

Vapor sample every 15 minutes and record below:

$$
\text { Differential Limit }
$$

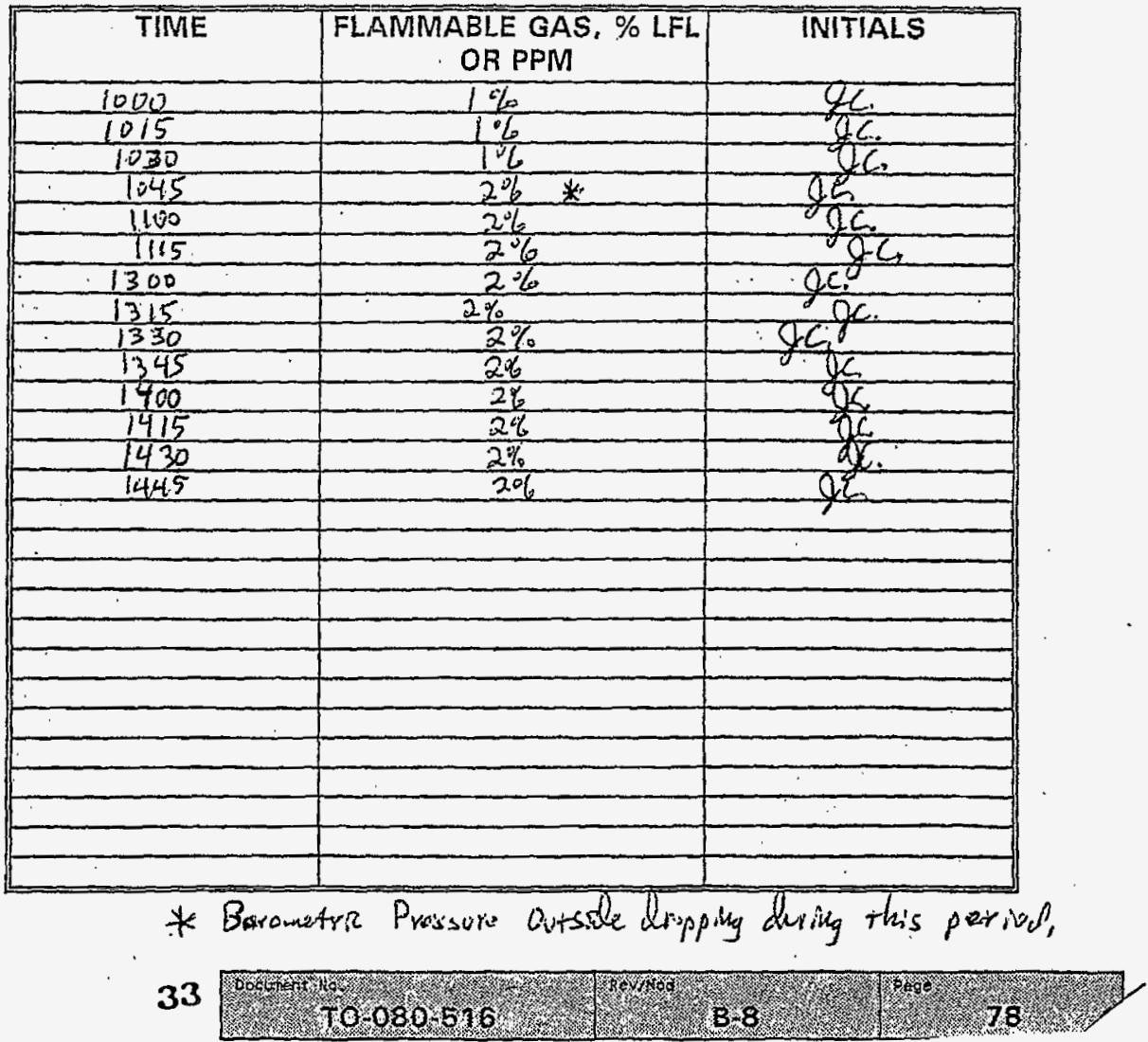




\section{WHC-SD-WM-DP-184, REV. 0}

\section{DATA SHEET 9 - IH\&S FLAMMABLE GAS MONITORING}
Tank No. $U-107$
Riser No.
7
Date $2-15-96$

NOTE- Vapor survey for single Shell Tanks with hand held combustible gas meter and probe per HASP or SHMS shal1 be recorded every 15 minutes.

NOTE- Vapor survey for Double Shell Tank in vent header or SHMS, if operable, recorded every 15 minutes with hand held combustible gas mèter per HASPO

Location (Vent Header on Dome Space

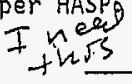

Vapor sample every 15 minutes and record below:

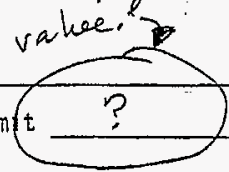

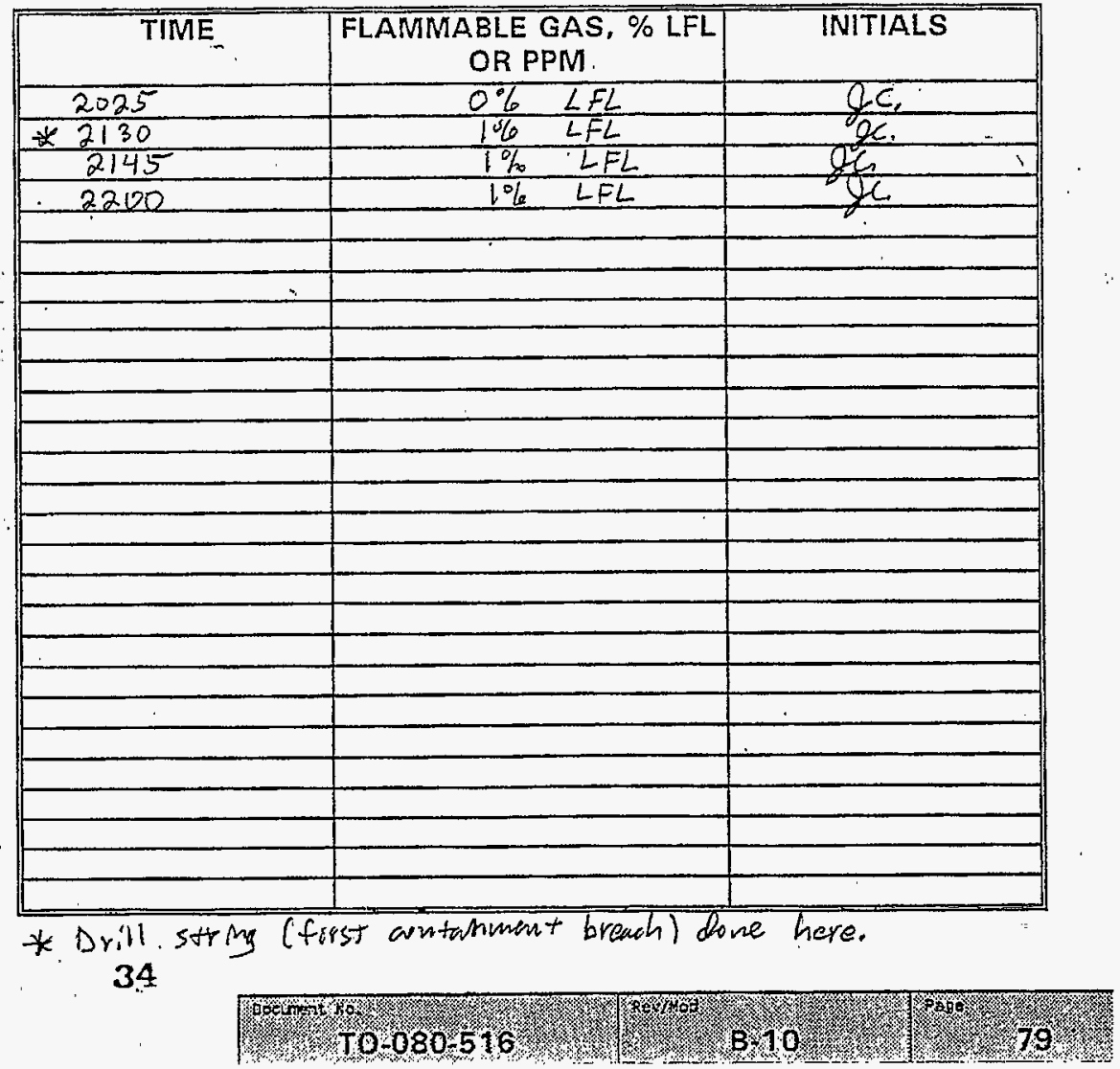




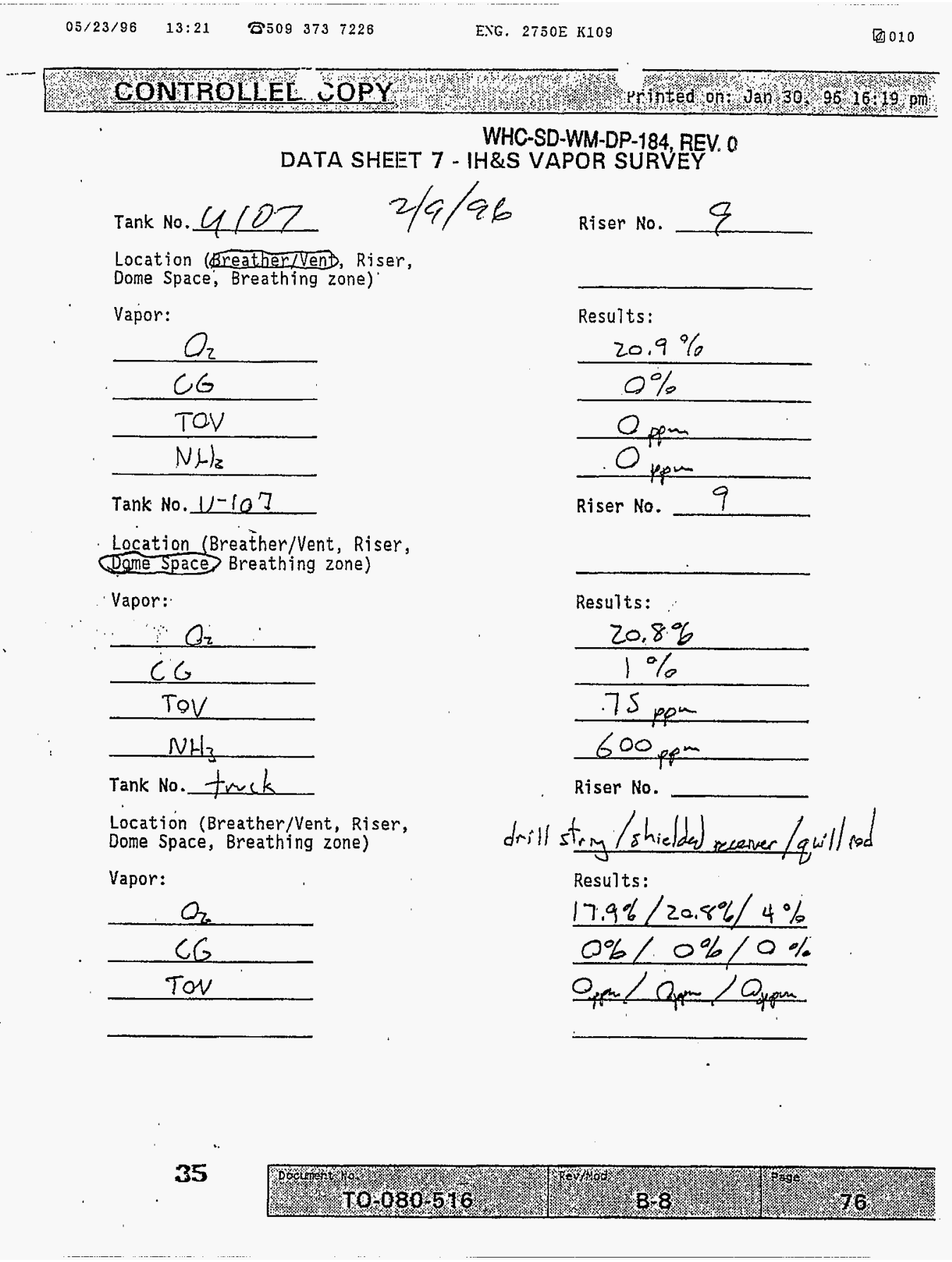




\section{WHC-SD-WM-DP-184, REV. 0}

\section{DATA SHEET 7 - IH\&S VAPOR SURVEY}

Tank No. $4-109$

Location Breathepyent, Riser,

Dome Space, Breathing zone)

Vapor:

$\frac{\frac{2 B L}{O 2}}{\frac{\mathrm{OOC}}{\mathrm{NH}_{3}}}$

Tank No. $\quad U-107$

Resu7ts:

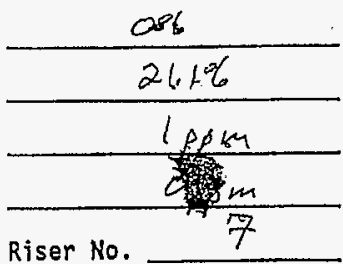

Location (Breather/Vent, Riser, one Space, Breathing zone)

Vapor:

Results:

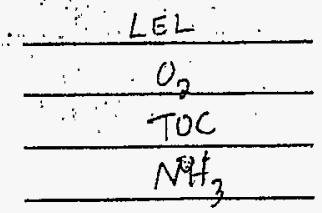

Tank No. $\quad U-107$

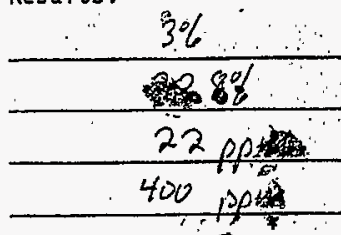

Riser No. . .

Location (Breather/Vent, Riser,

Dome Space, Breathing zone)

Vapor:

Irvach

$\frac{\frac{L E L}{\mathrm{R}_{2}}}{\frac{\mathrm{TOC}}{\mathrm{NH}_{3}}}$

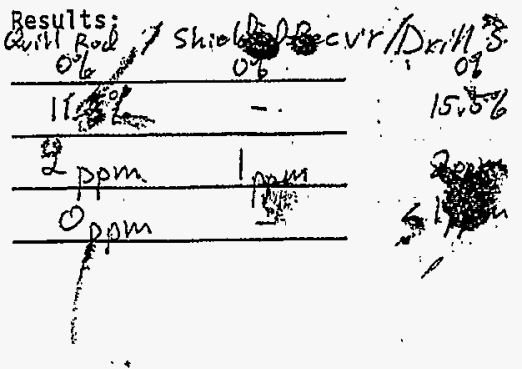




\section{WHC-SD-WM-DP-184, REV 0 \\ DATA SHEET 7 - IH\&S VAPOR SURVEY}

Tank No. $4-10 \%$. $2 / 21 / 9$

Location Breatheryvent, Riser,

Dome Space, Breatning zone)

Vapor:

$\frac{\frac{L E L}{O 2}}{\frac{O \mathrm{OC}}{\mathrm{NH}_{3}}}$

Tank No. $\quad U-1.07$

Location (Breather/Vent, Riser, (Dome Space Breathing zone)

Vapor:

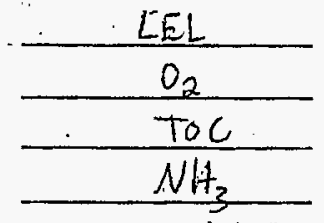

Tank No. $u-107$

Location (Breather/Vent, Riser, Dome Space, Breathing zone)

vapor: Truck

$\frac{\frac{\text { LEL }}{\mathrm{O}}}{\frac{\mathrm{TOC}}{\mathrm{NH}_{3}}}$

Riser No.

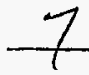

Results:

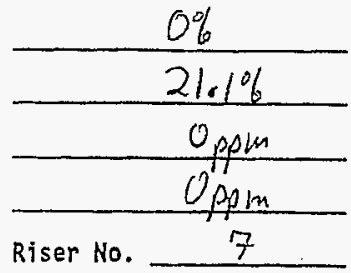

Riser No.

Results:

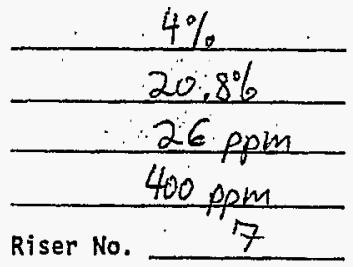

Riser No. $\frac{7}{7}$

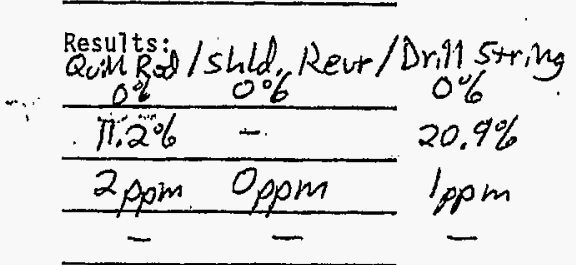


WHC-SD-WM-DP-184, REV. 0

STATISTICAL ANALYSIS

39 
WHC-SD-WH-DP-184, REV, 0

THIS PAGE WAS INTENTIONALLY LEFT BLANK 


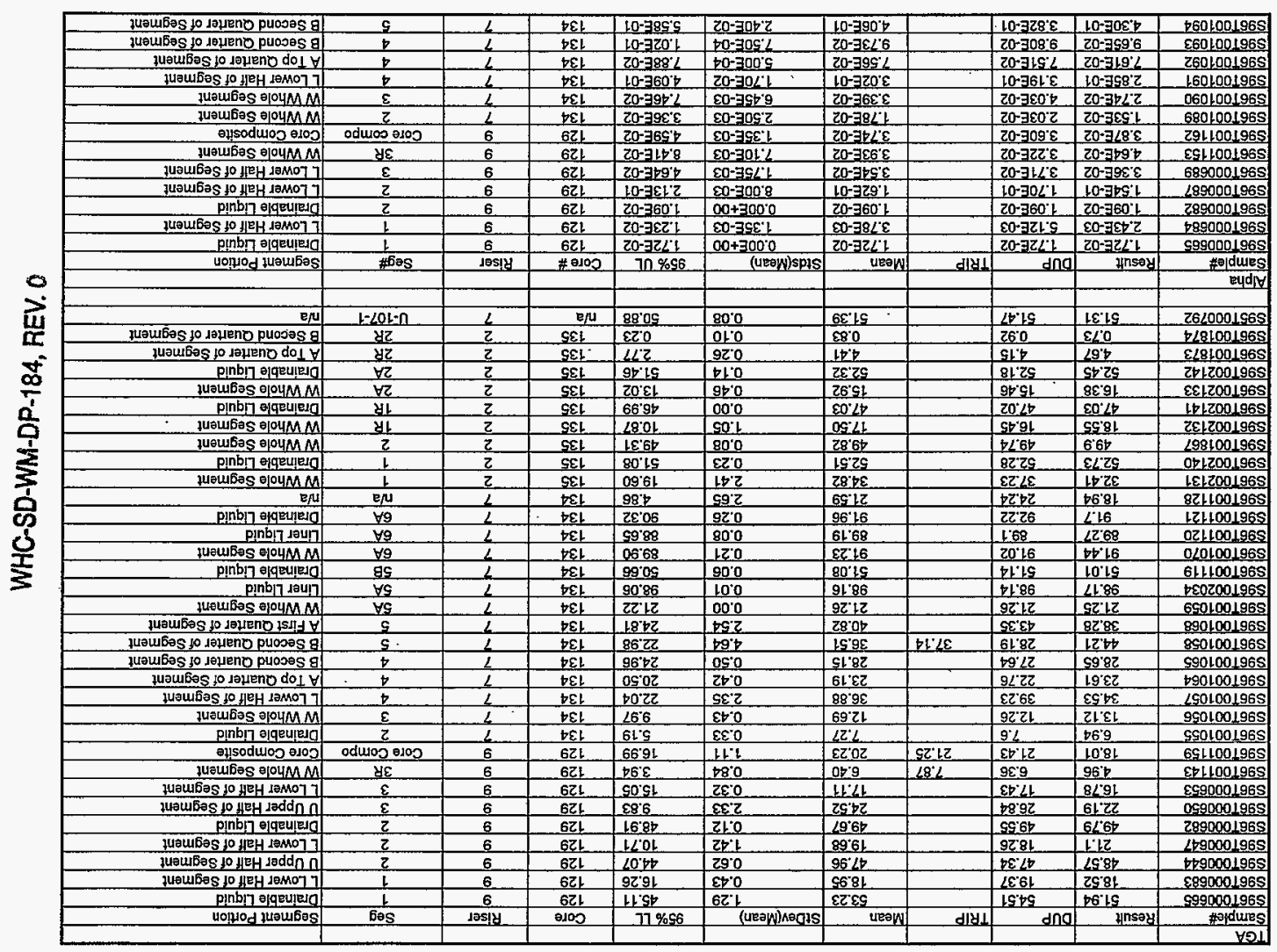




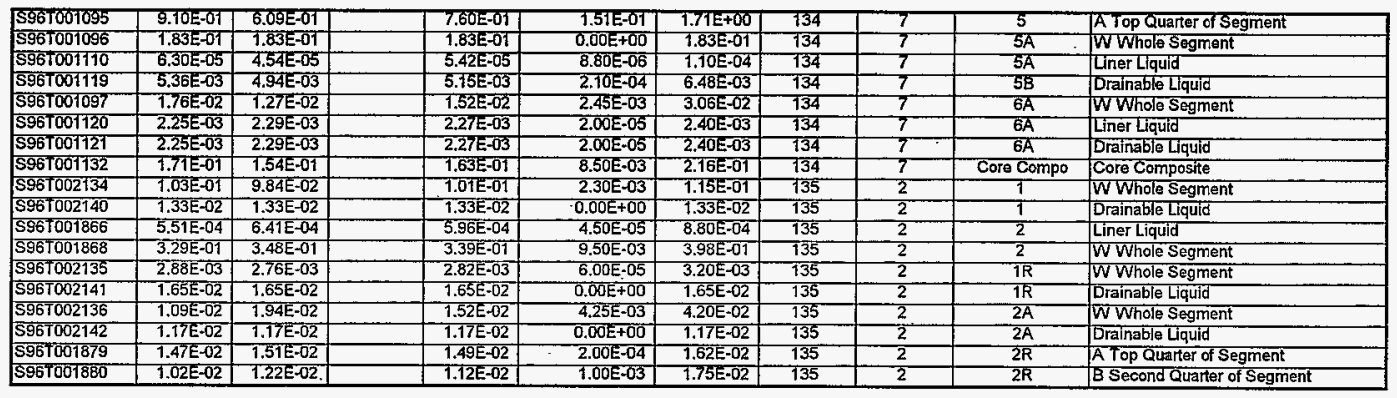

品 


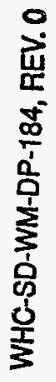

\begin{tabular}{|c|c|c|c|c|c|c|c|c|c|c|c|}
\hline poubas to jequeno puoves g & पे $z$ & 2 & $\mathrm{~s} \varepsilon_{1}$ & al7os & 000 & 000 & 000 & & D & 0 & $0 \angle 81001965$ \\
\hline fueubas jo dapeno do $\downarrow \forall$ & $8 z$ & $z$ & SEI & alios & $00^{\circ} 0$ & 000 & 000 & & D & 0 & E<81001955 \\
\hline pinblo өpqeulera & $\forall z$ & $z$ & SEl & ginoly & $00^{\circ} 0$ & 000 & 000 & & 0 & 0 & zF1z001969 \\
\hline ILaubas 키으MM & $\forall z$ & $z$ & SEl. & anos & $00^{\circ} 0$ & $00^{\circ} 0$ & $00^{\circ} 0$ & & 0 & 0 & EEl2001965 \\
\hline plnbl7 아queu|cia & 반 & 2 & SEI & ainoln & $868<9$ & 0912 & $09 \angle \varepsilon S$ & & 9t5 & 2699 & 1012001965 \\
\hline Juaubas פ & प्रI & $z$ & SEI & alnos & 00'0 & 000 & 000 & & 0 & 0 & Zदा200196s \\
\hline fueurbas 이이 MM & $z$ & $z$ & SEL. & anos & $8<\angle 96$ & OE'SE & Dstoz & & 9.602 & 2082 & 2981001965 \\
\hline pintig eiqquilar & $t$ & 2 & gEl & alnoin & $\angle 2<49$ & 58.81 & SL8SS & & 9446 & $66 E 9$ & $0+12001965$ \\
\hline 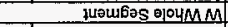 & $\frac{i}{2}$ & 2 & sel & anos & 000 & 000 & $00^{\prime} 0$ & & 0 & 0 & LELZ00196s \\
\hline RM & epu & L & $t \varepsilon t$ & alnos & $00^{\circ} 0$ & 000 & DOO & & 0 & 0 & $82+1001965$ \\
\hline pinbin ә中qeu!ejo & $\forall 9$ & 2 & $t \varepsilon i$ & olnoit & $00^{\circ} 0$ & $00^{\circ} 0$ & 000 & & 0 & 0 & LZLLO0196S \\
\hline pinbin taul] & $\forall 9$ & L & $+\varepsilon t$ & ainoin & $00^{\circ} 0$ & 00.0 & 000 & & 0 & 0 & 021.100196S \\
\hline juәu6as פ & $\forall 9$ & 4 & $\nabla \varepsilon i$ & al7os & $00^{\circ} 0$ & 000 & 000 & & 0 & 0 & $0<01001965$ \\
\hline 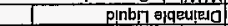 & g9 & 2 & $t \varepsilon t$ & alnolit & 000 & $00^{\circ} 0$ & 000 & & 0 & 0 & $6+11001965$ \\
\hline pinbri deu!n & $\forall s$ & $L$ & $b \varepsilon_{1}$ & alnol7 & 000 & $00^{\circ} 0$ & 000 & & 0 & 0 & $t$ EOZOOL96S \\
\hline jueu6es elouMM & $\forall s$ & $L$ & $t \varepsilon l$ & alnos & 000 & $00^{\circ} 0$ & $00^{\circ} 0$ & & 0 & 0 & 6501001965 \\
\hline 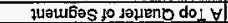 & $\frac{9}{2}$ & 6 & $t \varepsilon i$ & gizos & $00^{\circ} 0$ & 000 & $00^{\circ} 0$ & & 0 & 0 & 8901001965 \\
\hline zuaubes fo rapueno puoses g & $\mathrm{g}$ & -6 & $+\varepsilon 1$ & alnos & $98^{\circ} 991$ & S8.ZE & 76.09 & $226 \varepsilon$ & SSCI & 1281 & 8501001965 \\
\hline zuaubas po daueno puojas a & $t$ & 4 & tel & anos & $\infty 0^{\circ}$ & 000 & 000 & & 0 & 0 & S901001965 \\
\hline \}Uจนโอ & $t$ & 2 & tet & al7os & $\infty 0^{\circ}$ & $00^{\circ} 0$ & $00^{\circ} 0$ & & 0 & 0 & \$9100196S \\
\hline 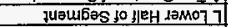 & 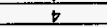 & $L$ & DEt & anos & GLPEZ & $08^{\circ} 0$ & $0<\varepsilon \varepsilon Z$ & & spez & $6 z \varepsilon z$ & $\angle 001001965$ \\
\hline 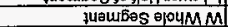 & $\varepsilon$ & $L$ & $t \varepsilon$ & alios & 000 & $00^{\circ} 0$ & 000 & & 0 & 0 & 9501001965 \\
\hline fualu6อS ӨjO4MM & 2 & 4 & $\nabla \varepsilon l$ & 멱os & conto & $00 \%$ & 000 & & 0 & 0 & S50100196s \\
\hline el!soduos ejos & oduroj eros. & 6 & 621 & glios: & OZSE & $a z \varepsilon$ & 00 c! & & 281 & 8.t. & 6511001965 \\
\hline puaubes epouM M & प⿺ & 6 & 621 & alnos & 000 & 000 & 000 & $\mathbf{0}$ & 0 & 0 & Ebll00196s \\
\hline juaw6əs to нен samo7 7 & $\varepsilon$ & 6 & 621 & alnos & $6 L \div \angle 8$ & 116 & 8692 & & $6 L^{-9} 9 \varepsilon$ & 6491 & ES90001965 \\
\hline 7นeแ & $\varepsilon$ & 6 & 621 & alnos & $98^{\circ} \mathrm{98}$ & 086 & $86 \varepsilon$ & & $8 L^{\circ}+1$ & $8 \angle \Omega \varepsilon$ & 0990001965 \\
\hline 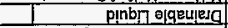 & 2 & 6 & 6Z1 & ainola & $\$ 6.2 E 1$ & SE' & sl'ezt. & & 9.9Z1 & $\angle 6 Z 1$ & 2890001965 \\
\hline 1 Hอw & 2 & 6 & $6 Z 1$ & allos & 4991 & $\angle 80$ & 8011 & & $12 \% 01$ & 96.11 & $4+90001965$ \\
\hline fuow & 2 & 6 & $6 Z 1$ & glios & $08<18$ & SLC & 59292 & & 825 & Eillz & $\$ 490001965$ \\
\hline Huaubas to HеH Jamon T & 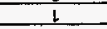 & 6 & 621 & onos & $49^{\prime} 81$ & 290 & $69+1$ & & $40 \div 1$ & E'St & E89000196S \\
\hline pinbig әgquiesa & 1 & 6 & 621 & ainon & $8 \mathrm{~g}^{\circ} \mathrm{ELI}$ & oz: & 00911 & & $8+4$ & $2<12$ & 5990001965 \\
\hline uolfod Hambas & \#官S & Ies!y & \#2100 & xu19n: & $7 n \% 56$ & (UeaVi)haCPIS & UEOW & & dna & Hnsay & 킴ues \\
\hline & & & & & & & & & & & 250 \\
\hline
\end{tabular}


WHC-SD-WM-DP-184, REV. 0

INORGANIC ANALYSES

44 
WHC-SD-WM-DP-184, REV. O

THIS PAGE WAS INTENTIONALLY LEFT BLANK 
worklistrpt Version $2.105 / 15 / 95$

WHC-SD-WM-DP-184, REV. 0

Page: I

02/26/96 07:21

LABCORE Data Entry Template for Worklist\#

5892

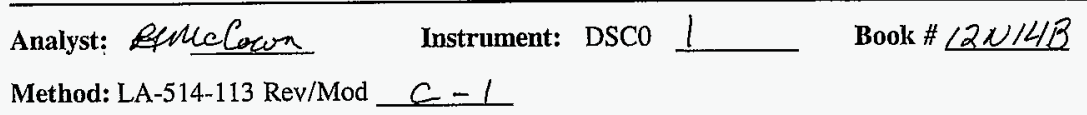

Worklist Comment: U-107 DSC RUN UNDER N2. RCJ

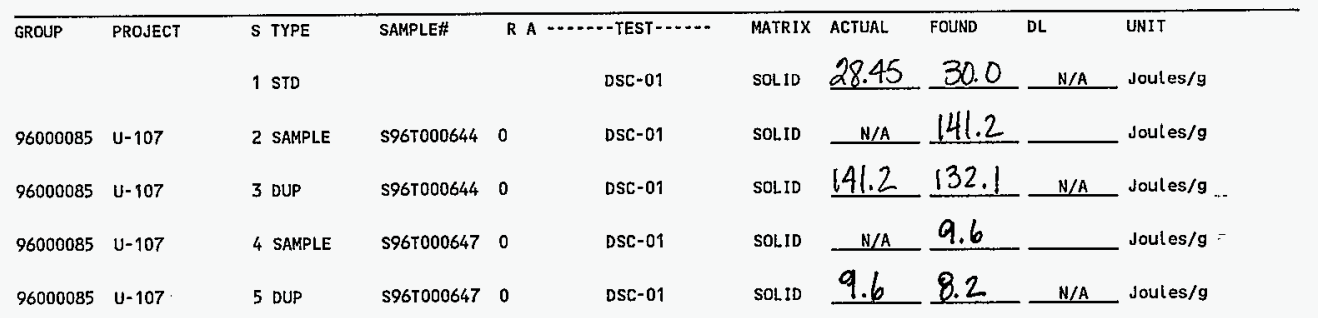

Final page for worklist \#

5892

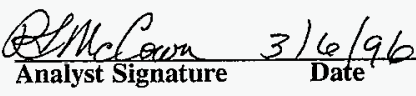

$$
\text { Analyst Signature Date }
$$

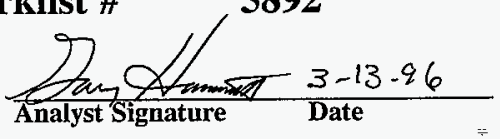
Verified by Blandina Valenzuela

Data Entry Comments:

5967000644 produced an endotherm at $125.3 \mathrm{C}$ with a delta $\mathrm{H}$ of $1243.8 \mathrm{~J} / \mathrm{g}$.

9967000647 produced two additional endothernus one at $136.22^{\circ} \mathrm{C}$ with a delta $H$ of $42 \mathrm{C}$ J lg and second at $303.2{ }^{\circ} \mathrm{C}$ with a delta 4 of $106.35 \mathrm{~F}$ $R=$ Replicate Number, $A=$ Aliquot Code.

46 


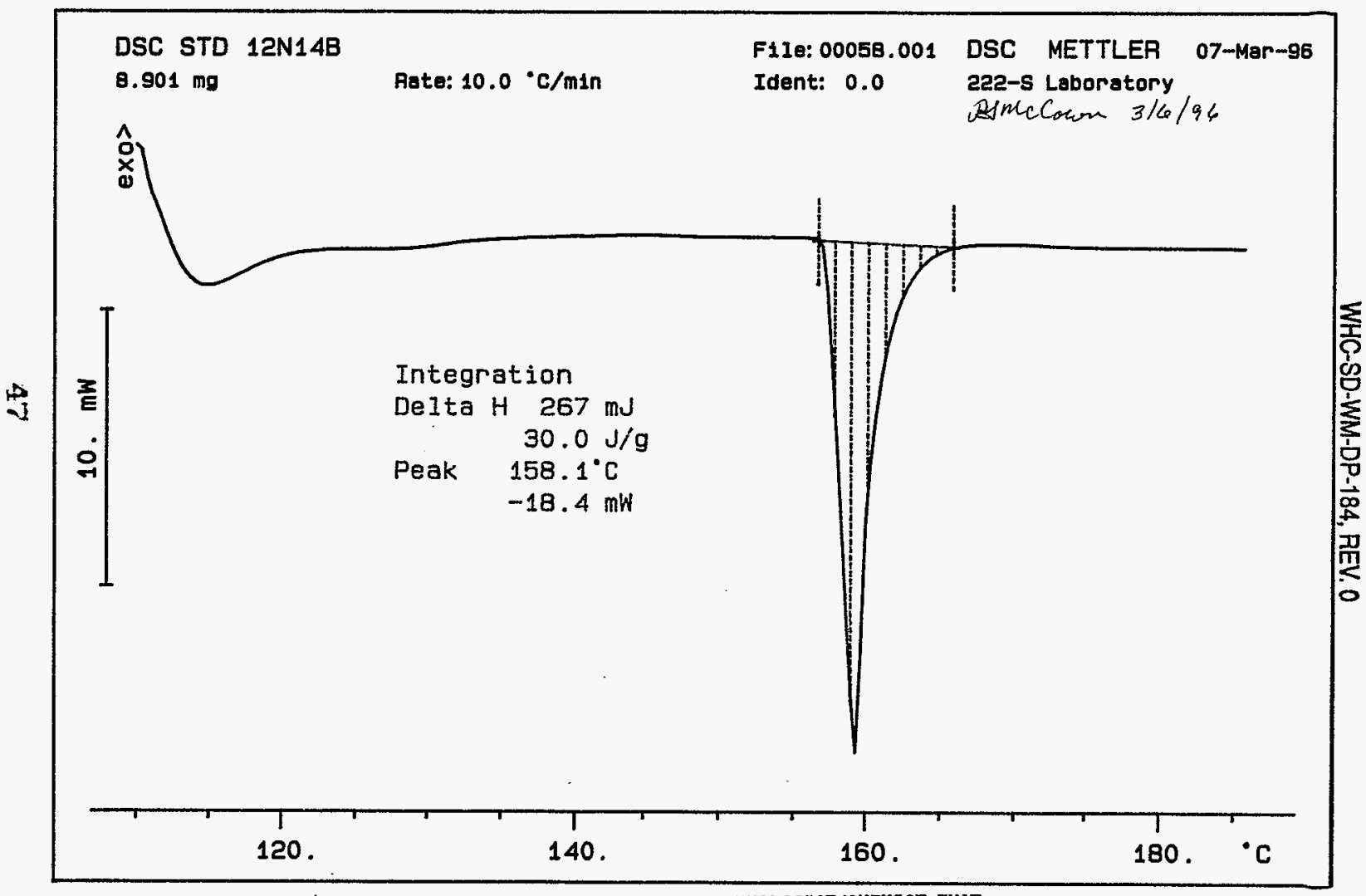

SIGNATURE ABOVE REPRESENTS CHEMICAL TECHNOLOGIST/CHEMIST THAT CONPLETED/VERIFIED THE CALIBRATION/ANALYSIS ON PAGES 47 TO 51. 
WHC-SD-WM-DP-184, REV. 0

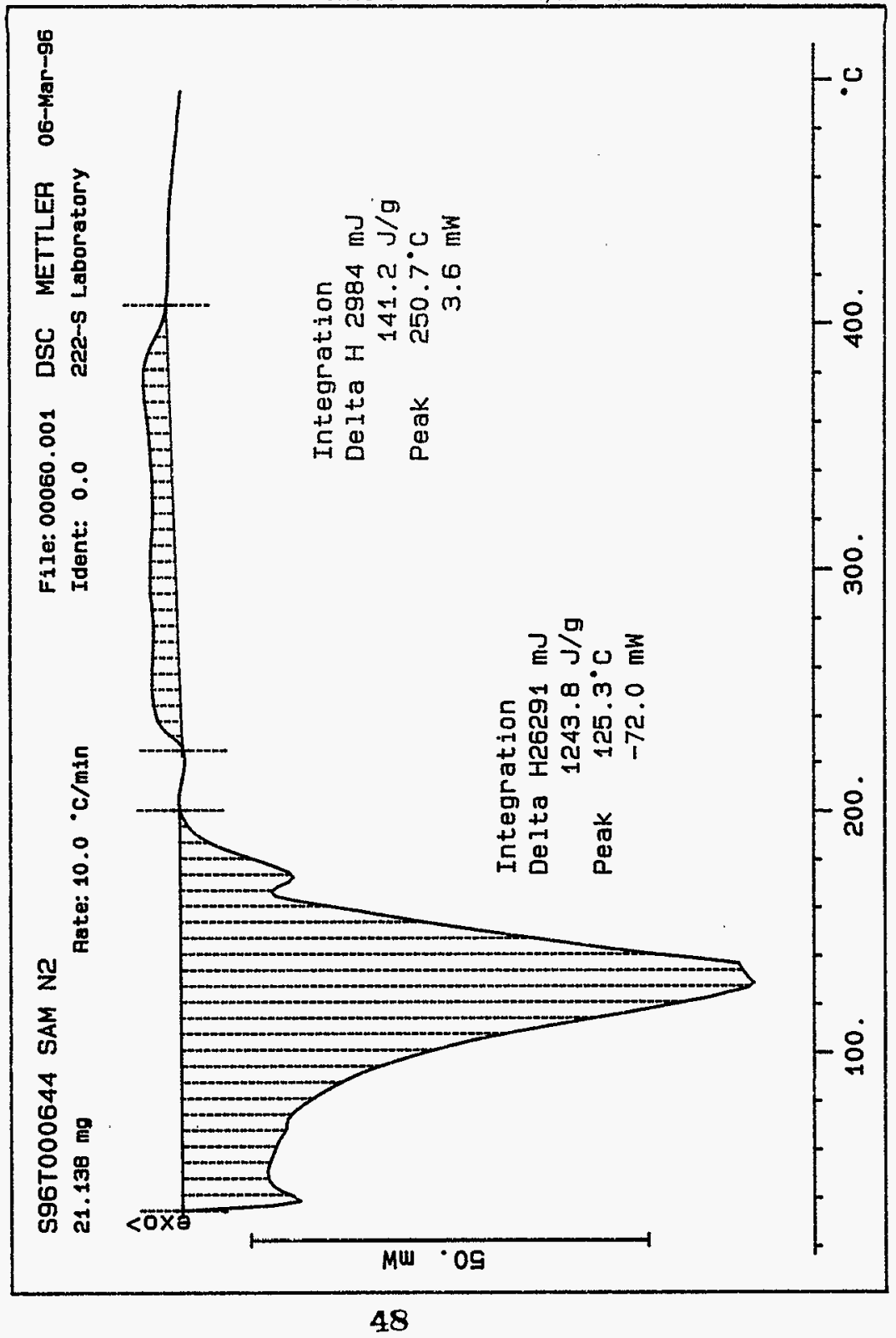




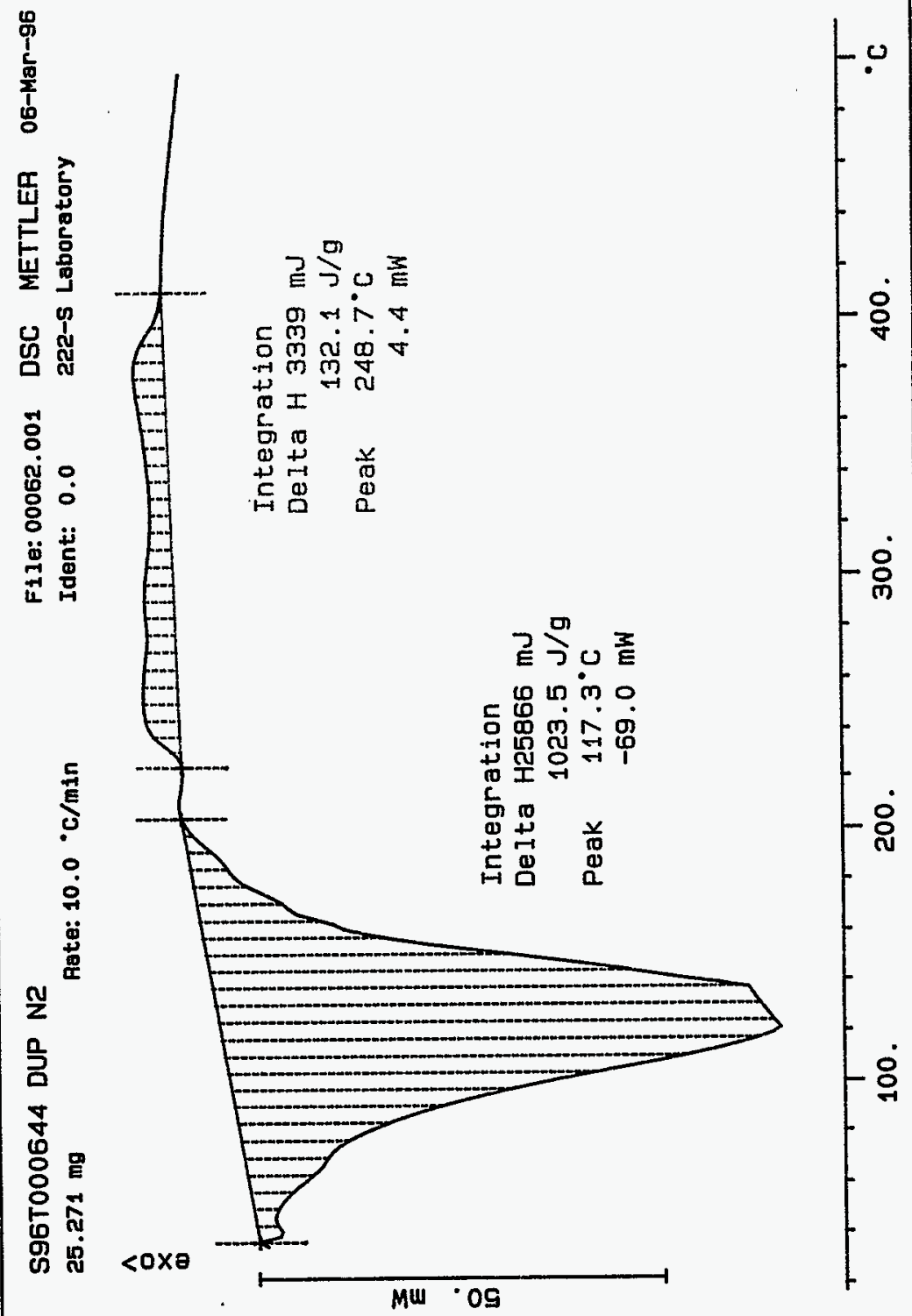




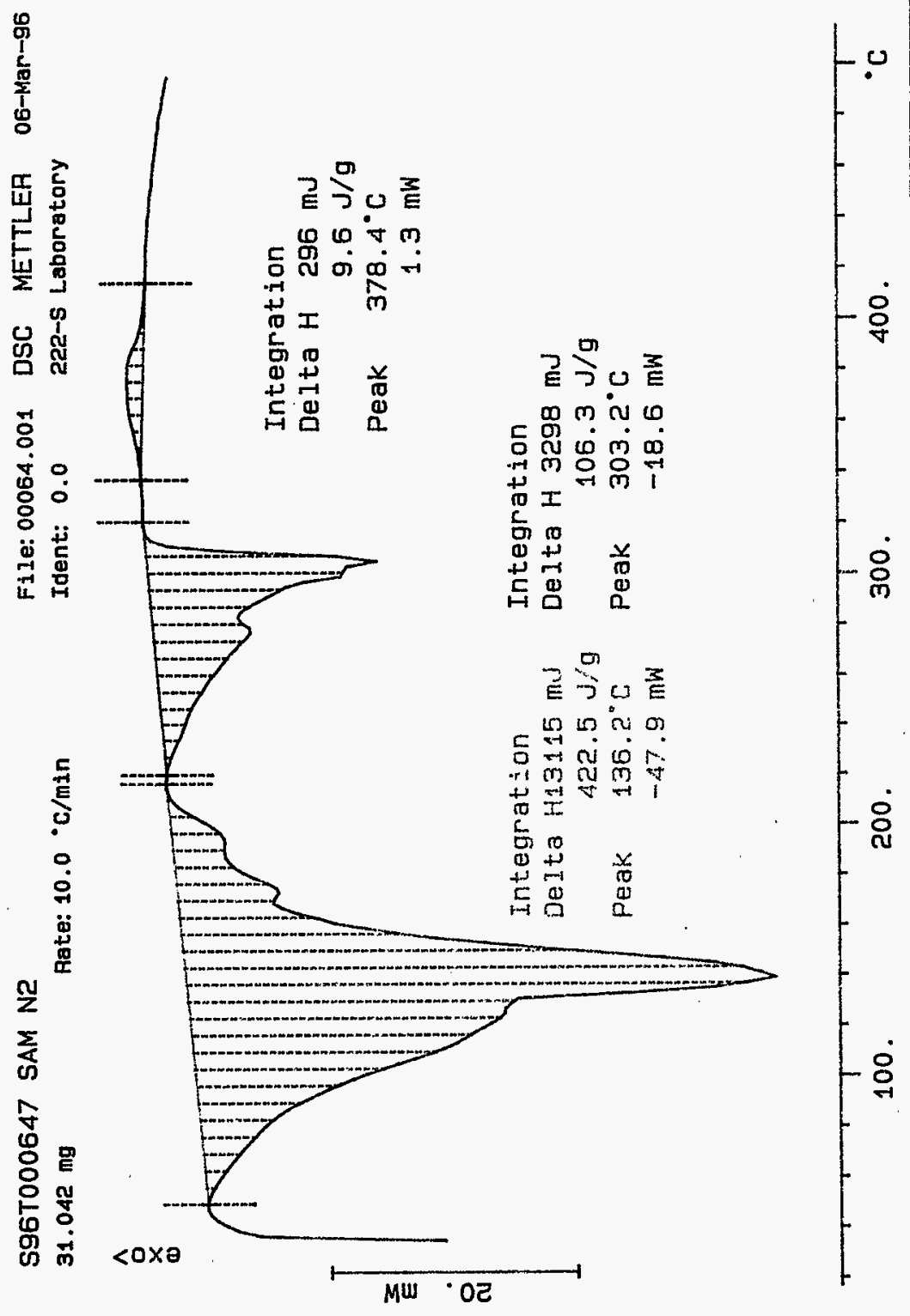


WHC-SD-WM-DP-184, REV. 0

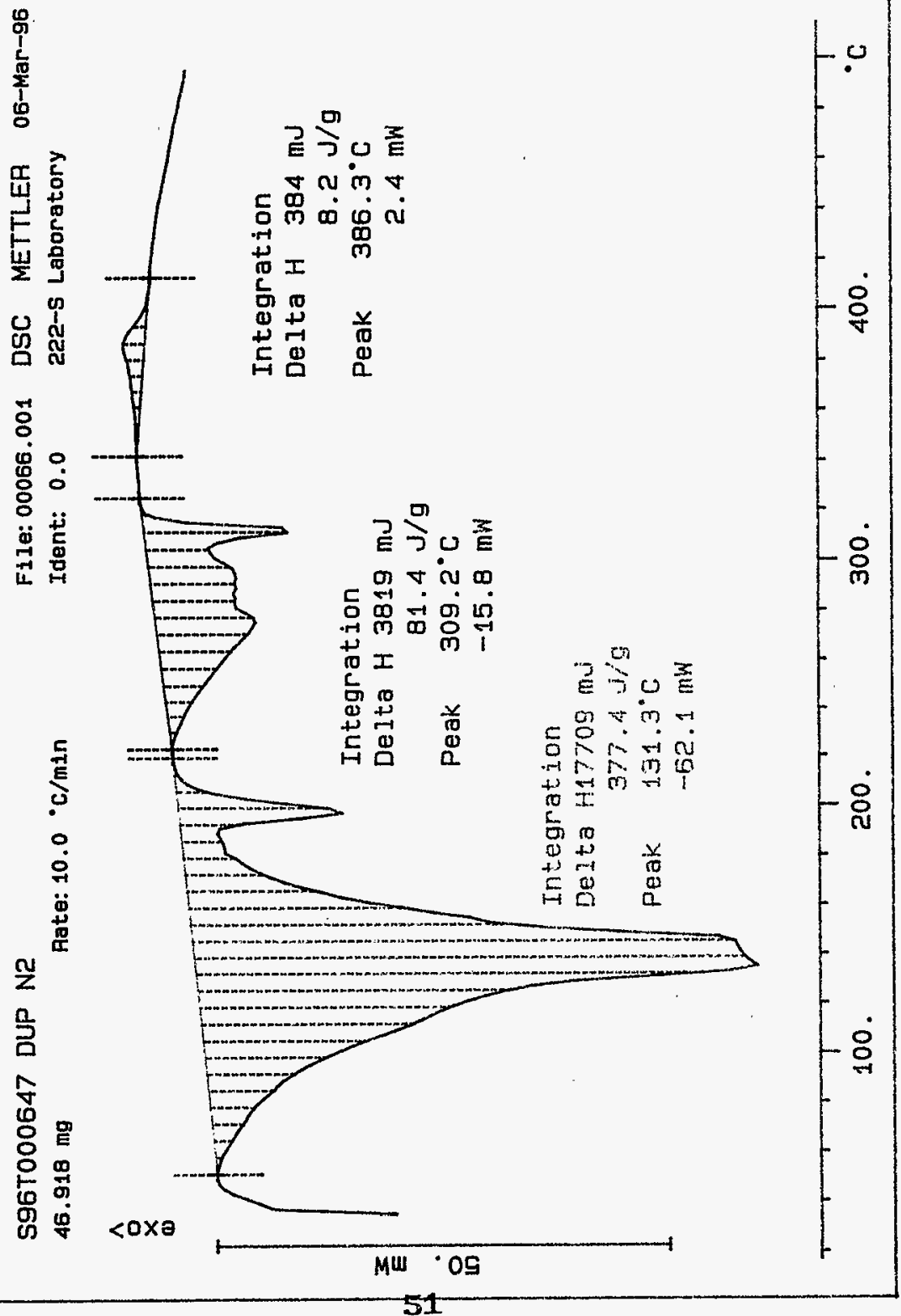


worklistrpt Version 2.1 05/15/95

WHC-SD-WM-DP-184, REV. 0

02/26/96 07:22

LABCORE Data Entry Template for Worklist\#

Page: I

5893

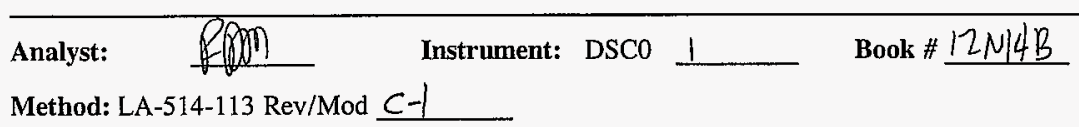

Worklist Comment: U-107 DSC RUN UNDER N2. RCJ

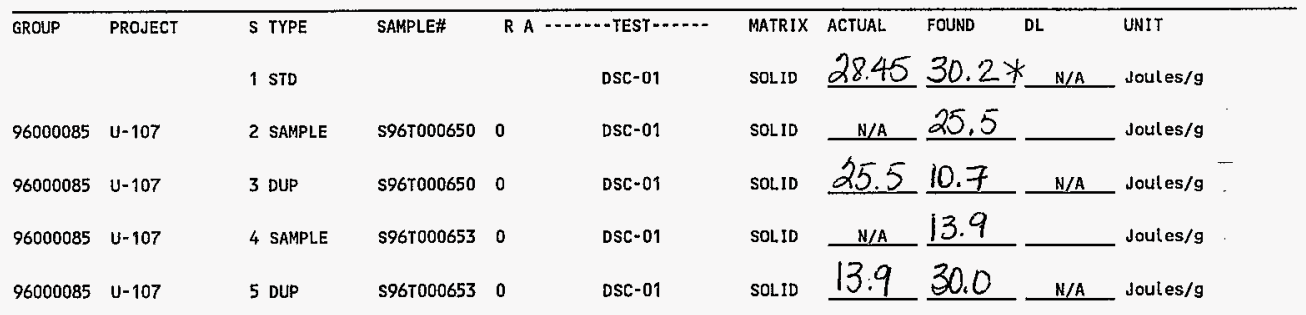

Final page for worklist \#

5893

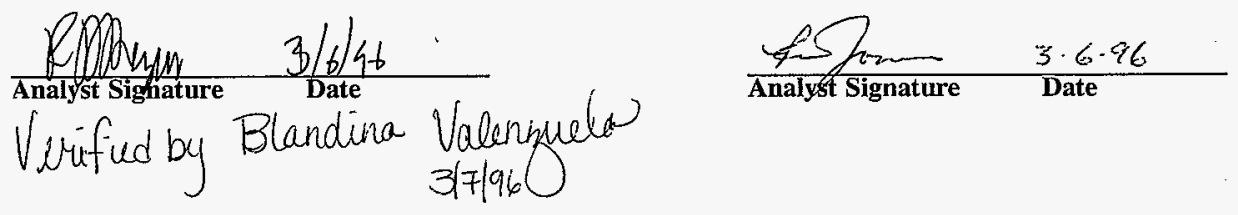

Data Entry Comments: S967000650 produced a large endotherm at $123.3^{\circ} \mathrm{C}$ with a delta 14 of $1074.5 \mathrm{~J} / \mathrm{g}$. $\frac{5967000653}{H}$ of $850.2 \mathrm{~J} / \mathrm{g}$. Units shown for $Q C$ (S FK \& STD) may not reflect the actual units. $D L=$ Detection Limit, $S=$ Worklist Slot Number, $R=$ Replicate Number, $A=$ Aliquot Code.

52 
SIGNATURE BELOW REPRESENTS CHEMICAL TECHNOLOGIST/CHEMIST THAT

COMPLETED/VERIFIED THE CALIBRATION/ANALYSIS ON PAGES 53 TO 57.

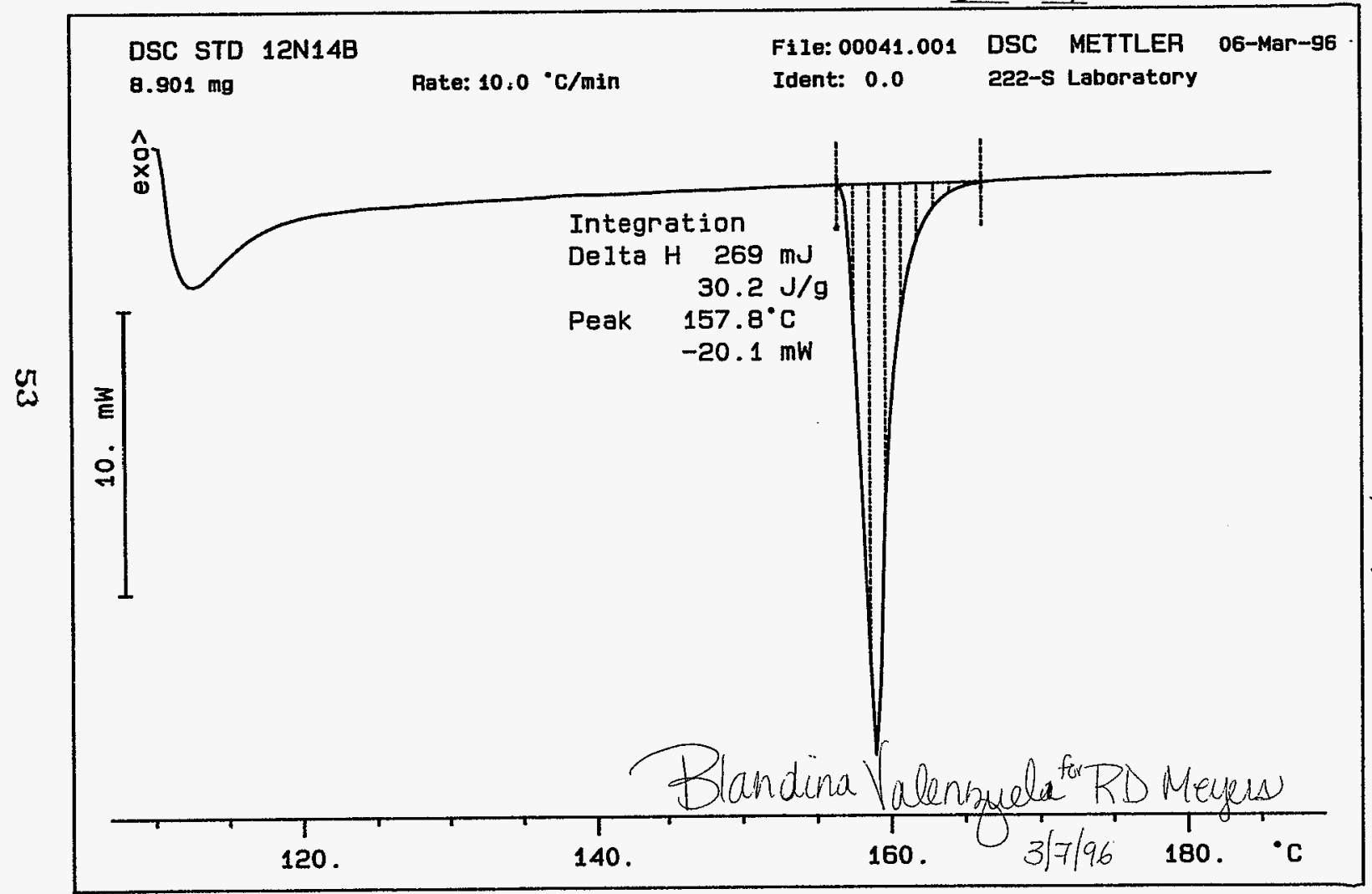


WHC-SD-WM-DP-184, REV. 0

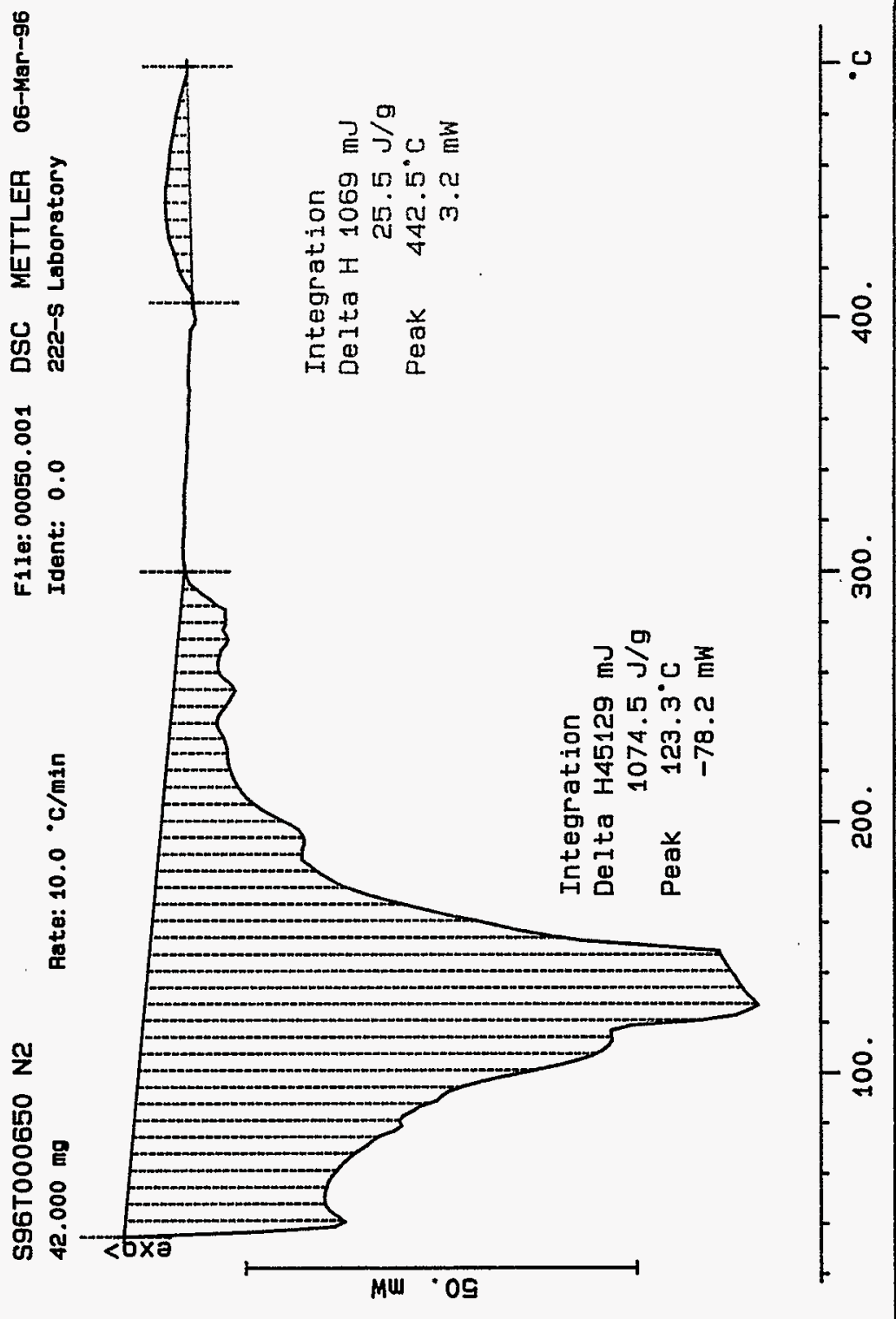


WHC-SD-WM-DP-184, REV 0

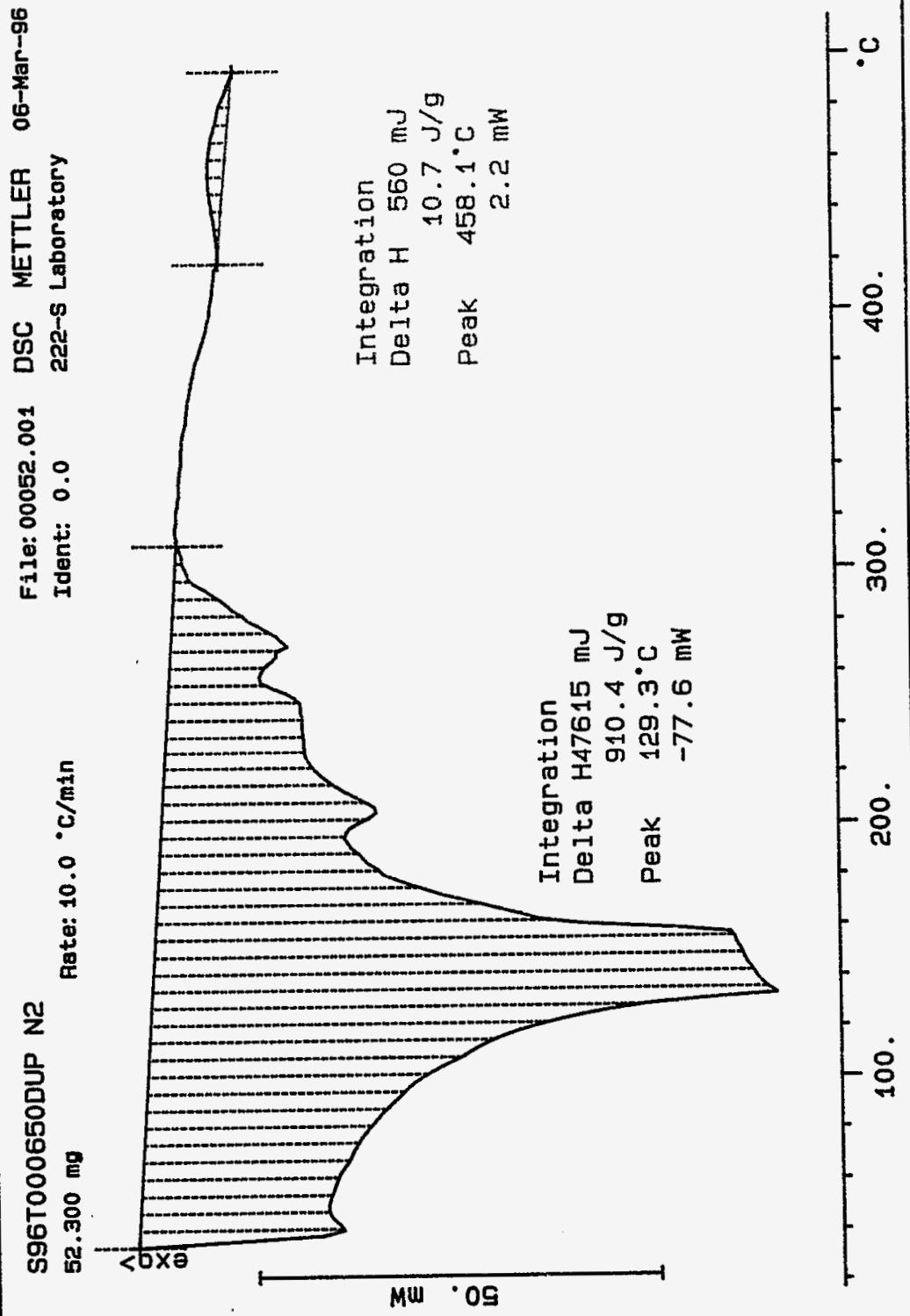


0
1
$\frac{1}{6}$
$\frac{1}{2}$
1
0
0

学

0 d d

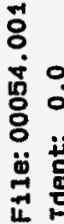

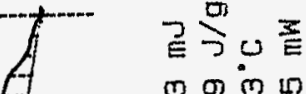

ᄃ

0 m $\mathrm{N} m$

$\rightarrow+1$ स

I

5

a +

$+\rightarrow$

$\pm 0$

$\frac{v}{\sigma o}$

$\rightarrow 0$



i

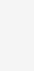

4

巳

I.

品

㟧

造蜔

号 
WHC-SD-WM-DP-184, REV. 0
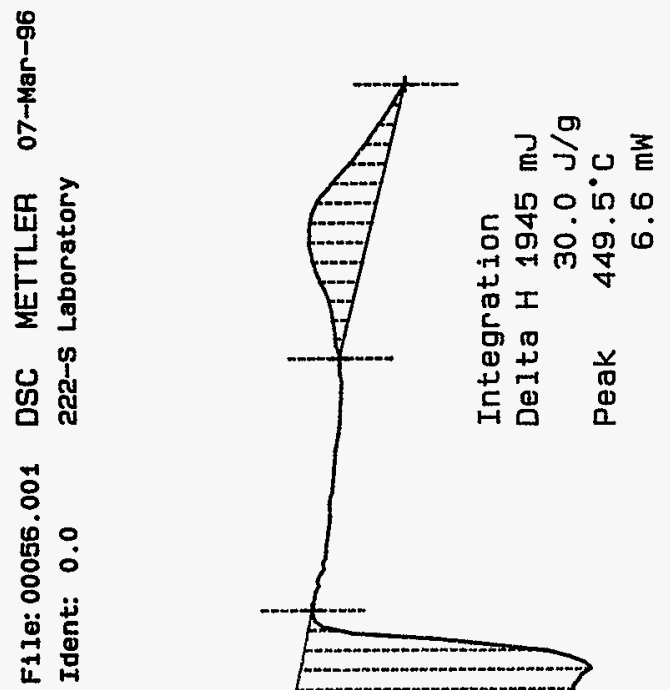

$\omega I$

ㄷ)

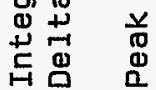

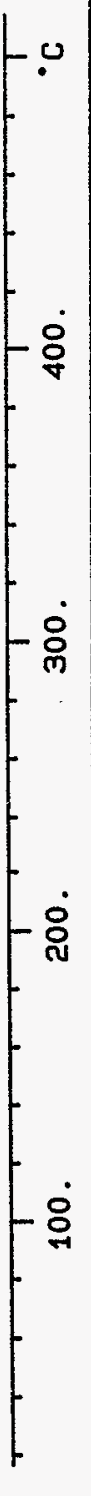


worklistrpt Version $2.105 / 15 / 95$

WHC-SD-WM-DP-184, REV. 0

Page: 1

02/29/96 14:17

LABCORE Data Entry Template for Worklist\#

6035

Analyst: SSMF Instrument: DSC0 I Book 12N14B

Method: LA-514-113 Rev/Mod C-1

Worklist Comment: U-107 DSC-01 RUN UNDER N2. RCJ

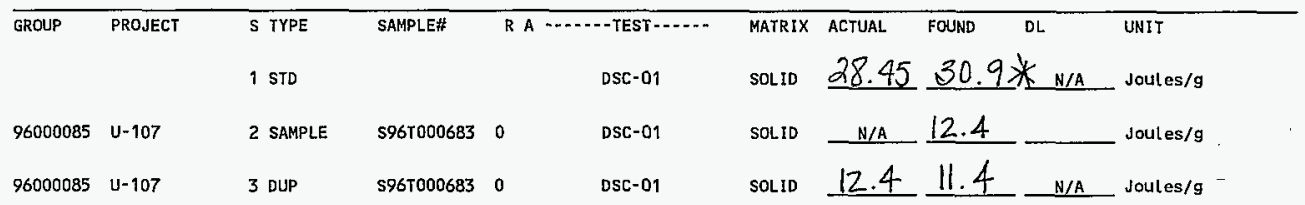

Final page for worklist \# 6035

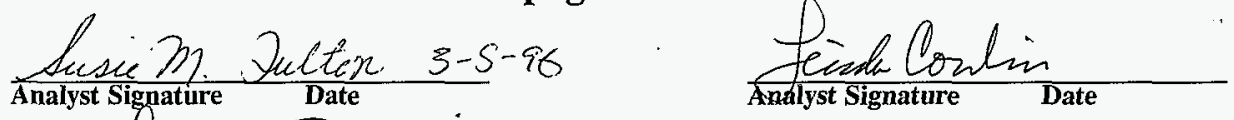

Veritua by Blandina Valenzuela 317196

Data Entry Comments: Sample produced two endotherm, one at $136.3^{\circ} \mathrm{C}$ with a delta $\mathrm{H}$ of $736.7 \mathrm{~J} / \mathrm{g}$ and second at $289.3^{\circ} \mathrm{C}$ with a delta $\mathrm{H}$ of $103.85 / g$

Units shown for QC (SPK \& STD) may not reflect the actual units. DL = Detection Limit, $S=$ Worklist Slot Number, $R=$ Replicate Number, $A=$ Aliquot Code.

58 
SIGNATURE BELOH REPRESENTS CHENICAL TECHNOLOGIST/CHEMIST THAT

COMPLETED/VERIFIED THE CALIBRATION/ANALYSIS ON PAGES 59 TO $6 /$.

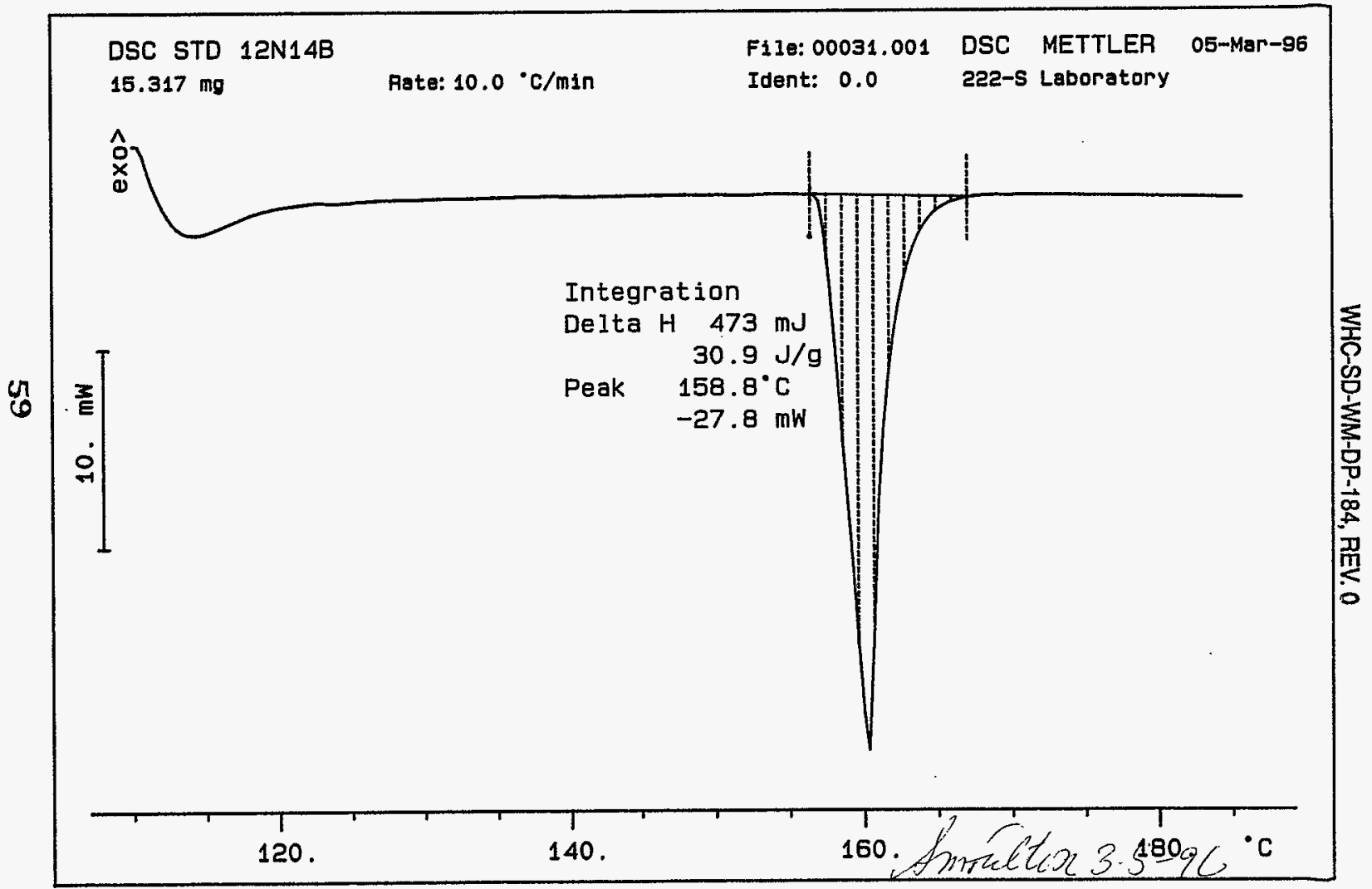


WHC-SD-WM-DP-184, REV. 0

9
0
1
5
$\frac{9}{2}$
$\vdots$
6

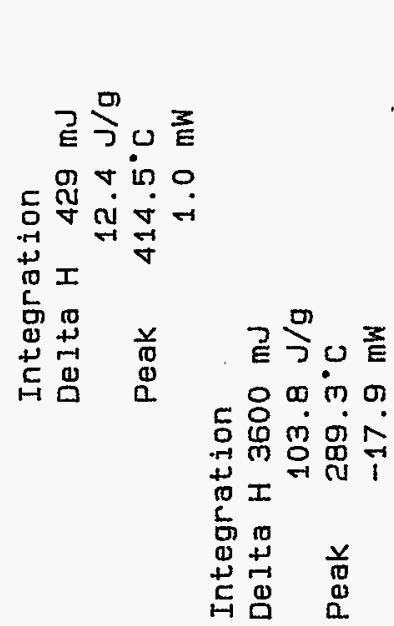

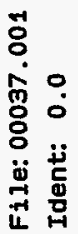

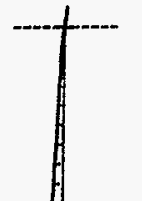

ำ

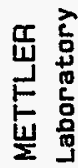

फ N

c)

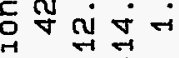

$+\nabla$

I I

吕苏

ह

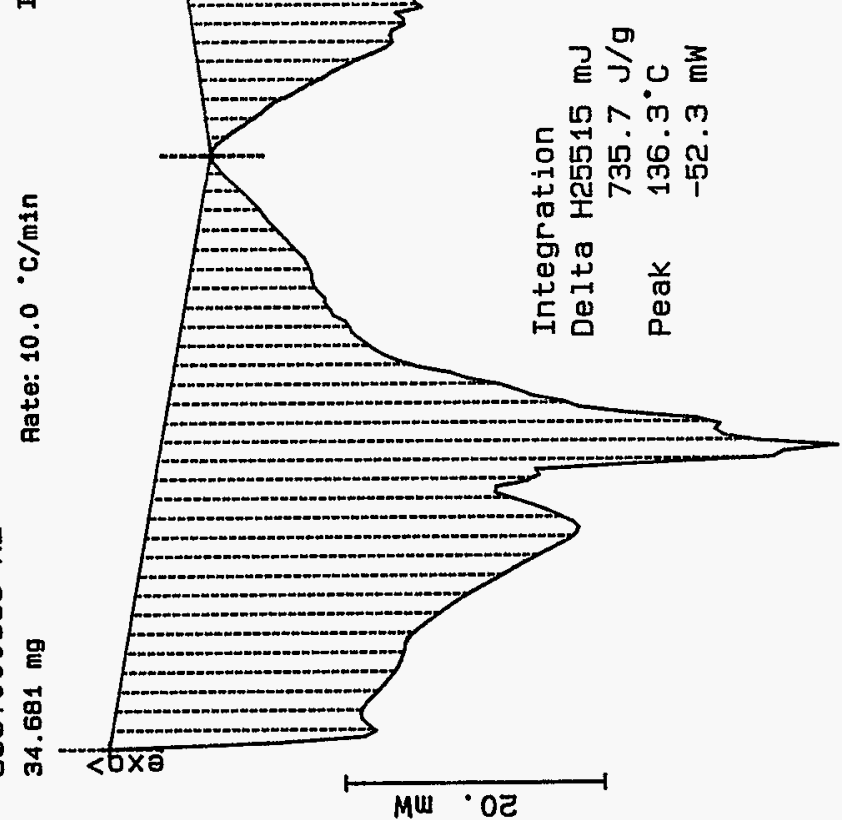

业 $\stackrel{2}{\rightarrow}$

10 I

占。

d

$+7$

둉

웅 
0
0
1
0
000
$\vdots$
$\vdots$
0

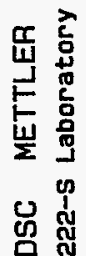

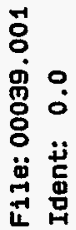
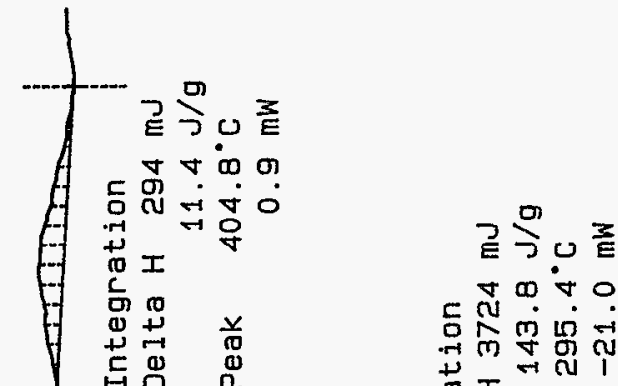

ष $\infty$ is 0

c N

$\rightarrow$ m响

म $\rightarrow$ त ।

ro

5

(2)

$\stackrel{4}{\rightarrow-1}$

ᄃ요

$\frac{1}{00}$
0
0
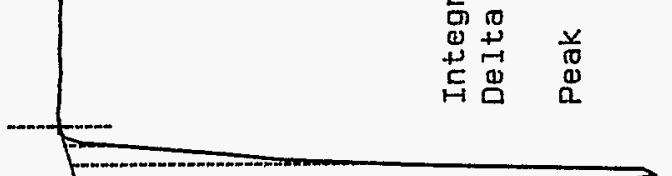

$-1$

r-manom

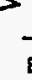

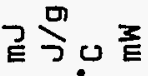

= ले था ?

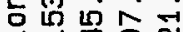

돌

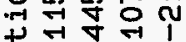

A.

$-1$

10

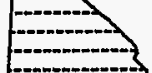

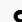

c.

吕

o

$+\rightarrow$

乭

$\frac{7}{10}$

0
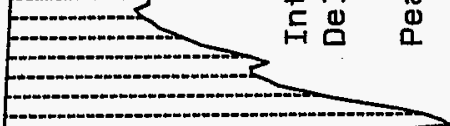

ญ
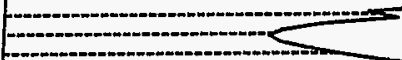

号

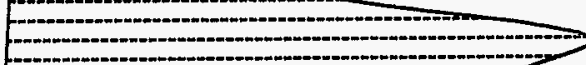

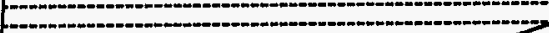

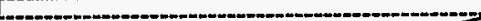

10

m
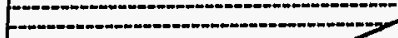

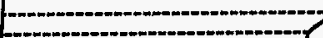

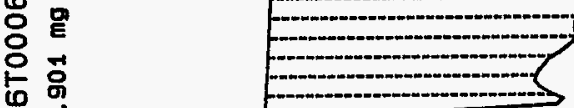

\begin{tabular}{lll}
8 & 0 \\
8 & 0 \\
\hline
\end{tabular}

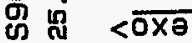

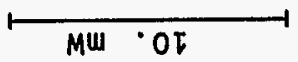


$04 / 18 / 96 \quad 10: 59 \quad 85093722929$

WESTINGHOUSE

W014

worklistrpt Version $2.105 / 15 / 95$

WHC-SD-WM-DP-184, REV. 0

04/16/96 15:46

LABCORE Data Entry Template for Worklist\#

Page: 1

6511

Analyst: SMF Instrument: DSC0 3 Book \#12N14B

Method: LA-514-114 Rev/Mod CC

Worklist Comment: U-107 DSC RUN UNDER N2. RCJ

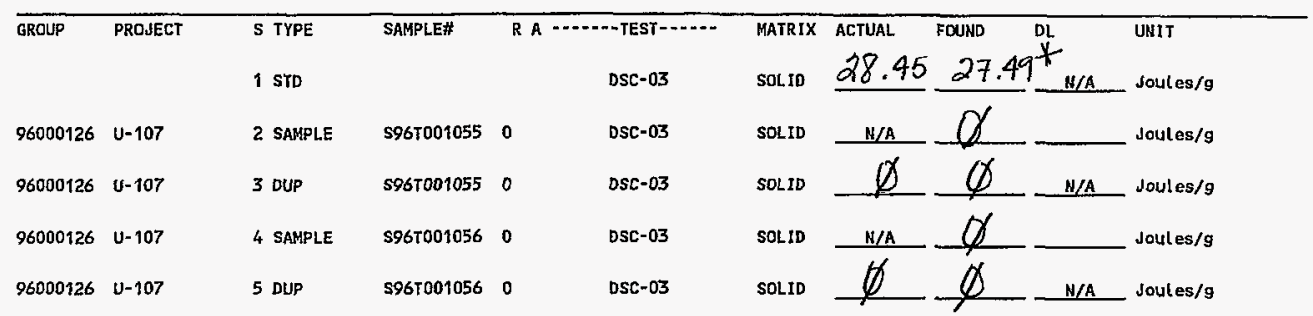

Final page for worklist \#

6511

See attached for signatures

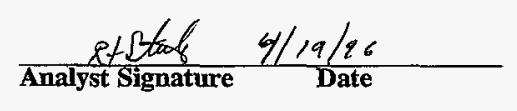

Verified br t

Jilanastos $4-22-96$

S96700 1055 produced two end therms, one at $109.3^{\circ} \mathrm{C}$ with a delta $H$ of $211.1 \mathrm{~J} / \mathrm{g}$ and second at $301.96^{\circ} \mathrm{C}$ with a delta $H$ of $178.75 \mathrm{Jg}$.

Data Entry Corners: $S 967001056$ produced two endotherm, one at $109.0^{\circ} \mathrm{C}$ with a delta $H$ of $296.4 \mathrm{Jg}$ and second at $290.76^{\circ} \mathrm{C}$ with a delft $\mathrm{H}$ of $91.1 \mathrm{~J} / \mathrm{g}$ Units shownifor $Q C$ (SPK \& STD) may not reflect the actual units. $D L=$ Detection Limit, $S=$ Worklist Slot Number,
$R=$ Replicate Number, $A=$ Aliquot Code.

62 
worklistrpt Version 2.1 05/15/95

WHC-SD-WM-DP-184, REV. 0

03/14/96 14:32

\section{LABCORE Data Entry Template for Worklist\#}

Analyst:

$S M F$

Instrument: DSCO

Book \#12N14B

Method: LA-514-113 Rev/Mod

Worklist Comment: U-107 DSC RUN UNDER N2, RCJ

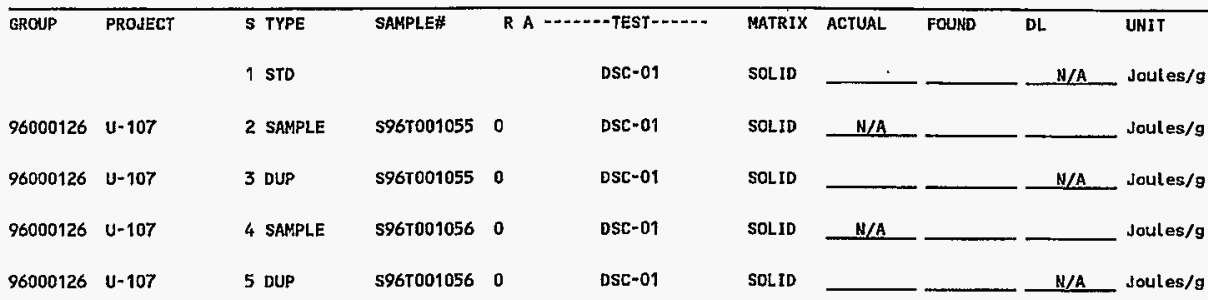

\section{Final page for worklist \# $\quad 6511$}

\section{Lusein. Buctorn $4 / 5-96$}

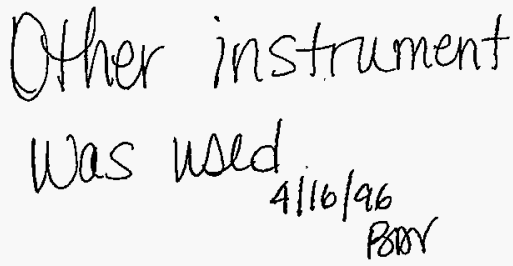

Data Entry Comments:

Units shown for QC (SPK \& STD) may not reflect the actual units. $D L=$ Detection Limit, $S=$ Worklist Slot Number, $R=$ Replicate Number, $A=$ Aliquot Code. 
Curve 1: DSC

File info: IND041501 Mon Apr 15 05:53:45 1996

Sanple Neight: 7.900 mg

12N14-B INDIUN AT 10CIMIN

SIGNATURE BELOW REPRESENTS CHEMICAL TECHNOLOGIST/CHEHIST THAT COHPLETED/VERIFIED THE CALIBRATION/ANALYSIS ON PAGES lOS TO $6 g$.

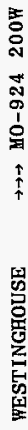

สิ

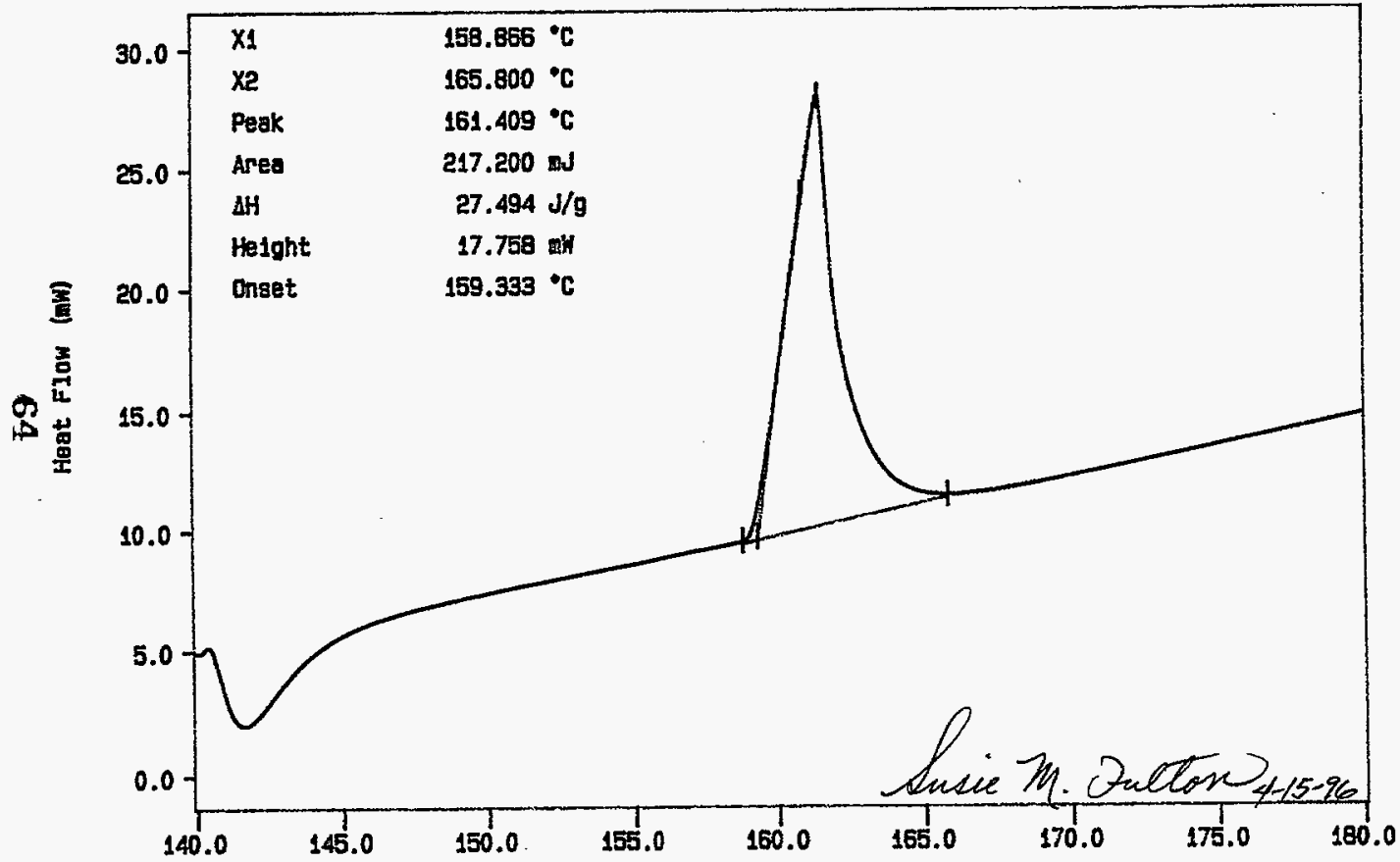

N2. EXOTHEAM DOHN

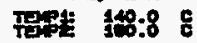

Temperature ( $\left.{ }^{\circ} \mathrm{C}\right)$

Sy FULTON

PERKIN-EIMEA

7 Series Thermal Analysis System

Mon Apr 15 05: 54: 43 1996 
Ftle info: SAM041504 Non Apr 15 07:30:07 1995

Sample Mejght: $26.740 \mathrm{mg}$

S96T001055

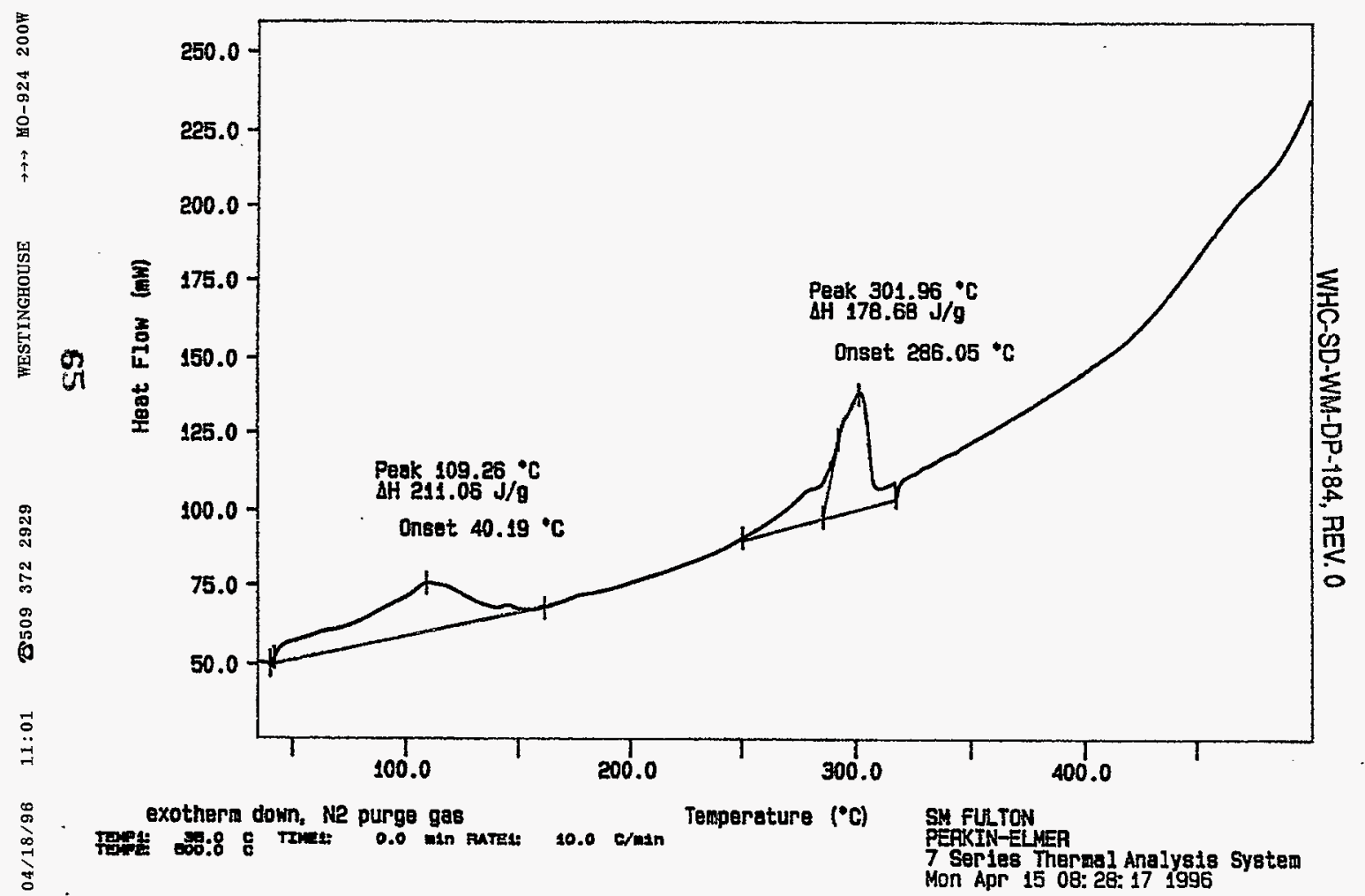


F1le info: SAM041505 Kon Apr 15 09: 23: 211996

Sample Height: 16.110

S96T001055 DUP

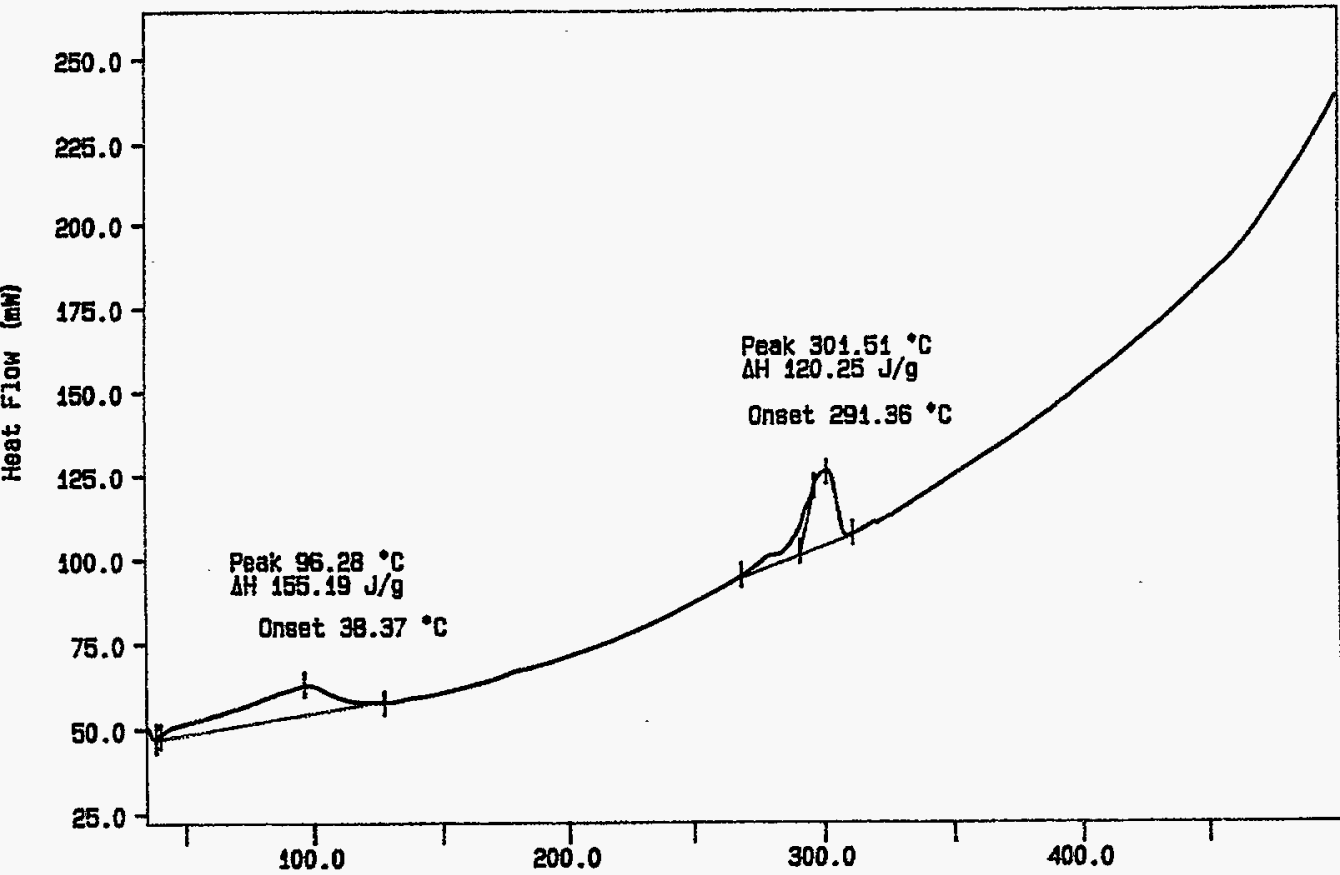


Curve 1: DSC

File info: SAM041506 Non Apr 15 13: 19:28 1995

Sample Weight: 16.800

ang

S96T001056

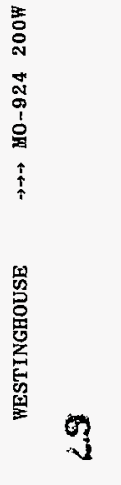

엉

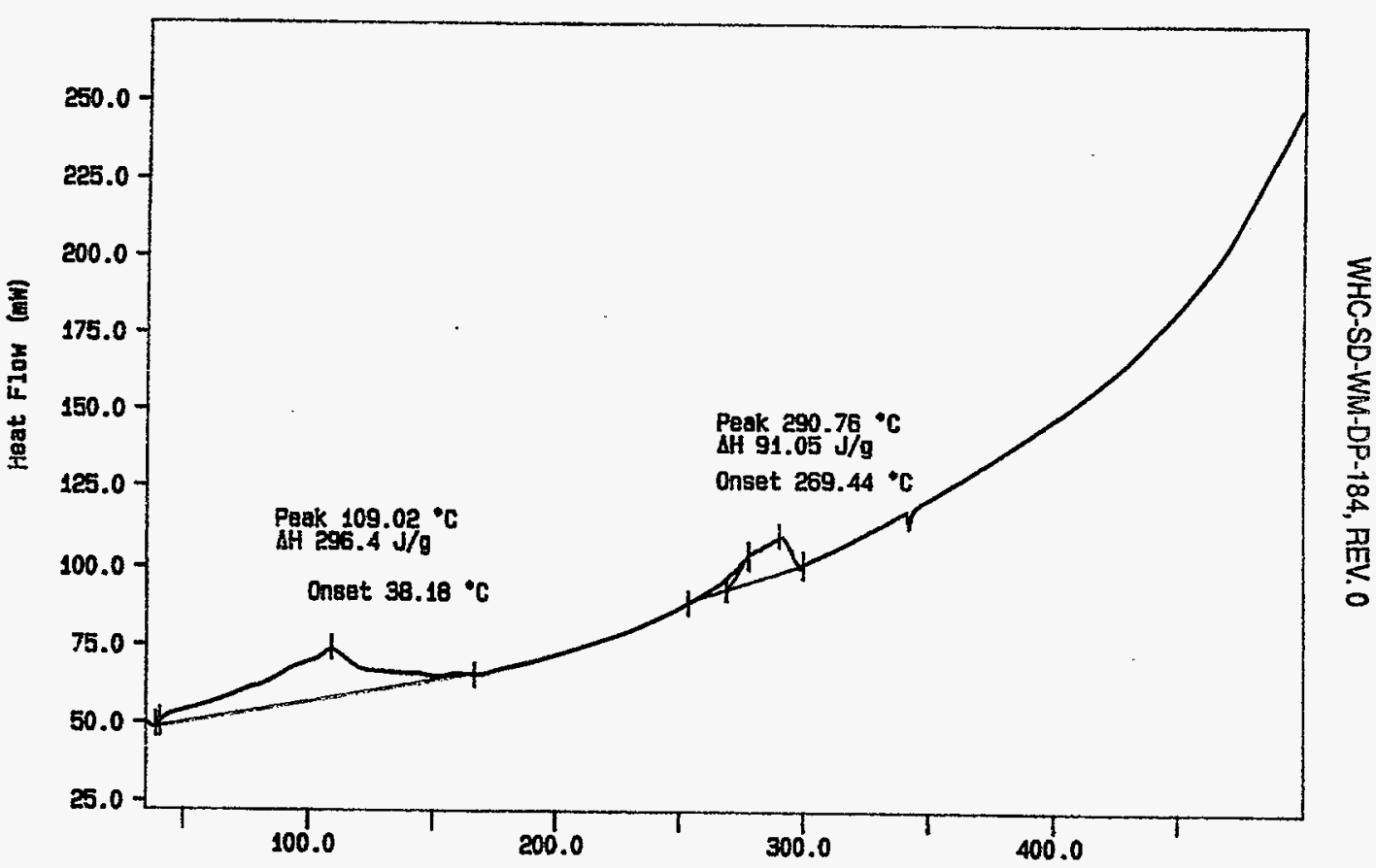

exotherm down, N2 purge gas

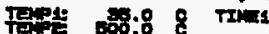

0.0 atin matels

10.0 orman

Temperature $\left({ }^{\circ} \mathrm{C}\right)$

SN FULTON

PERKIN-EIVEA

7 Sertes Therwel Anslysis Syste

Mon Apr 15 13: 29: 261996 


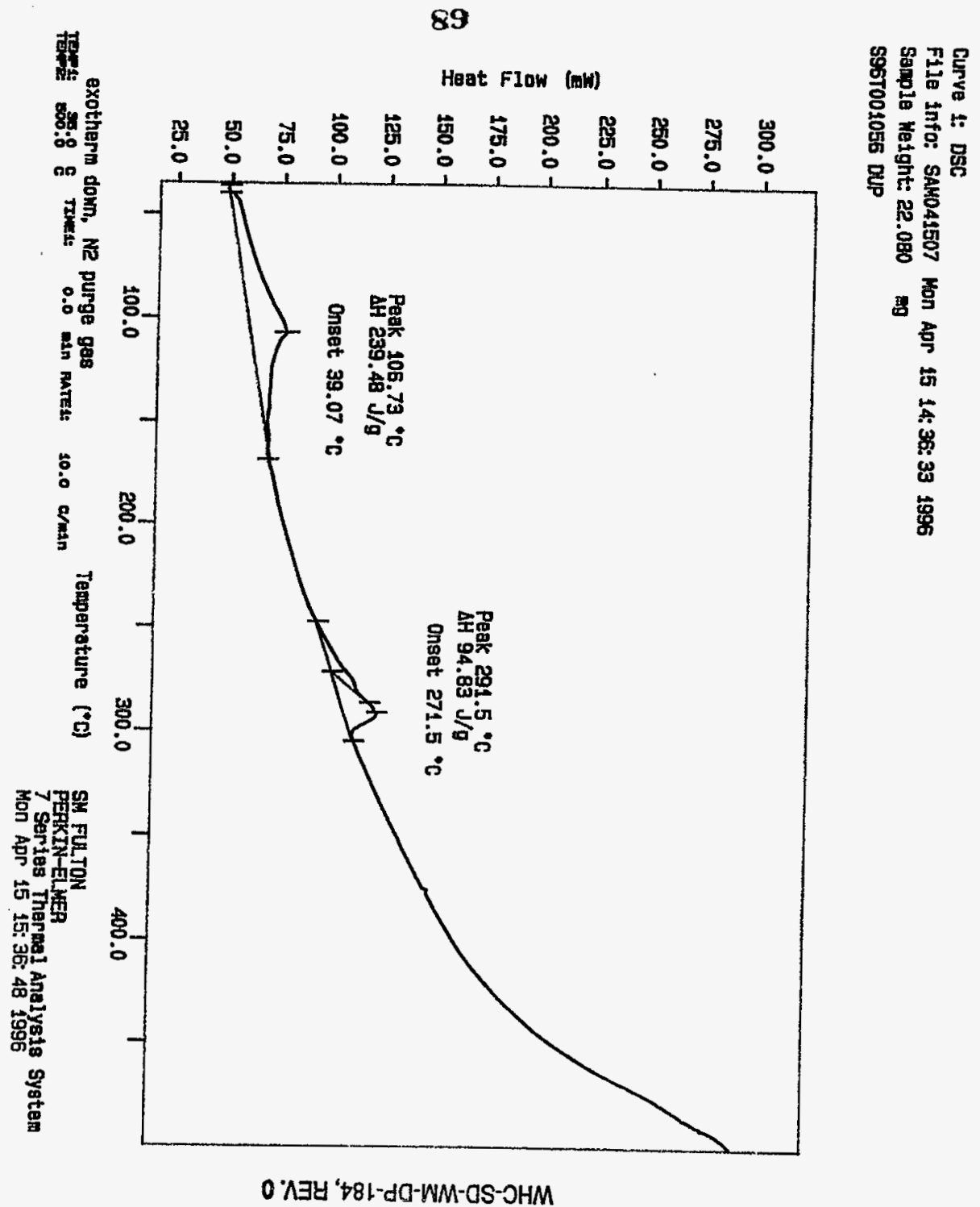


$04 / 17 / 96 \quad 14: 08 \quad B 5093722929 \quad$ WESTINGHOUSE $\ldots \rightarrow \rightarrow \rightarrow 0-924200 \mathrm{~W}$

worklistrpt Version 2.1 05/15/95 WHC-SD-WM-DP-184, REV. 0 .
04/177/96 11:51

LABCORE Data Entry Template for Worklist\#
Page: 1

6512

Analyst: JDS Instrument: DSCO I Book \# 12N14B

Method: LA -514-113 Rev/Mod Cl

Worklist Comment: U-107 DSC RUN UNDER N2. RCJ

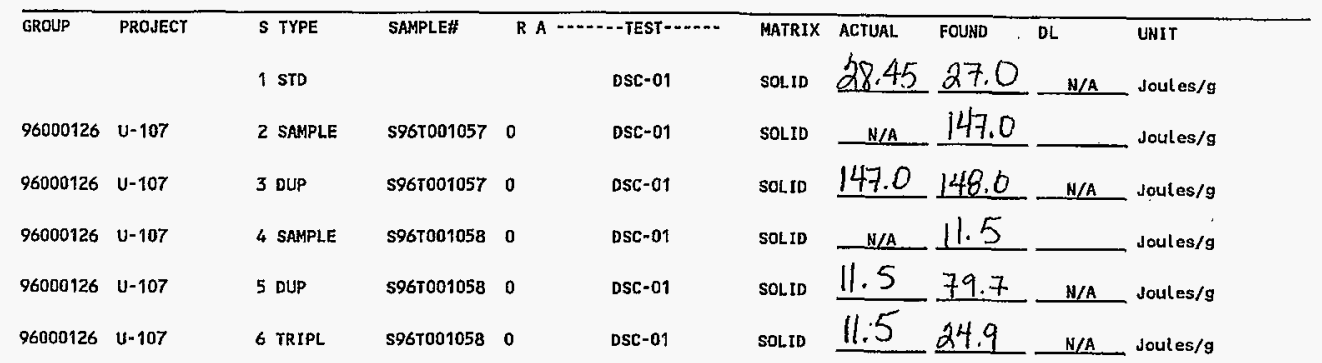

Final page for worklist \#

See attached for signatures

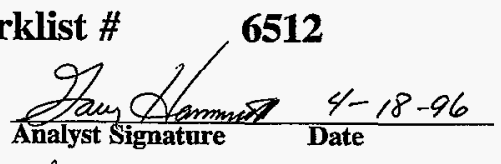

Verified by Blandina Valenzuela

Data Entry Comments: 5967001057 produced an endotherm at $114.5^{\circ} \mathrm{C}$ with a delta $\mathrm{H}$ of

$994.1 \mathrm{~J} / 9$ S96roviess produced two endotherm, one at $97.3^{\circ} \mathrm{C}$ with a delta $1 \mathrm{H}$ of $972.3 \mathrm{~J} / \mathrm{g}$ and Units shown for $Q C$ (SPK \& STD) may not reflect the actual units. $D L=$ Detection Limit, $S=$ Worklist Slot Number,

$R=$ Replicate Number, $A=$ Aliquot Code.
The triplicate Was run because the thermograms did not look alike. She dup and trip did not have two encl therms.

69 


\section{LABCORE Data Entry Template for Worklist\#}

Analyst: Jds Instrument: DSCO Book \# 12N140

Method: LA-514-113 Rev/Mod

Worklist Comment: U-107 DSC RUN UNDER N2. RCJ

\begin{tabular}{|c|c|c|c|c|c|c|c|c|c|c|}
\hline \multirow[t]{2}{*}{ GROUP } & \multirow[t]{2}{*}{ PROJECT } & S TYPE & \multirow[t]{2}{*}{ SAMPLE\# } & \multicolumn{2}{|c|}{ R A $\cdots \cdots$ TEST $\cdots$} & \multirow{2}{*}{$\begin{array}{l}\text { MATRIX } \\
\text { SOLID }\end{array}$} & \multirow[t]{2}{*}{ ACTUAL } & \multirow[t]{2}{*}{ FOUHD } & \multirow{2}{*}{$\begin{array}{l}\mathrm{DL} \\
\mathrm{N} / \mathrm{A} \\
\end{array}$} & \multirow{2}{*}{$\begin{array}{l}\text { INIT } \\
\text { toul es/g }\end{array}$} \\
\hline & & 1 STD & & & DSC-01 & & & & & \\
\hline 96000126 & U-107 & 3 DUP & S96T001057 & 0 & DSC-01 & SOLID & & & NIS & Joules/g \\
\hline 96000126 & $u-107$ & 5 DUP & s96T001058 & 0 & DSC-01 & SoLia & & & $\mathrm{N} / \mathrm{A}$ & Joules $/ 9$ \\
\hline
\end{tabular}

Final page for worklist \#

6512

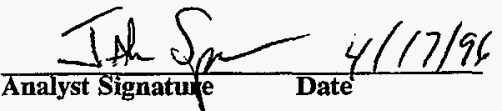

Analyst Signature Date

Data Entry Comments:

Ras

a Trip a

$596 T 601058$

Units shown for $Q C$ (SPK \& STD) moy not reflect the actual units. $D L=$ Detection Limit, $S=$ Worklist Slot Number, $R=$ Replicate Number, $A=$ Aliquot Code. 
SIGNATURE BELOW REPRESENTS CHEMICAL TECHNOLOGIST/CHEMIST THAT

CONPLETED/VERIFIED THE CALIBRATION/ANALYSIS ON PAGES $7 /$ TO 76 .

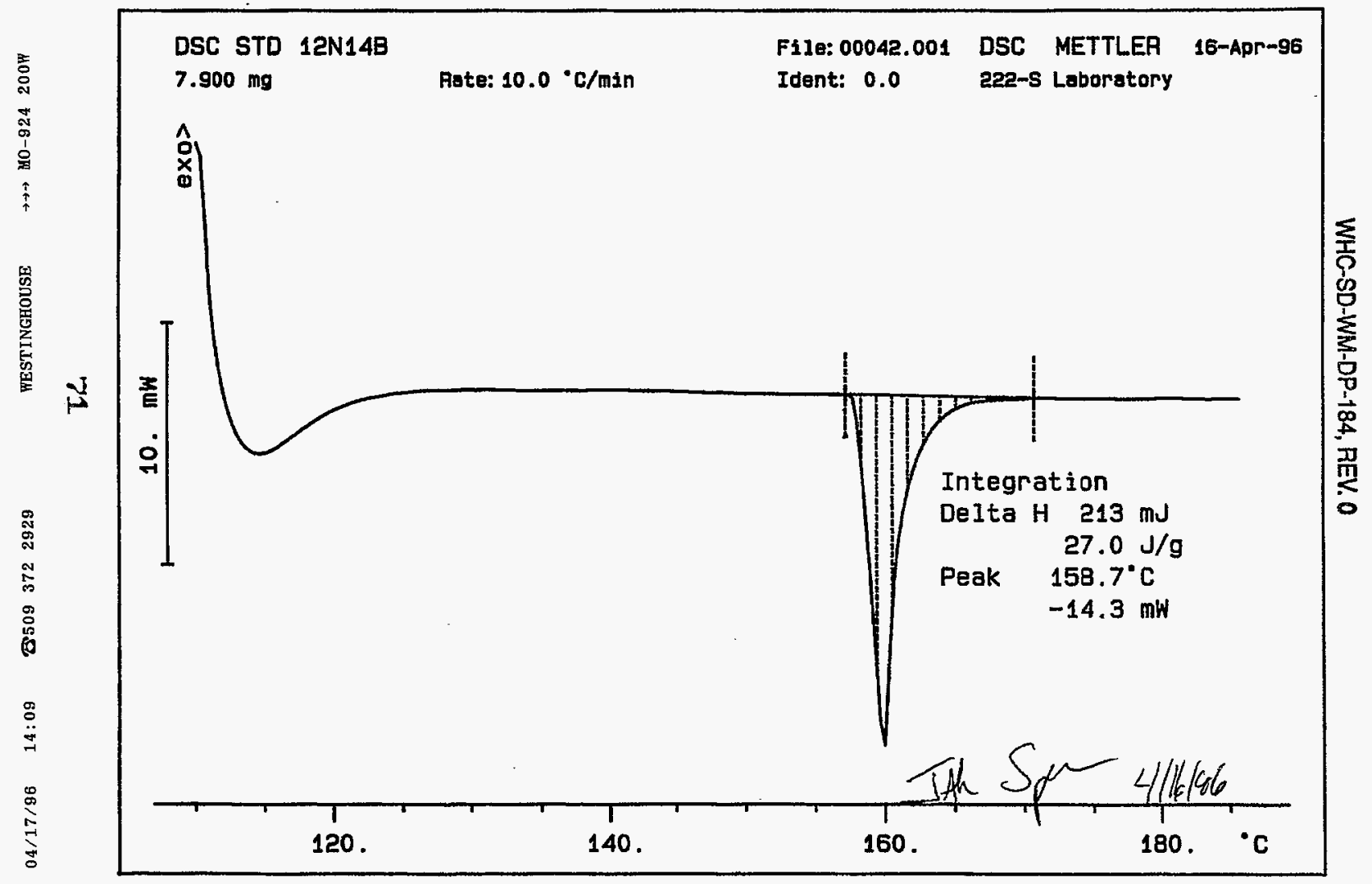




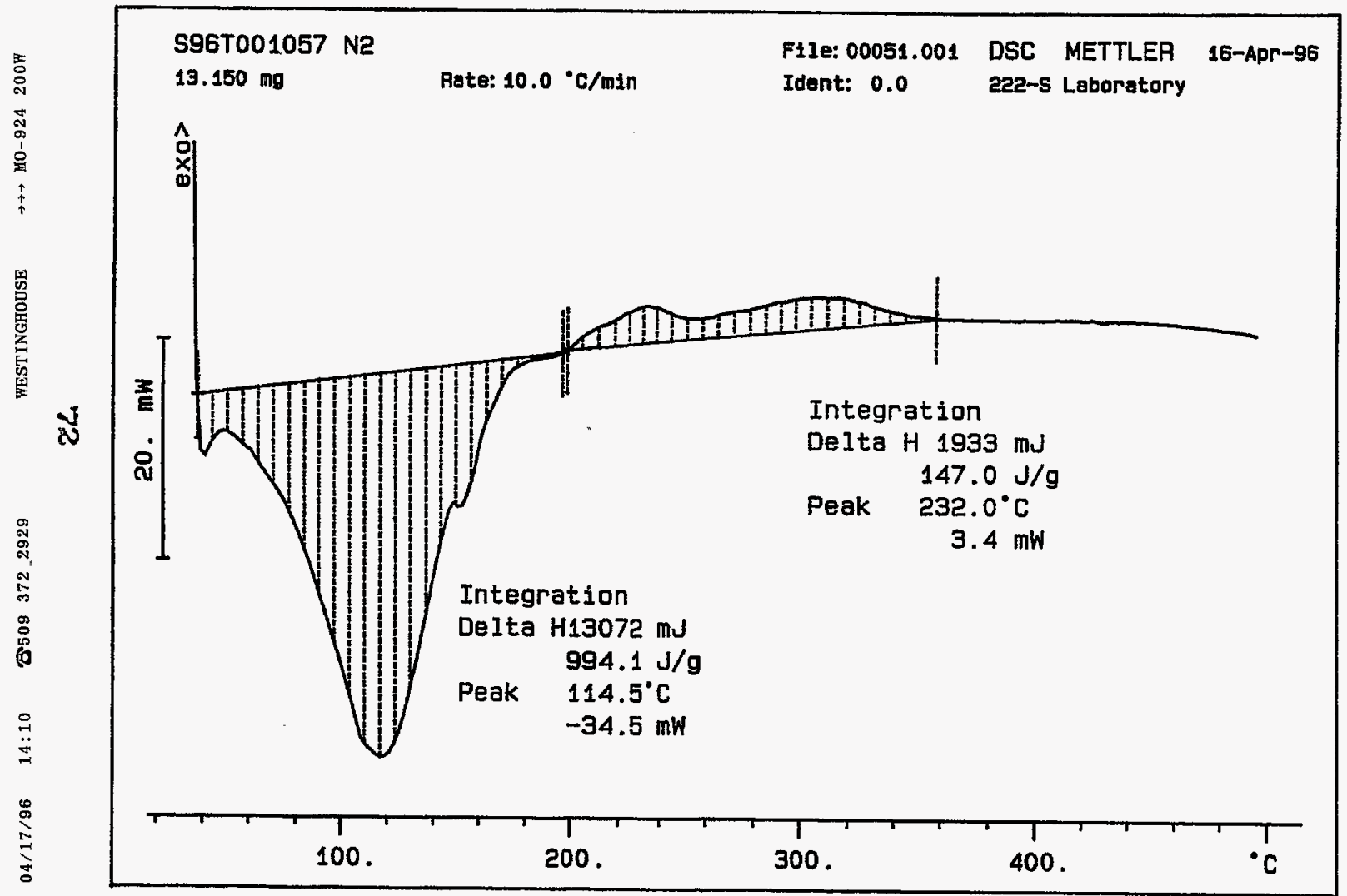




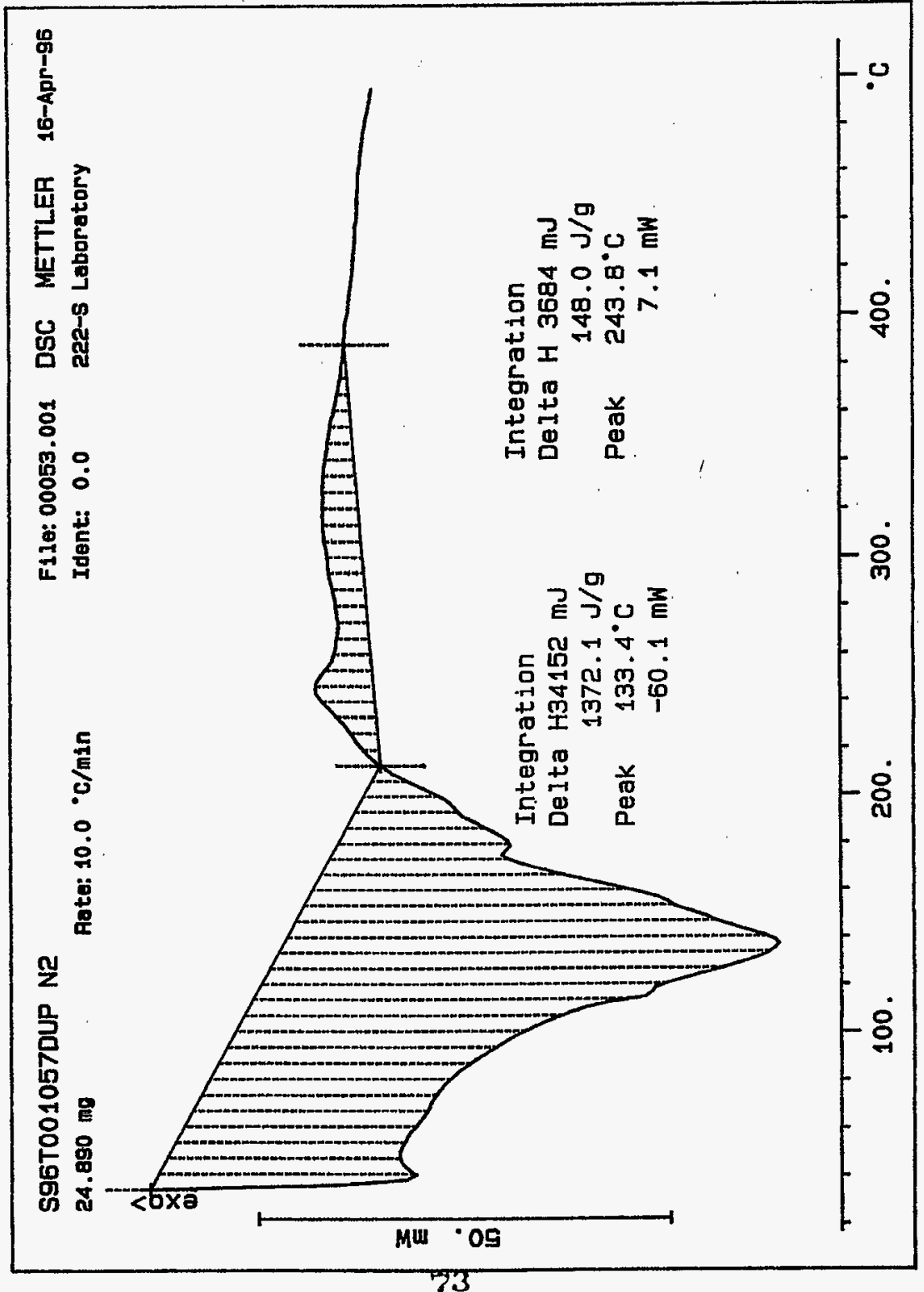


WHC-SD-WM-DP-184, REV. 0

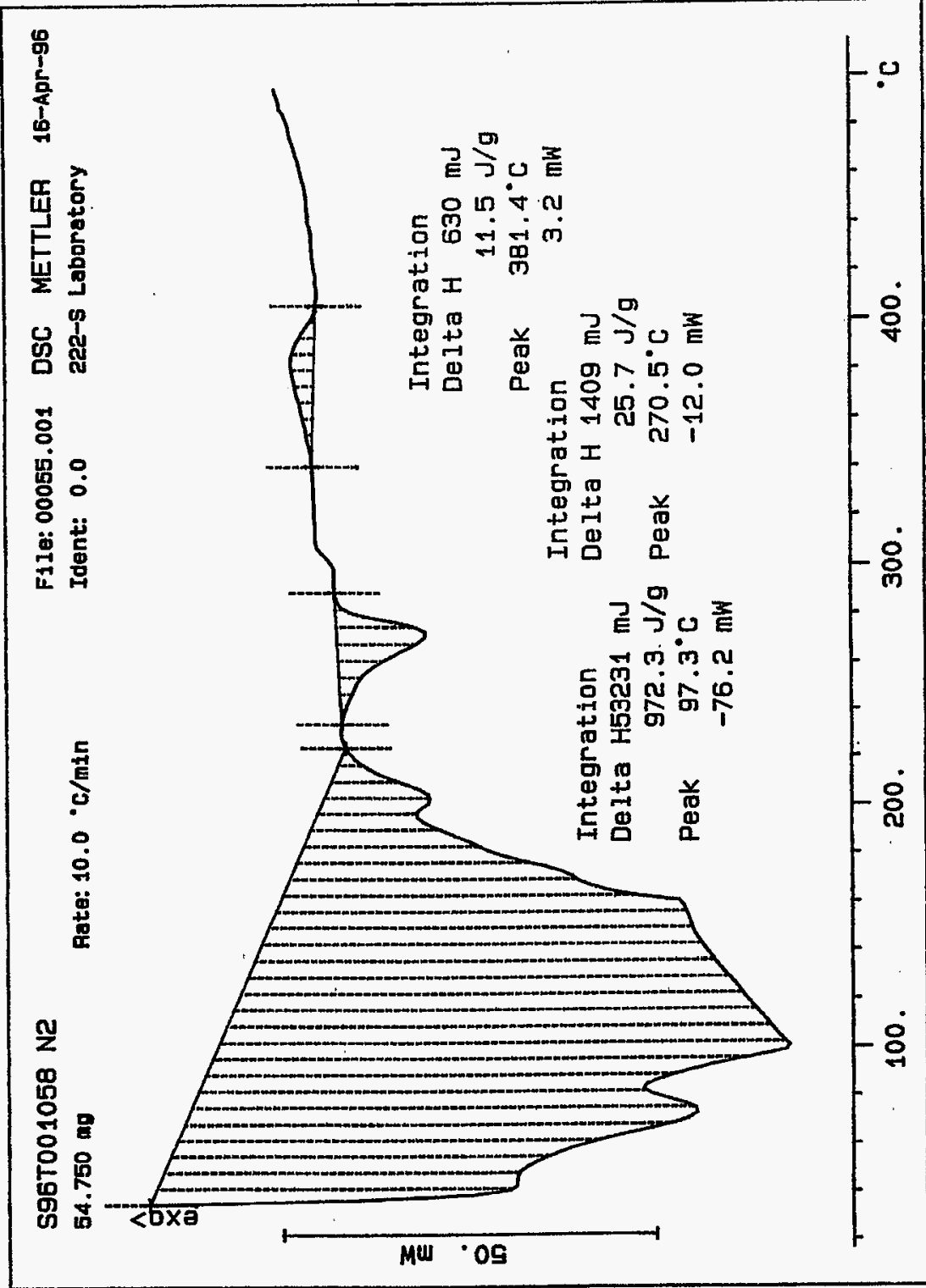


WHC-SD-WM-DP-184, REV. 0

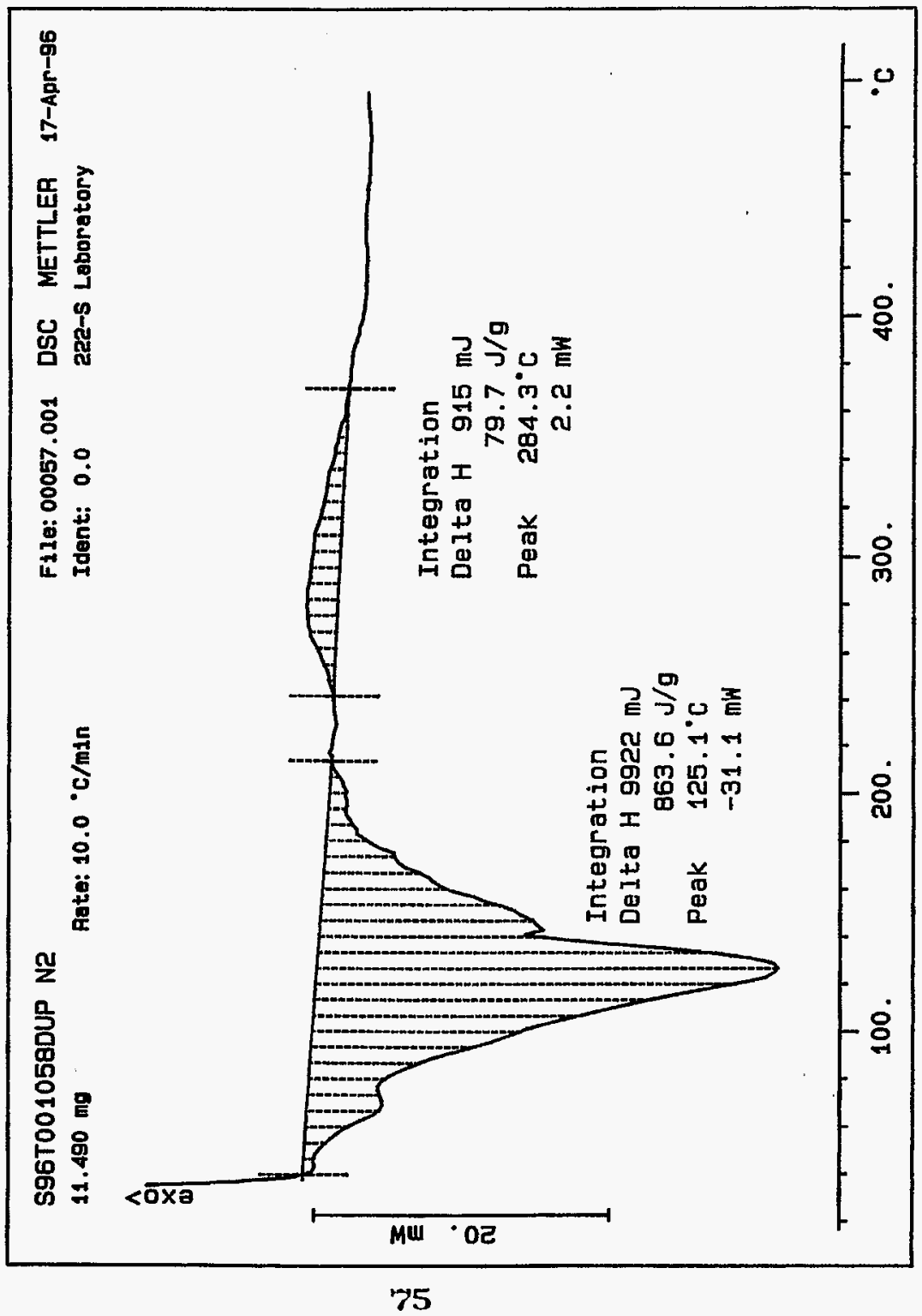


WHC-SD-WM-DP-184, REV. 0

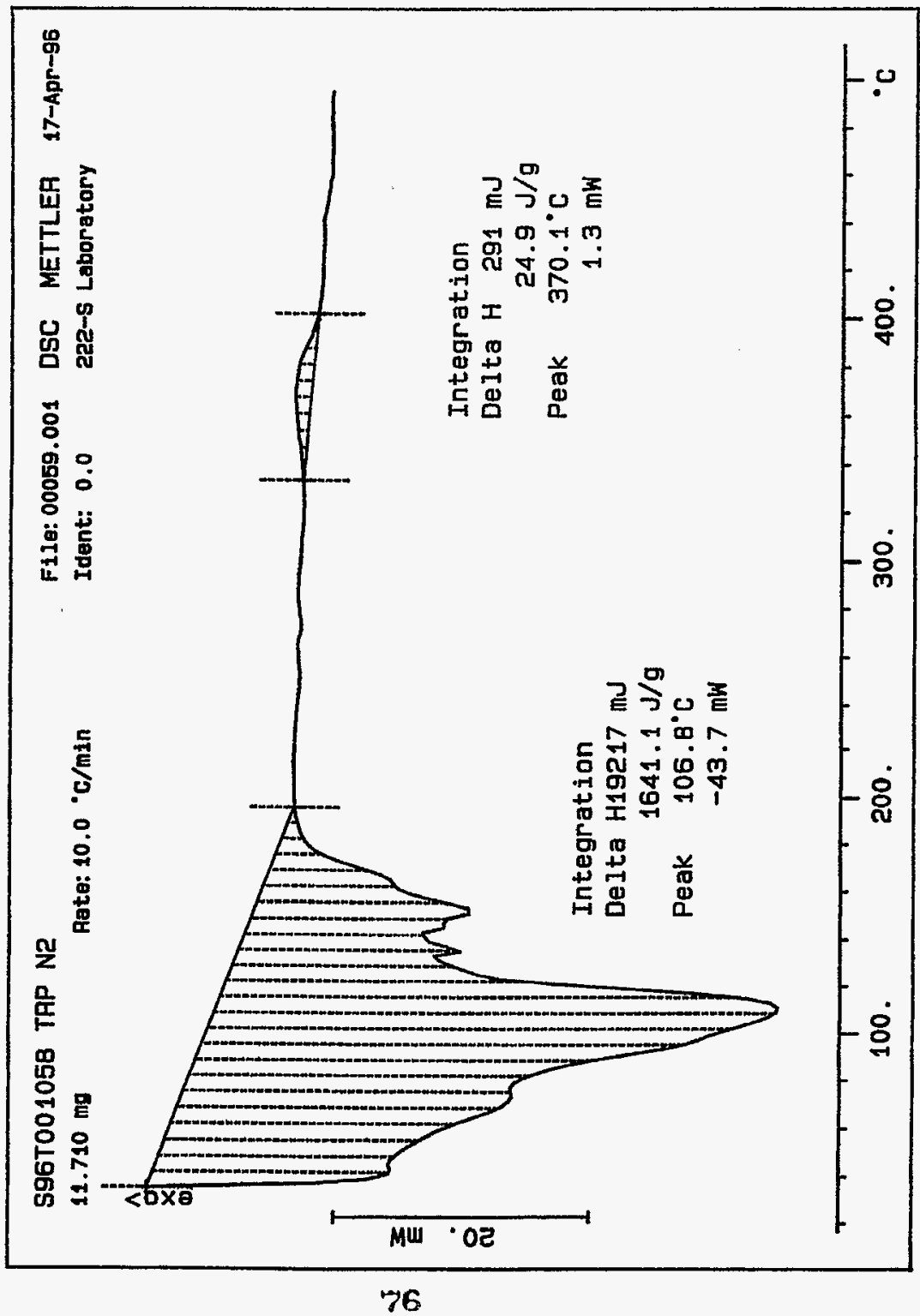




\section{LABCORE Data Entry Template for Worklist\#}

\section{Analyst: SMF Instrument: DSC0 I Book \# L IR: $14 B$}

Method: LA-514-113 Rev/Mod C -

Worklist Comment: U-107 DSC RUN UNDER N2. RCJ

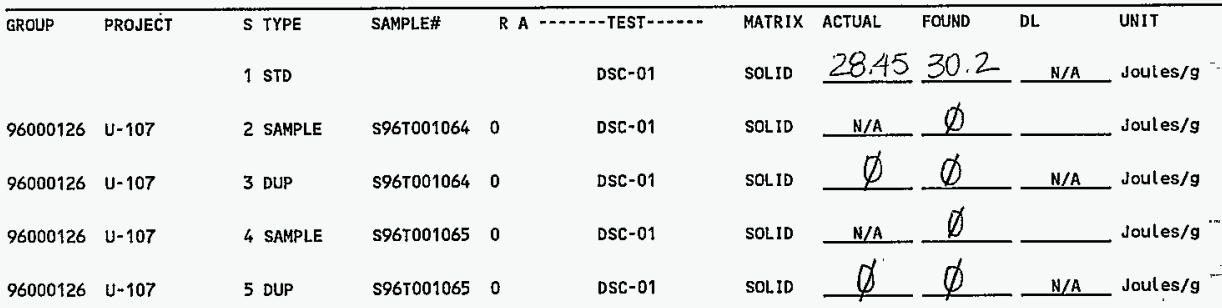

\section{Final page for worklist \# 6515}

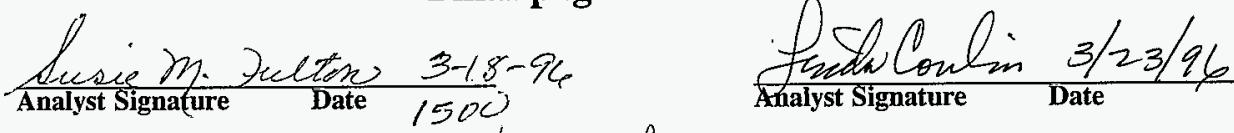

5967001064 produced a laige endotherm at $139.3^{\circ} \mathrm{C}$ with a delta $H$ witts 3 indar of $768.0 \mathrm{Jg}$.

Data Entry Comments: S96T001065 produced two endotherms one at $111.3^{\circ} \mathrm{C}$ with a delta $\mathrm{H}$ of $894.4 \mathrm{~J} / \mathrm{g}$ and second at $262.3 \mathrm{~K}^{\circ} \mathrm{C}$ with a delta Hor $41.3 \mathrm{~J} / \mathrm{g}$.

Units shown for QC (SPK \& STD) may not reflect the actual units. $D L=$ Detection Limit, $S=$ Worklist Slot Number, $R=$ Replicate Number, $A=$ Aliquot Code. 
SIGNATURE BELOH REPRESENTS CHEMICAL TECHNOLOGIST/CHEMIST THAT

COMPLETED/VERIFIED THE CALIBRATION/ANALYSIS ON PAGES $7 \&$ TO 82.

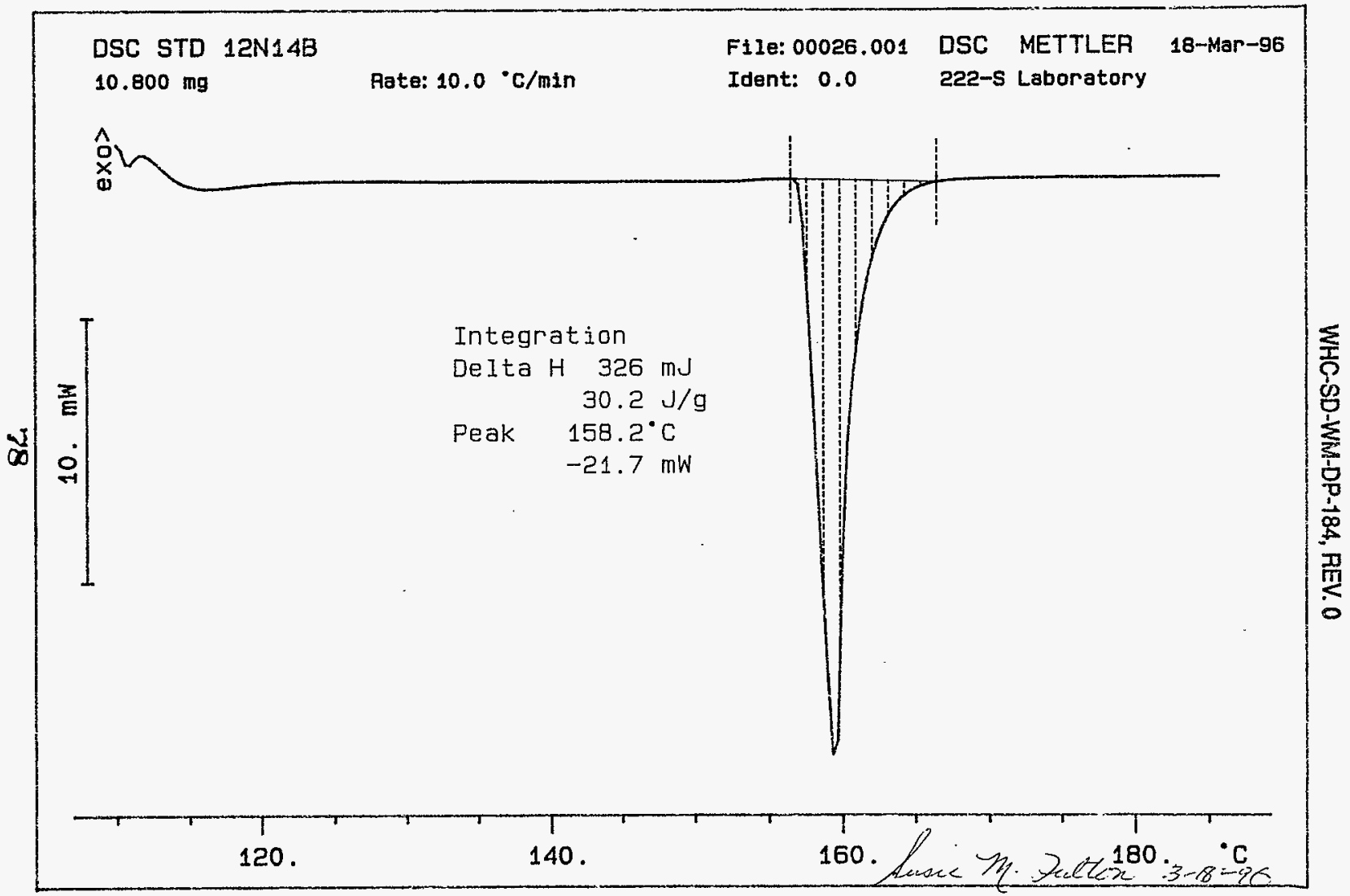




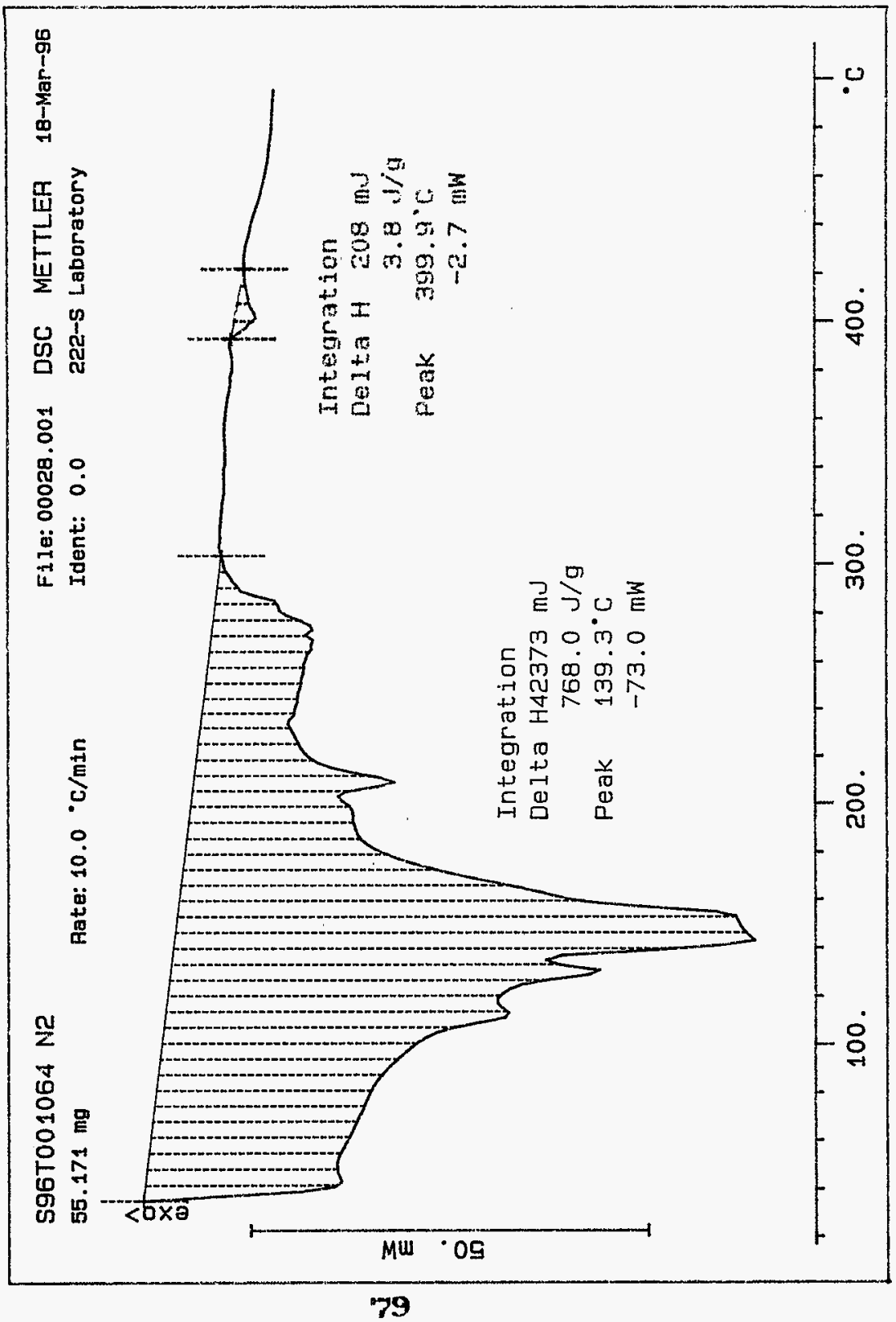


WHC-SD-WM-DP-184, REV. 0

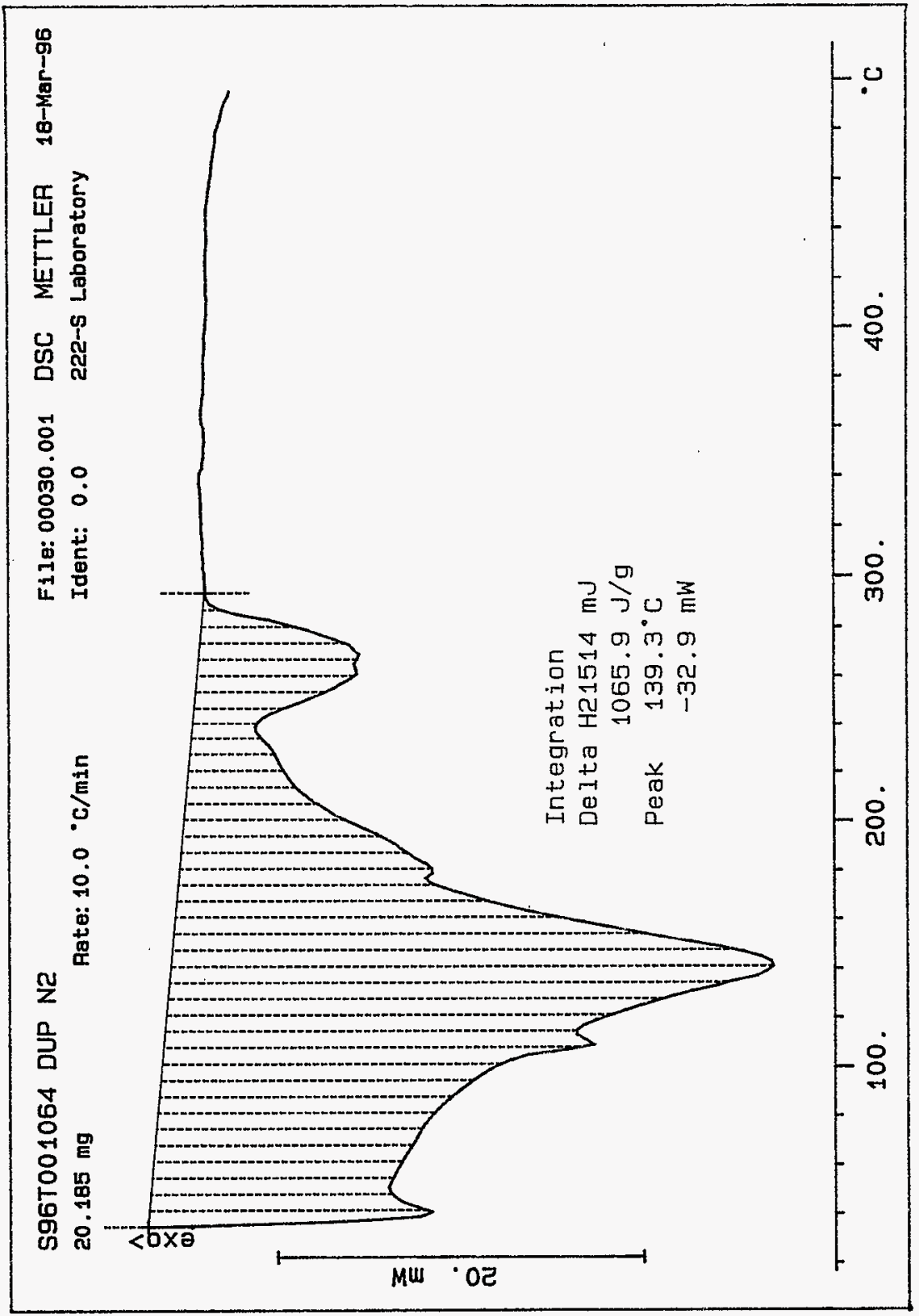


WHC-SD-WM-DP-184, REV. 0

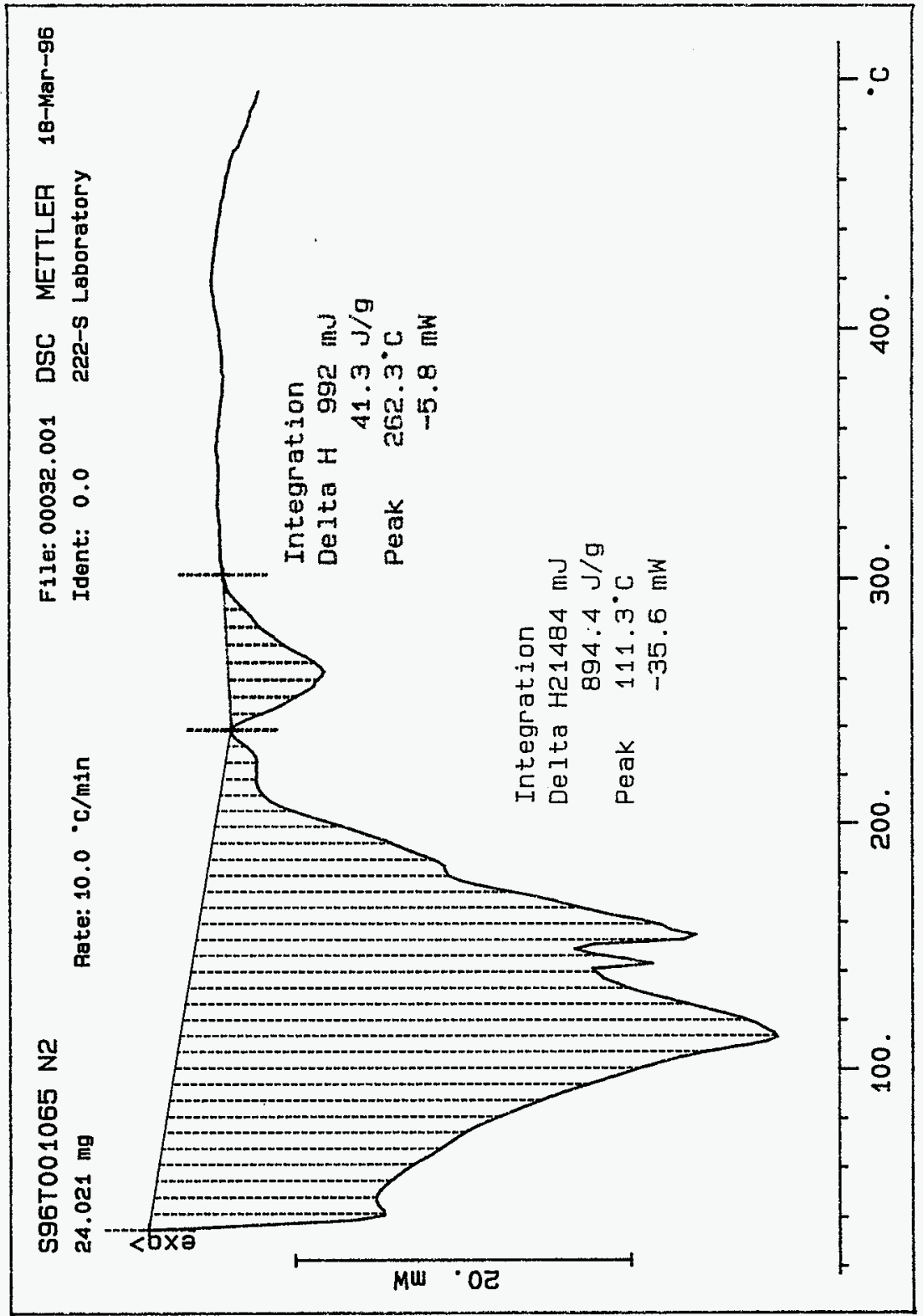




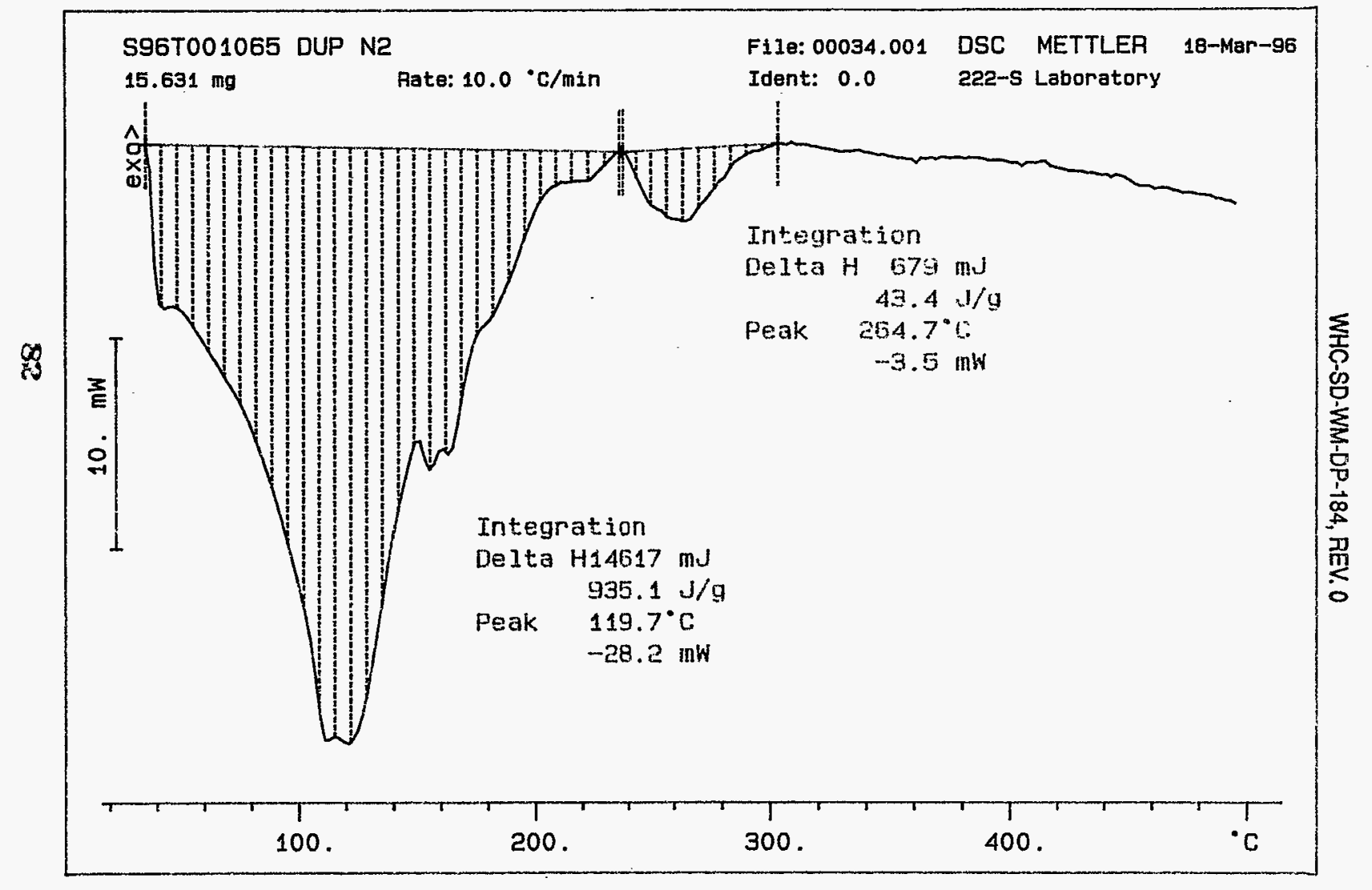


$04 / 23 / 96 \quad 10: 08 \quad 85093722929 \quad$ WESTINGHOUSE $\rightarrow \rightarrow \rightarrow$ MO -924 200W

016

worklistrpt Version $2.105 / 15 / 95$

WHC-SD-WM-DP-184, REV. 0

04/23/96 09:36

LABCORE Data Entry Template for Worklist\#

Page: 1

6516

Analyst: SMF Instrument: DSCO 3 Book \# $12 \mathrm{~N} 14 \mathrm{~B}$

Method: LA-514-114 Rev/Mod C -

Worklist Comment: U-107 DSC RUN UNDER N2. RCJ

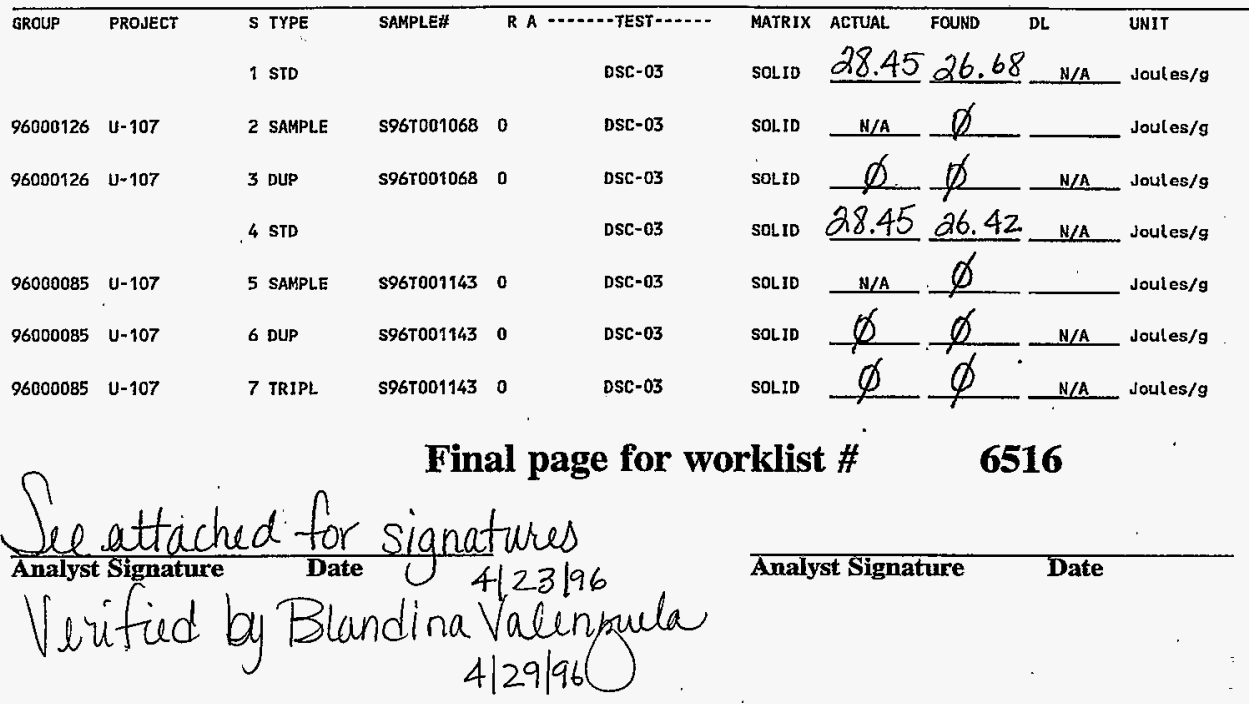

S96T001068 produced one large endotherm at $135.99^{\circ} \mathrm{C}$ with a delta $\mathrm{H}$ of $1218.6 \mathrm{~J} / \mathrm{g}$.

Data Entry Comments: 5967001143 produced two endotherm one at $101.99^{\circ} \mathrm{C}$ with a delta $\mathrm{H}$ of $141.82 \mathrm{~J} / \mathrm{g}$ and second at $304.95^{\circ} \mathrm{C}$ with a delta $\mathrm{H}$ of $134.18 \mathrm{~J} / \mathrm{g}$ the $A H$ was recalculated to include the correct samples weight.

Units shown for QC (SPK \& STD) may not reflect the actual units. $D L=$ Detection Limit, $S=$ Worklist Slot Number, $R=$ Replicate Number, $A=$ Aliquot Code.

83 


Analyst: SME Instrument: $\mathrm{DSCO}$

Book \#12N14B

Method: LA-514-113 Rev/Mod

Worklist Comment: U-107 DSC RUN UNDER N2. RCJ

\begin{tabular}{|c|c|c|c|c|c|c|c|c|c|c|}
\hline \multirow[t]{2}{*}{ GROUP } & \multirow[t]{2}{*}{ PROJECT } & S TYPE & \multirow[t]{2}{*}{ SAMPLE\# } & \multicolumn{2}{|c|}{ R A $\cdots$-TEST - } & \multirow{2}{*}{$\begin{array}{l}\text { MATRIX } \\
\text { SOLID }\end{array}$} & \multirow[t]{2}{*}{ ACTUAL } & \multirow[t]{2}{*}{ FBUHD } & DL & \multirow{2}{*}{$\begin{array}{l}\text { LNIT } \\
\text { Joules/g }\end{array}$} \\
\hline & & $1 \mathrm{STD}$ & & & DSC-01 & & & & N/A & \\
\hline 96000126 & U-107 & 2 SAMPLE & S96T0010ك8 & 0 & DSC-01 & SOLID & $H / A$ & & & Joules/g \\
\hline 96000126 & $v-107$ & 3 DUP & S967001068 & 0 & DSC-01 & SOLID & & & N/A & Joules/g \\
\hline 96000085 & U-107 & 4 SAMPLE & S967001143 & 0 & DSC-01 & SOLID & N/A & & & Joul es/g \\
\hline 96000085 & $u-107$ & 5 DUP & S96r001143 & 0 & DSC-01 & sol.tD & & & N/A & Joules/g \\
\hline
\end{tabular}

\section{Final page for worklist \# 6516}
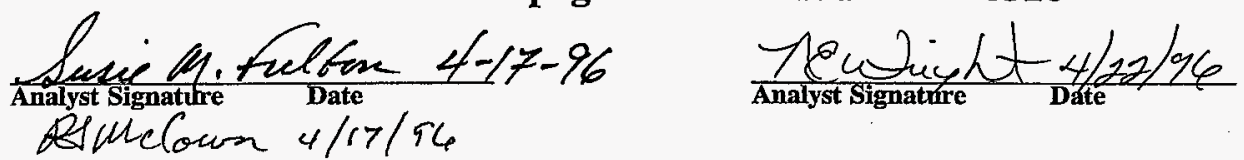

Data Entry Comments:

Units shown for $Q C$ (SPK \& STD) may not reflect the actual units. $D L=$ Detection Limit, $S=$ Worklist Slot Number, $R=$ Replicate Number, $A=$ Aliquot Code. 
Curye 1: DSC

F1]e info: INDO41701 Wed Apr 17 05:57:58 1935

Sampla Veight: $7.900 \mathrm{mg}$

IEN14-B INDIUNA AT 10CWMIN

SIGNATURE BELOH REPRESENTS CHEHICAL TECHNOLOGIST/CHEMIST THAT

COMPLETED/VERIFIED THE CALIBRATION/ANALYSIS ON PAGES \&S TO $\%$.

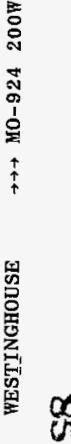

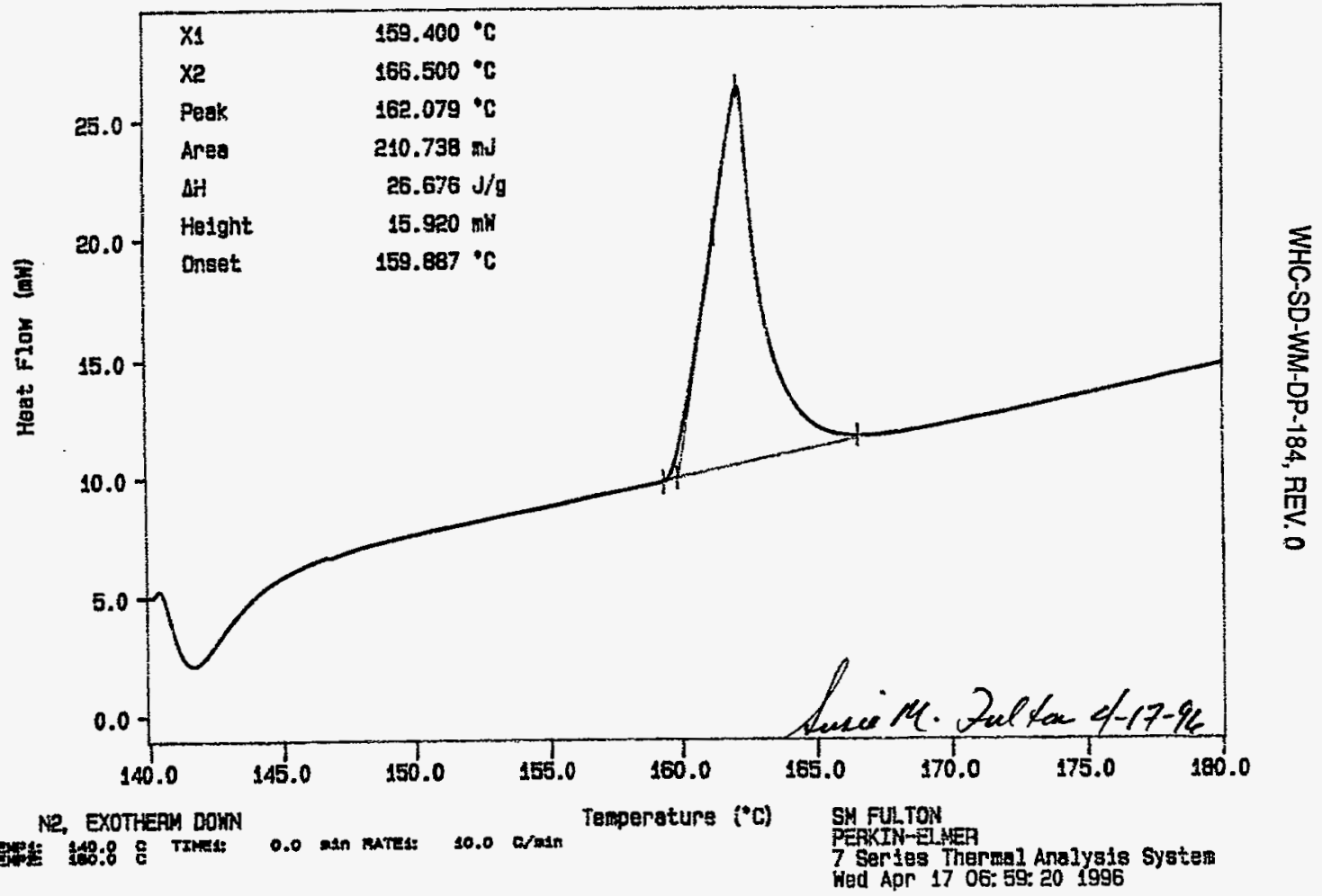


Curve 1: DSC

F1le info: SAH041701 Hed App 17 07:49:42 1996

Sample fieight: 37.540

$\operatorname{lng}$

S56T001068

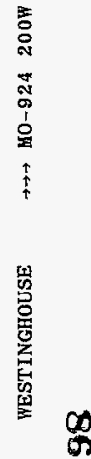

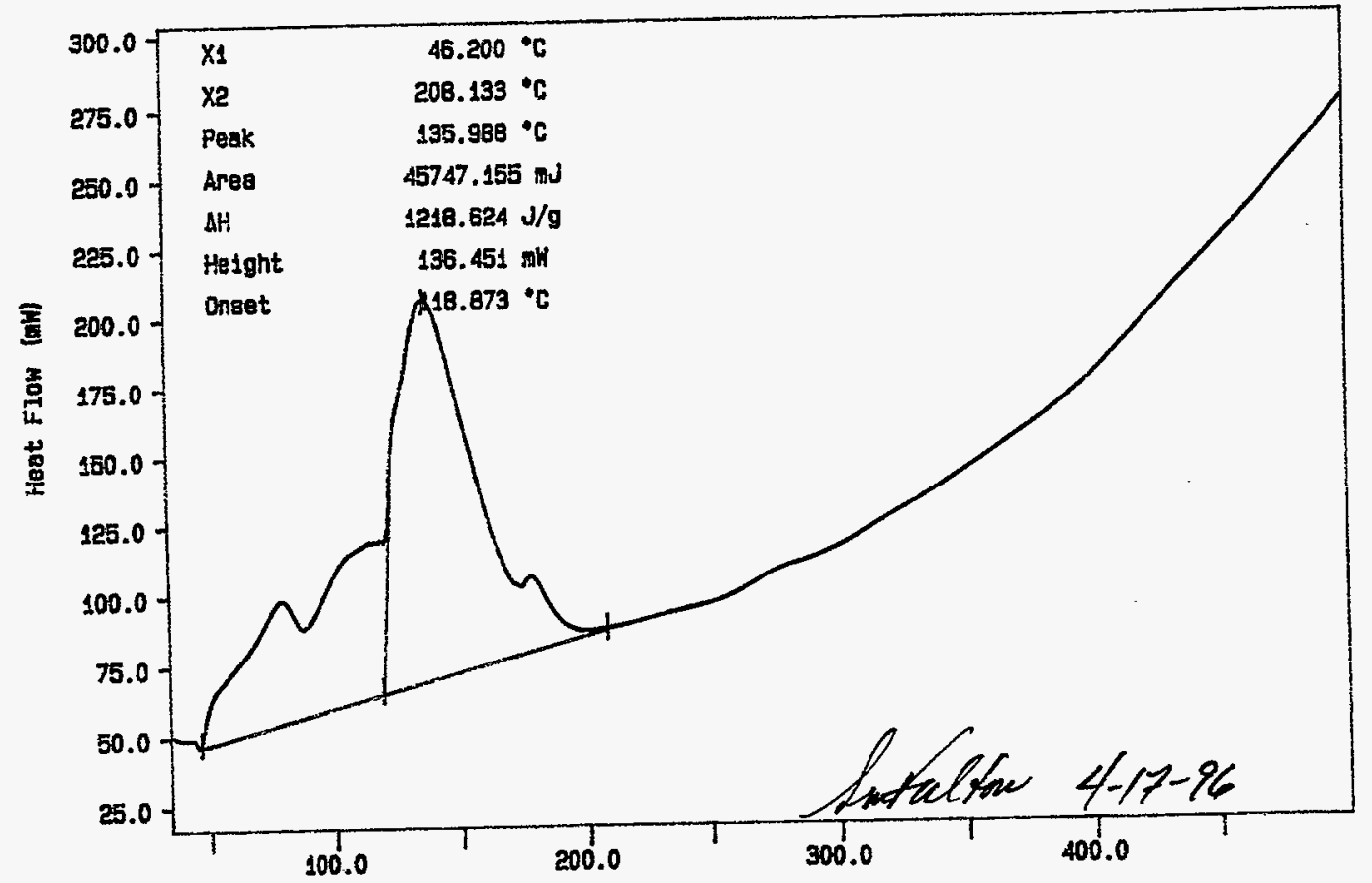

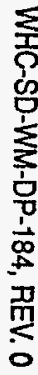

exotherw down, Ne purge gas

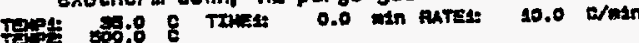

Temparature $\left({ }^{\circ} \mathrm{C}\right)$ SM FULTON

7 Sertes Thermal Anelysis Systen

Wed Apr 17 O8: 32531996 
Curve 1: DSC

F110 info: SAH041702 Hed Apr 17 09;29;00 1996

Sangle Weight: 17.320

ang

s967001068 DLP

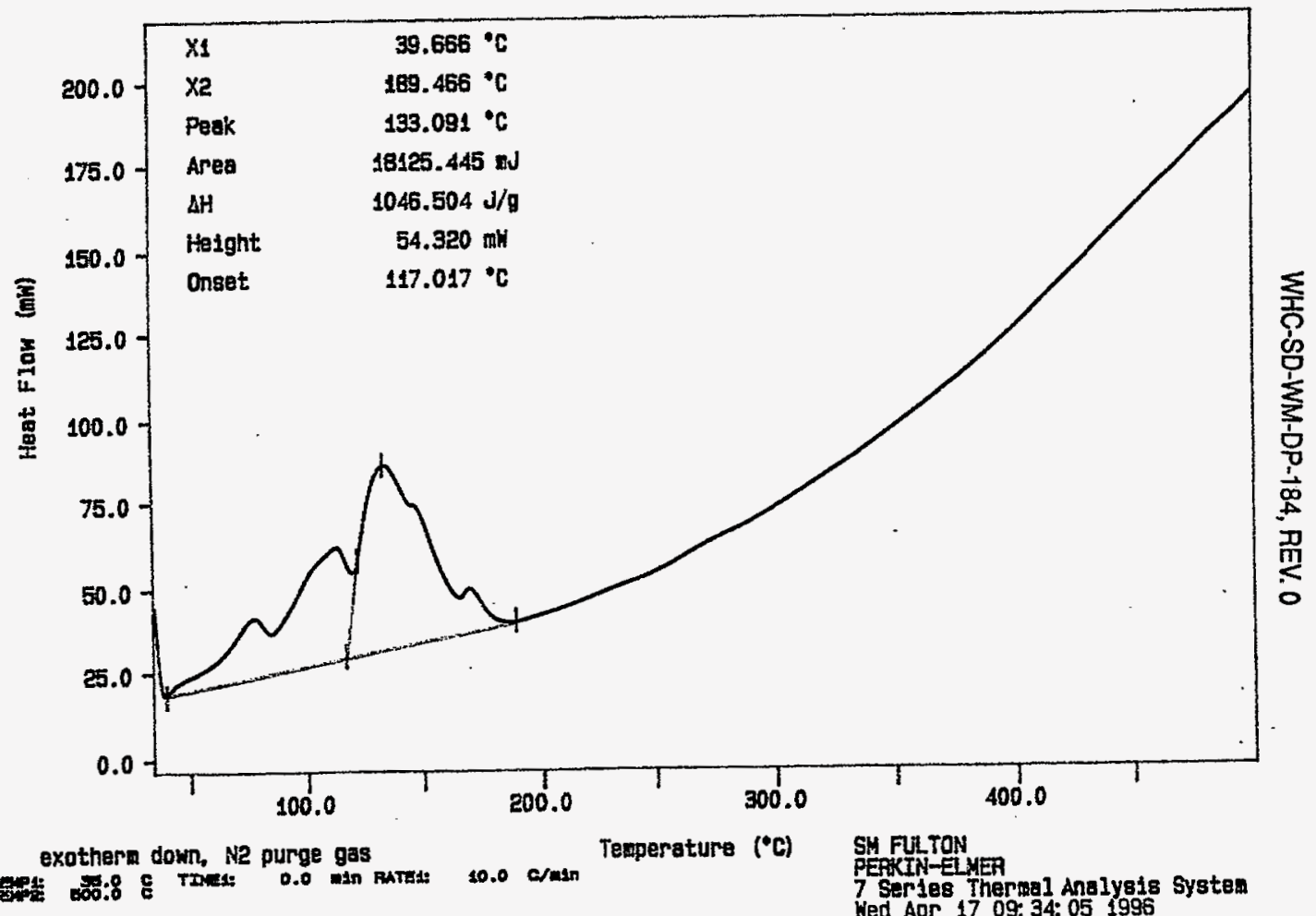


Curve 1: DSC

F13e infa: INDO4t705 hed Apr 17 1E:29: $17: 996$

Semple Height: 7.300 ag

12114-8 IHDIUN AT 10EXMTH

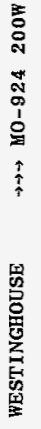

$\infty$

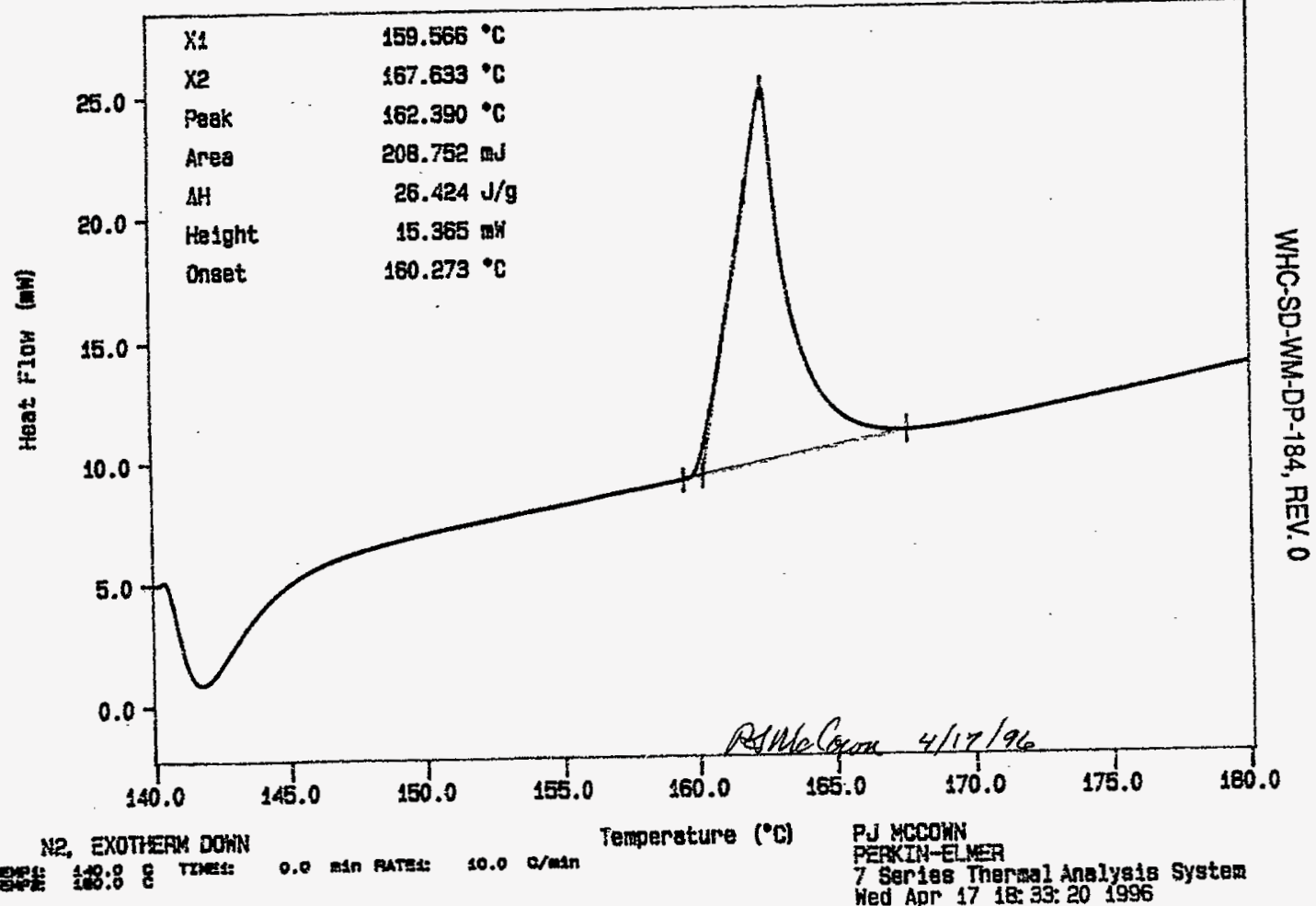


WHC-SD-WM-DP-184, REV. 0

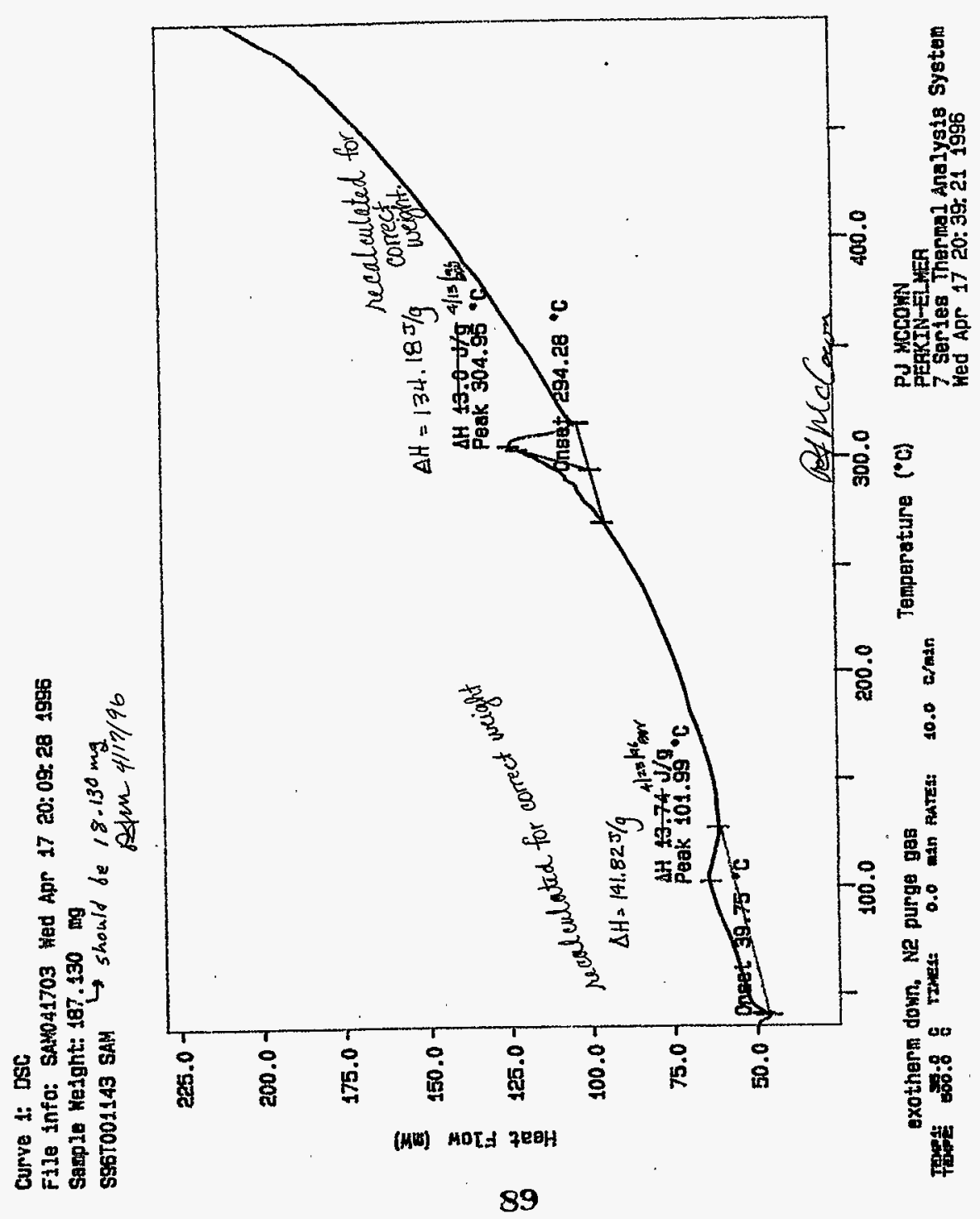


Curve 1: DSC

Flle Info: SAM0A3704 Wed Apr i7 21: 41:59 1996

Sample Keight: 19.070

ang

S96TO01143 DUP

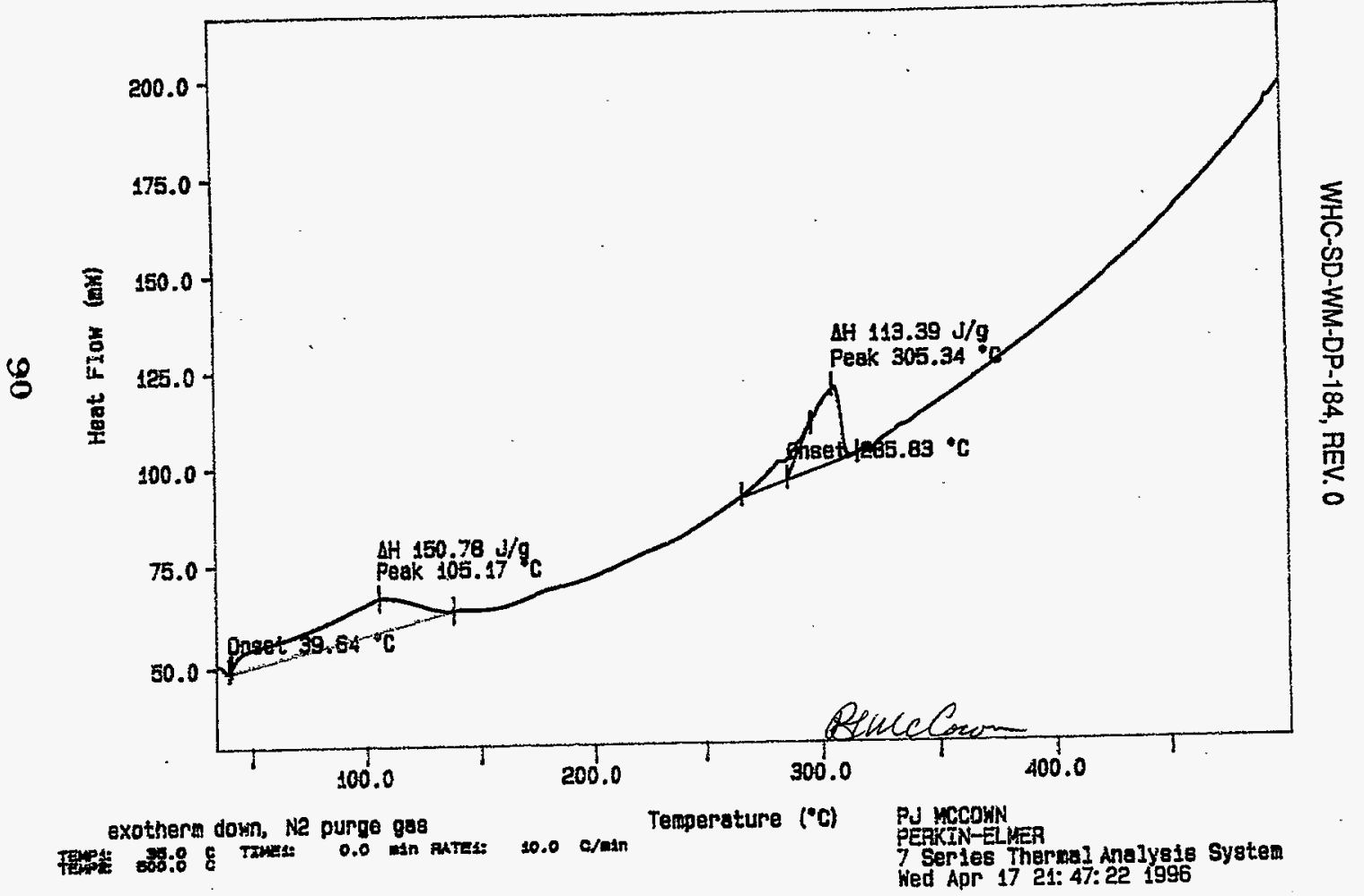




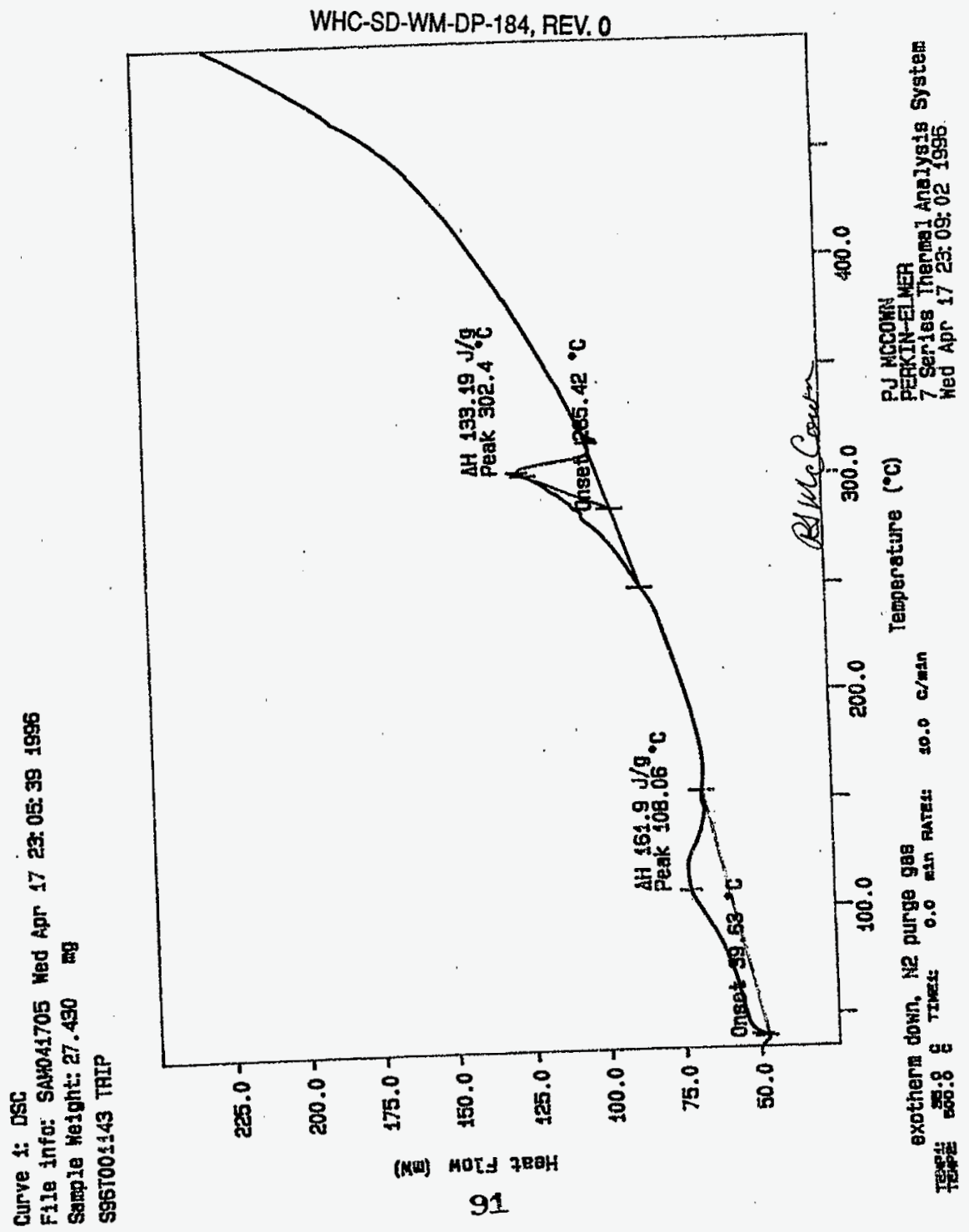


$04 / 08 / 96=14: 54 \quad 05093722929$

WESTINGHOUSE

001

worklistrpt Version 2.1 05/15/95

WHC-SD-WM-DP-184, REV. 0

Page: $\quad I$

$04 / 08 / 9612: 07$

LABCORE Data Entry Template for Worklist\#

6624

Analyst: RDM Instrument: $\operatorname{DSC} 03-$ Book \# 12 N N IL

Method: LA-514-114 Rev/Mod C-1

Worklist Comment: U-107 DSC RUN UNDER N2. RCJ

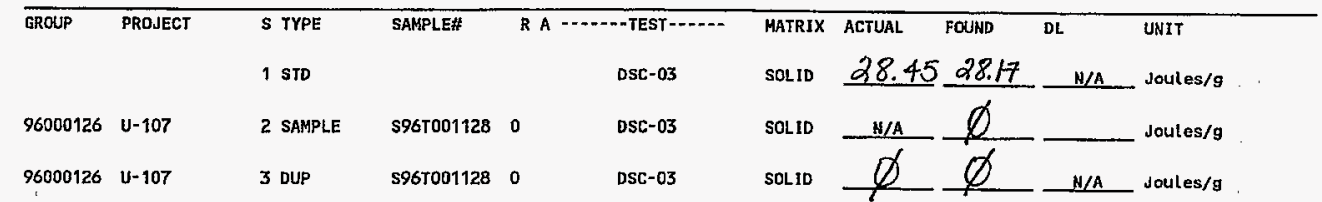

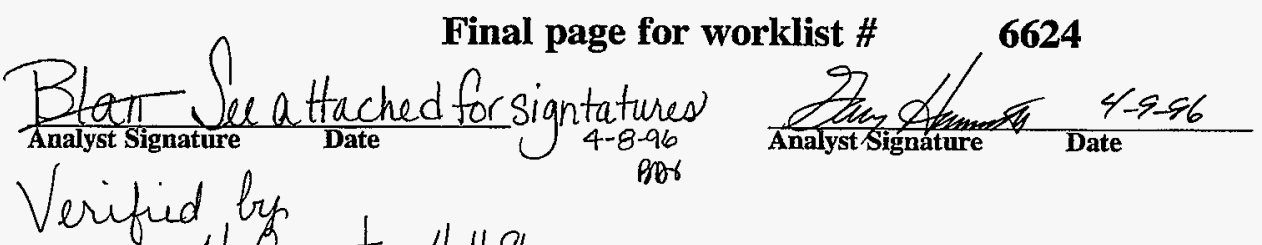

Verified by

13. Enactor 4-11-96

This is a composite sample of 6-3-96

Data Entry comments: Sample produced two endotherm one at $145.45 \mathrm{C}$ with a delta H of $423.48 \mathrm{~J} / \mathrm{g}$ and second at $281.6 \mathrm{C}$ with a delta $H$ of $73.16 \mathrm{~J} / \mathrm{g}$.

Units shown for $Q C$ (SPK \& STD) may not reflect the actual units. $D L=$ Detection Limit, $S=$ Worklist Slot Number, $R=$ Replicate Number, $A=$ Aliquot Code.

92 


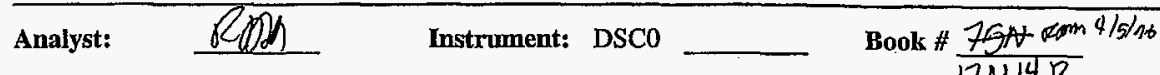

Method: LA-514-113 Rev/Mod C.

Worklist Comment: U-107 DSC RUN UNDER N2. RCJ

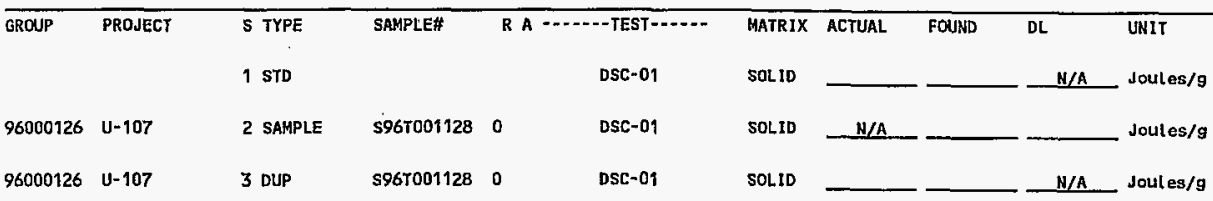

\section{Final page for worklist \# $\quad 6624$}

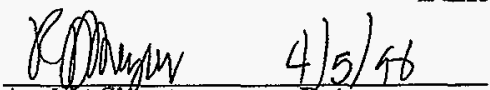

Data Entry Comments:

Units shown for QC (SPK \& STD) may not reflect the actual units. $D L=$ Detection Limit, $S=$ Worklist Slat Number, $R=$ Replicate Number, $A=$ Aliquot Code. 
File info: IND040401 Thu Apr 4 06: 40: 501996

Sample Keight: $7.900 \mathrm{mg}$

12N14-B INDIUM AT 10C \MIN

SIGNATURE BELOH REPRESENTS CHEMICAL TECHNOLOGIST/CHENIST THAT COMPLETED/VERIFIED THE CALIBRATION/ANALYSIS ON PAGES $\rightarrow 4$. TO

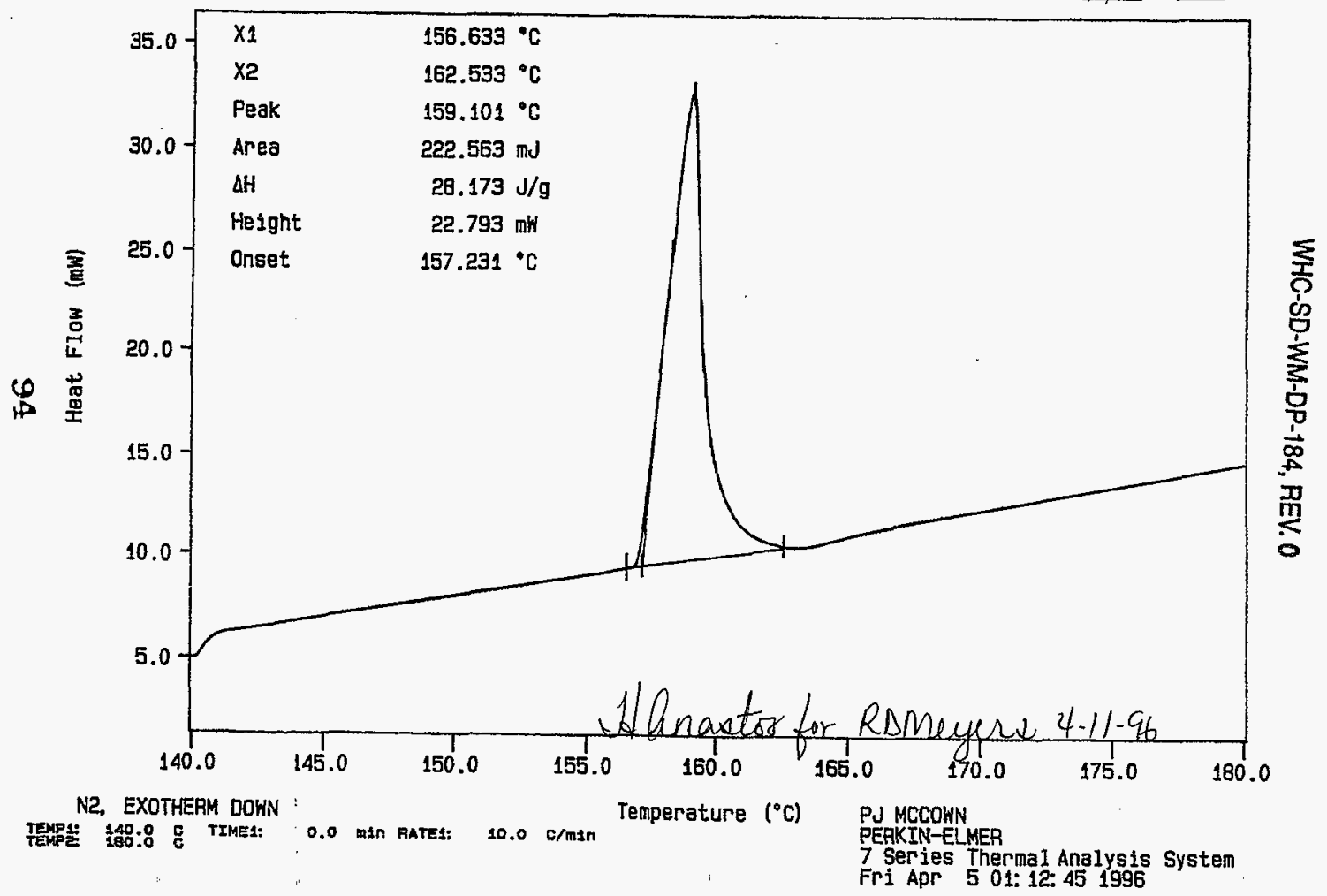


Curve 1: DSC

File info: sam040401 Thu Apr 4 22: 24:09 1996

Sample Weight: $50.100 \mathrm{mg}$

S96TO01128

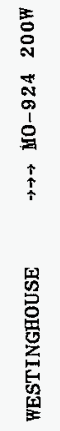

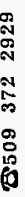

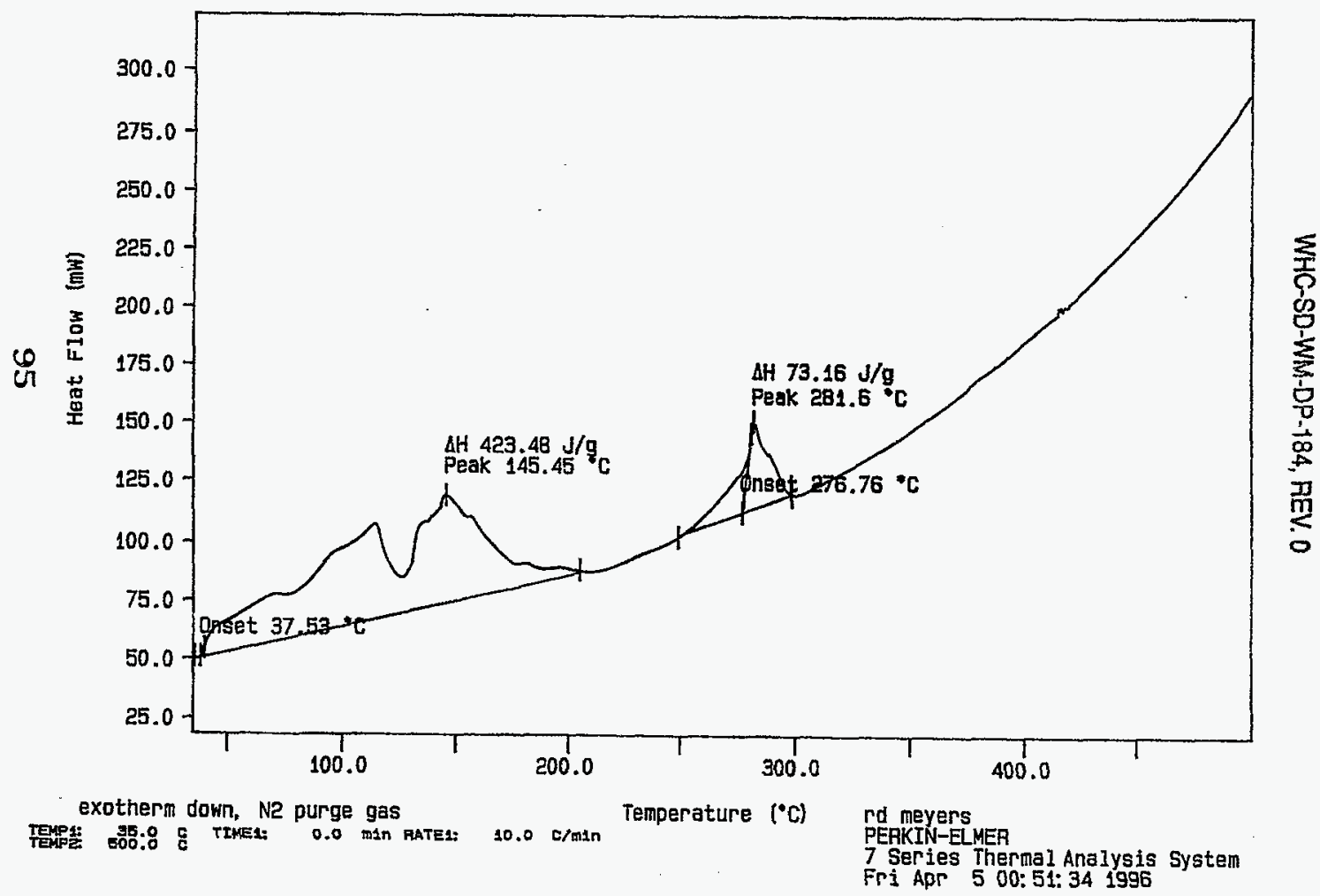


Curve 1: DSC

File info: sam040501 Fri Apr 5 01: $42: 461996$

Sample Height: 45.360

Ing

S96TO01128 DUP

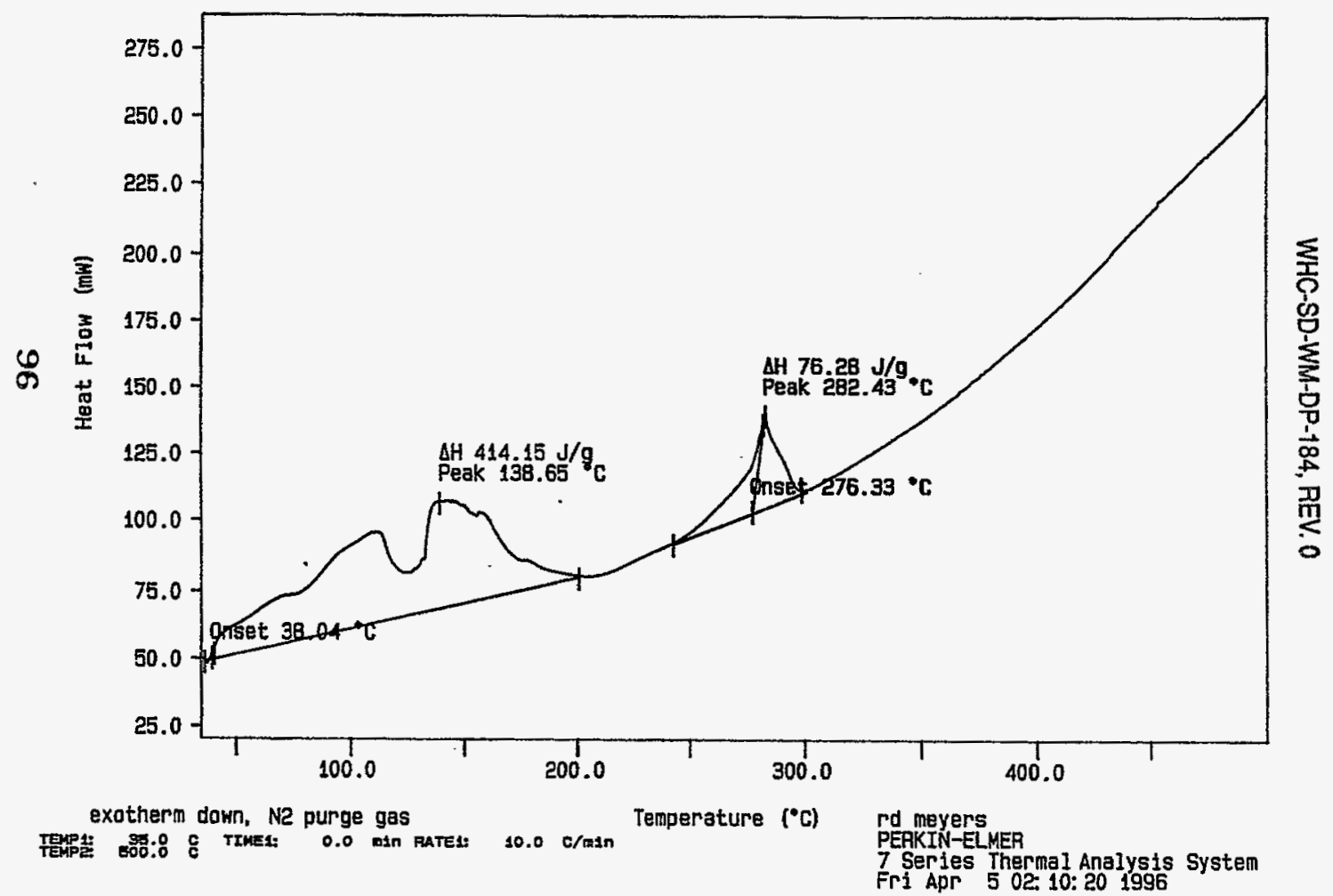


WHC-SD-WM-DP-184, REV. 0 04/03/96 11:38

\section{LABCORE Data Entry Template for Worklist\#}

Page: 1

Analyst: $\quad$ PDM $\quad$ Instrument: $\mathrm{DSCO} \perp$ Book \#12N14B

Method: LA-514-113 Rev/Mod C-1

Worklist Comment: U-107 DSC RUN UNDER N2. RCJ

\begin{tabular}{|c|c|c|c|c|c|c|c|c|c|}
\hline GROUP & PROJECT & $\begin{array}{l}5 \text { TYPE } \\
1 \text { STD }\end{array}$ & SAMPLE\# & R A & $\begin{array}{l}\text { DSEST- }-\cdots \\
\text { DSC-D1 }\end{array}$ & $\begin{array}{l}\text { MATRIX } \\
\text { SOLID }\end{array}$ & $\begin{array}{l}\text { ACTUAL FOUMD } \\
28.45 \quad 32,0 \\
\end{array}$ & $\begin{array}{l}\text { DL } \\
N / A \\
\end{array}$ & Joules/g \\
\hline \multirow[t]{2}{*}{96000126} & U-107 & 2 SAMPLE & s96T001059 & 0 & DSC-01 & SOL ID & $N / A$ & & Joules/g \\
\hline & & 3 STD & & & DSC-01 & SOL.ID & $38.45 \quad 32.4$ & H/A & Joules/g \\
\hline \multirow[t]{2}{*}{96000126} & U-107 & 4 DuP & S96T001059 & 0 & DSC-01 & SOL ID & $\phi$ & $N / A$ & Joules/g \\
\hline & & $5 \mathrm{STD}$ & & & DSC-01 & SOL.ID & 28.4532 .4 & N/A & Joules/g \\
\hline 96000126 & U-107 & 6 SAMPLE & s96t001070 & o & DSc-01 & SOLID & W/A & & Joules/g \\
\hline 96000126 & U-107 & 7 DUP & S96T001070 & 0 & DSC-01 & SOLID & $\not$ & H/A & Joules/g \\
\hline
\end{tabular}

\section{Final page for worklist \# 6627} See Qffached tir Siguatures Vinfiud by Blandina Valengaela

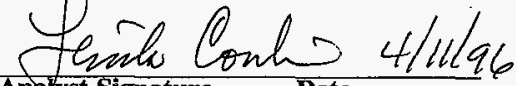

Anałyst Signature
Date 


\section{LABCORE Data Entry Template for Worklist\#}

Analyst: RXOM Instrument: $\mathrm{DSCO}$ Book \#12N)4.B

Method: LA-514-113 Rev/Mod C-

Worklist Comment: U-107 DSC RUN UNDER N2. RCJ

\begin{tabular}{|c|c|c|c|c|c|c|c|c|c|}
\hline GROUP & PROJECT & $\begin{array}{l}\text { S TYPE } \\
\text { STD }\end{array}$ & SAMPLE\# & R A & DSC-01 & $\begin{array}{l}\text { MATRIX } \\
\text { SOLID }\end{array}$ & 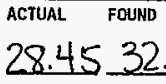 & $\begin{array}{l}\mathrm{DL} \\
\mathrm{N} / \mathrm{A} \\
\end{array}$ & $\begin{array}{l}\text { UNIT } \\
\text { Joules/g }\end{array}$ \\
\hline 96000126 & $U-107$ & 2 SAMPLE & S96T001059 & 0 & DSC-0t & SOLID & $\mathrm{N} / \mathrm{A}$ & & Joules/g \\
\hline 96000126 & $U-107$ & 3 DUP & s967001059 & 0 & DSC-01 & SOLID & . & N/A & Joules/g \\
\hline 96000126 & $v-107$ & 4 SAMPLE & s96t001070 & 0 & DSC-01 & SOL.ID & N/A & 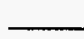 & Joules/s \\
\hline 96000126 & $U-107$ & 5 DUP & s96r001070 & 0 & DSC-01 & SOLID & & $N / A$ & Joules/g \\
\hline
\end{tabular}

\section{Final page for worklist \# $\quad 6627$}

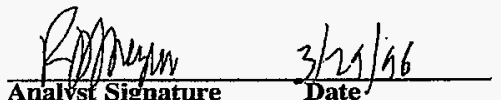




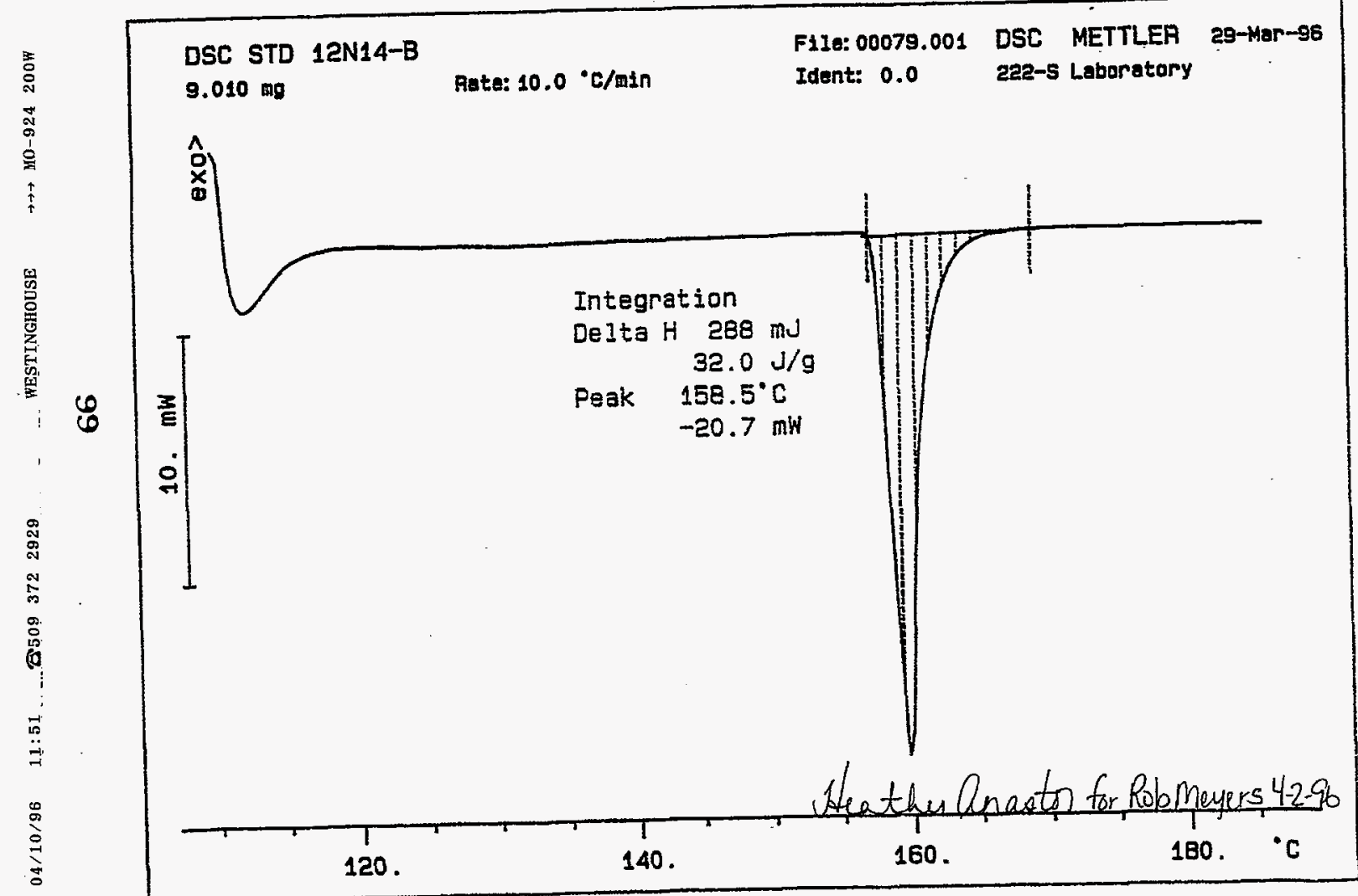


WHC-SD-WM-DP-184, REV. 0

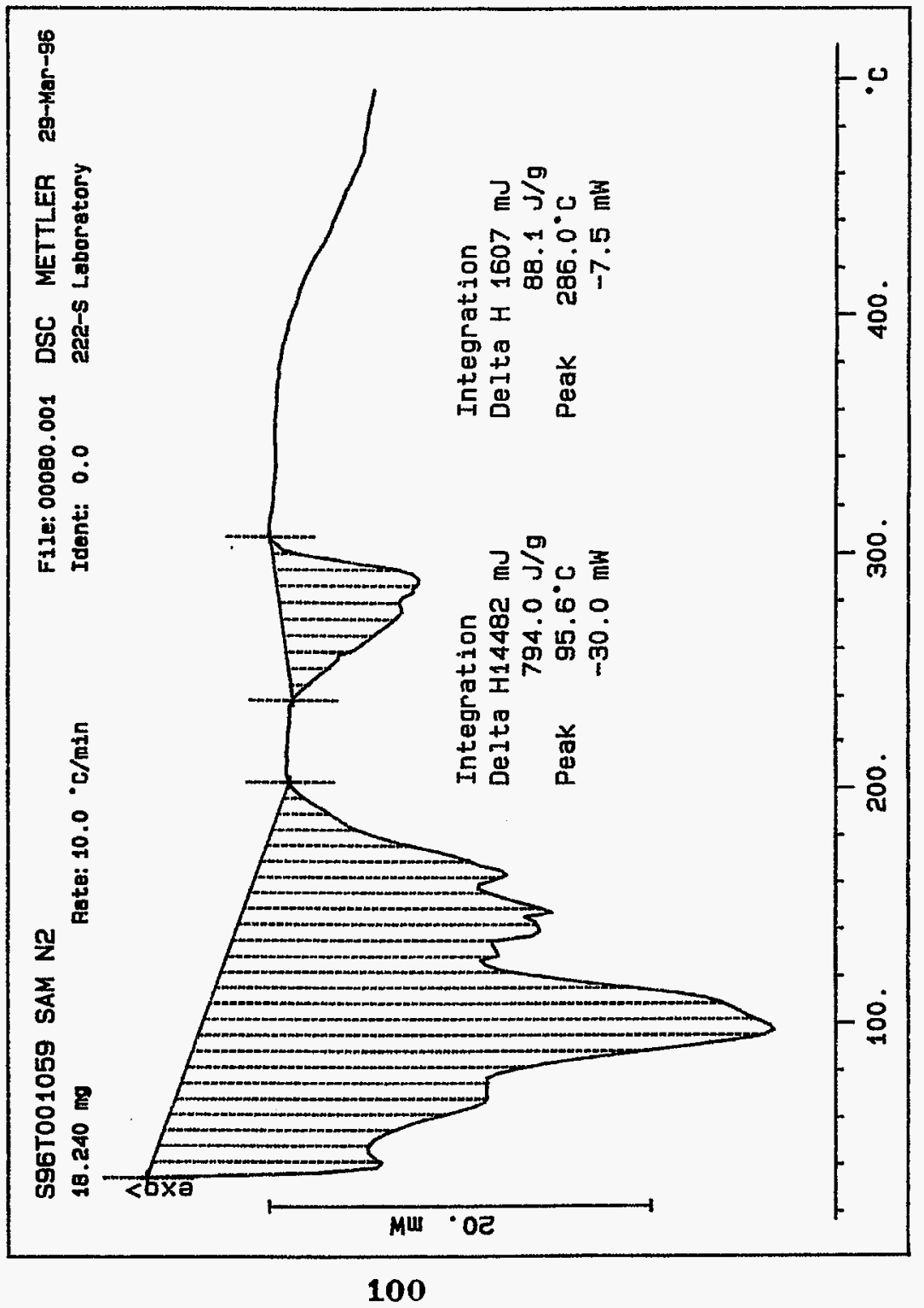




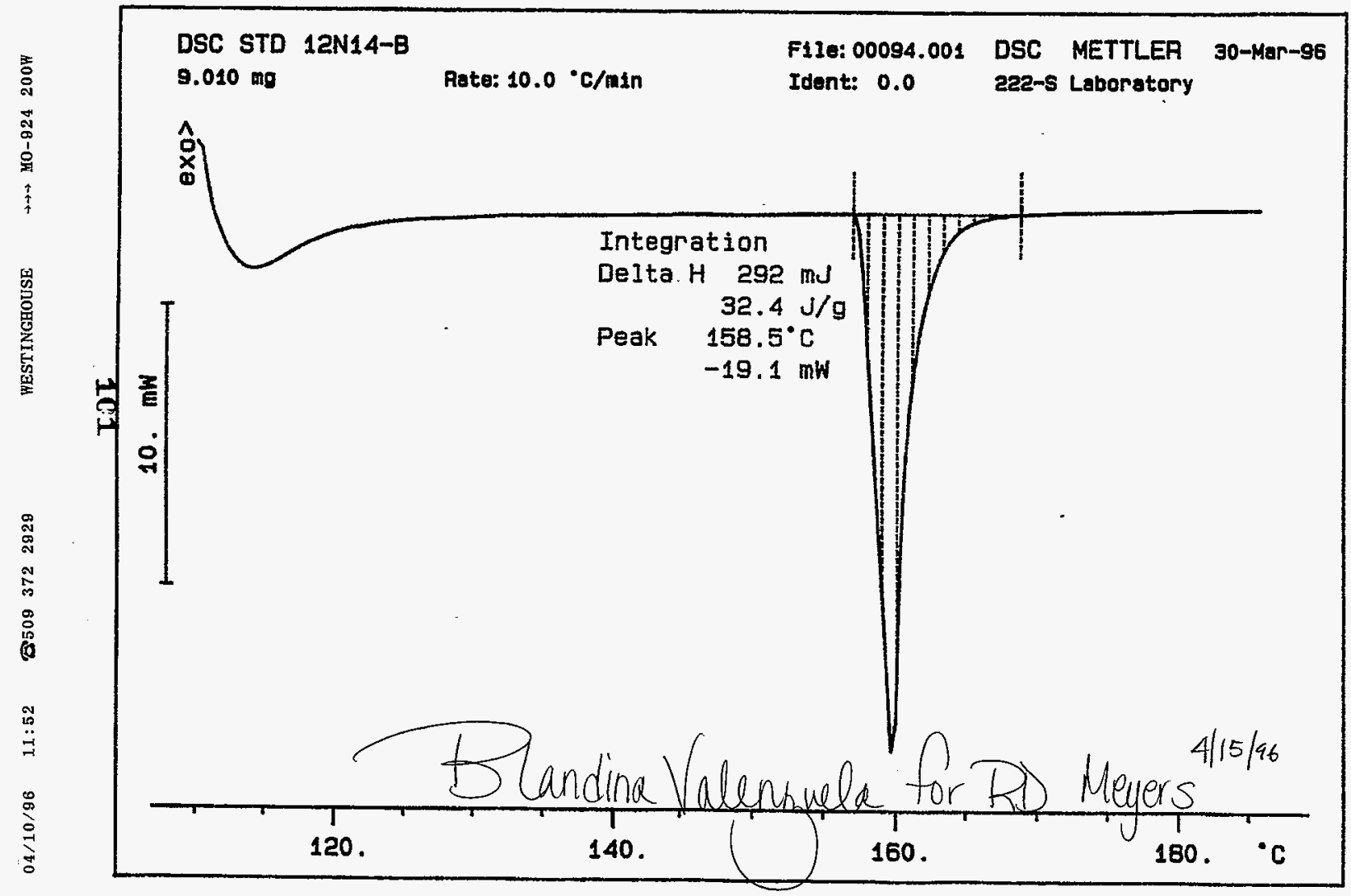


WHC-SD-WM-DP-184, REV . 0

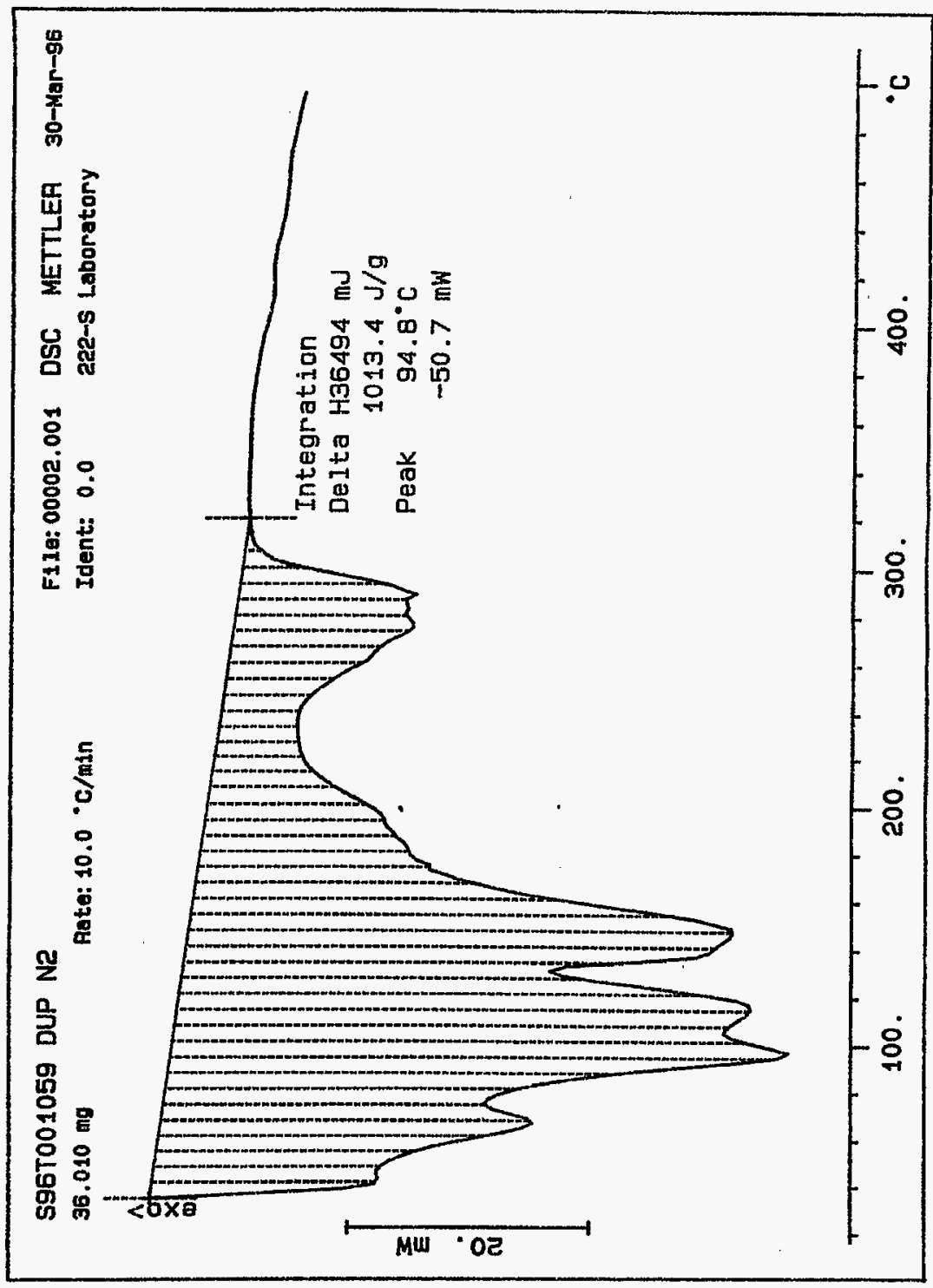




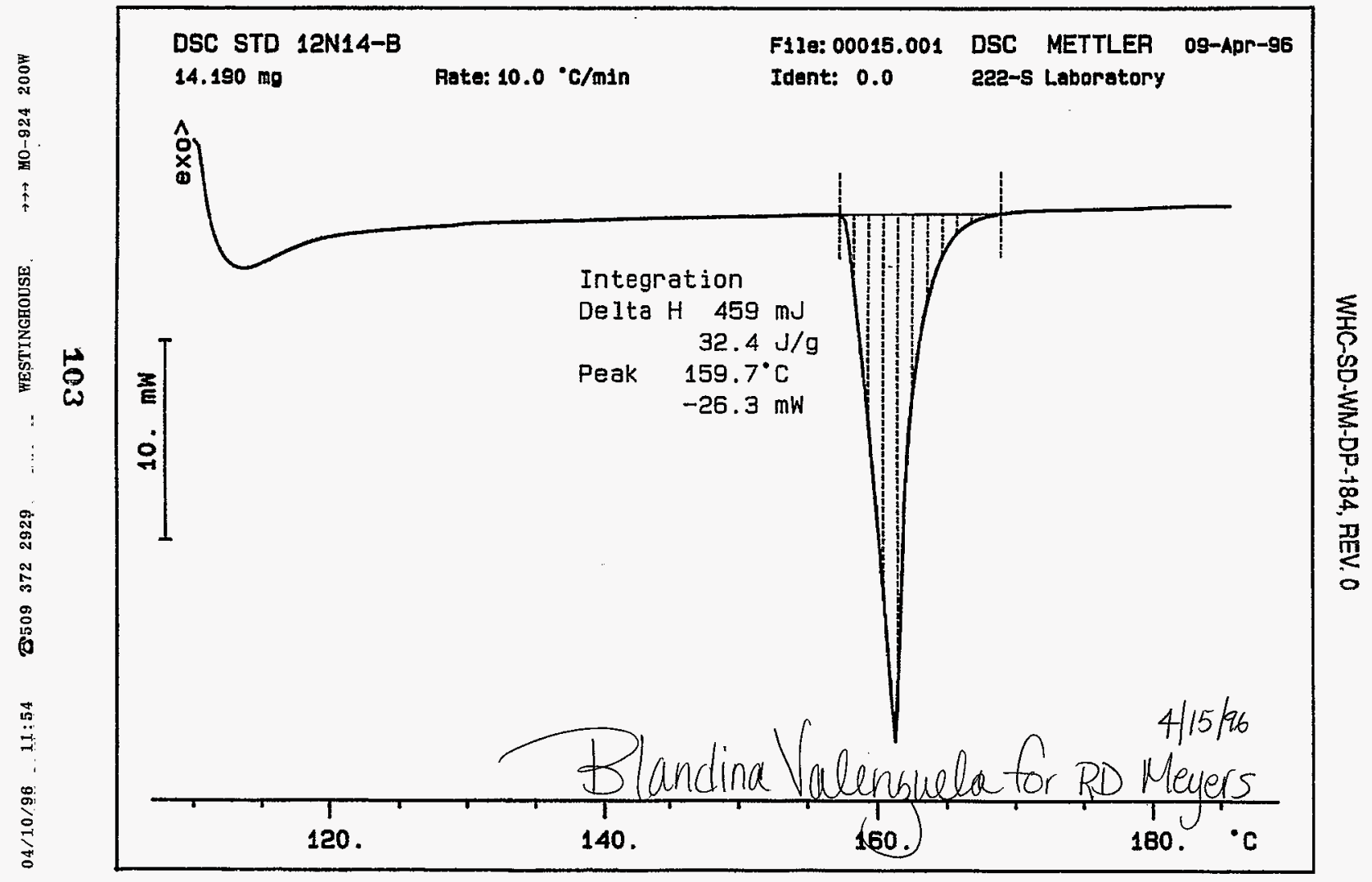


WHC-SD-WM-DP-184, REV. 0

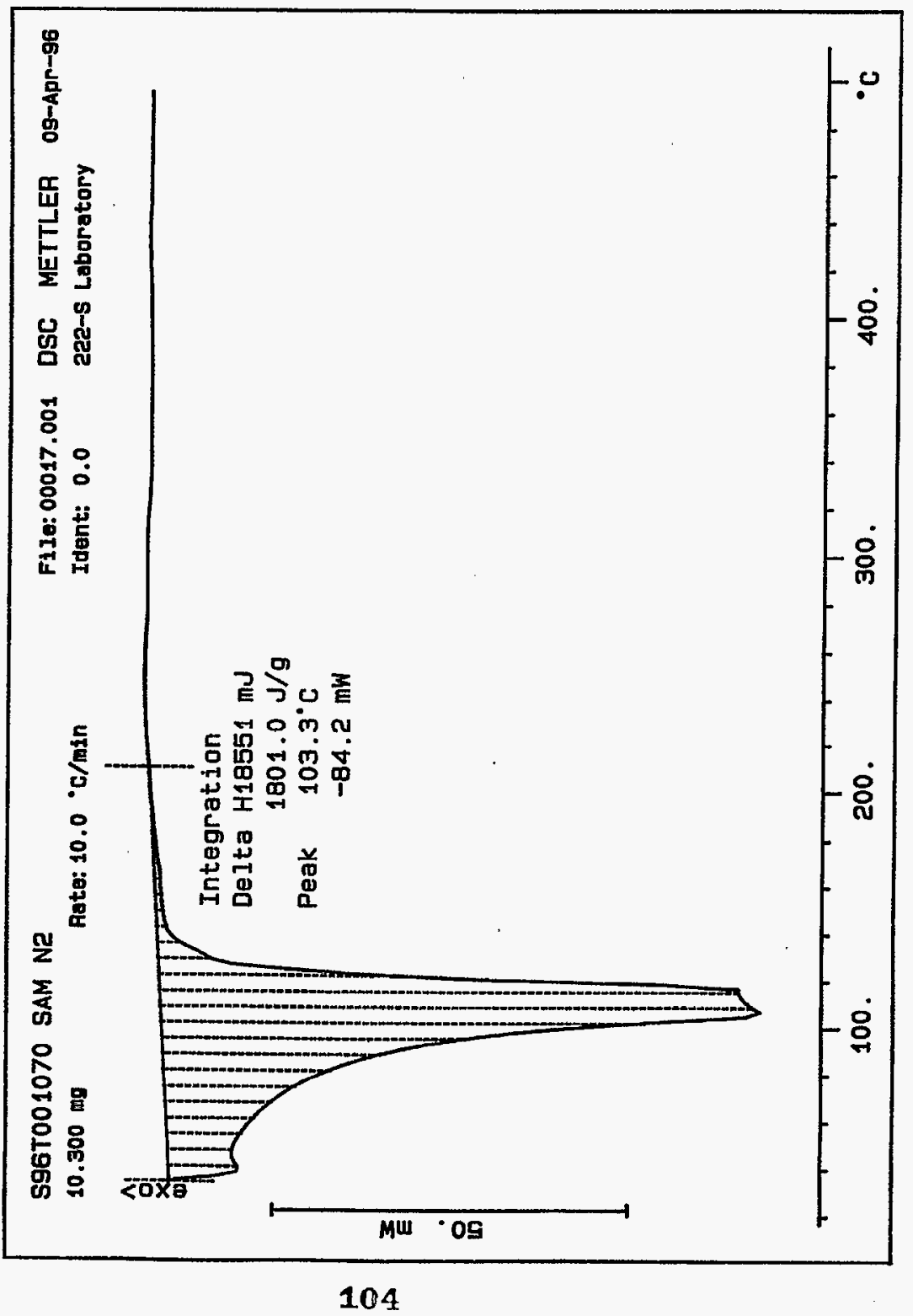


WHC-SD-WM-DP-184, REV. 0

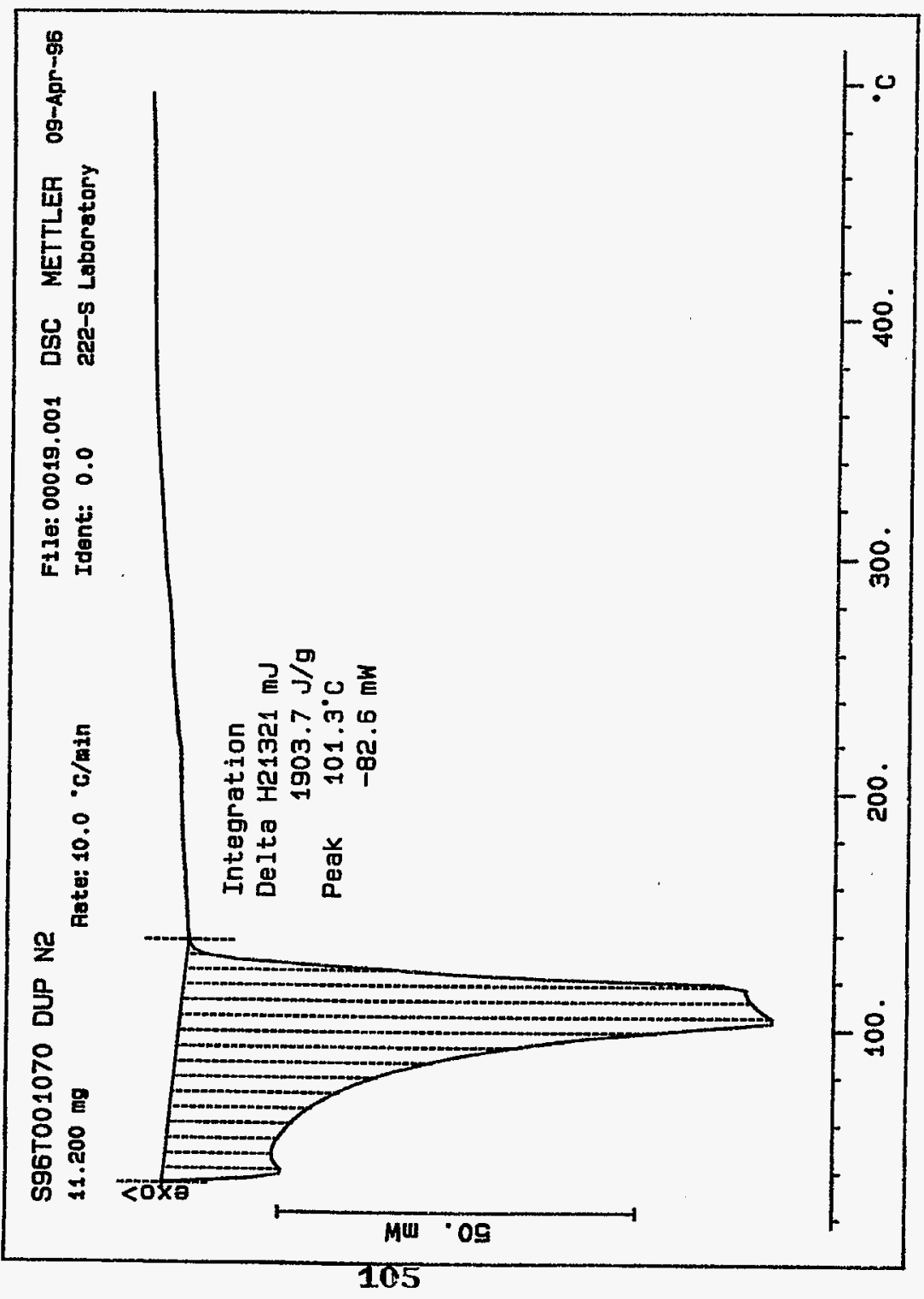


$04 / 18 / 96 \quad 10: 40 \quad 85093722929$

Q 001

WHC-SD-WIV-DP-184, REV. 0

worklistrpt Version 2.1 05/15/95

LABCORE Data Entry Template for Worklist\#

Page: 1

03/18/96 14:39

Analyst: $\quad$ SMF Instrument: ${ }^{2}$ I SC 1 Book $12 N / 4 B$

Method: LA-514-113 Rev/Mod $\mathrm{C}-1$

Worklist Comment: U-107 DSC RUN UNDER N2. RCJ

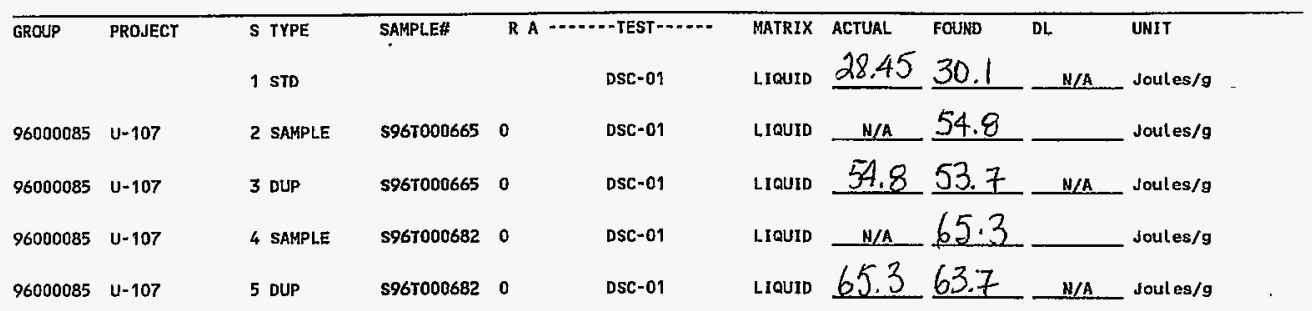

Final page for worklist \# 6655

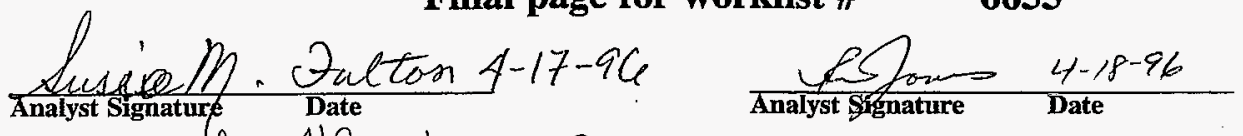

Verified by Hilnasto 4-22-96

Data Entry Comments: 5967000665 produced an endotherm at $117.6 \mathrm{C}$ with a delta $1 \mathrm{of} 1008.2 \mathrm{~J} / \mathrm{g}$ The results given are the sum of two exotherms.

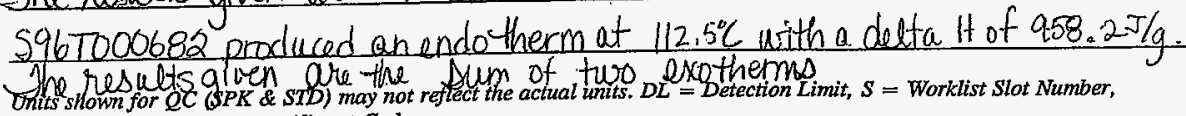
$R=$ Replicate Number, $A=$ Aliquot Code.

106 
SIGNATURE BELOW REPRESENTS CHEMICAL TECHNOLOGIST/CHEMIST THAT

COMPLETED/VERIFIED THE CALIBRATION/ANALYSIS ON PAGES $/ C 7 T O M$ TO

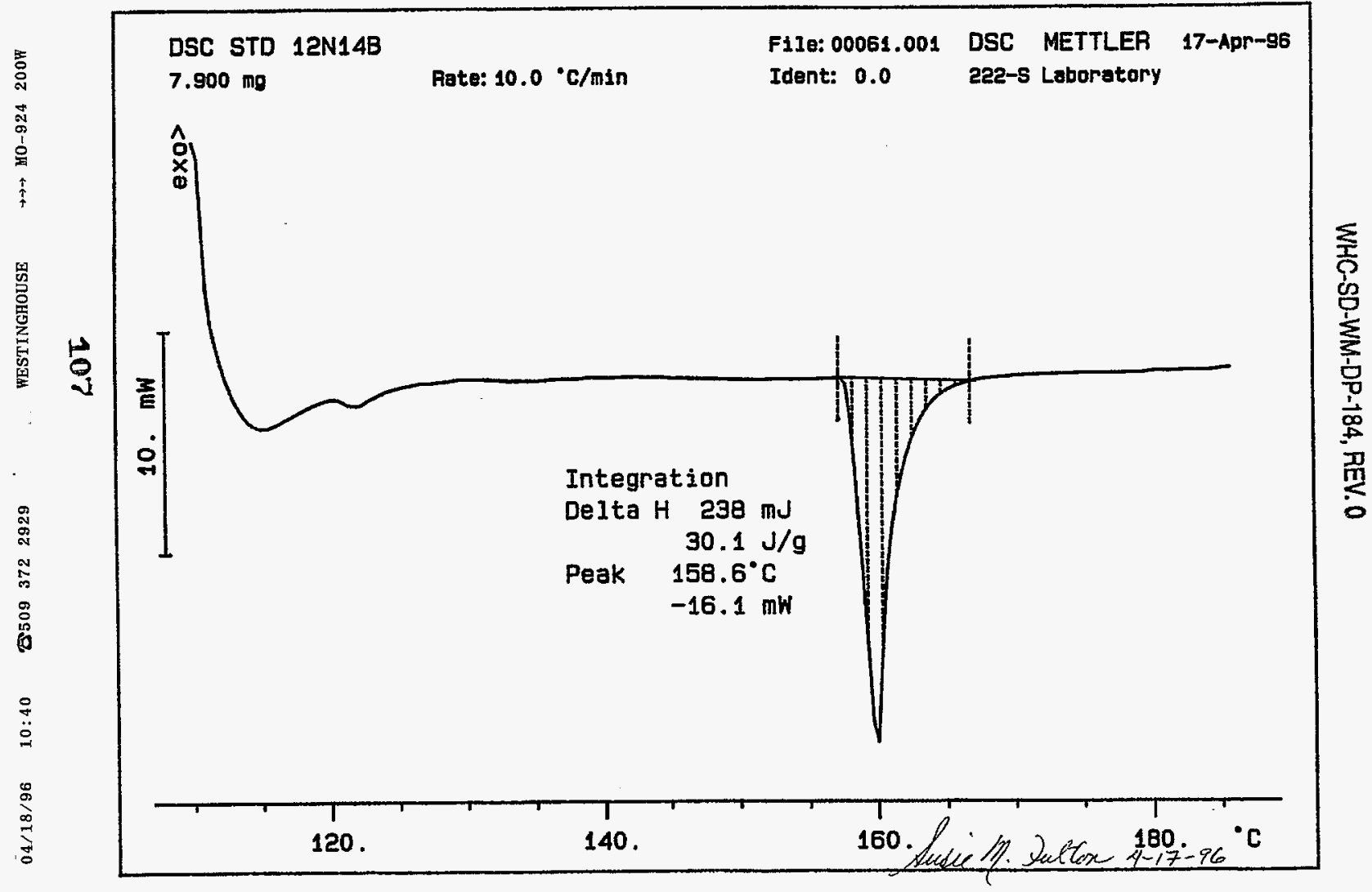




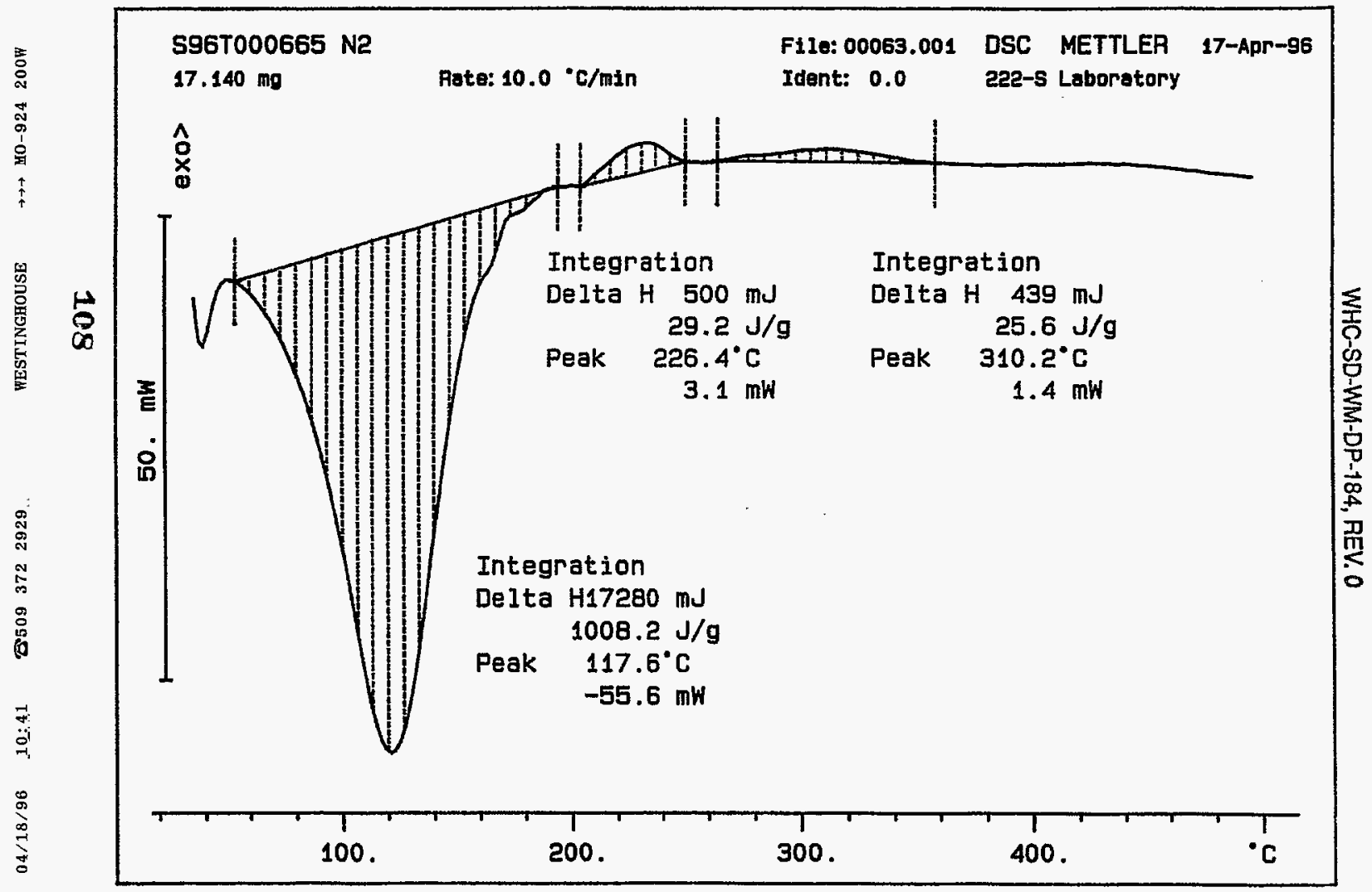




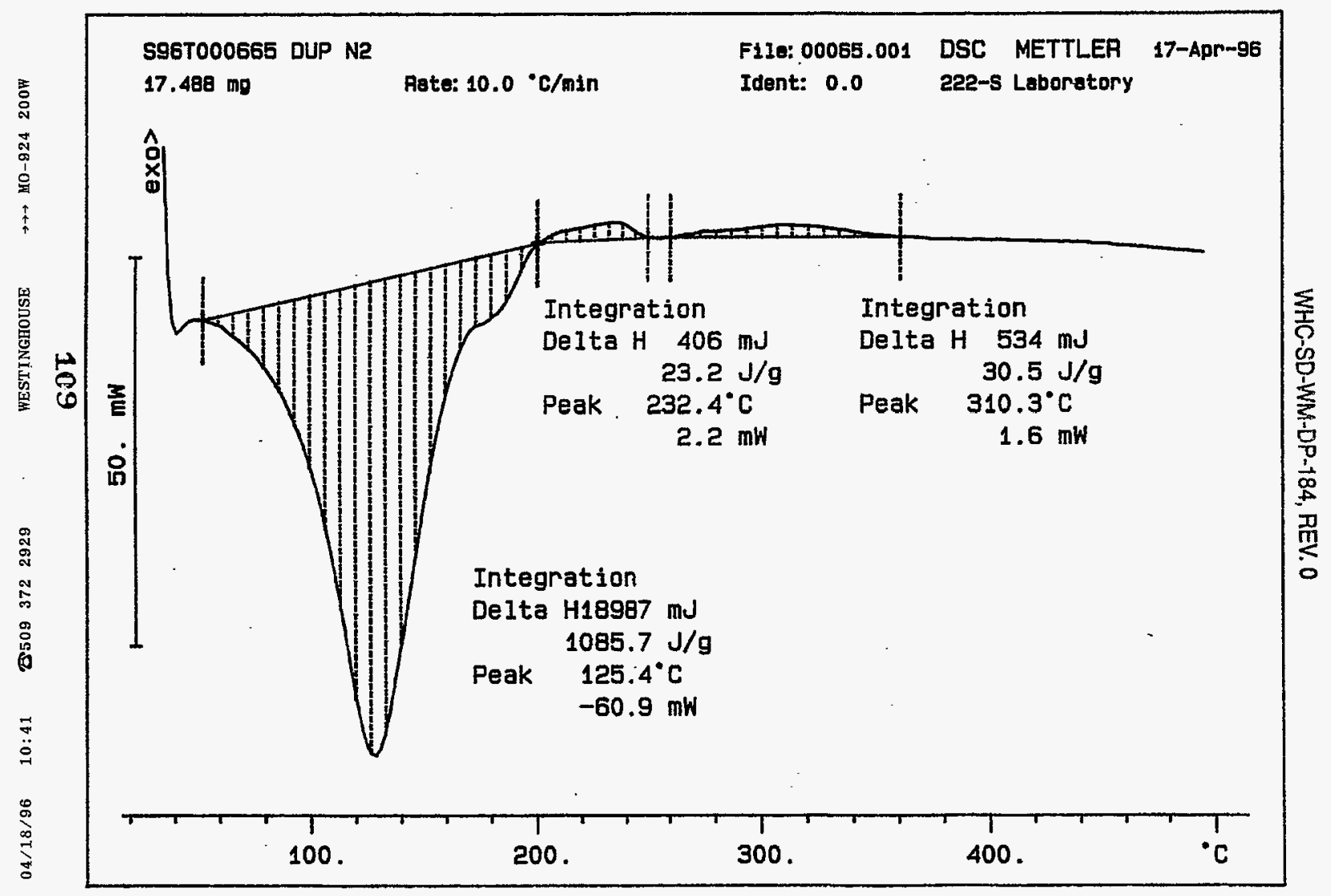


WHC-SD-WM-DP-184, REV. 0

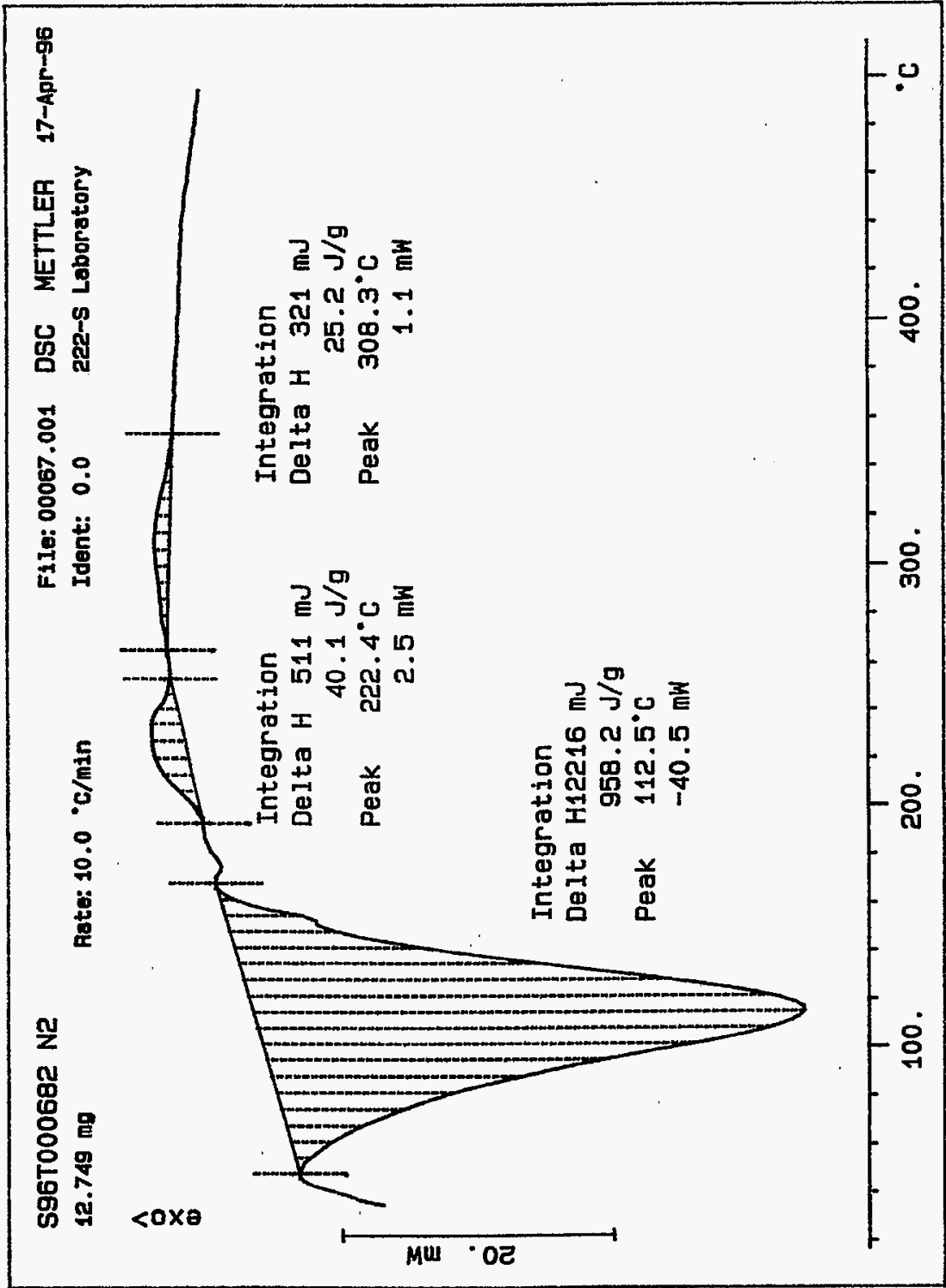




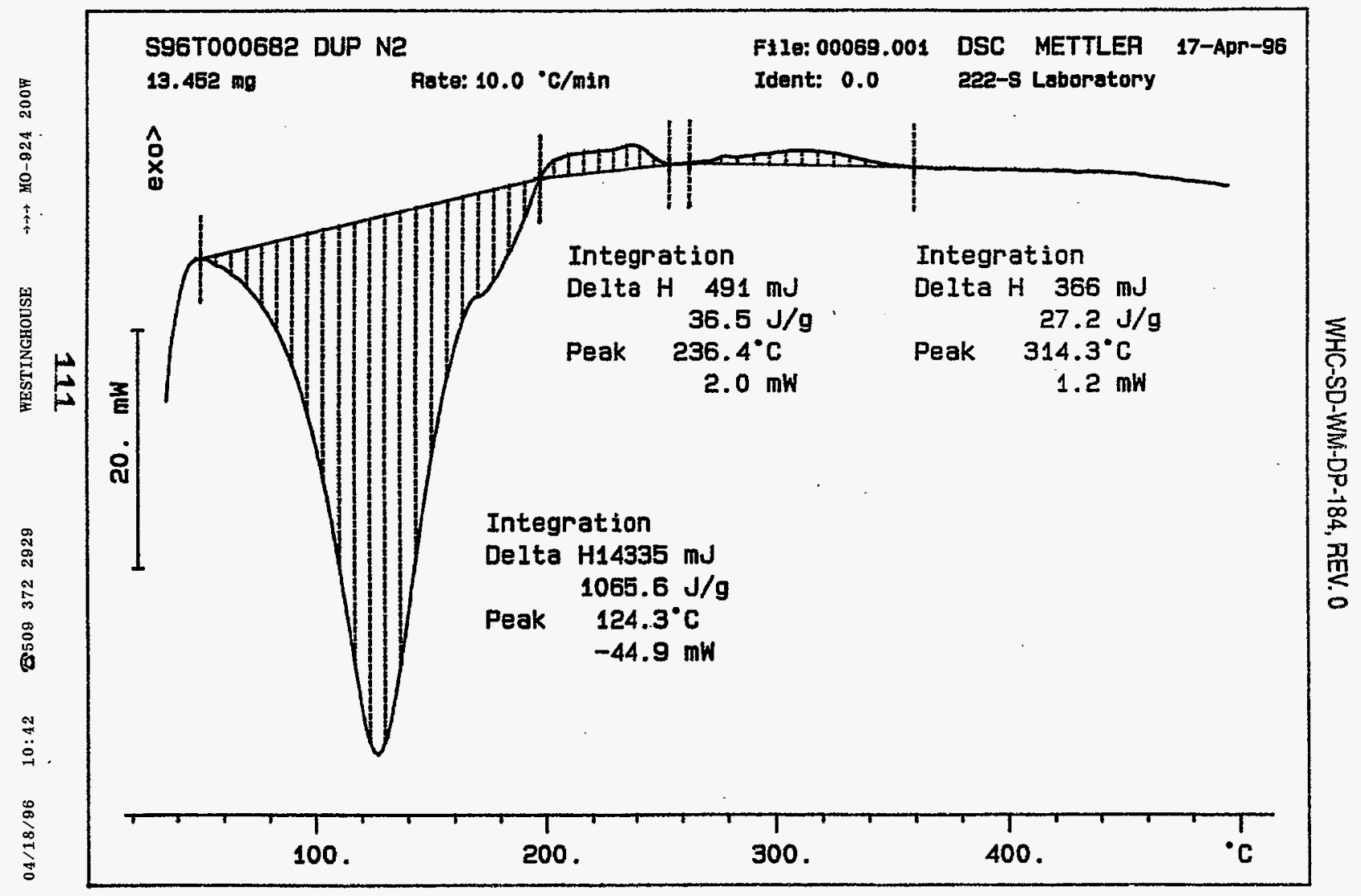


$\underline{\ldots}=04 / 24 / 96 \quad 06: 32 \quad 85093722929$.

WESTINGHOUSE $\rightarrow \rightarrow \rightarrow$ MO-

003

worklistrpt Version 2.1 05/15/95

WHC-SD-WM-DP-184, REV. 0

$03 / 2019611: 13$

LABCORE Data Entry Template for Worklist\#

Page: $\quad I$

6753

Analyst: $\quad 27 / 2$ Instrument: $\mathrm{DSCO} \perp$ Book $\# 12 h / 4-\mathcal{B}$

Method: LA-514-113 Rev/Mod C- L

Worklist Comment: U-107 FOR DSC-01 PLEASE RUN UNDER N2 RTS!

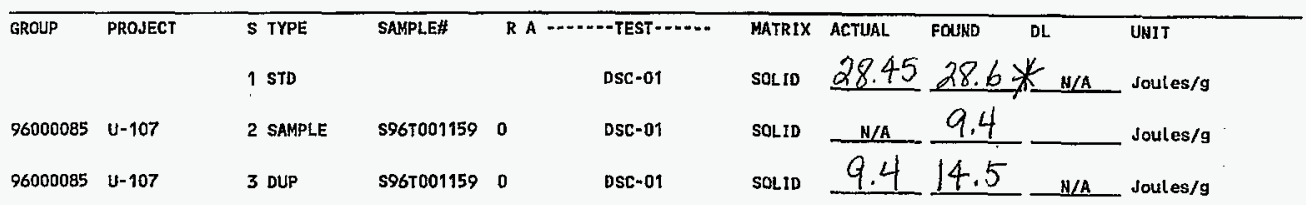

Final page for worklist \# $\quad 6753$

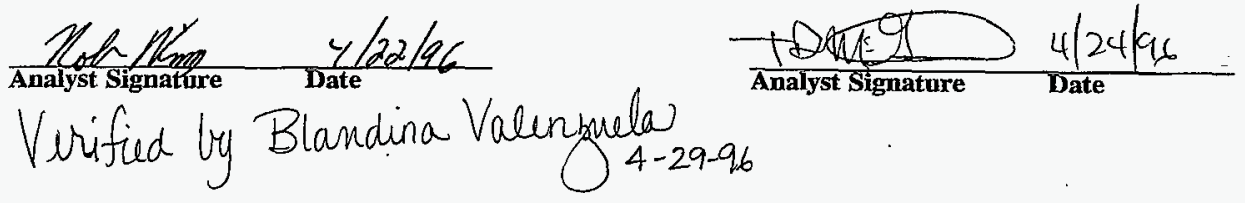

This sample is a composite sample gq- 6-3-96

Data Entry Comments: Sample produced two endothermic regions, one with a peakat $145.4^{\circ} \mathrm{C}$ with a delta $H$ of $582.4 \mathrm{Jg}$ and second at $283.9 \mathrm{i}$ with a delta $H$ of $88.1 \mathrm{~J} / \mathrm{g}$.

Units shown for QC (SPK \& STD) may not reflect the actual units. $D L=$ Detection Limit, $S=$ Worklist Slot Number, $R=$ Replicate Number, $A=$ Aliquot Code.

112 


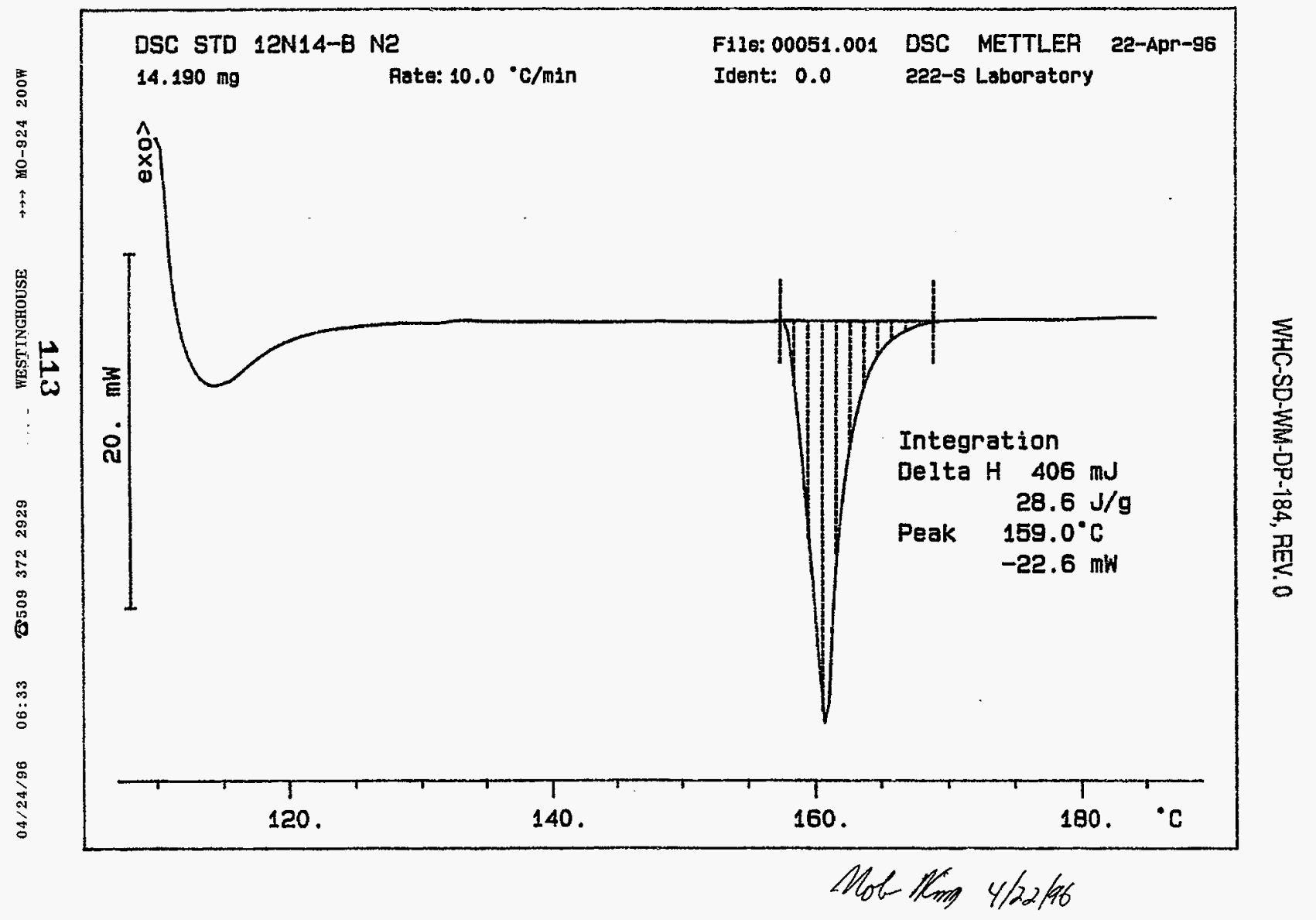




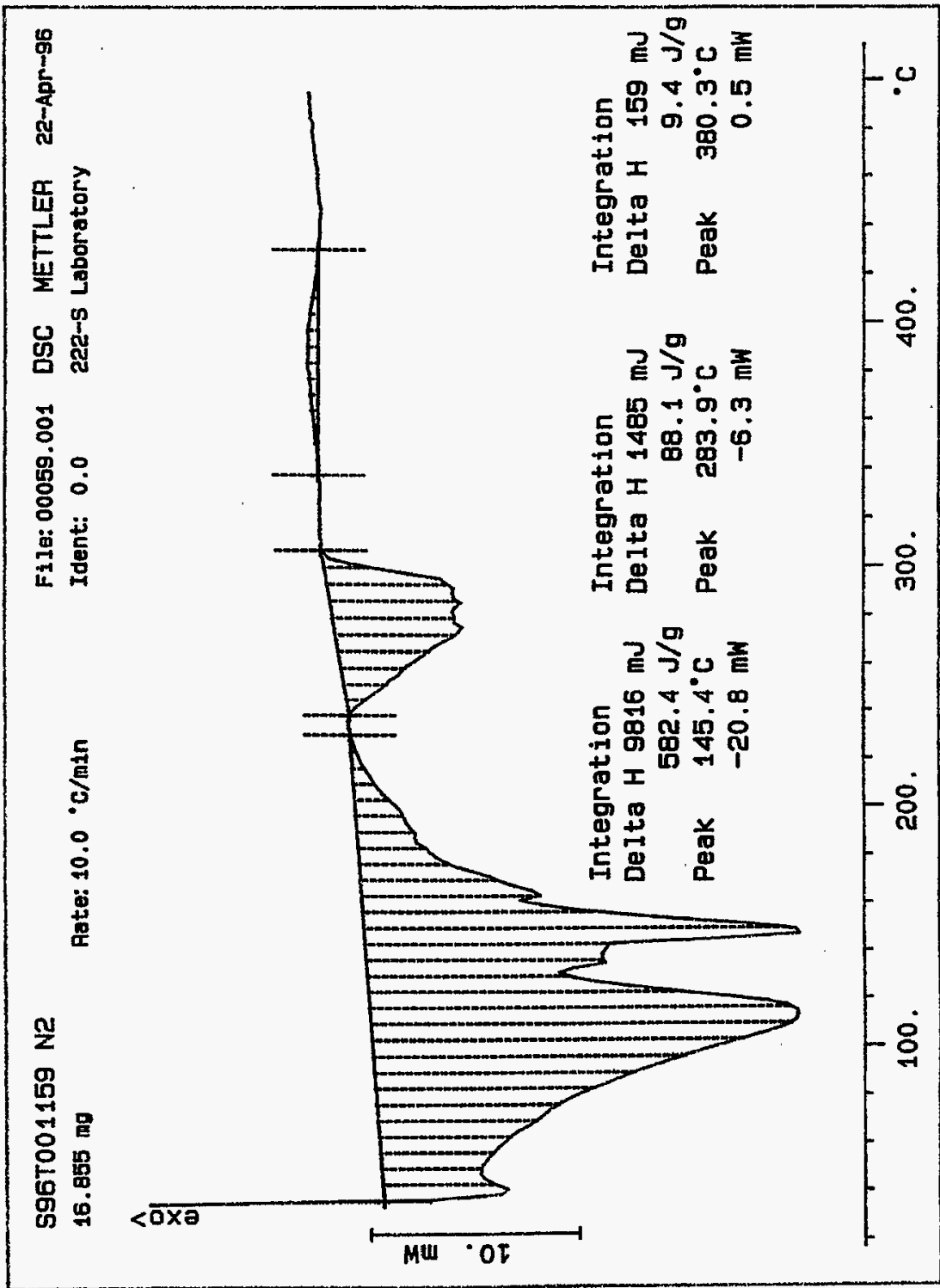

रे

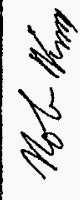




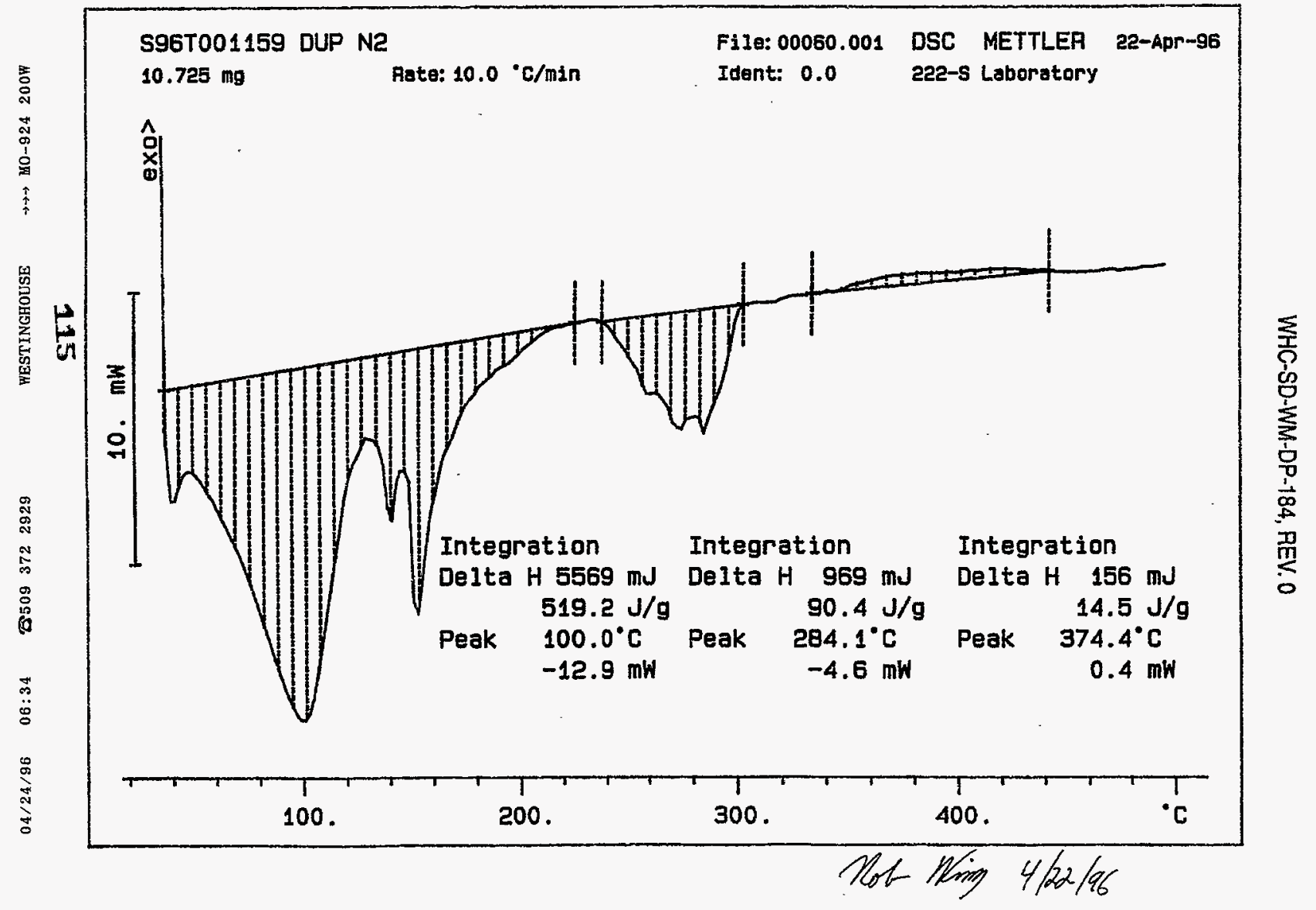




\section{LABCORE Data Entry Template for Worklist\#}

Page: $\quad 1$

7587

Analyst: SMF Instrument: $\mathrm{DSCO} 3$ Book \#12N14B

Method: LA-514-114 Rev/Mod C-1

Worklist Comment: U-107 DSC RUN UNDER N2. RCJ

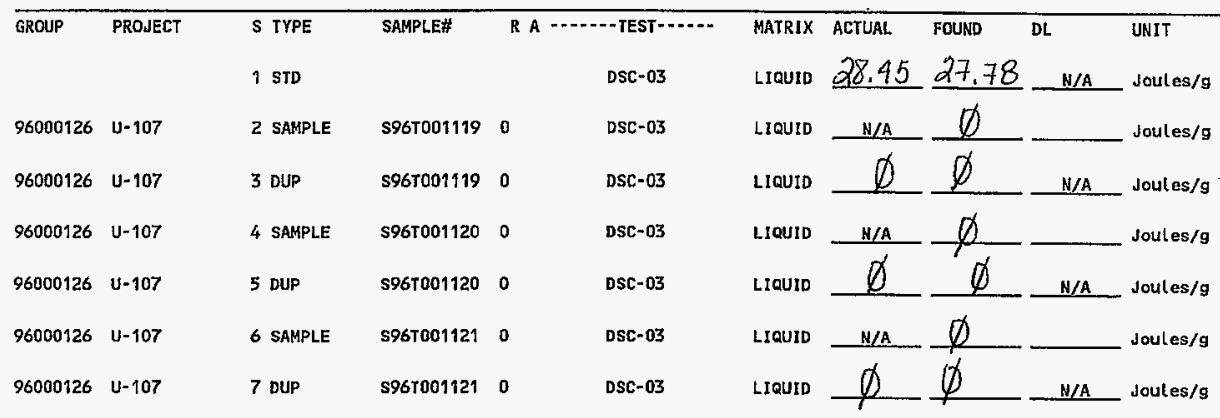

\section{Final page for worklist \# 7587}

\section{Se attached for signatures} Analyst Signature Date $4 / 17 / 96$

Verified by Hnasto 4-22-96

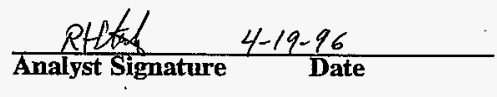

5967001119 produced an endotherm at $124.5^{\circ} \mathrm{C}$ with a delta $\mathrm{H}$ of $1094.9 \mathrm{Jg}$.

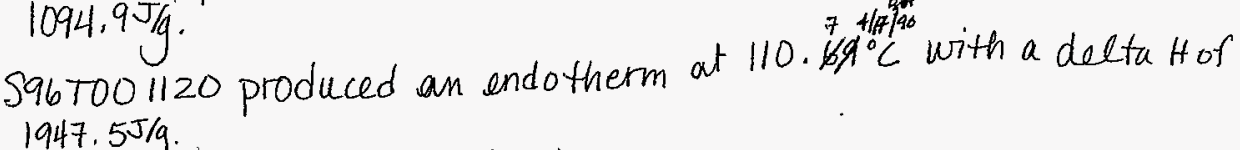

Data Enry Comnets:s: $\$ 967001121$ produced two endo therms one at $113.8^{\circ} \mathrm{C}$ With a delta $\mathrm{H}$ of $2097.76 \mathrm{~J} / \mathrm{g}$ and second at $301.5^{\circ} \mathrm{C}$ with a delta $\mathrm{H}$

Units shown for QC (SPK \& STD) may not reflect the actual units. DL = Detection Limit, $S=$ Worklist Slot Number, $R=$ Replicate Number, $A=$ Aliquot Code. 


\section{LABCORE Data Entry Template for Worklist\#}

Analyst: $\quad$ SMF Instrument: DSC0

Book $\# 12 N 14 B$

Method: LA-514-113 Rev/Mod

Worklist Comment: U-107 DSC RUN UNDER N2. RCJ

\begin{tabular}{|c|c|c|c|c|c|c|c|c|c|c|}
\hline \multirow[t]{2}{*}{ GROUP } & \multirow[t]{2}{*}{ PROJECT } & S TYPE & \multirow[t]{2}{*}{ SAMPLE\# } & \multicolumn{2}{|c|}{ R A $\cdots$ TEST $\cdots$} & \multirow{2}{*}{$\begin{array}{l}\text { MATRIX } \\
\text { LIOUID }\end{array}$} & \multirow[t]{2}{*}{ ACTUAL } & \multirow[t]{2}{*}{ FOUND } & \multirow[t]{2}{*}{ DL } & \multirow{2}{*}{$\begin{array}{l}\text { UNIT } \\
\text { Joules/g }\end{array}$} \\
\hline & & 1 STo & & & DSC-01 & & & & & \\
\hline 96000126 & $U-107$ & 2 SAMPLE & S967001110 & $\mathbf{0}$ & DSC-01 & LIQUID & $\mathrm{N} / \mathrm{A}$ & & & Joul es/g \\
\hline 96000126 & $U-107$ & 3 DUP & s96T001110 & 0 & DSC-01 & LIQUID & & & N/A & Joules/g \\
\hline 96000126 & $U-107$ & 4 SAMPLE & 5967001119 & 0 & DSC-01 & LIRUID & $N / A$ & & & Joules/g \\
\hline 96000126 & $U-107$ & 5 DUP & S96T001719 & $\mathbf{0}$ & DSC-01 & LIQUID & & & N/A & Joules/g \\
\hline 96000126 & $U-107$ & 6 SAMPLE & s96T001120 & 0 & DSC-01 & LIQUID & $N / A$ & & & Joules/g \\
\hline 96000126 & $U-107$ & 7 Dup & S96T001120 & 0 & DSC-01 & LIQUID & & & N/A. & Joules/g \\
\hline 96000126 & $U-107$ & B SAMPLE & S96T001121 & 0 & DSC-01 & LIQUID & $\mathrm{N} / \mathrm{A}$ & & - & Joules/g \\
\hline 96000126 & $U-107$ & 9 DUP & s96t001121 & 0 & Dsc-01 & LIQUID & & & N/A & Joules/g \\
\hline
\end{tabular}

\section{Final page for worklist \# 7587}

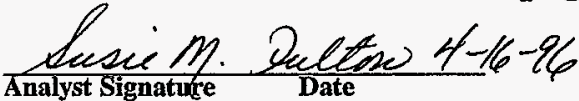

Analyst Signature

Data Entry Comments:

$596 T 001110-\operatorname{enp} P Y$

Units shown for QC (SPK \& STD) may not reflect the actual units. $D L=$ Detection Limit, $S=$ Worklist Slot Number, $R=$ Replicate Number, $A=$ Aliquot Code. 
Curve 1: DSC

Ftle Info: INB041601 Tue Apr 16 06: 46; 251996

Sample kejght: 7.900 ang

12N14-B INDILN AT 10CWMIN

SIGNATURE BELOH REPRESENTS CHEMICAL TECHNOLOGIST/CHEHIST, THAT

COMPLETED/VERIFIED THE CALIBRATION/ANALYSIS ON PAGES $/ / 8$ TO/23.1

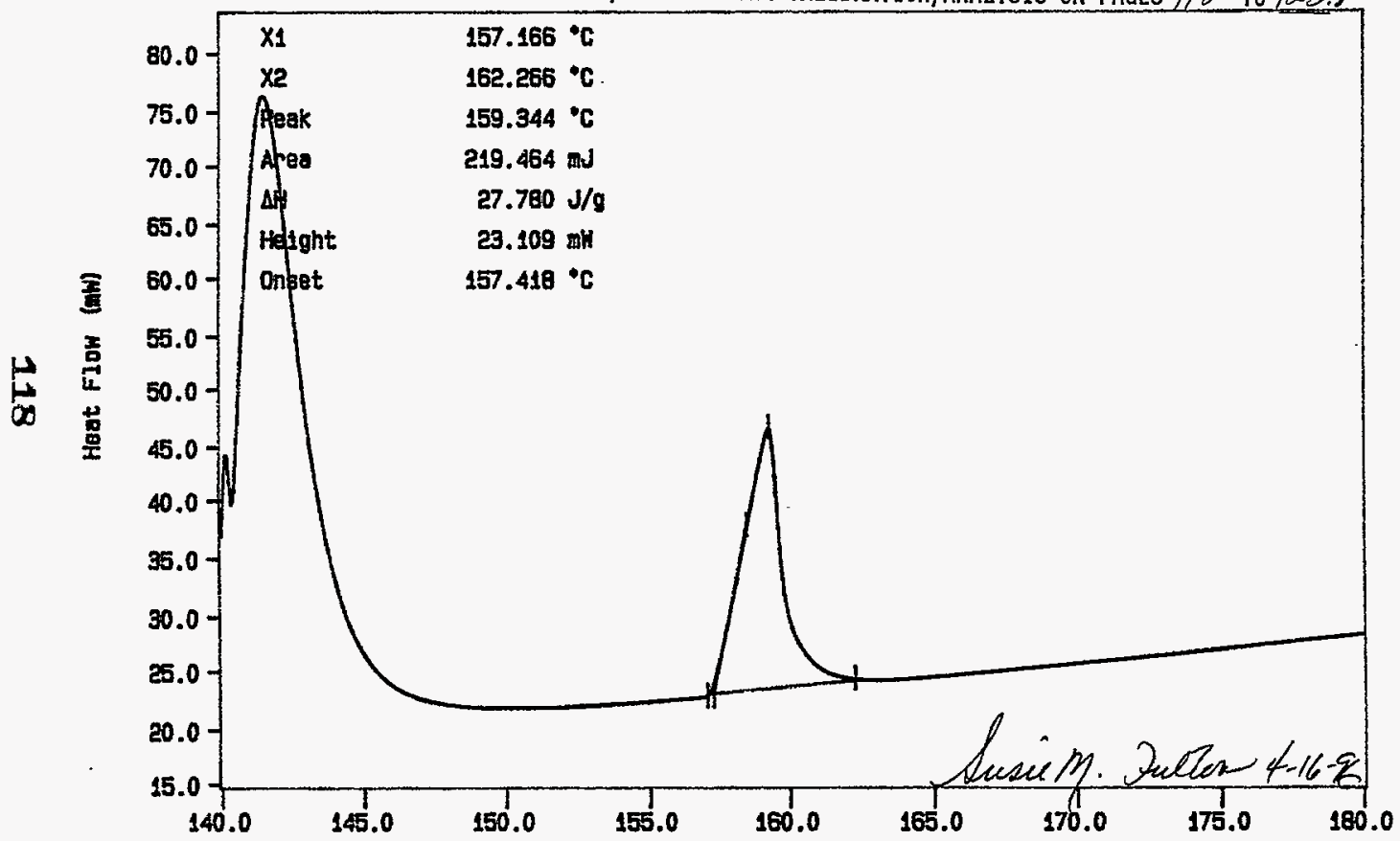

Ne. EXOTHEPY DOWN

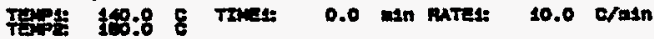


Curve 1: DSC

F1le Info: SAM041601 Tue Apr 16 09:20:00 1996

Sample Haight: $15.240 \mathrm{mg}$

S967001119

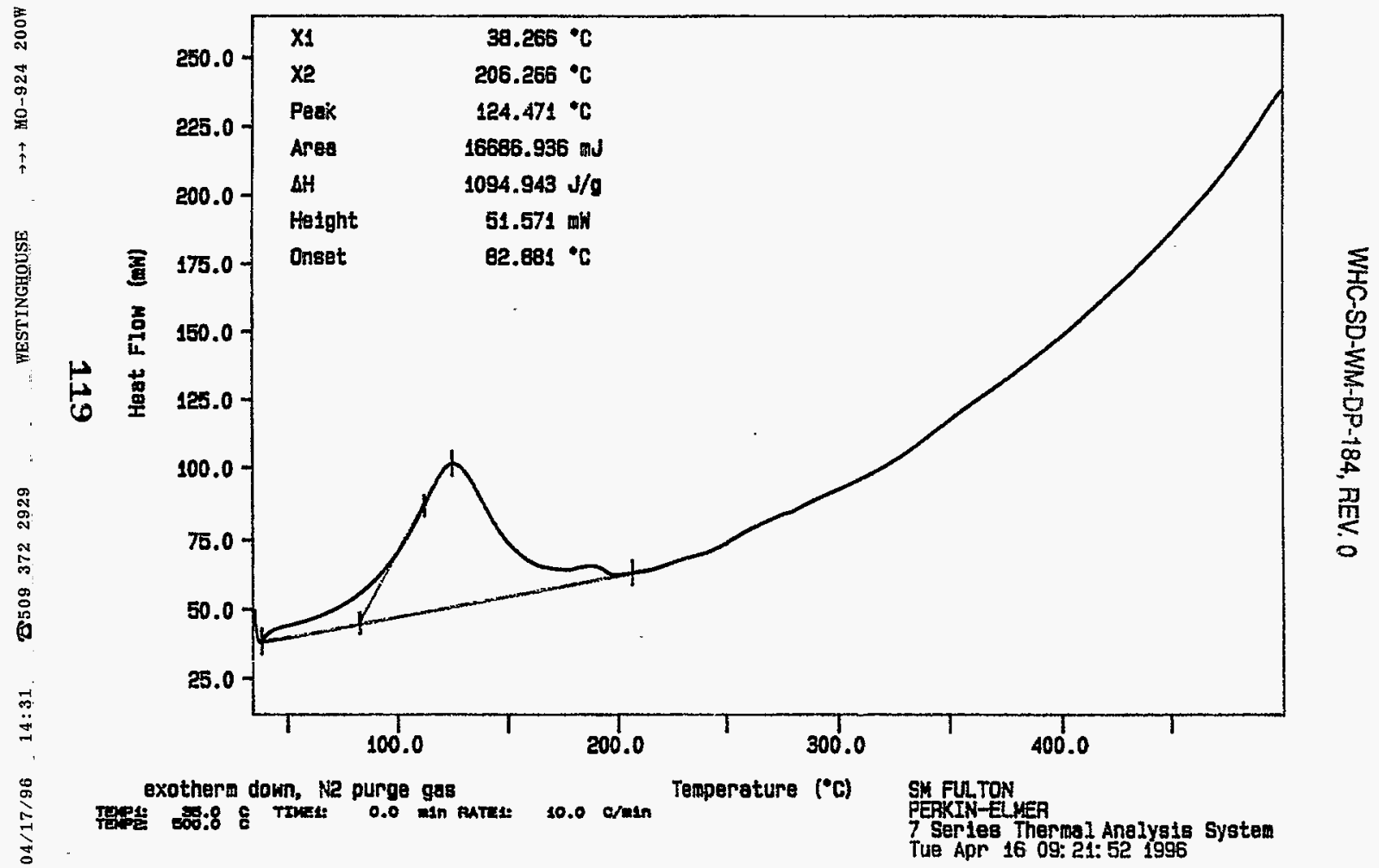


WHC-SD-WM-DP-184, AEV. 0

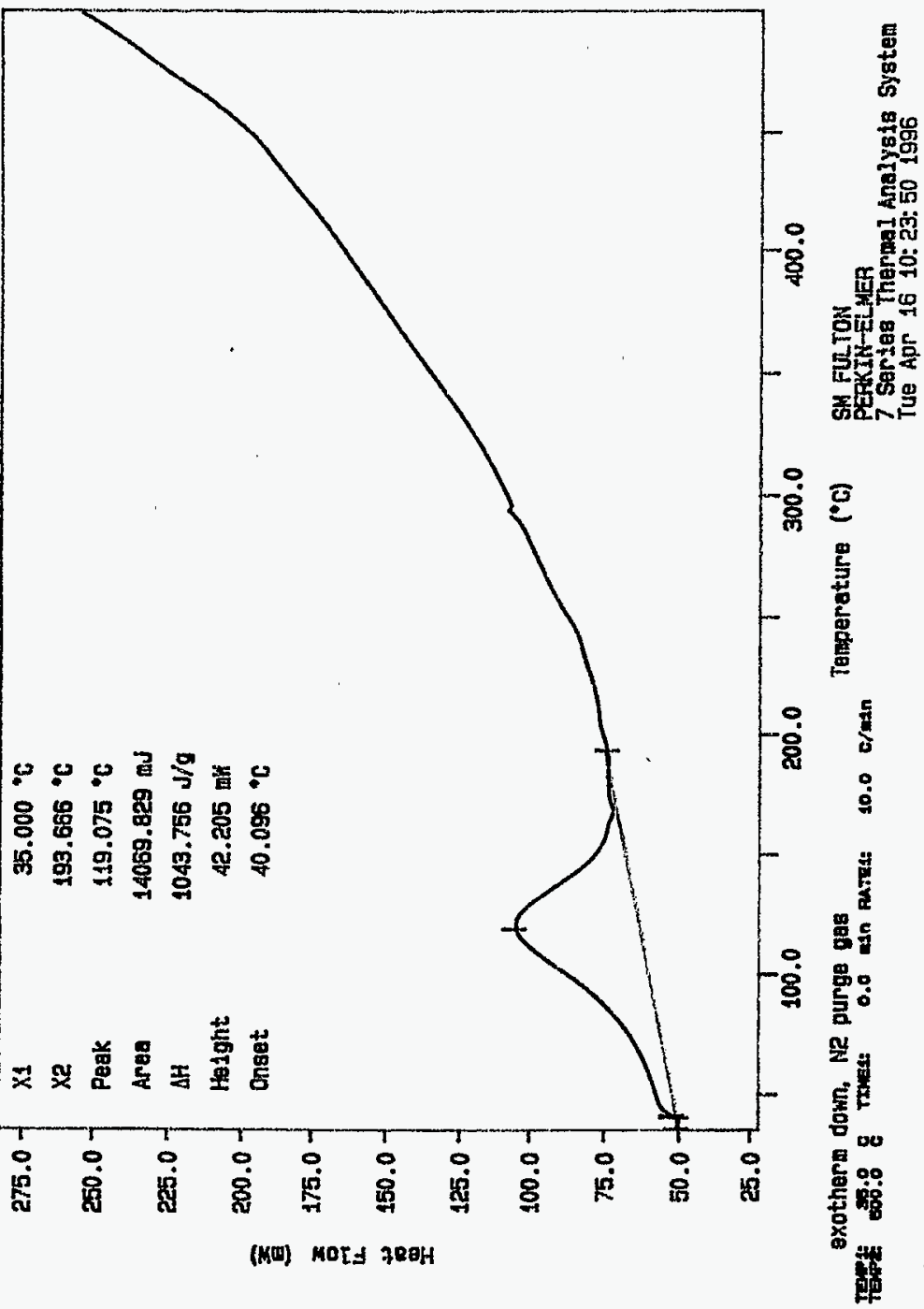

120 
ํ. Curve 1: DSC

F11e info: SAM041603 TuB Apr 16 11: 28: 171996

Sample Netght: 10.900

$4 \mathrm{~g}$

S96T001120

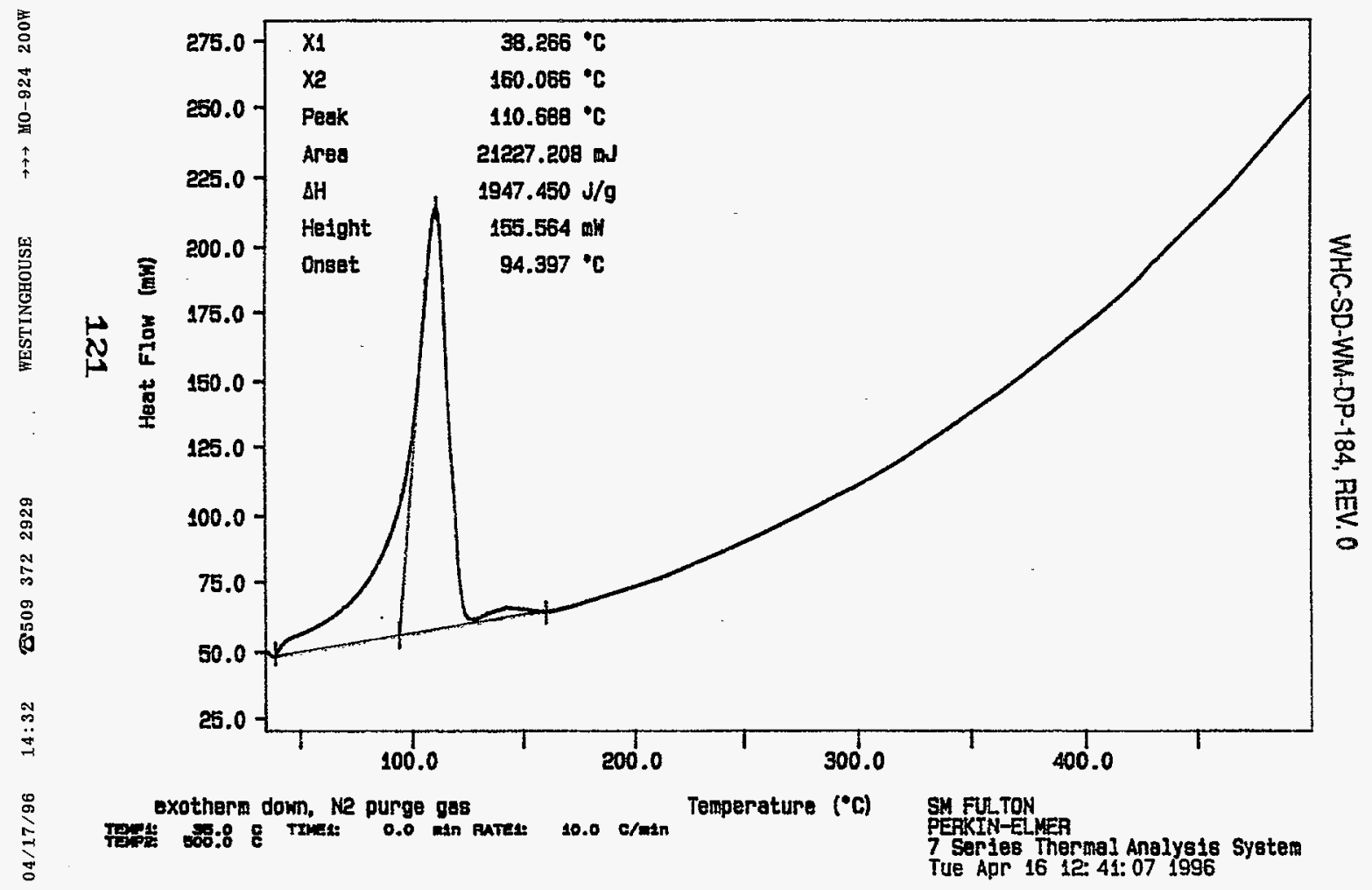


File Info: SAN041604 Tue Apr 16 13:38: 491996

Sample Weight: 10.780 mg

s96T001120 DUP

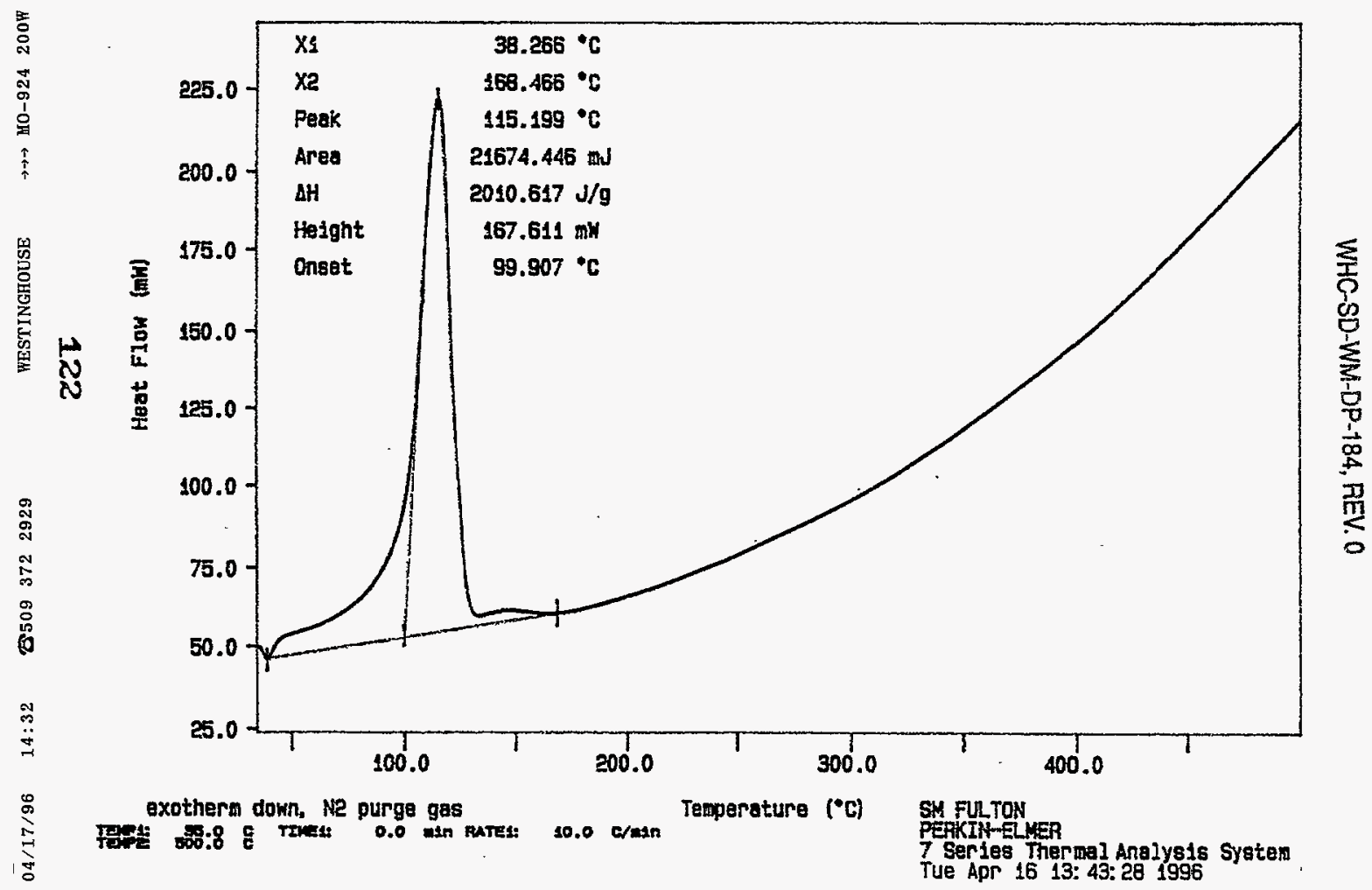


WHC-SD-WM-DP-184, REV. 0

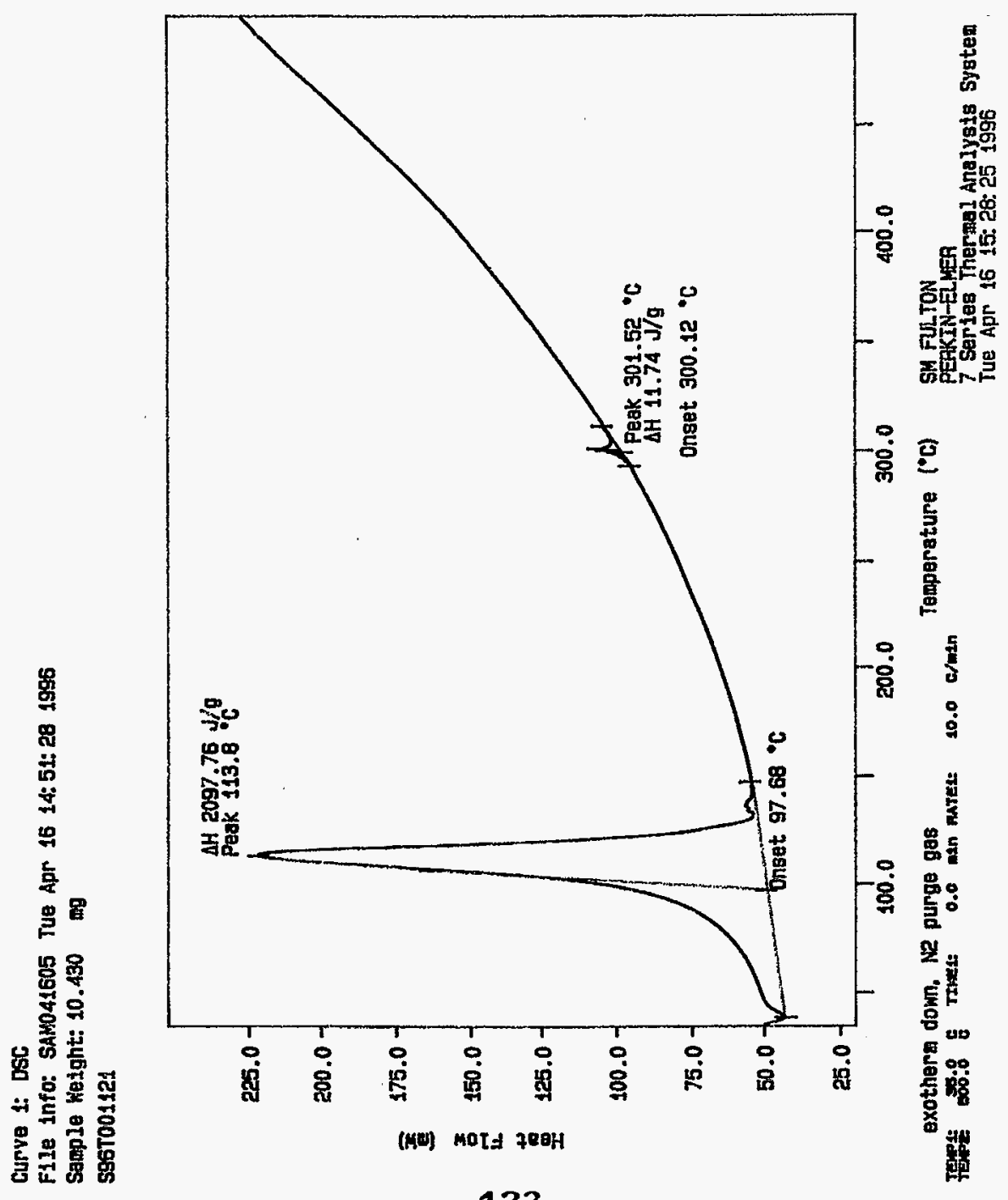

\section{3}


Curve 1: DSC

F1le info: SAM041606 Tue Apr 16 16: 27:03 1996

Sample Weight: 10.040 ang

SSGT001121 DUP

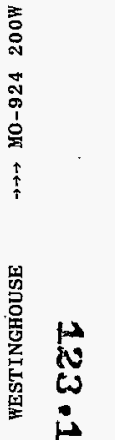

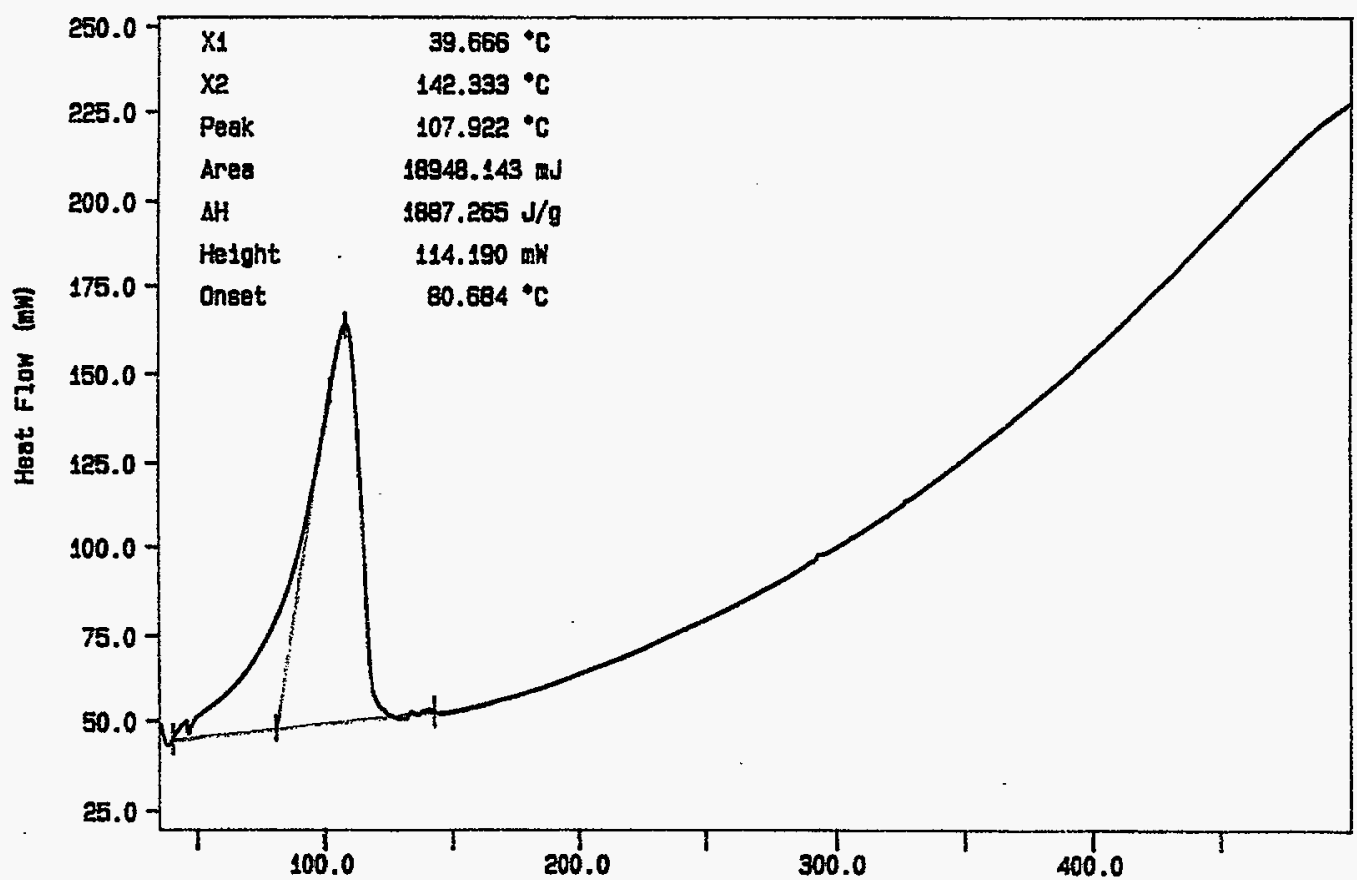

exotherw down, Ne purge gas

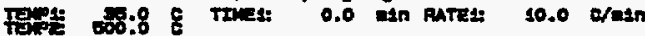

Temperature $\left({ }^{\circ} \mathrm{C}\right)$ SM FULTON

PERIKIN-ELMEA

7 Sertes Thermel Analys is Syaten Tue ApR 16 16: 28: 32 1996 


\section{LABCORE Data Entry Template for Worklist\#}

\section{Analyst: KRM Instrument: DSC0 _ Book\#12N14B}

Method: LA-514-113 Rev/Mod $\mathrm{C}-1$

Worklist Comment: U-107, Run under Nitrogen. new

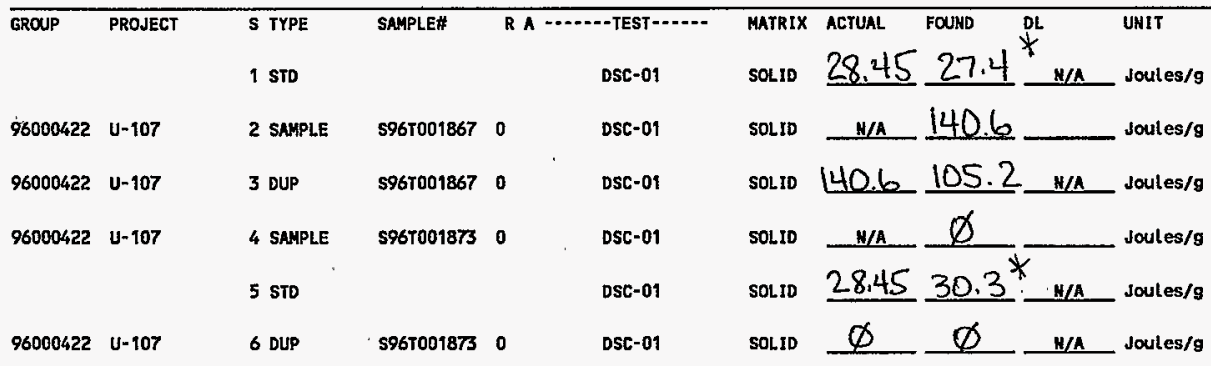

\section{Final page for worklist \# $\quad \mathbf{8 0 2 5}$}

See attached for Signatures
Analyst Signature
Date
by dinastor 5-13-96

Units shown for $Q C$ (SPK \& SID) may not reflect the actual units. $D L=$ Detection Limit, $S=$ Worklist Slot Number, $R=$ Replicate Number, $A=$ Aliquot Code. 


\section{LABCORE Data Entry Template for Worklist\#}

Analyst: $\quad K R M \quad$ Instrument: $D S C 0 \ldots$ Book \# $12 \mathrm{~N} / 4 \mathrm{~B}$

Method: LA-514-113 Rev/Mod C.I

Worklist Comment: U-107, Run under Nitrogen. new

\begin{tabular}{|c|c|c|c|c|c|c|c|c|c|c|}
\hline \multirow[t]{2}{*}{ GROUP } & \multirow[t]{2}{*}{ PROJECT } & S TYPE & SAMPLEA & \multicolumn{2}{|c|}{ R A } & MATRIX & ACTUAL & FOUND & DL & UNIT \\
\hline & & 1 STO & . & & DSC-01 & SOLID & & & & Joules/g \\
\hline 96000422 & $U-107$ & 2 SAMPLE & S967001867 & 0 & DSC-01 & SOLID & $N / A$ & & & Joules/g \\
\hline 96000422 & $u-107$ & 3 DUP & s967001867 & 0 & DSC-01 & SOLID & & & & Joules/g \\
\hline 96000422 & $i-107$ & $\begin{array}{l}4 \text { SAMPLE } \\
\text { STO }\end{array}$ & \$967001873 & 0 & DSC-01 & SOLID & $\mathbf{N} / \mathbf{A}$ & & & Joules/g \\
\hline 96000422 & $v-107$ & 5 Dup & \$967001873 & 0 & DSE-01 & SOLID & . & & & Joules/g \\
\hline
\end{tabular}

\section{Final page for worklist \# $\quad 8025$}
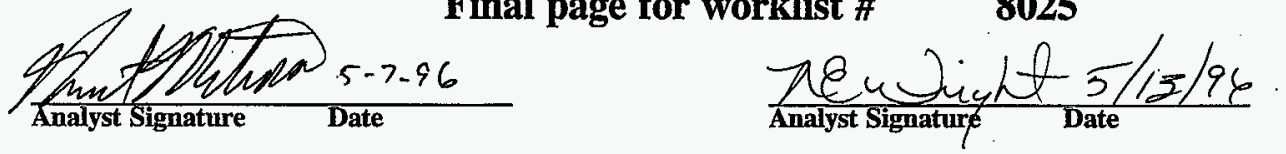

Data Entry Comments:

Units shown for $Q C$ (SPK \& STD) may not reflect the actual units. $D L=$ Detection Limit, $S=$ Worklist Slot Number, $R=$ Replicate Number, $A=$ Aliquot Code. 
SIGNATURE BELOH REPRESENTS CHEMICAL TECHNOLOGIST/CHEHIST THAT

COMPLETED/VERIFIED THE CALIBRATION/ANALYSIS ON PAGES $/ 2 \mathrm{~K}$, TO $/ 3 \%$

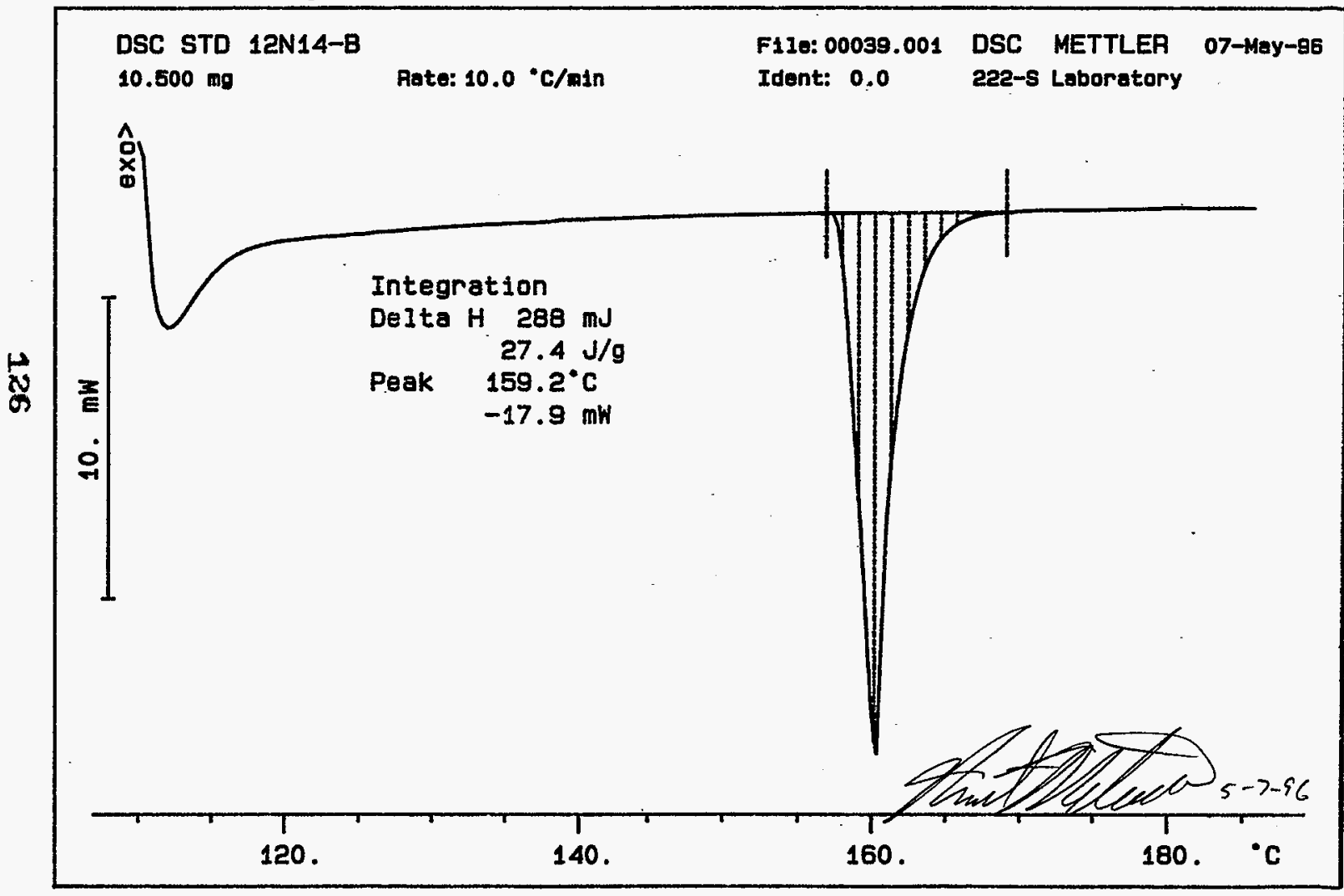


WHC-SD-WM-DP-184, REV. 0

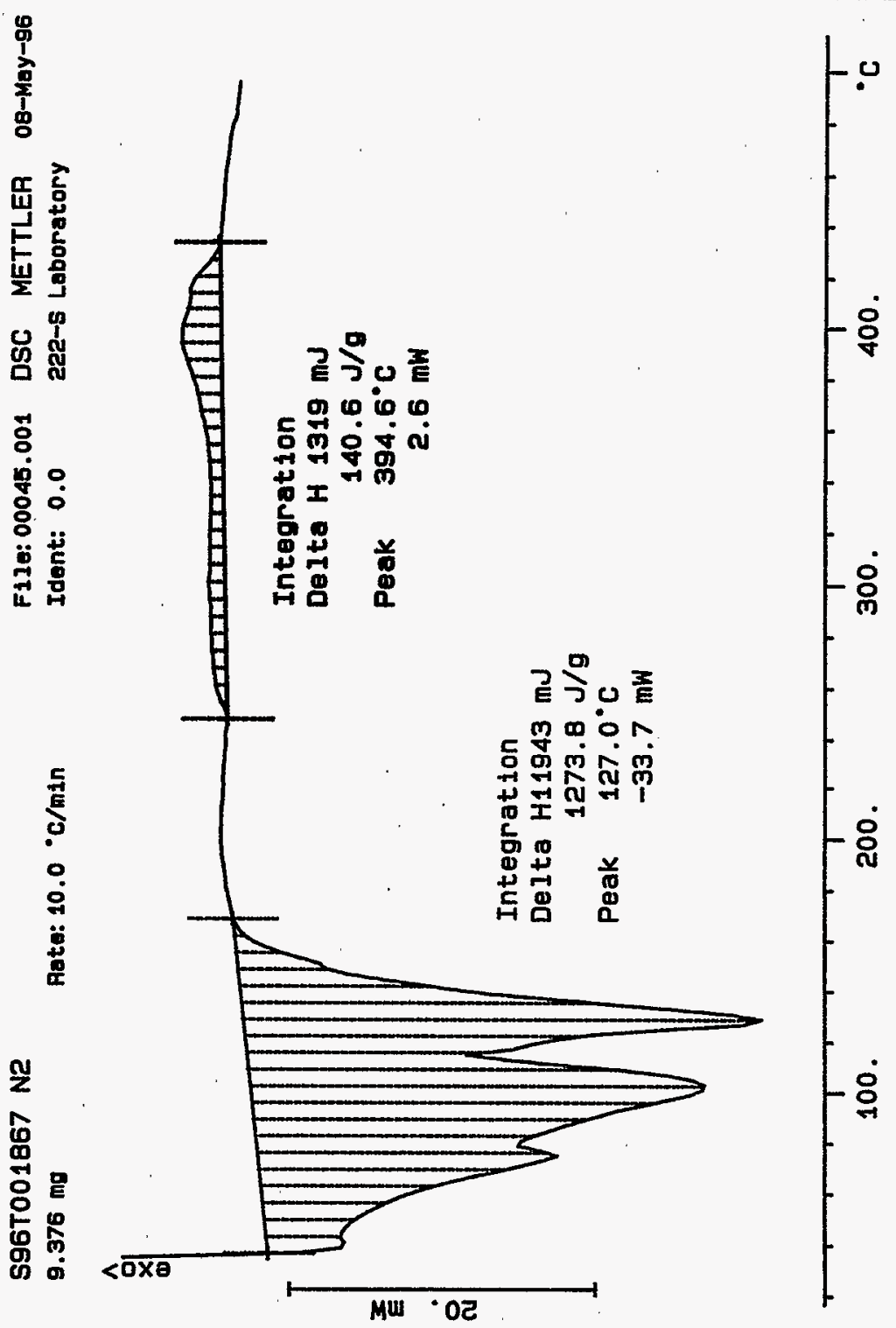

127 


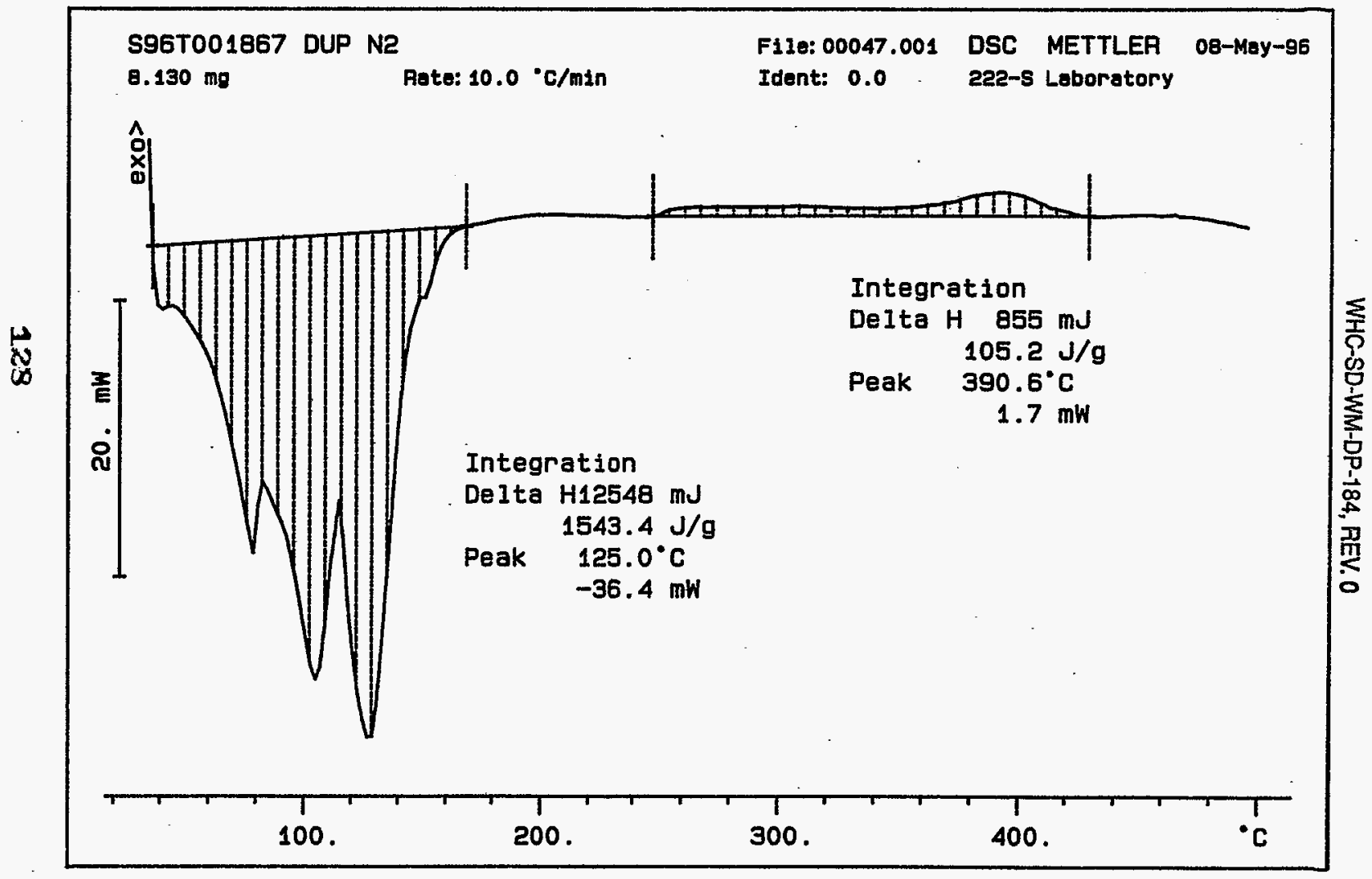


WHC-SD-WM-DP-184, REV. 0

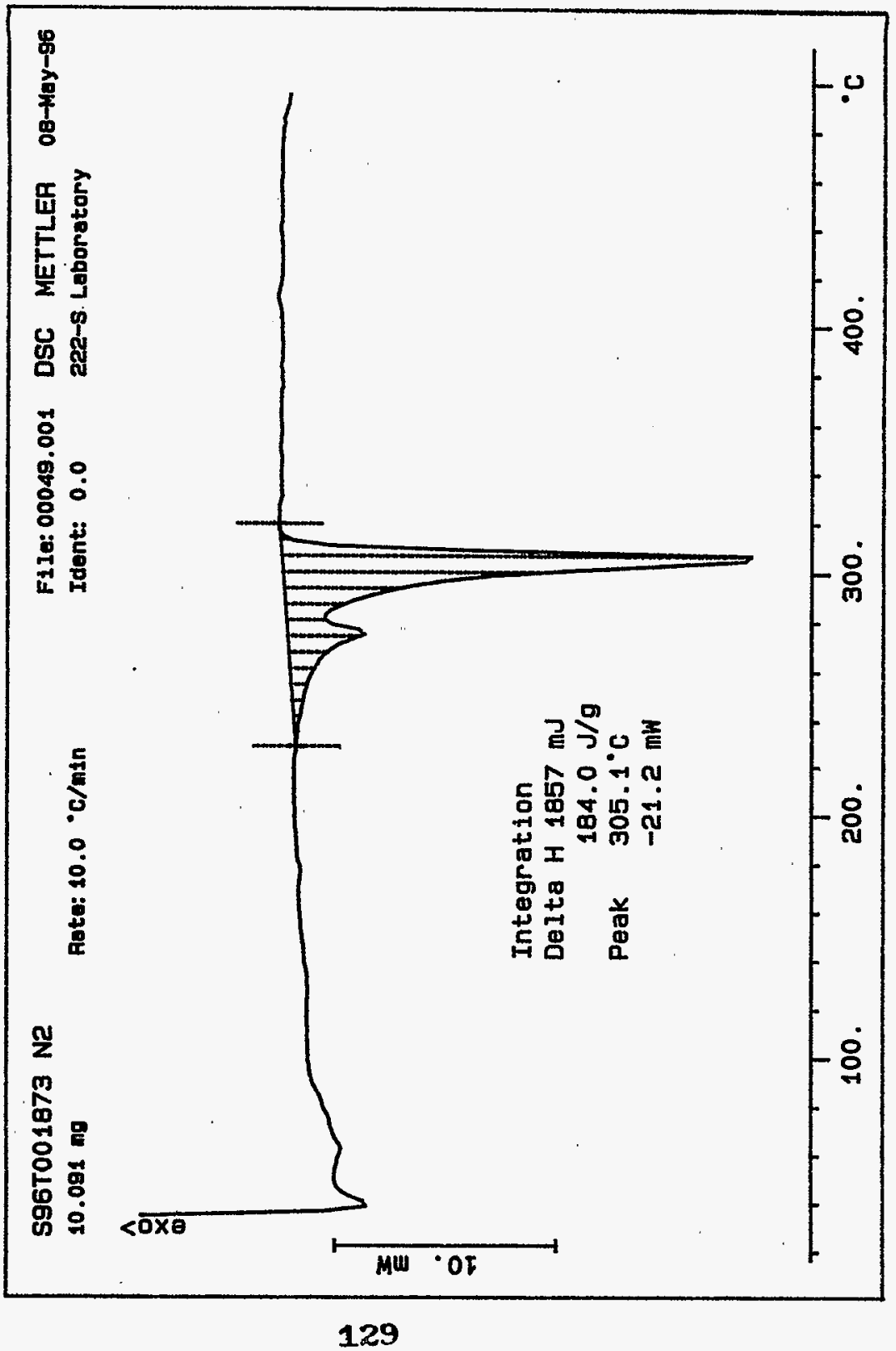


WHC-SD-WM-DP-184, REV. 0

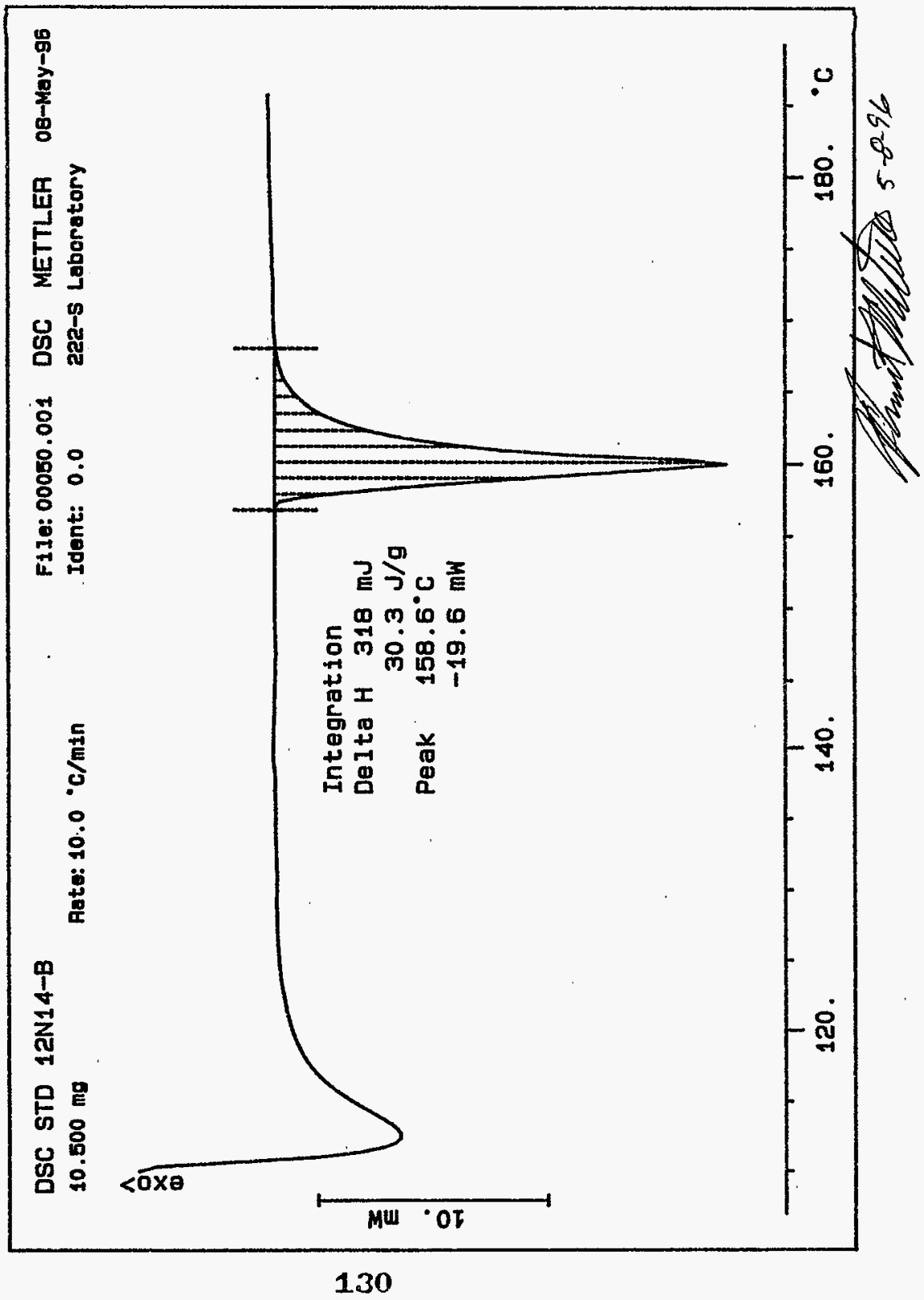


WHC-SD-WM-DP-184, REV. 0

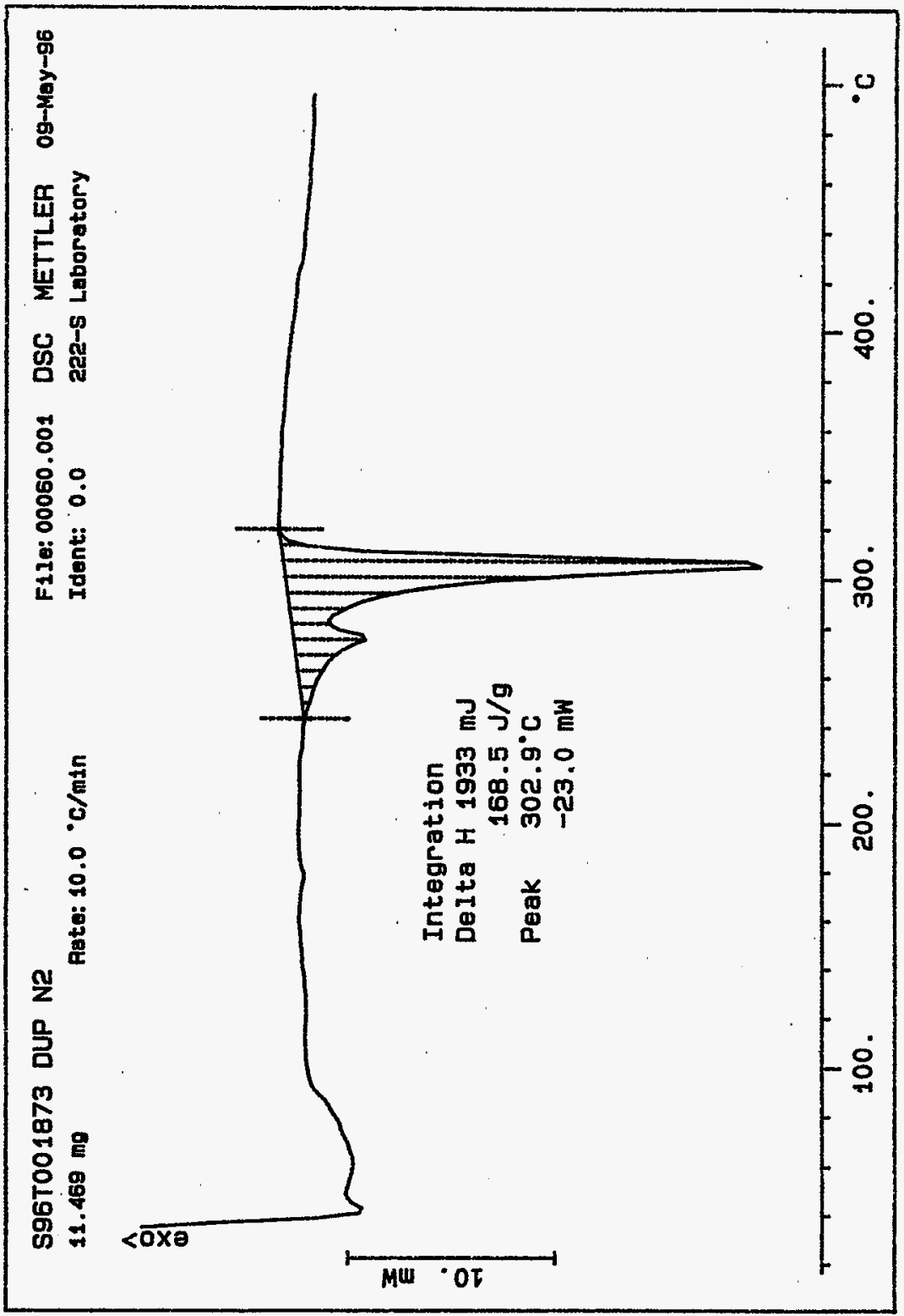




\section{LABCORE Data Entry Template for Worklist\#}

Analyst: ADP Instrument: DSC0 I_ Book\#12N14 B

Method: LA-514-113 Rev/Mod C-I

Worklist Comment: U-107. Run under nitrogen. new

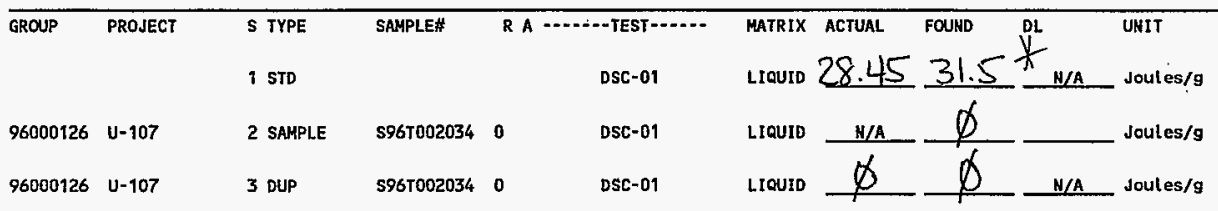

Final page for worklist \#

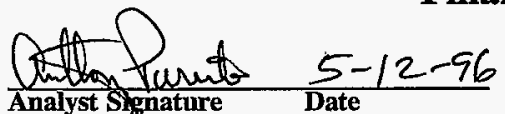

Analyst Spnature Date

Validated by Hanastos 5-14-96

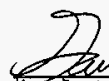

Analyst Signature
$5-1396$

Date

Data Entry Comments:

Units shown for $Q C$ (SPK \& STD) may not reflect the actual units. $D L=$ Detection Limit, $S=$ Worklist Slot Number, $R=$ Replicate Number, $A=$ Aliquot Code. 
SIGNATURE BELOW REPRESENTS CHEMICAL TECHNOLOGIST/CHEMIST THAT

COMPLETED/VERIFIED THE CALIBRATION/ANALYSIS ON PAGES 133 TO 135.

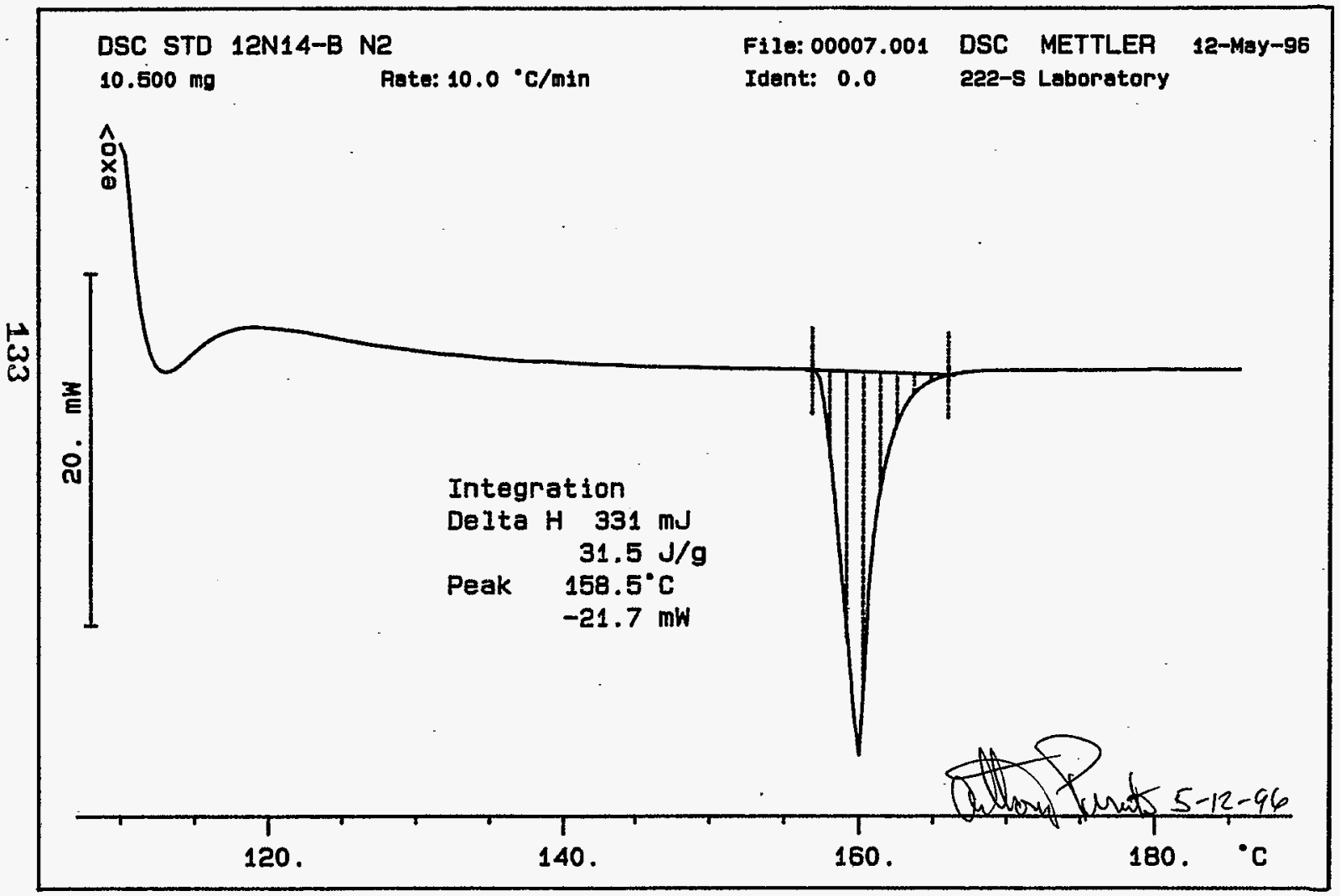




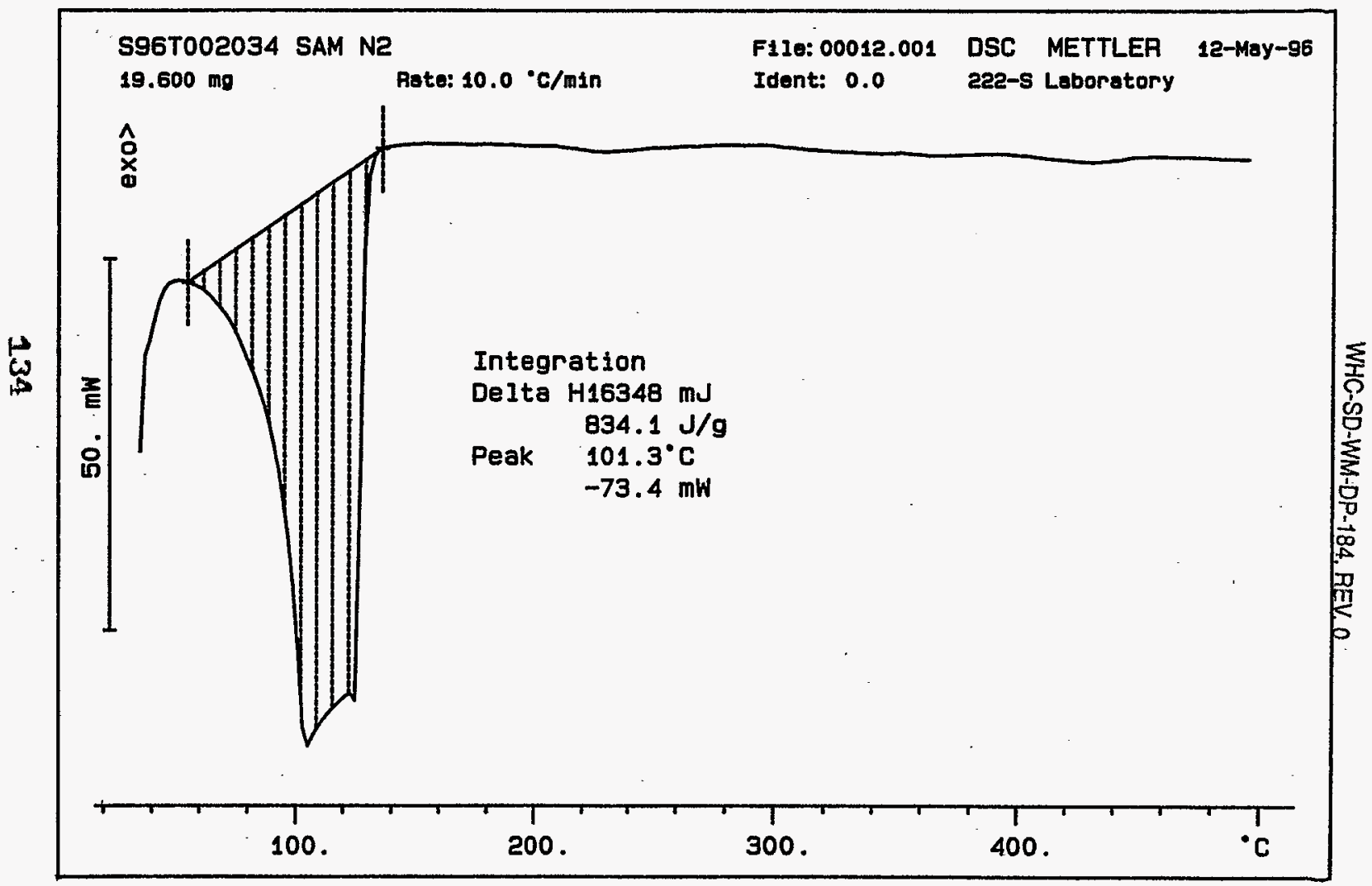




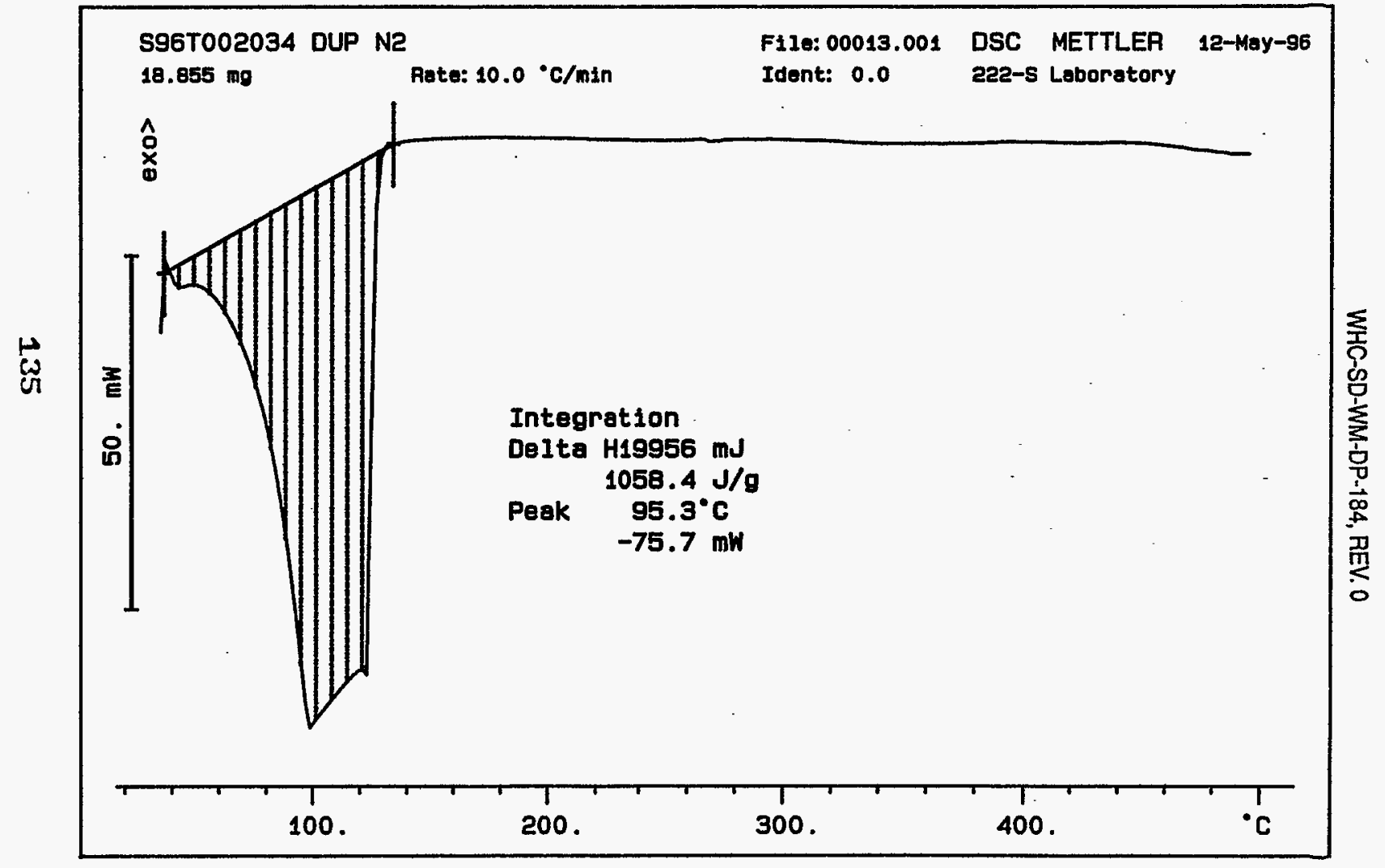




\section{LABCORE Data Entry Template for Worklist\#}

Analyst: $\quad$ ADP $\quad$ Instrument: DSCO 3 Book \#12N14B

Method: LA-514-114 Rev/Mod $C-1$

Worklist Comment: U-107. Run under nitrogen. new

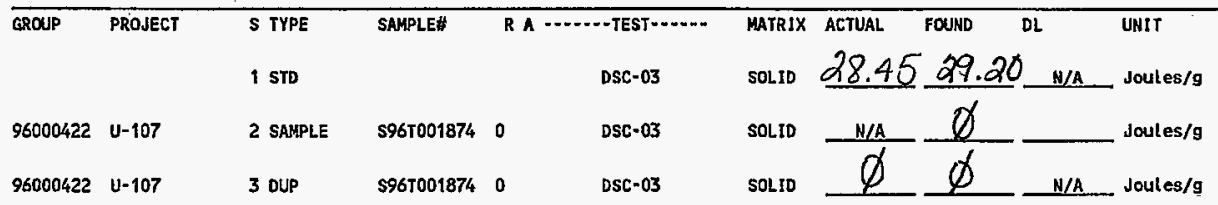

Ile attached for sianatures Analyst Signature Date $\bigcup 5-21-96$ Validated by Nhnastor 5.22 .96

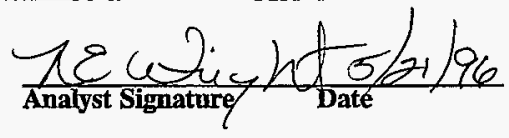

Data Entry Comments:

Units shown for $Q C$ (SPK \& STD) may not reflect the actual units. $D L=$ Detection Limit, $S=$ Worktist Slot Number, $R=$ Replicate Number, $A=$ Aliquot Code. 
worklistpt Version 2.1 05/15/95

WHC-SD-WM-DP-184, REV. 0

05/01/96 14:21

LABCORE Data Entry Template for Worklist\#

Page: 1

8230

Analyst: $A D P$ instrument: DSCO $3 \quad$ Book \#/2N/4B

Method: LA-514-113 Rev/Mod C-1

Worklist Comment: U-107. Run under nitrogen. new

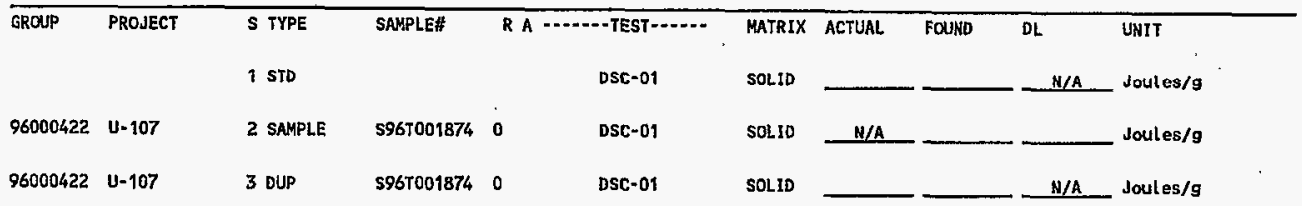

Final page for worklist \# $\quad \mathbf{8 2 3 0}$

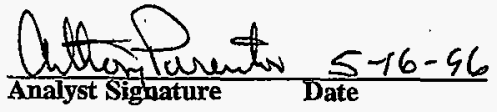

Analyst Signature Date

Other Instrument was used.

Data Entry Comments:

Units shown for $Q C$ (SPK \& STD) may not reflect the actual units. $D L=$ Detection Limit, $S=$ Worklist Slot Number, $R=$ Replicate Number, $A=$ Aliquot Code.

137 
Curve 1: DSC

File Info: IND051603 Thu May 16 22: 33: 501996

Sample Weight: $11.620 \mathrm{mg}$

12N14-B INDIUM AT 10CWIN

SIGNATURE BELOW REPRESENTS CHEMICAL TECHNOLOGIST/CHEMIST THAT

COMPLETED/VERIFIED THE CALIBRATION/ANALYSIS ON PAGES $/ 39$ TO $/ 40$.

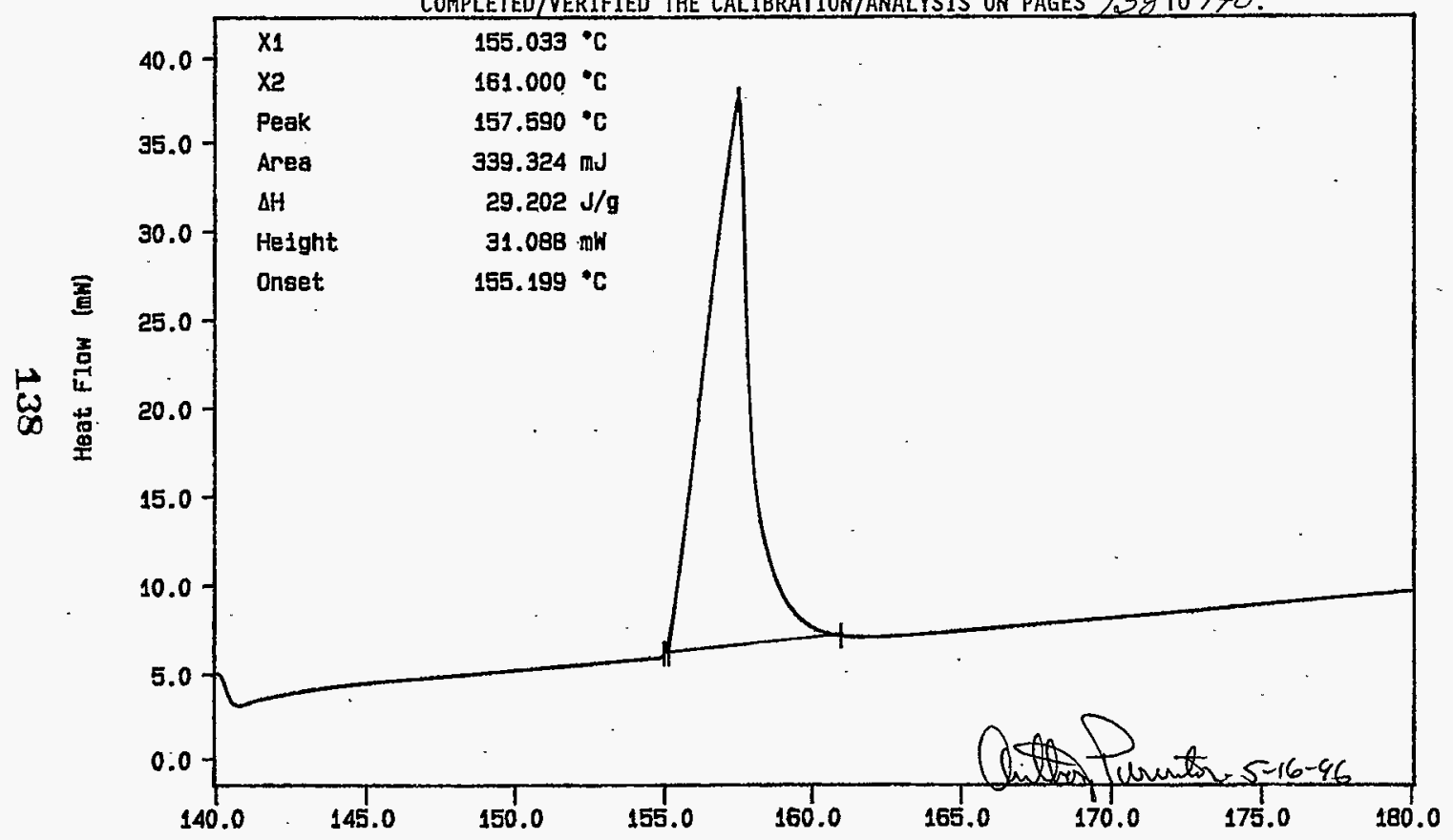

N2. EXOTHERM DOWN

TERPR: 140:8 $\mathrm{C}$ TIMES:

Temperature $\left({ }^{\circ} \mathrm{C}\right)$

AD PUATNTON

PERKIN-ELMER

7 Sertes Thermal Analysis System

Thu May 16 22 39: 44 1996 
Curve 1: DSC

F1le info: SAM051601 Thu May 16 23: 51: 231996

Sample Welght: $28.110 \mathrm{ng}$

S96T001874 SAM

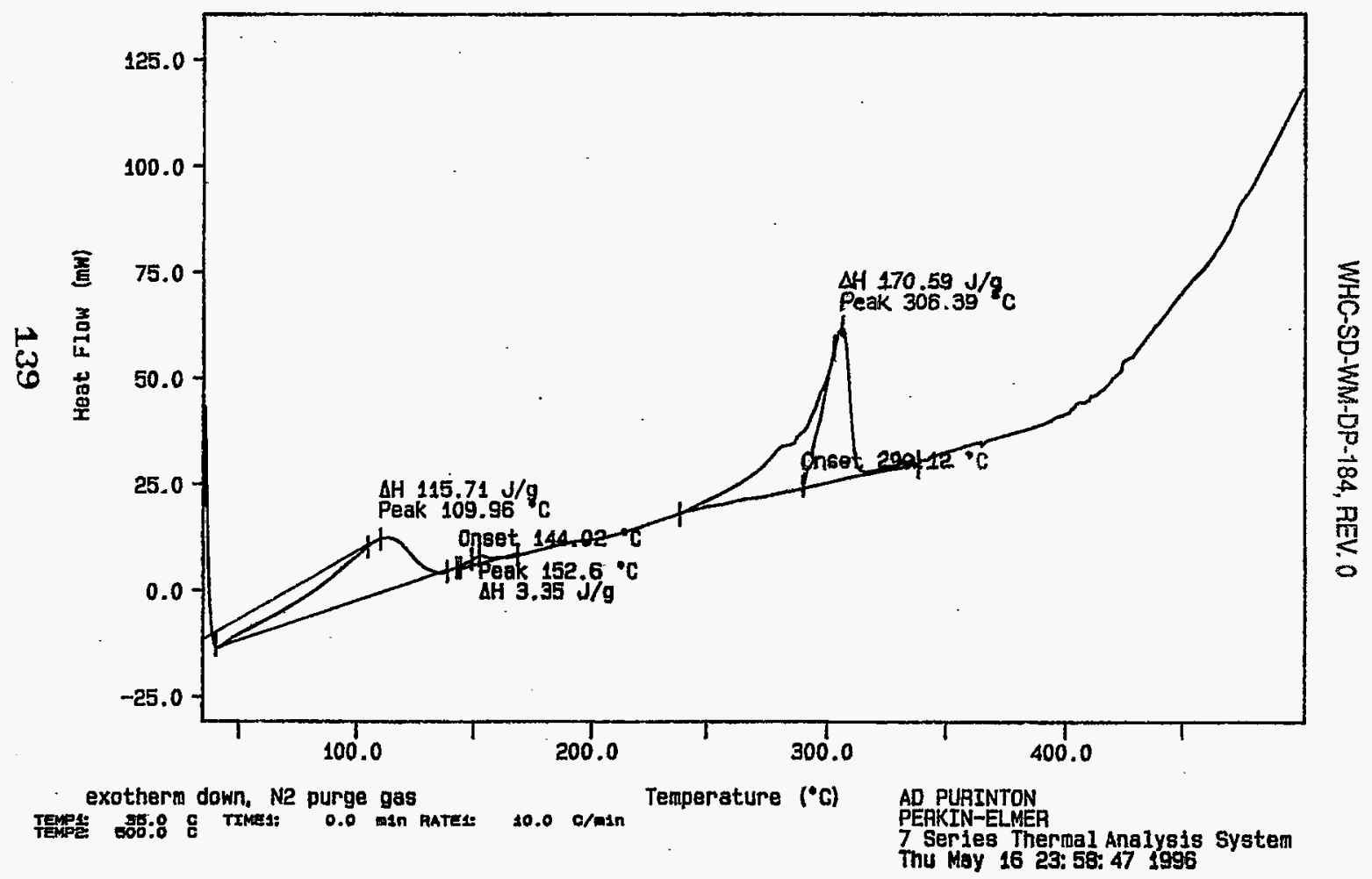




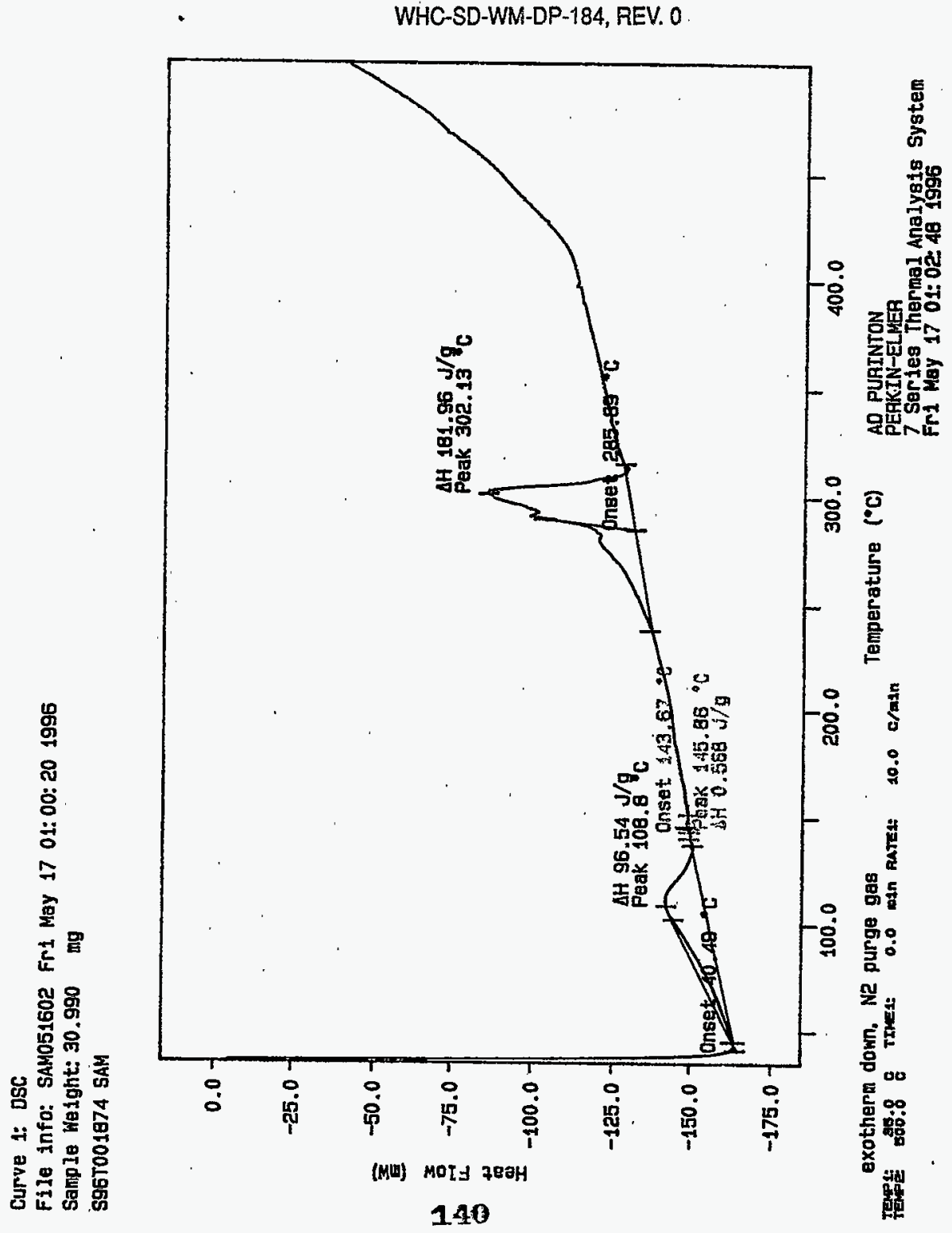


worklistrpt Version 2.1 05/15/95

WHC-SD-WM-DP-184, REV. 0

Page: 1

05/21/96 09:00

LABCORE Data Entry Template for Worklist\#

8245

\begin{tabular}{lll}
\hline Analyst: $\quad$ ADP & Instrument: & SC $\frac{71}{5 \cdot 2-96}$
\end{tabular} Book \#12N14B

Worklist Comment: U-107. Run under nitrogen. new

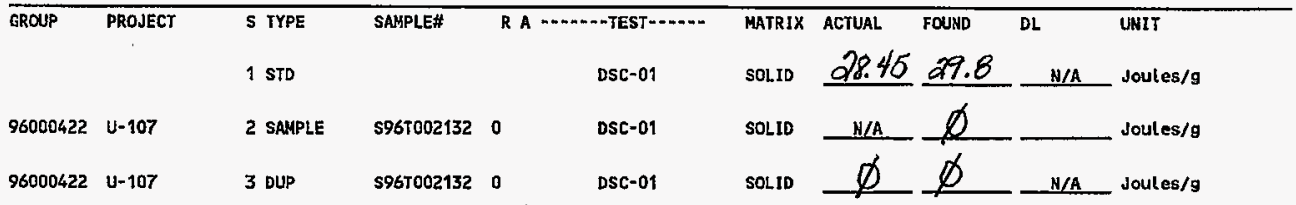

Final page for worklist \# $\quad \mathbf{8 2 4 5}$

Final page
Analyst Signature for signatures
5-2l-96
Dor

Validated by Hanasts 5.22.96

Data Entry comments: The second endotherm on the thermogram maybe due to Aluminum Hydroxide.

Units shown for $Q C$ (SPK \& STD) may not reflect the actual units. $D L=$ Detection Limit, $S=$ Worklist Slot Number, $R,=$ Replicate Number, $A=$ Aliquot Code.

141 


\section{LABCORE Data Entry Template for Worklist\#}

\section{Analyst: AlP Instrument: $\mathrm{DSCO} \longrightarrow$ Book \# 12 N $14 \mathrm{~B}$}

Method: LA-514-113 Rev/Mod C-1

Worklist Comment: U-107. Run under nitrogen, new

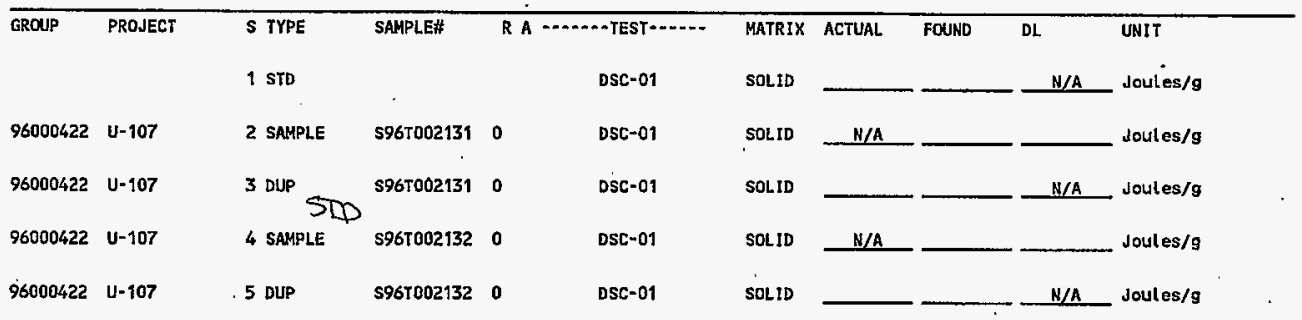

\section{Final page for worklist \# $\quad \mathbf{8 2 4 5}$}
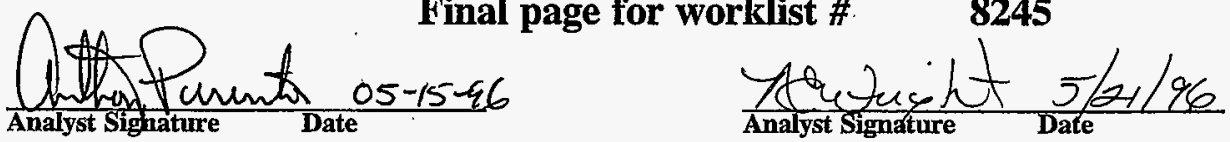

S96TO02131 Moved to wL \#9028 b1

Data Entry Comments:

Units shown for QC (SPK \& STD) may not reflect the actual units. $D L=$ Detection Limit, $S=$ Worklist Slot Number, $R=$ Replicate Number, $A=$ Aliquot Code. 


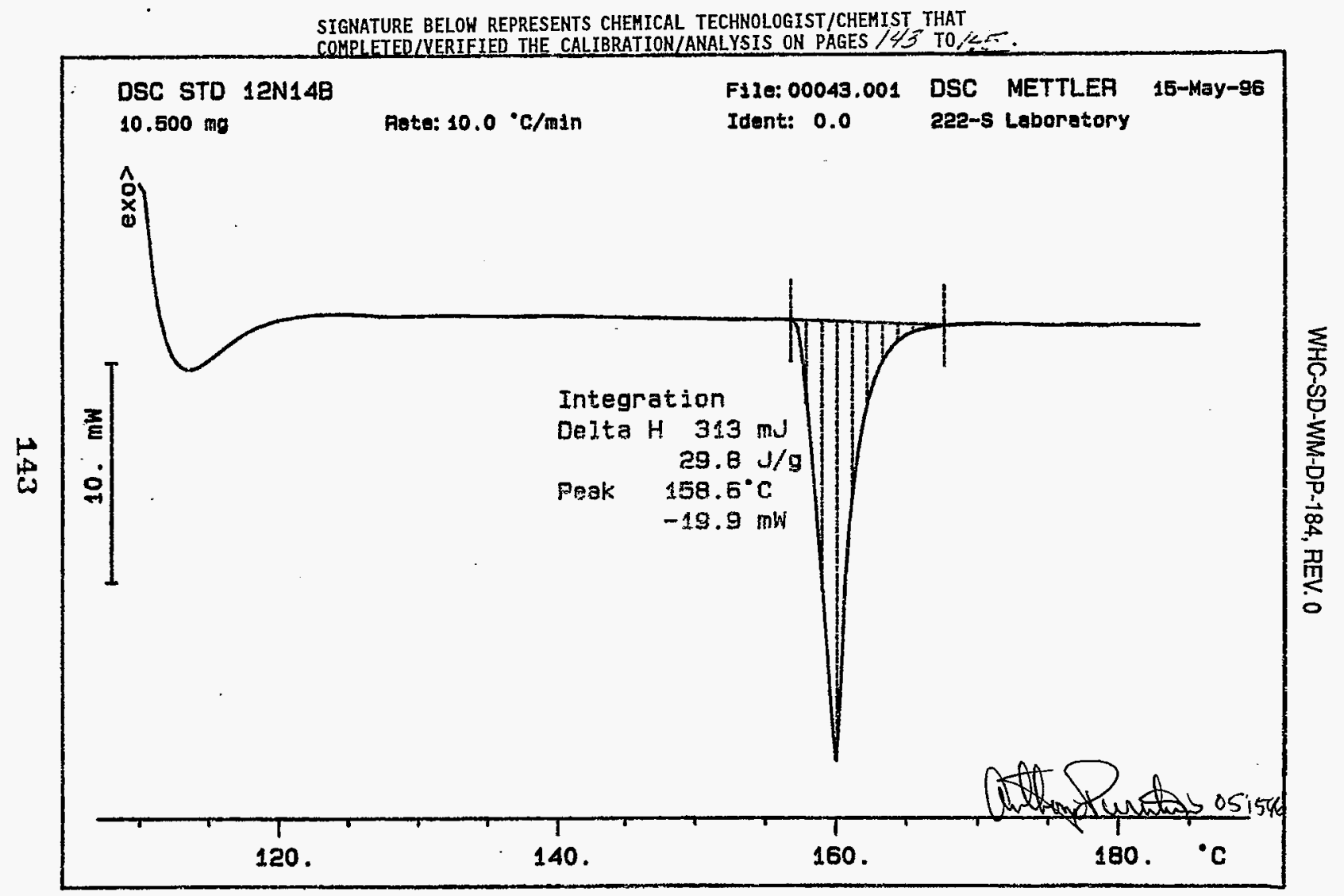


WHC-SD-WM-DP-184, REV. 0

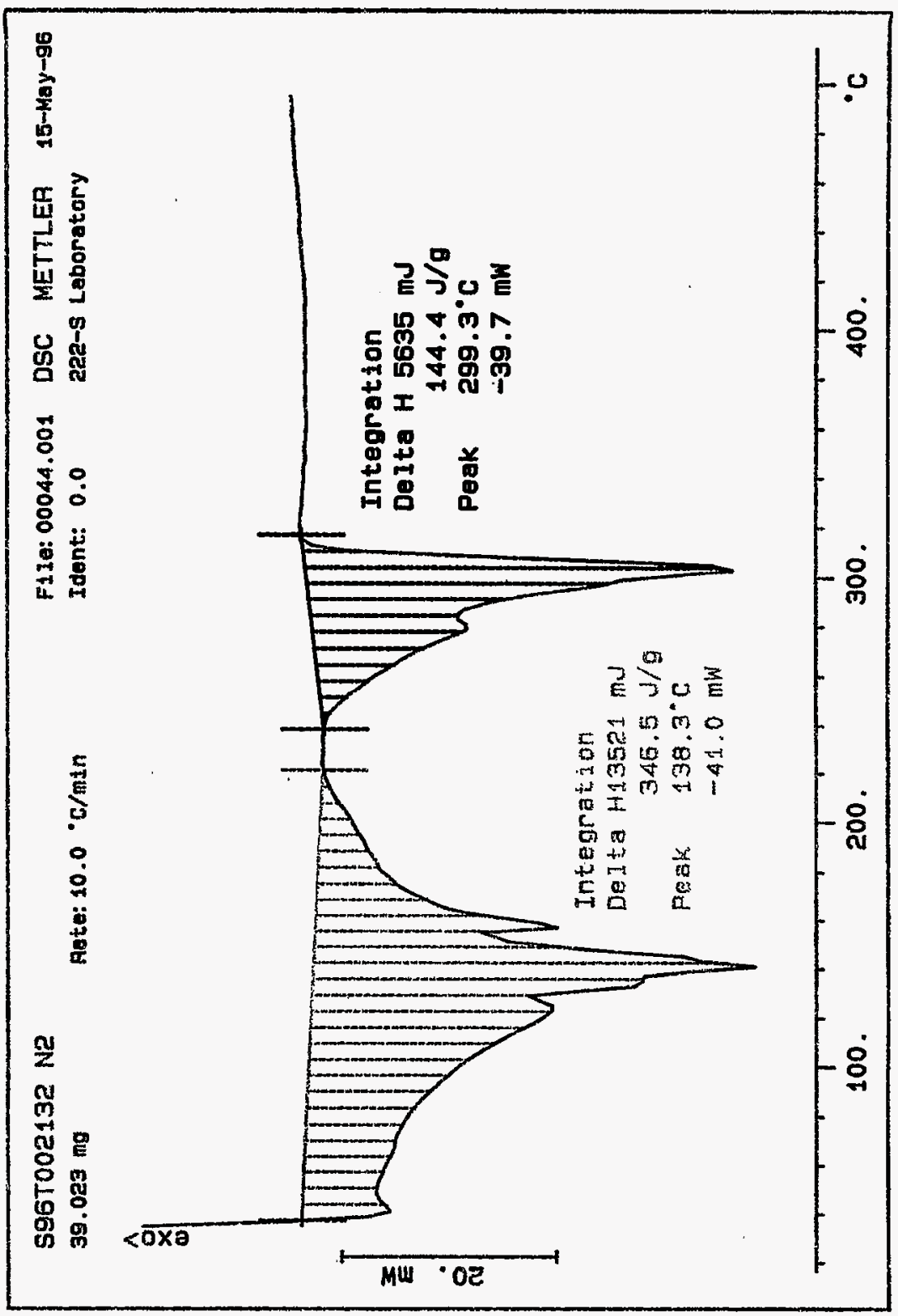

144 


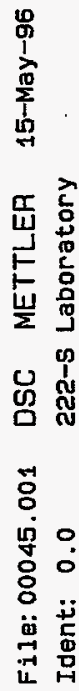

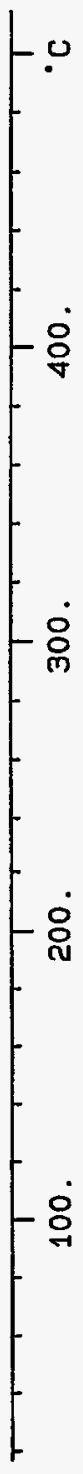

Mu $\bullet \mathrm{OZ}$ 
Analyst: $\quad$ XPM Instrument: DSCO __ Book \# 12 N $14 . B$

Method: LA-514-113 Rev/Mod C-1

Worklist Comment: U-107 DSC RUN UNDER N2. RCJ

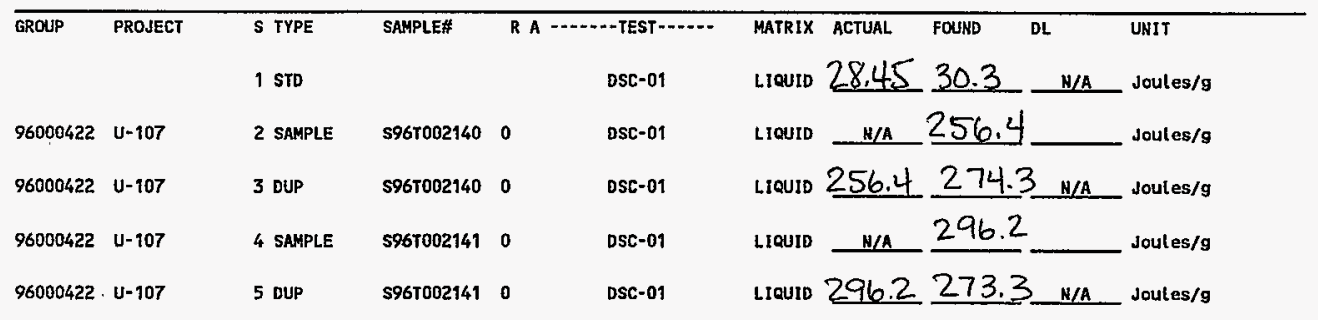

\section{Final page for worklist \#}

\section{7}

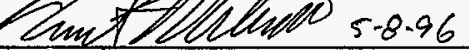

Analyst Signature Date Validated bif 1 llnaston $5.14-96$

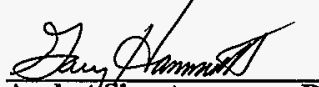

Analyst Signature

Data Entry Comments:

Units shown for $Q C$ (SPK \& STD) may not reflect the actual units. $D L=$ Detection Limit, $S=$ Worklist Slot Number,

$R=$ Replicate Number, $A=$ Aliquot Code. 
SIGNATURE BELOW REPRESENTS CHEHICAL TECHNOLOGIST/CHEMIST THAT

COHPLETED/VERIFIED THE CALIBRATION/ANALYSIS ON PAGES $1470 / 5 /$.

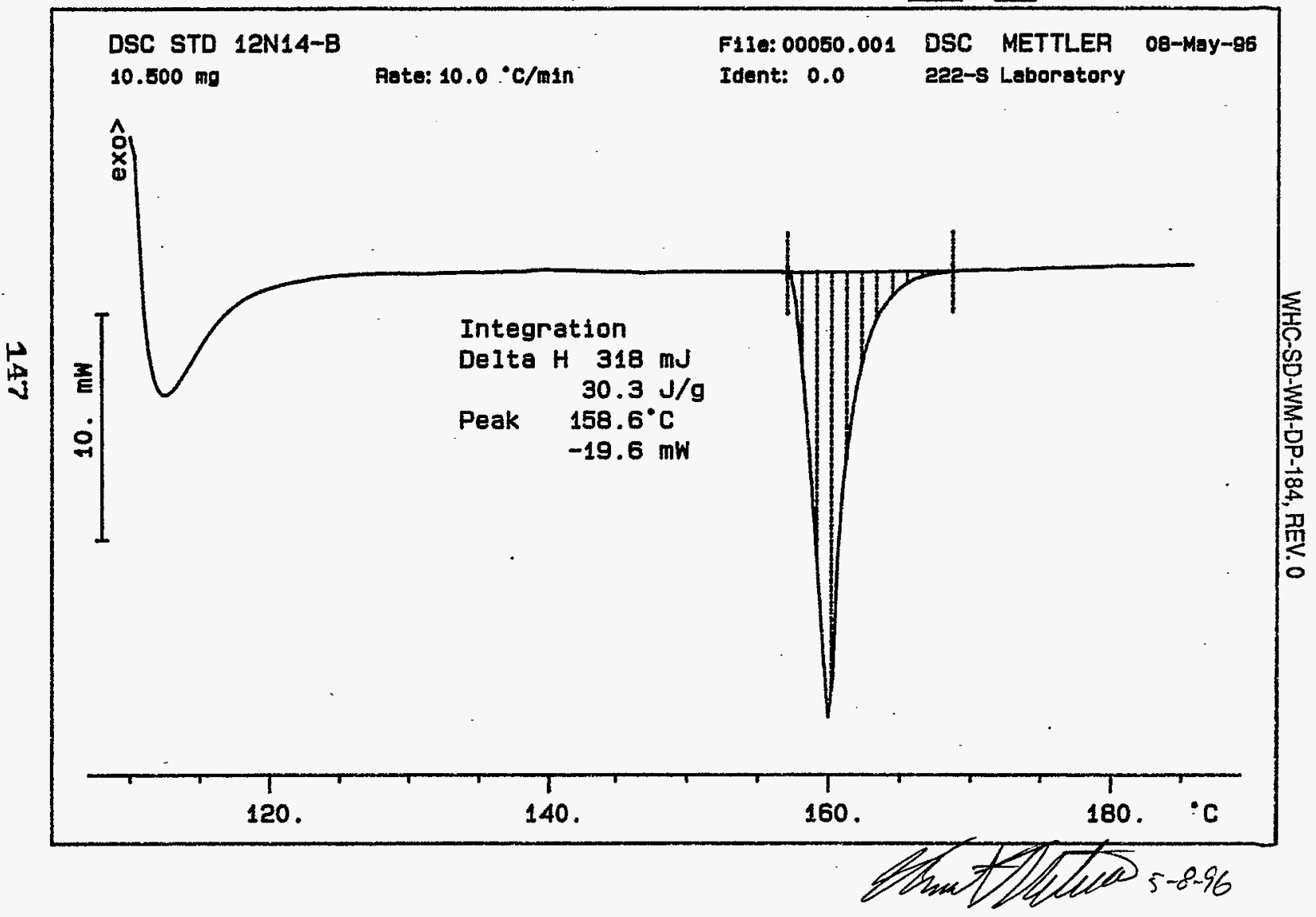




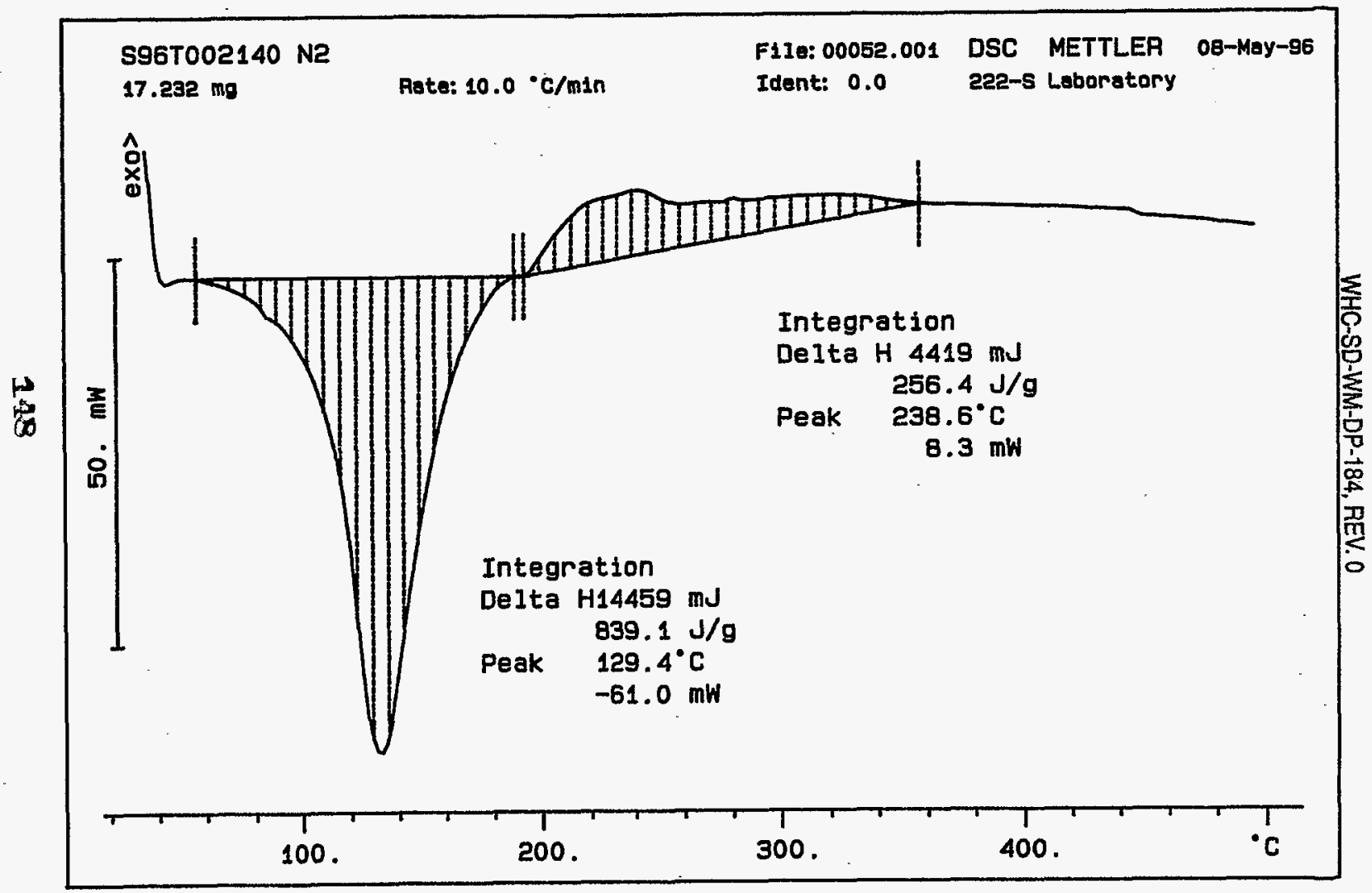




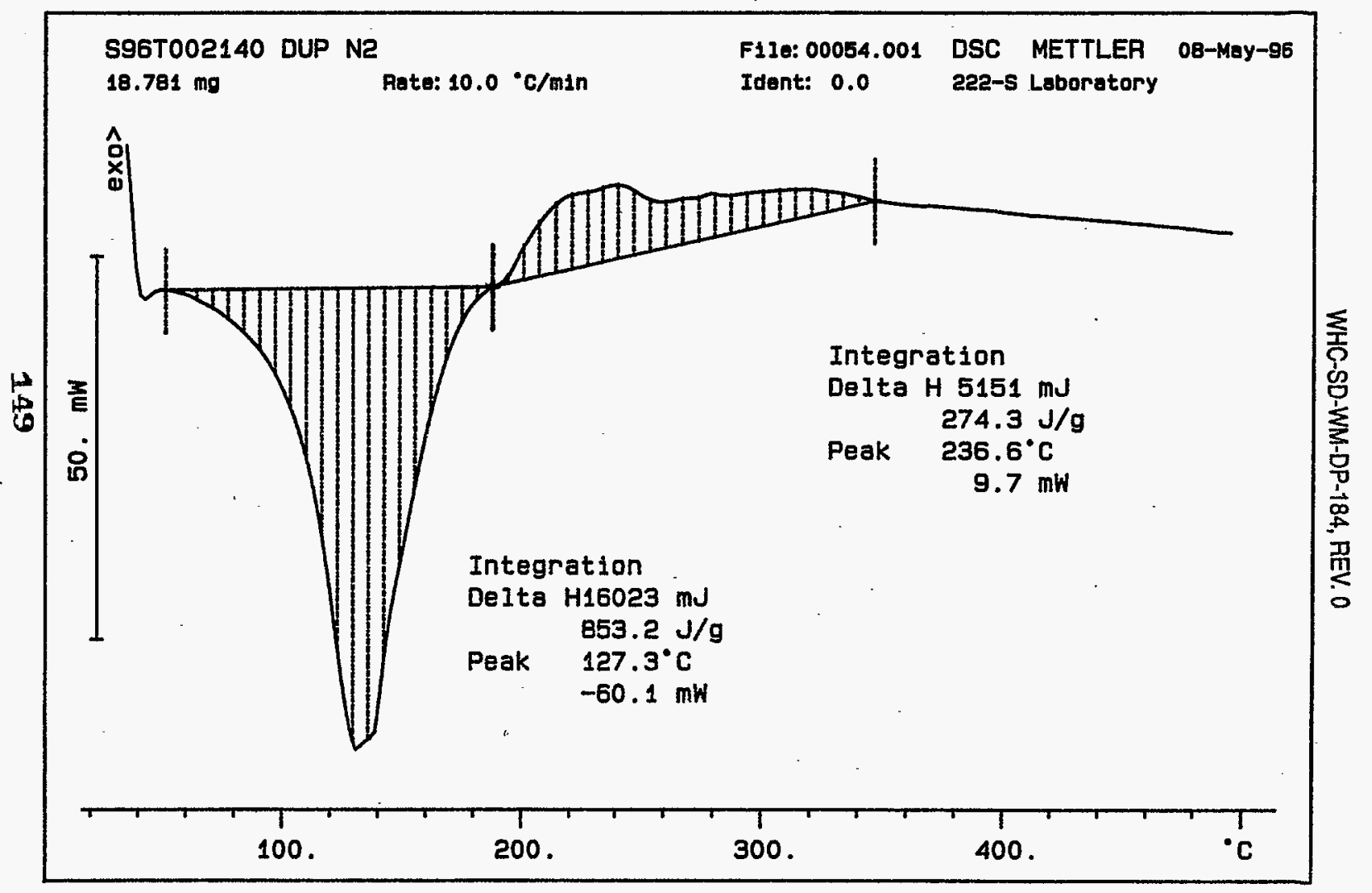




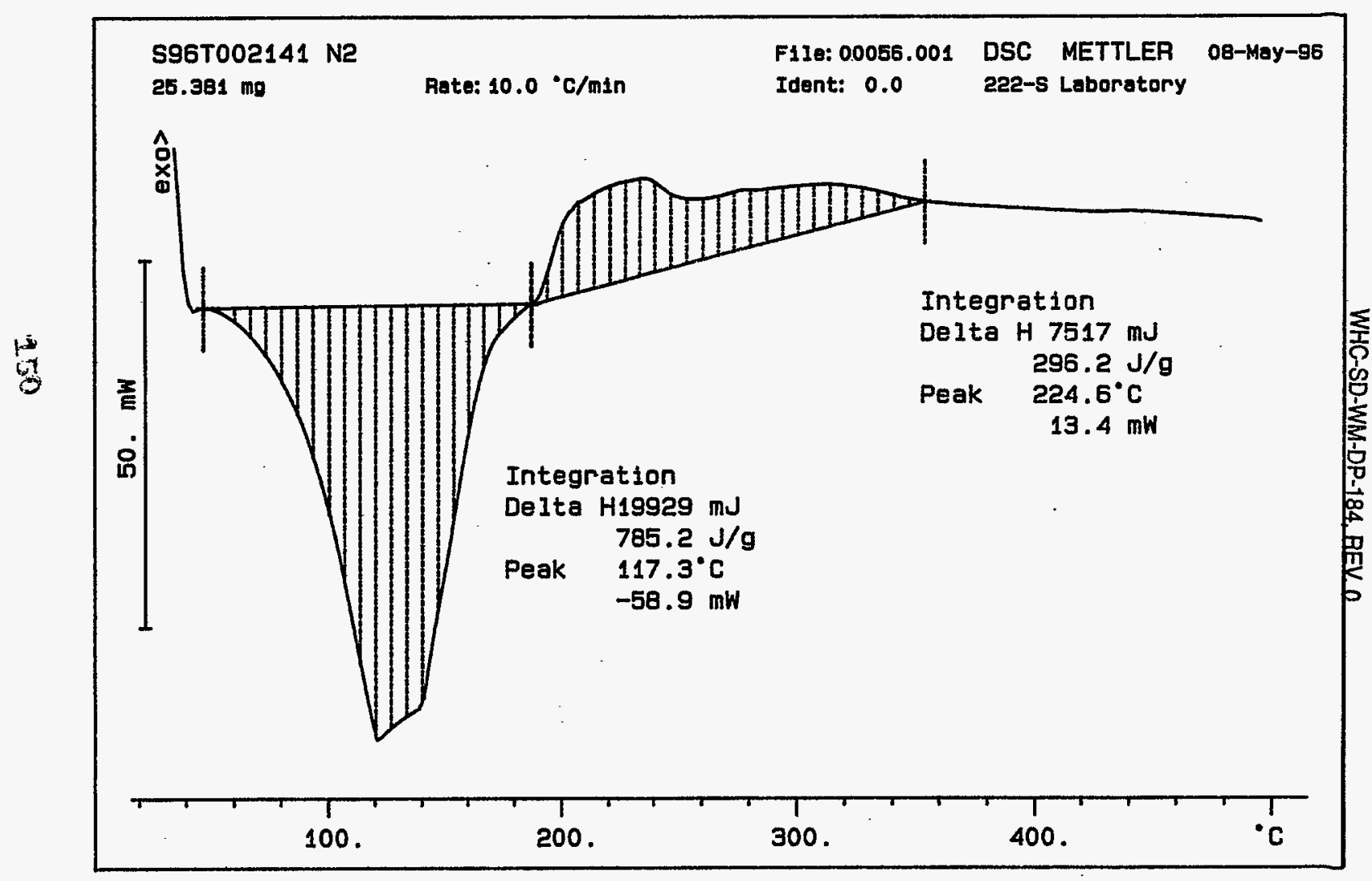




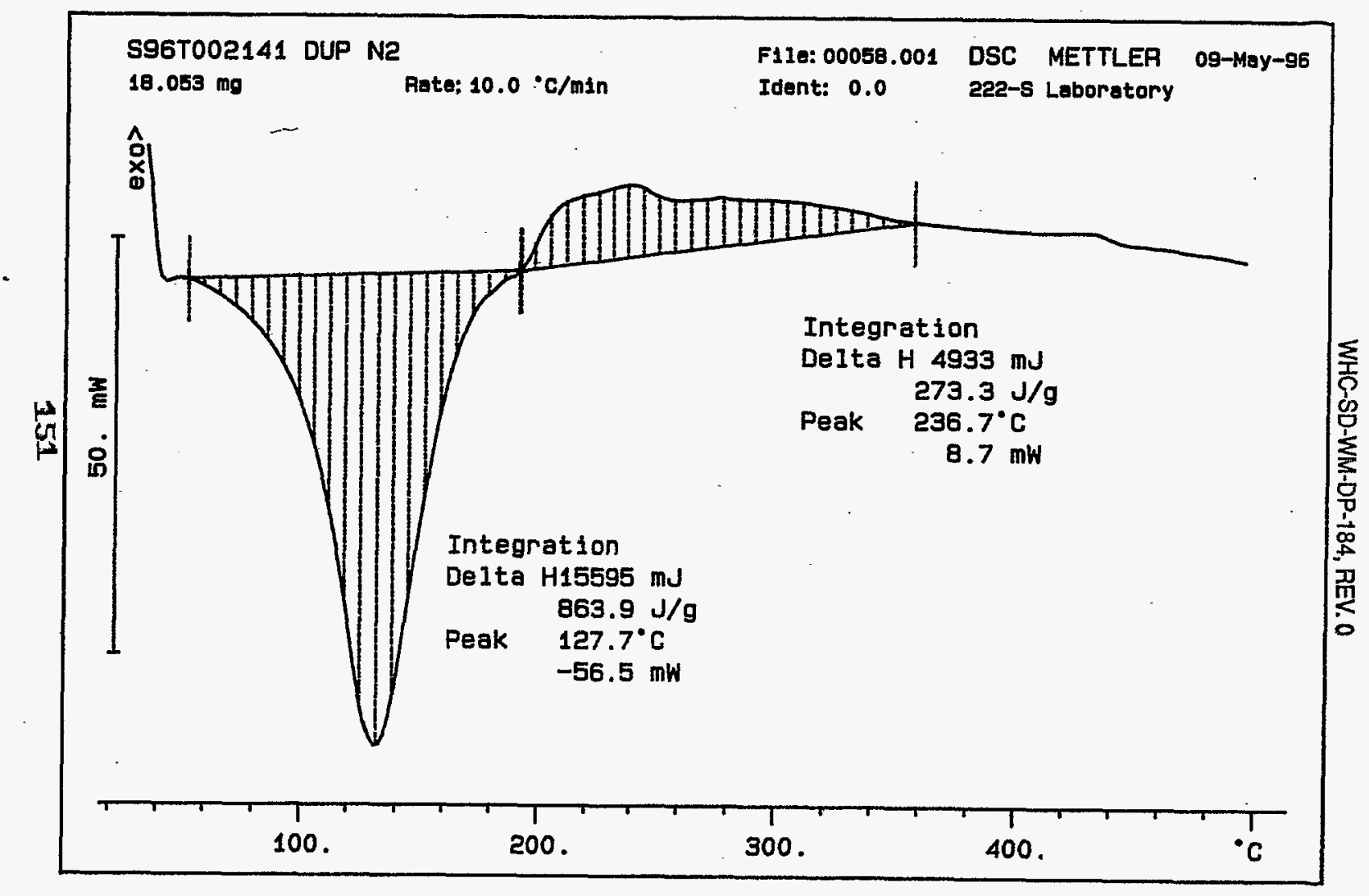




\section{LABCORE Data Entry Template for Worklist\#}

Analyst: $\quad$ ADP $\quad$ Instrument: $D S C 0$ Book \# 12 N14 B

Method: LA-514-114 Rev/Mod C - 1

Worklist Comment: U-107 DSC RUN UNDER N2. RCJ

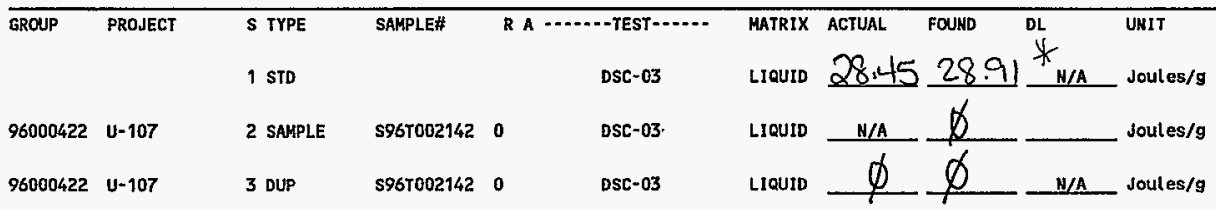

\section{Final page for worklist \# $\mathbf{8 4 3 8}$}

See Qttacled In Signatrus Analyst Signature Validated by Nonaston 5-15.96 elfifif 5.14.86 Analyst Signature Date

Data Entry Comments:

Units shown for $Q C$ (SPK \& STD) may not reflect the actual tunits. $D L=$ Detection Limit, $S=$ Worklist Slot Number, $R=$ Replicate Number, $A=$ Aliquot Code. 


\section{LABCORE Data Entry Template for Worklist\#}

Analyst: ADP Instrument: DSC0 3. Book \# 12 N $14 \mathrm{~B}$

Method: LA-514-113 Rev/Mod C-1

Worklist Comment: U-107 DSC RUN UNDER N2. RCJ

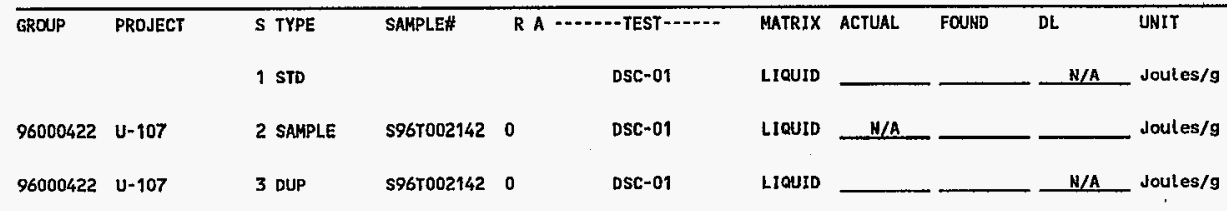

\section{Final page for worklist \# 8438}

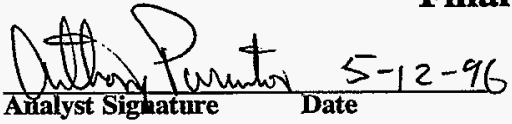

Analyst Signature Date

$$
\text { Other Instrument was used. }
$$

Data Entry Comments:

Units shown for $Q C$ (SPK \& STD) may not reflect the actual units. $D L=$ Detection Limit, $S=$ Worklist Slot Number, $R=$ Replicate Number, $A=$ Aliquot Code. 
Curve 1: DSC

File info: IND051201 Sun May 12 05: 48:54 1996

Sample Weight: $12.080 \mathrm{mg}$

12N14-B INDIUM AT 10CIMIN

SIGNATURE BELOW REPRESENTS CHEMICAL TECHNOLOGIST/CHEMIST THAT

COMPLETED/VERIFIED THE CALIBRATION/ANALYSIS ON PAGES 154 TO $/ 56$.

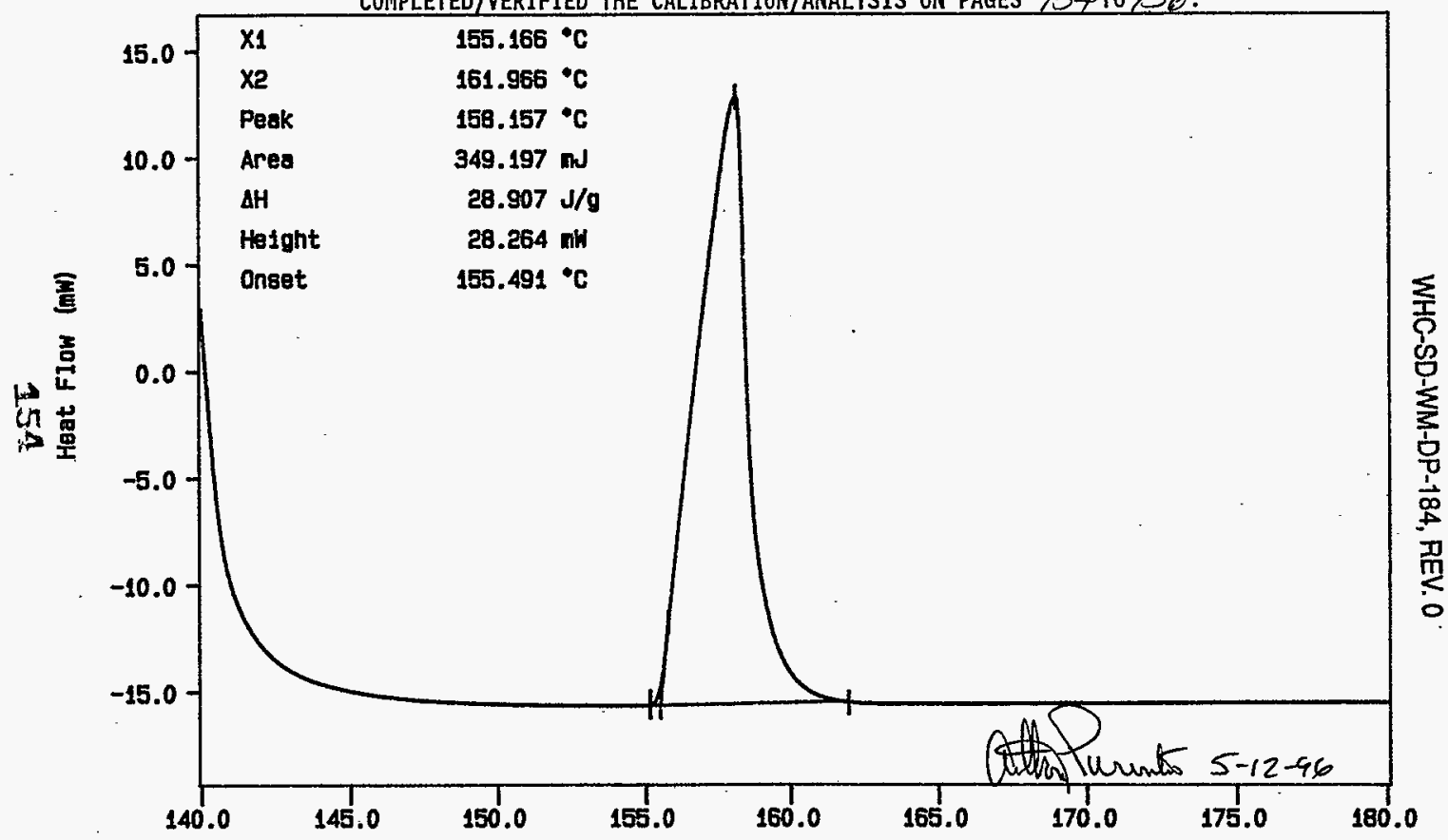

N2, EXDTHERM DOHN

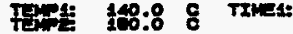

0.0 aln RAtet: $10.0 \mathrm{c} / \mathrm{min}$

Temperature $\left({ }^{\circ} \mathrm{C}\right)$

AD PUAINTON

PEAKIN-ELMER

7 Series Thermsl Analysis Syatem

Sun May 12 06: $54: 25$ 1996 
Curve 1: DSC

F1le info: SAMO51208 Sun May 12 14: 08: 351996

Sample Weight: 15.800

S96T002142 SAM

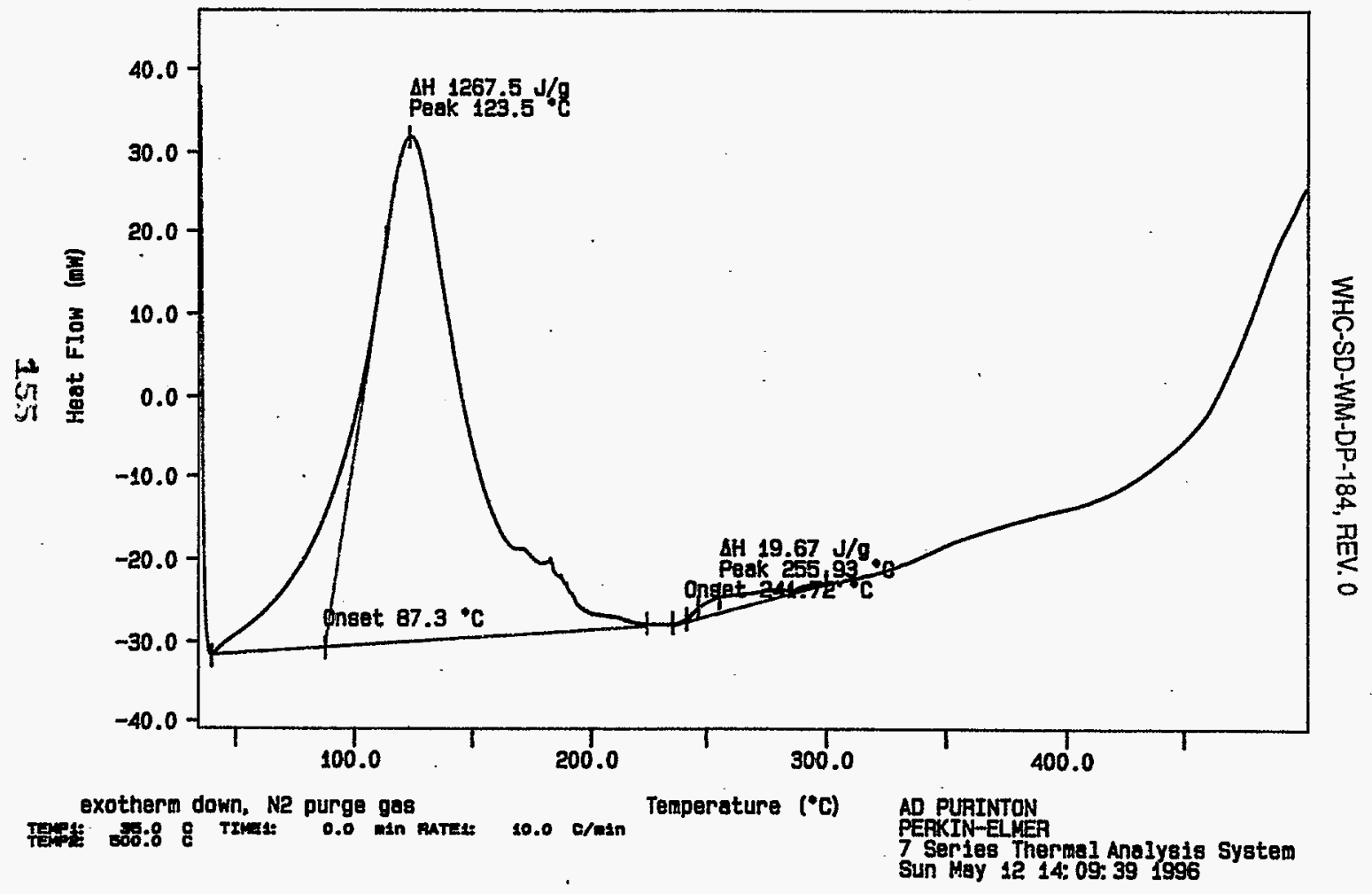


Curve 1: DSC

File info: SAM051209 Sun May 12 15: 04: 101996

Sample Neight: 29.510

ming

S96T002142 DUP

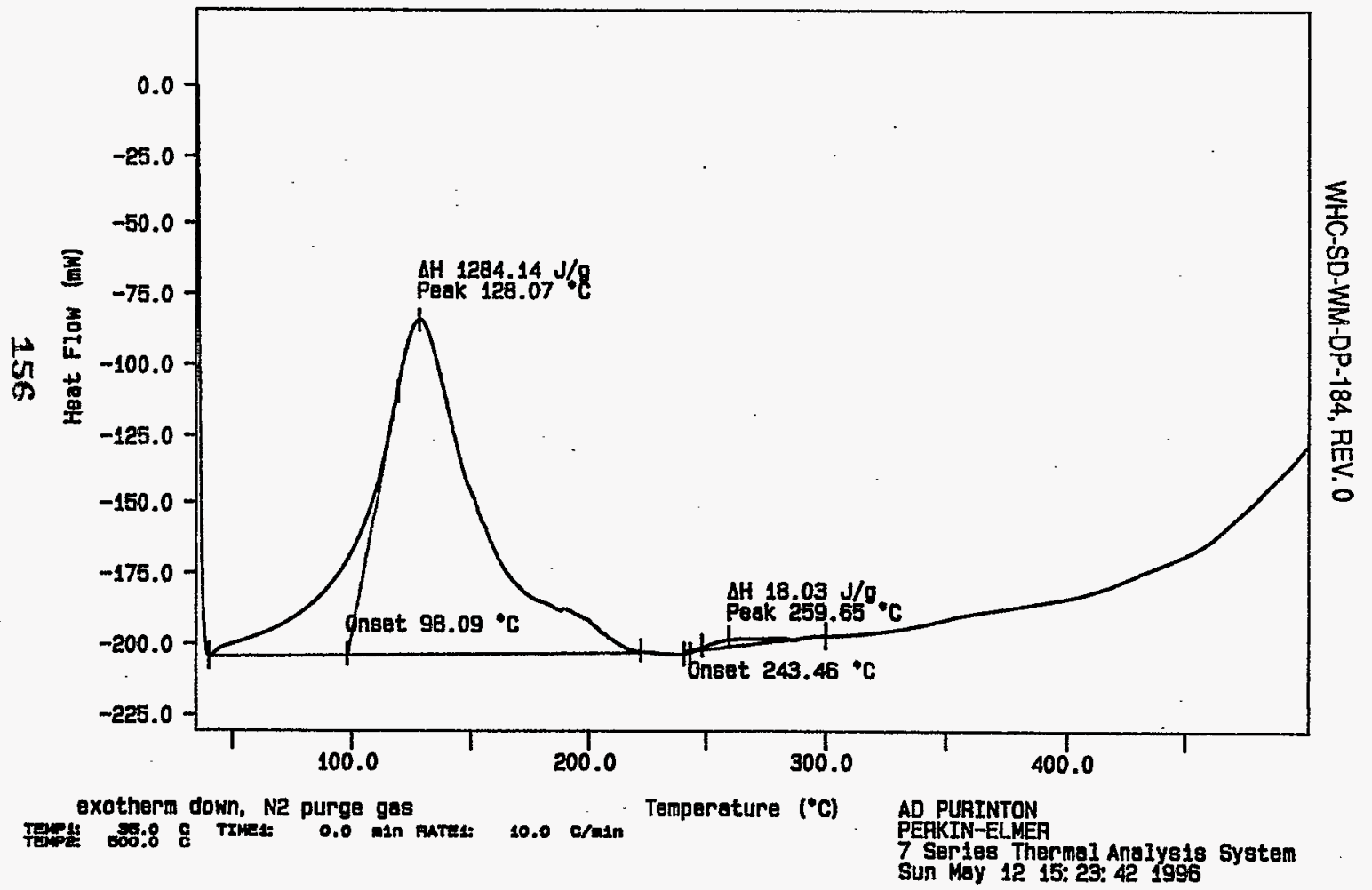


worklistrpt Version 2.1 05/15/95 05/07/96 11:17

LABCORE Data Entry Template for Worklist\#
Page: I

8439

Analyst: $\quad$ AP $\quad$ Instrument: $D S C 01 \ldots$ Book \# 12 N14B

Method: LA-514-113 Rev/Mod $C$-1

Worklist Comment: U-107 DSC RUN UNDER N2. RCJ

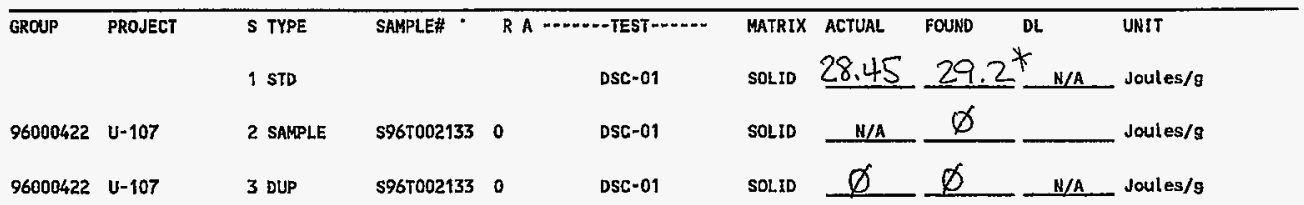

Final page for worklist \#

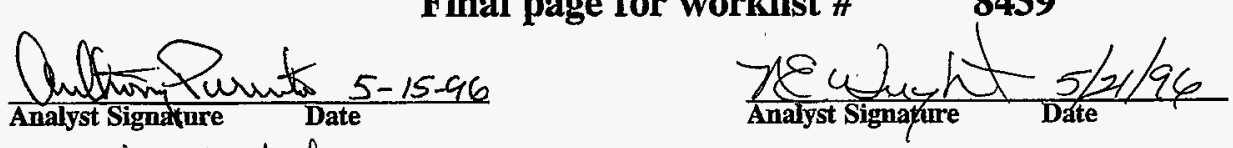

Validated by 1 lanootos 5.22-96.

Data Entry Comments:

Units shown for QC (SPK \& STD) may not reflect the actual units. $D L=$ Detection Limit, $S=$ Worklist Slot Number, $R=$ Replicate Number, $A=$ Aliquot Code.

157 
SIGNATURE BELOW REPRESENTS CHEHICAL TECHNOLOGIST/CHEMIST THAT

COMPLETED/VERIFIED THE CALIBRATION/ANALYSIS ON PAGES $/ 5 Z$ TO TO $/ \$ 0$.

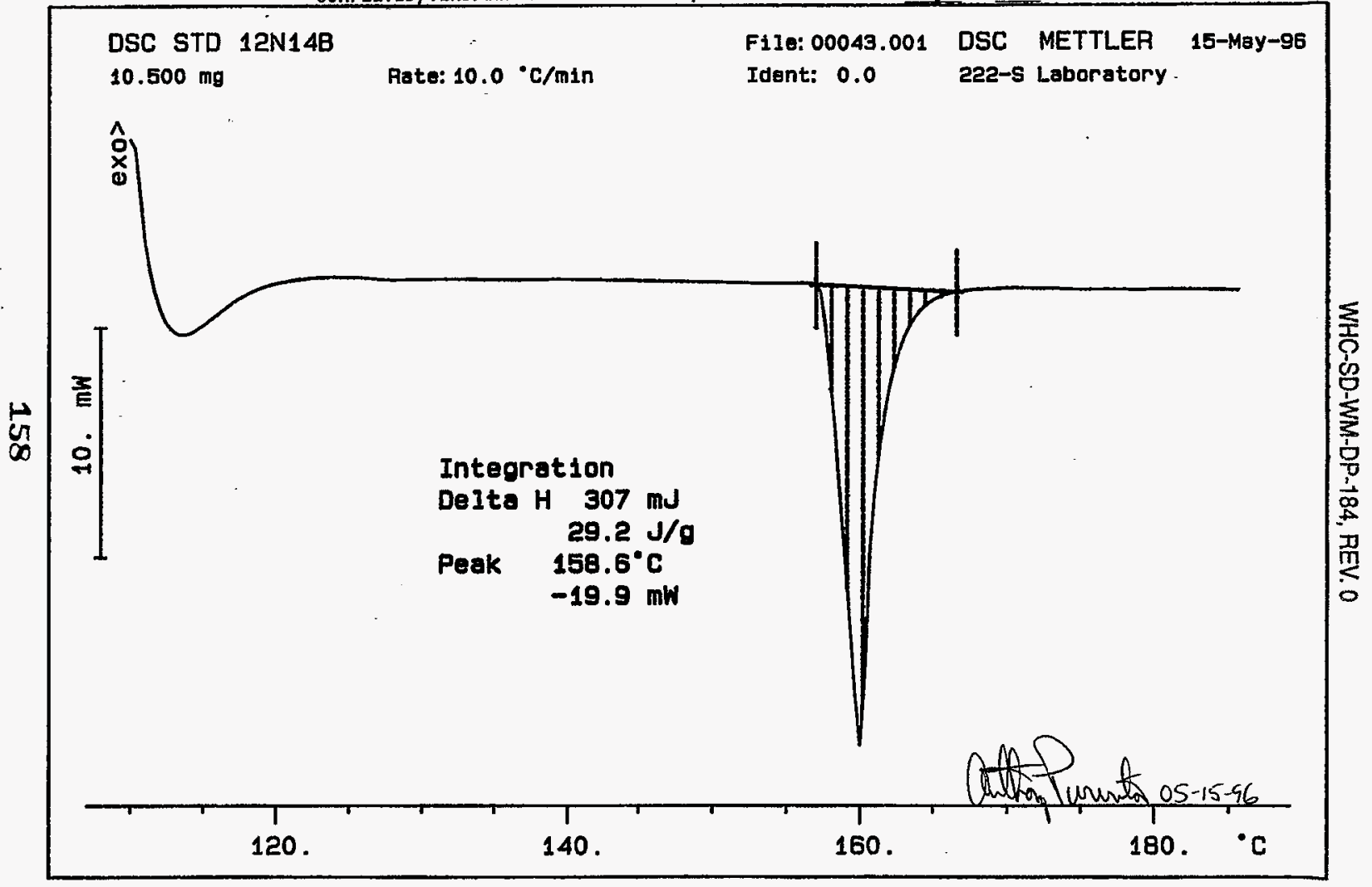




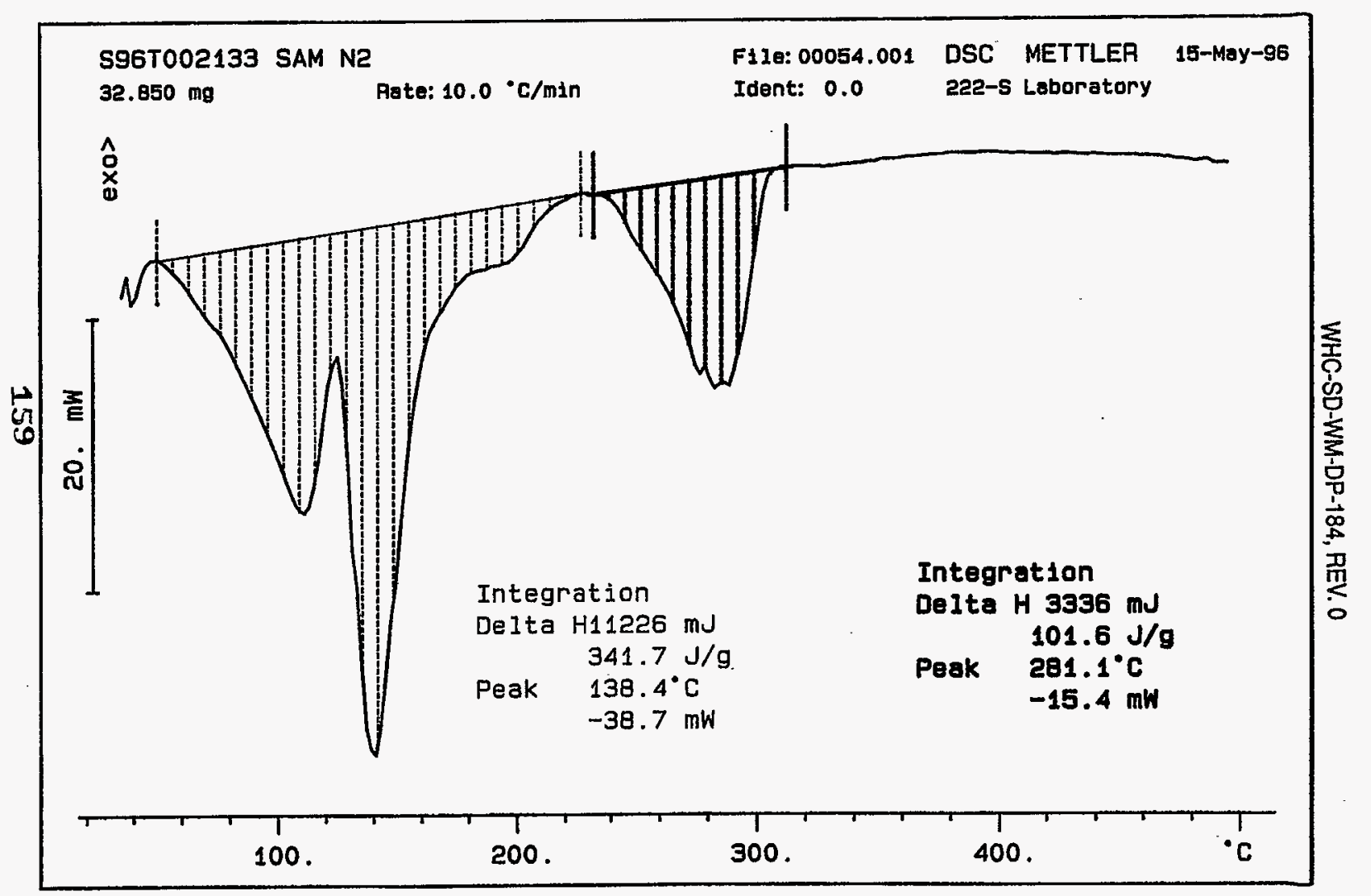


WHG SD WALOP-184, REV O

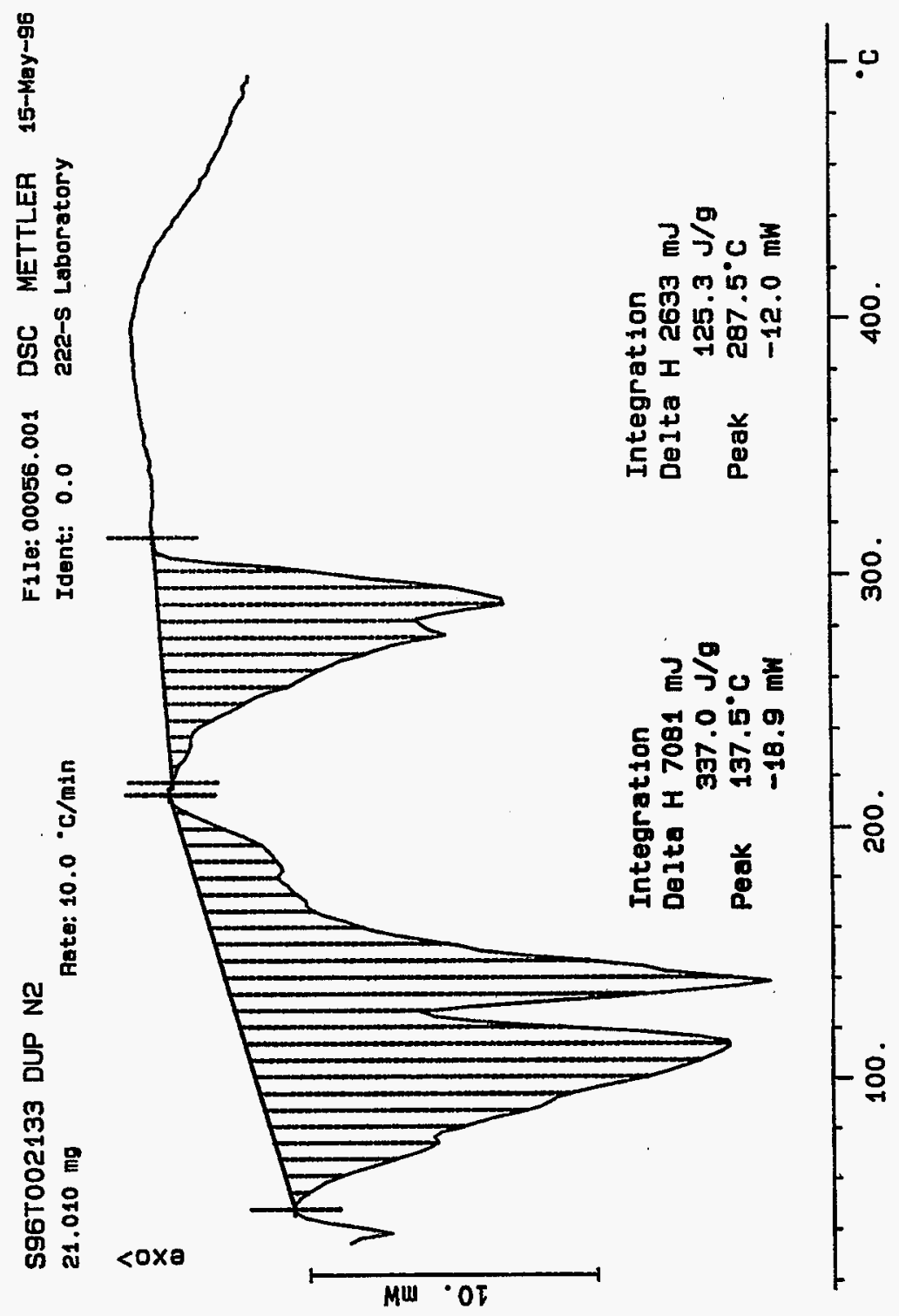

$66^{2}$ 
worklistrpt Version 2.1 05/15/95

05/20/96 11:08

LABCORE Data Entry Template for Worklist\#

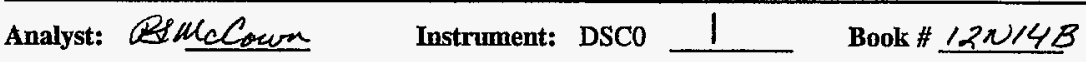

Method: LA-514-113 Rev/Mod $C-1$

Worklist Comment: U-107 FOR DSC-01 RUN UNDER N2 ～RTS!

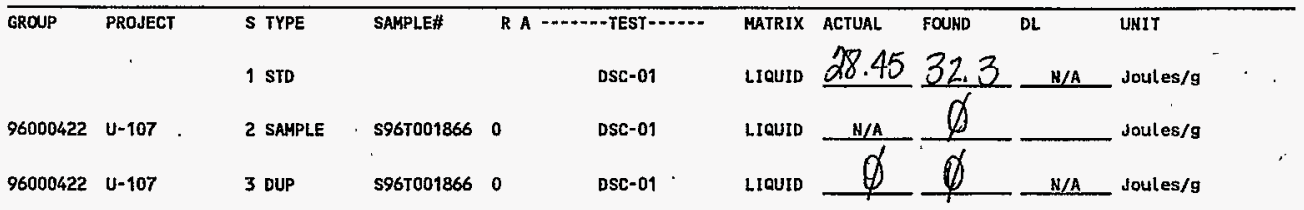

Final page for worklist \#

8949

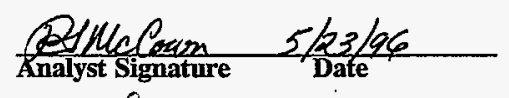

Verified/ralidated by

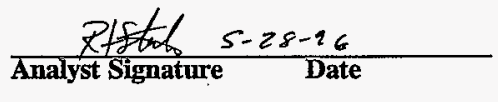

Banding Vacenpula

$5-31-96$

Data Entry Comments:

Units shown for QC (SPK \& STD) may not reflect the actual units. $D L=$ Detection Limit, $S=$ Worklist Slot Number, $R=$ Replicate Number, $A=$ Aliquot Code.

161 


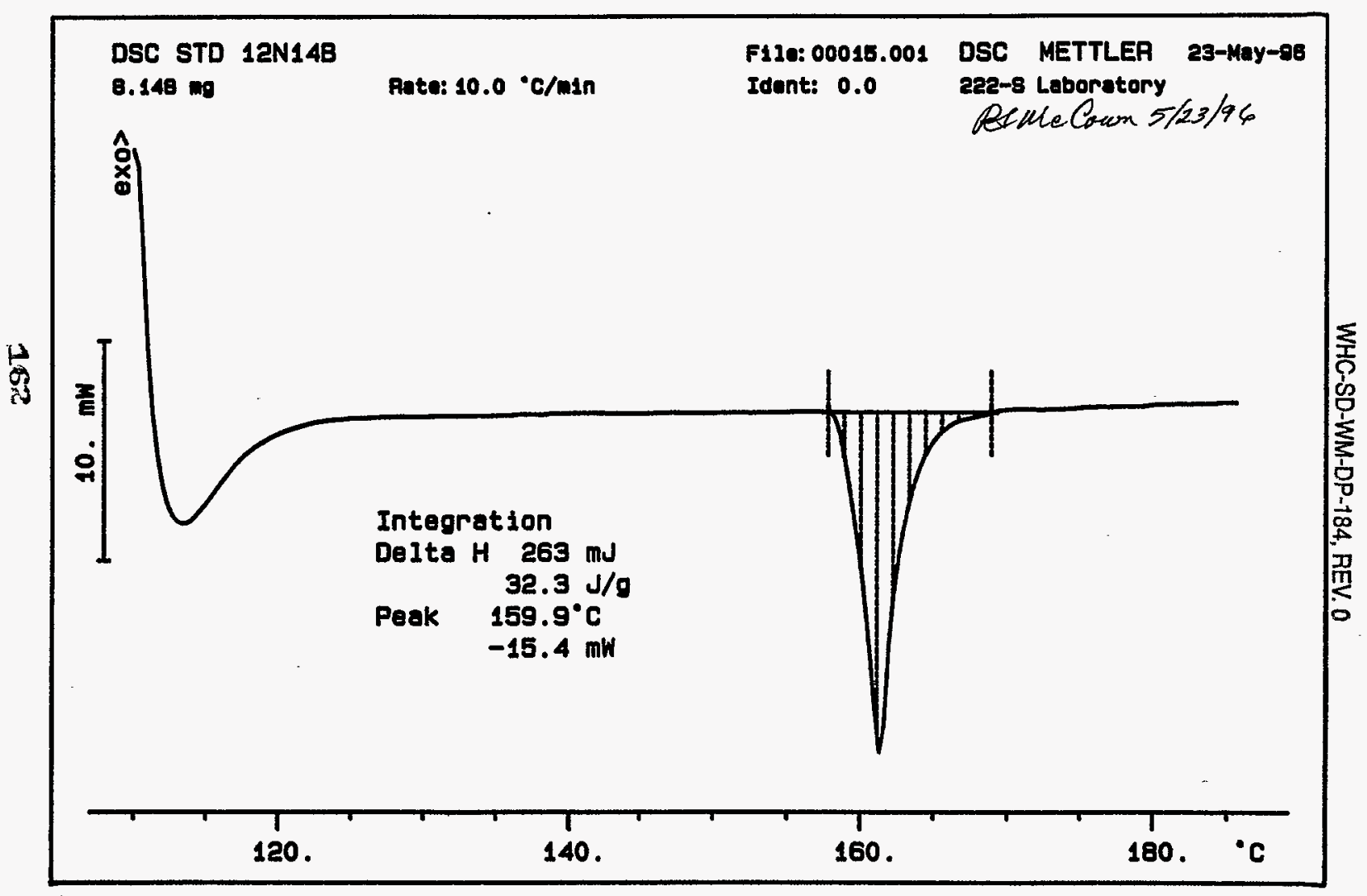




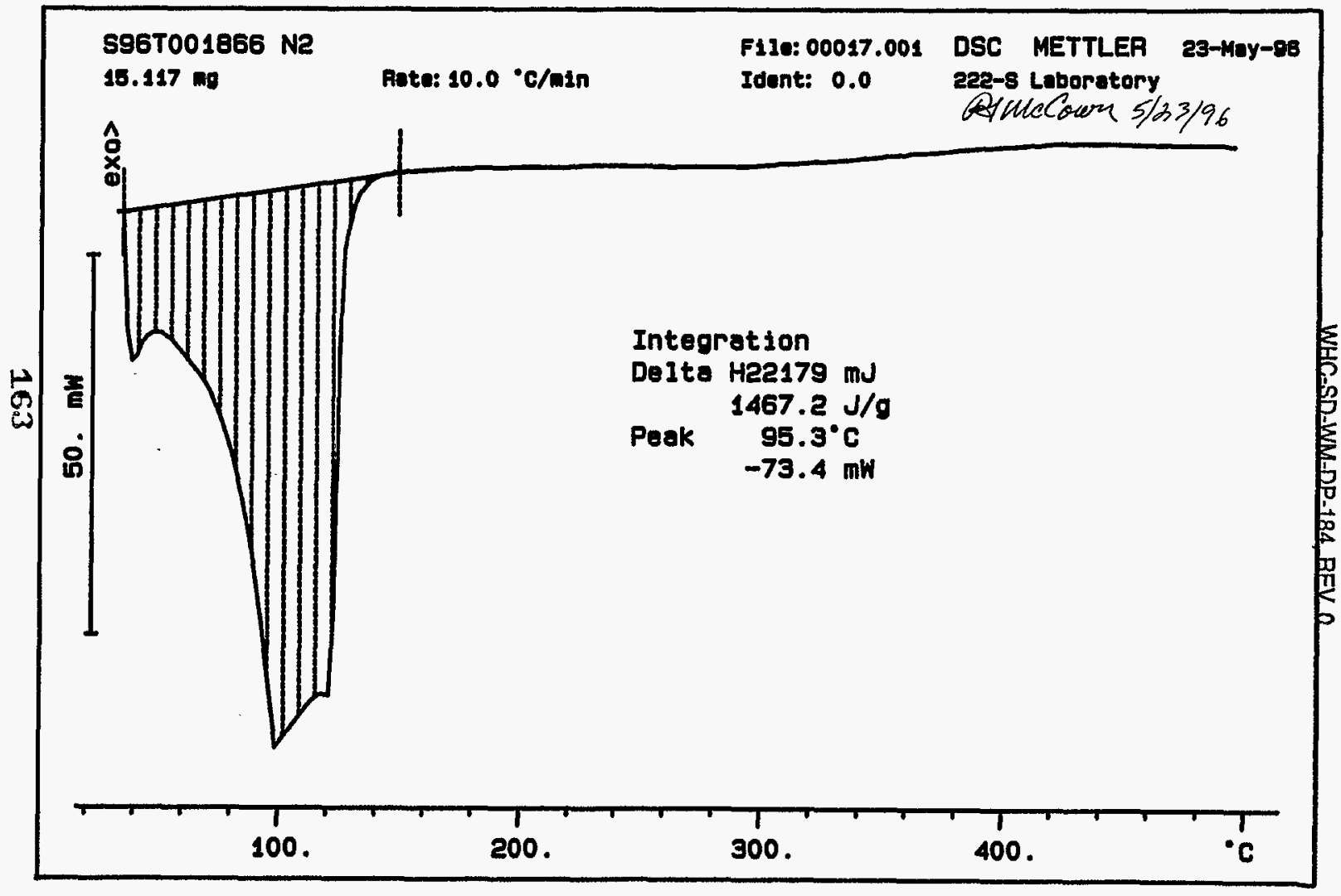




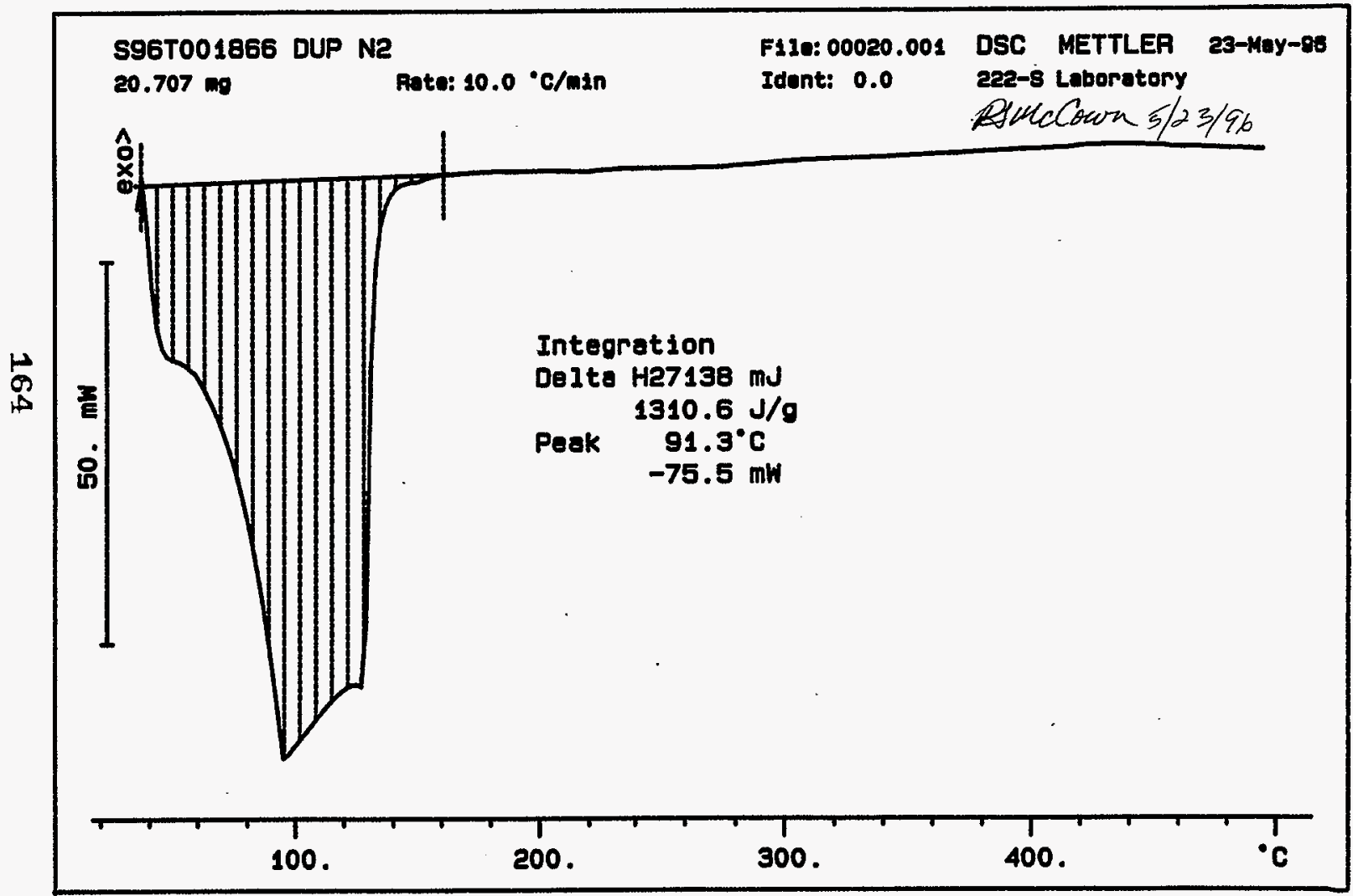




\section{Analyst: BOr Instrument: $\mathrm{DSC} 01$}

Book \#

Method: LA-514-113 Rev/Mod

Worklist Comment: Dry DSC for U-107. bdv

\begin{tabular}{|c|c|c|c|c|c|c|c|c|c|c|}
\hline GROLP & PROJECT & S TYPE & SAMPLE\# & R A & W-N-OTEST $-\cdots$ & MATRIX & ACTUAL & FOUND & $\mathrm{DL}$ & UNIT \\
\hline 96000085 & $U-107$ & 1 SAMPLE & 596T000665 & 0 & DSC-02 & LIQUID & $\mathrm{N} / \mathrm{A}$ & 117.2 & $\dot{.}$ & Joules/g Dry \\
\hline 96000085 & U-107 & 2 DUP & S96T000665 & 0 & DSC-02 & LIQUID & 117.2 & 114.8 & $N / A$ & Joules/g Dry \\
\hline 96000085 & $U-107$ & 3 SAMPLE & S96T000682 & 0 & DSC-02 & LIQUID & N/A. & 129.7 & & Joules/g Dry \\
\hline 96000085 & $v-107$ & 4 DUP & S96T000682 & 0 & DSC- 02 & LIQUID & 129.7 & 126.6 & N/A & soules/g Dry \\
\hline 96000422 & $u-107$ & 5 SAMPLE & s96r002140 & 0 & DSC-02 & LIQUID & $N / A$ & 539.9 & & Joules/g Dry \\
\hline 96000422 & $\mathrm{U}-107$ & 6 DUP & S96T002140 & 0 & DSC-02 & LIQUID & 539.9 & 577.6 & $\mathrm{~N} / \mathrm{A}$ & Joules/g Dry \\
\hline 96000126 & $\mathrm{u}-107$ & 7 SAMPLE & \$967001119 & 0 & DSC-02 & LIQUID & N/A & $\varnothing$ & & Joules/g Dry \\
\hline 96000126 & $\mathrm{u}-107$ & 8 DUP & S96T001119 & 0 & DSC-02 & LIQUID & $\varnothing$ & & $N / A$ & Joules/g Dry \\
\hline 96000126 & $\mathrm{u}-107$ & 9 SAMPLE & S96T001120 & 0 & DSC -02 & LIQUID & $\mathrm{N} / \mathrm{A}$ & & & Joules/g Dry \\
\hline 96000126 & $u=107$ & 10 DUP & $\$ 967001120$ & 0 & Dsc-02 & LIQUID & $\varnothing$ & $\varnothing$ & $\mathrm{N} / \mathrm{A}$ & Joules/g Dry \\
\hline 96000126 & $u-107$ & 11 SAMPLE & \$96T001121 & 0 & DSC-02 & LIQUID & $\mathrm{N} / \mathrm{A}$ & & & Joules/g Dry \\
\hline 96000126 & U-107 & 12 DUP & S96T001121 & 0 & DSC-02 & LIQUID & $\varnothing$ & & $\mathrm{N} / \mathrm{A}$ & Joules/g Dry \\
\hline 96000.126 & $U-107$ & 13 SAMPLE & S96T002034 & 0 & Dsc-02 & LIQUID & $N / A$ & $\varnothing$ & & Joules/g Dry \\
\hline 96000126 & $\mathrm{v}-107$ & 14 DUP & s967002034 & 0 & DSC-02 & LIQUID & $\varnothing$ & $\varnothing$ & N/A & Joules/g Dry \\
\hline 96000422 & $u-107$ & 15 SAMPLE & S96T002141 & 0 & DSC-02 & LIQUID & N/A & 559.2 & & Joules/g Dry \\
\hline 96000422 & $\mathrm{U}-107$ & 16 DUP & s96T002141 & 0 & DSC-02 & LIQUID & 559.2 & 516.0 & N/A & Joules/g Dry \\
\hline 96000422 & $U-107$ & 17 SAMPLE & S96T002142 & 0 & DSC-02 & LIQUID & N/A & $\not$ & & Joules/g Dry \\
\hline 96000422 & U- 107 & 18 DUP & $S 96 T 002142$ & 0 & DSC-02 & LIQUID & $\varnothing$ & $\varnothing$ & N/A & Joules/g Dry \\
\hline
\end{tabular}

Data Entry Comments:

Units shown for $Q C$ (SPK \& STD) may not reflect the actual units. $D L=$ Detection Limit, $S=$ Worklist Slot Number, $R=$ Replicate Number, $A=$ Aliquot Code. 


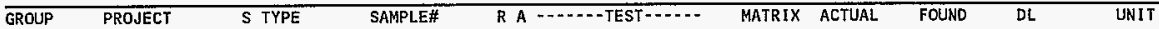

\section{Final page for worklist \# $\quad 9021$}

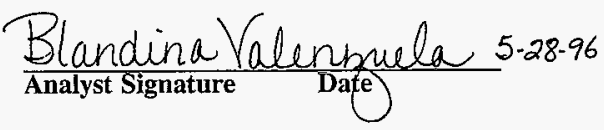

Analyst Signature Date

Data Entry Comments:

Units shown for $Q C$ (SPK \& STD) may not reflect the actual units. $D L=$ Detection Limit, $S=$ Worklist Slot Number, $R=$ Replicate Number, $A=$ Aliquot Code. 
U. $1 C^{\circ} 7$ WHC-SD-WM-DP-184, REV.O

\begin{tabular}{|c|c|c|c|}
\hline \multicolumn{4}{|c|}{ CALCULATED DRY DSC } \\
\hline SAMPLE NO. & DSC RESULT $(\mathrm{J} / \mathrm{g})$ & TGA RESULT (\% water) & DRY DSC RESULT \\
\hline $596 \pi 000605$ & 54.8 & 5.3 .23 & 117.2 \\
\hline 6650 & 53.7 & 53.23 & $1 / 4.8$ \\
\hline 682 & 65.3 & 49.67 & $512^{22} 139 .+129.7$ \\
\hline 6820 & 63.7 & 49.67 & 126.6 \\
\hline 1866 & D.29 DOSE & DOSE & \\
\hline \multicolumn{4}{|l|}{18660} \\
\hline 2140 & 256.4 & 52.51 & 539.9 \\
\hline 21400 & 274.3 & $52.5 !$ & 577.6 \\
\hline 1119 & $\phi$ & - & $\varnothing$ \\
\hline 111911 & $\varnothing$ & - & $\varnothing$ \\
\hline 1120 & $\alpha$ & - & $\alpha$ \\
\hline $112 C D$ & 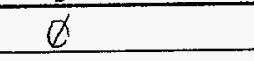 & $=$ & $\not x$ \\
\hline 1121 & $\varnothing$ & $=$ & $\varnothing$ \\
\hline 11210 & $\not 4$ & - & $\varnothing$ \\
\hline 20.34 & $\phi$ & 98.16 & $\not$ \\
\hline 20.340 & $\varnothing$ & 98.16 & $\varnothing$ \\
\hline 2141 & 296.2 & 47.03 & 559.2 \\
\hline $2141 D$ & 273.3 & 47.03 & 516.0 \\
\hline 2142 & $\emptyset$ & $=$ & $\varnothing$ \\
\hline $2142 D$ & $\varnothing$ & - & $\phi$ \\
\hline & & & \\
\hline & & , & \\
\hline & & & \\
\hline
\end{tabular}


Analyst: BBV Instrument: DSC01

Book \#

Method: LA-514-113 Rev/Mod

Worklist Comment: Dry DSC for U-107. bdv

\begin{tabular}{|c|c|c|c|c|c|c|c|c|c|c|}
\hline GROUP & PROJECT & S TYPE & SAMPLE\# & R A & $\because$ TEST $-\ldots .$. & MATRIX & ACTUAL & FOUND & $D L$ & UNIT \\
\hline 96000085 & $U-107$ & 1 SAMPLE & $\$ 96 T 000650$ & 0 & DSC -02 & SOLID & N/A & 33.78 & & Joules/g Dry \\
\hline 96000085 & $U-107$ & 2 DUP & $596 \mathrm{~T} 000650$ & 0 & DSC-02 & SOLID & 38.78 & 14.18 & N/A & Joules/g Dry \\
\hline 96000085 & $U-107$ & 3 SAMPLE & s96T000653 & 0 & DSC-02 & SOLID & N/A & 16.77 & & Joules/g Dry \\
\hline 96000085 & $U-107$ & 4 DUP & s96T000653 & 0 & DSC-02 & SOLID & 16.77 & 36.19 & $N / A$ & Joules/g Dry \\
\hline 96000085 & $U-107$ & 5 SAMPLE & $596 \mathrm{~T} 000644$ & 0 & DSC-02 & SOLID & $\mathrm{N} / \mathrm{A}$ & 271.3 & & Joules/g Dry \\
\hline 96000085 & $U-107$ & 6 DUP & 5967000644 & $\mathfrak{0}$ & $\mathrm{DSC}-02$ & SOLID & 271.3 & 253.8 & $\mathrm{~N} / \mathrm{A}$ & Joules/g Dry \\
\hline 96000085 & $U-107$ & 7 SAMPLE & 596r000647 & 0 & DSC -02 & SOLID & $\mathrm{N} / \mathrm{A}$ & 11.95 & & Joules/g Dry \\
\hline 96000085 & $U-107$ & 8 DUP & S96r000647 & 0 & DSC-02 & SOLID & 11.95 & 10.21 & $\mathrm{~N} / \mathrm{A}$ & Joules/g Dry \\
\hline 96000085 & $U-107$ & 9 SAMPLE & 5967000683 & 0 & DSC -02 & SOLID & $\mathrm{N} / \mathrm{A}$ & 15.30 & & Joules/g Dry \\
\hline 96000085 & $U-107$ & 10 DUP & 5961000683 & 0 & DSC-02 & SOLID & 15.30 & 14.07 & $\mathrm{~N} / \mathrm{A}$ & Joules/g Dry \\
\hline 96000126 & $v-107$ & 11 SAMPLE & S96T001055 & 0 & DSC-02 & SOLID & N/A & $\varnothing$ & & Joules/g Dry \\
\hline 96000126 & $U-107$ & 12 DUP & S96T001055 & 0 & DSC-02 & SOLID & $\varnothing$ & $\varnothing$ & $\mathrm{N} / \mathrm{A}$ & Joules/g Dry \\
\hline 96000126 & $U-107$ & 13 SAMPLE & 5967001128 & 0 & DSC -02 & SOLID & N/A & $\varnothing$ & & Joules/g Dry \\
\hline 96000126 & $\mathrm{u}-107$ & 14 DuP & s96T001128 & 0 & DSC-02 & SOLID & $\phi$ & $\varnothing$ & $\mathrm{N} / \mathrm{A}$ & Joules/g Dry \\
\hline 96000085 & $U-107$ & 15 SAMPLE & S96T001143 & 0 & DSC-02 & SOLID & N/A & & & Joules/g Dry \\
\hline 96000085 & $\mathrm{U}-107$ & 16 DUP & S96T001143 & 0 & DSC-02 & SOL.ID & $\not$ & $\varnothing$ & N/A & Joules/g Dry \\
\hline 96000085 & $U-107$ & 17 TRIPL & s967001143 & 0 & DSC-02 & SOLID & $\varnothing$ & & $\mathrm{N} / \mathrm{A}$ & Joules/g Dry \\
\hline 96000085 & $U-107$ & 18 SAMPLE & \$96T001159 & 0 & DSC-02 & SOLID & $N / A$ & 11.80 & & Joules/g Dry \\
\hline
\end{tabular}

Data Entry Comments:

Units shown for $Q C$ (SPK \& STD) may not reflect the actual units. $D L=$ Detection Limit, $S=$ Worklist Slot Number, $R=$ Replicate Number, $A=$ Aliquot Code. 


\section{LABCORE Data Entry Template for Worklist\#}

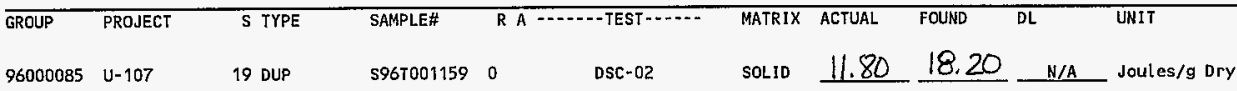

\section{Final page for worklist \# 9022}

Blandina Valenisuela 5-28-96 Analyst Signature 


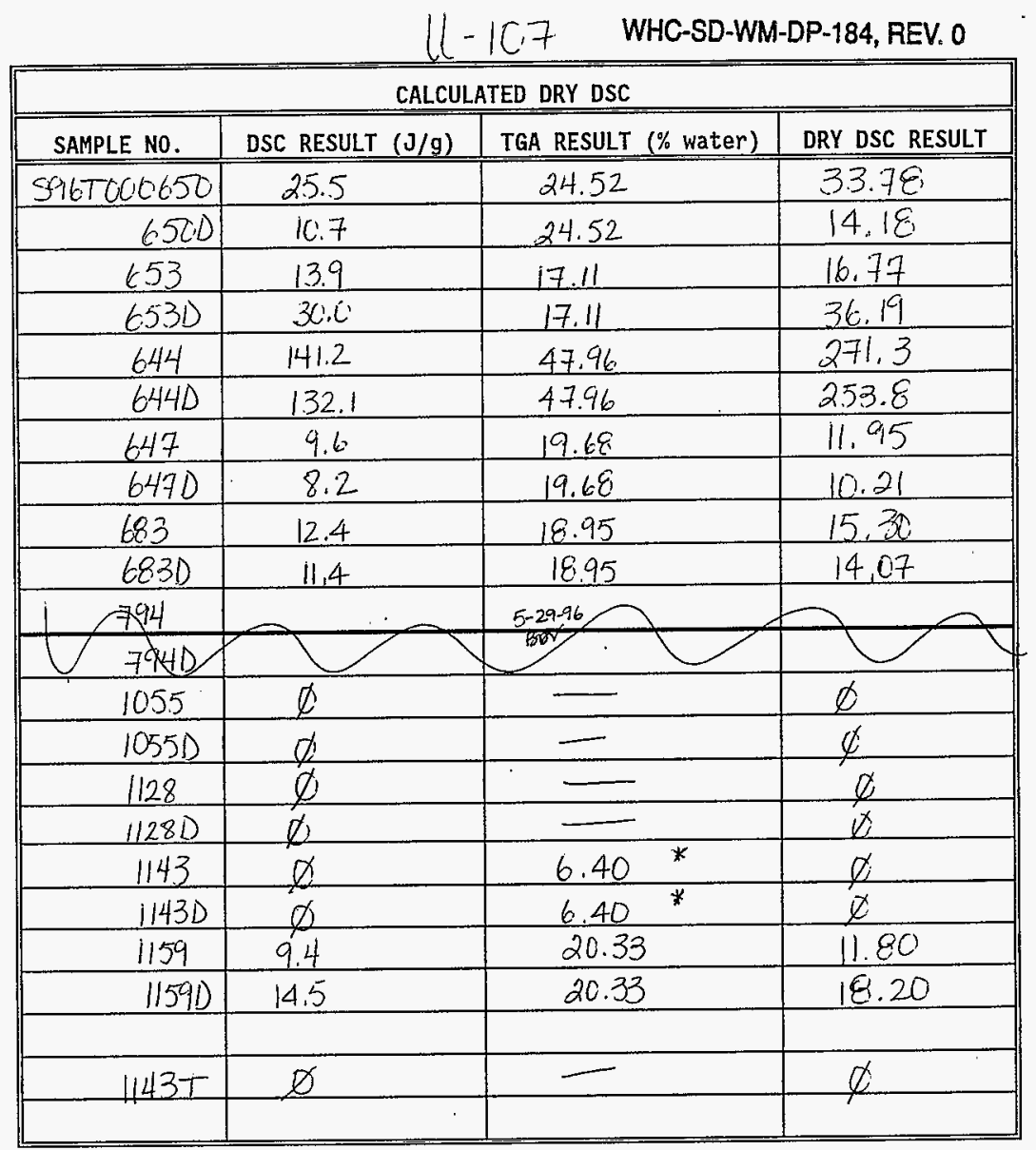

* Avg of 3 results 
Analyst: BOV

Instrument: DSC01

Book \#

Method: LA-514-113 Rev/Mod

Worklist Comment: Dry DSC for U-107. bdv

\begin{tabular}{|c|c|c|c|c|c|c|c|c|c|c|}
\hline GROUP & PROJECT & S TYPE & SAMPLE\# & $\mathrm{RA}$ & $-\cdots$ TEST $\cdots$ & MATRIX & ACTUAL & FOUND & $\mathrm{DL}$ & UNIT \\
\hline 96000422 & $u-107$ & 1 SAMPLE & S96T002131 & 0 & DSC-02 & SOLID & $\mathrm{N} / \mathrm{A}$ & & & Joules/g Dry \\
\hline 96000422 & $U-107$ & 2 DUP & S96T002131 & 0 & DSC-02 & SOLID & $\phi$ & $\varphi$ & N/A & Joules/g Dry \\
\hline 96000126 & $\mathrm{U}-107$ & 3 SAMPLE & S96T001056 & 0 & DSC-02 & SOLID & $N / A$ & & & Joules/g Dry \\
\hline 96000126 & $u-107$ & 4 DUP & \$96T001056 & 0 & DSC-02 & SOLID & $\varnothing$ & $\phi$ & $N / A$ & Joules/g Dry \\
\hline 96000126 & $U-107$ & 5 SAMPLE & s96T001057 & 0 & DSC-02 & SOLID & $\mathrm{N} / \mathrm{A}$ & 232.9 & & Joules/g Dry \\
\hline 96000126 & $u-107$ & 6 DUP & S96T001057 & 0 & DSC-02 & SOLID & 232.9 & 234.5 & N/A & Joules/g Dry \\
\hline 96000126 & $U-107$ & 7 SAMPLE & S96T001058 & 0 & DSC-02 & SOL.ID & N/A & 18.11 & & Joules/g Dry \\
\hline 96000126 & $u-107$ & 8 DUP & $\$ 967001058$ & 0 & DSC-02 & SOLID & 18.11 & 125.5 & N/A & Joules/g Dry \\
\hline 96000126 & U- 107 & 9 TRIPL & S96T001058 & 0 & DSC-02 & SOLID & 18.11 & 39.22 & $N / A$ & Joules/g Dry \\
\hline 96000126 & $\mathrm{U}-107$ & 10 SAMPLE & S96T001059 & 0 & DSC-02 & SOLID & $\mathrm{N} / \mathrm{A}$ & $\varnothing$ & & Joules/g Dry \\
\hline 96000126 & $U-107$ & 11 DUP & S961001059 & 0 & DSC-0Z & SOL ID & $\varnothing$ & $\varnothing$ & N/A & Joules/g Dry \\
\hline 96000126 & $U-107$ & 12 SAMPLE & \$961001064 & 0 & $\mathrm{DSC}-02$ & SOLID & & & & Joules/g Dry \\
\hline 96000126 & U- 107 & 13 DUP & S96T001064 & 0 & DSC-02 & SOLID & & & N/A & Joules/g Dry \\
\hline 96000126 & $U-107$ & 14 SAMPLE & S96T001065 & 0 & DSC-02 & SOLID & & & & Joules/g Dry \\
\hline 96000126 & $\mathrm{U}-107$ & 15 DUP & 596T001065 & 0 & $\mathrm{DSC}-02$ & SOLID & & & $\mathrm{N} / \mathrm{A}$ & Joules/g Dry \\
\hline 96000126 & $U-107$ & 16 SAMPLE & 596T001068 & 0 & DSC-02 & SOL.ID & & & & Joules/g Dry \\
\hline 96000126 & $U-107$ & 17 DUP & S967001068 & 0 & DSC.02 & SOLID & & & $\mathrm{N} / \mathrm{A}$ & Joules/g Dry \\
\hline 96000126 & $u-107$ & 18 SAMPLE & \$96T001070 & 0 & DSC-02 & SOLID & N/A & & & Joules/g Dry \\
\hline
\end{tabular}

Data Entry Comments:

Units shown for $Q C$ (SPK \& STD) may not reflect the actual units. $D L=$ Detection Limit, $S=$ Worklist Slot Number, $R=$ Replicate Number, $A=$ Aliquot Code. 


\section{LABCORE Data Entry Template for Worklist\#}

\begin{tabular}{|c|c|c|c|c|c|c|c|c|c|c|}
\hline GROUP & PROJECT & S TYPE & SAMPLE\# & $\mathrm{RA}$ & $-\cdots+-$ TEST $\ldots \ldots$ & MATRIX & ACTUAL & FOUND & DL & UNIT \\
\hline 96000126 & $U-107$ & 19 DUP & S96T001070 & 0 & DSC-02 & SOLID & & & $\mathrm{N} / \mathrm{A}$ & Joules/g Dry \\
\hline
\end{tabular}

Final page for worklist \#

9023

Blandina Valensulla 5-28-96 Analyst Signature 
U-107 WHG-SD-WM-DP-184, REV.O

\begin{tabular}{|c|c|c|c|}
\hline \multicolumn{4}{|c|}{ CALCULATED DRY DSC } \\
\hline SAMPLE NO. & DSC RESULT $(\mathrm{J} / \mathrm{g})$ & TGA RESULT (\% water) & DRY DSC RESULT \\
\hline 5967002131 & $\varnothing$ & - & $\varnothing$ \\
\hline 21310 & Q & - & $\varnothing$ \\
\hline 1056 & $\varnothing$ & - & $\varphi$ \\
\hline 10560 & $\varnothing$ & - & $\varnothing$ \\
\hline 1057 & 147.0 & 36.88 & 232.9 \\
\hline $1057 D$ & 148.0 & 36.88 & 234,5 \\
\hline 1058 & 11.5 & $36.57 *$ & 18.11 \\
\hline $1058 \mathrm{D}$ & 79.7 & $36.51 *$ & 125.5 \\
\hline 1059 & $q$ & 21.26 & $\varnothing$ \\
\hline 10590 & $\varnothing$ & 21.26 & $\varnothing$ \\
\hline 1004 & $\varnothing$ & - & $\not$ \\
\hline 10640 & $\varnothing$ & - & $\notin$ \\
\hline 1065 & $\varnothing$ & - & $\phi$ \\
\hline 10651 & $\not C$ & $=$ & $\varnothing$ \\
\hline 1068 & $\varnothing$ & 40.82 & 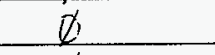 \\
\hline 10680 & $\phi$ & 40.8 .2 & $\varnothing$ \\
\hline 1070 & $\varnothing$ & 91.23 & $\not \varnothing$ \\
\hline 10700 & $\not$ & 91.23 & $\varnothing$ \\
\hline & & & \\
\hline $1058 \mathrm{~T}$ & 24.9 & $36.57 *$ & 39.22 \\
\hline & & & \\
\hline & & & \\
\hline
\end{tabular}

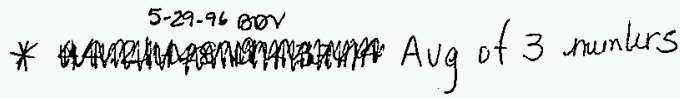


Analyst: $\quad B O V$

Instrument: DSC01

Book \#

Method: LA-514-113 Rev/Mod

Worklist Comment: Dry DSC for U-107. bdv

\begin{tabular}{|c|c|c|c|c|c|c|c|c|c|c|}
\hline GROUP & PROJECT & S TYPE & SAMPLE\# & R A & - - TEST & MATRIX & ACTUAL & FOUND & $\mathrm{DL}$ & UNIT \\
\hline 96000422 & $U-107$ & 1 SAMPLE & \$96T001867 & 0 & DSC -02 & SOL1D & $N / A$ & 280.2 & & Joules/g Dry \\
\hline 96000422 & $U-107$ & 2 DUP & S96T001867 & 0 & DSC -02 & SOLID & 280.2 & 207.6 & N/A & Joules/g Dry \\
\hline 96000422 & $u-107$ & 3 SAMPLE & S96T001873 & 0 & DSC-02 & SOLIO & $\mathrm{N} / \mathrm{A}$ & & & Joules/g Dry \\
\hline 96000422 & $U-107$ & 4 DUP & \$967001873 & 0 & DSC-02 & SOLID & 0 & & $\mathrm{~N} / \mathrm{A}$ & Joules/g Dry \\
\hline 96000422 & U-107 & 5 SAMPLE & S96T001874 & 0 & DSC -02 & SOLID & $\mathrm{N} / \mathrm{A}$ & $Q$ & & Joules/g Dry \\
\hline 96000422 & U-107 & 6 DUP & S96T001874 & 0 & DSC -02 & SOLID & $\varphi$ & & N/A & Joules/g Dry \\
\hline 96000422 & $U-107$ & 7 SAMPLE & S96T002132 & 0 & DSC -02 & SOLID & $N / A$ & & & Joules/g Dry \\
\hline 96000422 & $U-107$ & 8 DUP & S96T002132 & 0 & DSC -02 & SOLID & $\emptyset$ & & N/A & Joules/g Dry \\
\hline 96000422 & U-107 & 9 SAMPLE & S96T002133 & 0 & DSC -02 & SOLID & N/A. & $\varnothing$ & & Joules/g Dry \\
\hline 96000422 & $U-107$ & 10 DUP & S96T002133 & 0 & $D S C-02$ & SOLID & $D$ & & $\mathrm{~N} / \mathrm{A}$ & Joules/g Dry \\
\hline
\end{tabular}

\section{Final page for worklist \#}

9024

Blandina Valenncela) 5/22196 Analyst Signature Date

Analyst Signature Date

Data Entry Comments:

Units shown for $Q C$ (SPK \& STD) may not reflect the actual units. $D L=$ Detection Limit, $S=$ Worklist Slot Number, $R=$ Replicate Number, $A=$ Aliquot Code. 
WHC-SD-WM-DP-184, REV O

\begin{tabular}{|c|c|c|c|}
\hline \multicolumn{4}{|c|}{ CALCULATED DRY DSC } \\
\hline SAMPLE NO. & DSC RESULT $(\mathrm{J} / \mathrm{g})$ & IGA RESULT (\% water) & DRY DSC RESULT \\
\hline 5967001867 & 140.6 & 49.82 & 280.2 \\
\hline $1867 D$ & 105.2 & 49.82 & 209,6 \\
\hline 1873 & $\varnothing$ & - & $\varnothing$ \\
\hline 18730 & $\varnothing$ & - & $\notin$ \\
\hline 1874 & $\phi$ & $=$ & $\varnothing$ \\
\hline 18740 & $\not$ & - & $\varnothing$ \\
\hline 2132 & $\varnothing$ & - & $\varnothing$ \\
\hline 21320 & $\varnothing$ & - & $\varnothing$ \\
\hline 2133 & $\varnothing$ & 15.92 & $\varnothing$ \\
\hline 21331 & $\varnothing$ & 15.92 & $\varnothing$ \\
\hline & & & \\
\hline & & & \\
\hline & & & \\
\hline & & & $\because$ \\
\hline & & & \\
\hline & & & \\
\hline & & & 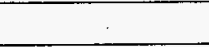 \\
\hline & & & \\
\hline & & & \\
\hline & & & \\
\hline & & & \\
\hline & & & \\
\hline & & & \\
\hline
\end{tabular}




\section{LABCORE Data Entry Template for Worklist\#}

Analyst: ADP Instrument: DSCO 3 Book \# 12 N14B

Method: LA-514-114 Rev/Mod C - /

Worklist Comment: U-107. Run under N2.

\begin{tabular}{|c|c|c|c|c|c|c|c|c|c|}
\hline GROUP & PROJECT & $\begin{array}{l}\text { S TYPE } \\
3 \text { STD }\end{array}$ & SAMPLE\# & $\begin{array}{l}\text { DASC } 03 \\
\text { DSC }\end{array}$ & $\begin{array}{l}\text { MATRIX } \\
\text { SOLID }\end{array}$ & $\begin{array}{l}\text { ACTUAL } \\
28.45 \\
\end{array}$ & $\begin{array}{l}\text { FCUND } \\
28.94 \\
\end{array}$ & $*_{\text {N/A }}^{*}$ & $\begin{array}{l}\text { UNIT } \\
\text { Joules/g }\end{array}$ \\
\hline 96000422 & U- 107 & 2 SAMPLE & S96T002131 & -DSC-03 & SOLID & N/A & $\varnothing$ & & Joules/g \\
\hline 96000422 & U-107 & 3 DUP & 596T002131 & DSC $=03$ & SOLID & $\varnothing$ & $\varnothing$ & N/A & Joules/g \\
\hline
\end{tabular}

\section{Final page for worklist \# 9028} Validated by Hanaston 5.22 .96

Data Entry Comments:

Units shown for $Q C$ (SPK \& STD) may not reflect the actual units. $D L=$ Detection Limit, $S=$ Worklist Slot Number, $R=$ Replicate Nimber, $A=$ Aliquot Code. 
Curye 1: asc

F138 info: Ifo051502 Wed May 45 18: 29: 241996

Sample Veight: 11.620 ng

12N14-B INDTUM AT 10C $M$ HIN

SIGNATURE BELOW REPRESENTS CHEMICAL TECHNOLOGIST/CHEMIST THAT

CONPLETED/VERIFIED THE CALIBRATION/ANALYSIS ON PAGES $17 / 7$ TO $17 \%$.

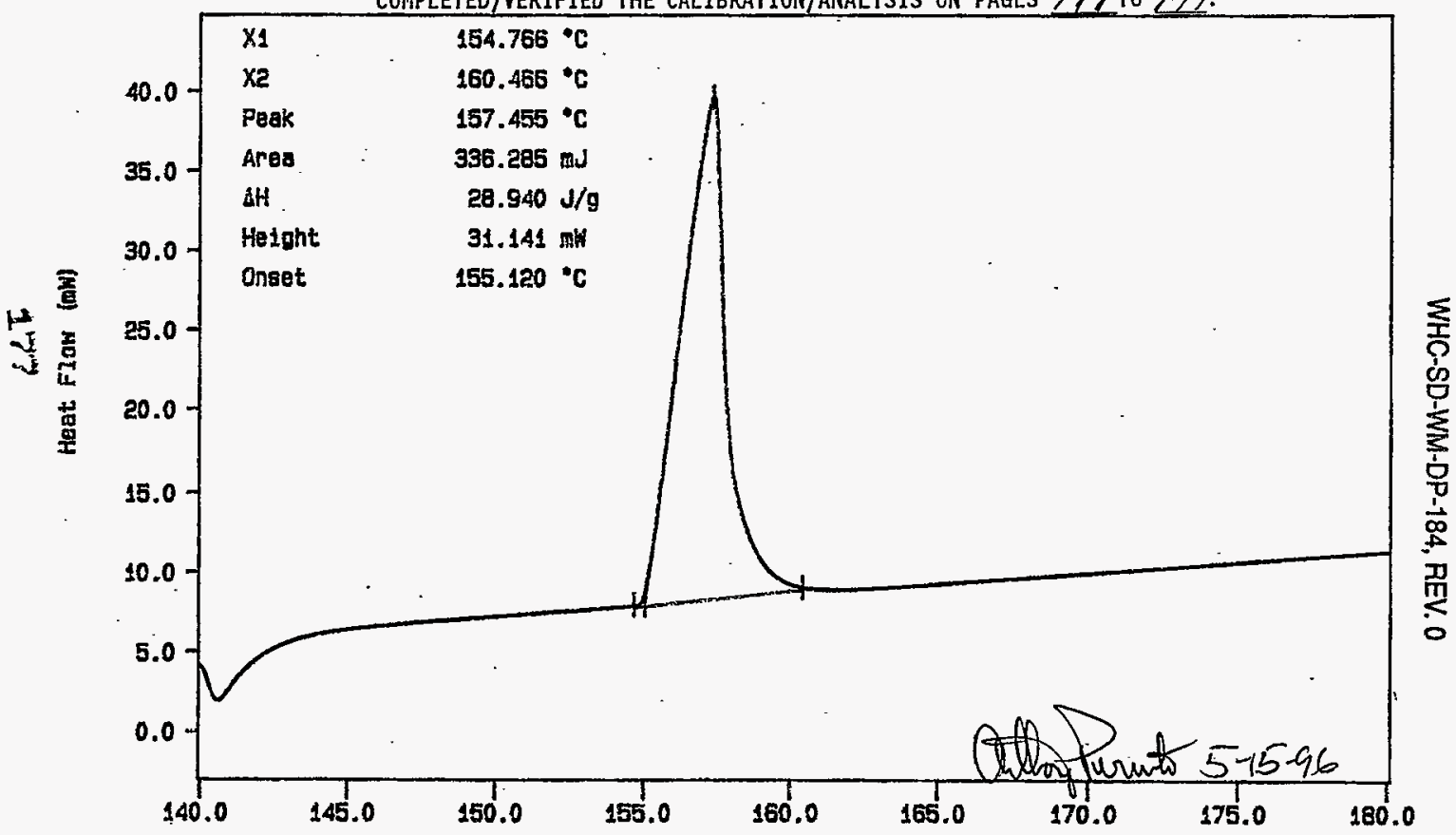

N2, EXOTHERM DOHN

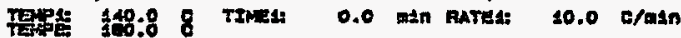

Temperature $\left({ }^{\circ} \mathrm{C}\right)$

$A D$ PUAIMTON

PEAKIN-ELMEA

7 Sertes Thermel Analysis Systen Hed May 15 16:32 151996 
Curve 1: DSC

File info: SAMQ54505 hed Moy 15 21: 10:51 1996

Sampis Height: $42.390 \mathrm{mg}$

S96T002131 SAM

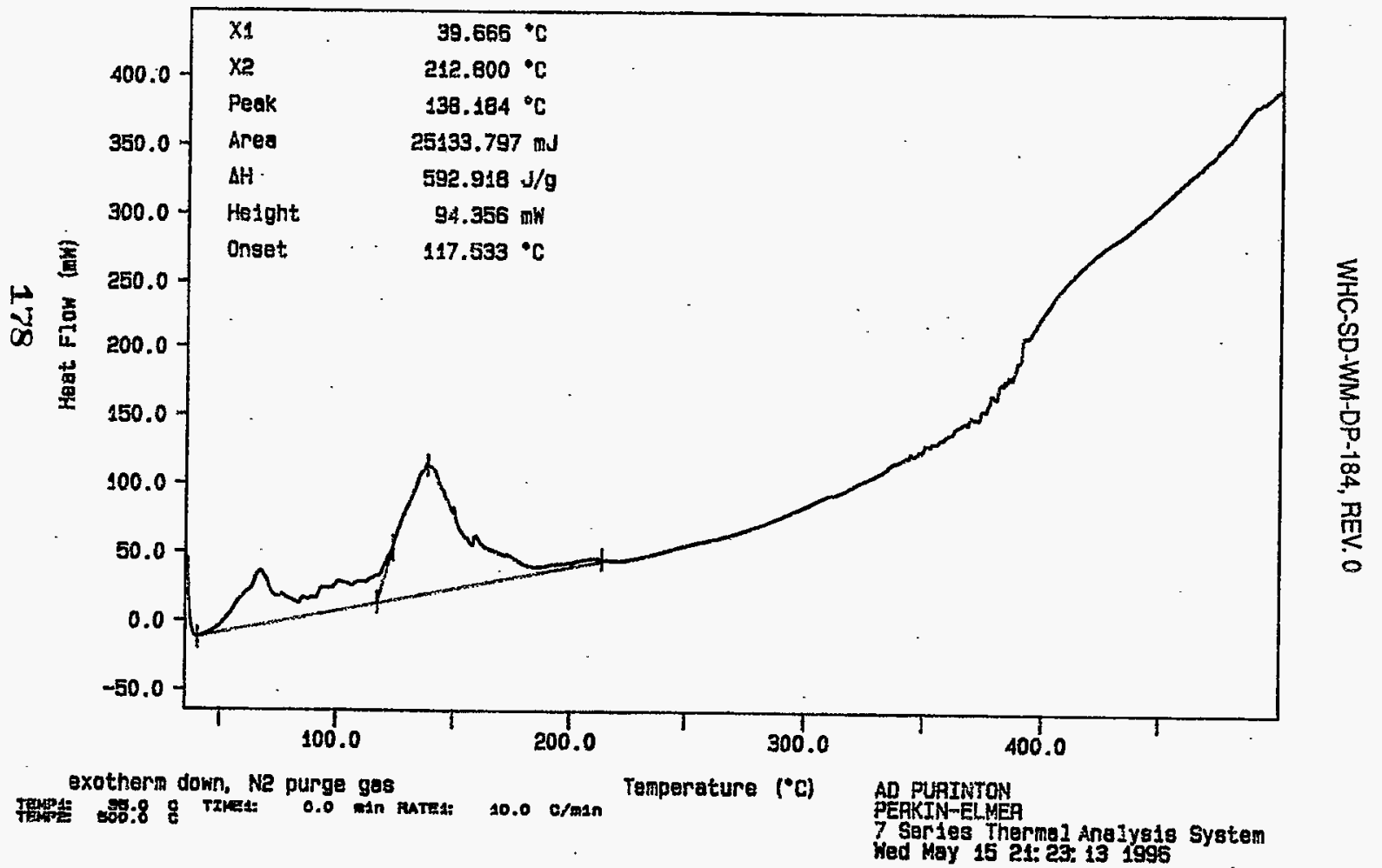


Curve 1: 050

File info: SAM051506 Hed May 15 22: 19; 471996

Sample Naight: $29.090 \mathrm{mg}$

S967002131 DUP

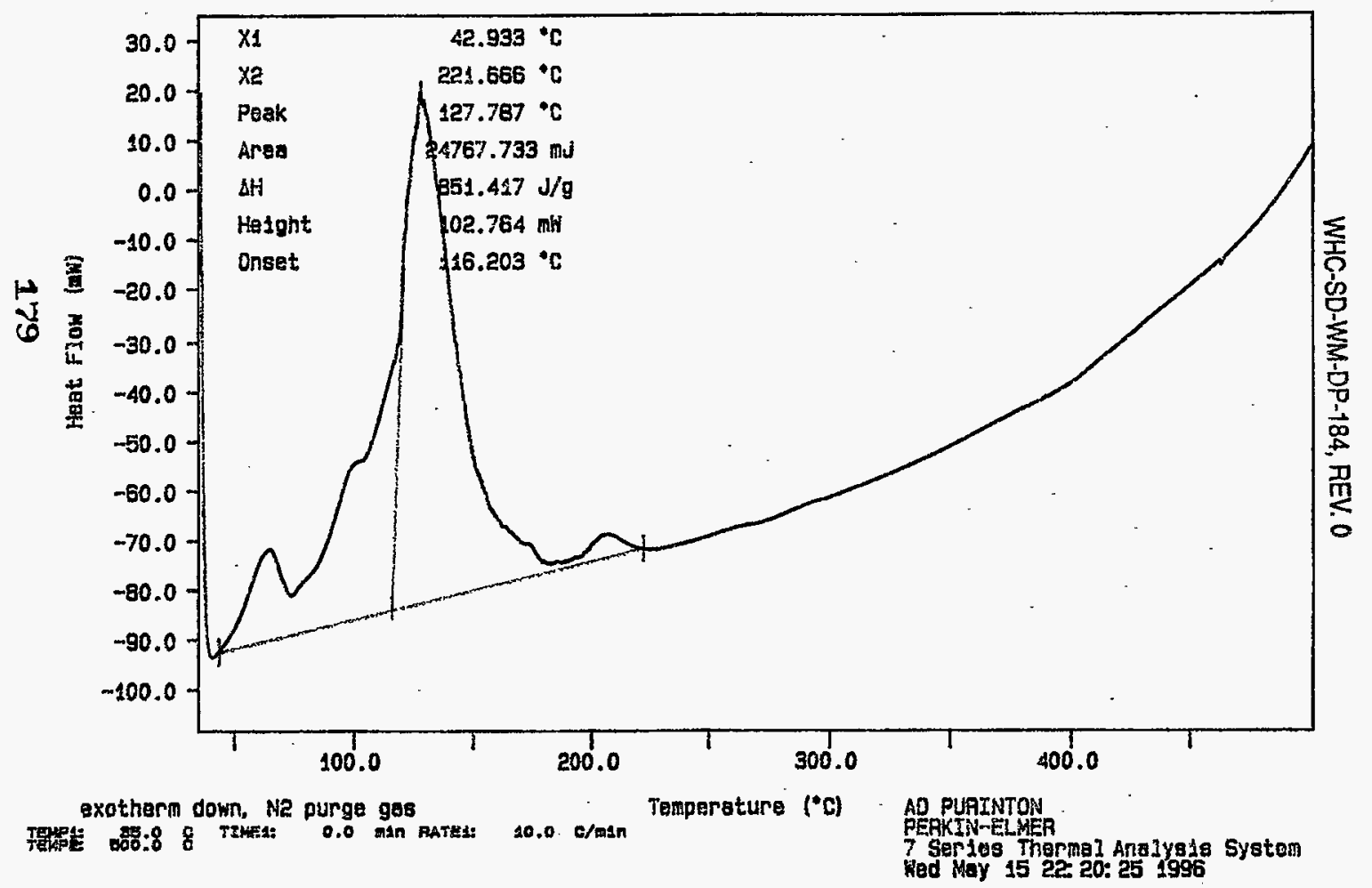




\section{LABCORE Data Entry Template for Worklist\#}

Analyst: $\quad$ BSW $\quad$ Instrument: $\mathrm{DSC} \quad$ Book \# _-

Method: LA-514-113 Rev/Mod

Worklist Comment: Dry DSC for U-107. bdv

\begin{tabular}{|c|c|c|c|c|c|c|c|c|c|c|}
\hline GROUP & PROJECT & S TYPE & SAMPLE\# & $R$ & A $\cdots---$ TEST $-\cdots$ & MATRIX & ACTUAL & FOUND & $\mathrm{DL}$ & UNIT \\
\hline 96000422 & $\mathrm{U}-107$ & 1 SAMPLE & s96T001866 & 0 & DSC-02 & LIQUID & $N / A$ & $\phi$ & & Joules/g Dry \\
\hline 96000422 & U-107 & 2 DUP & S96T001866 & 0 & DSC-02 & LIQUID & $\infty$ & $(\infty$ & $\mathrm{N} / \mathrm{A}$ & Joules/g Dr \\
\hline
\end{tabular}

Cata entered + verified by Final page for worklist \# 9283 Blandina Valenbuela, Analyst Signature 
worklistrpt Version 2.1 05/15/95

WHC-SD-WM-DP-184, REV. 0

Page: 1 02/26/96 07:17

LABCORE Data Entry Template for Worklist\#

5890

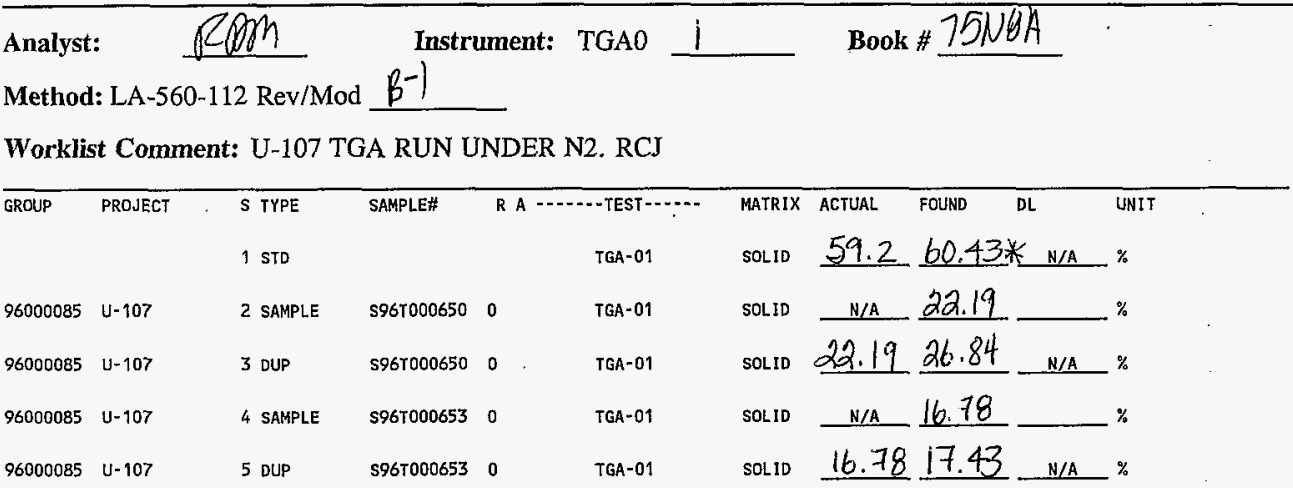

Final page for worklist \# $\mathbf{5 8 9 0}$
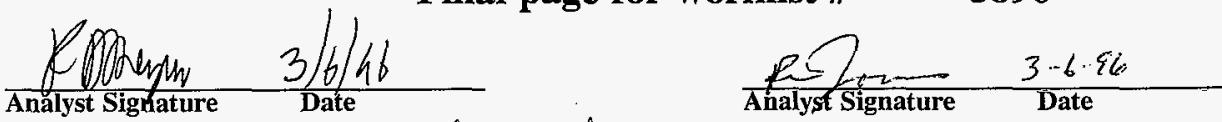

Verified by Blandina Valenzuela $\$ 17 / 96$

Data Entry Comments: S967000650 results are the sum of two $\$$. wright

Units shown for $Q C$ (SPK \& STD) may not reflect the actual units. $D L=$ Detection Limit, $S=$ Worklist Slot Number, $R=$ Replicate Number, $A=$ Aliquot Code.

181 


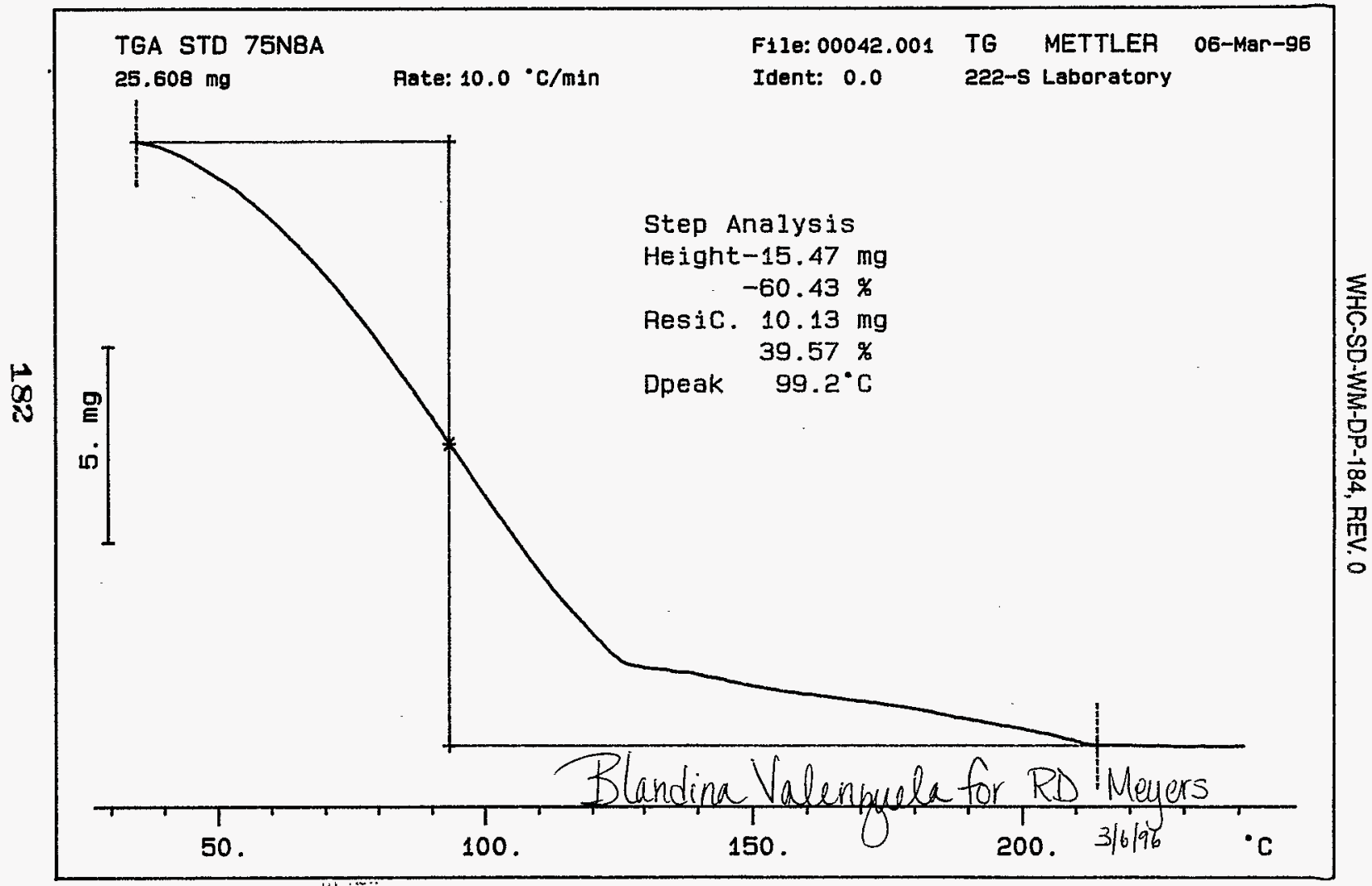

SIGNATURE ABOVE REPRESENTS CHEMICAL TECHNOLOGIST/CHEHIST THAT COMPLETED/VERIFIED THE CALIBRATION/ANALYSIS ON PAGES \&TO/\&6. 


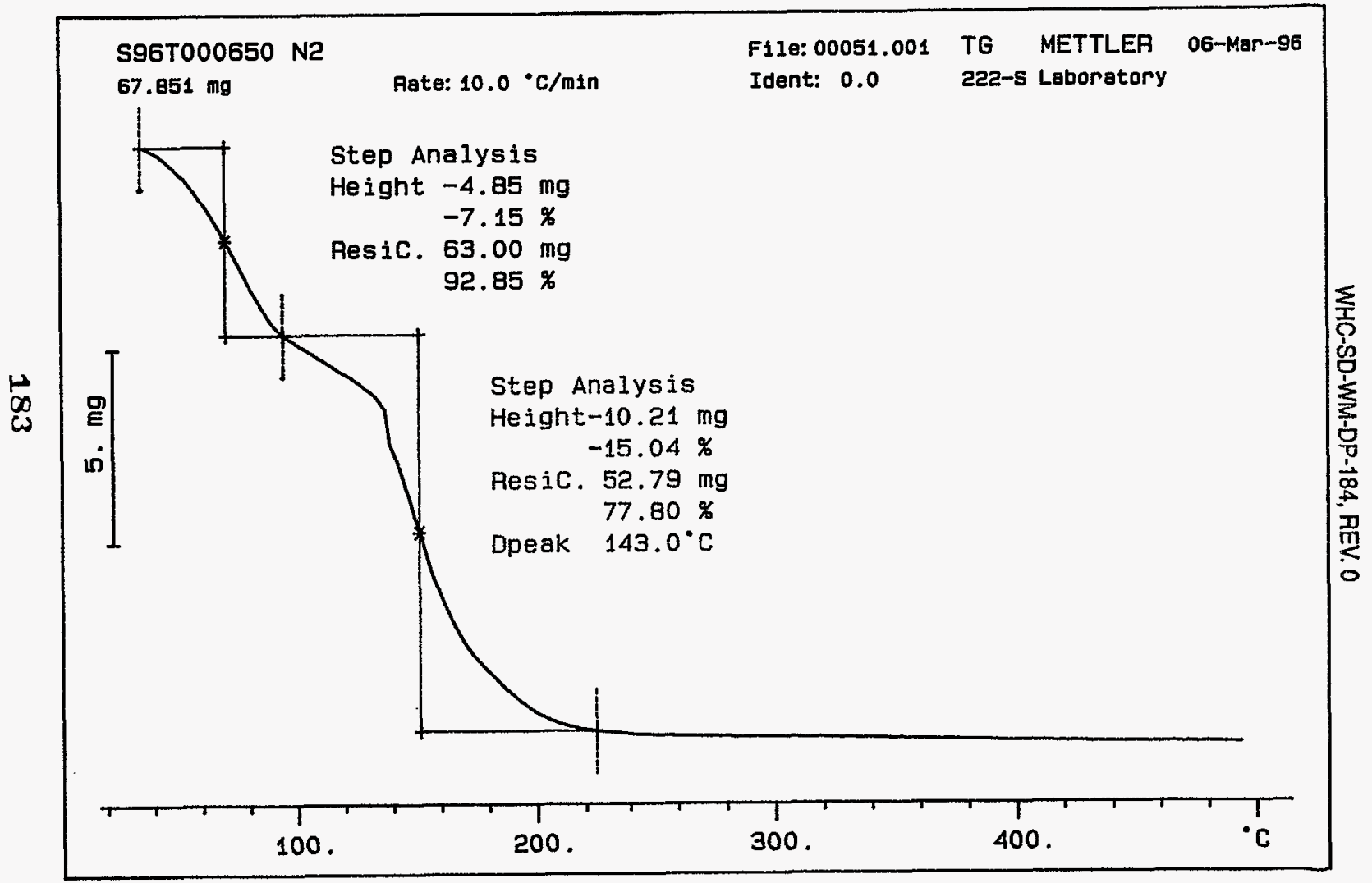




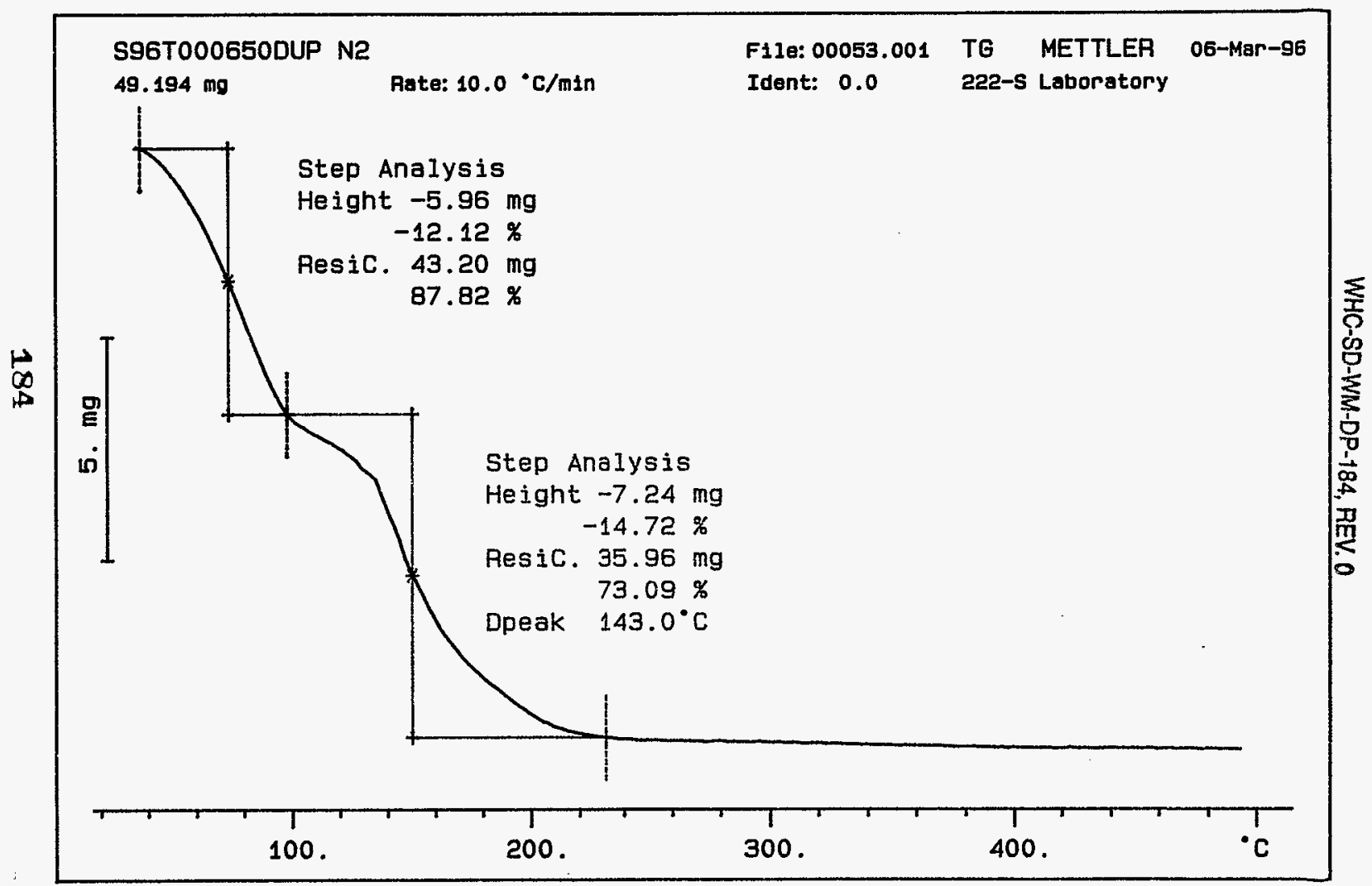




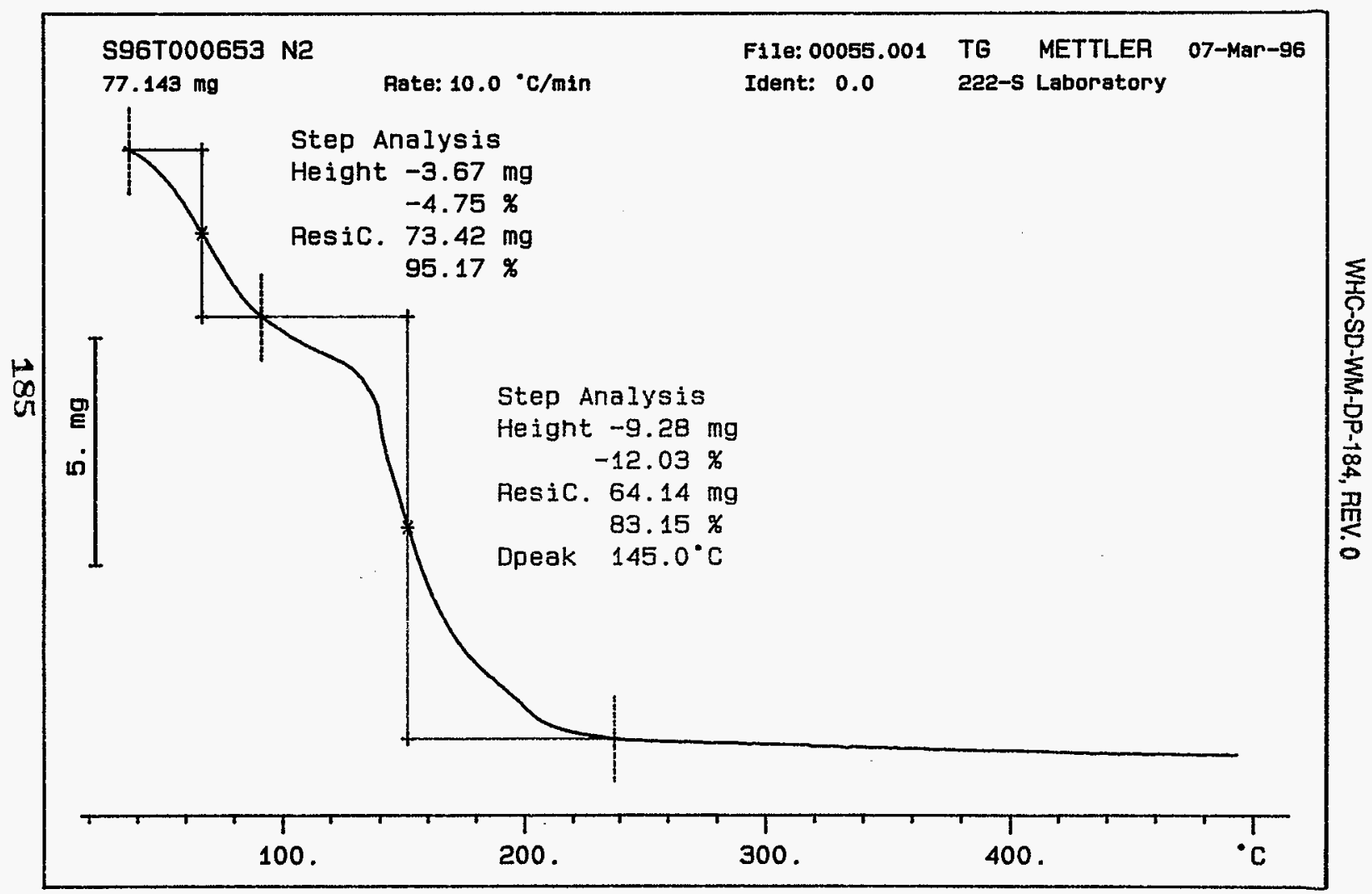




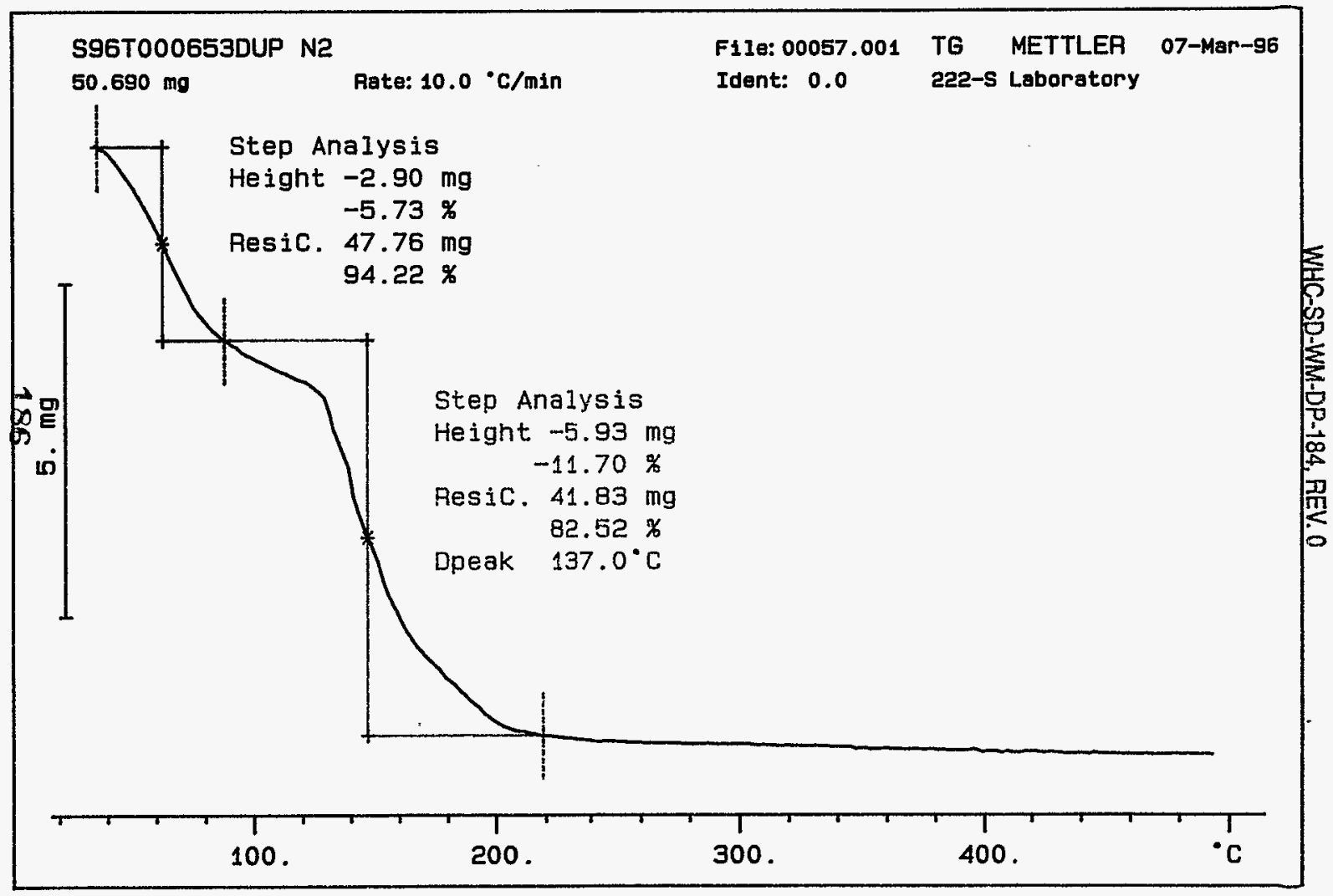


Analyst: HfMclowx Instrument: TGA0 I Book \# 7508A

Method: LA-560-112 Rev/Mod $B-1$

Worklist Comment: U-107 TGA RUN UNDER N2. RCJ

\begin{tabular}{|c|c|c|c|c|c|c|c|c|}
\hline GROUP & PROJECT & $\begin{array}{l}\text { S TYPE } \\
1 \text { STD }\end{array}$ & SAMPLE\# & $\mathrm{RA}$ & TGA-01 & $\begin{array}{l}\text { MATRIX } \\
\text { SOLID }\end{array}$ & 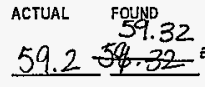 & DL \\
\hline 96000085 & U-107 & 2 SAMPLE & S96T000644 & 0 & TGA-01 & SOLID & 48.57 & \\
\hline 96000085 & $\mathrm{U}-107$ & 3 DUP & S96T000644 & 0 & TGA-01 & SOLID & 47.34 & N/A \\
\hline 96000085 & U-107 & 4 SAMPLE & S96T000647 & 0 & TGA-01 & SOLID & 21.1 & \\
\hline 96000085 & U-107 & 5 DUP & S96T000647 & 0 & TGA-01 & SOLID & 18.26 & N/A \\
\hline
\end{tabular}

Final page for workligt \# 5891
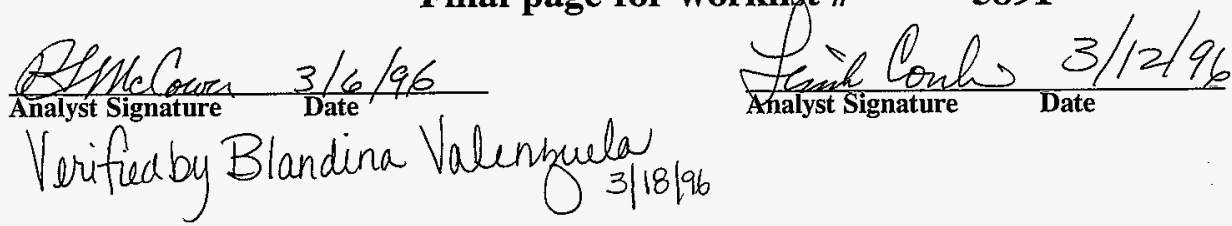

Data Entry Commens: S96T000644 results are the sum of two weight loss steps 5967000647 results are the sum of two wright loss steps

Units shown for $Q C$ (SPK \& STD) may not reflect the actual units. $D L=$ Detection Limit, $S=$ Worklist Slot Number, $R=$ Replicate Number, $A=$ Aliquot Code. 


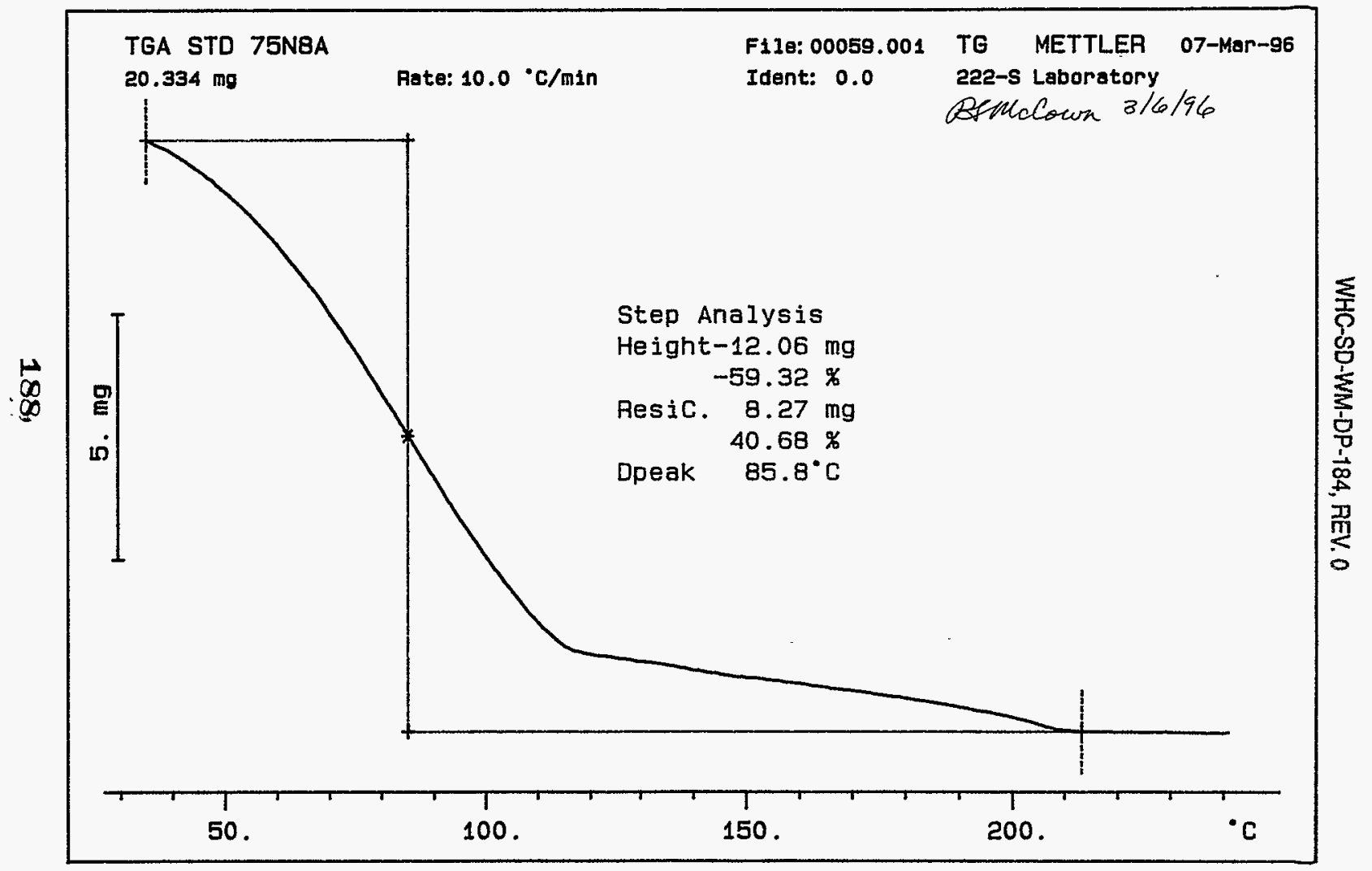




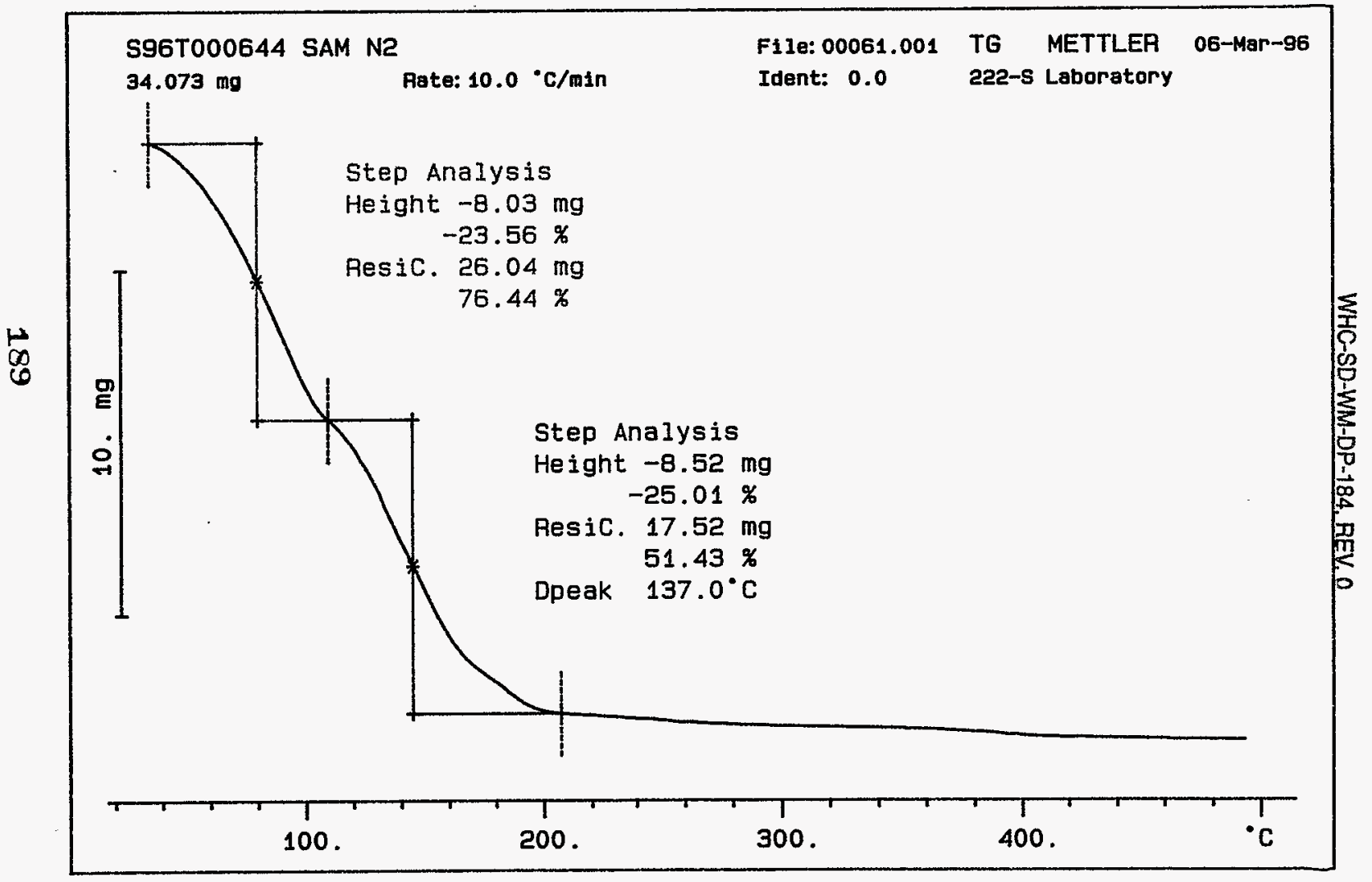




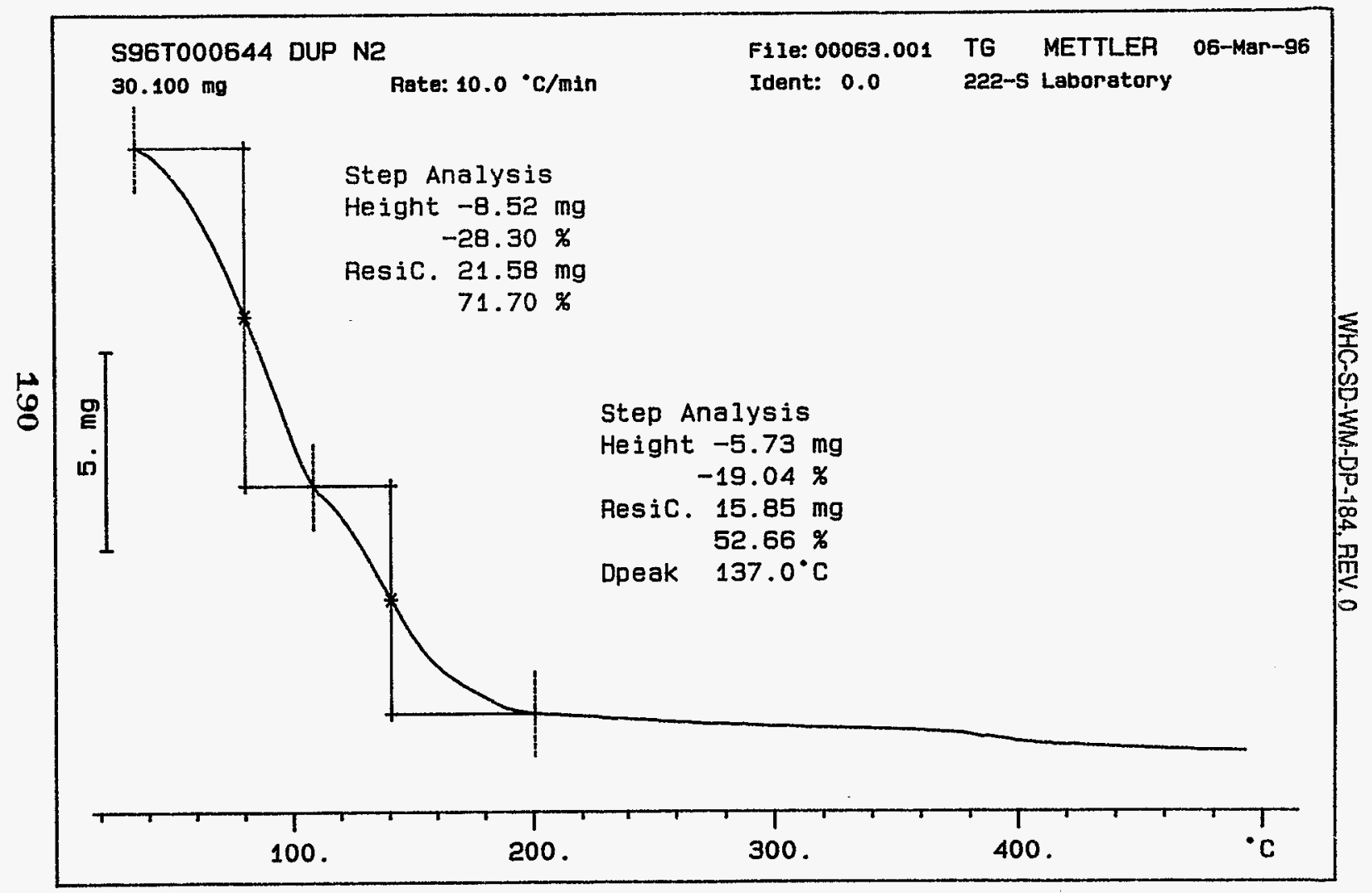


WHC-SD-WM-DP-184, REV. 0

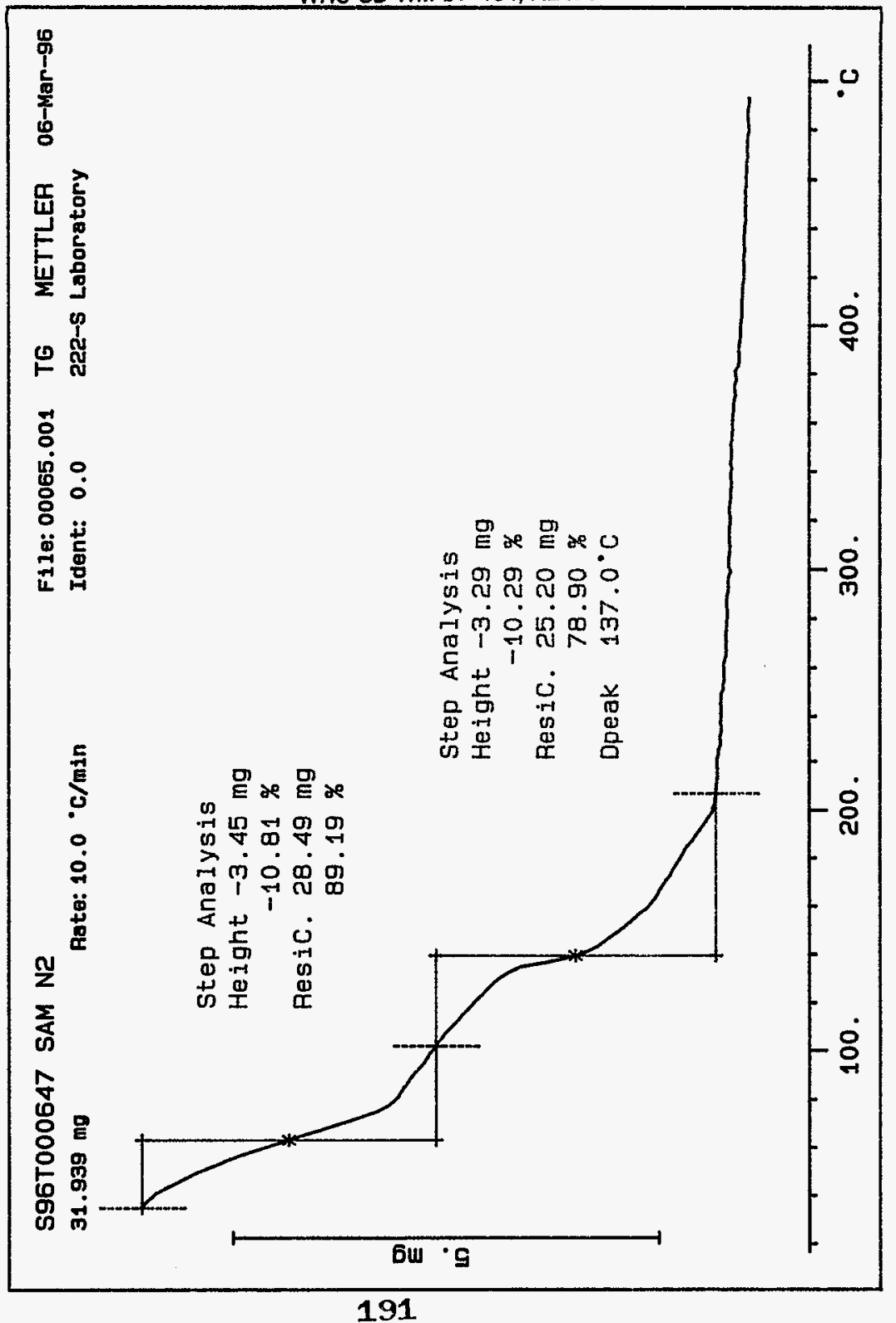




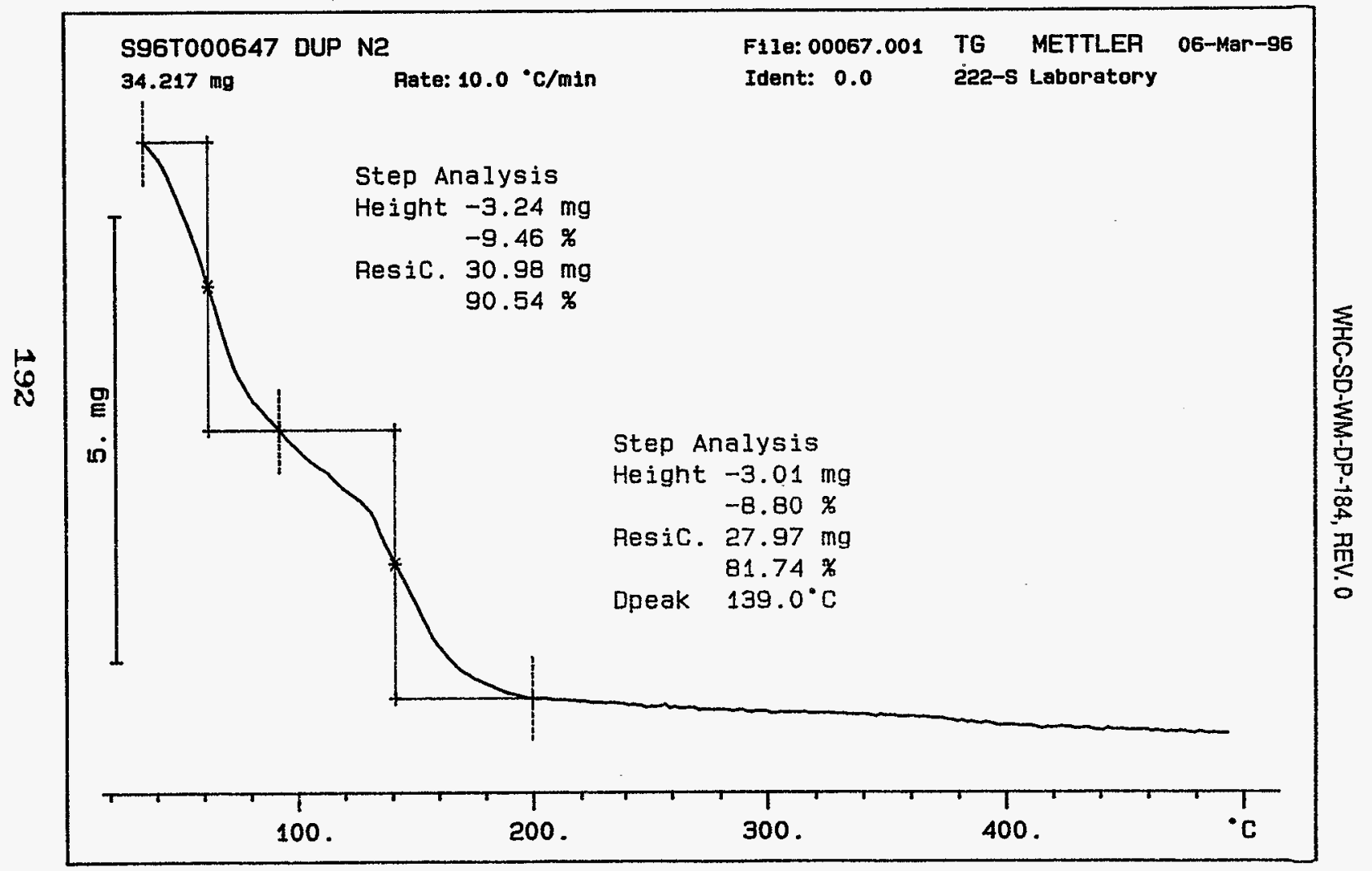




\section{LABCORE Data Entry Template for Worklist\#}

Analyst: SMF Instrument: TGA0 I Book \# $75 \mathrm{~N} 8 \mathrm{~A}$

Method: LA-560-112 Rev/Mod B-1

Worklist Comment: U-107 TGA-01 RUN UNDER N2. RCJ

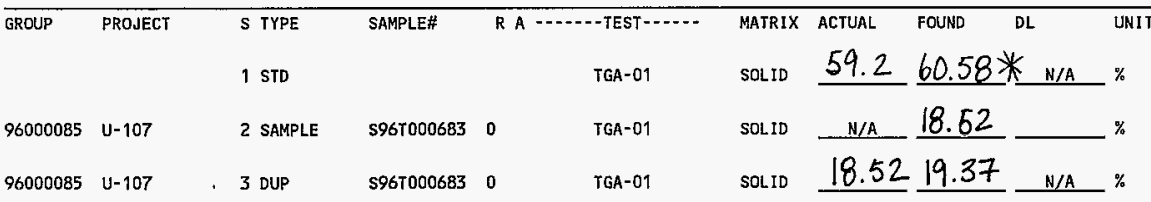

\section{Final page for worklist \#}

Luse 77 . Zultor 3-5-76 Analyst Signature

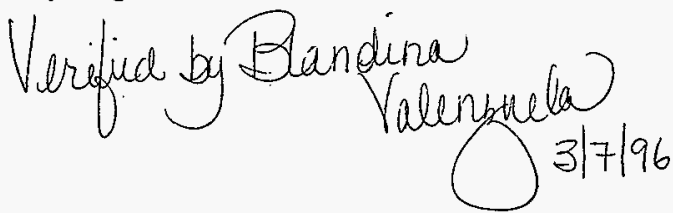

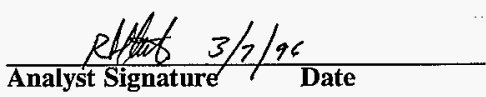

Data Entry Comments: She results given are the sum of two weight loss steps.

Units shown for $Q C$ (SPK \& STD) may not reflect the actual units. $D L=$ Detection Limit, $S=$ Worklist Slot Number, $R=$ Replicate Number, $A=$ Aliquot Code. 
SIGNATURE BELOH REPRESENTS CHEHICAL TECHNOLOGIST/CHEHIST THAT

COMPLETED/VERIFIED THE CALIBRATION/ANALYSIS ON PAGES 94 TO 19.5 .

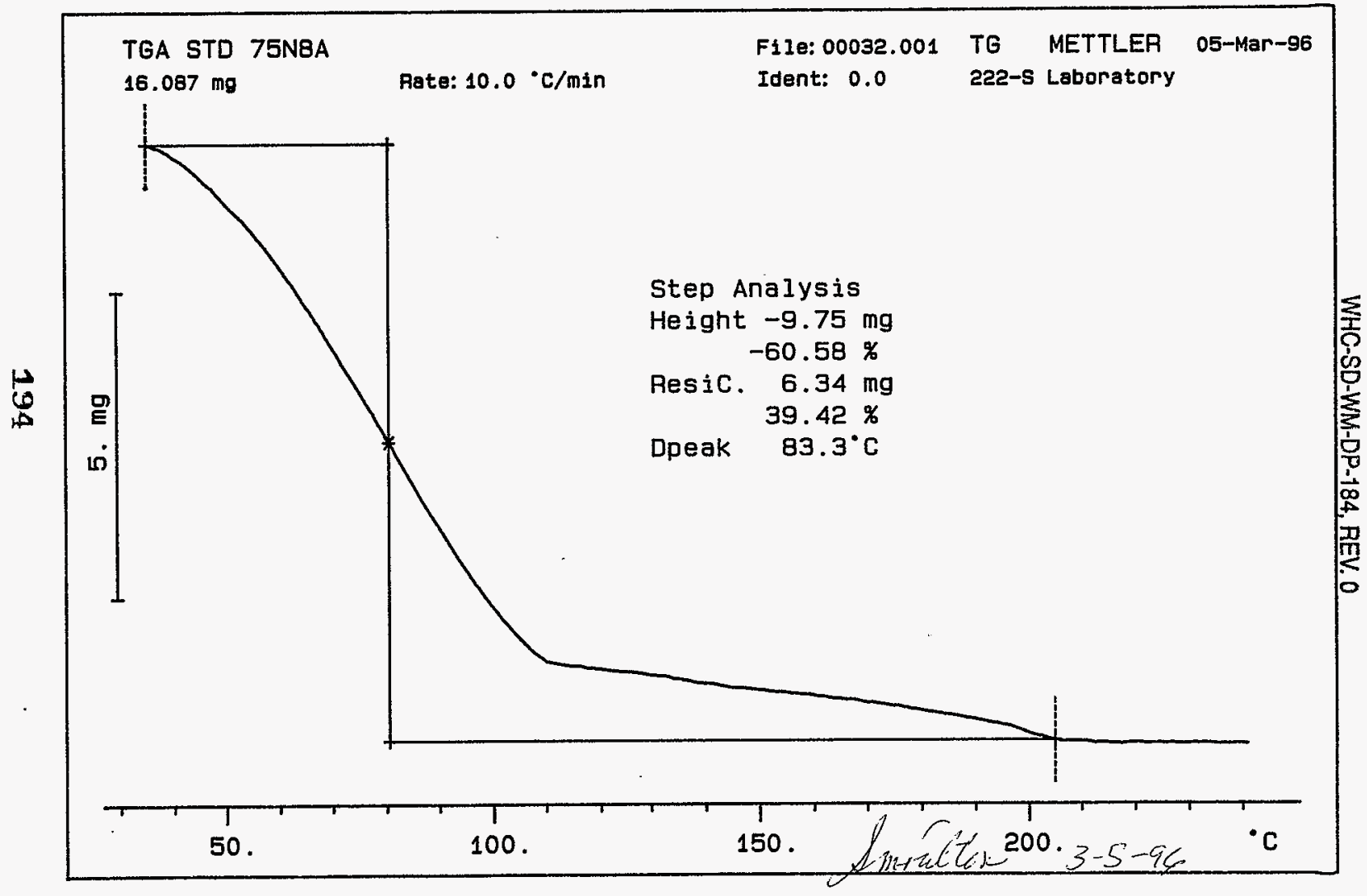




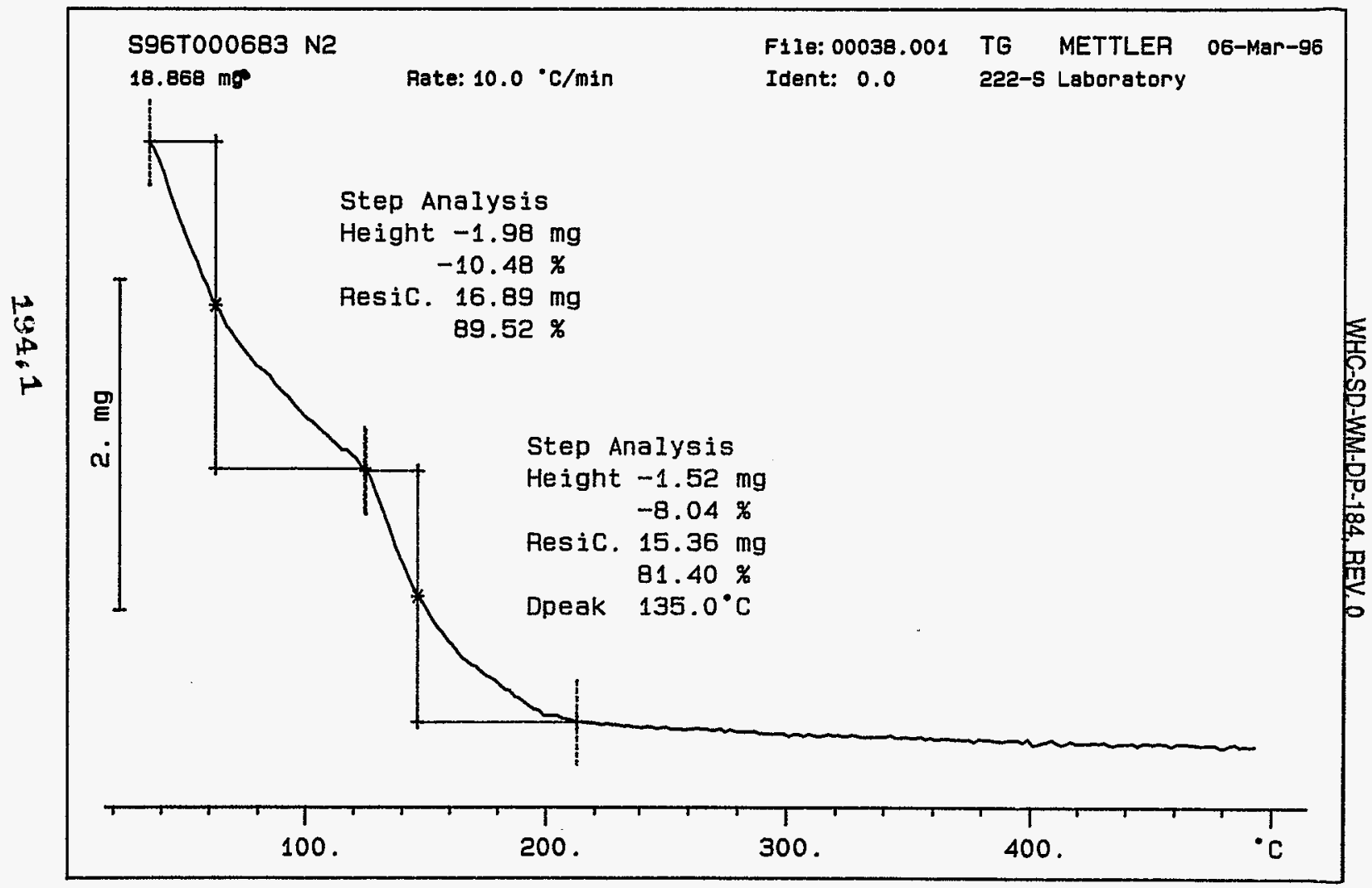




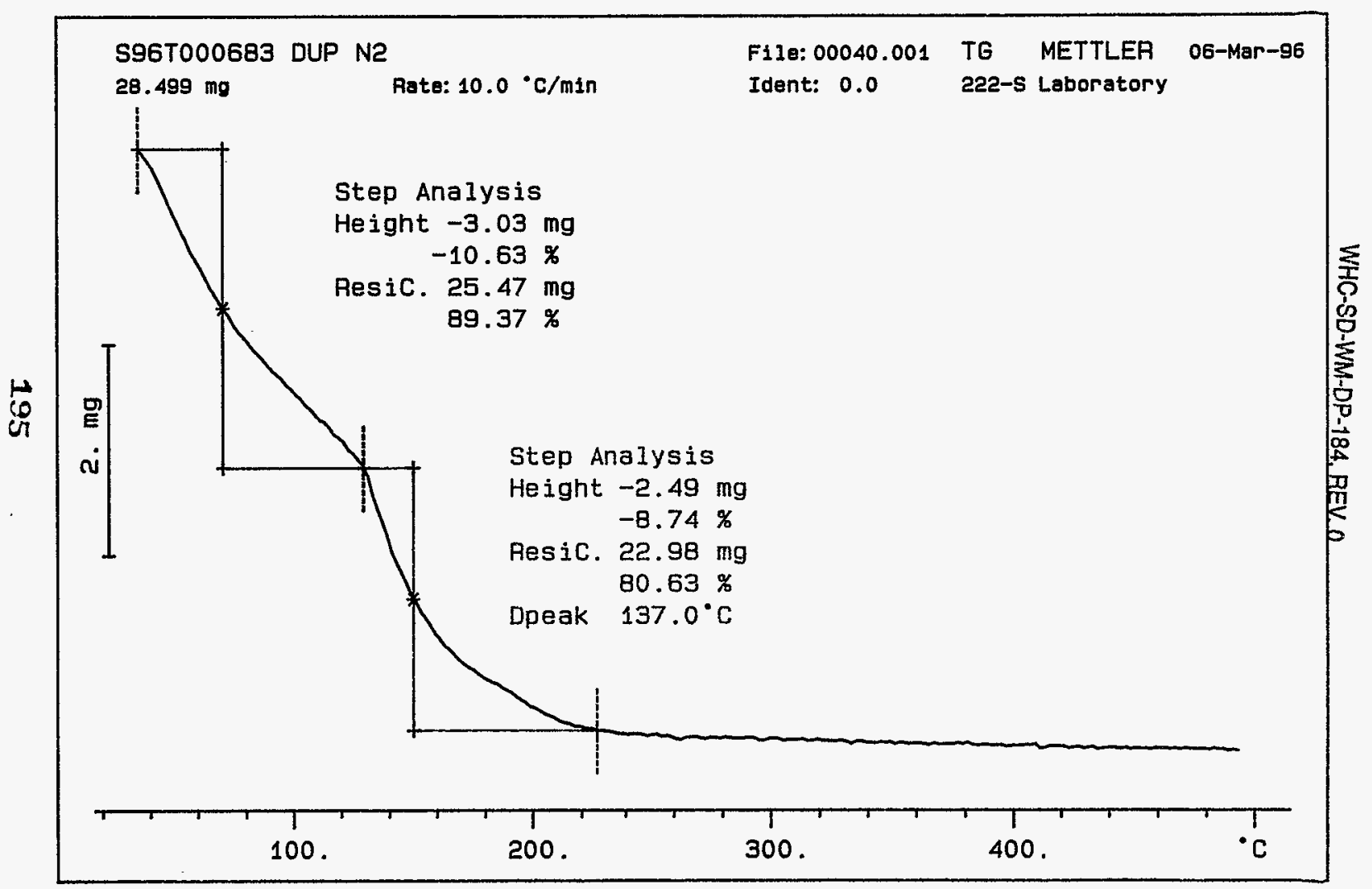


Analyst: $\quad S M F$ Instrument: TGAO 3 Book \# $82 \mathrm{~N} 8 \mathrm{~A}$

Method: LA-514-114 Rev/Mod $\mathrm{C}-1$

Worklist Comment: U-107 TGA RUN UNDER N2. RCJ

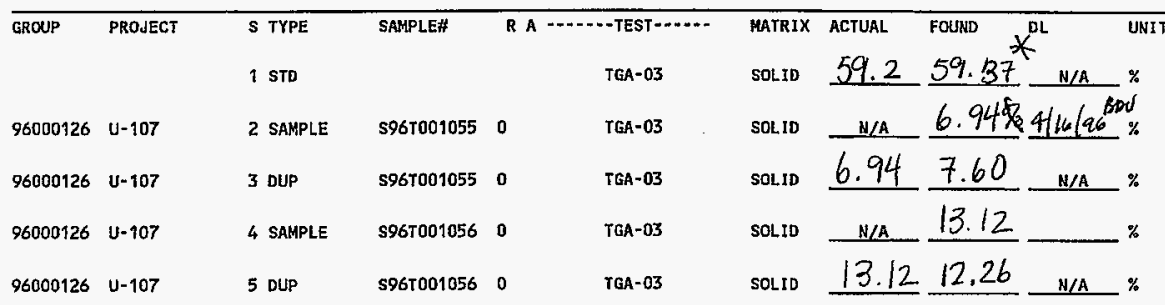

\section{Final page for worklist \#}

6499

Se attached for sianattures

Analyst Signature Date $4116 / 96$ Verified HQnaston $4-22-96$

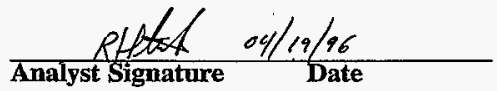

Data Entry Comments:

Units shown for $Q C$ (SPK \& STD) may not reflect the actual units. $D L=$ Detection Limit, $S=$ Worklist Slot Number, $R=$ Replicate Number, $A=$ Aliquot Code. 


\section{LABCORE Data Entry Template for Worklist\#}

Analyst: SMF Instrument: TGA0 Book B

Method: LA-560-112 Rev/Mod

Worklist Comment: U-107 TGA RUN UNDER N2. RCJ

\begin{tabular}{|c|c|c|c|c|c|c|c|c|c|c|}
\hline \multirow[t]{2}{*}{ GROLP } & \multirow[t]{2}{*}{ PROJECT } & S TYPE & \multirow[t]{2}{*}{ SAMPLE\# } & \multicolumn{2}{|c|}{ R A $\cdots$ TEST $\cdots$} & \multirow{2}{*}{$\begin{array}{l}\text { MATRIX } \\
\text { SOLID }\end{array}$} & \multirow[t]{2}{*}{ ACTUAL } & \multirow[t]{2}{*}{ FOUND } & \multirow[t]{2}{*}{ DL } & \multirow{2}{*}{$\begin{array}{l}\text { UNI } \\
\%\end{array}$} \\
\hline & & 1 STD & & & TEA-01 & & & & & \\
\hline 96000126 & $U-107$ & 2 SAMPLE & S96T001055 & 0 & TEA-01 & SOLID & $N / A$ & & & $\%$ \\
\hline $96000 \uparrow 26$ & $U-107$ & 3 DUP & 596T001055 & 0 & TEA-01 & SOLID & & & & $\%$ \\
\hline 96000126 & u- 107 & 4 SAMPLE & \$96T001056 & 0 & rGA-01 & SOLID & N/A & & & $\%$ \\
\hline 96000126 & $U-107$ & 5 DUP & S96r001056 & 0 & $T G A-01$ & SOLID & & & & $\%$ \\
\hline
\end{tabular}

\section{Final page for worklist \#}

6499

Susum. chetore 4-15-96

Analyst Signature Date
Analyst Signature Date

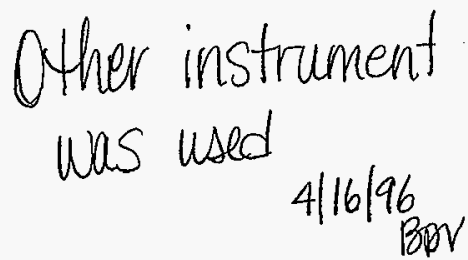

Data Entry Comments:

Units shown for $Q C$ (SPK \& STD) may not reflect the actual units. $D L=$ Detection Limit, $S=$ Worklist Slot Number, $R=$ Replicate Number, $A=$ Aliquot Code. 
Curve 1: TEA

F11e Info: TERO41501 Mon Apr 15 06: 12: 101996

Sanple Height: 21.445 ang

TGA STD BENB-A

SIGNATURE BELOH REPRESENTS CHEMICAL TECHNOLOGIST/CHEMIST THAT COHPLETED/VERIFIED THE CALIBRATION/ANALYSIS ON PAGES $19 \&$ TO ZCZ

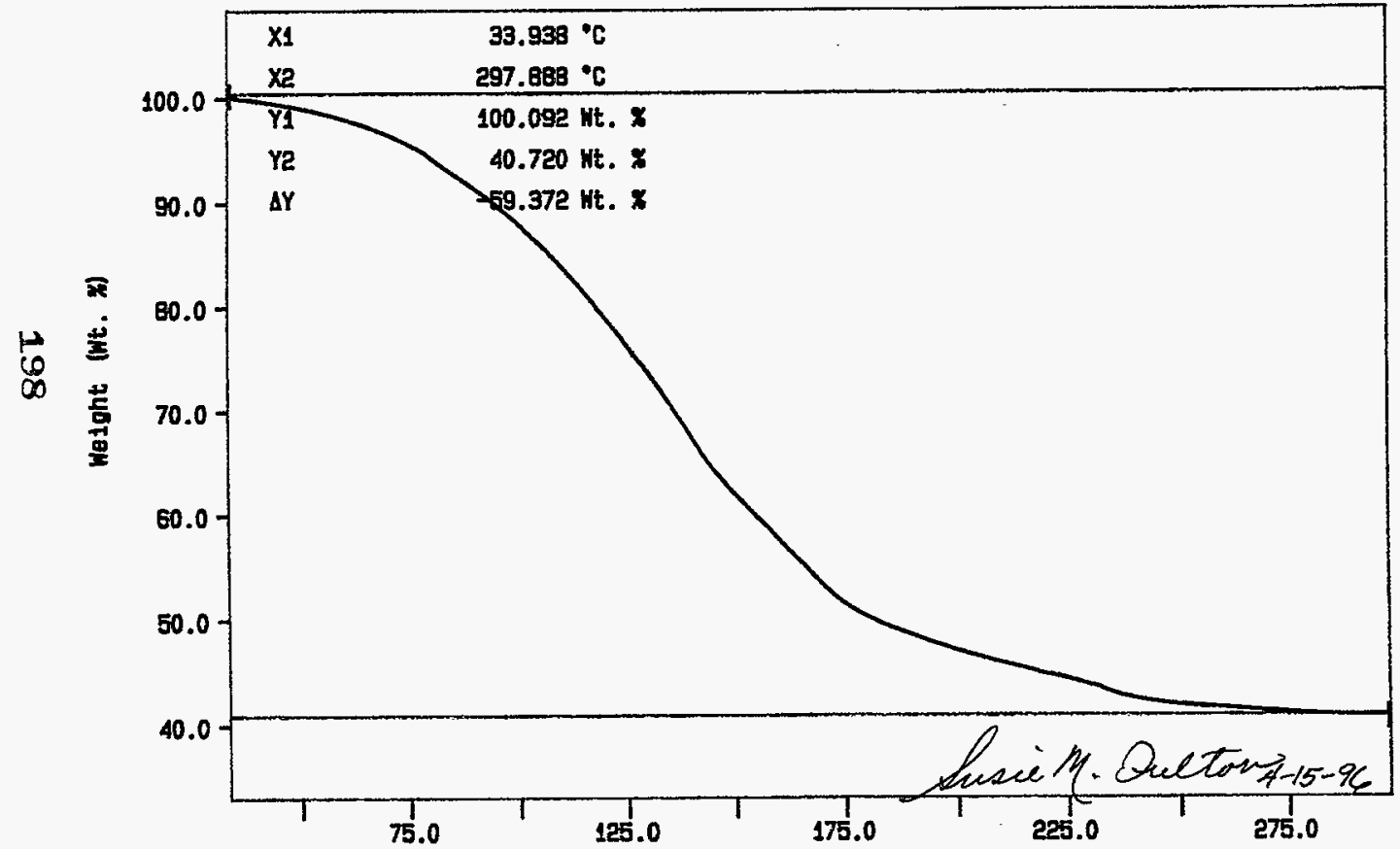


용 Curve 1: TEA

FIle into: SAM041504 Won Apr 15 07:35: 481995

Serple Height: 12.179 mg

S95T001055
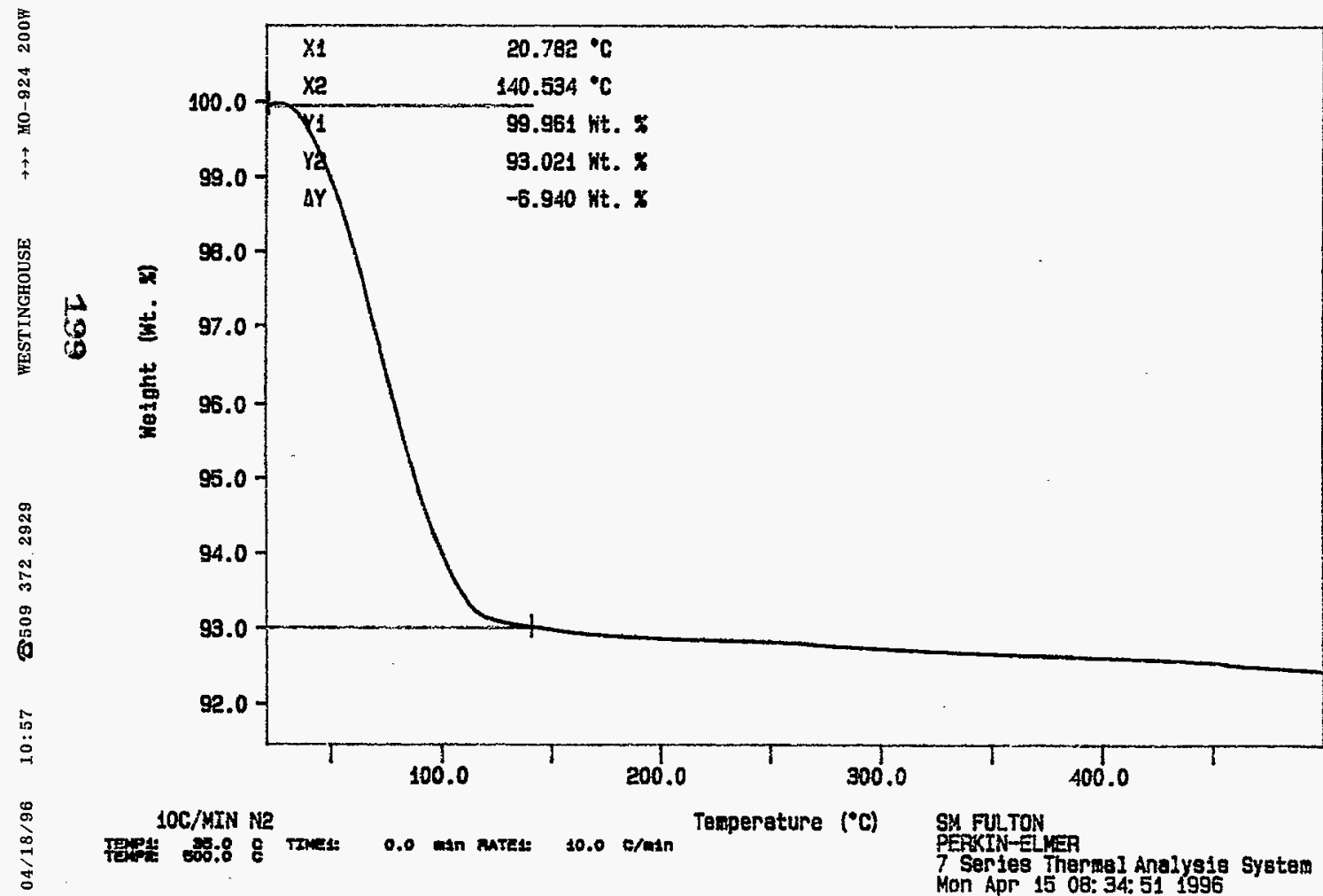

$\stackrel{8}{\circ}$

is 
Curve 1: TGA

File info: SAy041505 Mon Apr 15 09: 27:07 1996

Sample Neight: 21.256 ag

S96T001055 DUP

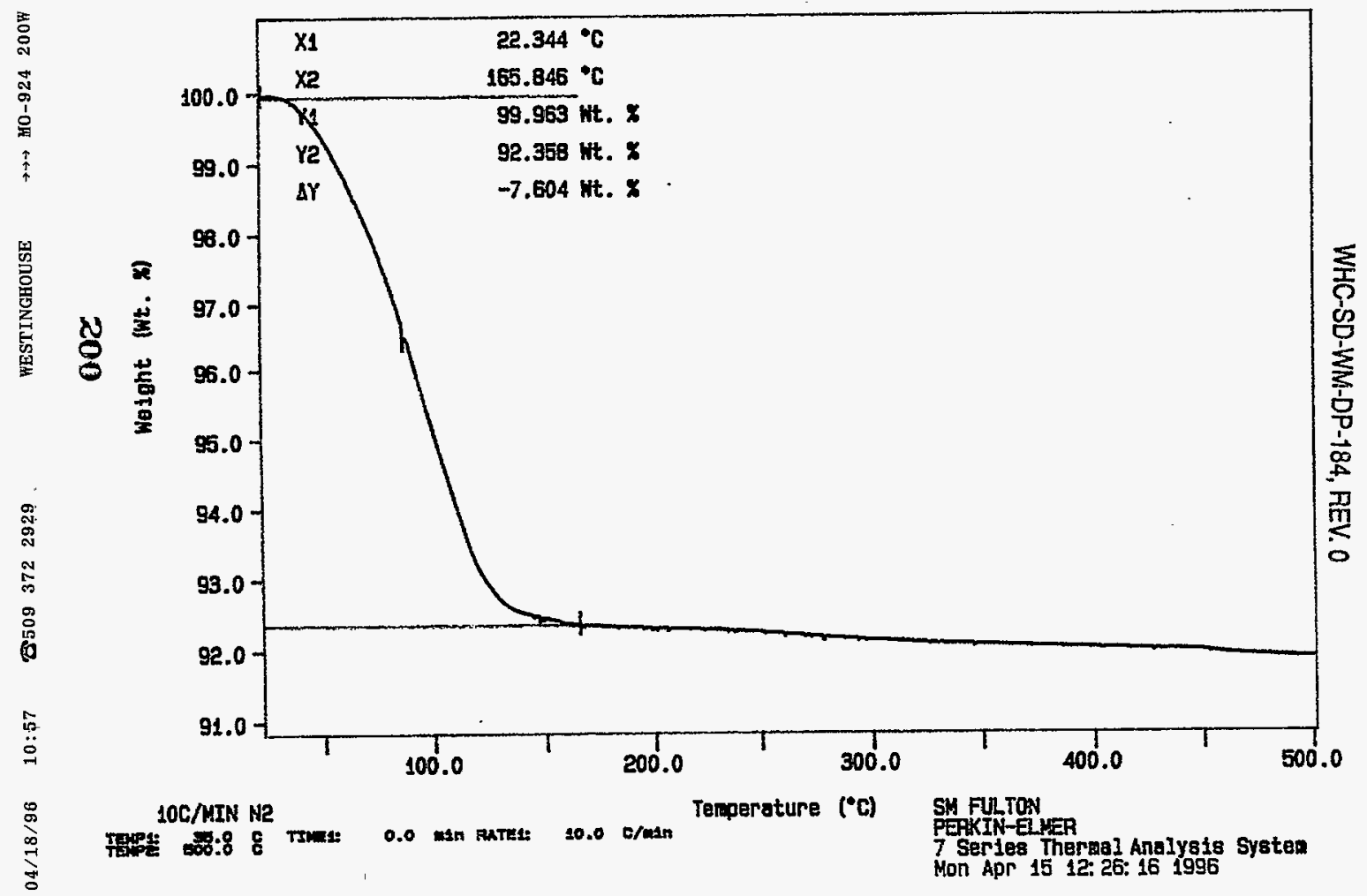


File info: SAY041506 Kon Apr 15 13:24:51 1996

Sample Weight: 22.822 ag

S967001056

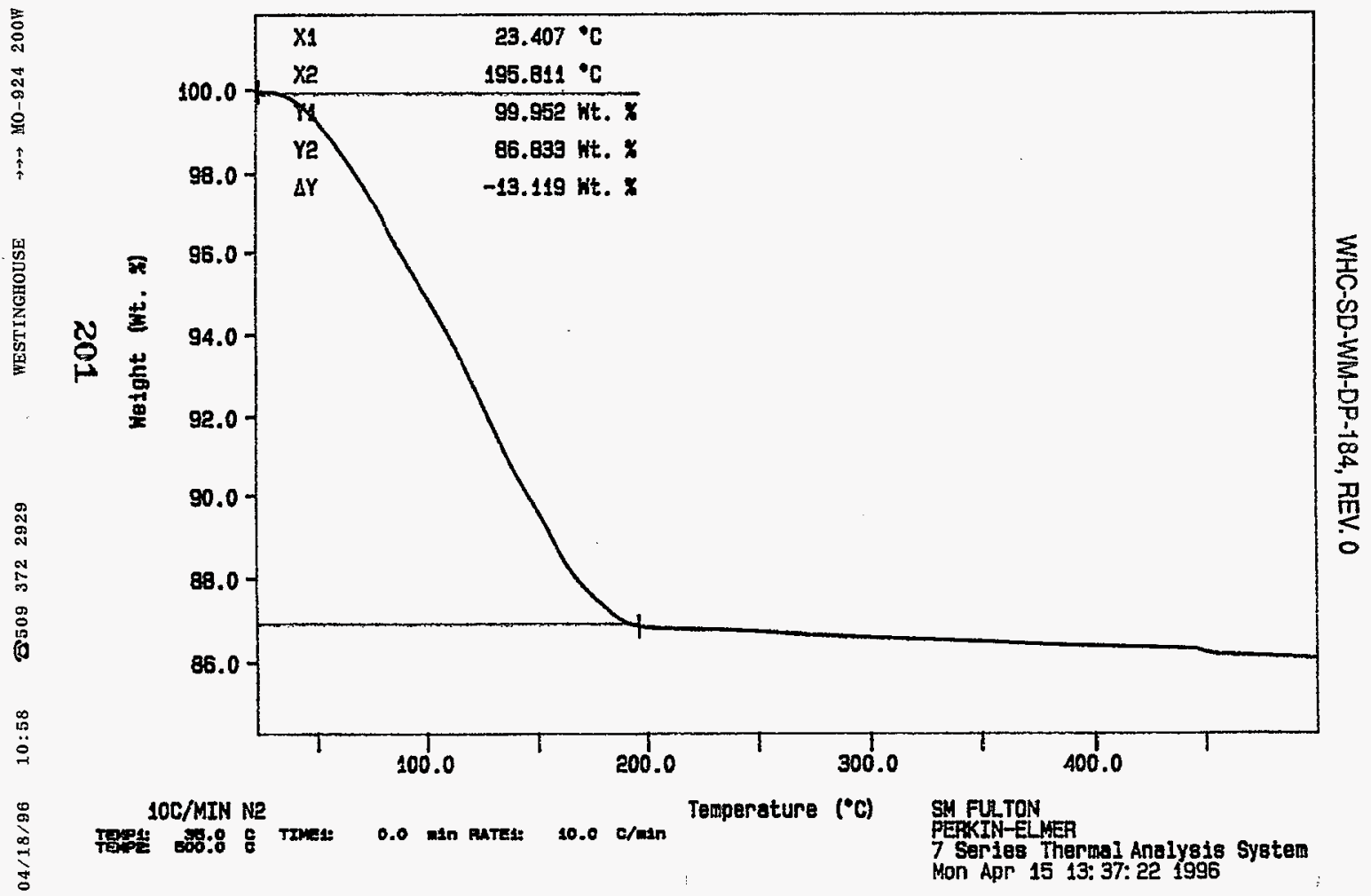




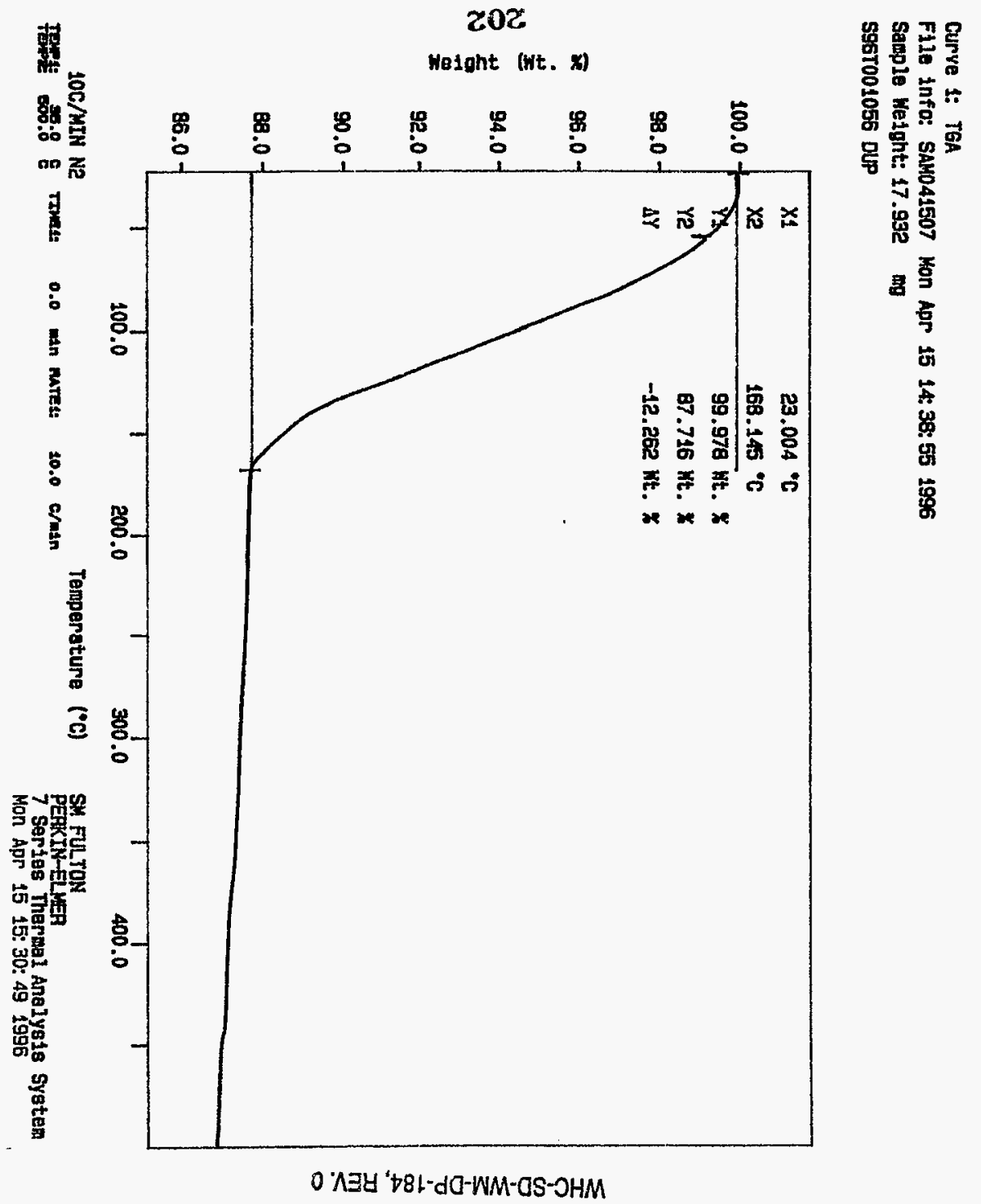


$04 / 17 / 96 \quad 14: 13 \quad Z 5093722929 \quad \ldots$ WESTINGHOUSE $\rightarrow \rightarrow$ HO -924 $200 \mathrm{~W}$

@023

worklistrpt Version 2.1 0S/15/95

WHC-SD-WM-DP-184, REV. 0

Page: 1 04/17/96 11:54

LABCORE Data Entry Template for Worklist\#

6500

Analyst: $\quad$ IDS Instrument: TGAO $\quad$ Book \# 82N8A

Method: LA-560-112 Rev/Mod B-1

Worklist Comment: U-107 TGA RUN UNDER N2. RCJ

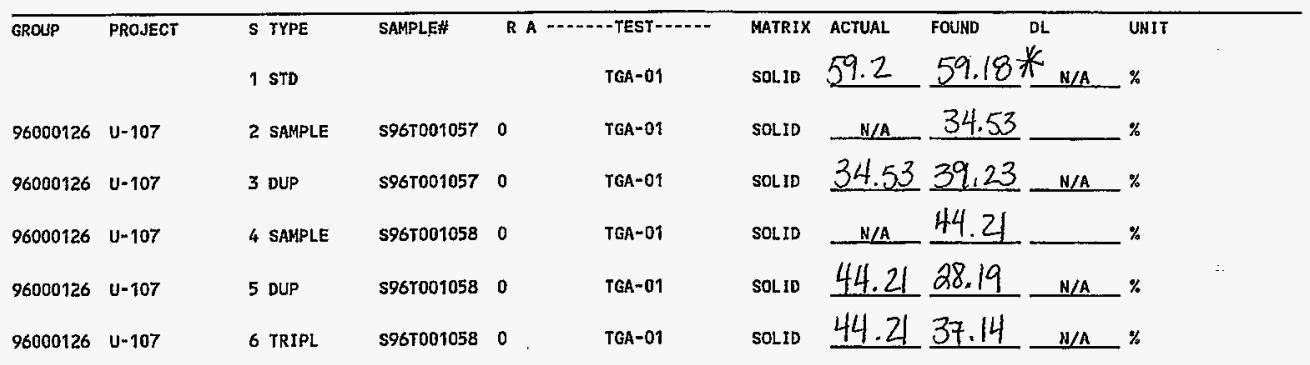

Final page for worklist \# 6500

See attached for signatures

Verified by Blandina Valenzuela.

Data Entry Comments: S96700 1057 produced an additional we right loss step of $1.67 \%$ 5967001058 result ore the sum of two wright loss steps. Atrip wars run because of the difference bturn themograms.

Units shown for $Q C$ (SPK \& STD) may not reflect the actual units. $D L=$ Detection Limit, $S=$ Worklist Slot Number, $R=$ Replicate Number, $A=$ Aliquot Code.

203 
worklistrpt Version $2.105 / 15 / 95$

WHC-SD-WM-DP-184, REV. 0 03/14/96 13:31

\section{LABCORE Data Entry Template for Worklist\#}

Analyst: Jids Instrument: TGA0 Book \# 82/18 8

Method: LA-560-112 Rev/Mod

Worklist Comment: U-107 TGA RUN UNDER N2. RCJ

\begin{tabular}{|c|c|c|c|c|c|c|c|c|c|c|}
\hline \multirow[t]{2}{*}{ GROUP } & \multirow[t]{2}{*}{ PROJECT } & $S$ TYPE & \multirow[t]{2}{*}{ SAMPLE\# } & \multicolumn{2}{|r|}{ 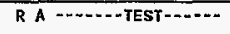 } & \multirow{2}{*}{$\begin{array}{l}\text { MATRIX } \\
\text { SOLID }\end{array}$} & \multirow[t]{2}{*}{ AGTUAL } & \multirow[t]{2}{*}{ FOUND } & \multirow[t]{2}{*}{$\overline{D L}$} & \multirow{2}{*}{$\begin{array}{l}\text { UNIT } \\
\%\end{array}$} \\
\hline & & 1 STD & & & TGA-01 & & & & & \\
\hline 96000126 & $v-107$ & 2 SAMPLE & S96T001057 & o & TGA-01 & SOL1D & N/A & & & $\%$ \\
\hline 96000126 & $u=107$ & 3 DUP & \$26T001057 & 0 & $T G A-01$ & SOLID & & & & $\%$ \\
\hline 96000126 & $y=107$ & 4 SAMPLE & $\$ 967001058$ & 0 & TEA-01 & SOLID & N/A & & & $\%$ \\
\hline 96000126 & $U-107$ & 5 DUP & S96T00105B & 0 & TEA-01 & SOLID & & & & $\%$ \\
\hline
\end{tabular}

\section{Final page for worklist \# $\quad 6500$}

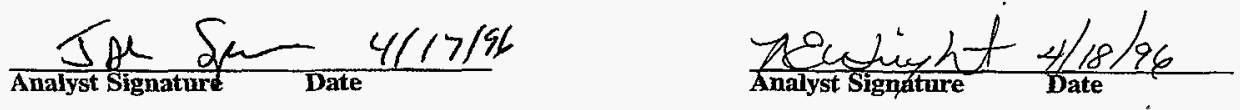

Data Entry Comments:

$\operatorname{Ran}$ en 5967001058

Units shown for $Q C$ (SPK \& STD) may not reflect the actual units. $D L=$ Detection Limit, $S=$ Worklist Slot Number, $R=$ Replicate Number, $A=$ Aliquot Code. 
COHPLETED/VERIFIED THE CALIBRATION/ANALYSIS ON PAGES 205 TO $2 / 0$

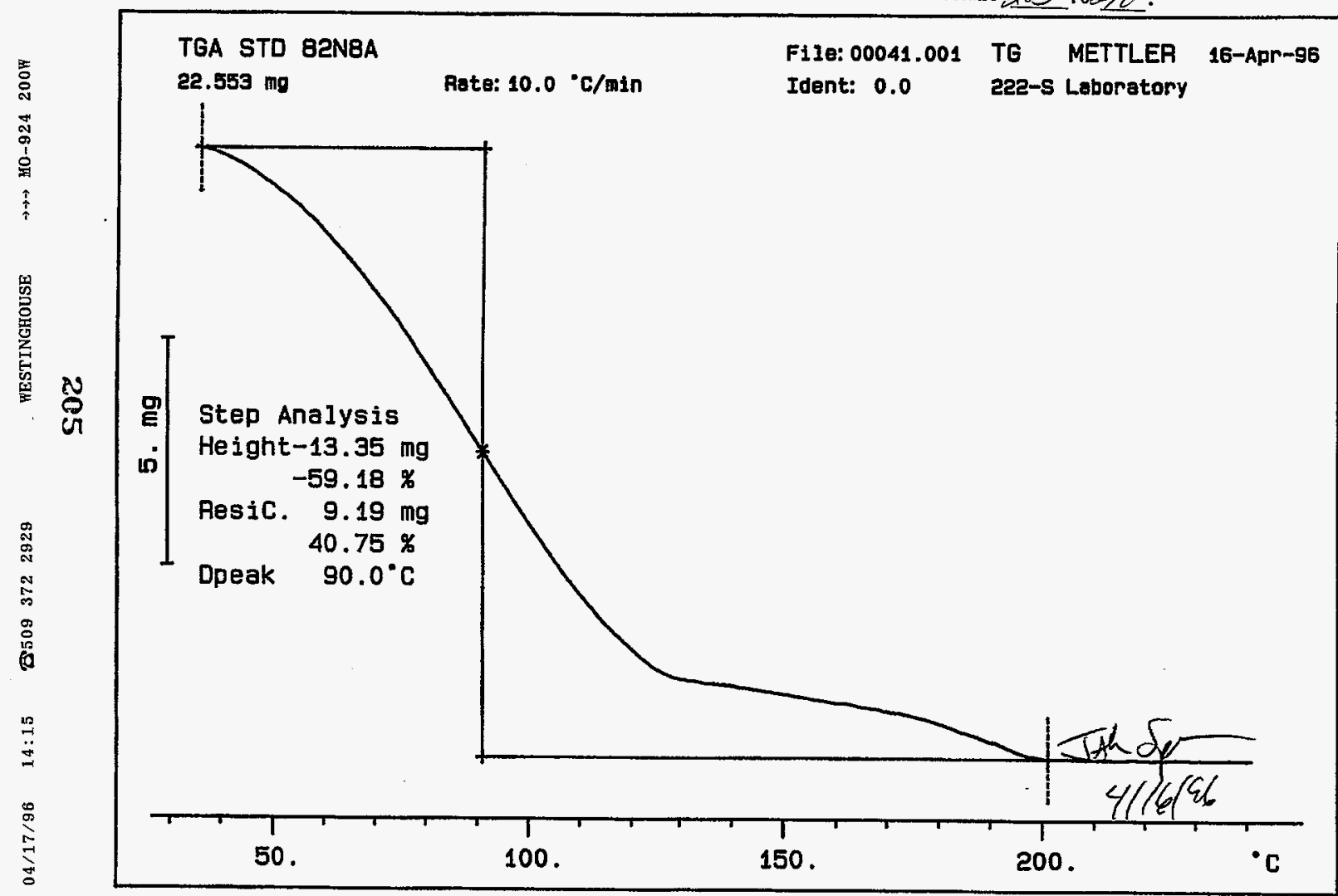

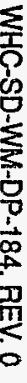




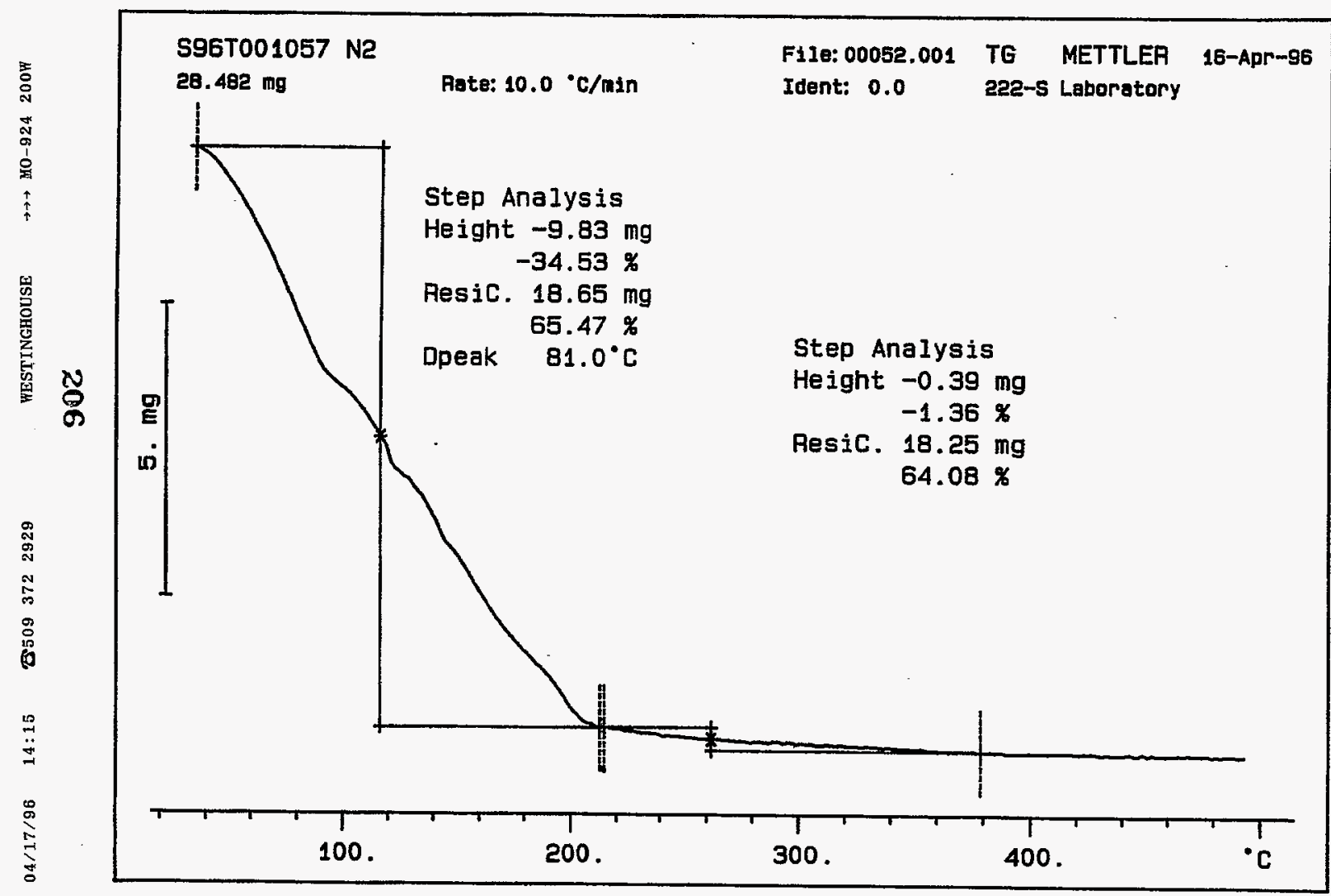




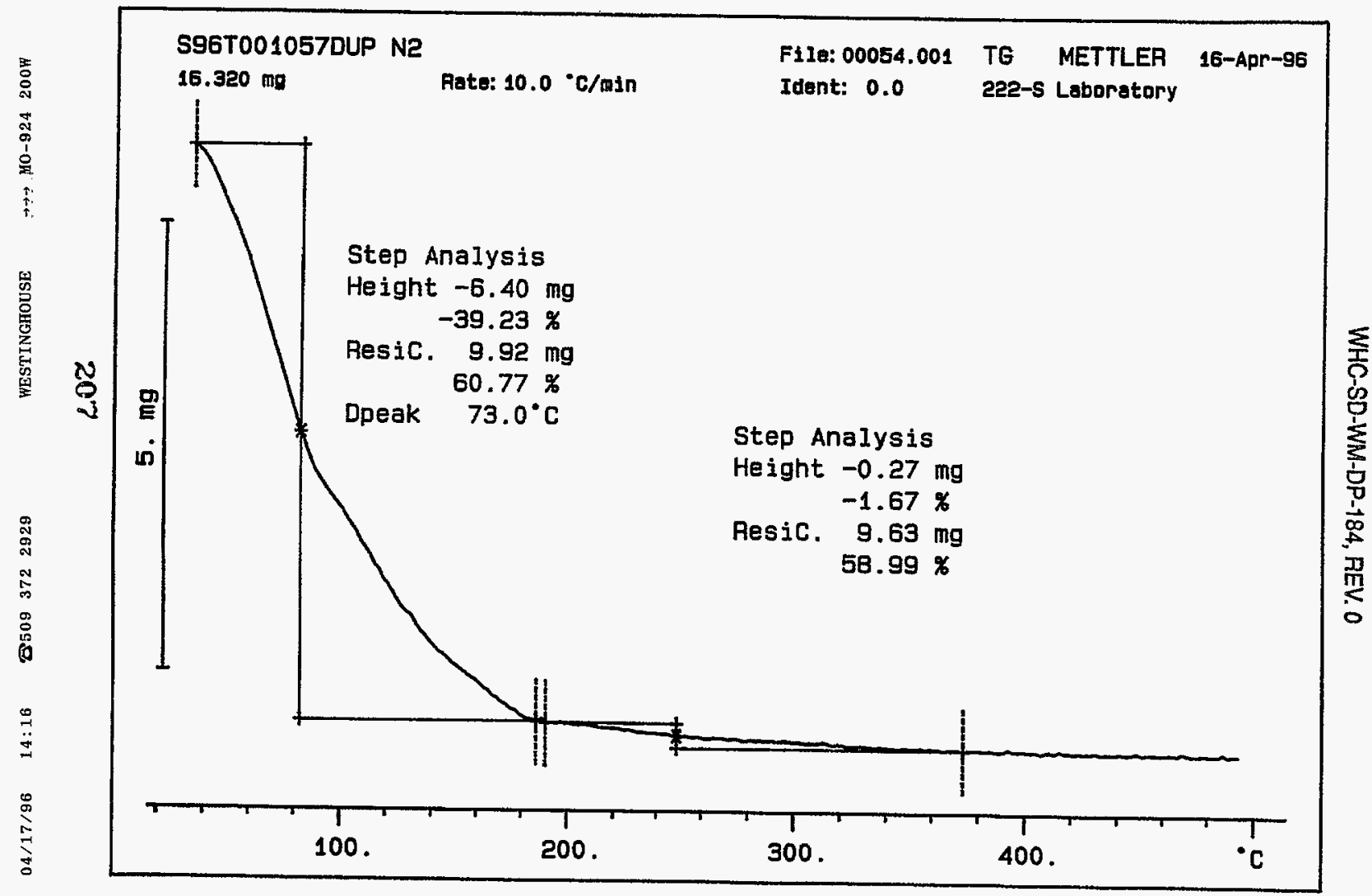


WHC-SD-WM-DP-184, REV. 0

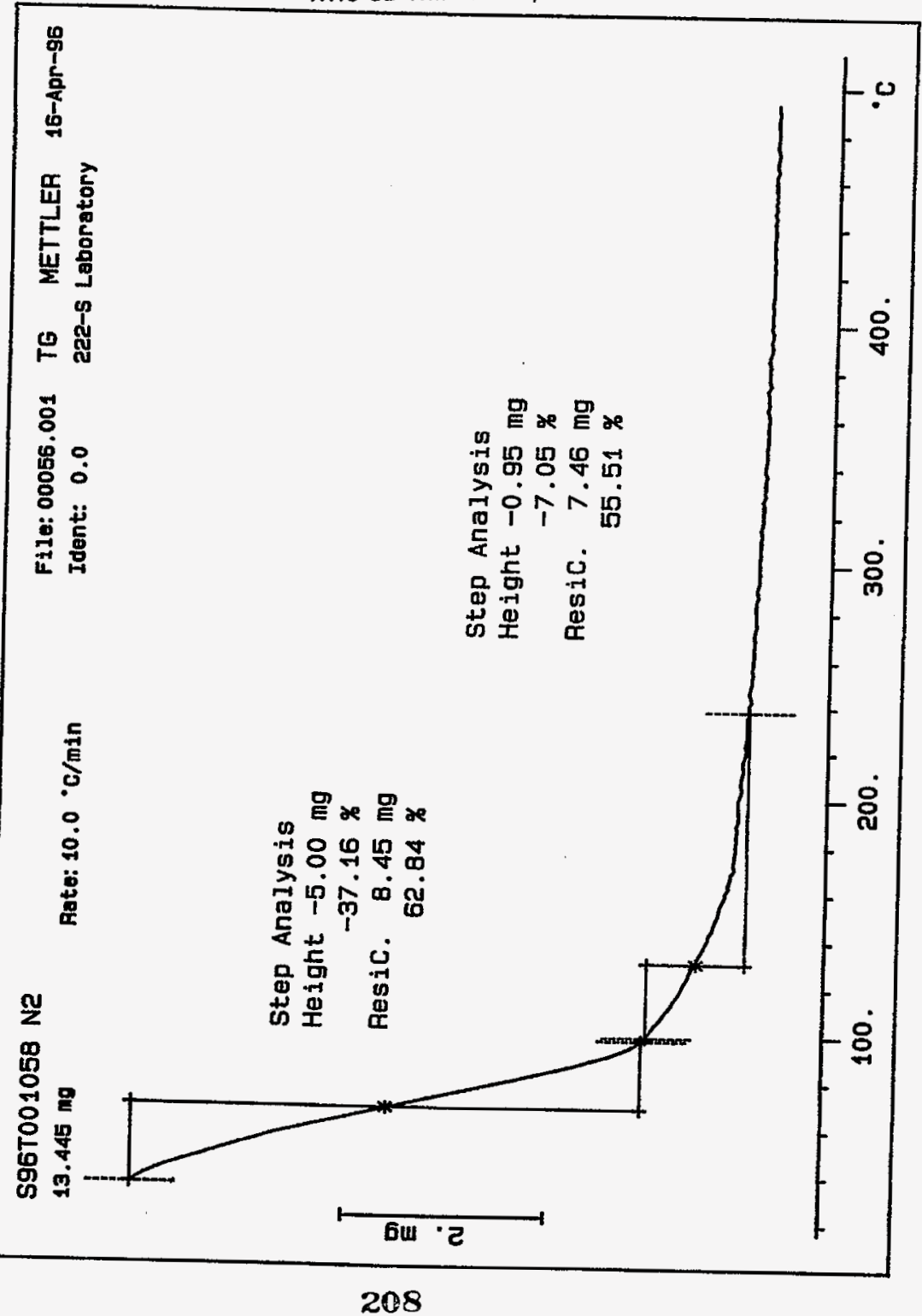




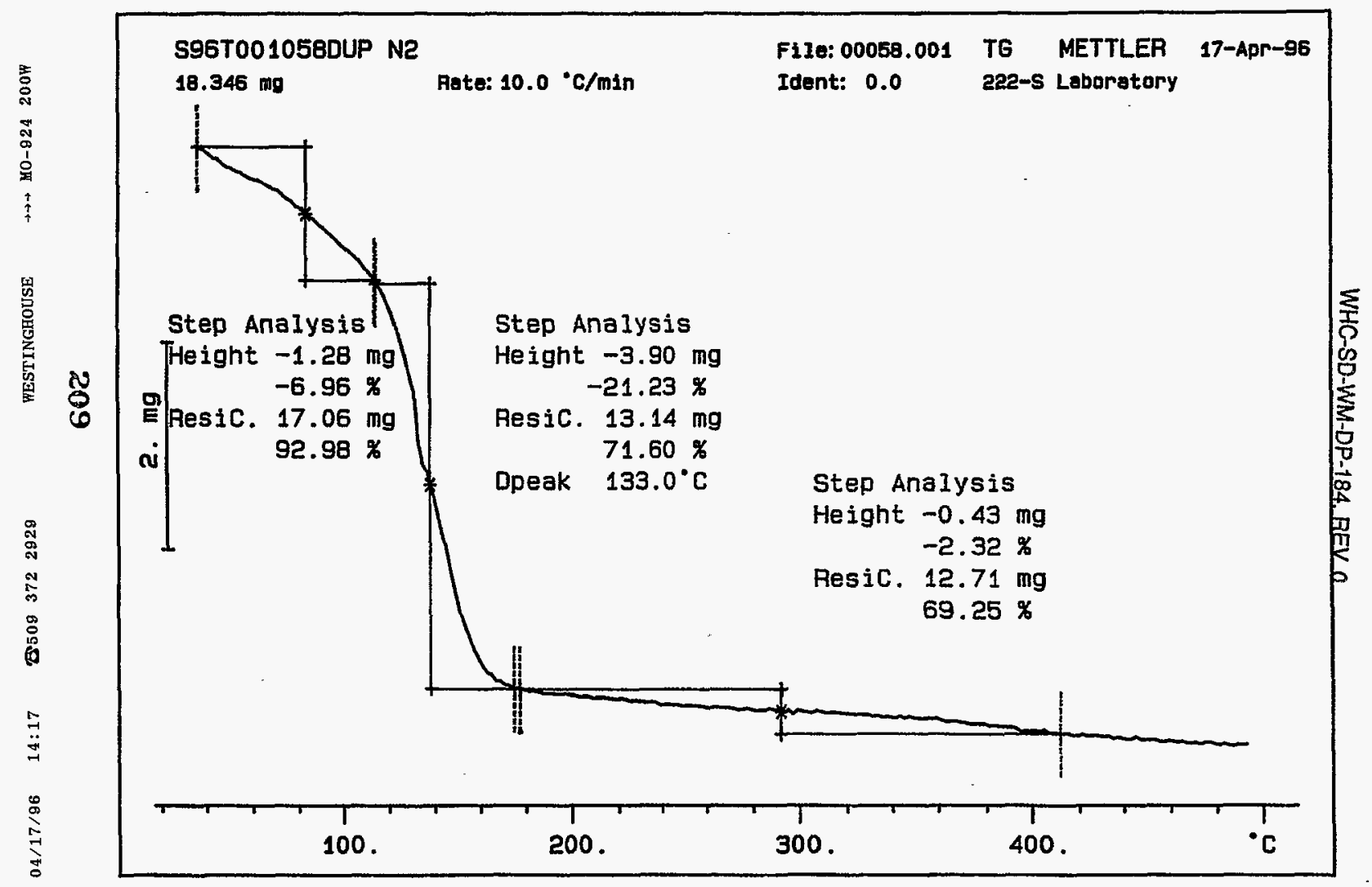




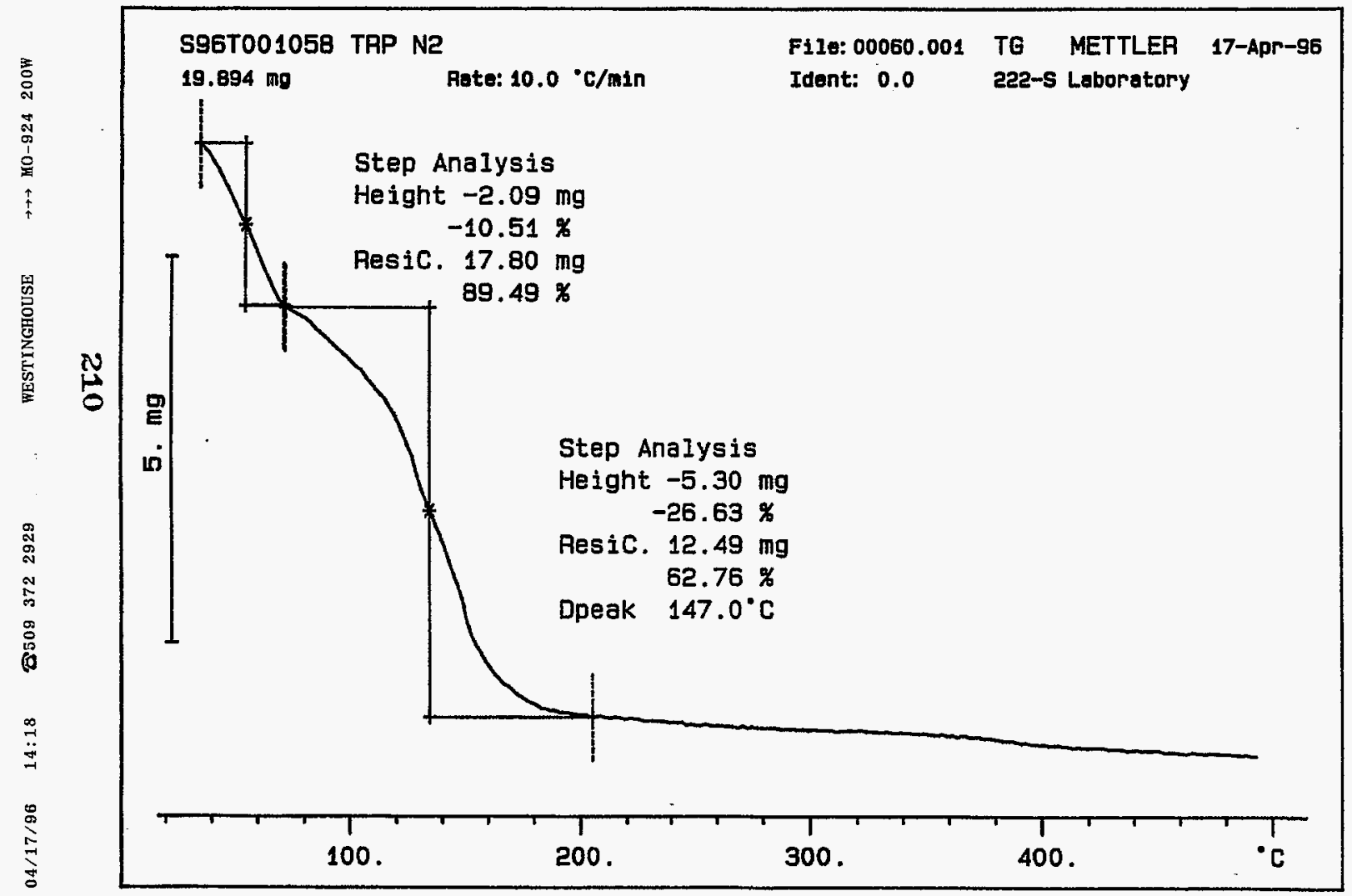




\section{LABCORE Data Entry Template for Worklist\#}

Analyst: SMF Instrument: TGA0 1 Book\# 75N8-A

Method: LA-560-112 Rev/Mod $\beta-1$

Worklist Comment: U-107 TGA RUN UNDER N2. RCJ

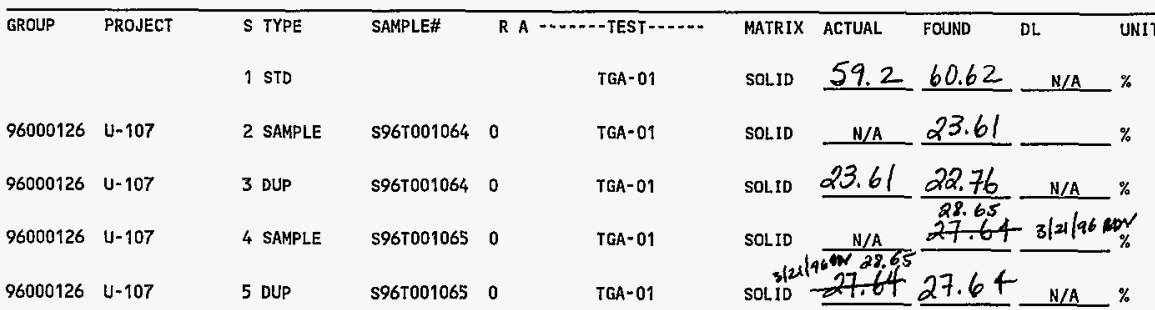

\section{Final page for worklist \# $\quad \mathbf{6 5 0 1}$}
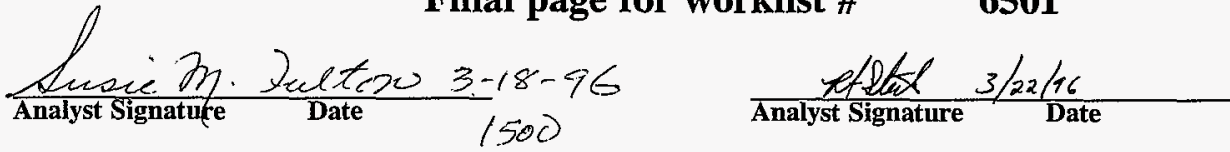

Verified by thather Anastos 3.25 .96

Data Entry Commens: S957001065 results are the sum of three weight lossisteps. 
SIGNATURE BELOW REPRESENTS CHEMICAL TECHNOLOGIST/CHEMIST THAT

COMPLETED/VERIFIED THE CALIBRATION/ANALYSIS ON PAGES $2 / 2$ T0 $2 / 5$.

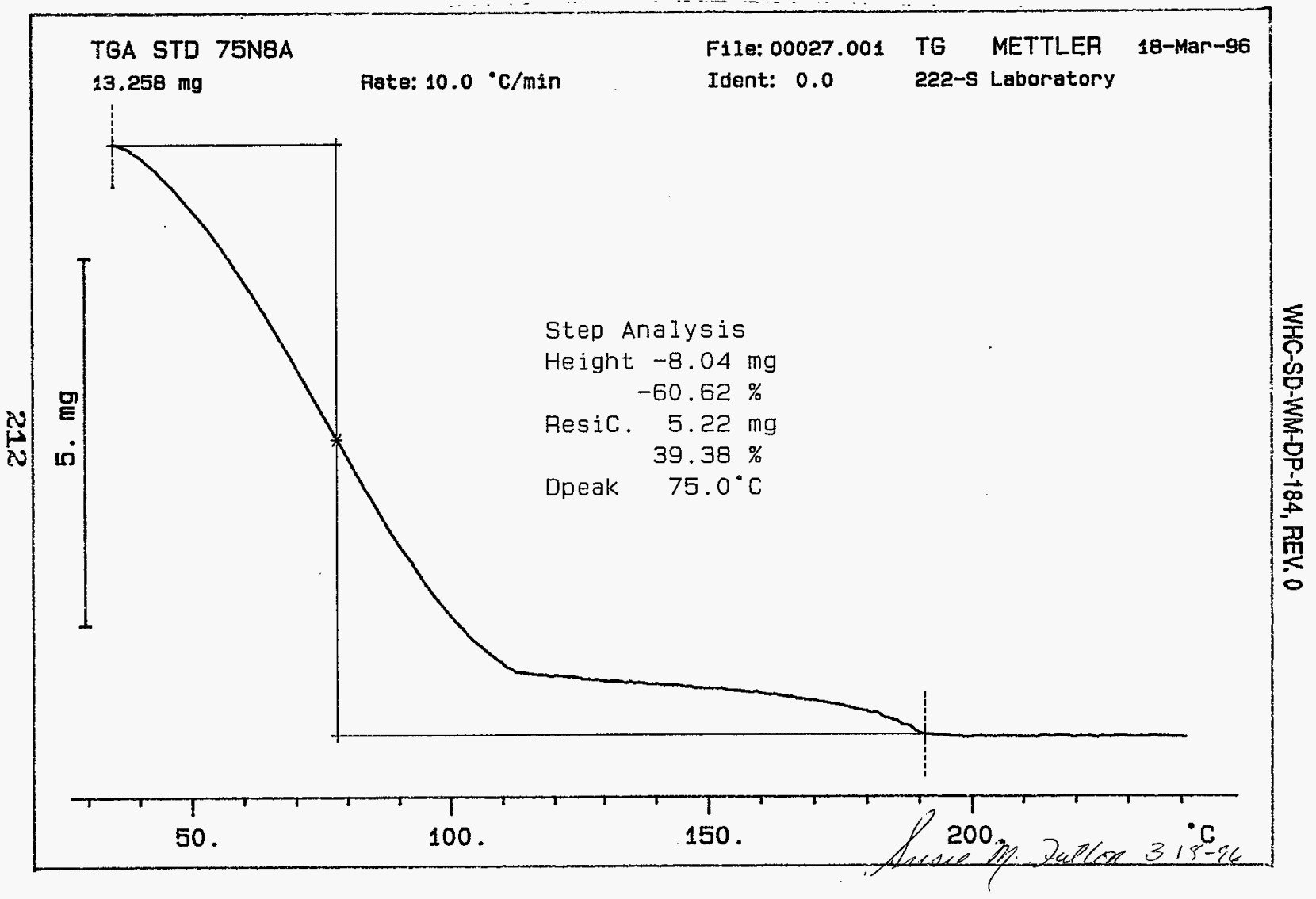




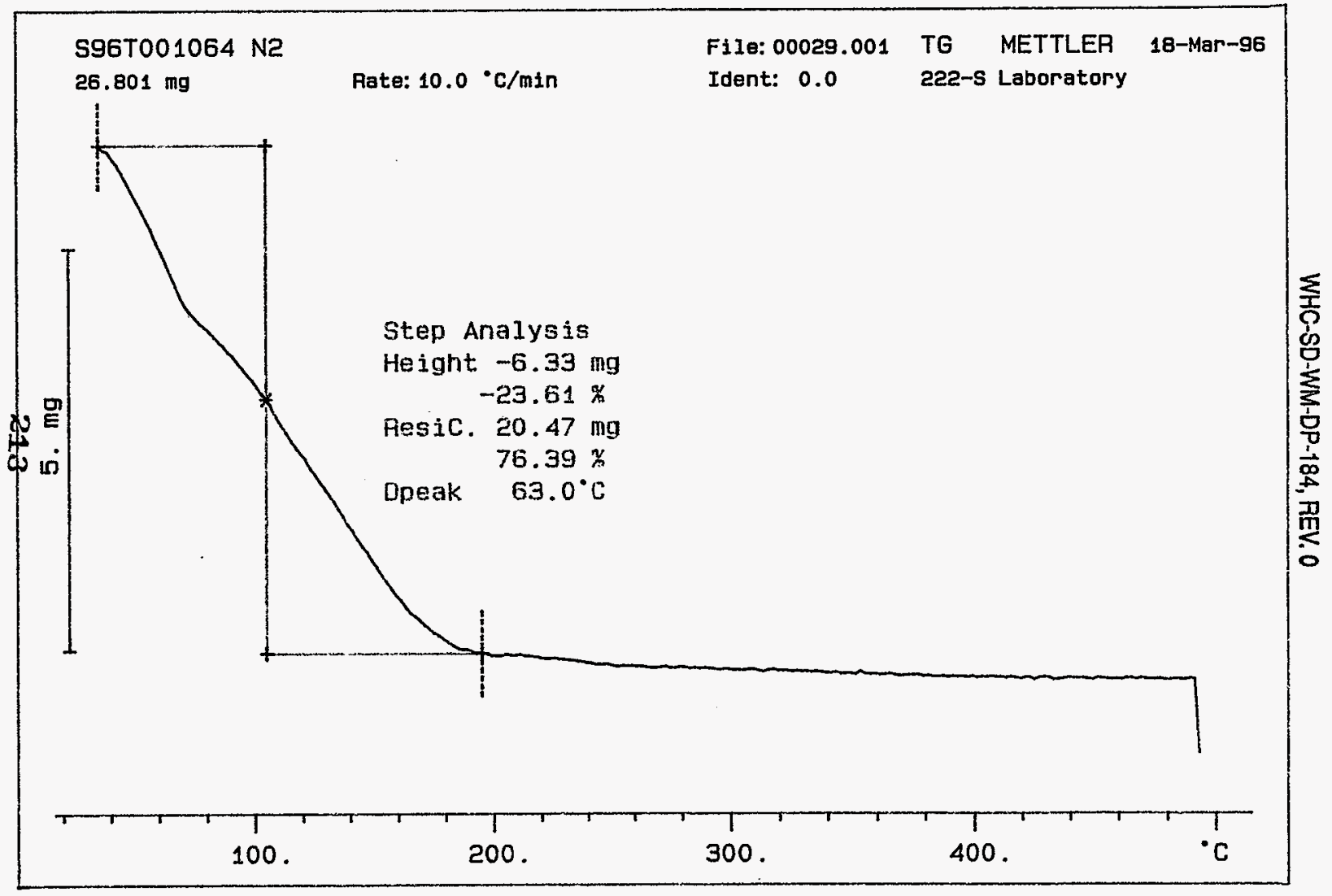


WHC-SD-WM-DP-184, REV. 0

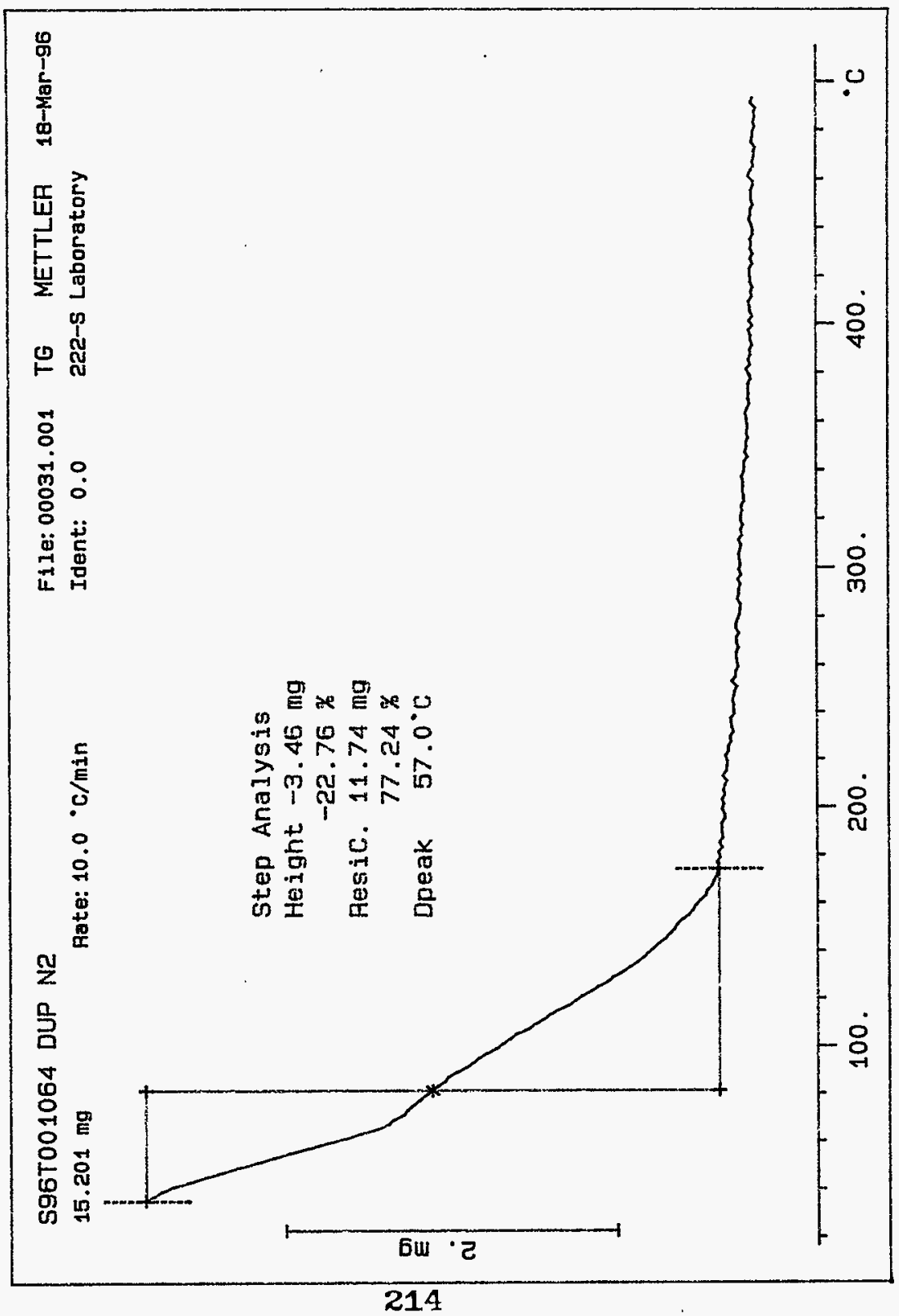



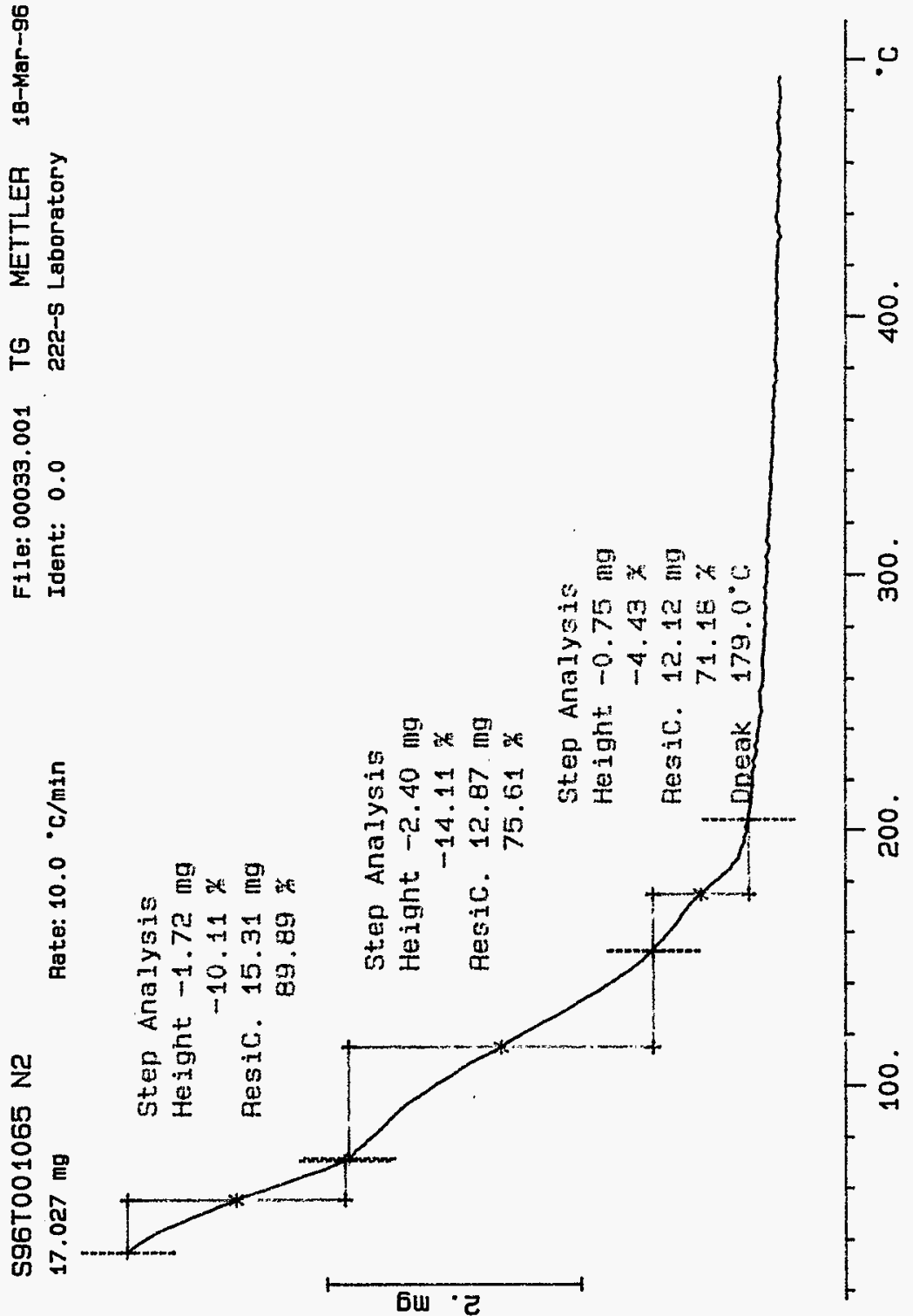

214.1 

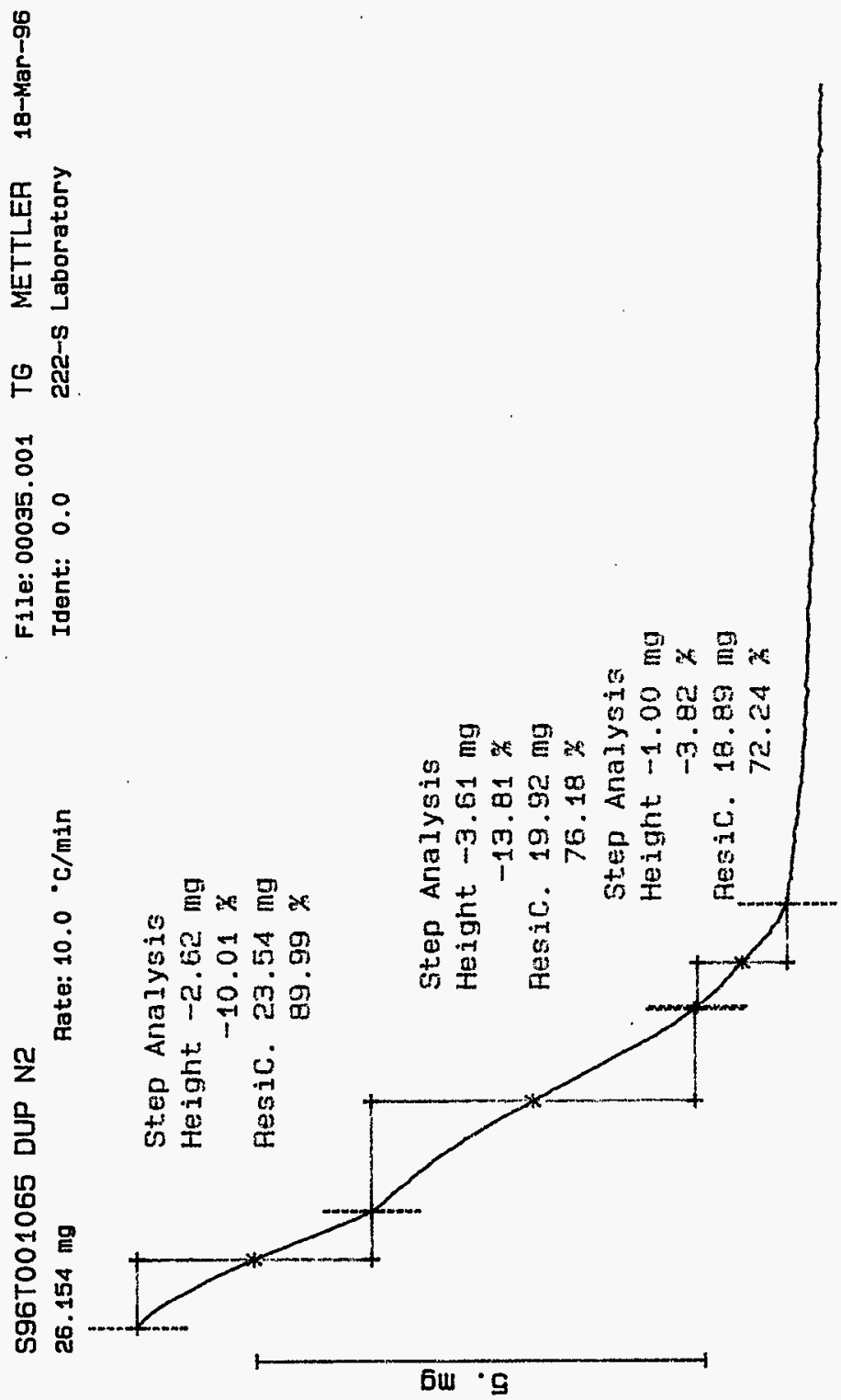

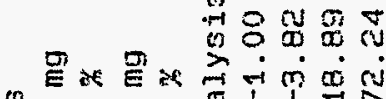
ही काष 舟 iा 1 . 茄娄 c 产 U

.

모

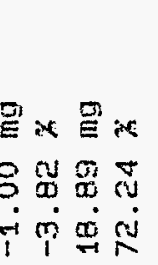

正要 
worklistrpt Version 2.1 05/15/95

WHC-SD-WM-DP-184, REV. 0

\section{LABCORE Data Entry Template for Worklist\#}

Analyst: SMF Instrument: TGA0 3 Book\# 82N8A

Method: LA-514-114 Rev/Mod C - 1

Worklist Comment: U-107 TGA RUN UNDER N2. RCJ

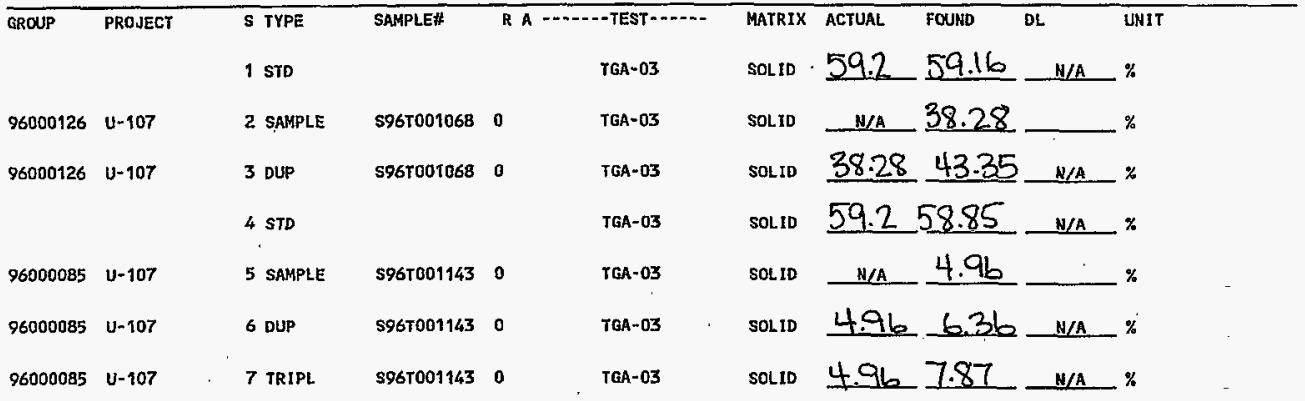

\section{Final page for worklist \# $\quad 6502$}

Sueattached fn Siguatures.

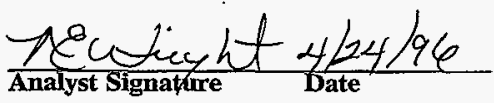

S96T001068 nesults are the sum of two weight loss steps $(9.57+28.71 \%)$.

verified by il Rnaotor 4-24-96

Data Entry Comments:

Units shown for $Q C$ (SPR \& STD) may not reflect the actual units. $D L=$ Detection Limit, $S=$ Worklist Slot Number, $R=$ Replicate Number, $A=$ Aliquot Code. 


\section{SMF Instrument: TGAO _ Book $8 \% 1 / 84$

Analyst: SMF Instrument: TGA0 _ Book $8 \% 18 A$

Method: LA-560-112 Rev/Mod

Worklist Comment: U-107 TGA RUN UNDER N2, RCJ

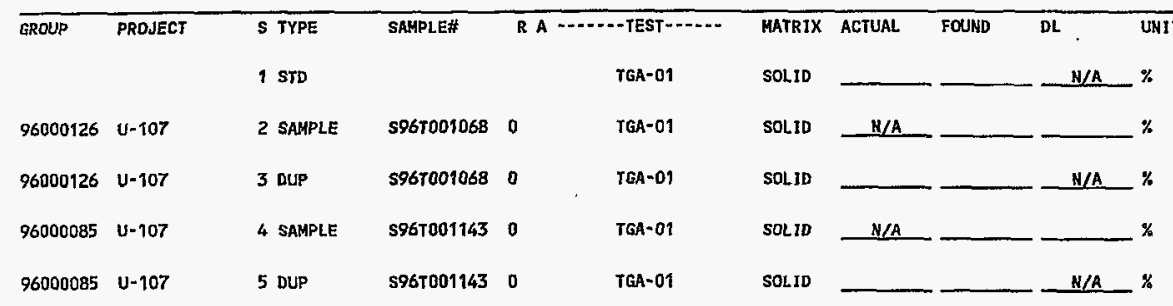

\section{Final page for worklist \# $\quad 6502$}

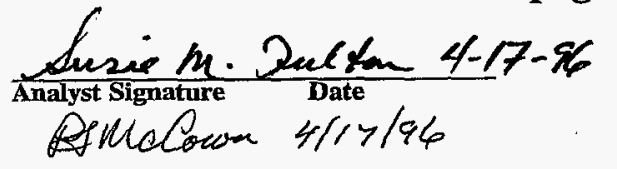

\section{Analyst Signature Date}

Other lustrument was Used.

Data Entry Comments:

Units shown for $Q C$ (SPK \& STD) may not reflect the actual units. $D L=$ Detection Limit, $S=$ Worklist Slot Number, $R=$ Replicate Number, $A=$ Aliquot Code. 
WHC-SD-WM-DP-184, REV. 0

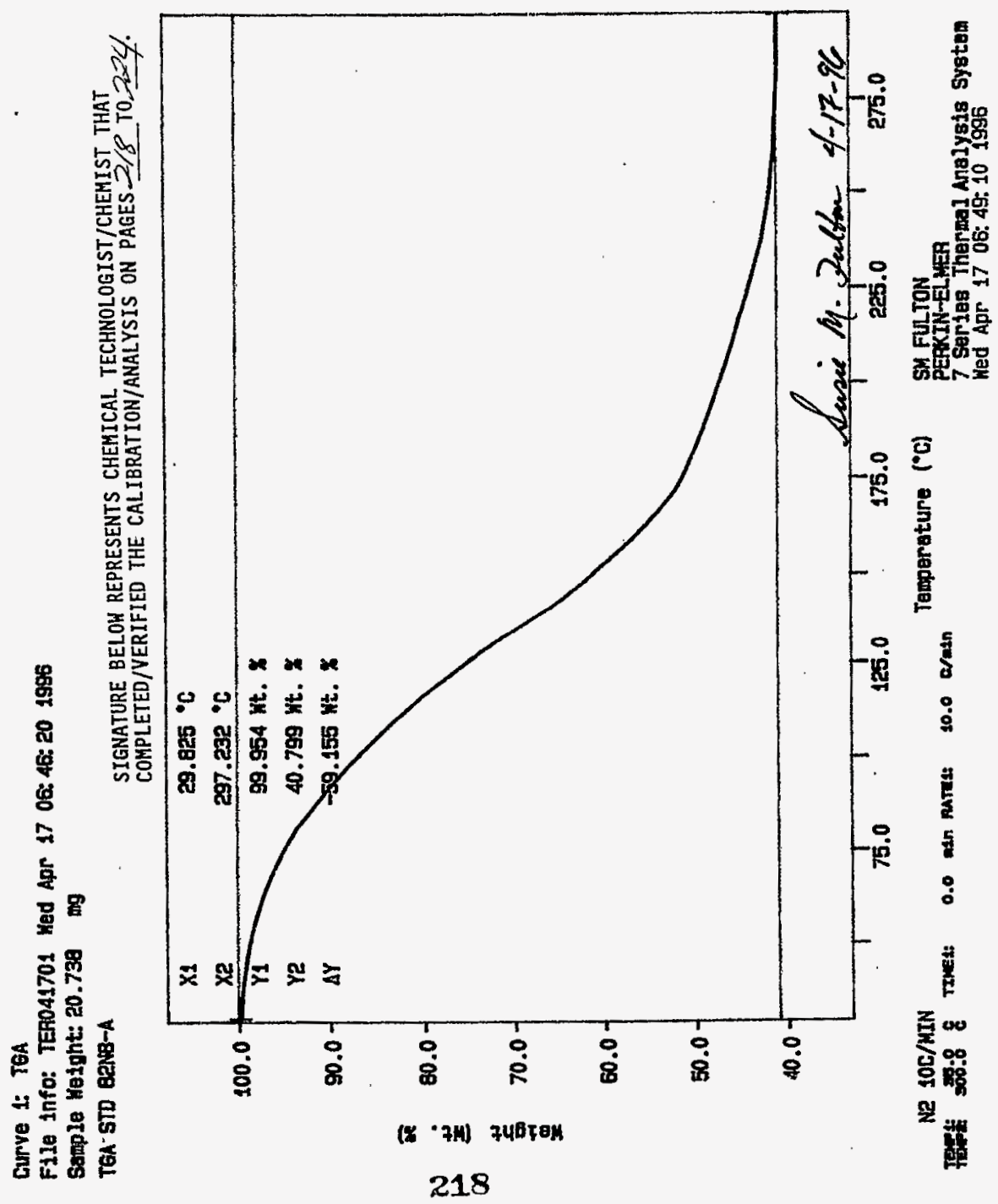


Curve 1: TGA

File info: SAk041701 Ked Apr 17 07:52:09 1996

Sapple Kaight: 30.536 ag

s9670010s8

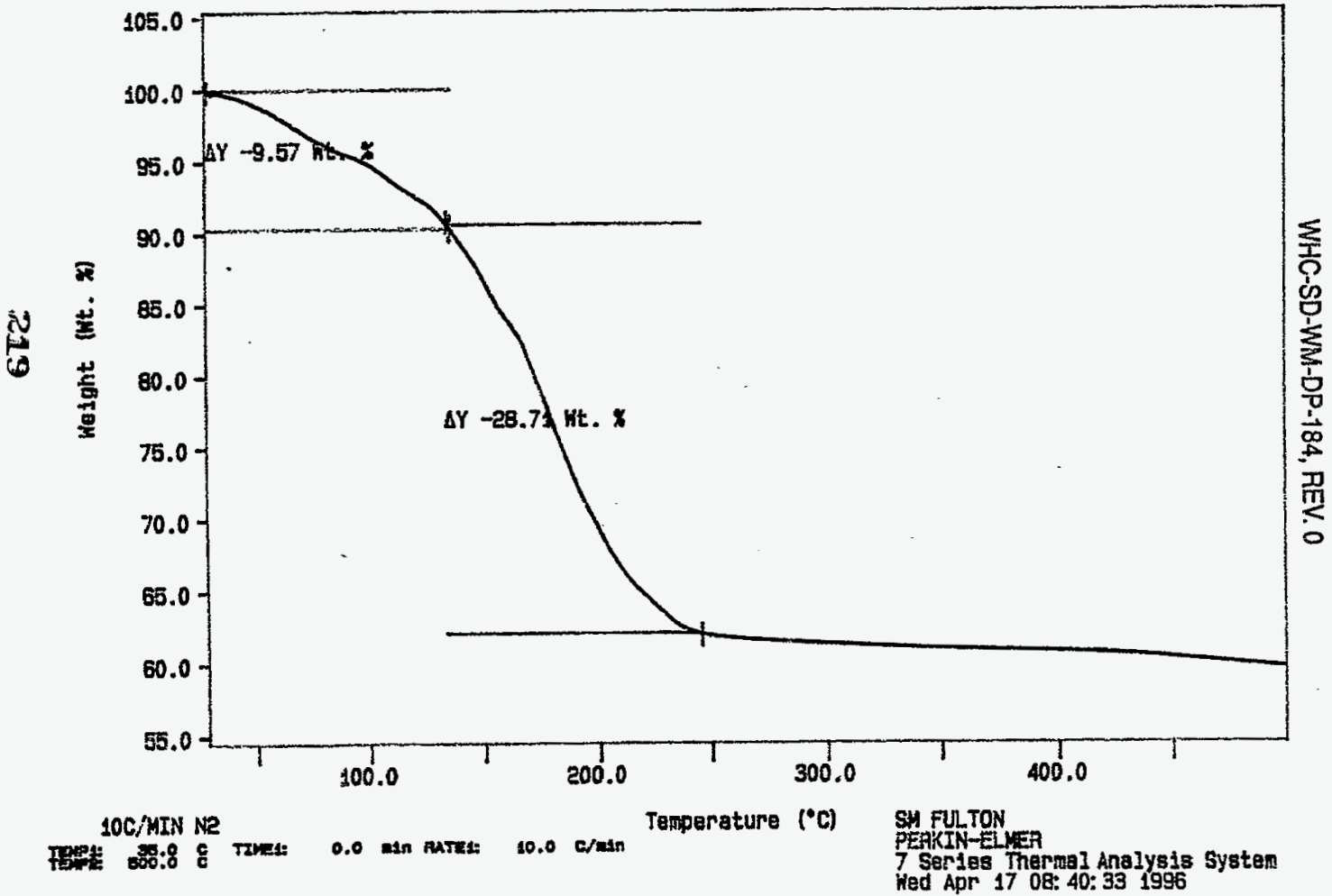


curve 1: TGA

File Info: SAMO41702 Wed Apr 17 09,39: 261996

Seiple Keight: 19.384

S96T0010E0 DUP

里

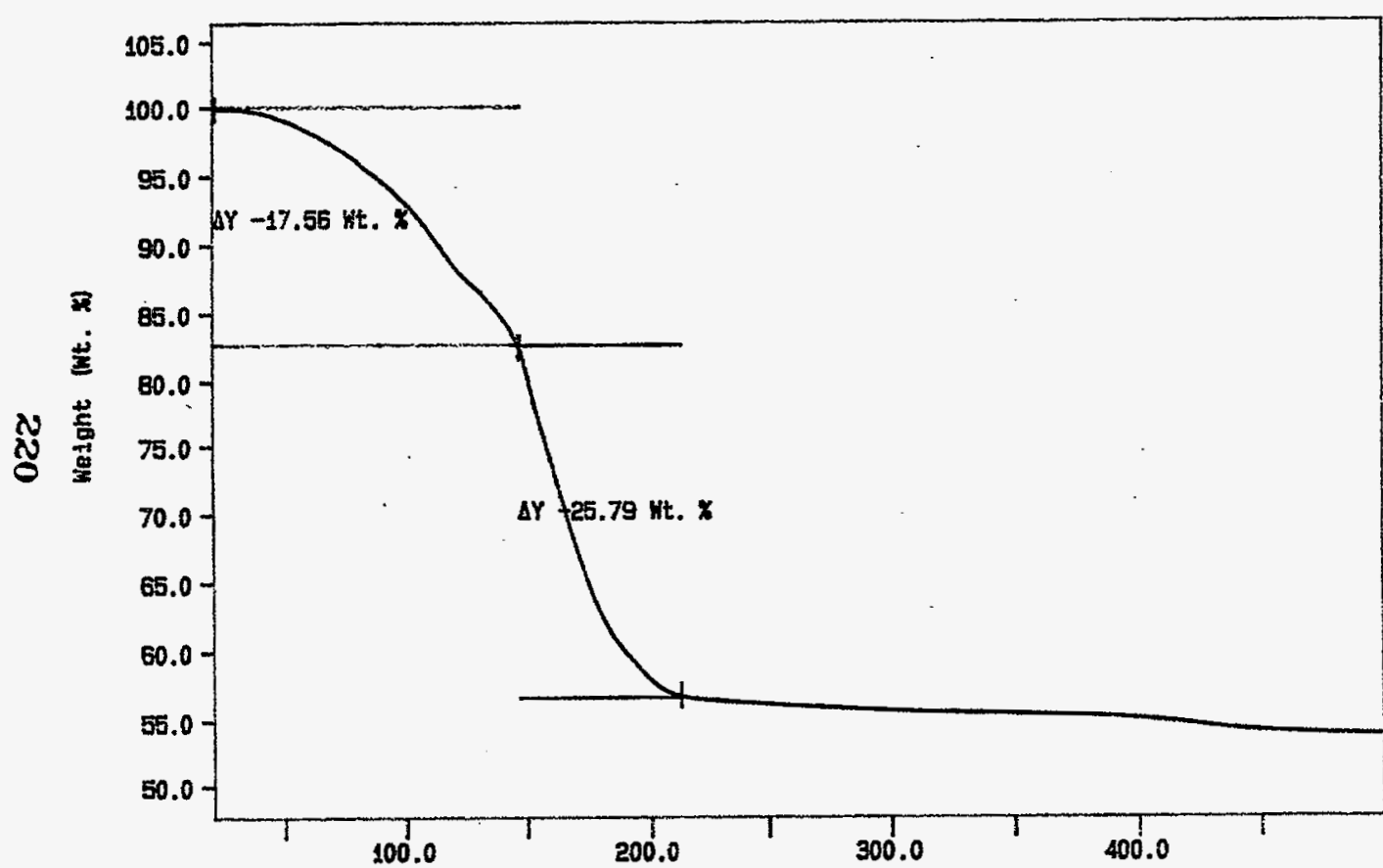




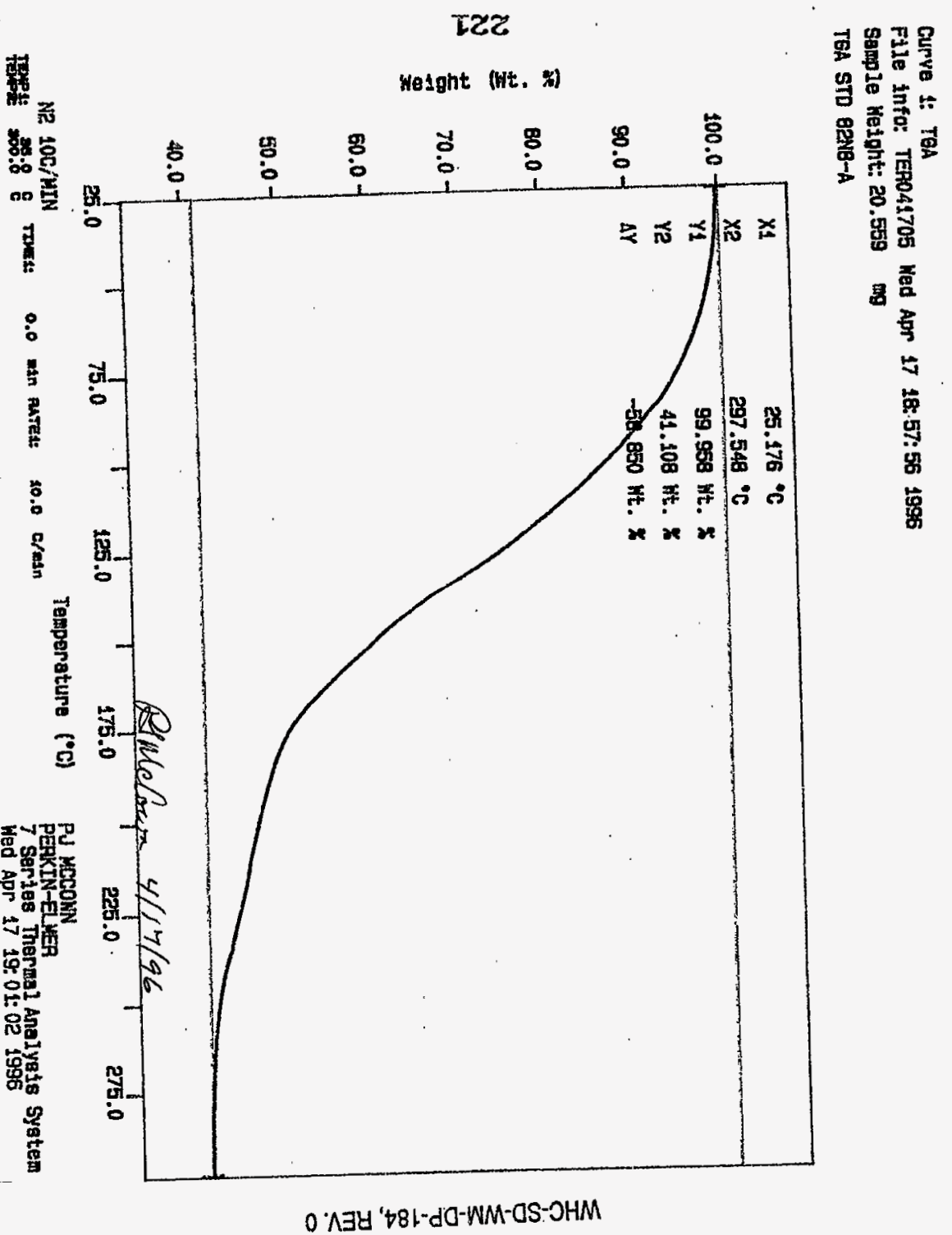


WHC-SD-WM-DP-184, REV. 0

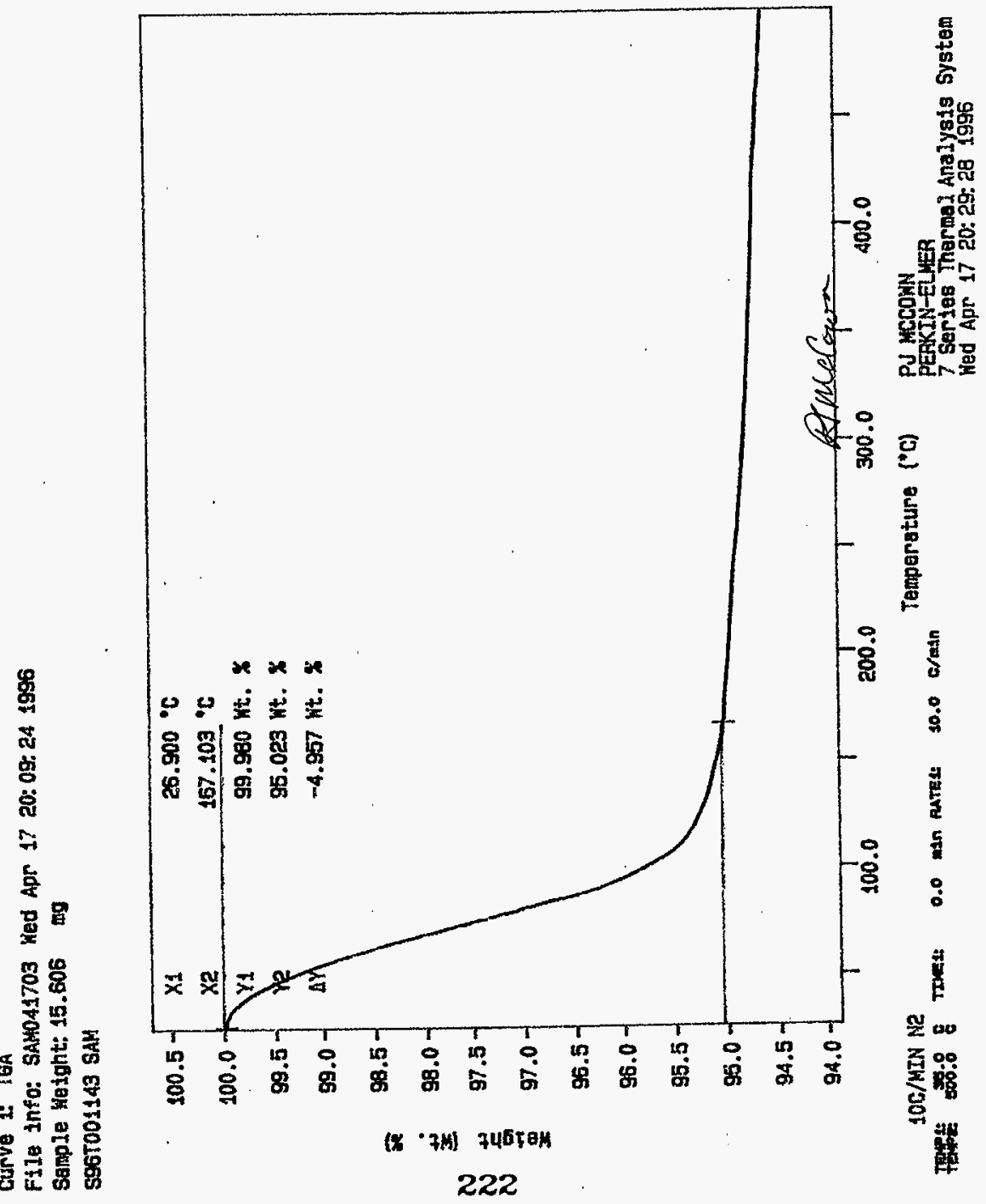




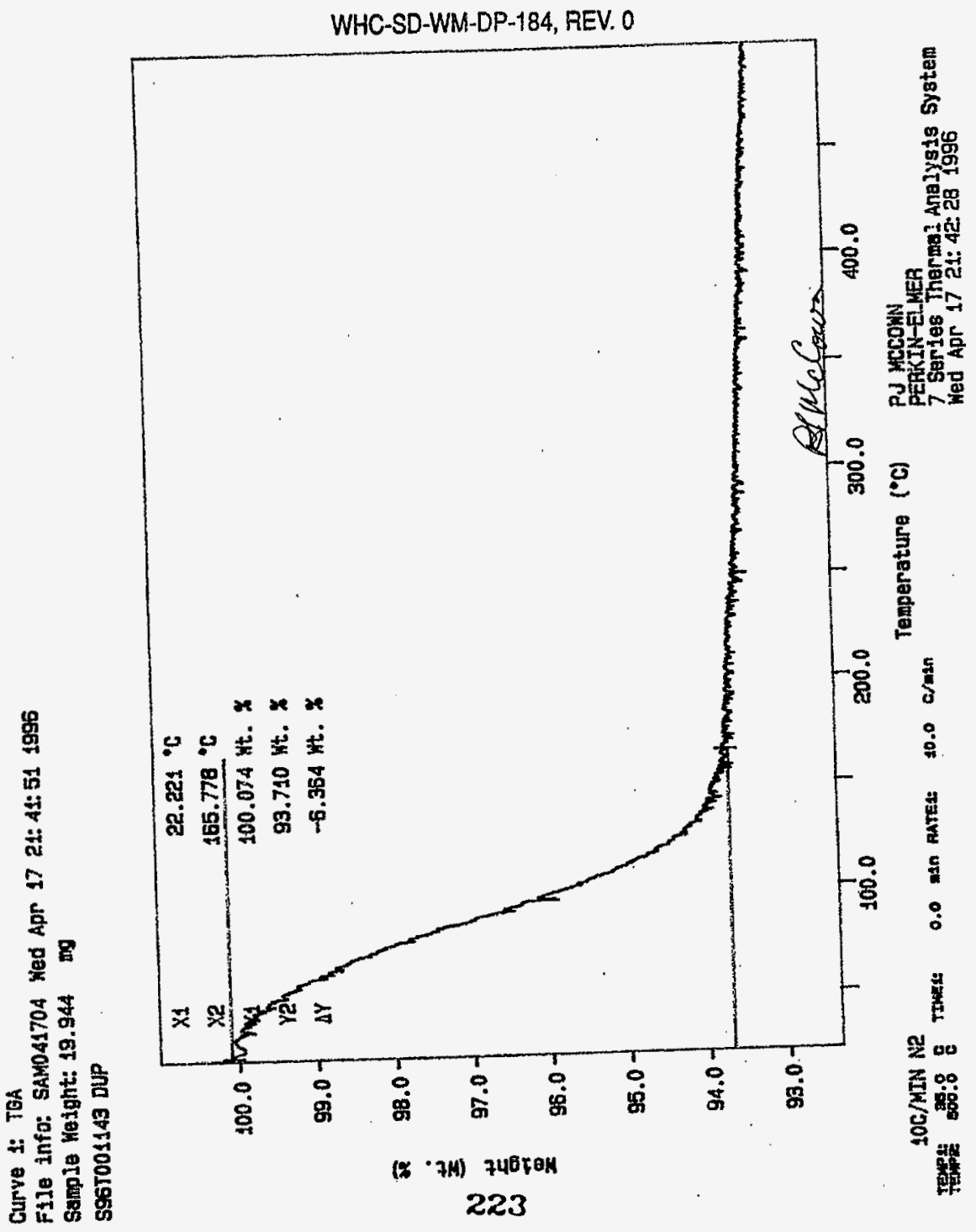


WHC-SD-WM-DP-184, REV. 0

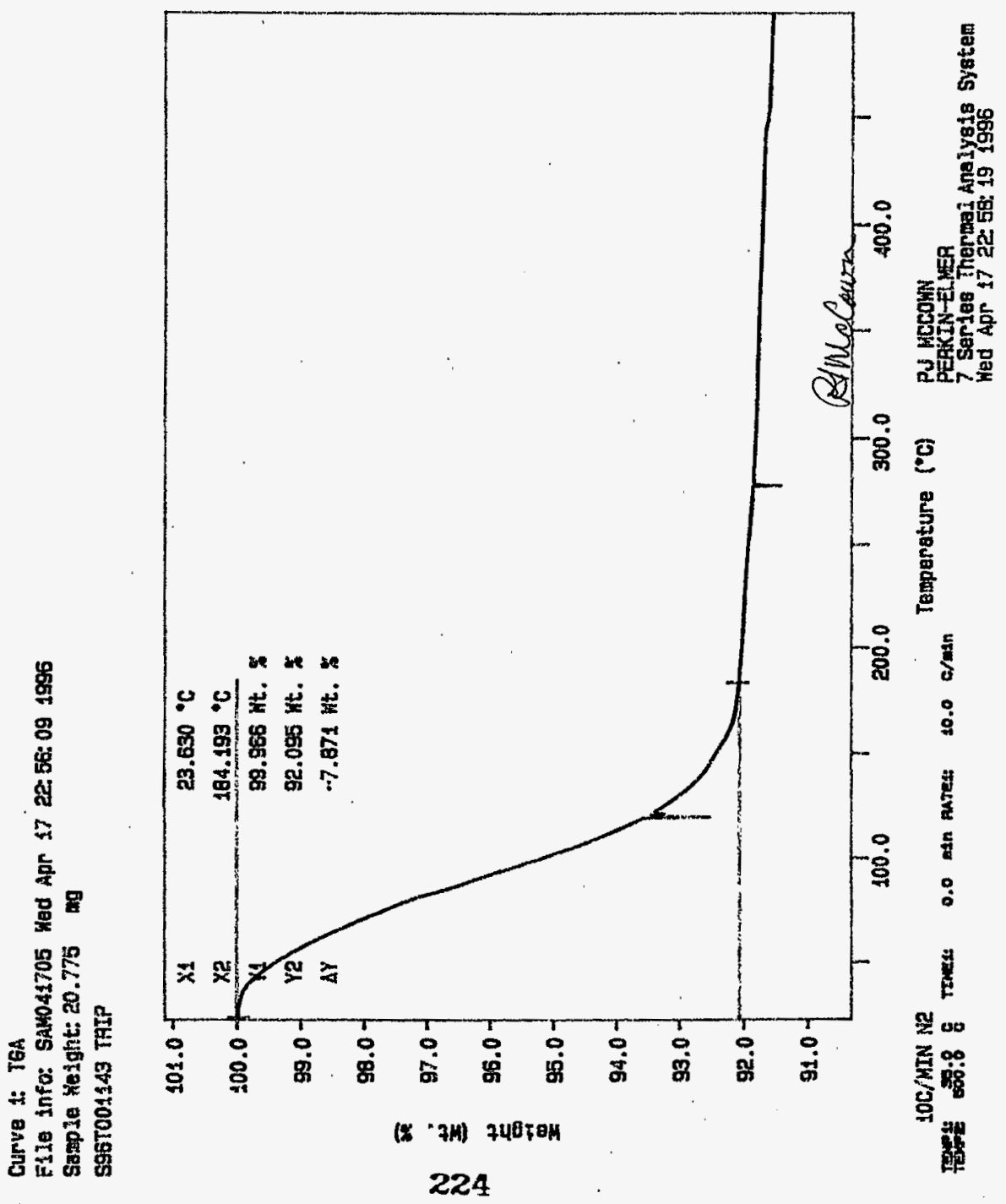


$04 / 18 / 96 \quad 10: 52 \quad 8509 \quad 372 \quad 2929$

WEST INGHOUSE

MO-924 200W

[1 001

worklistrpt Version $2.105 / 15 / 95$ WHC-SD-WM-DP-184, REV. 0 $03 / 18 / 9613: 50$

LABCORE Data Entry Template for Worklist\#

Page: 1

Analyst: $\quad$ Golf Instrument: TGAO I Bonk\# $82 N 8 / 7$

Method: LA-560-112 Rev/Mod B-1

Worklist Comment: U-107 FOR TGA (RUN UNDER N2) RTS

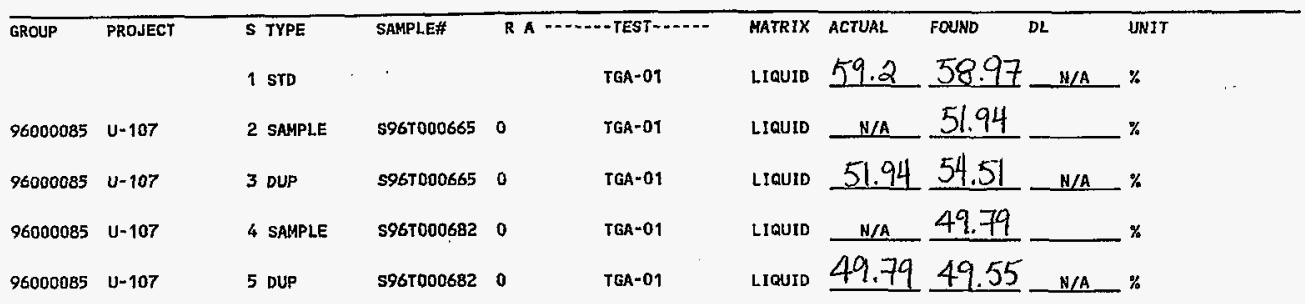

Final page for worklist \# 6633

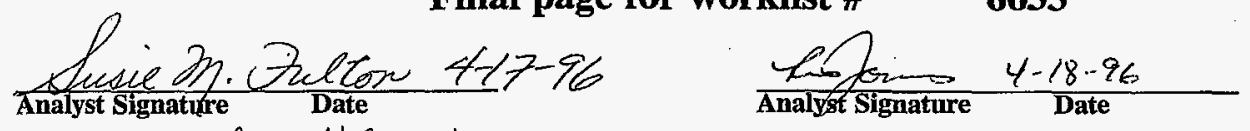

Verified by tHanatos

$4-22-96$

Data Entry Comments: S967000665 results are the sum of two weight loss steps.

5967000682 results are the sum of two wright loss steps

Units shown for QC (SPK \& STD) may not reflect the actual units. DL = Detection Limit, $S=$ Worklist Slot Number, $R=$ Replicate Number, $A=$ Aliquot Code.

225 


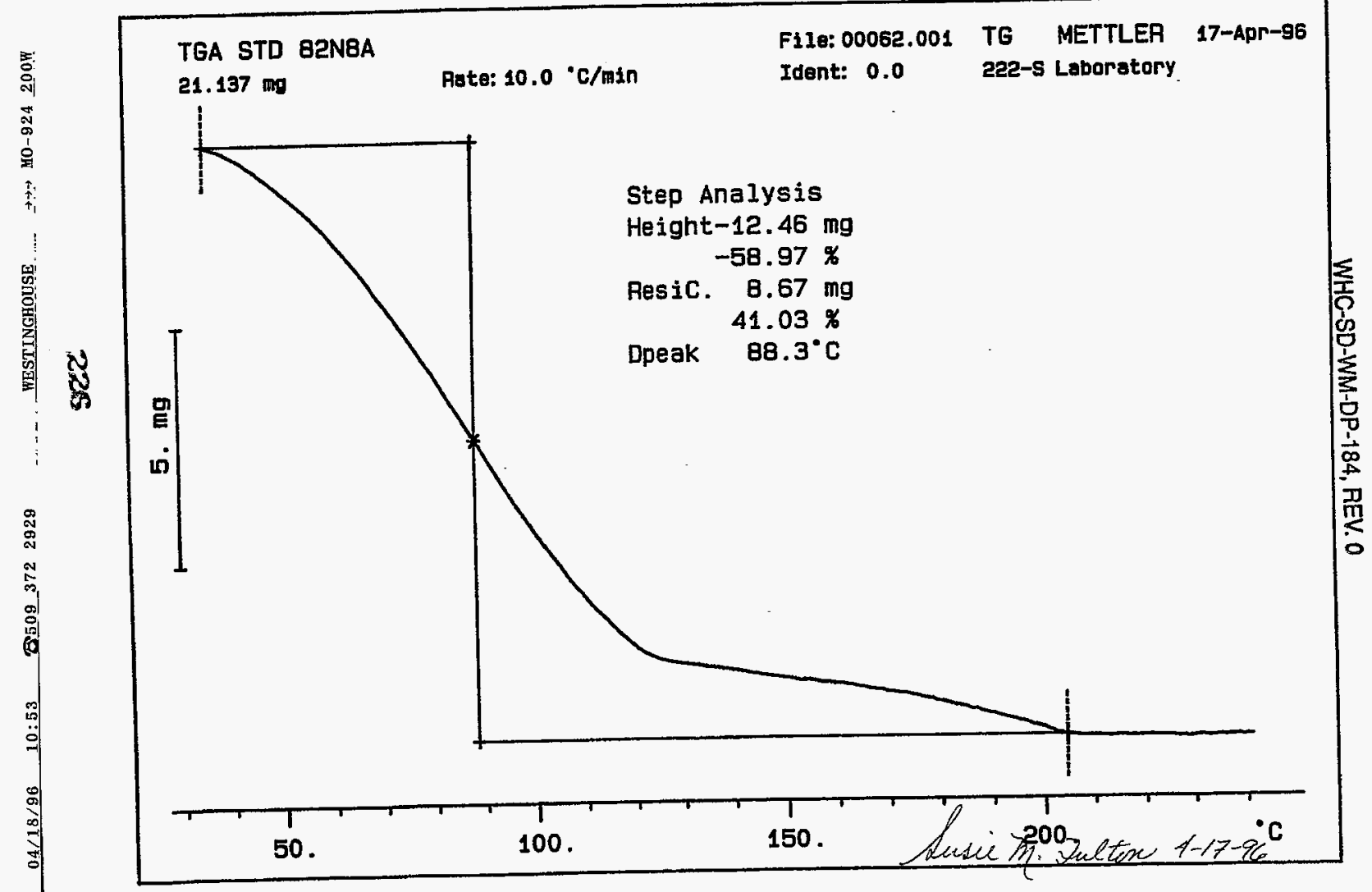




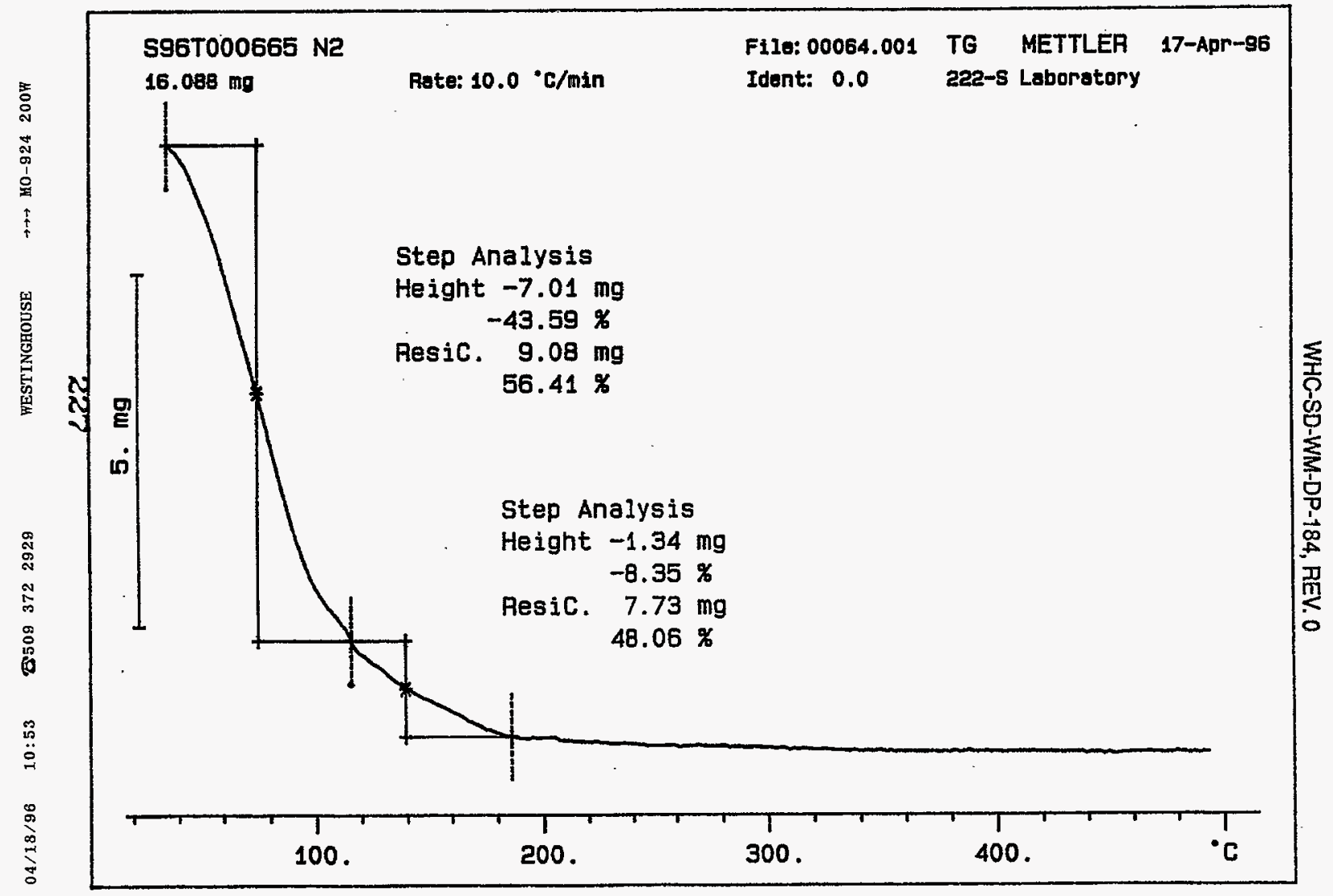




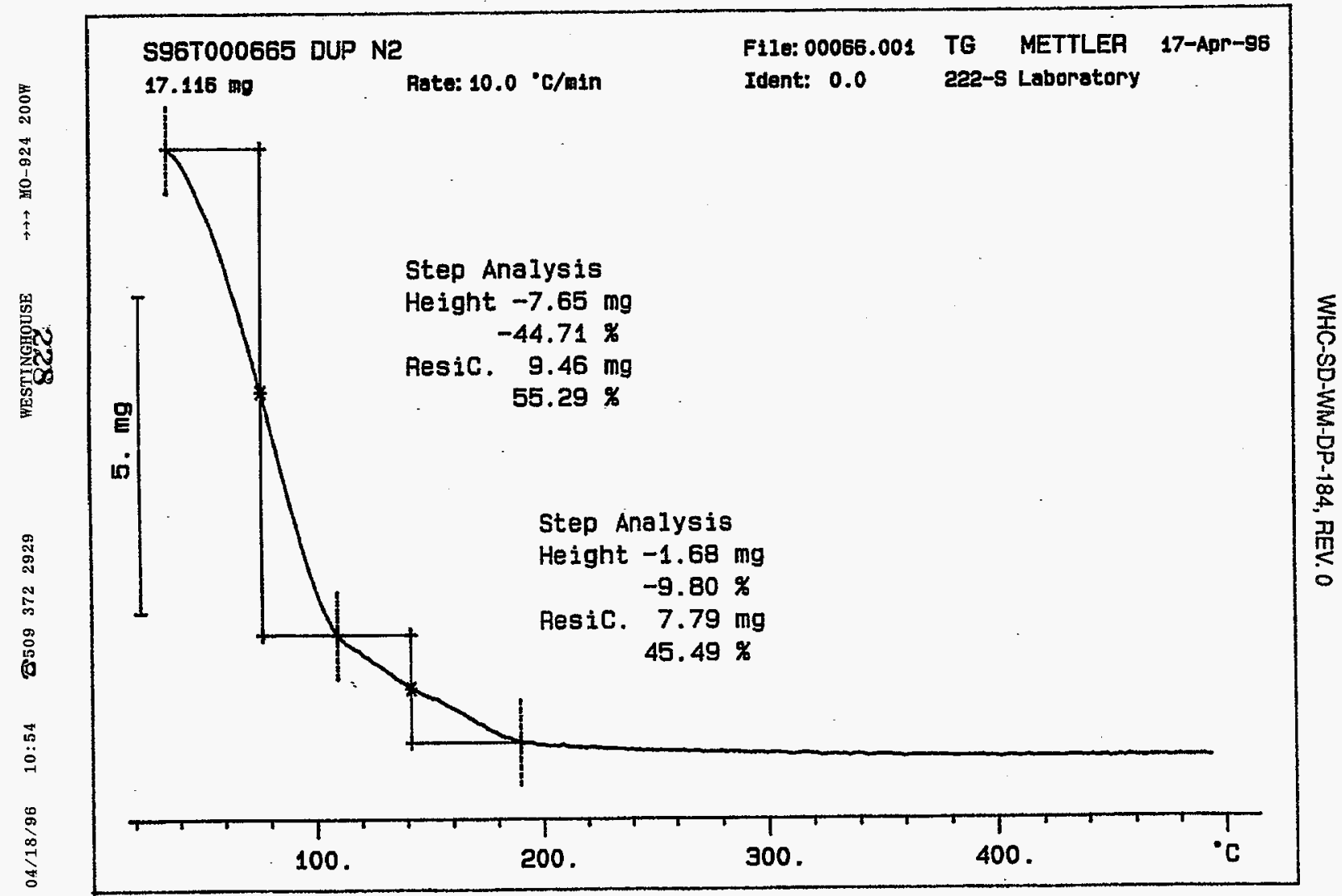


号

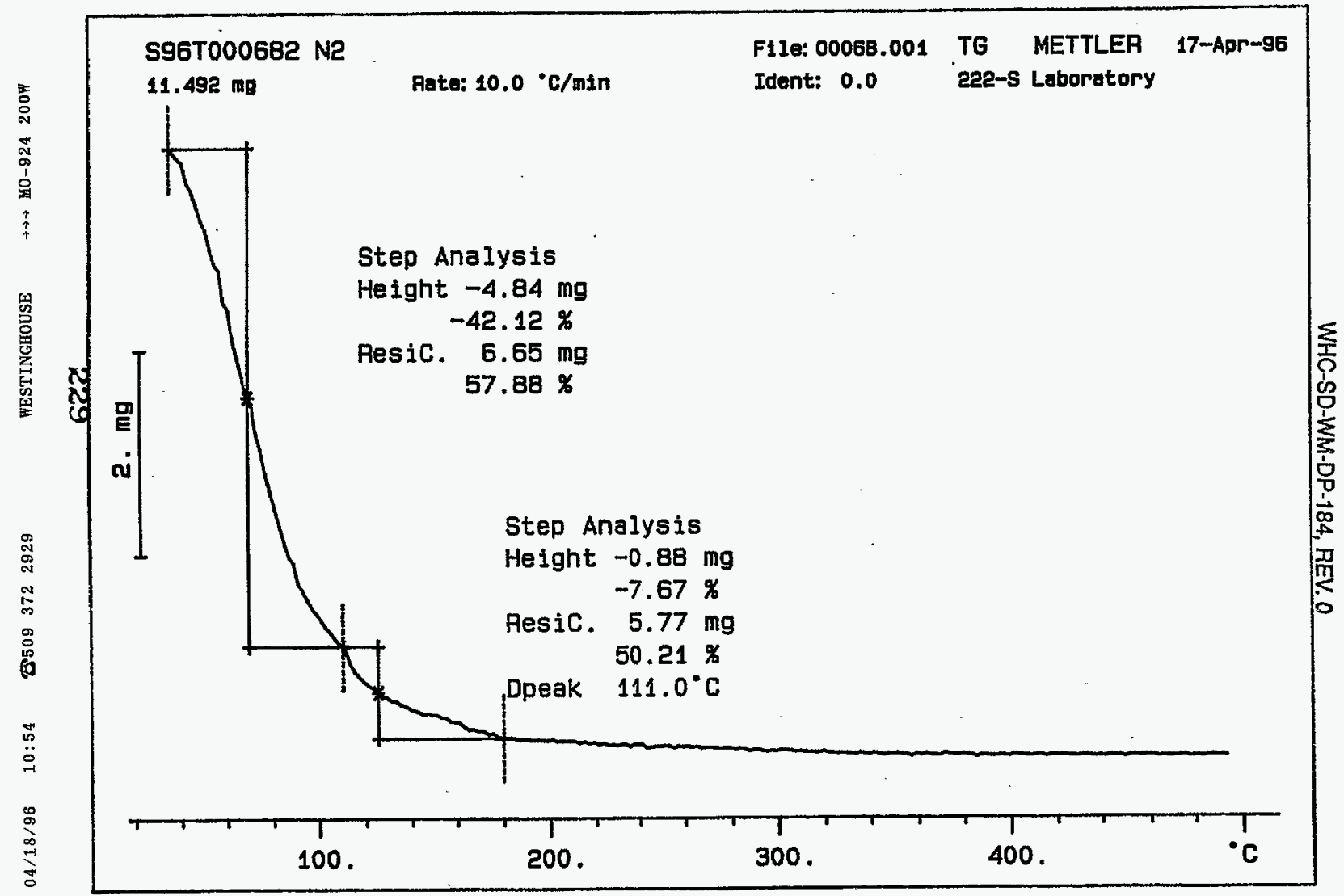




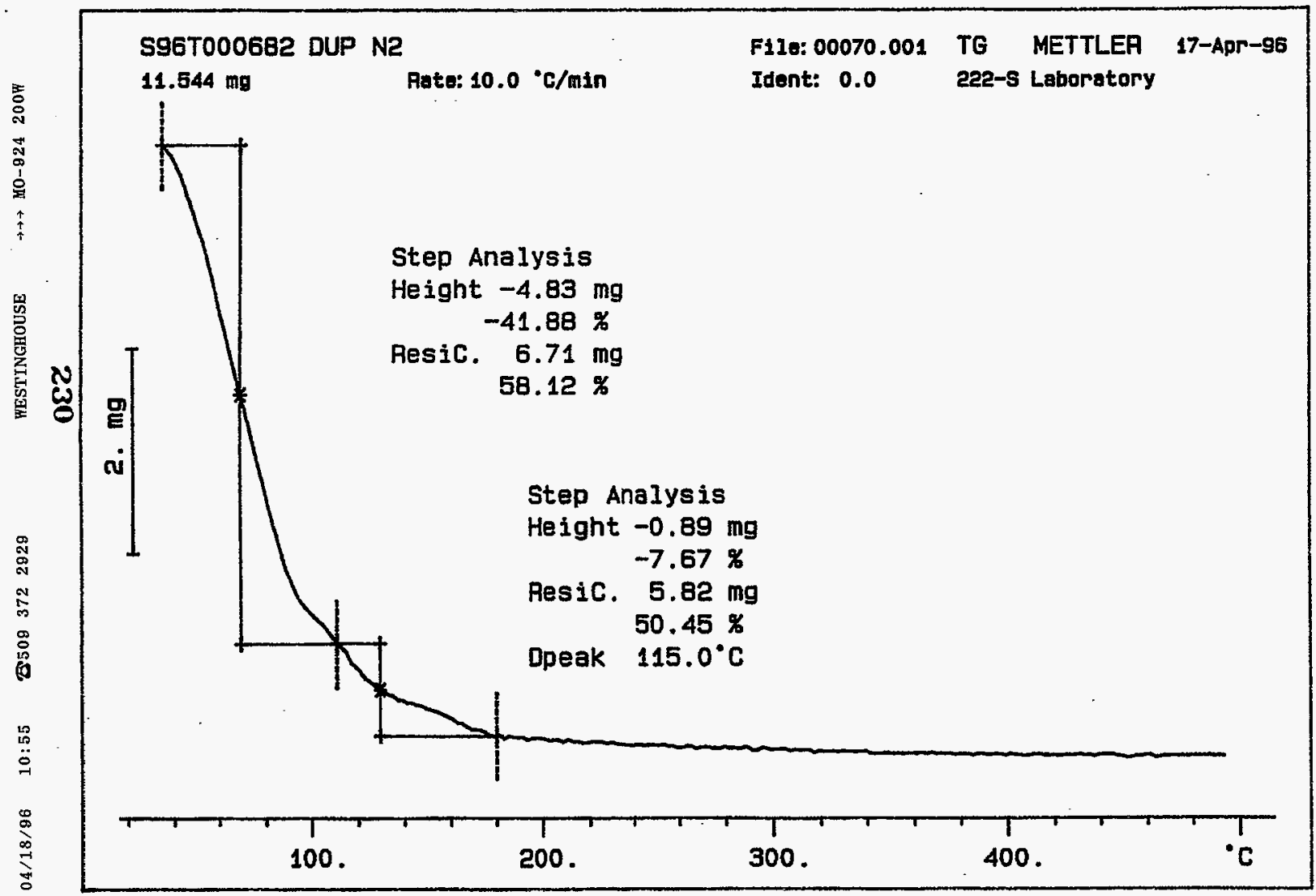




\section{LABCORE Data Entry Template for Worklist\#}

Analyst: RDM Instrument: TGAO I Book\#100CN2

Method: LA-560-112 Rev/Mod B-1

Worklist Comment: U-107 FOR TGA (RUN UNDER N2) RTS!

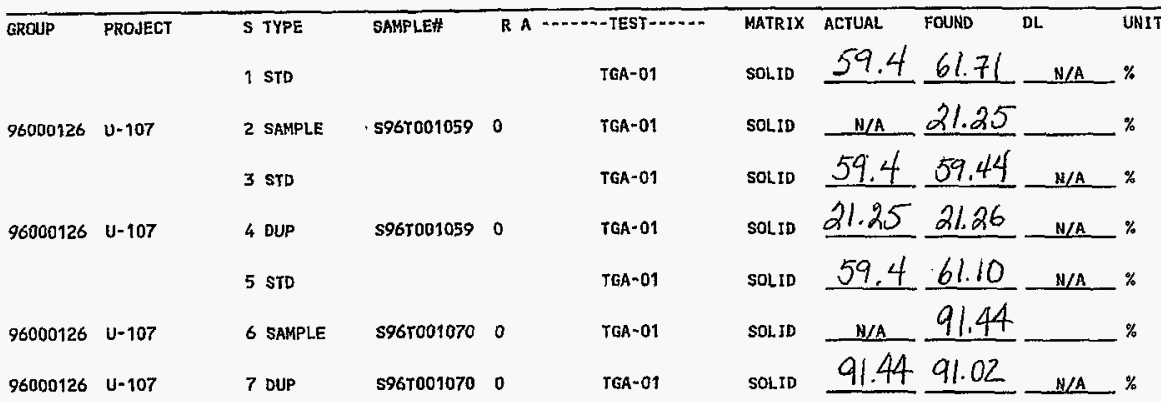

\section{Final page for worklist \# 6648}

See Qttached Ln Siguatures Venfiad by Reandina Valeinzuela

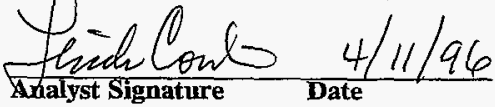

Data Entry Comments:

Units shown for $Q C$ (SPK \& STD) may not reflect the actuat units. $D L=$ Detection Limit, $s=$ Worklist Slot Number, $R=$ Replicate Number, $A=$ Aliguat Code. 
worklistrpt Version 2.1 05/15/95

WHC-SD-WM-DP-184, REV. 0 03/18/96 14:19

\section{LABCORE Data Entry Template for Worklist\#}

Page: $\quad l$

\section{Analyst: ROMM Instrument: TGAO Book \# DOLNZ}

Method: LA-560-112 Rev/Mod B-1

Worklist Comment: U-107 FOR TGA (RUN UNDER N2) RTS!

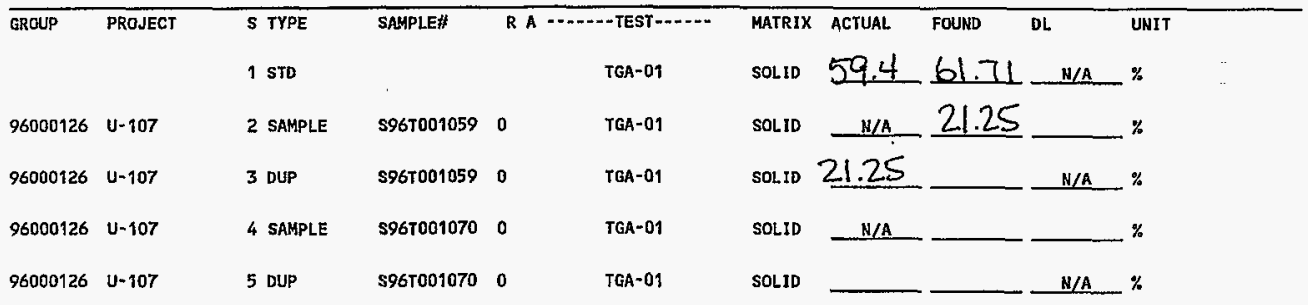

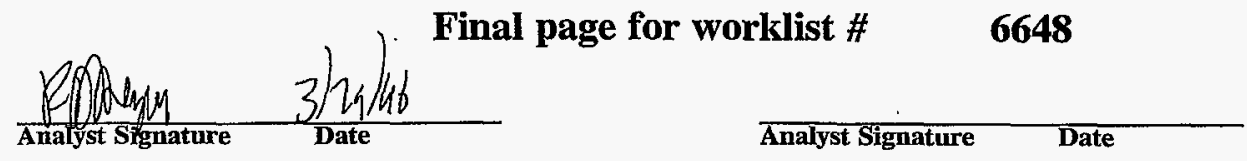

Data Entry Comments:

Units shown for $Q C$ (SPK \& STD) may not reflect the actual units. $D L=$ Detection Limit, $S=$ Worklist Slot Number, $R=$ Replicate Number, $A=$ Aliquot Code. 
SIGNATURE BELOH REPRESENTS CHEMICAL TECHNOLOGIST/CHEHIST THAT

COMPLETED/VERIFIED THE CALIBRATION/ANALYSIS ON PAGES $233 T 023 \%$.

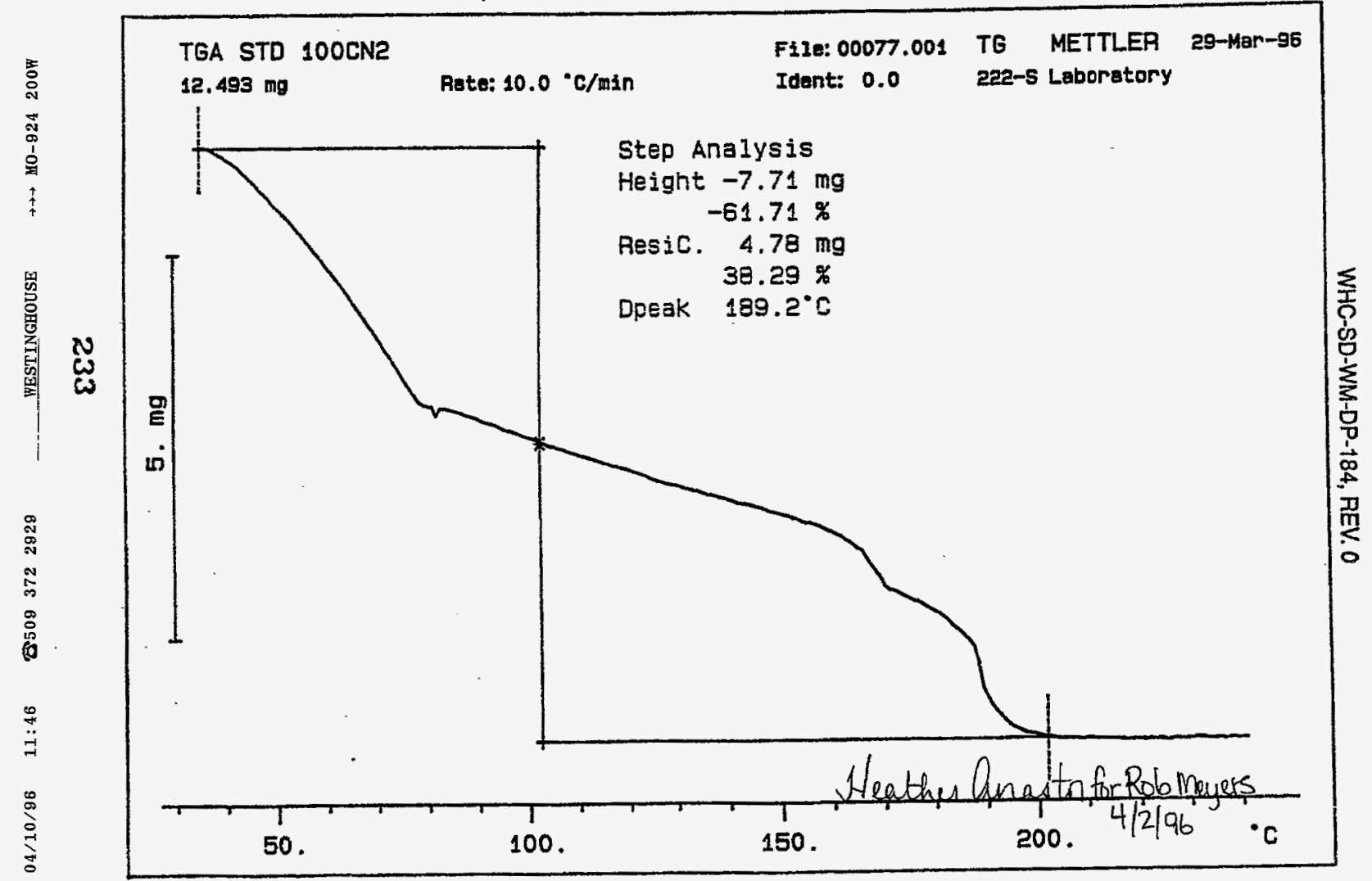




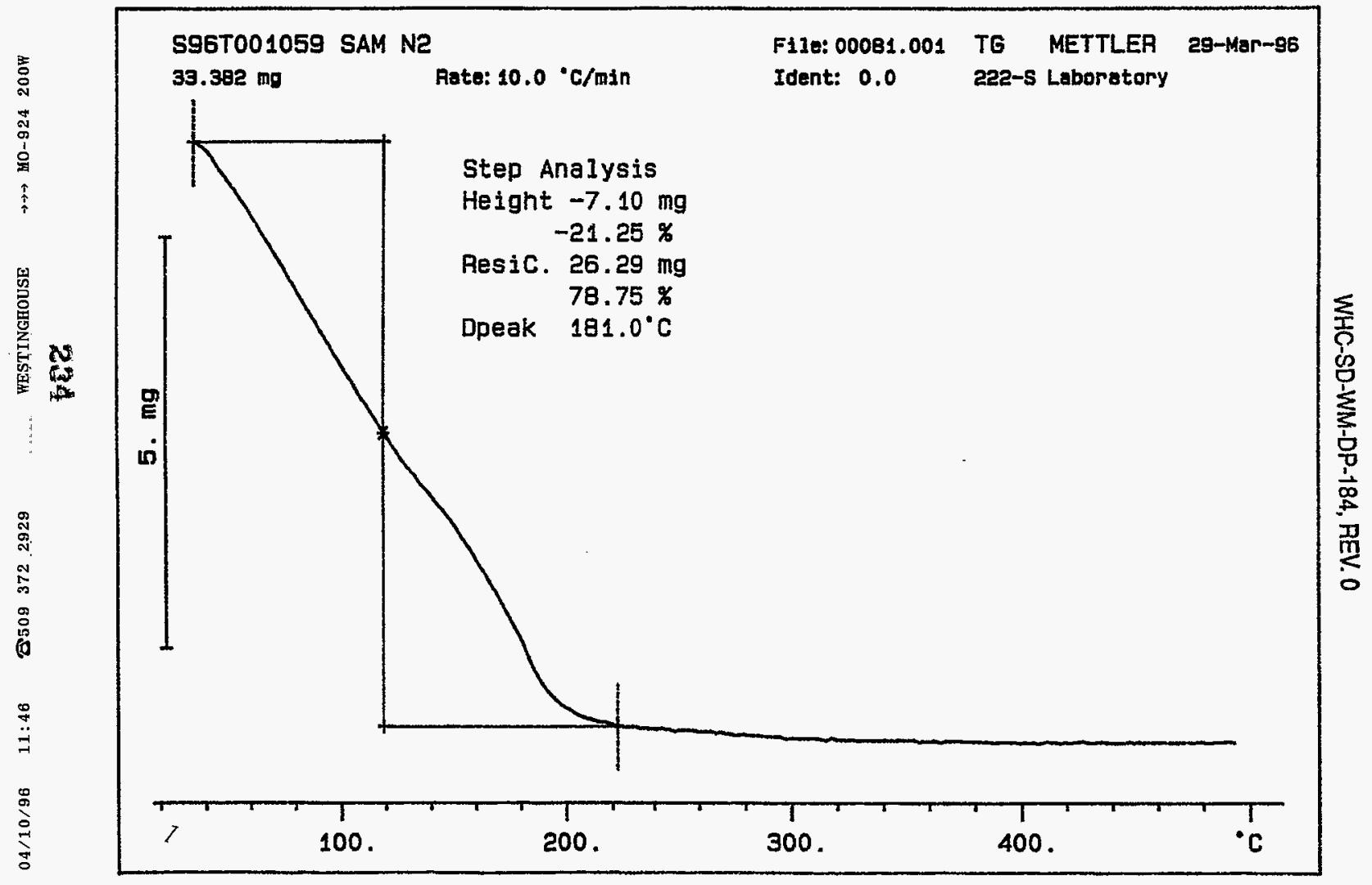




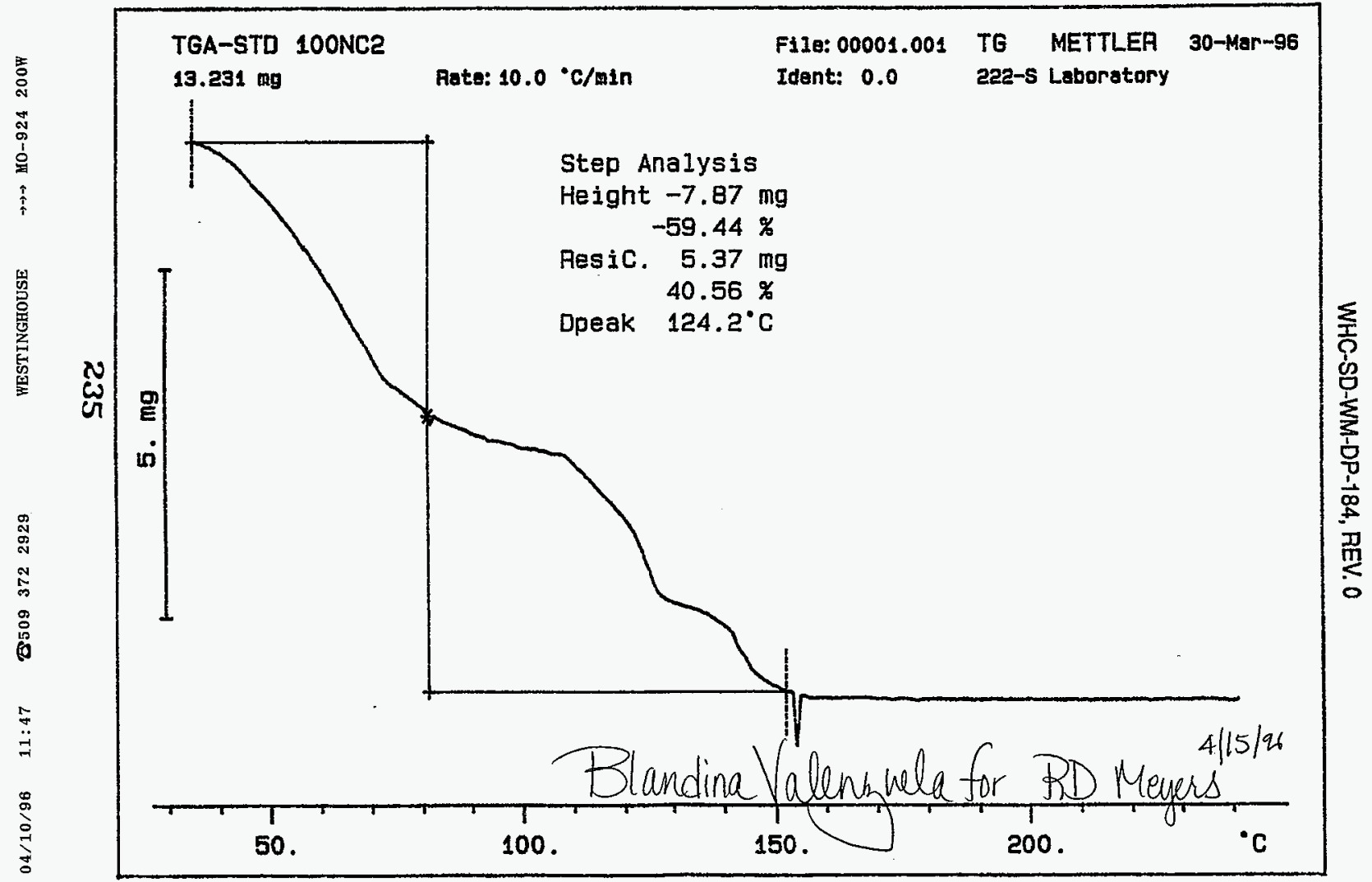




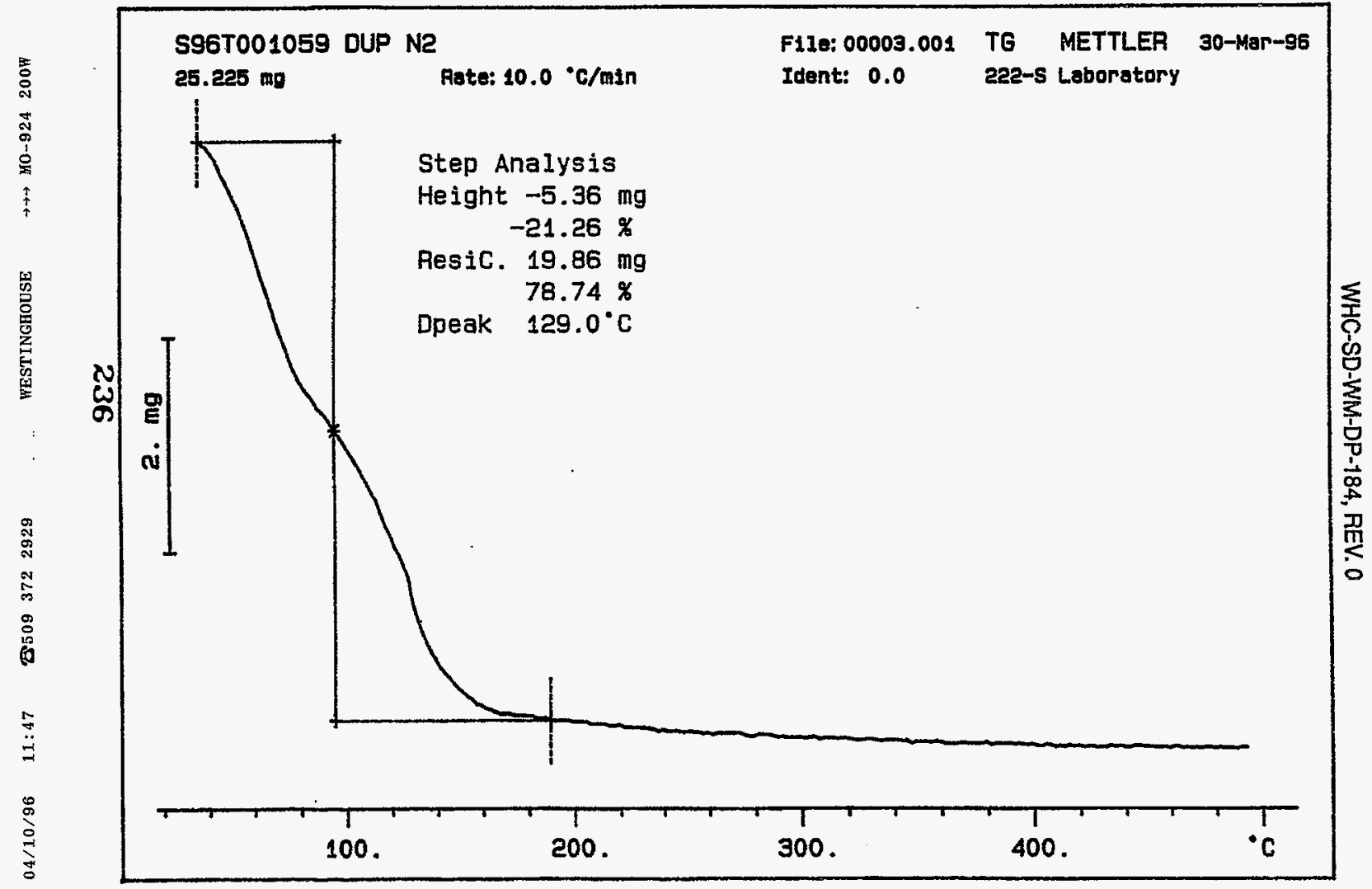


WHC-SD-WM-DP-184, BEV O

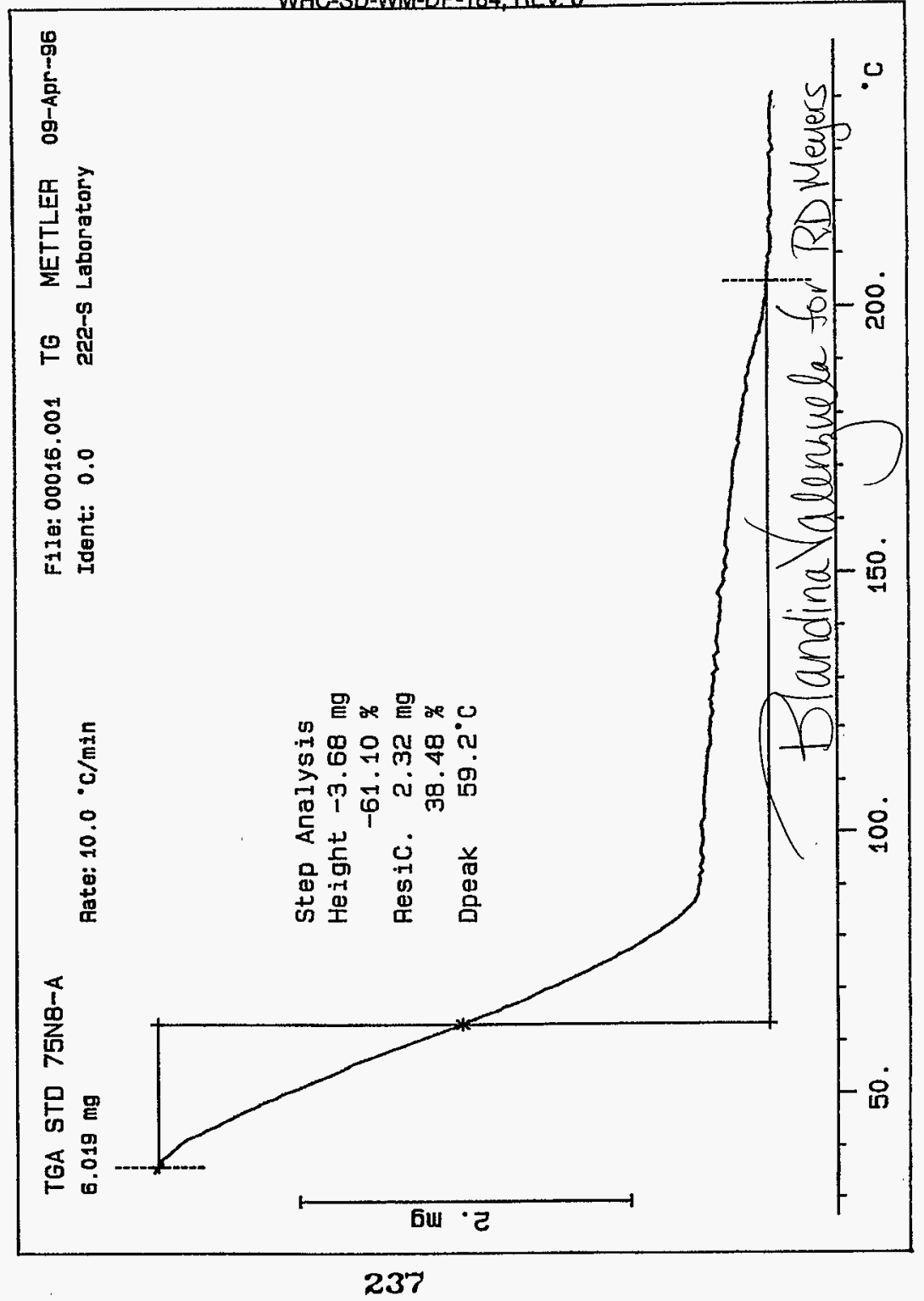




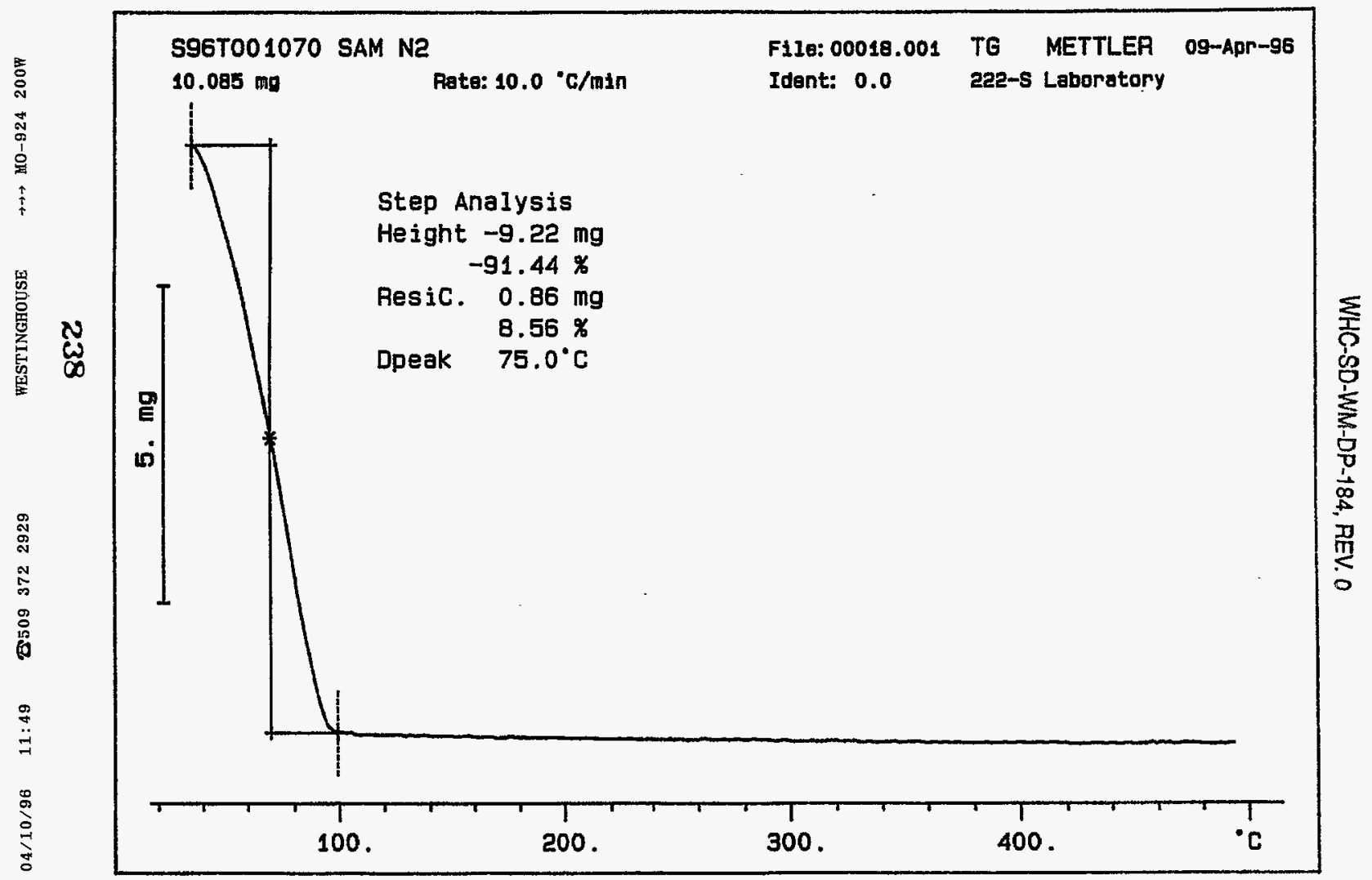




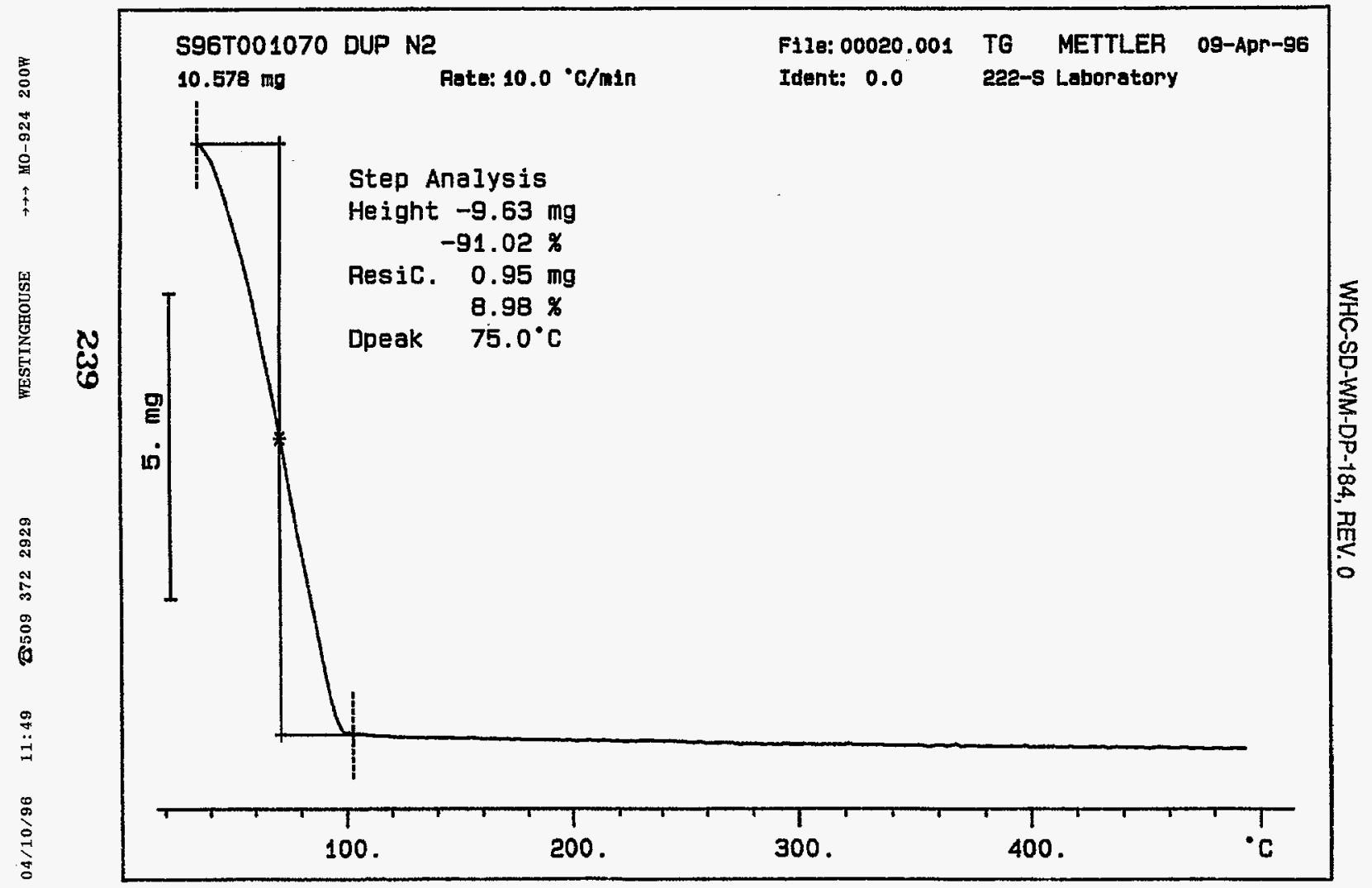




\section{LABCORE Data Entry Template for Worklist\#}

Analyst: $\quad$ RDM Instrument: TGA0/ 3 Book \# 75 N8A

Method: LA-514-114 Rev/Mod $C-1$

Worklist Cowment: U-107 FOR TGA (RUN UNDER N2 PLEASE!) RTS!

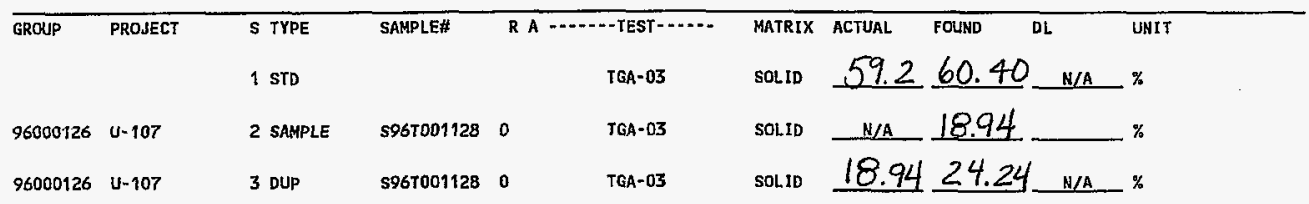

\section{Final page for worklist \#}

See attached for signatures Analyst Signature Date 4-896 Verified by Hanastor 4-11-96

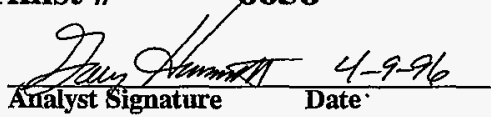

Data Entry Comments:

Units shown for $Q C$ (SPK \& STD) moy not reflect the actual units. $D L=$ Detection Limit, $S=$ Worklist Slot Number, $R=$ Replicate Number, $A=$ Aliquot Code. 


\section{LABCORE Data Entry Template for Worklist\#}

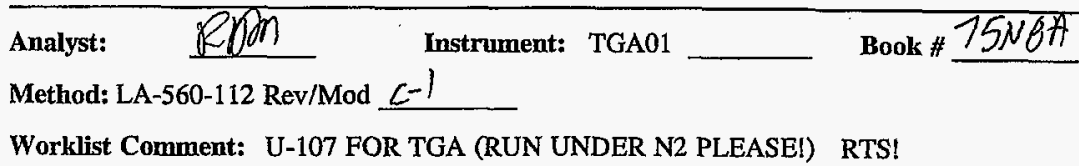

\begin{tabular}{|c|c|c|c|c|c|c|c|c|c|c|}
\hline \multirow[t]{2}{*}{ GROUP } & \multirow[t]{2}{*}{ PROJECT } & S TYPE & \multirow[t]{2}{*}{ SAMPLE\# } & \multicolumn{2}{|r|}{ R A $\ldots-\ldots$ TEST $\cdots \cdots$} & \multirow[t]{2}{*}{ MATRIX } & \multirow[t]{2}{*}{ ACTUAL } & \multirow[t]{2}{*}{ FOUND } & \multirow[t]{2}{*}{ DL } & UNIT \\
\hline & & 1 STD & & & $T G A-01$ & & & & & $\%$ \\
\hline 96000126 & $U-107$ & 2 SAMPLE & s96T00า128 & 0 & TGA-01 & SOLIO & $N / A$ & & & $y$ \\
\hline 96000126 & $u-107$ & 3 DuP & S96T001728 & 0 & $T G A=01$ & SOLID & & & & $x$ \\
\hline
\end{tabular}

Final page for worklist \# $\quad 6656$

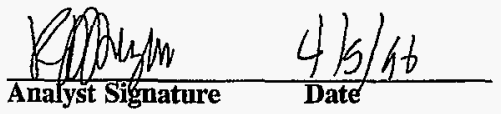

Analyst Signature Date

Data Entry Comments:

Units shown for $Q C$ (SPK \& STD) may not reflect the actual tnits. $D L=$ Detection Limit, $S=$ Worklist Slot Number, $R=$ Replicate Number, $A=$ Aliquot Code. 
File info: TER040401 Thu Apr 4 07:03:08 1996

Sample Weight: 22.156

TGA STD 75NB-A

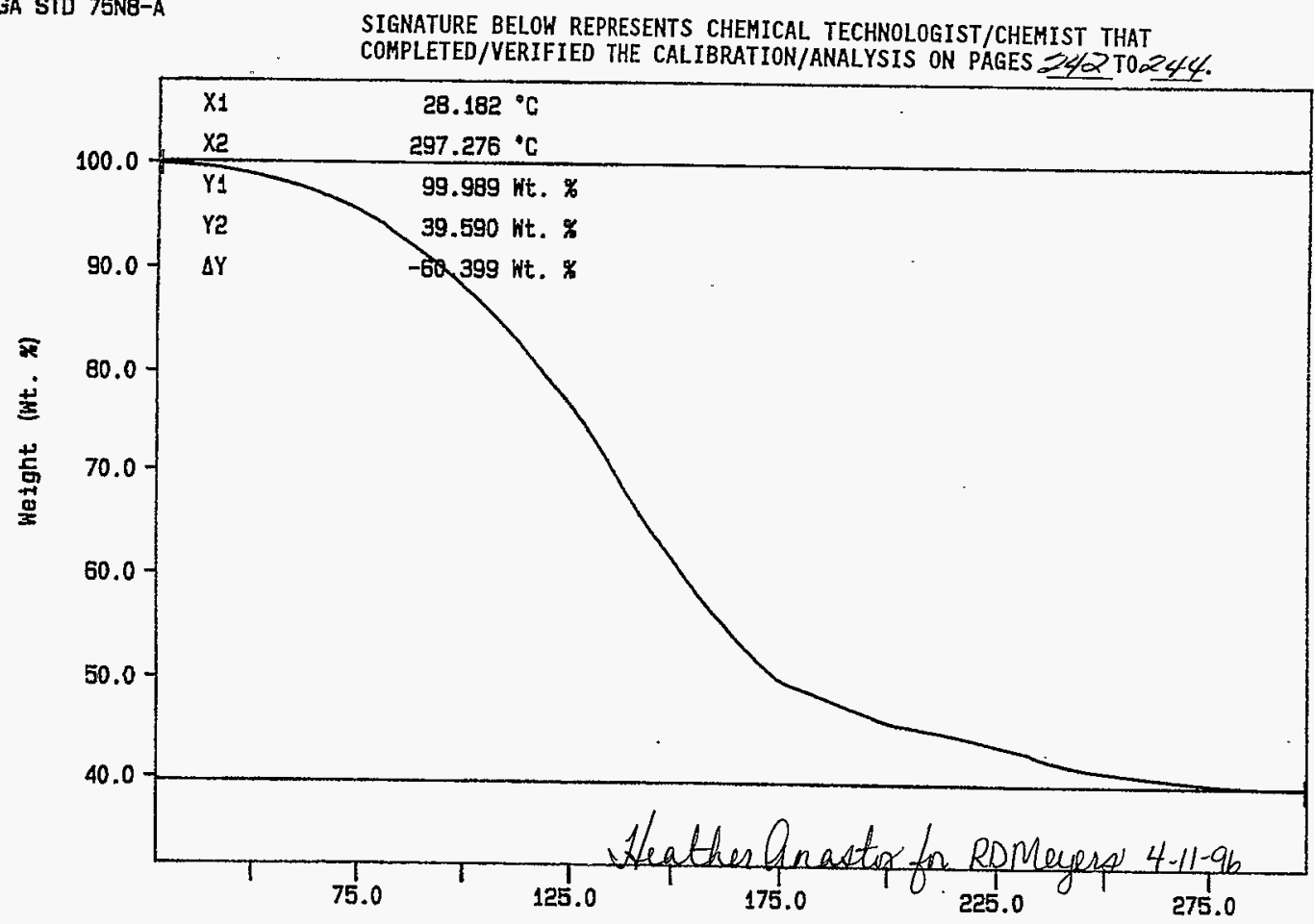

SIGNATURE BELOW REPRESENTS CHEMICAL. TECHNOLOGIST/CHEMIST THAT

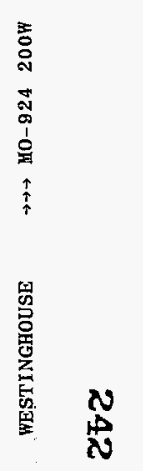

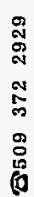

N2 10C/MIN

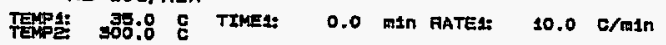

Temperature ('C)

PJ MCCOWN

PEAKIN-ELMER

7 Sertes Thermal Analysis System

Fri Apr 5 01:21: 22 1996 
Curve 1: TGA

Ftle info: sam040401 Thu Apr 4 22: 29: 481996

Sample Kaight: $32.221 \mathrm{mg}$

S96T001128

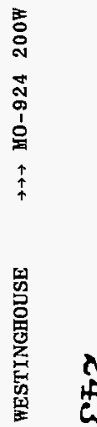

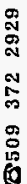

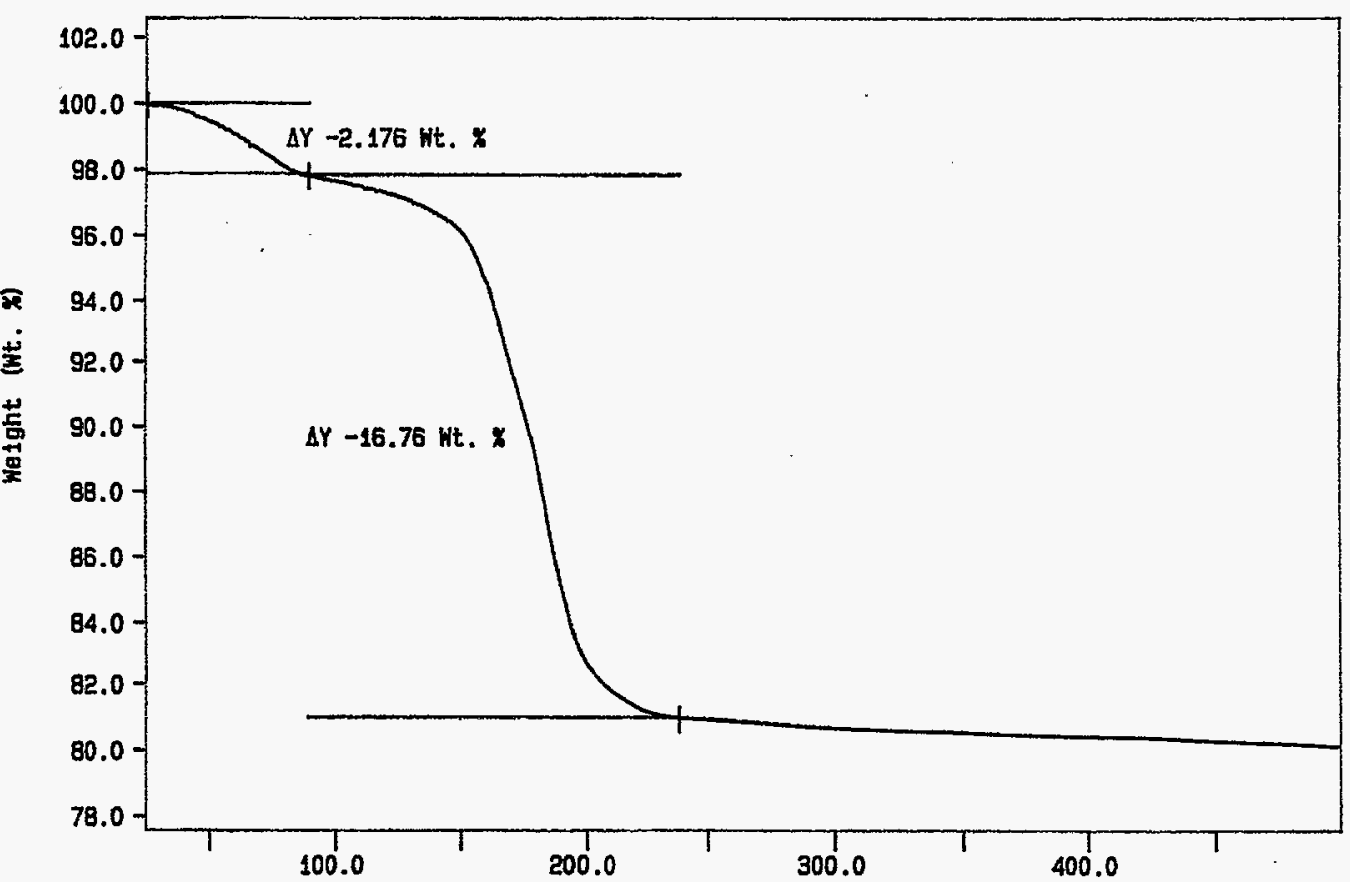

300.0

$\stackrel{\circ}{\circ} \quad 10 \mathrm{C} / \mathrm{MIN}$ N2

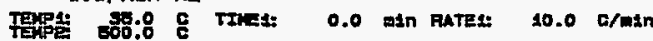

Temperature ("c)

rd meyers

PERKIN-ELMER

7. Sertes Thermal Analysis System

Mon ApP B 11: 29: 47 1996 
File info: sam040501 Fri Apr 5 04:54: 251996

Sample Keight: 9.630

S96T001128 DUP

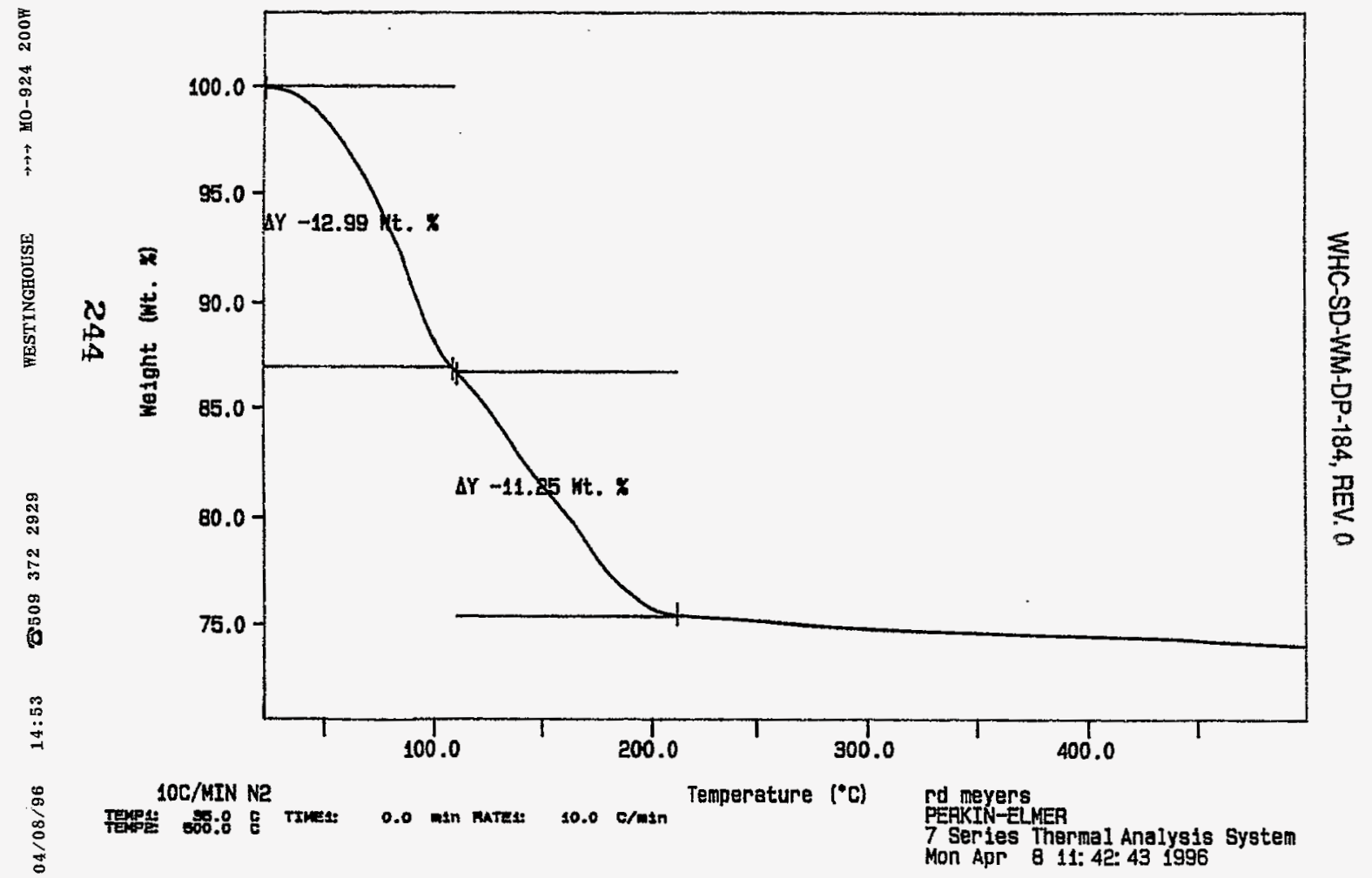


$04 / 24 / 96 \quad 07: 29 \quad B 5093722929 \quad \ldots$. WESTINGHOUSE $\rightarrow \rightarrow$ HO -924 $200 \mathrm{~W}$

ه20

worklistrpt Version 2.1 05/15/95

WHC-SD-WM-DP-184, REV. 0

LABCORE Data Entry Template for Worklist\#

Page: 1 04/23/96 11:30

6758

Analyst: $\quad$ PJM Instrument: TGA0 I $\quad$ Book \# 82N8A

Method: LA-560-112 Rev/Mod B-1

Worklist Comment: U-107 FOR TGA-01 PLEASE RUN UNDER N2 RTS!

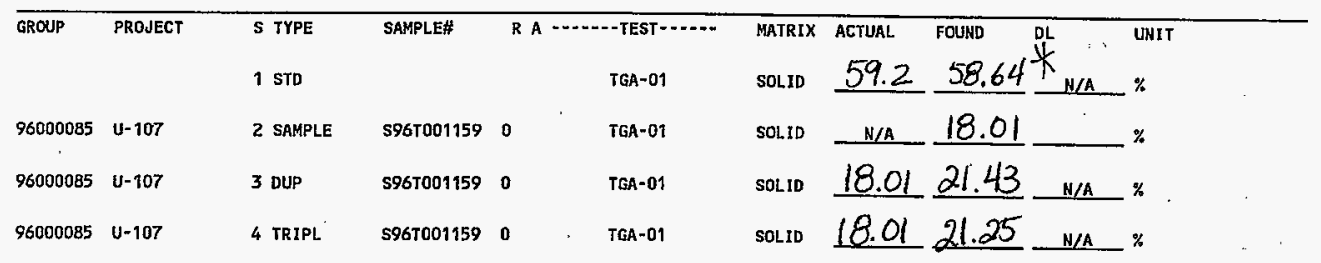

Final page for worklist \# 6758
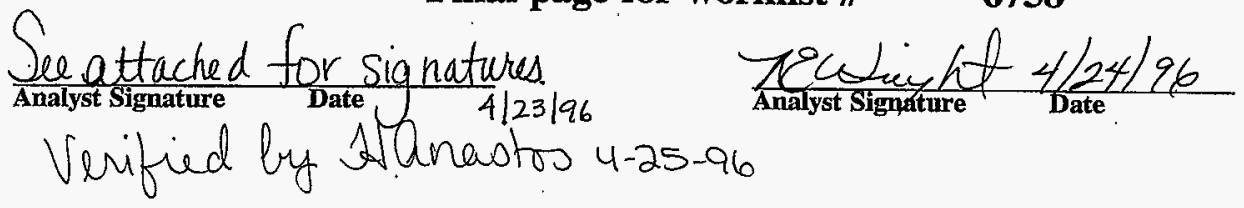

Data Entry Comments: Sample results are the sum of two weight loss steps

Units shown for QC (SPK \& STD) may not reflect the actual units. $D L=$ Detection Limit, $S=$ Worklist Slot Number, $R=$ Replicate Number, $A=$ Aliquot Code.

$$
245
$$


worklistrpt Verston $2.105 / 15 / 95$ $03 / 20 / 9611: 32$

\section{LABCORE Data Entry Template for Worklist\#}

Analyst: RueCown Instrument: TGA0 — Book\# 82N8A

Method: LA-560-112 Rev/Mod B-1

Worklist Comment: U-107 FOR TGA-01 PLEASE RUN UNDER N2 RTS!

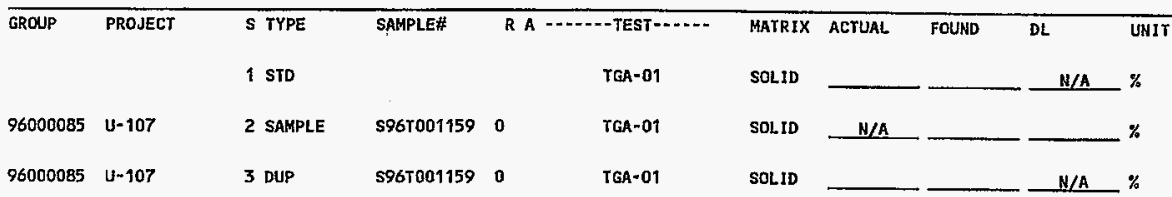

\section{Final page for worklist \# $\quad 6758$}

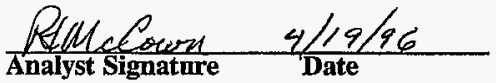

Analyst Signature Date

Data Entry Comments:

Units shown for QC (SPK \& STD) may not reflect the actual units. $D L=$ Detection Limit, $S=$ Worklist Slot Number, $R=$ Replicate Number, $A=$ Aliquot Code. 
:

SIGNATURE BELOW REPRESENTS CHEMICAL TECHNOLOGIST/CHEHIST THAT

CONPLETED/VERIFIED THE CALIBRATION/ANALYSIS ON PAGES $\$ 47 T 0250$.

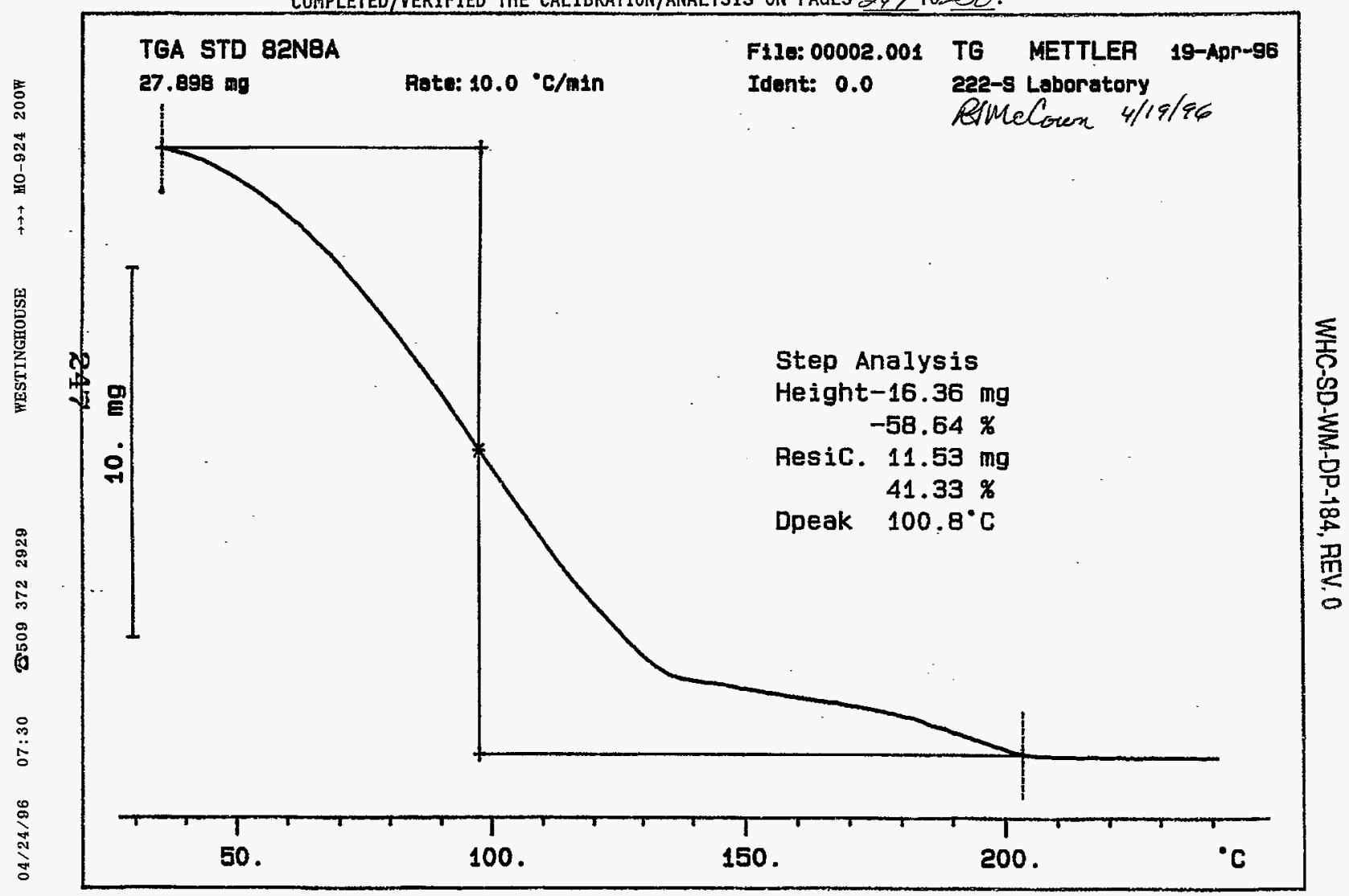




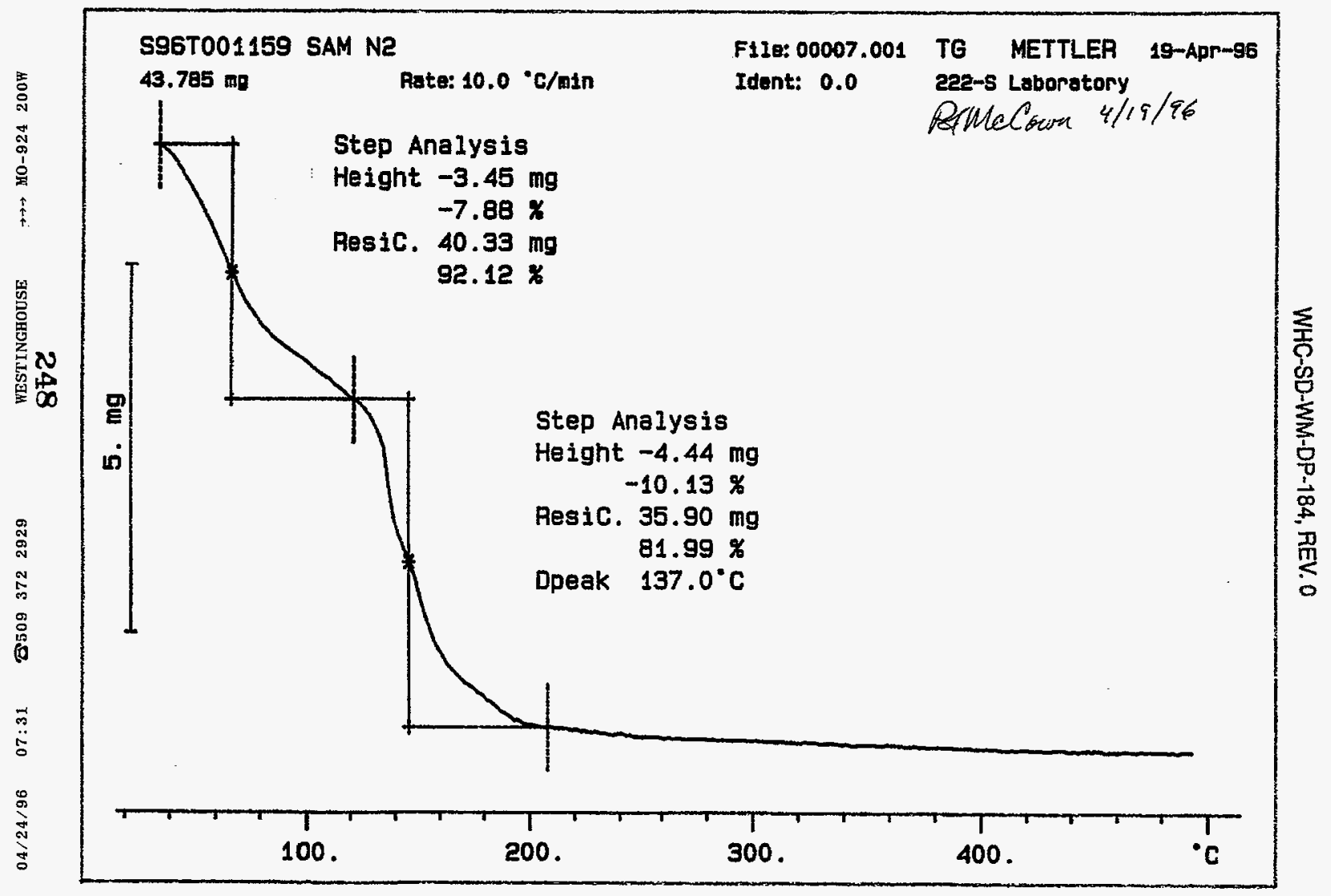




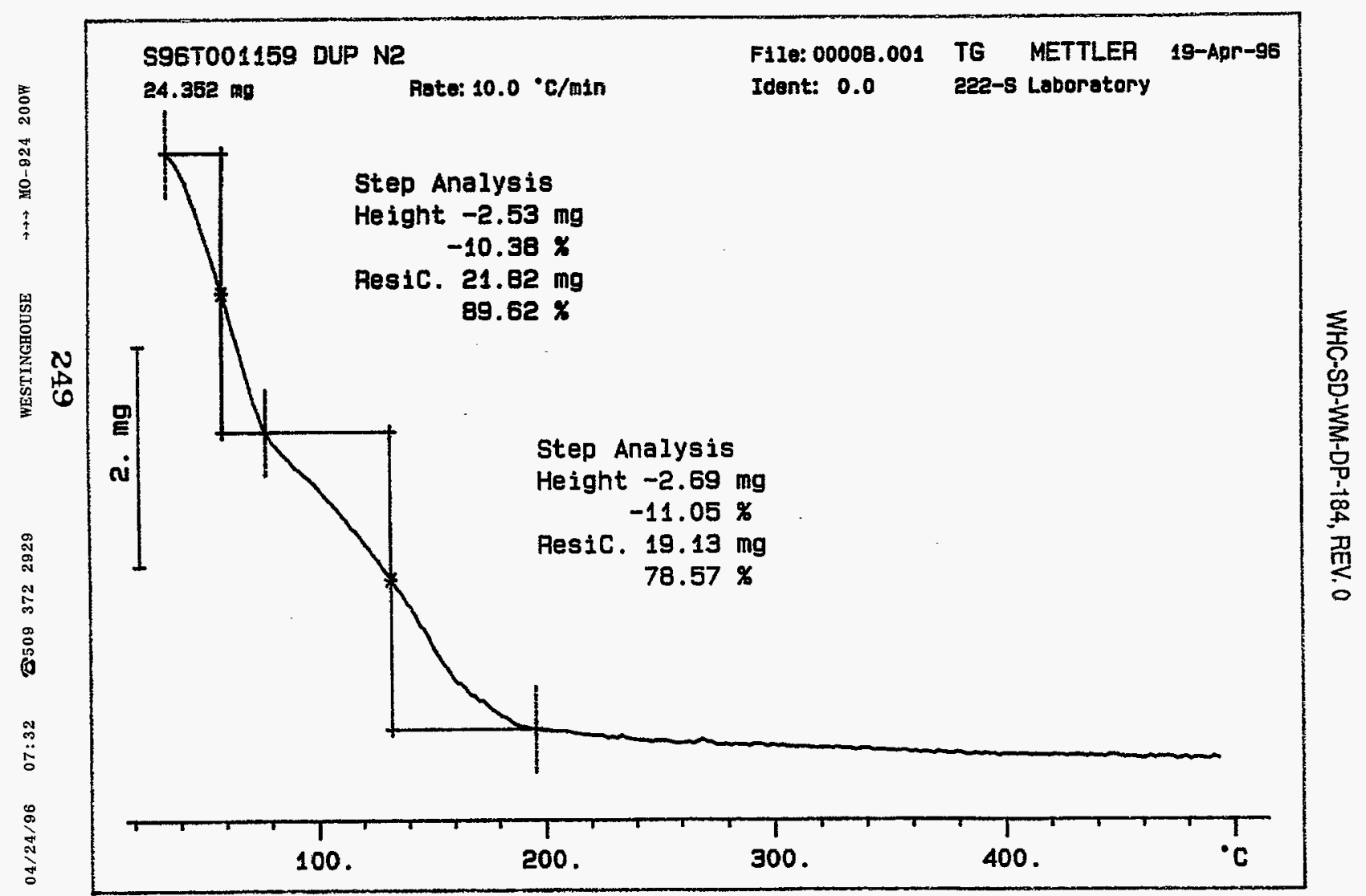




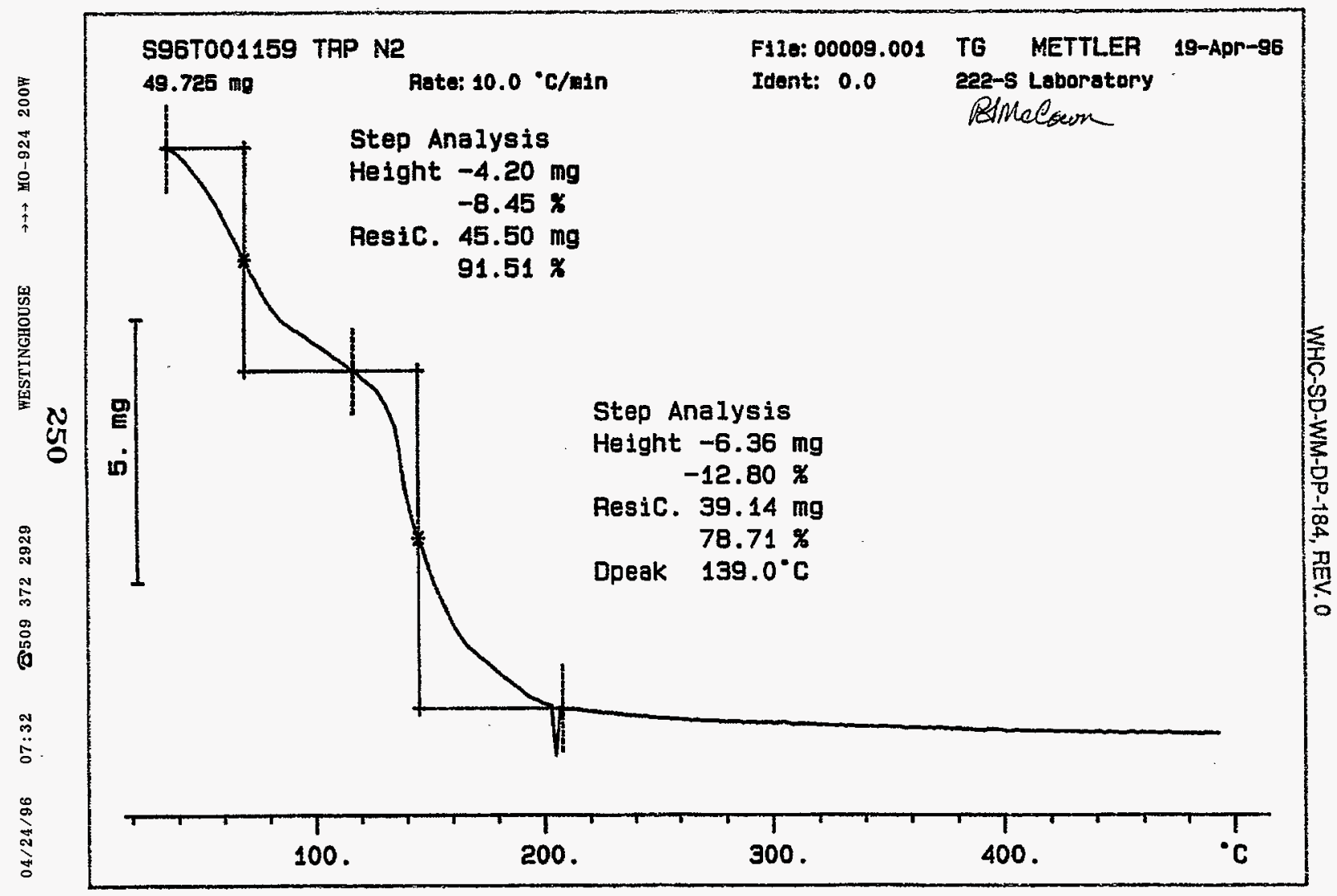




Analyst: $\quad$ SMF $\quad$ Instrument: TGA0 3 Book \# $82 \mathrm{~N} 8 \mathrm{~A}$

Method: LA-514-114 Rev/Mod C - 1

Worklist Comment: U-107 TGA RUN UNDER N2. RCJ

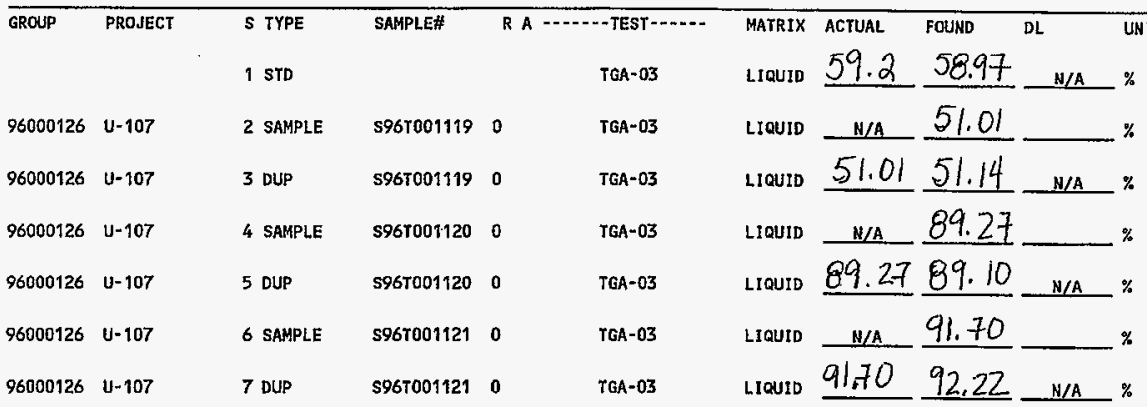

\section{Final page for worklist \# . $\mathbf{7 5 8 5}$} $\frac{\text { De attached for signatures }}{\text { Analyst signature }}$

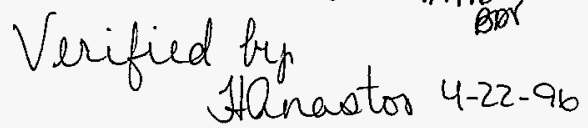

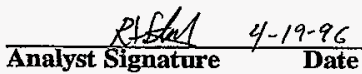

Data Entry Comments:

Units shown for QC (SPK \& STD) may not reflect the actual units. DL = Detection Limit, $S=$ Worklist Slot Number, $R=$ Replicate Number, $A=$ Aliquot Code. 
worklistrpt Version 2.1 05/15/95

04/11/96 09:54

\section{LABCORE Data Entry Template for Worklist\#}

Analyst:

SMF Instrument: TGAO

Book \# $8208 A$

Method: LA-560-112 Rev/Mod

Worklist Comment: U-107 TGA RUN UNDER N2. RCJ

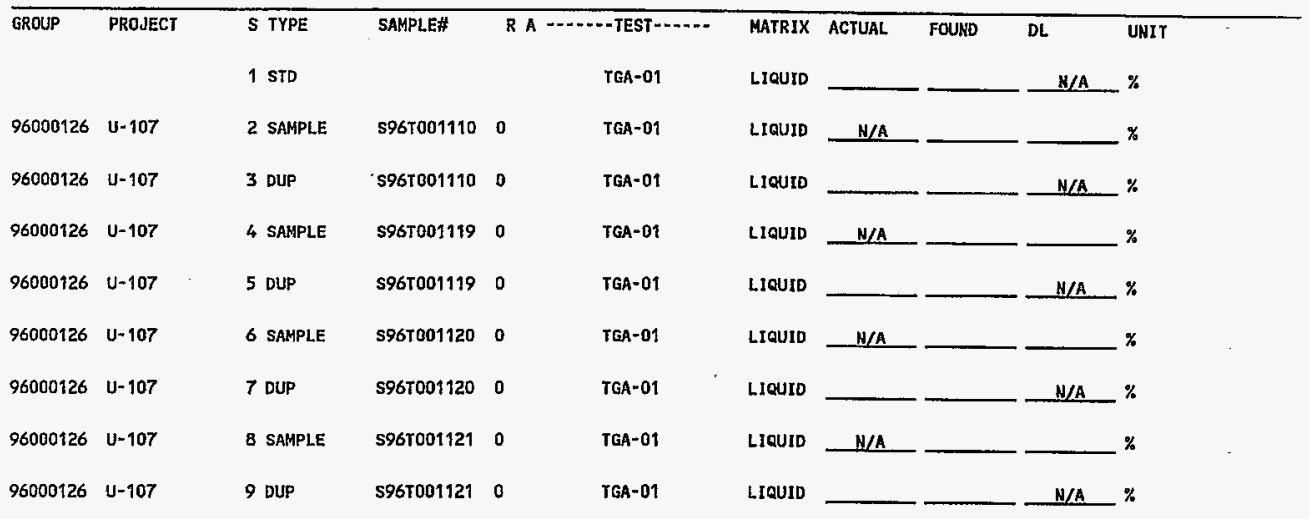

\section{Final page for worklist \# 7585}

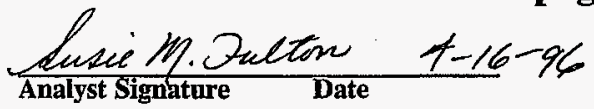

\section{Analyst Signature Date}

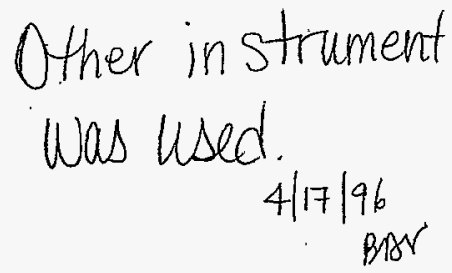

Data Entry Comments:

S94TOOH10- EMPTY

Units shown for $Q C$ (SPK \& STD) may not reflect the actual units. $D L=$ Detection Limit, $S=$ Worklist Slot Number, $R=$ Replicate Number, $A=$ Aliquot Code. 
F110 Info: TEP041601 TUE Apr 16 07: 24: 331996 Sample height: 24.029

由g

TGA STD BQNB-A

SIGNATURE BELOW REPRESENTS CHENICAL TECHNOLOGIST/CHEMIST THAT

COMPLETED/VERIFIED THE CALIBRATION/ANALYSIS ON PAGES $253 T 025 \%$

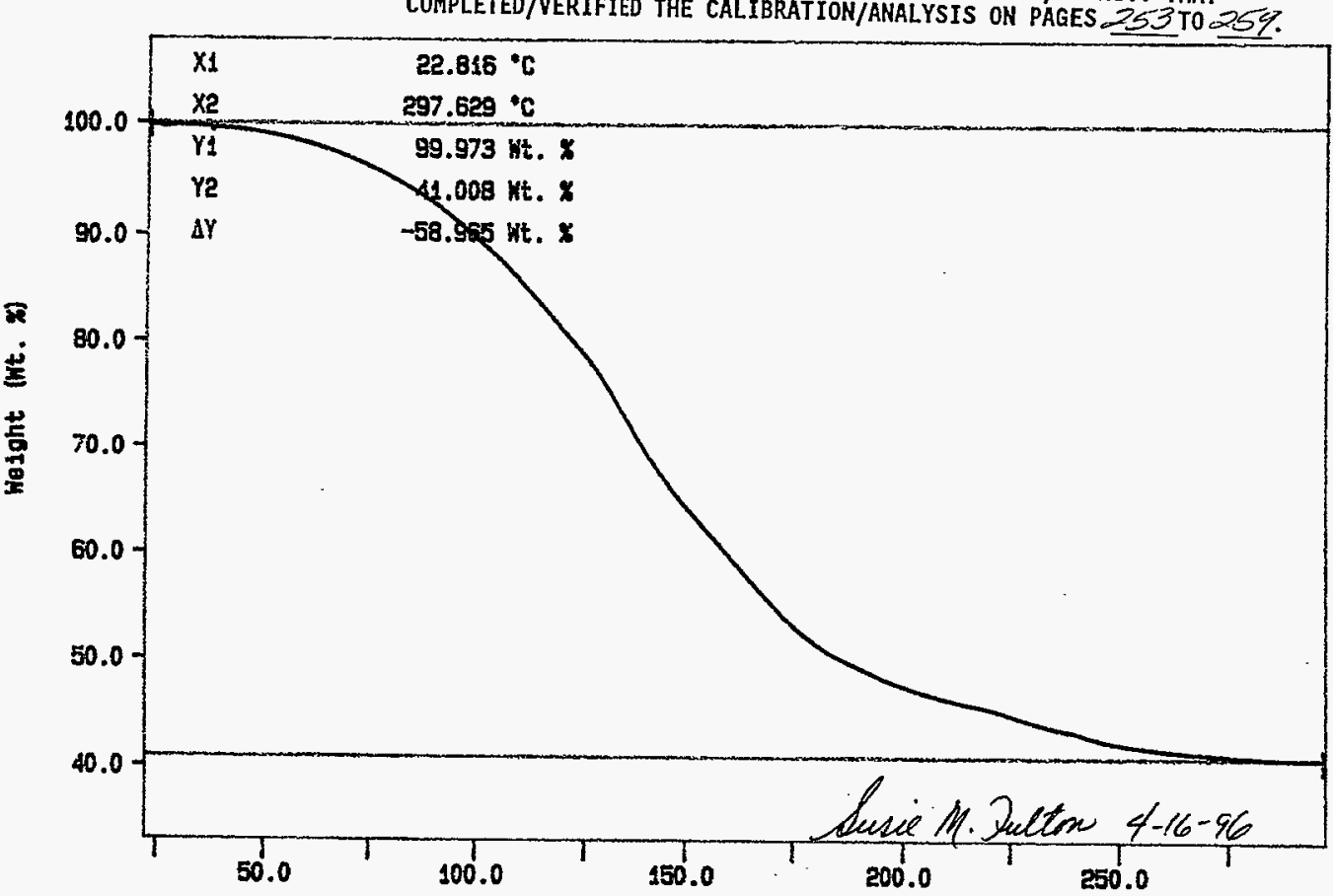

10.0 oratn

Temperature ('C) sy FULToN

PERKIN-CLLER 7 Sertes Thernal Analysis system
Tue Apr 16 07: $25: 27$ 1996 
File info: SAM041601 Tue Apr 16 09: 22: 171996

Sanple Veight: $14.242 \mathrm{mg}$

S96T001119

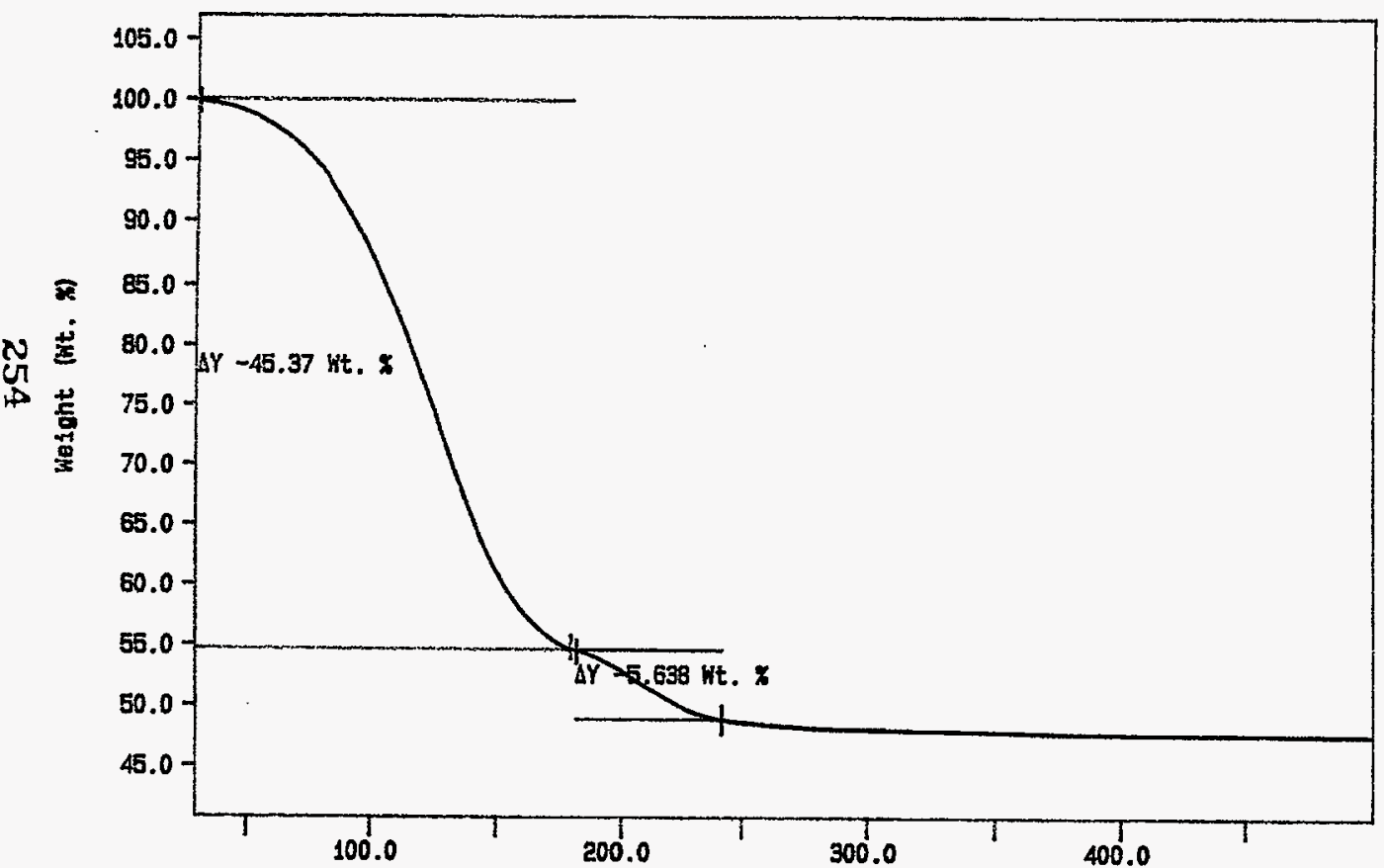

10C/NIH He

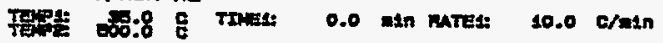

Temperature $\left({ }^{\circ} \mathrm{C}\right)$ SM FULTOH 
File Info: SAM041602 Tue Apr 16 10: $29: 171996$

Semple Neight: 15.617 mg

S95T001119 DUP

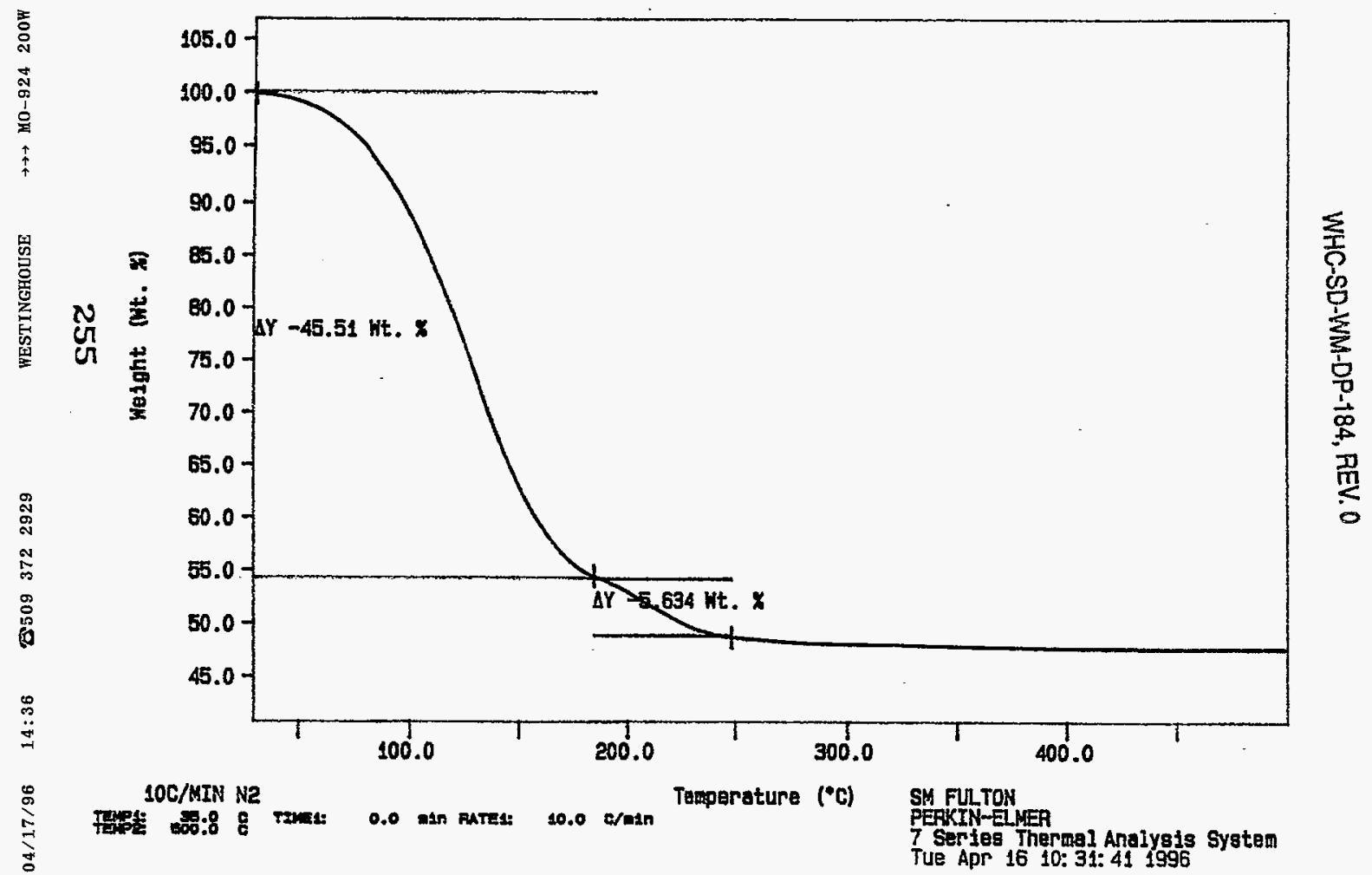




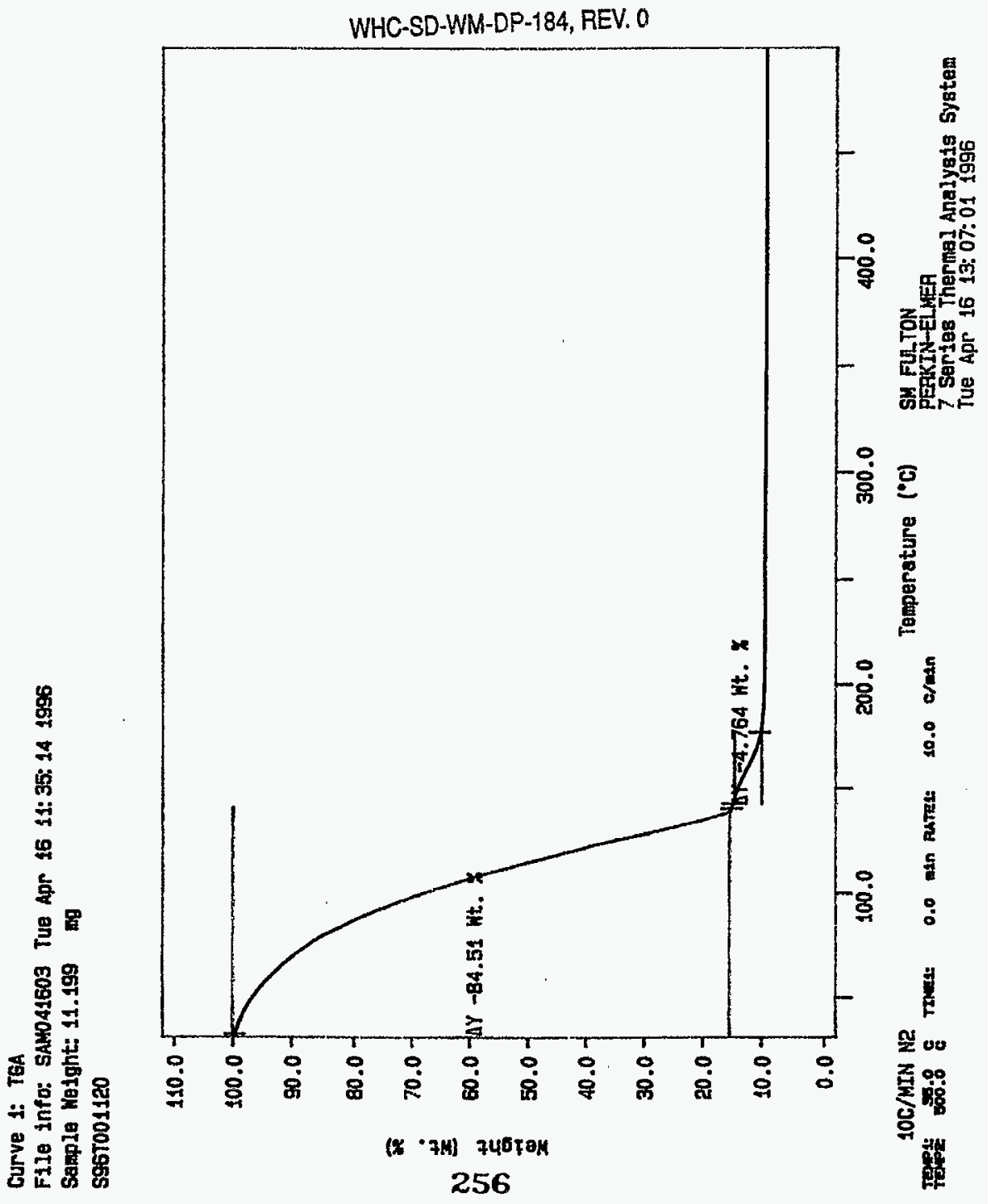


WHC-SD-WM-DP-184, REV. 0

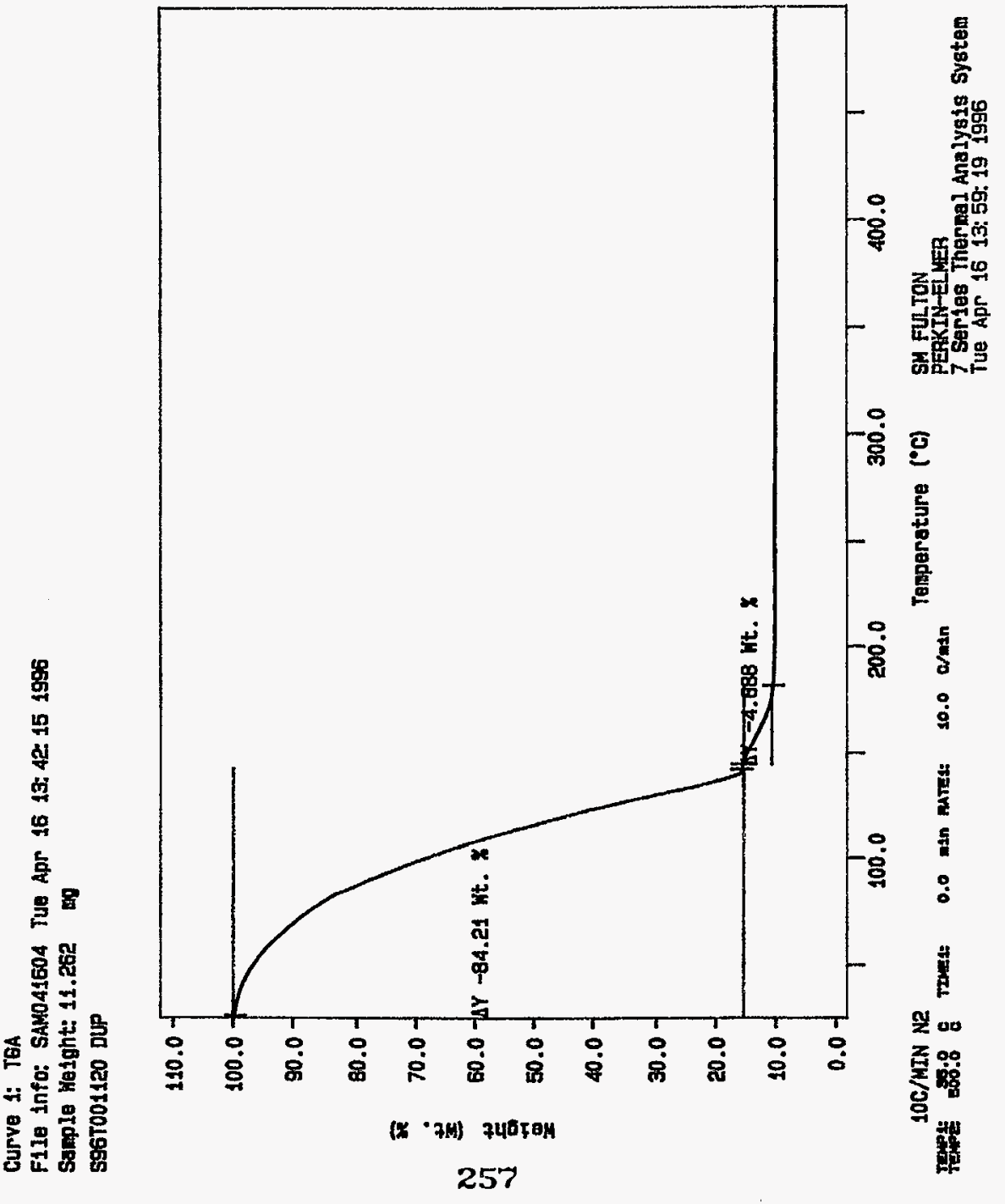


Curye 1: TEA

F1le Info: SAM041605 Tue Aps 16 14:53; 411996

Sample Keight: 10.095

aig

S96T001121

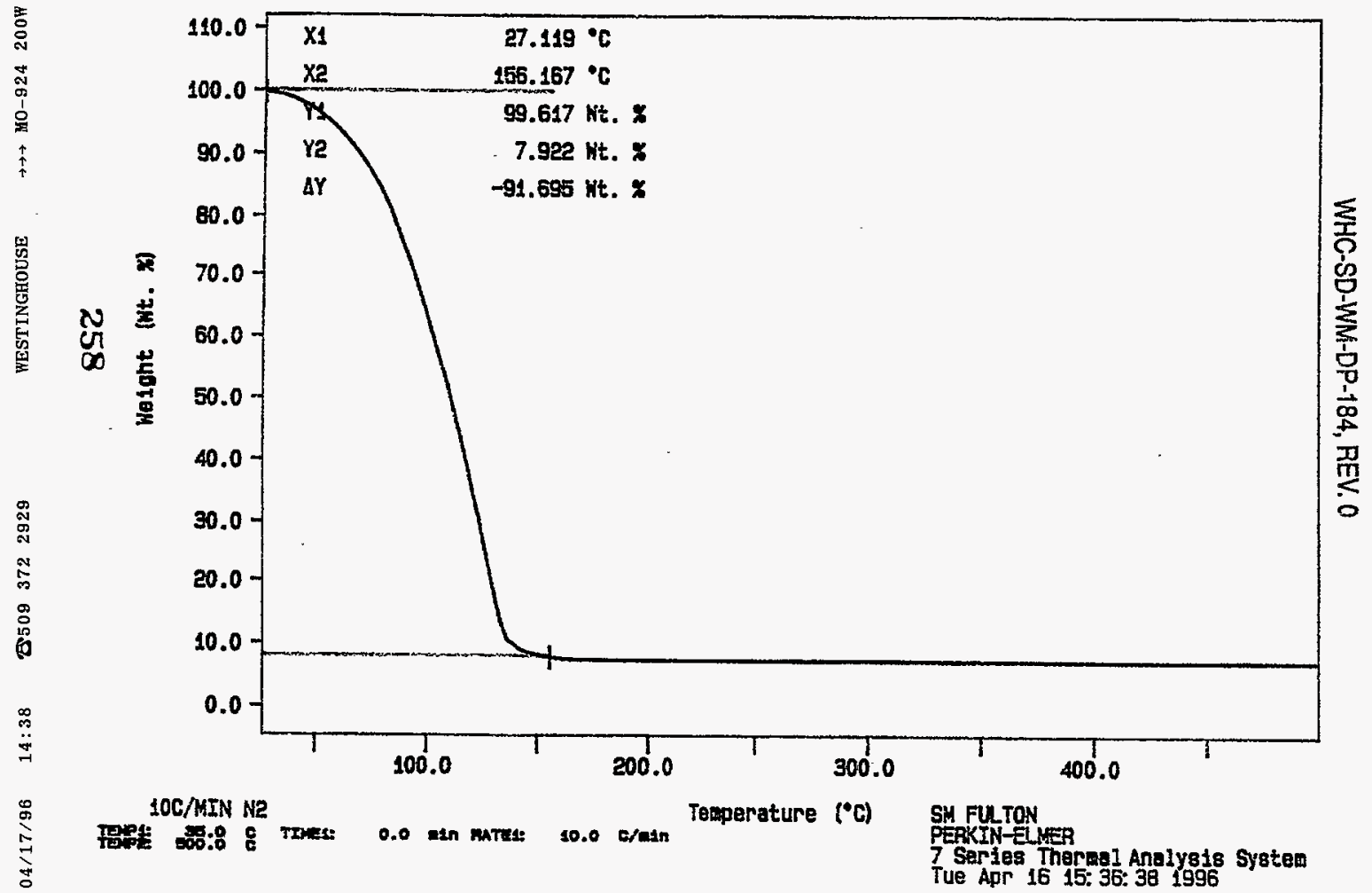


Curve 1: TEA

F118 info: SAM041606 Tua Apr 16 16: 28: 231996

Sample Vetght: 10.279

ag

S96T001121 DUP

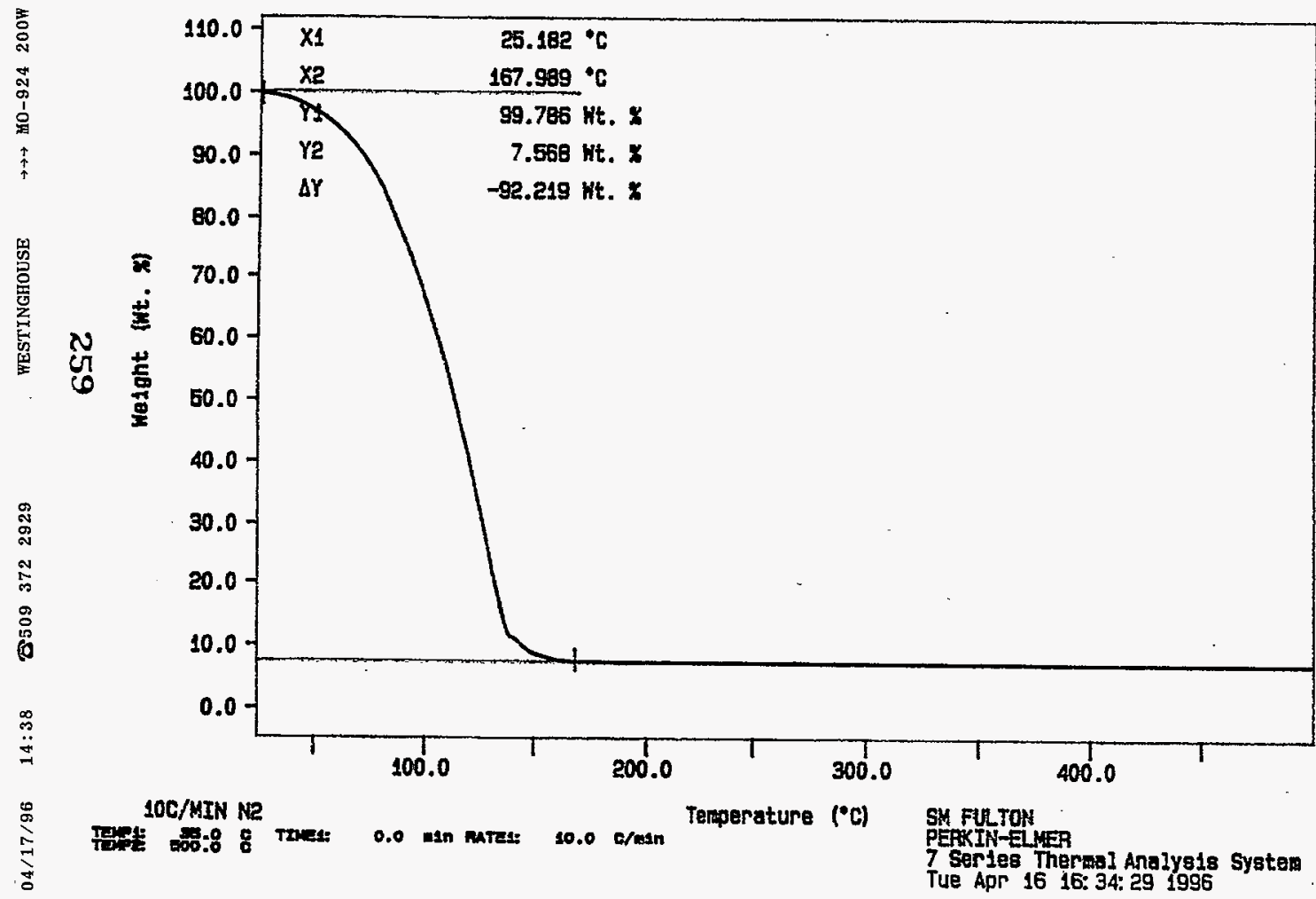




\section{LABCORE Data Entry Template for Worklist\#}

Analyst: SMF Instrument: TGA0 I Book\# 82N8A

Method: LA-560-112 Rev/Mod B-1

Worklist Comment: U-07 for tga-01 please run under N2 RTS!

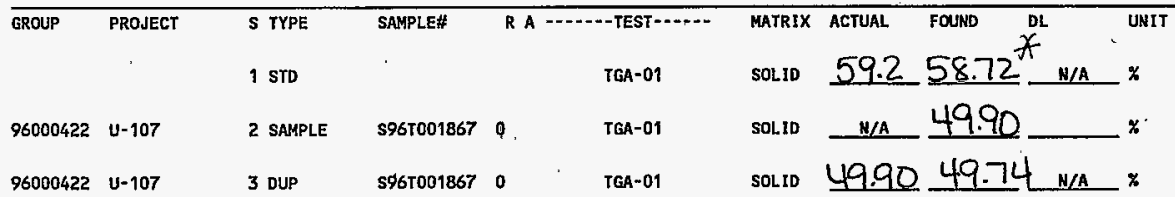

\section{Final page for worklist \#}

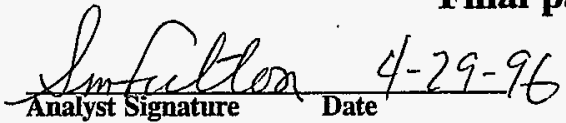

Verified by wanastos 5-6-96

Units shown for QC (SPK \& STD) may not reflect the actual units. $D L=$ Detection Limit, $S=$ Worklist Slot Number, $R=$ Replicate Number, $A=$ Aliquot Code. 


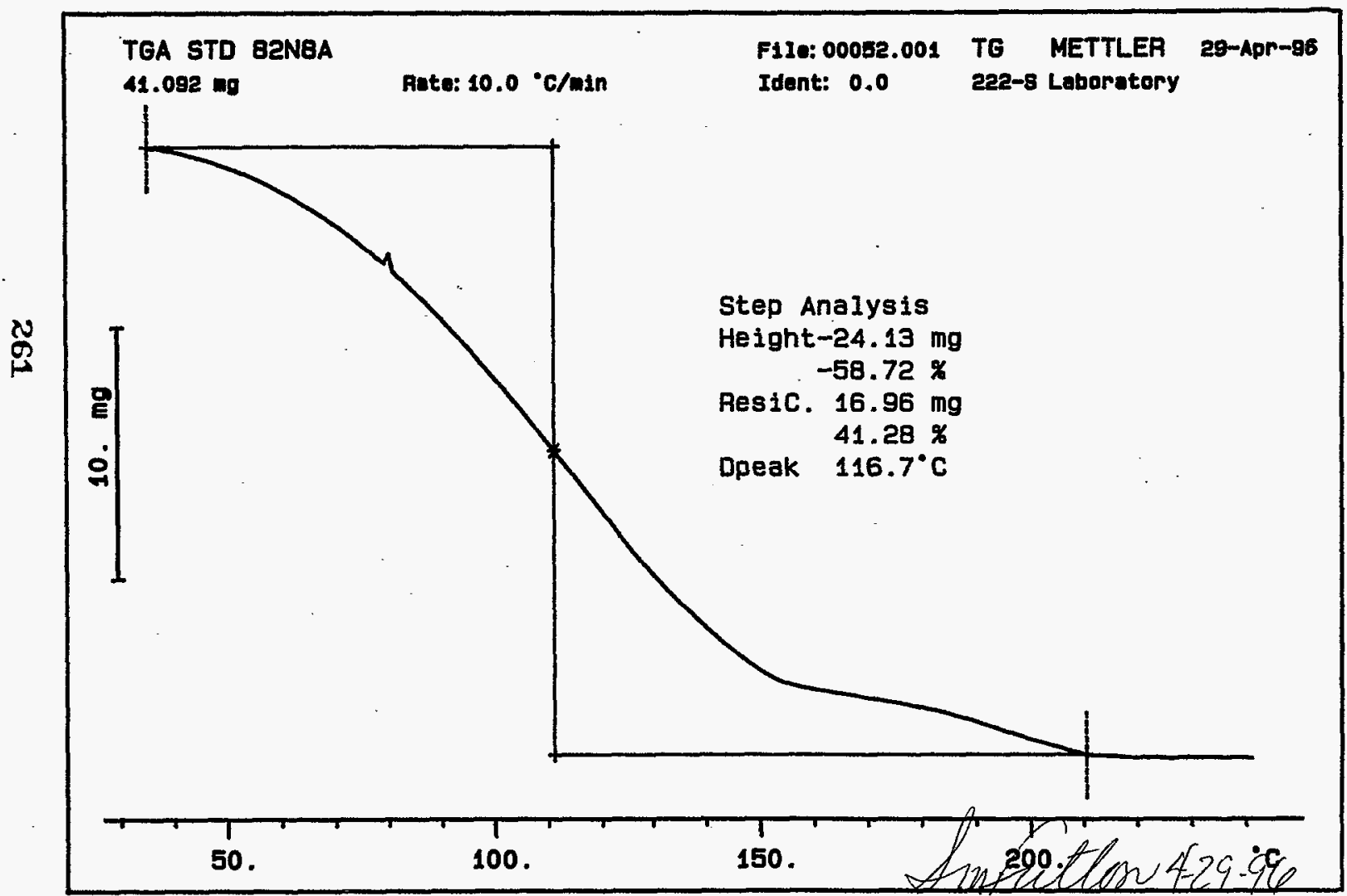

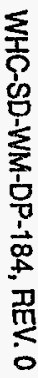

SIGNATURE ABOVE REPRESENTS CHEMICAL TECHNOLOGIST/CHEMIST THAT

SIGNATUR ABOTEFTER THF CAI TRRATINN/ANAI YSTS ON PAGES 26/TO $2 \% \zeta$. 


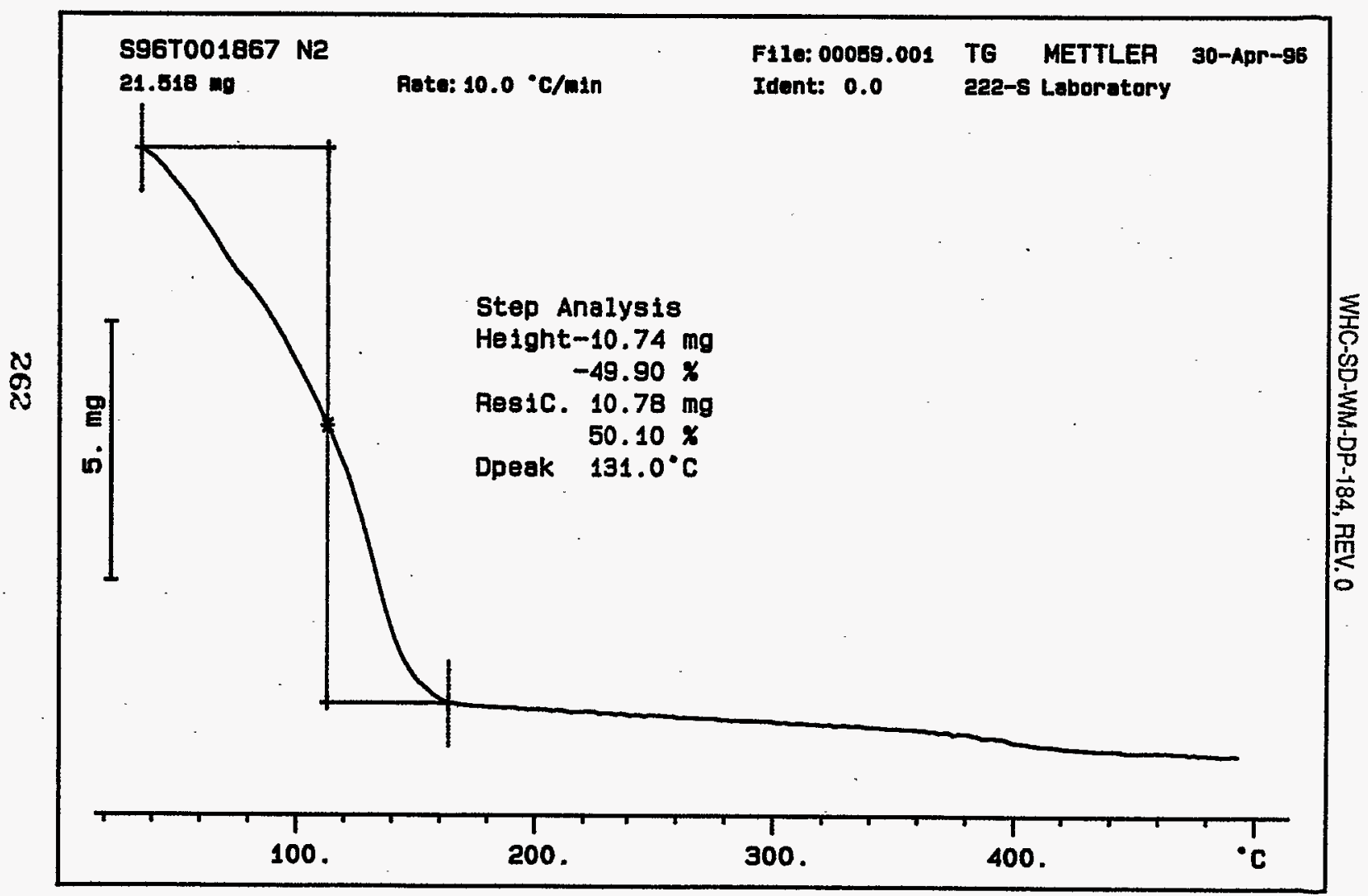




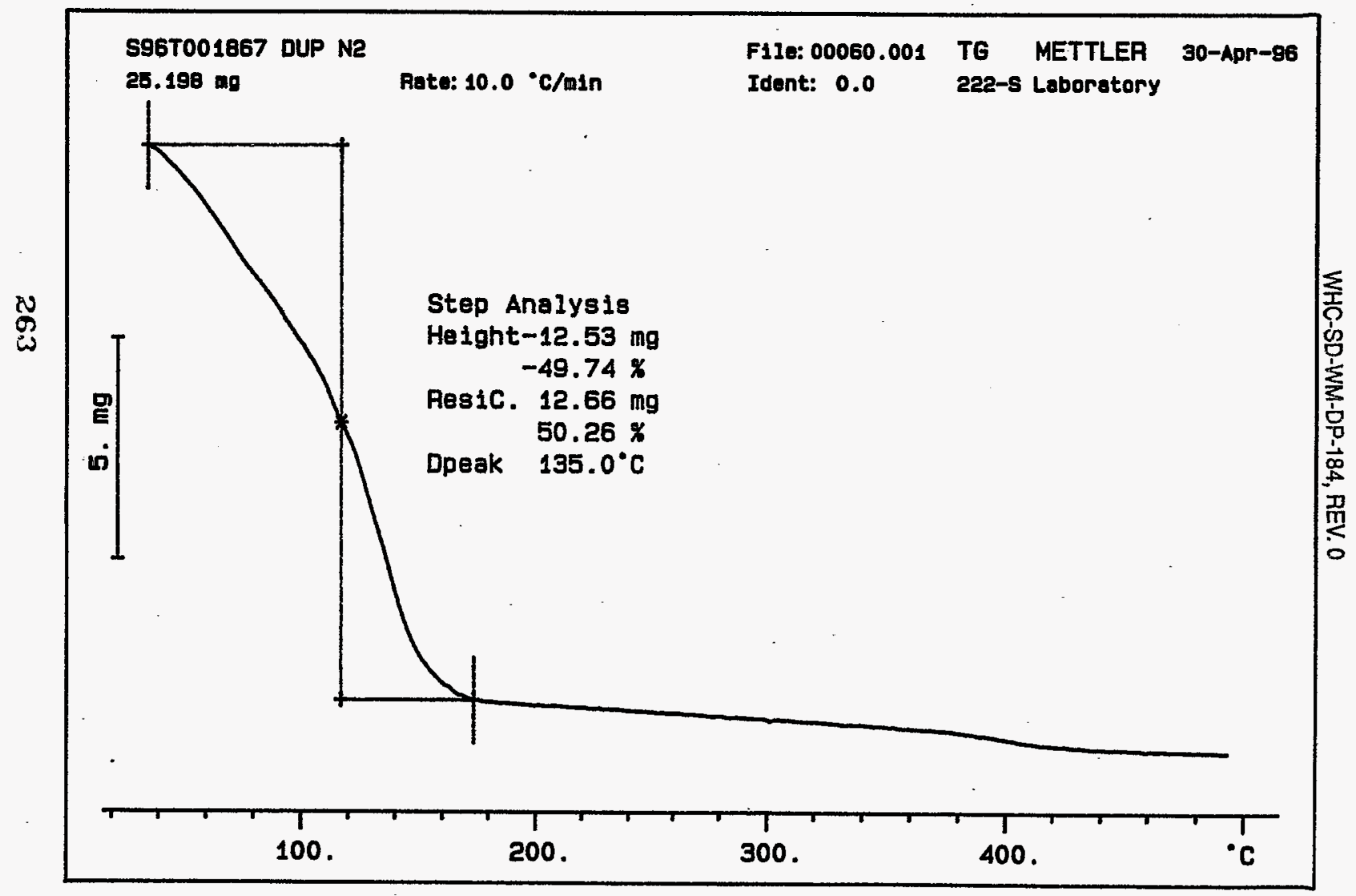




\section{LABCORE Data Entry Template for Worklist\#}

\section{Analyst: $\quad$ SMF Instrument: TGAO I_ Book \# 82N8-A}

Method: LA-560-112 Rev/Mod B-1

Worklist Comment: U-107 TGA RUN UNDER N2. RCJ

\begin{tabular}{|c|c|c|c|c|c|c|c|c|c|}
\hline GROUP & PRDJECT & $\begin{array}{l}\text { S TYPE } \\
1 \text { STD }\end{array}$ & SAMPLE\# & $R A$ & TGR-01 & $\begin{array}{l}\text { MATRIX } \\
\text { SOLID }\end{array}$ & $\begin{array}{l}\text { ACTUAL } \\
59.2 \\
\end{array}$ & $\begin{array}{l}\text { FOUND } \\
58.74 \\
\end{array}$ & $\begin{array}{l}D t \\
K_{N / A}\end{array}$ \\
\hline 96000422 & $\downarrow-107$ & 2 SAMPLE & $\$ 967001873$ & 0 & $T G A=01$ & SOLtD & $N / A$ & 4.67 & \\
\hline 96000422 & $U-107$ & 3 DUP & \$967001873 & 0 & TGA-01 & SOLID & & 4.15 & $N / A$ \\
\hline
\end{tabular}

\section{Final page for worklist \# 8037}
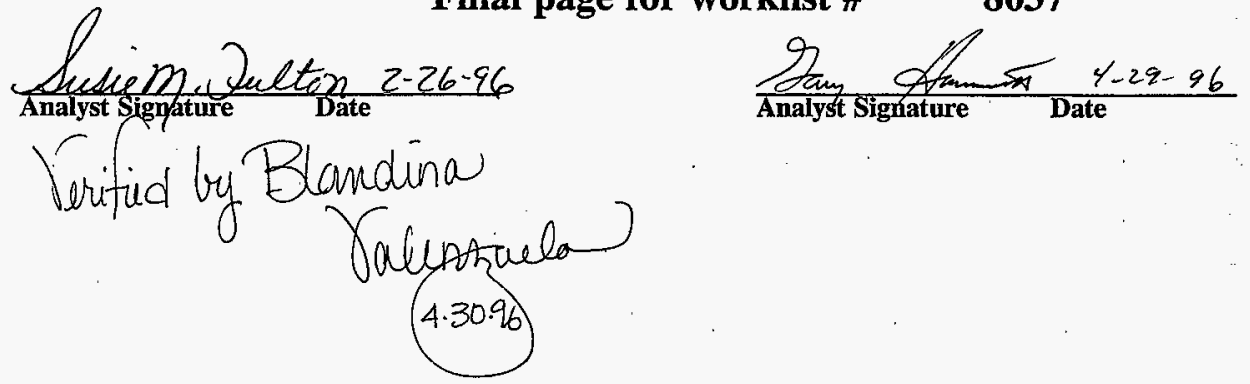

Units shown for QC (SPK \& STD) may not reflect the actual units. DL = Detection Limit, $S=$ Worklist Slot Number, $R=$ Replicate Number, $A=$ Aliquot Code.

$100-100 / 22: 21-62-70-96 / \quad 264=-/=-$


SIGNATURE BELOW REPRESENTS CHEMICAL TECHNOLOGIST/CHEMIST THAT

COMPLETED/VERIFIED THE CALIBRATION/ANALYSIS ON PAGES $\chi 45$ TO 247

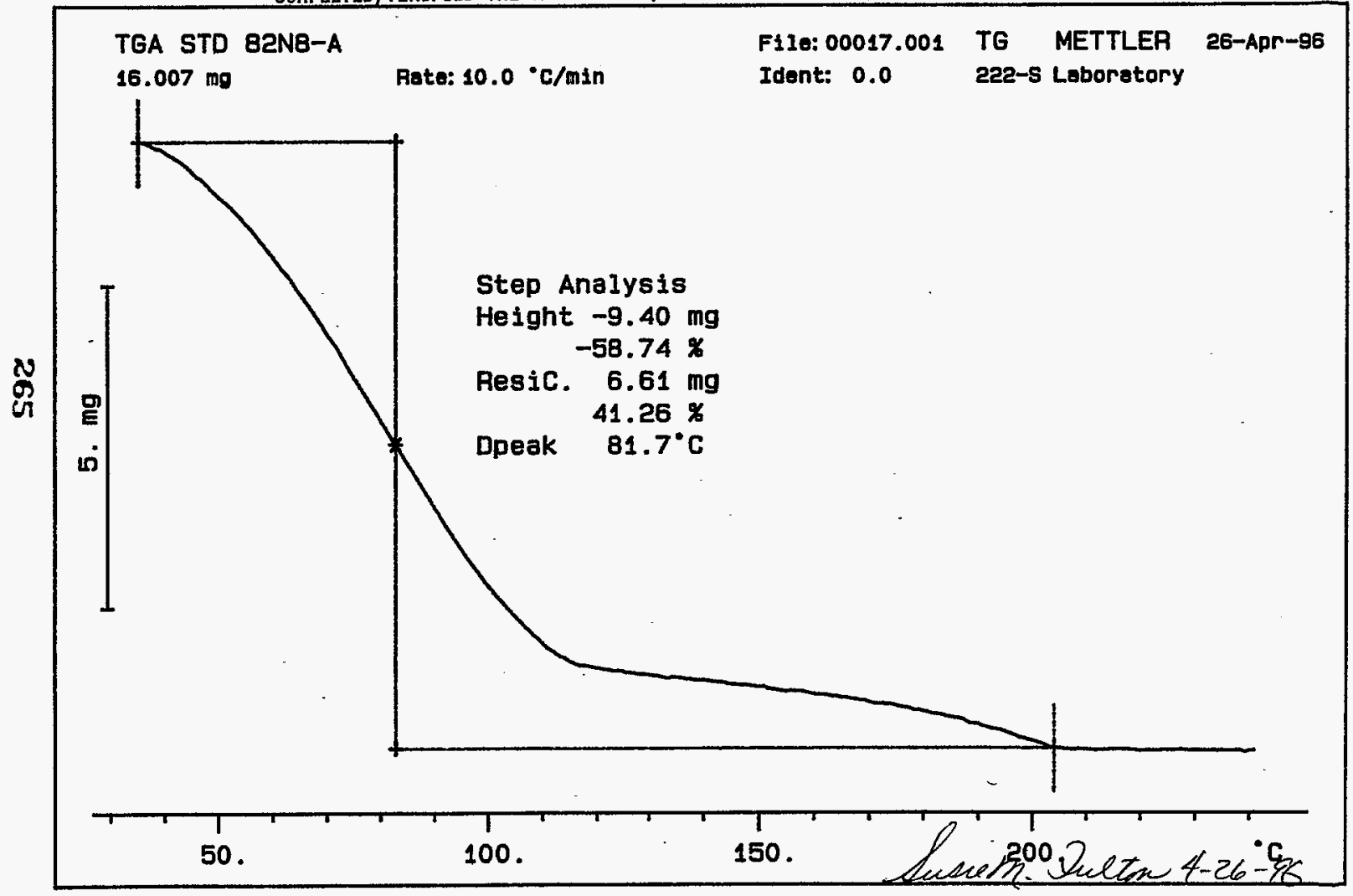




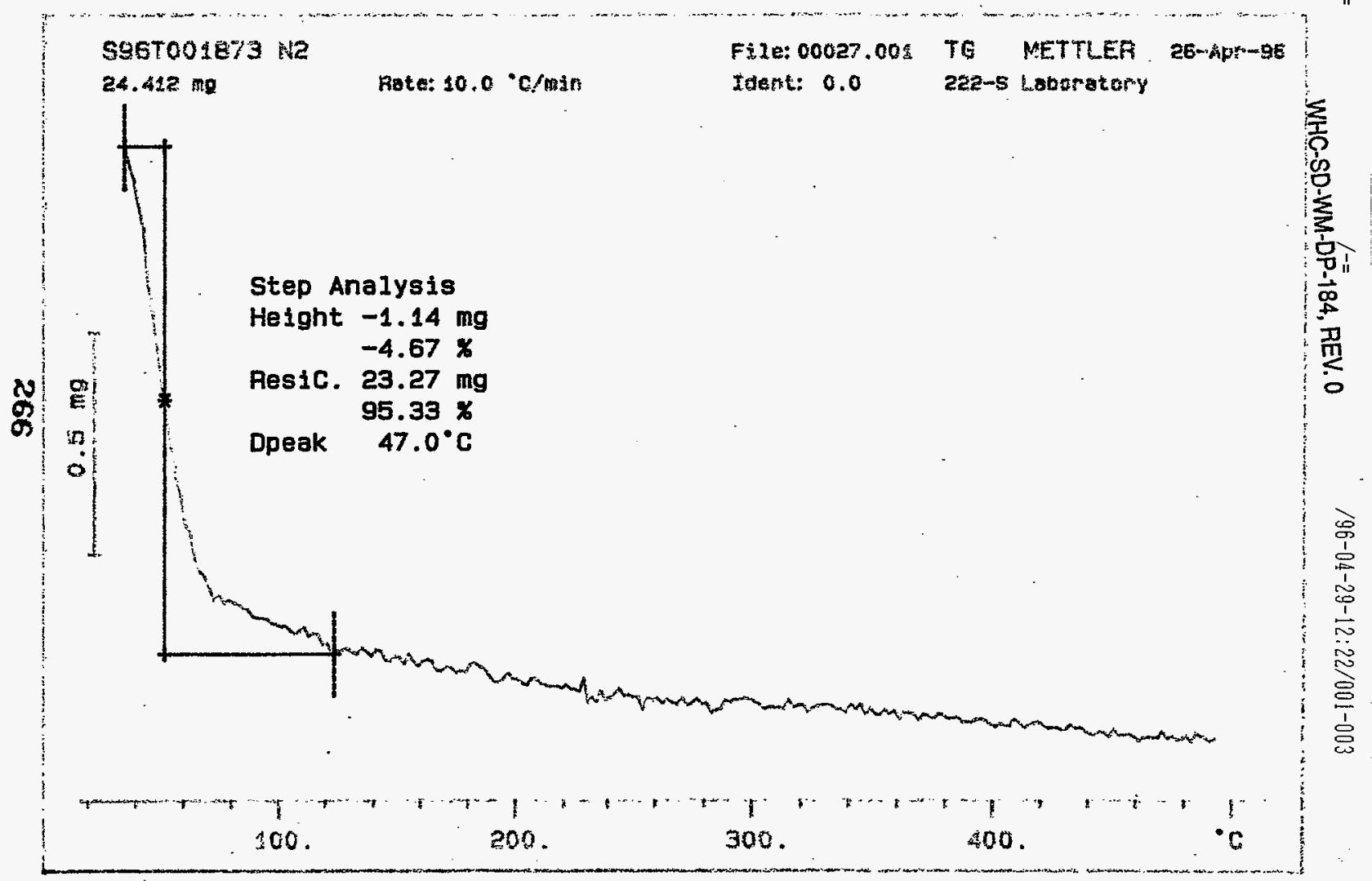




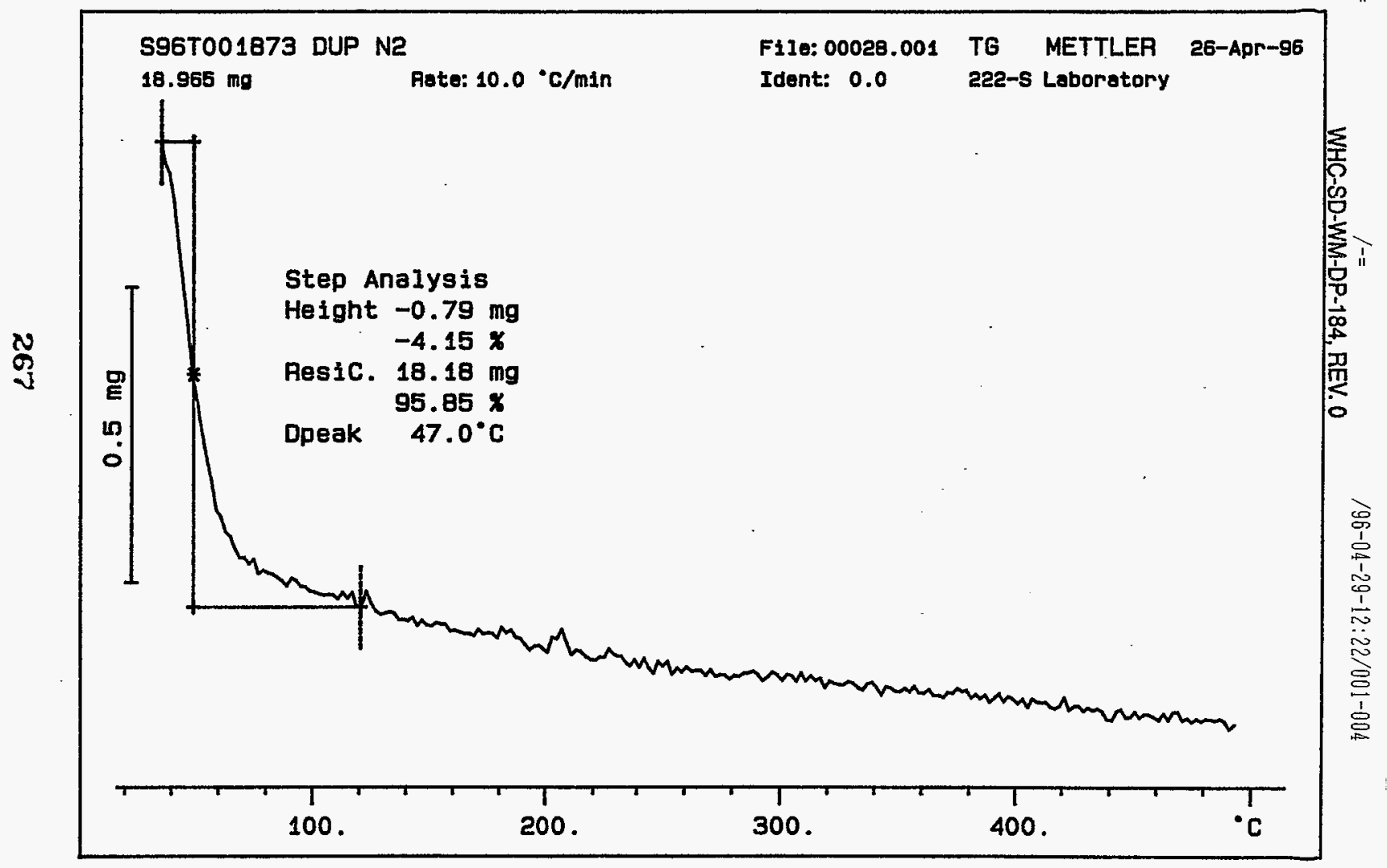


worklistrpt Version 2.1 05/15/95

WHC-SD-WM-DP-184, REV. 0

05/01/96 13:58

LABCORE Data Entry Template for Worklist\#

Page: $\quad I$

8223

Analyst: Jd s Instrument: TGA0 I Book \# $82 \times 18 \mathrm{~A}$

Method: LA-560-112 Rev/Mod B-1

Worklist Comment: U-107. Run under nitrogen. new

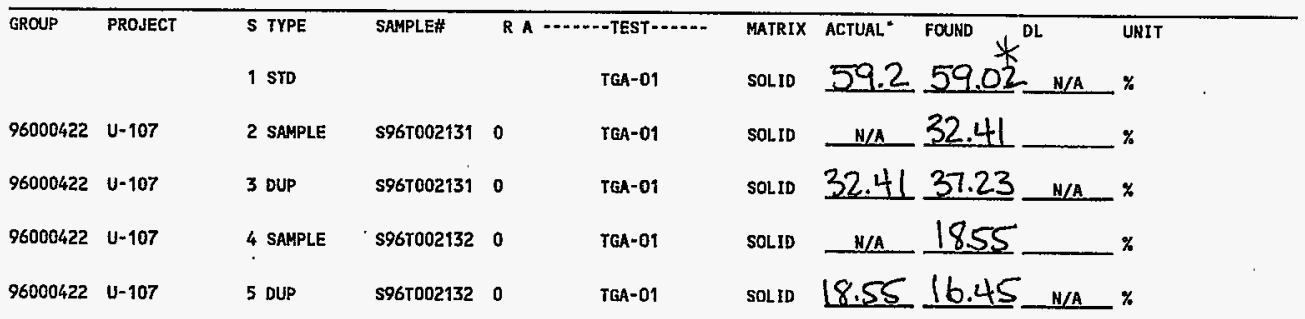

Final page for worklist \#

$\frac{\text { Sal Refire }}{\text { Analyst Signature }} 5-1-96$

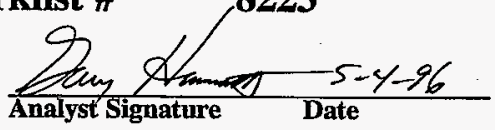

Verified by Manastor

$5 \cdot 6 \cdot 96$

S96T002131 also produced a second weight loss step of 2.16\%. However, the dup only showed one weight loss step.

S96T002132 results ane the sum of three weight loss steps $(8.78,1.55,+8.22 \%)$.

Data Entry Comments:

Units shown for QC (SPK \& STD) may not reflect the actual units. $D L=$ Detection Limit, $S=$ Worklist Slot Number, $R=$ Replicate Number, $A=$ Aliquot Code.

268 
SIGNATURE BELOW REPRESENTS CHEMICAL TECHNOLOGIST/CHEMIST THAT

COMPLETED/VERIFIED THE CALIBRATION/ANALYSIS ON PAGES $2 \angle \%$ TO 273 .

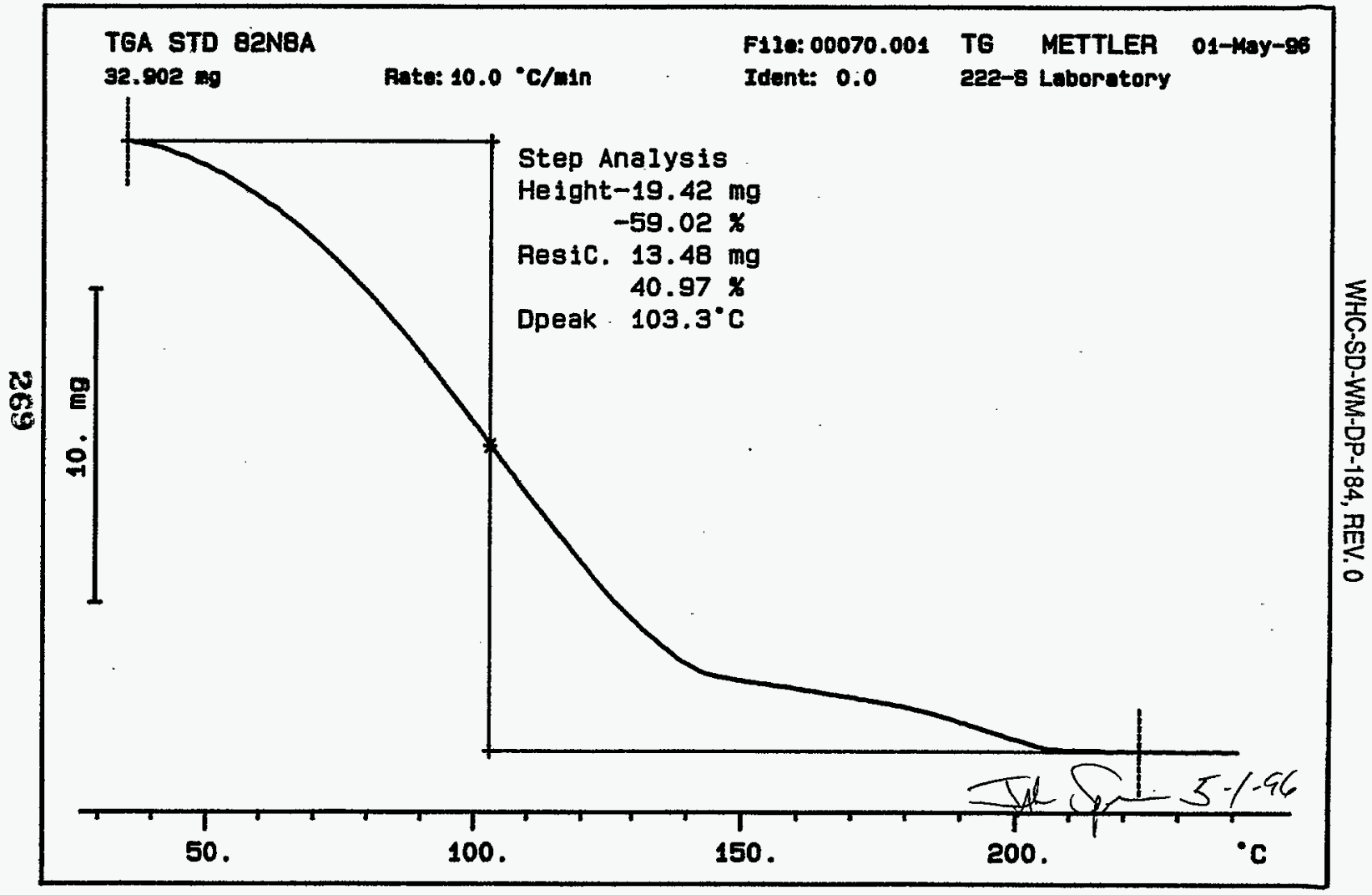




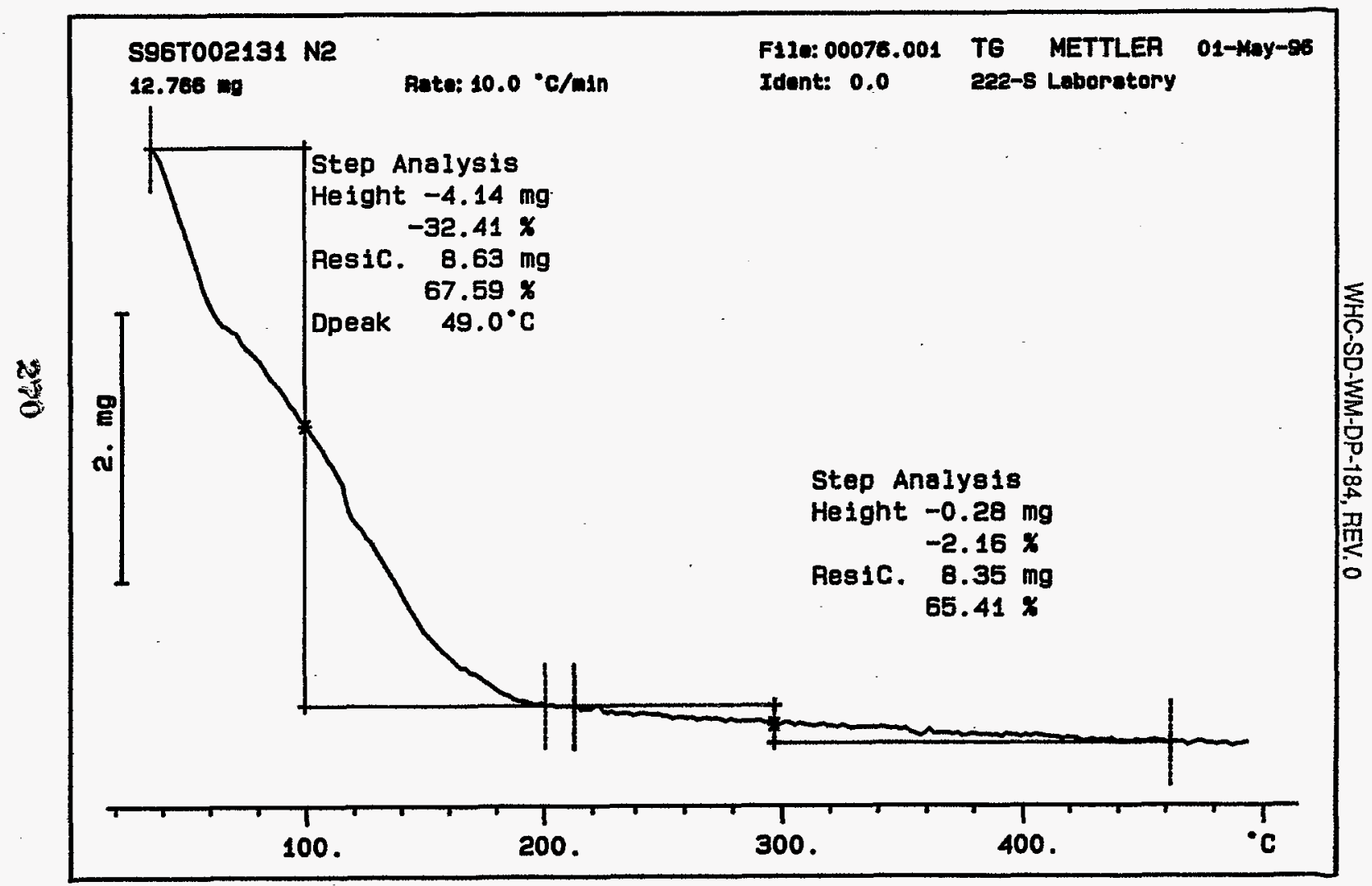




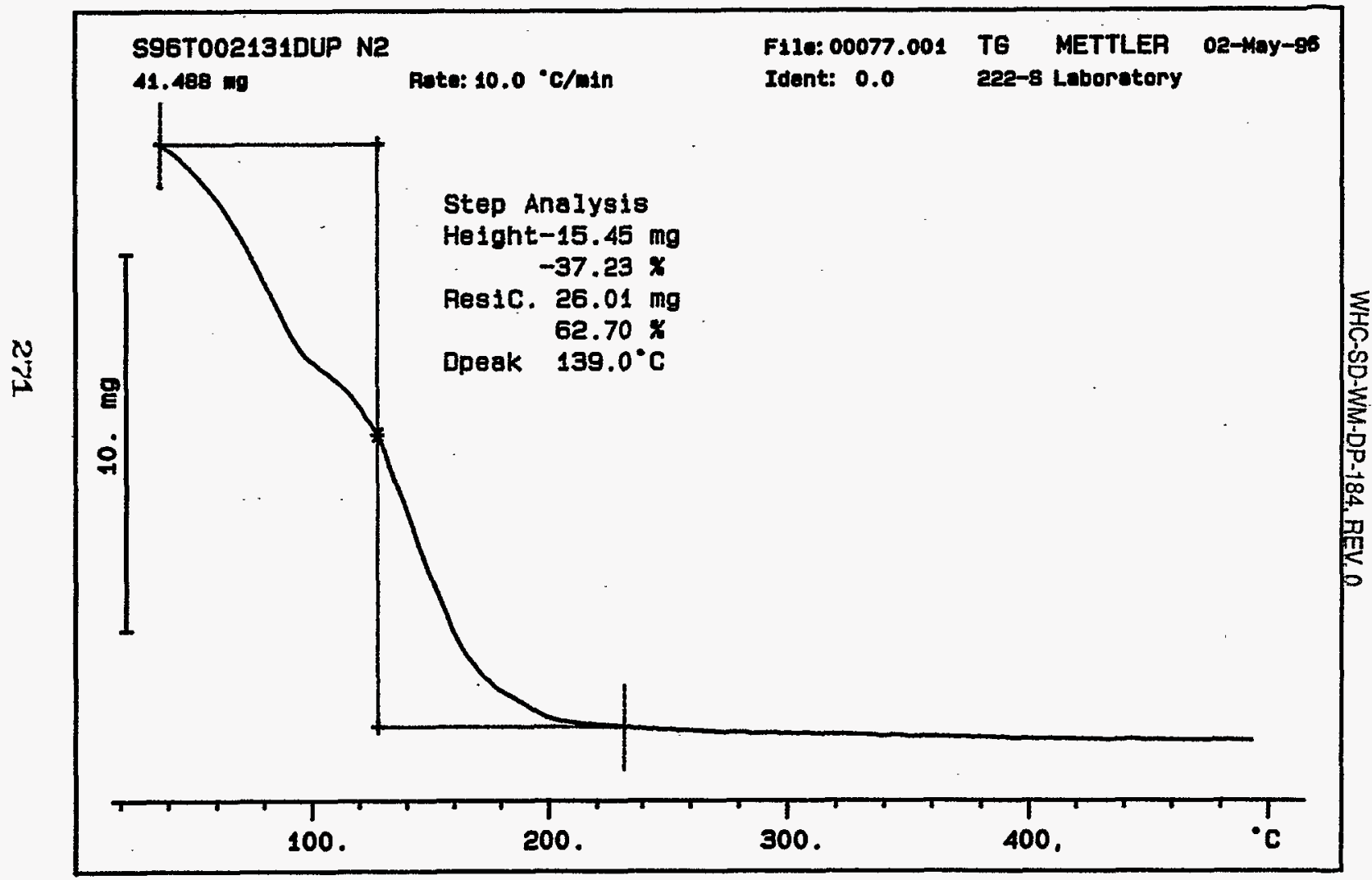




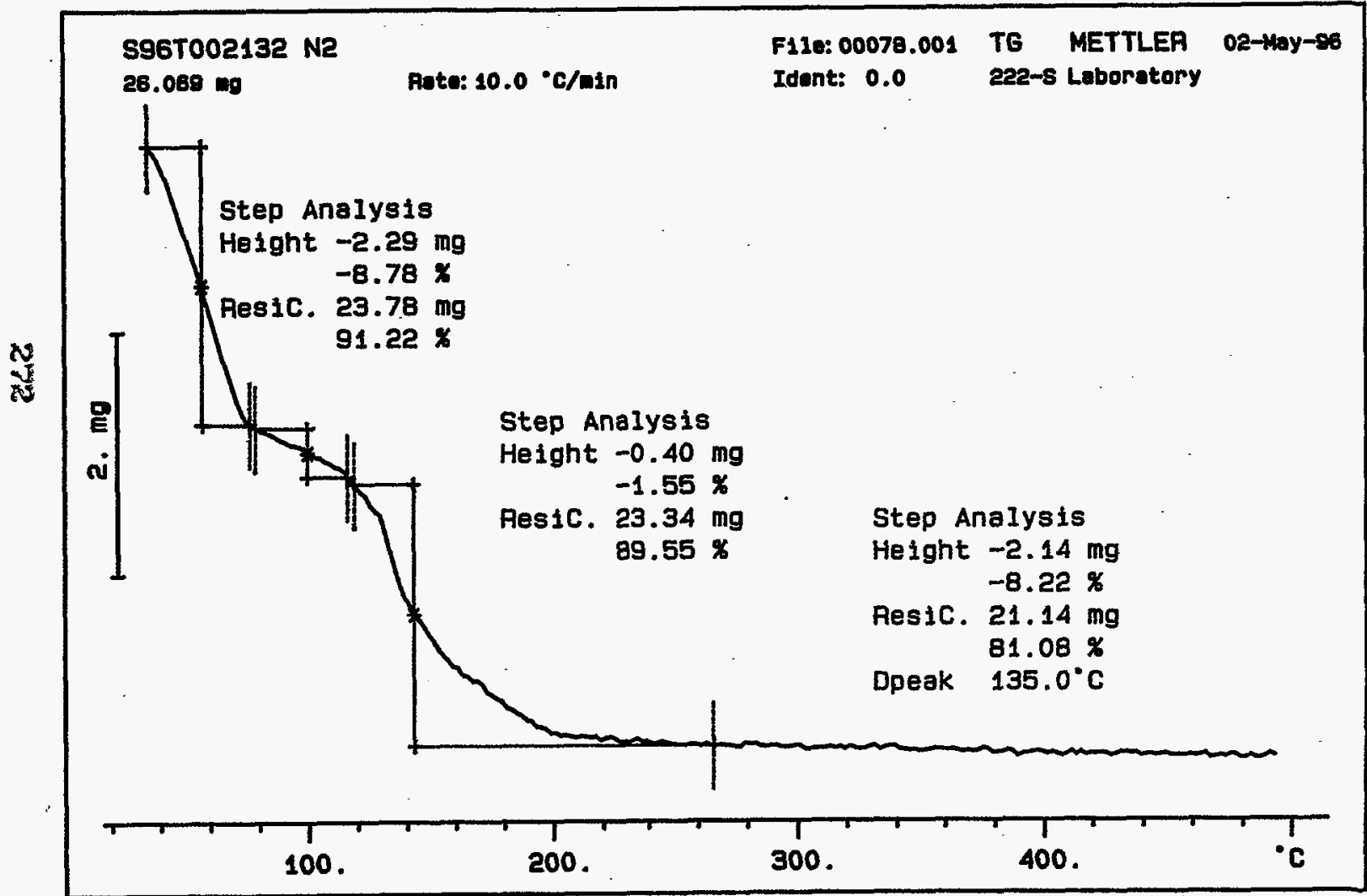




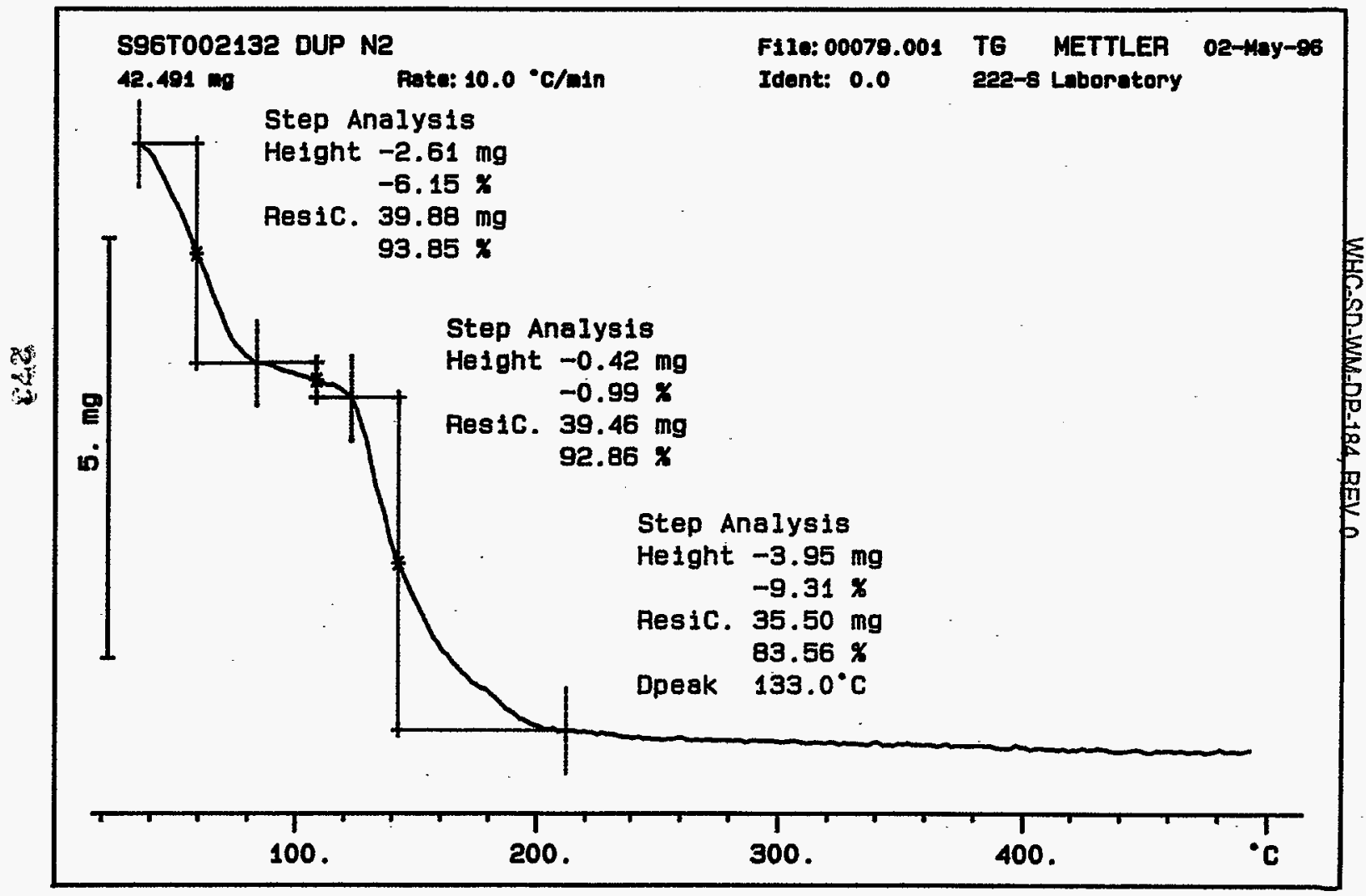




\section{LABCORE Data Entry Template for Worklist\#}

Analyst: $J d S$ Instrument: TGA0 3 Book\# $82 \mathrm{NSA}$

Method: LA-514-114 Rev/Mod C-1

Worklist Comment: U-107. Run under nitrogen. new

\begin{tabular}{|c|c|c|c|c|c|c|c|c|c|c|}
\hline GROUP & PROJECT & $\begin{array}{l}\text { S TYPE } \\
\text { I STD }\end{array}$ & SAMPLE\# & & TGA-03 & $\begin{array}{l}\text { MATRIX } \\
\text { SOLID }\end{array}$ & $\begin{array}{l}\text { ACTUAL } \\
59.2\end{array}$ & $\begin{array}{l}\text { FOUND } \\
58.5\end{array}$ & $*^{\mathrm{DL}}$ & $\begin{array}{l}\text { UNIT } \\
\%\end{array}$ \\
\hline 96000422 & $v-107$ & 2 SAMPLE & $596 \mathrm{~T} 001874$ & 0 & $T G A-03$ & SOL.TD & $H / A$ & 0.73 & & $\%$ \\
\hline 96000422 & U-107 & 3 DUP & \$967001874 & 0 & $T G A-03$ & SOLID & 0 & 0.97 & H/A & $\%$ \\
\hline
\end{tabular}

\section{Final page for worklist \#} See attached fn Signatures.

Verified by Hanaston 56-96

Sample showed quite a lit of moise and drift. Pue to the low level of moisture in it.

Data Entry Comments:

Units shown for QC (SPK \& STD) may not reflect the actual units. $D L=$ Detection Limit, $S=$ Worklist Slot Number, $R=$ Replicate Number, $A=$ Aliquot Code. 


Analyst: Jods Instrument: TGA0 Book\# 82 N/84

Method: LA-560-112 Rev/Mod

Worklist Comment: U-107. Run under nitrogen. new

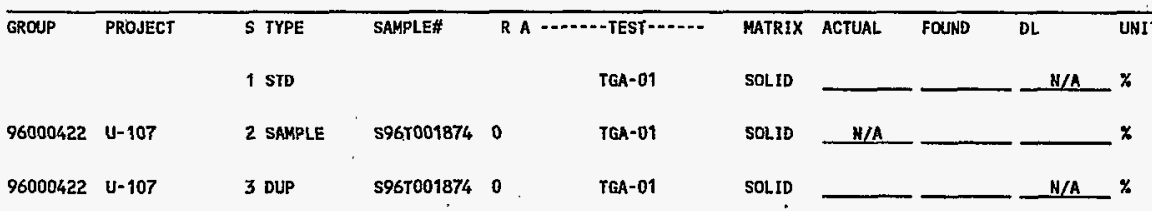

\section{Final page for worklist \# $\quad \mathbf{8 2 2 4}$}

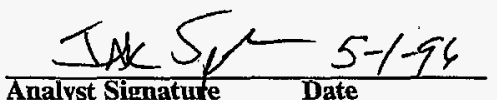

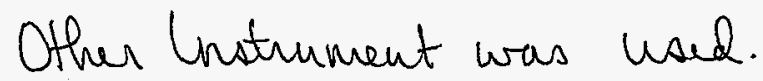

Data Entry Comments:

Units shown for $Q C$ (SPK \& STD) may not reflect the actual units. $D L=$ Detection Limit, $S=$ Warklist Slot Number, $R=$ Replicate Number, $A=$ Aliquot Code. 
Curve 1: TGA

File info: TER050101 hed May 1 08:59:10 1996

Sample Neight: 22.801 mg

TGA STD BENB-A

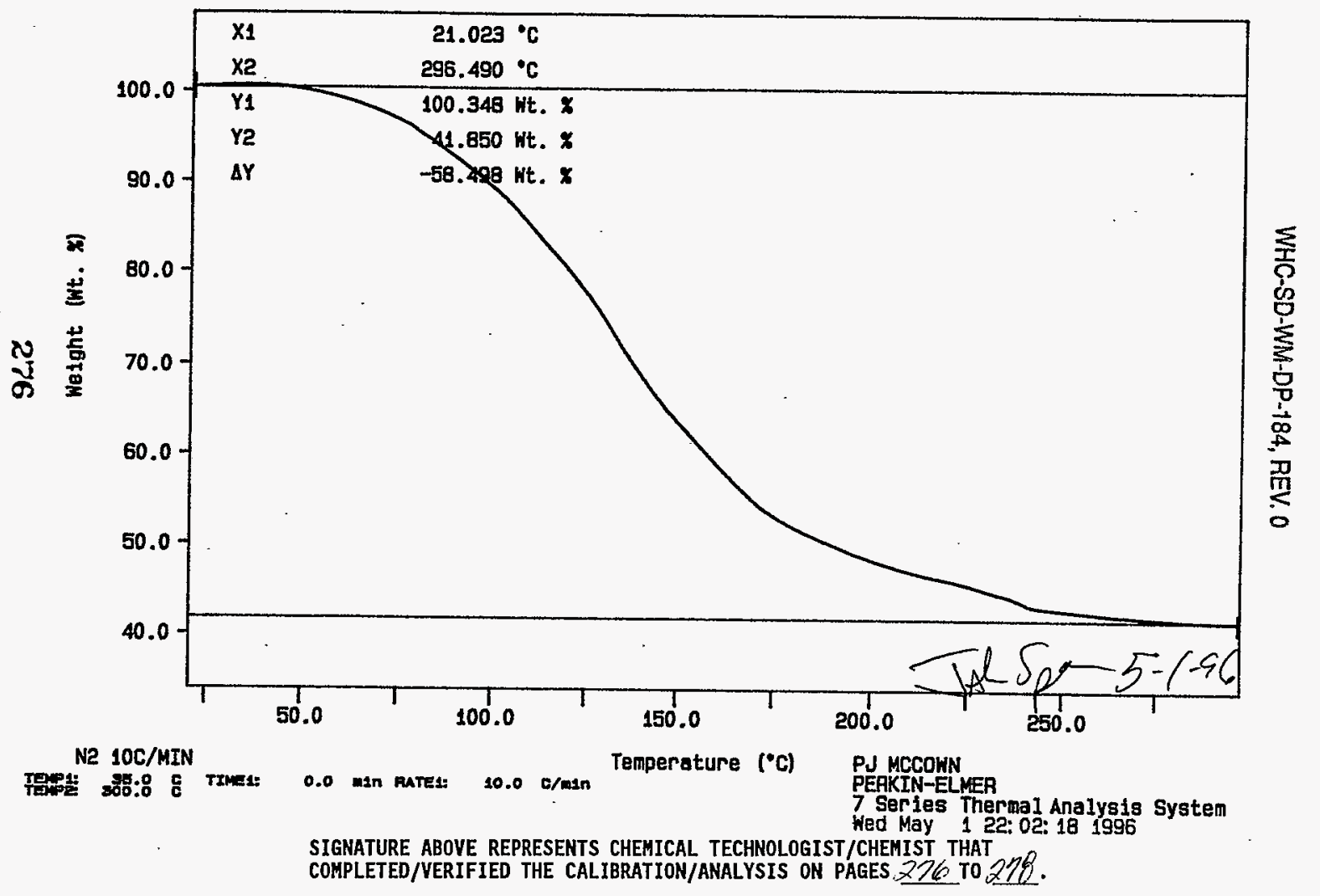


Curve 1: TGA

F1le info: SAM050108 Hed May 1 23:09:25 1996

Sample Weight: 23.471

S96T000835 1874 SAM

ing

$5 / 3 / 96 \mathrm{ov}$

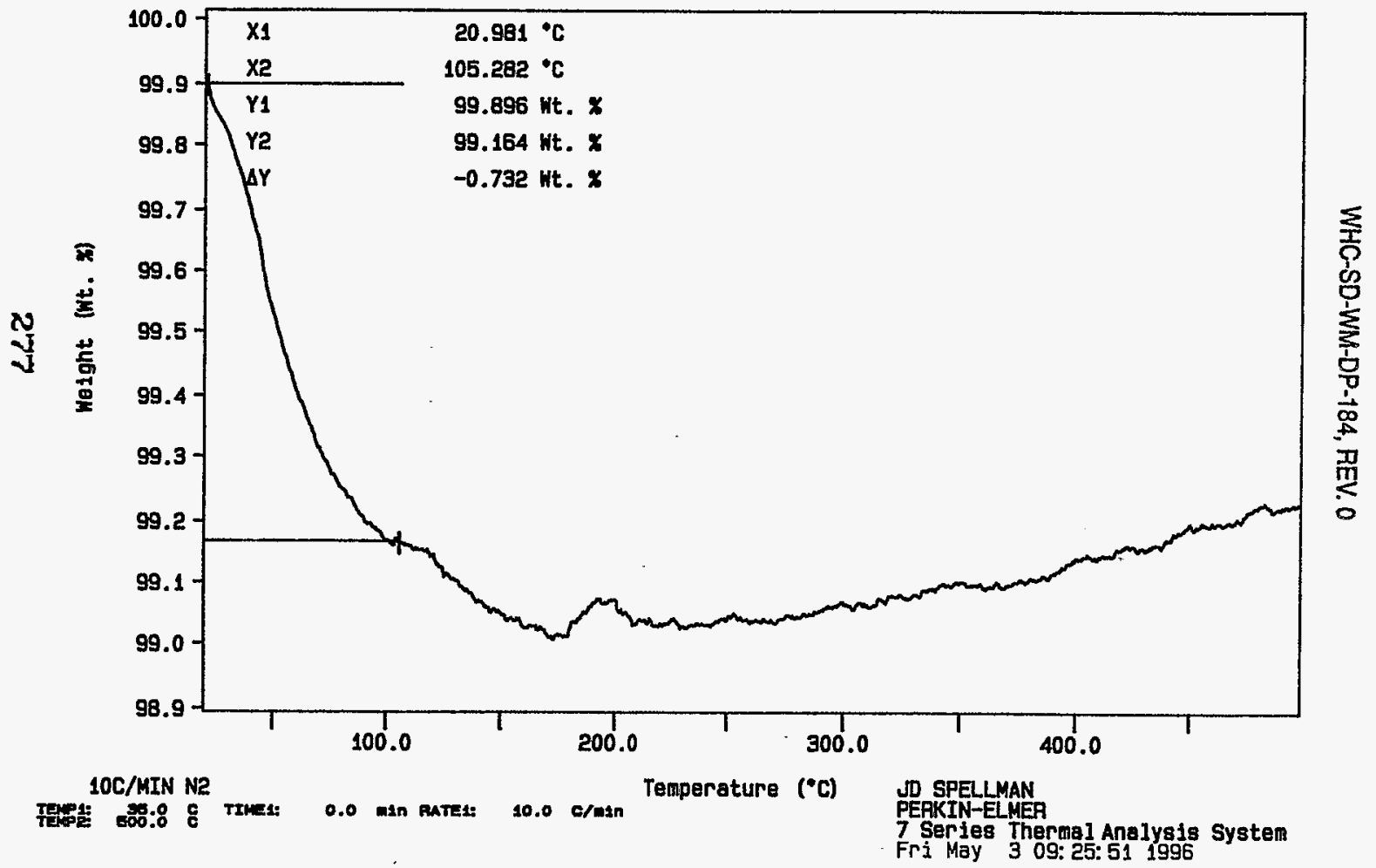




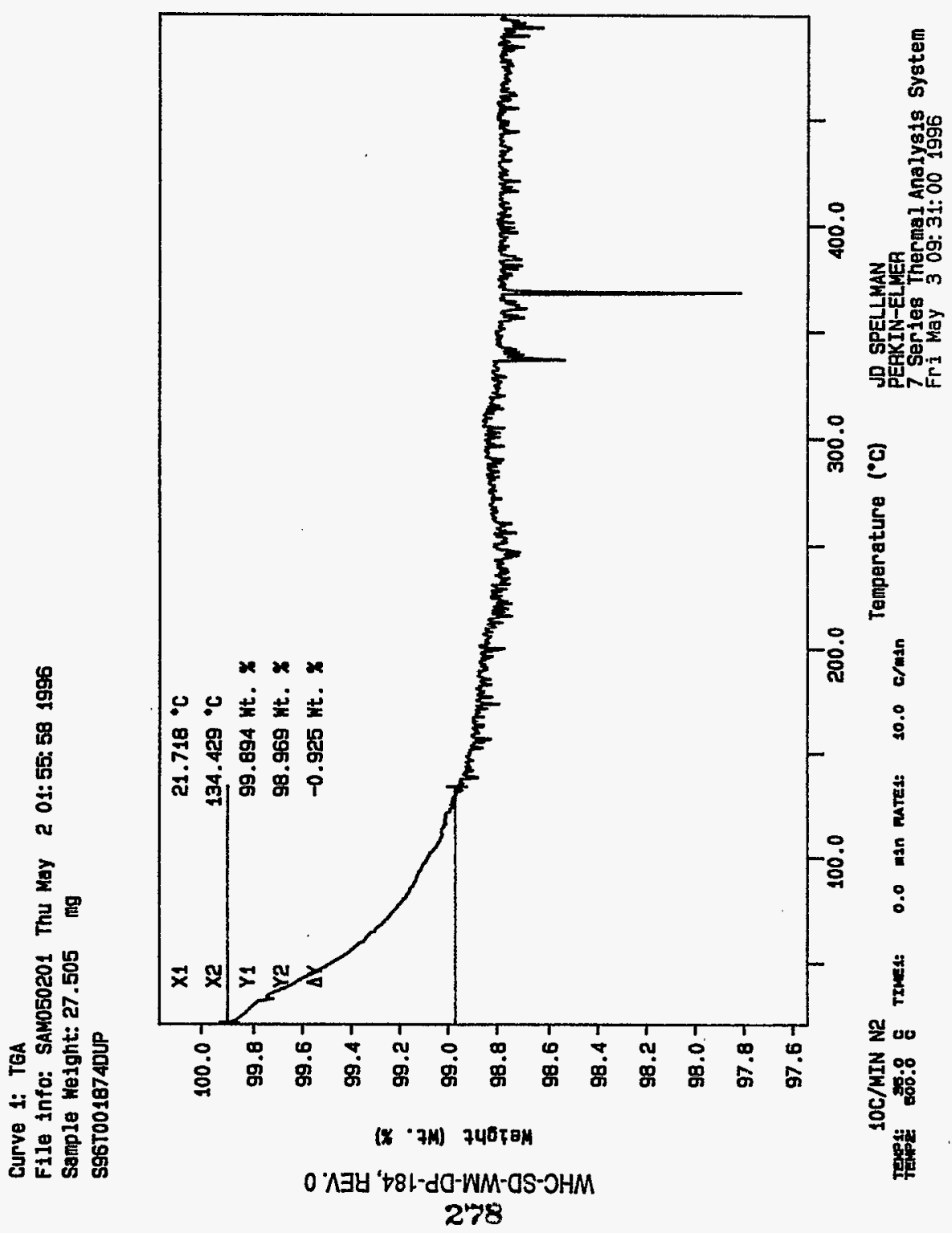


Analyst: Pinle Cocen Instrument: TGA0 I Book \#2008A

Method: LA-560-112 Rev/Mod B-/

Worklist Comment: U-107. Run under nitrogen. new

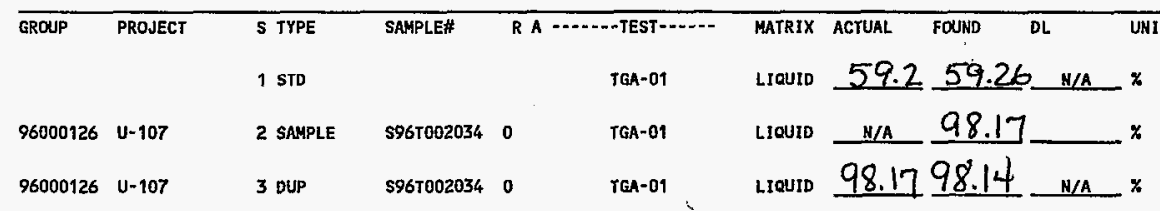

\section{Final page for worklist \#}

8226
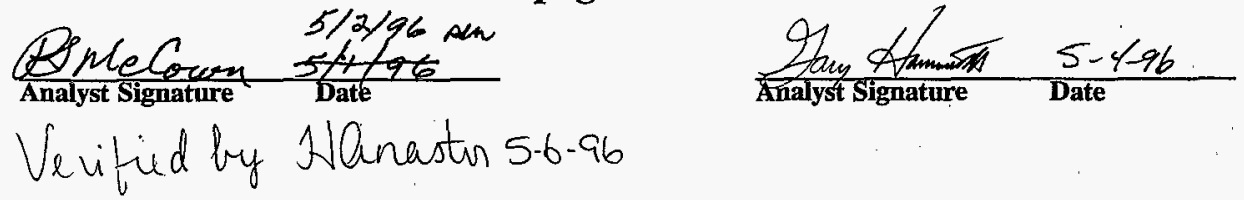

Data Entry Comments:

Units shown for QC (SPK \& STD) may not reflect the actual units. $D L=$ Detection Limit, $S=$ Worklist Slot Number, $R=$ Replicate Number, $A=$ Aliquot Code. 


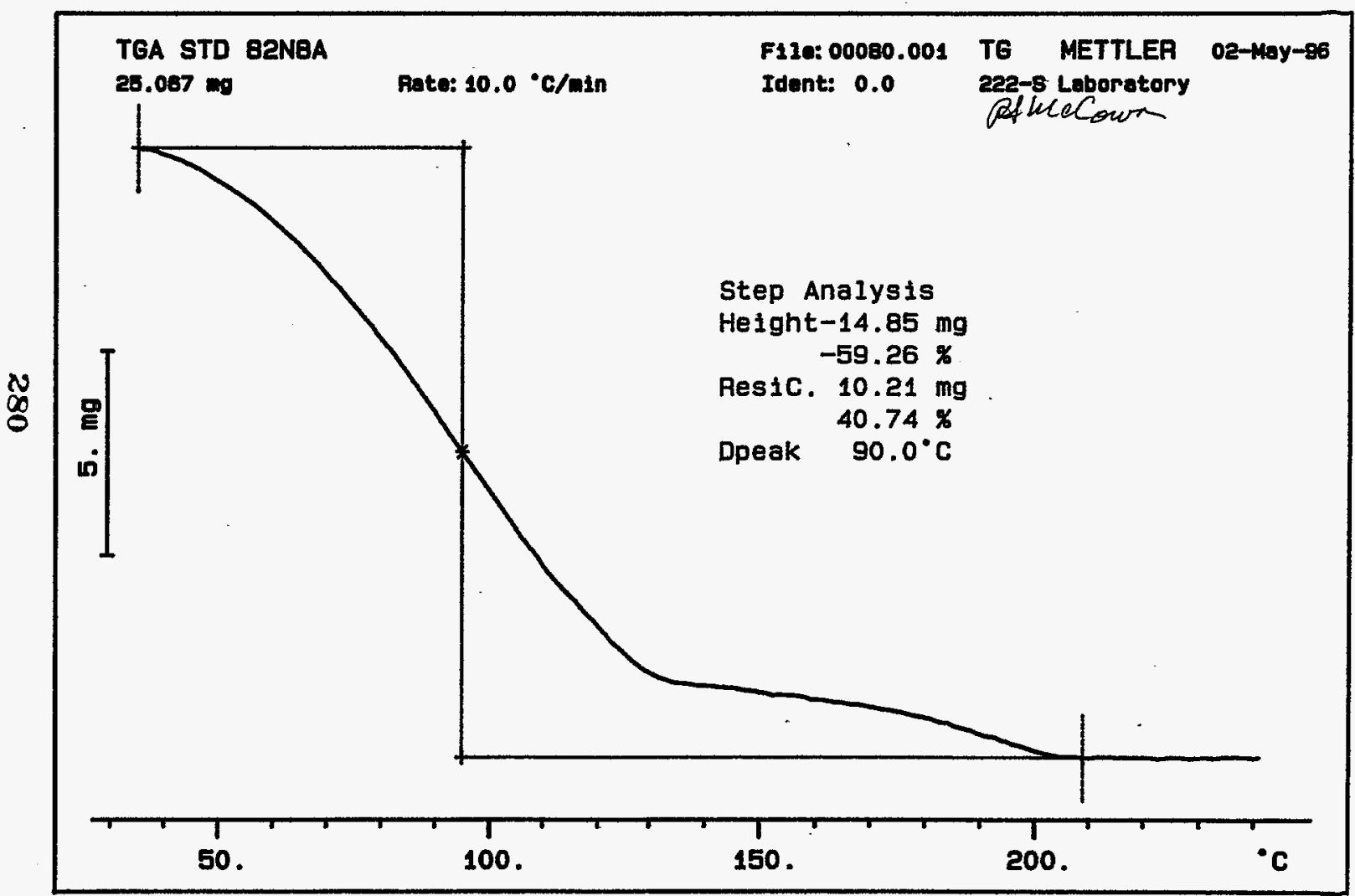




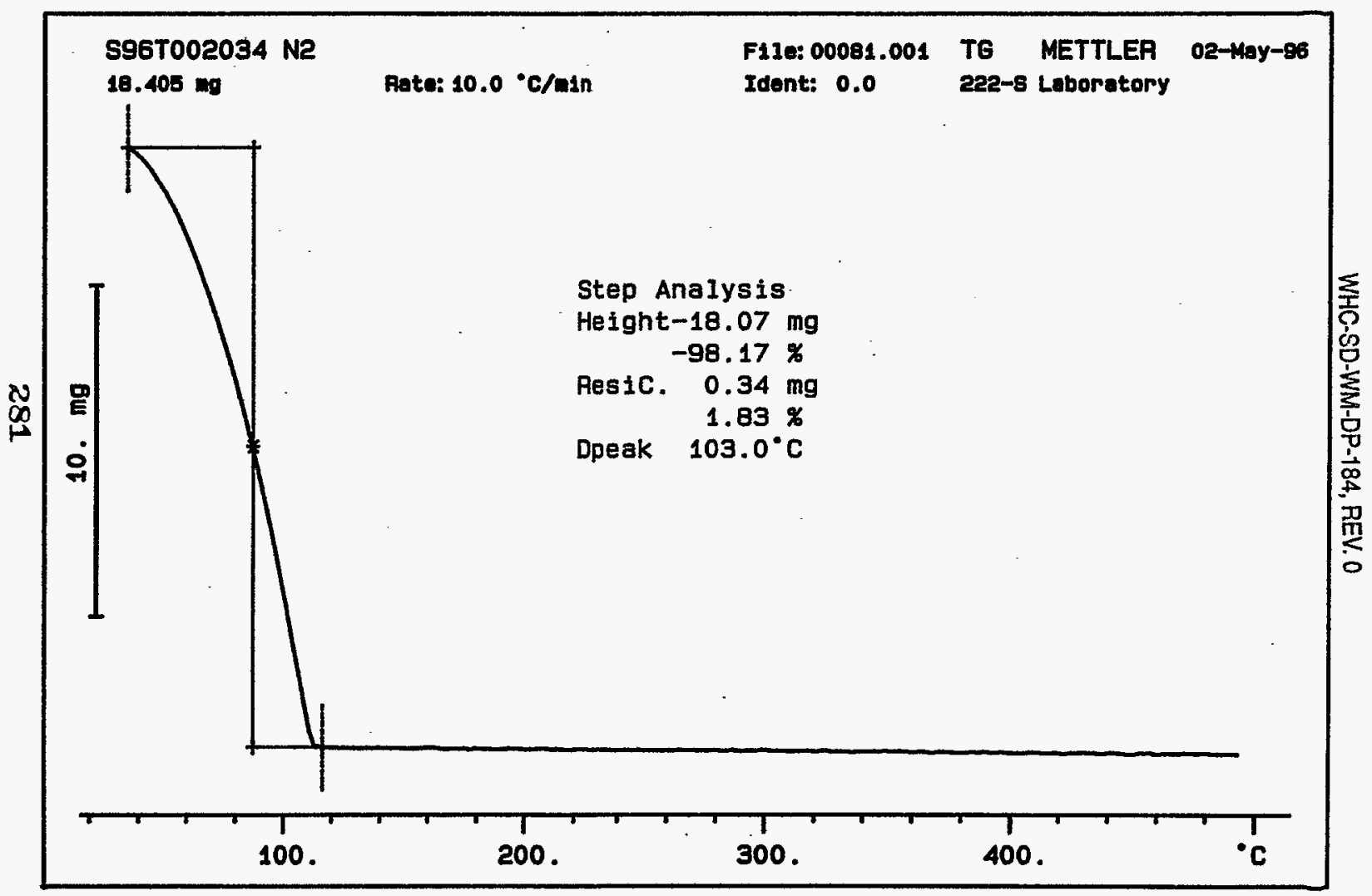




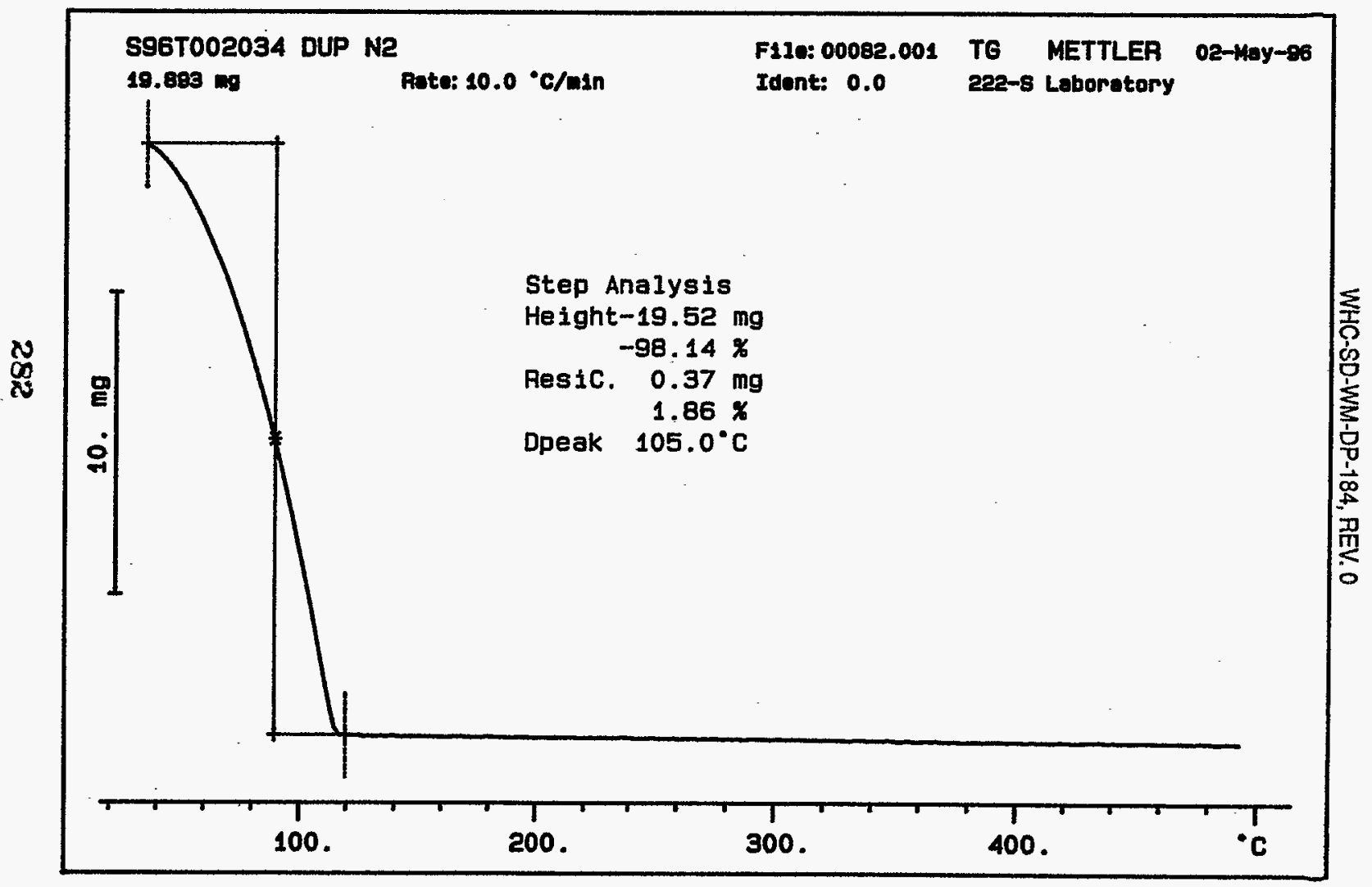


Analyst: $K R M$ Instrument: TGA0 1 Book\# \&2a \&.A

Method: LA-560-112 Rev/Mod B-/

Worklist Comment: U-107 TGA RUN UNDER N2. RCJ

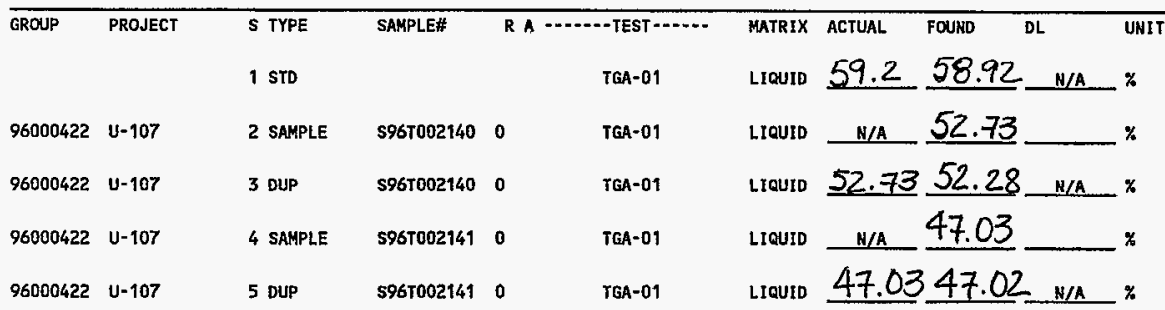

\section{Final page for worklist \#}

8434

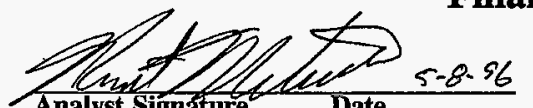

Analyst Signáture Date

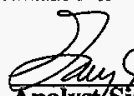

Analyst/Signature

Validated by Hanastos 5.14.96

Data Entry Comments:

Units shown for $Q C$ (SPK \& STD) may not reflect the actual units. $D L=$ Detection Limit, $S=$ Worklist Slot Number, $R=$ Replicate Number, $A=$ Aliquot Code. 
SIGNATURE BELOW REPRESENTS CHEMICAL TECHNOLOGIST/CHEMIST THAT

COMPLETED/VERIFIED THE CALIBRATION/ANALYSIS ON PAGES ZE\&TO 28.

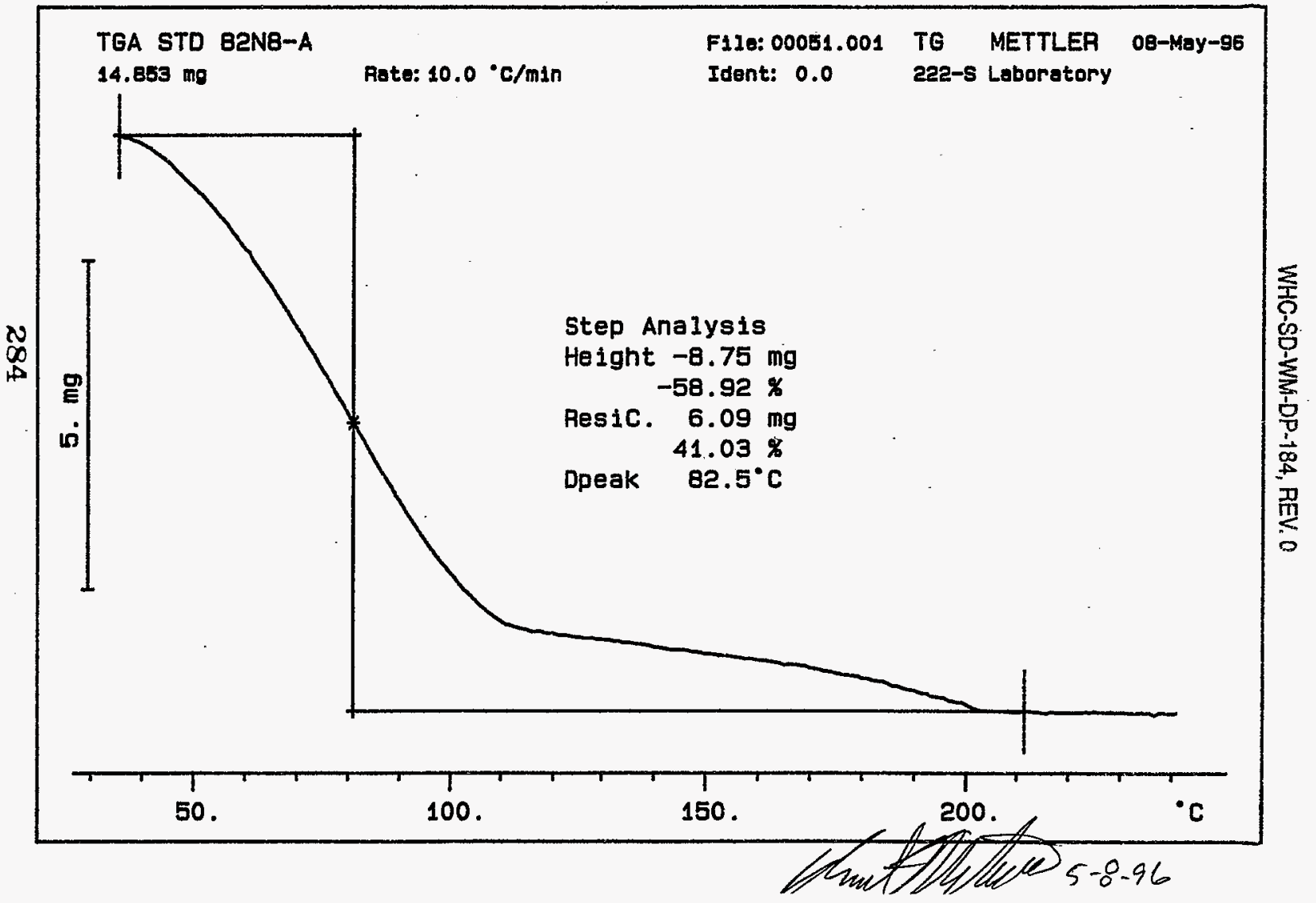




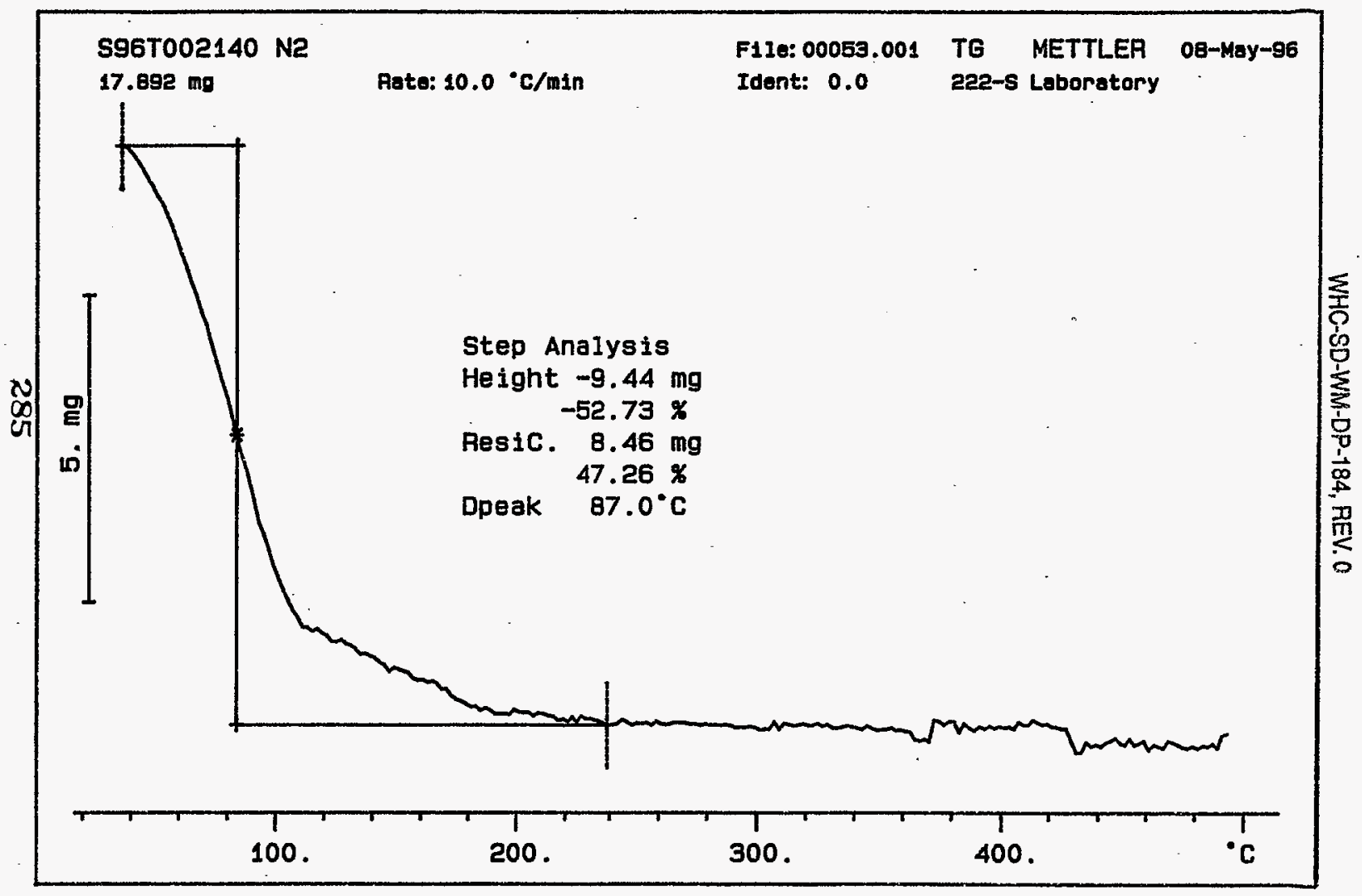



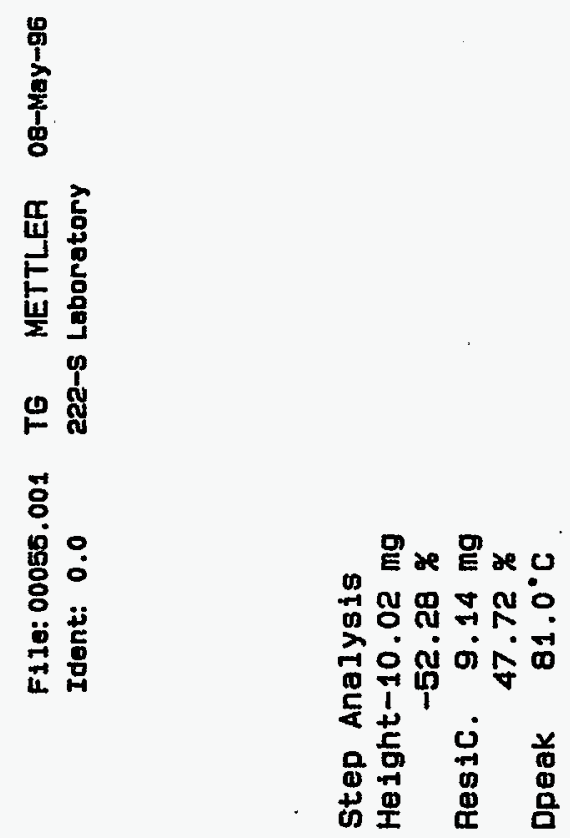

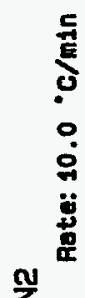

몽

웅

:

$\rightarrow$

용

요

of

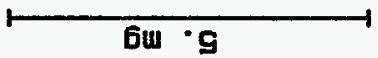




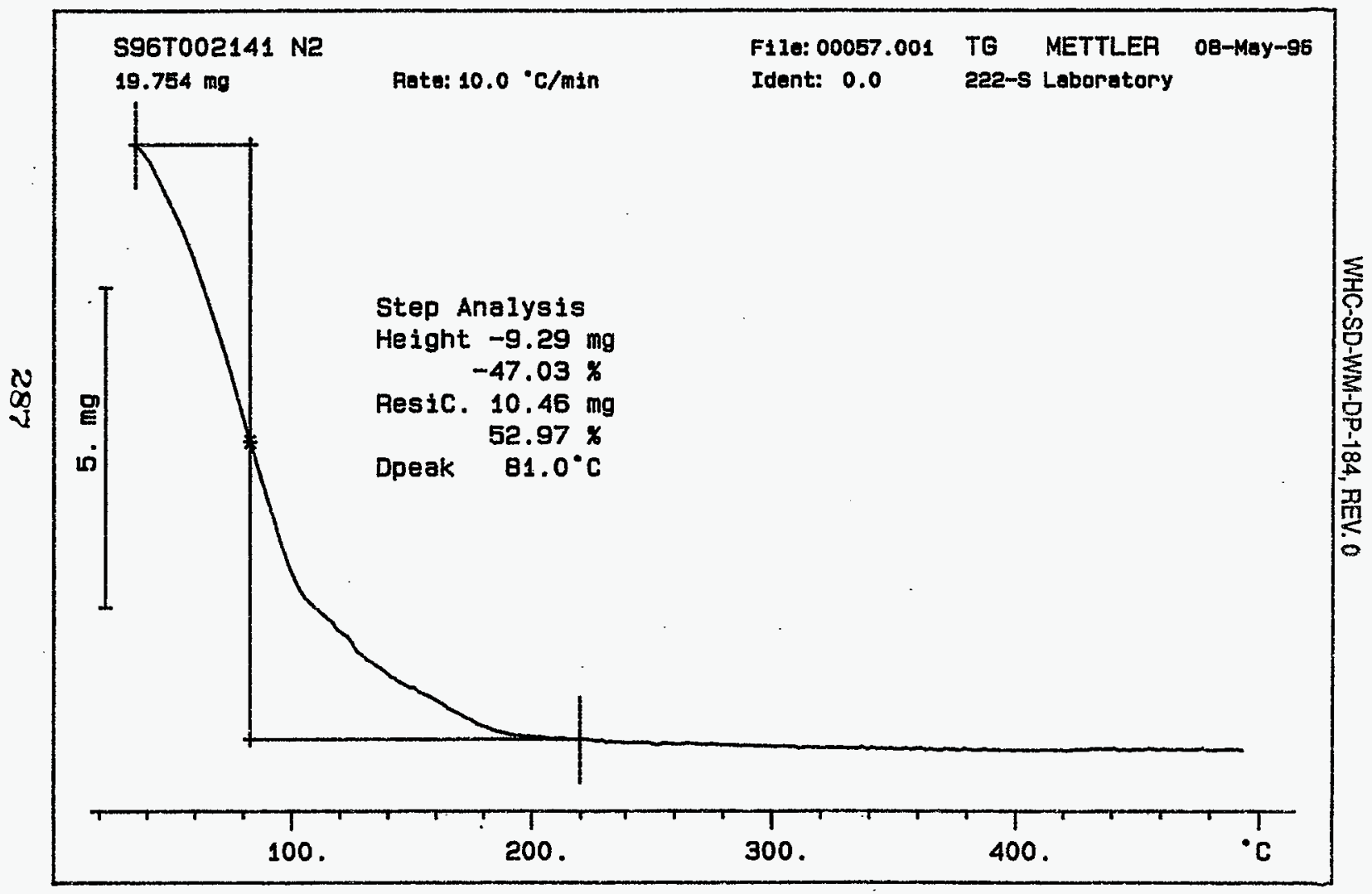




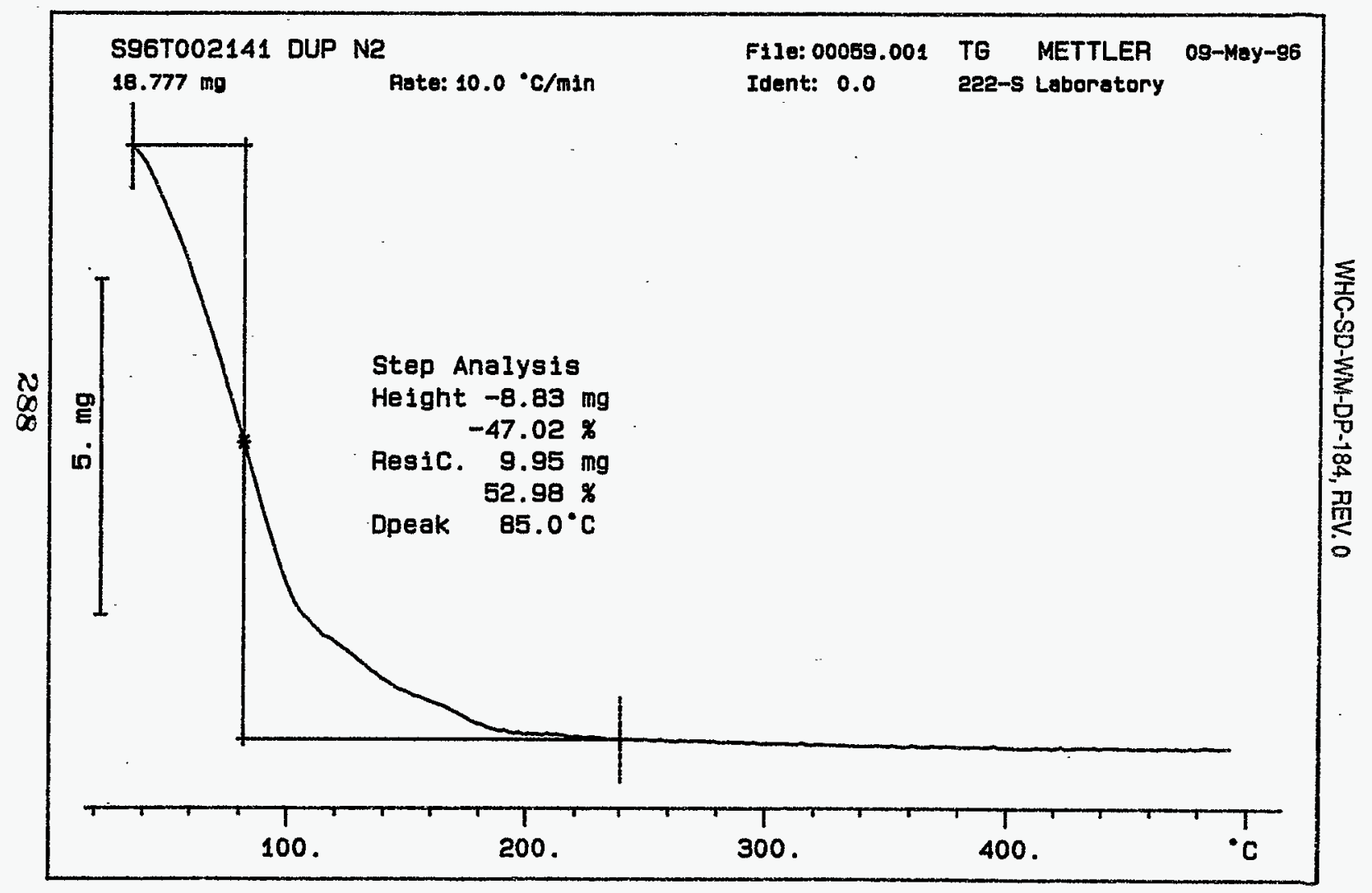




\section{LABCORE Data Entry Template for Worklist\#}

Analyst: $\quad K R M \quad$ Instrument: TGA0 L Book \# $8208 \mathrm{~A}$

Method: LA-560-112 Rev/Mod B-I

Worklist Comment: U-107 TGA RUN UNDER N2. RCJ

\begin{tabular}{|c|c|c|c|c|c|c|c|c|c|c|}
\hline GROUP & PROJECT & $\begin{array}{l}S \text { TYPE } \\
1 \text { STD }\end{array}$ & SAMPLE\# & & $\begin{array}{c}\text { A } \cdots \text { TEST } \cdots-1 \\
\text { TGA-01 }\end{array}$ & $\begin{array}{l}\text { MATRIX } \\
\text { LIOUID }\end{array}$ & $\begin{array}{l}\text { ACTUAL } \\
59.2\end{array}$ & $59.13^{*}$ & N/A & UNIT \\
\hline 96000422 & $u-107$ & 2 SAMPLE & S96T002142 & 0 & TEA-DI & LIQUID & W/A & 52,45 & & $x$ \\
\hline 96000422 & $U-107$ & 3 DUP & s96T002142 & 0 & TGA-01 & LIQUID & 52,45 & 52.18 & N/A & $\%$ \\
\hline
\end{tabular}

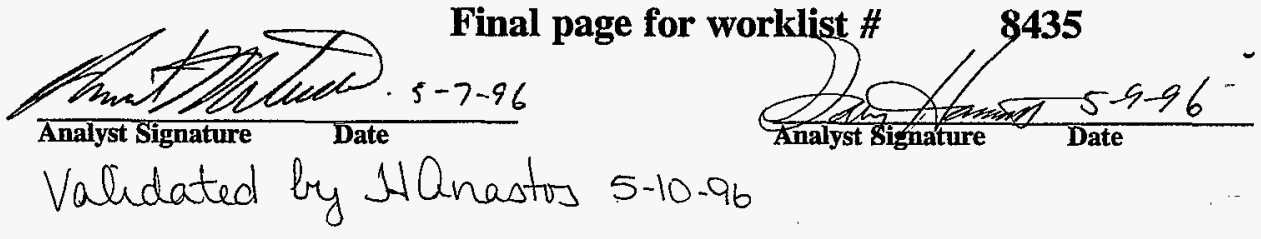

Data Entry Comments:

Units shown for QC (SPK \& STD) may not reflect the actual units. DL = Detection Limit, $S=$ Worklist Slot Number, $\boldsymbol{R}=$ Replicate Number, $A=$ Allquot Code. 
SIGNATURE BELOW REPRESENTS CHEMICAL TECHNOLOGIST/CHEMIST THAT

COMPLETED/VERIFIED THE CALIBRATION/ANALYSIS ON PAGES X9OTO 292

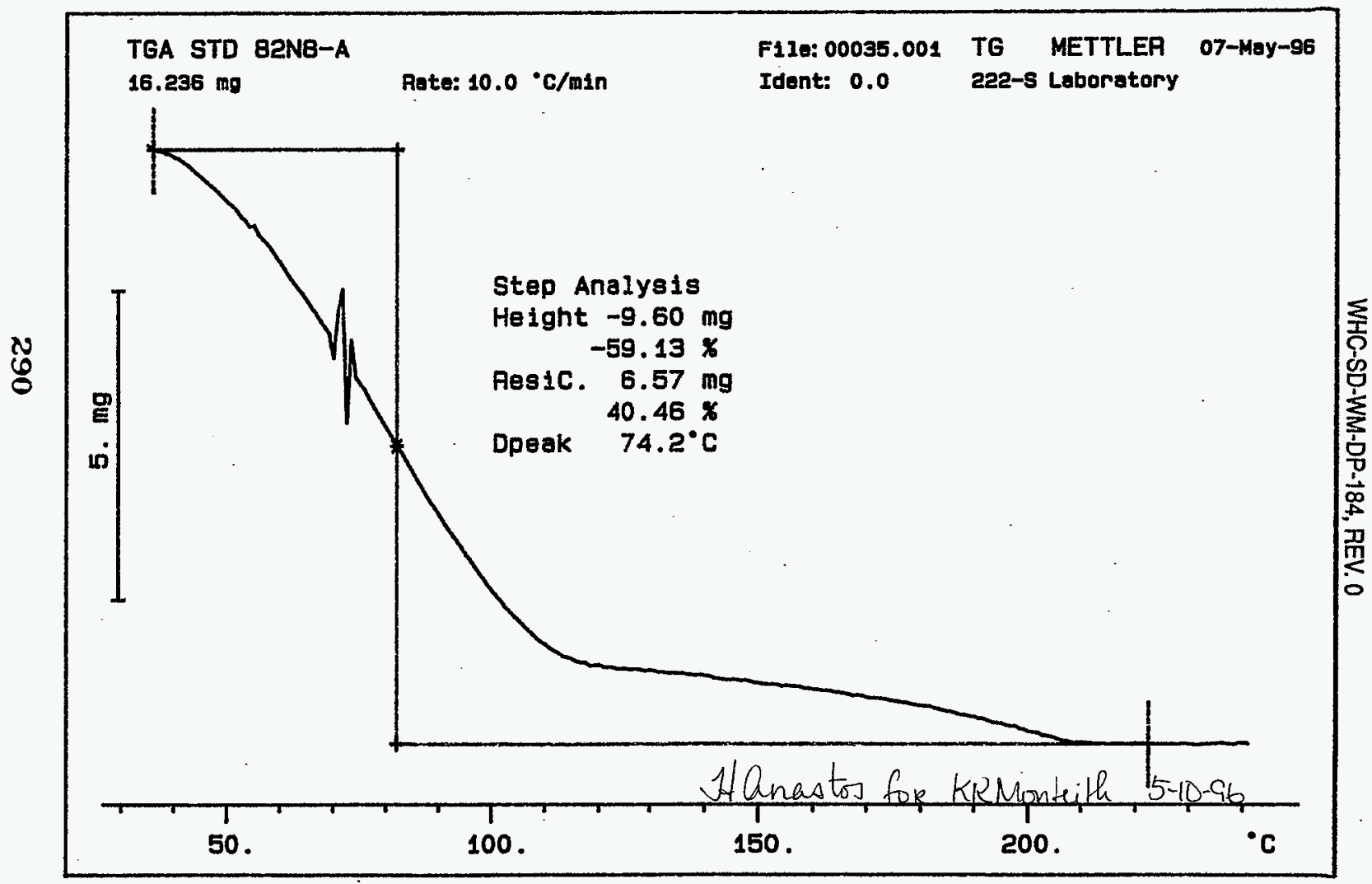




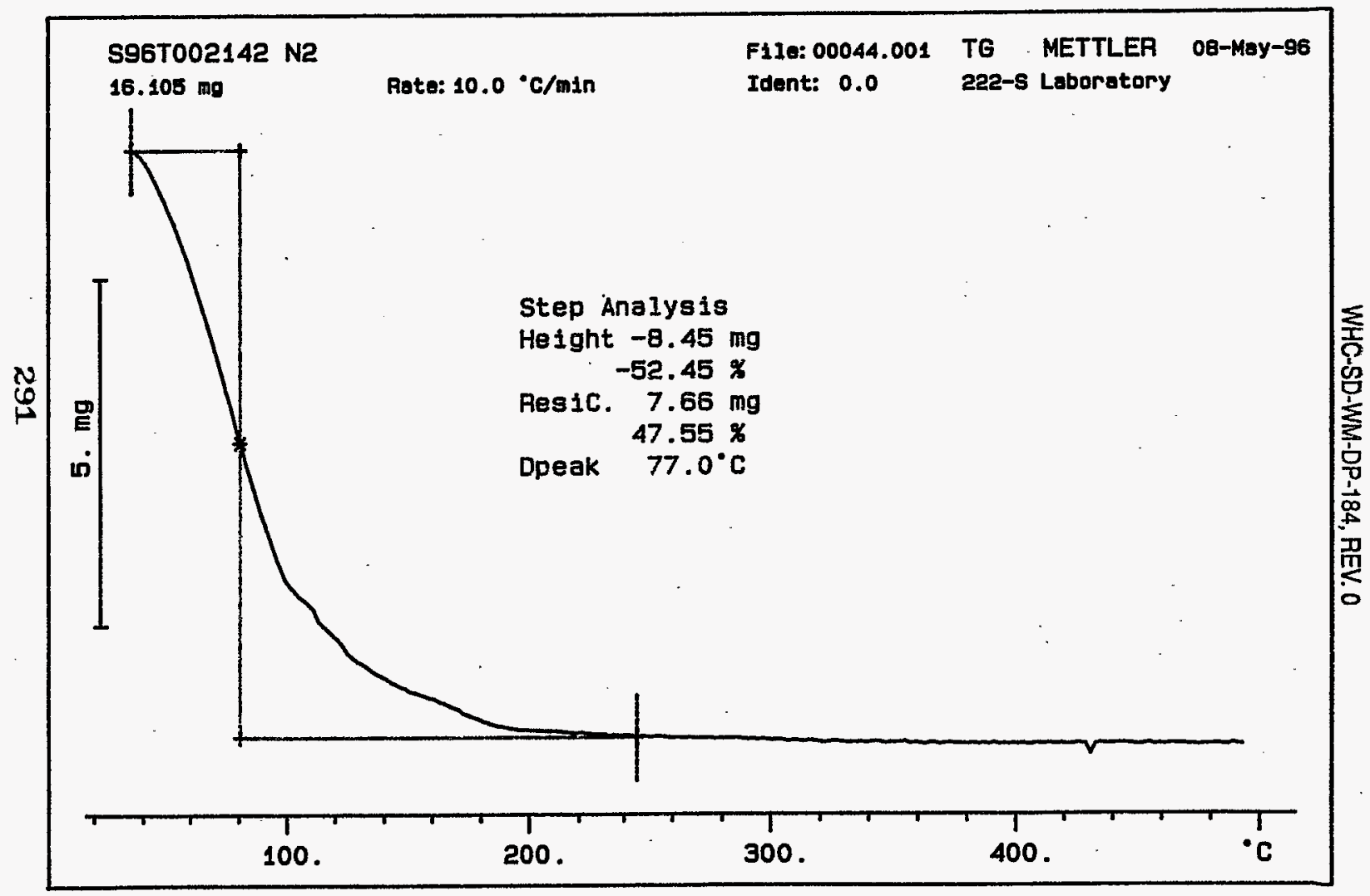




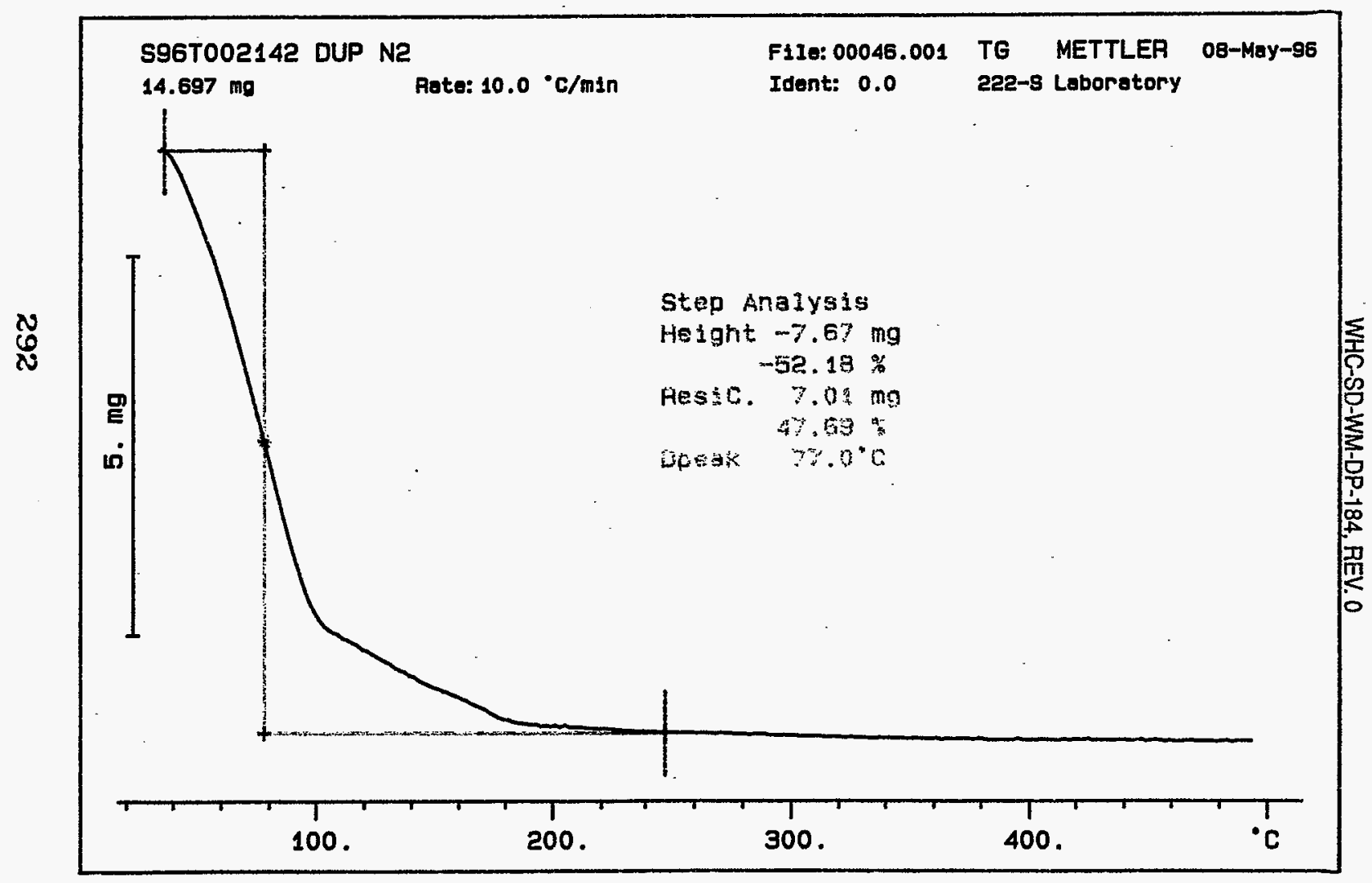


WHC-SD-WM-DP-184, REV. 0

worklistrpt Version 2.1 05/15/95

Analyst: KRM Instrument: TGAO I Book\# 82N8A

Method: LA-560-112 Rev/Mod B-l

Worklist Comment: U-107 TGA RUN UNDER N2. RCJ

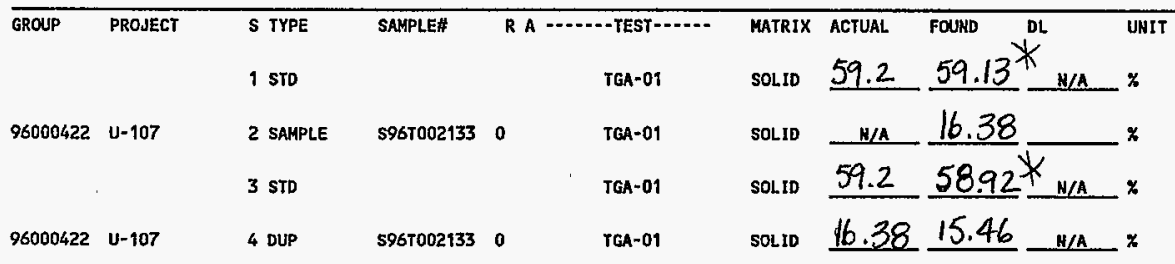

\section{Final page for worklist \#}

See attached for sianatures Analyst Signature Datey 5-13-96

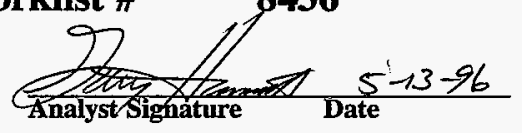
Validated by Nanastos 5-14-96 


\section{LABCORE Data Entry Template for Worklist\#}

Analyst: $\quad K R m$ Instrument: TGAO Book \# $82 \mathrm{NEA}$

Method: LA-560-112 Rev/Mod B- I

Worklist Comment: U-107 TGA RUN UNDER N2. RCJ

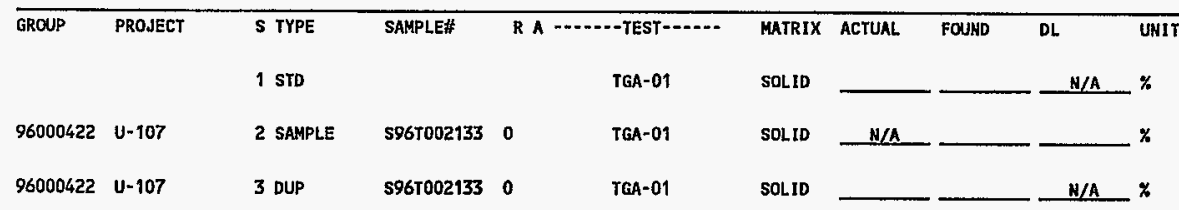

\section{Final page for worklist \# $\quad \mathbf{8 4 3 6}$}

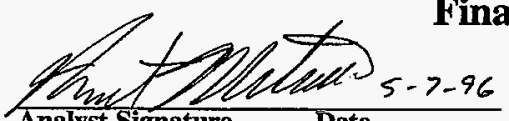

Analyst Signature Date

Analyst Signature Date

Data Entry Comments:

Units shown for QC (SPK \& STD) may not reflect the actual units. $D L=$ Detection Limit, $S=$ Worklist Slot Number, $\boldsymbol{R}=$ Replicate Number, $A=$ Aliquot Code. 


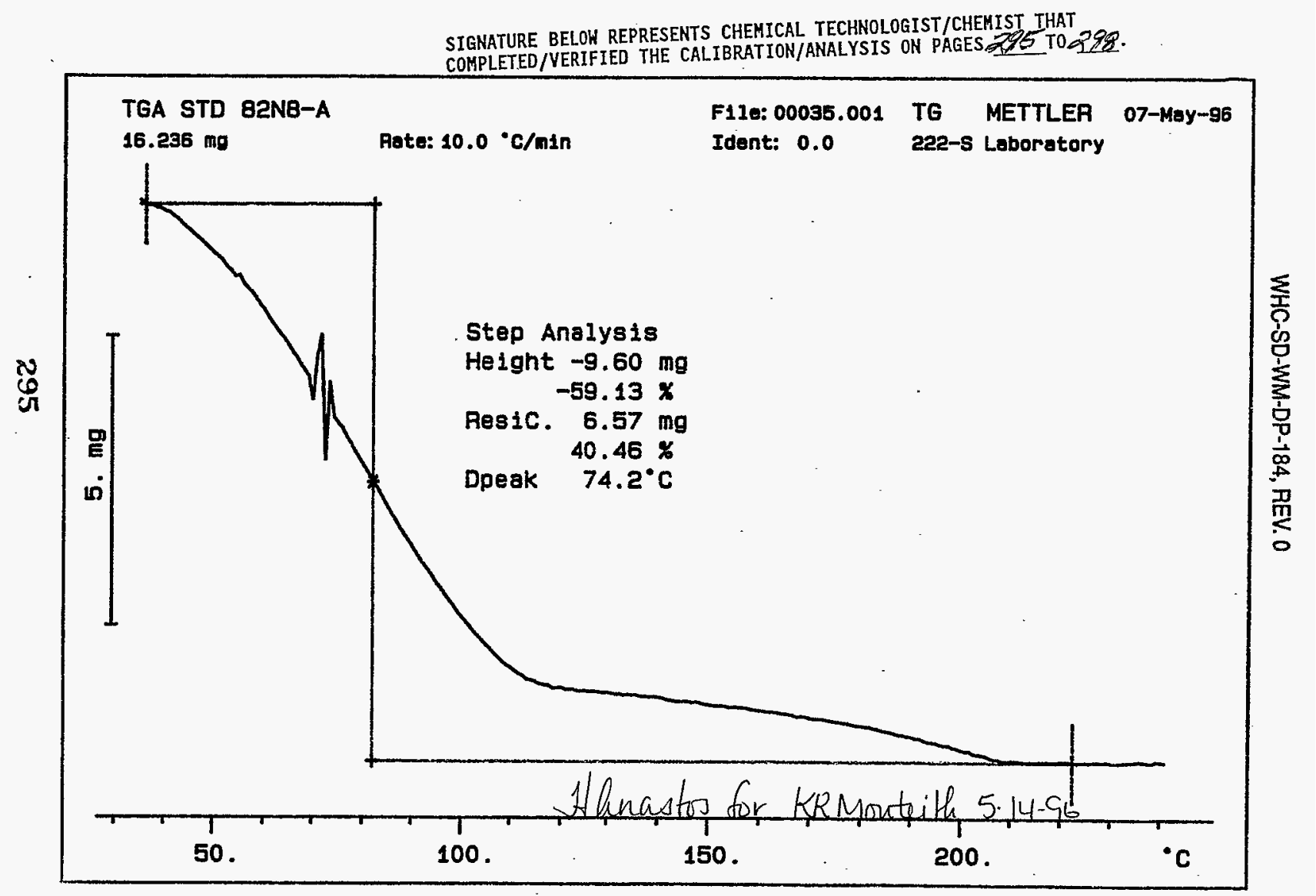




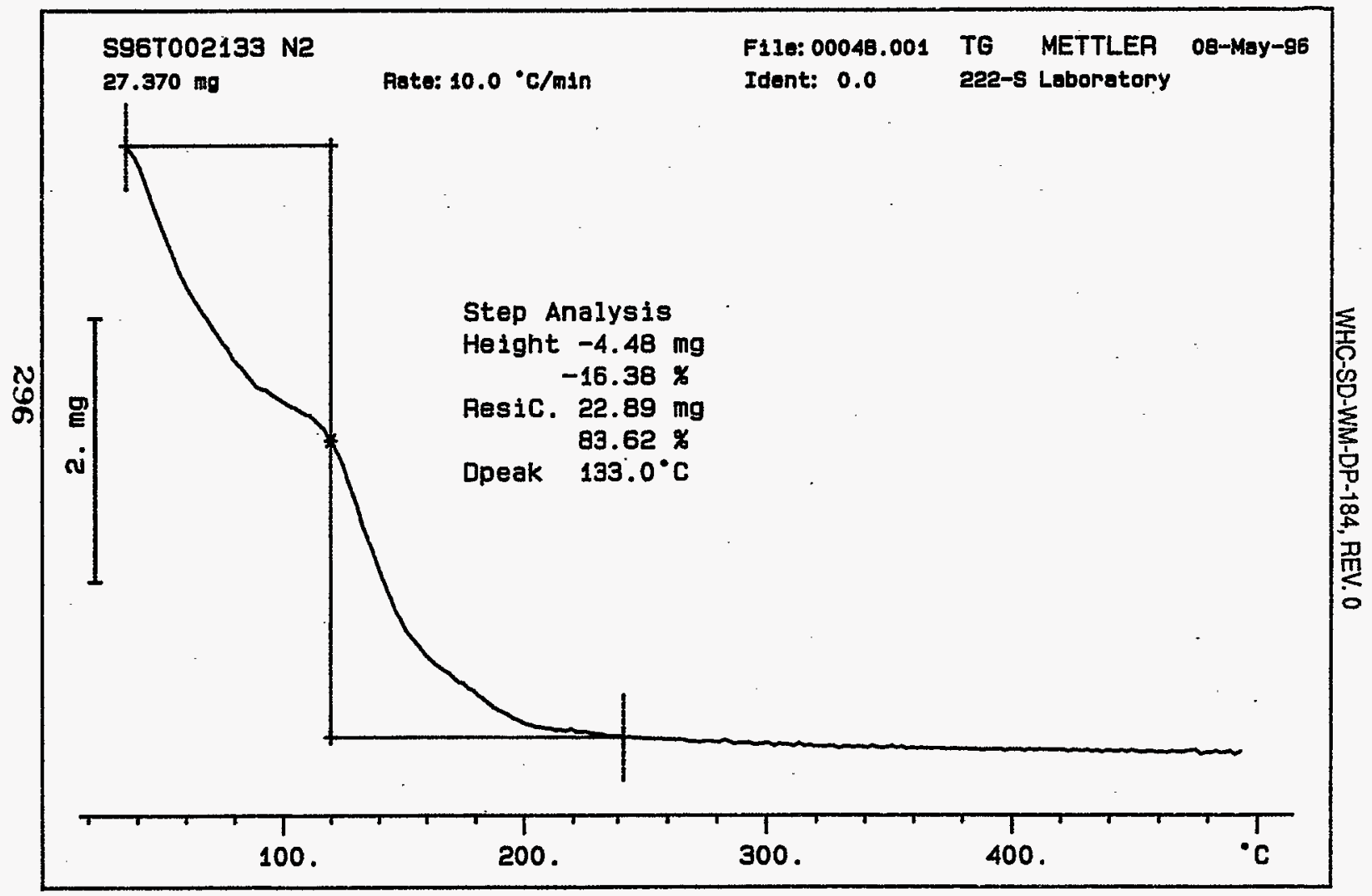


WHC-SD-WM-DP-184, REV. 0

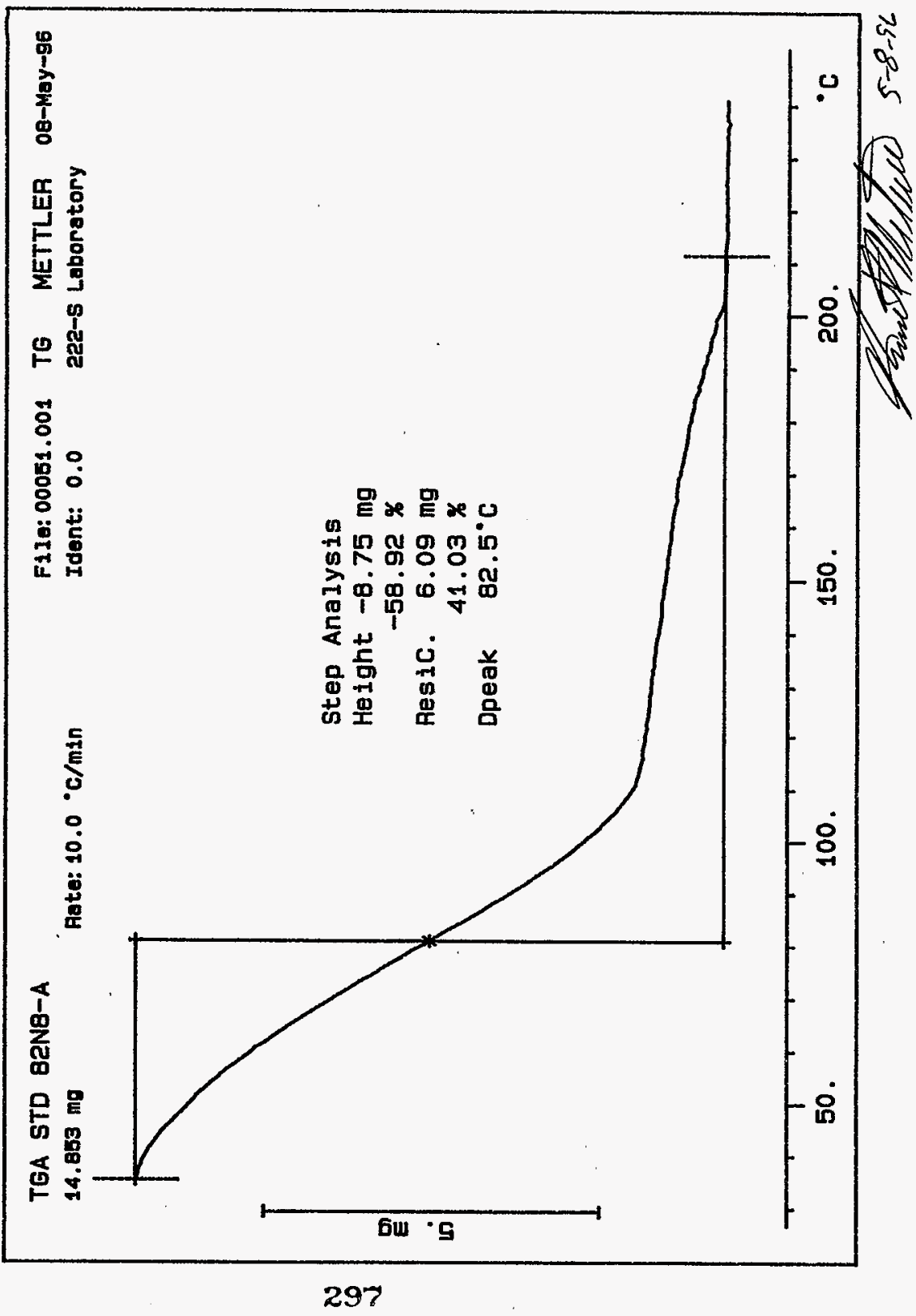




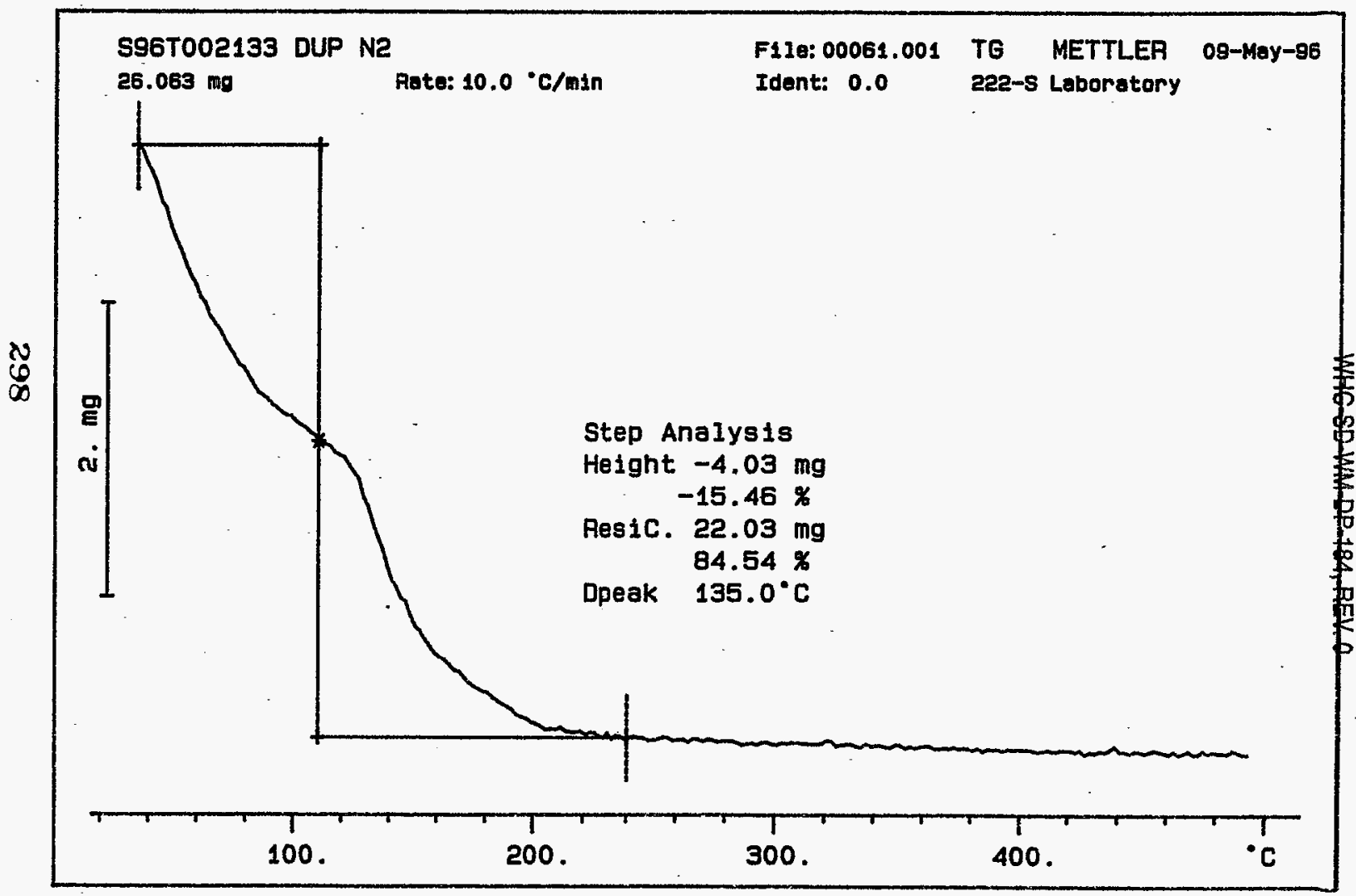




\section{LABCORE Data Entry Template for Worklist\#}

Analyst: RMcCoon Instrument: TGAO I_ Book \#2N8A

Method: LA-560-112 Rev/Mod $B-/$

Worklist Comment: U-107 TGA-01 RUN UNDER N2 RTS!

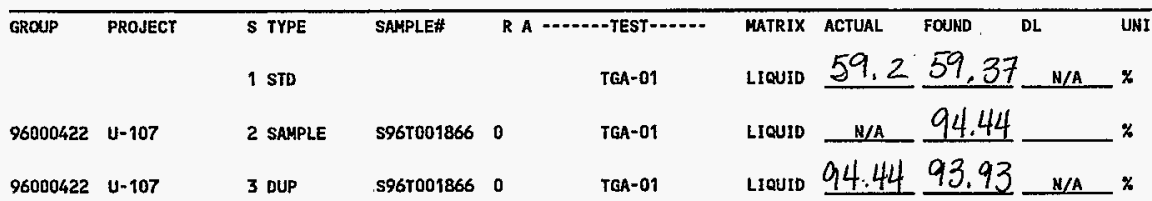

\section{Final page for worklist \# $\quad \mathbf{8 9 4 7}$}

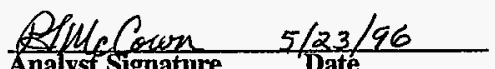

Analyst Signature Date

Verified / Validated bry

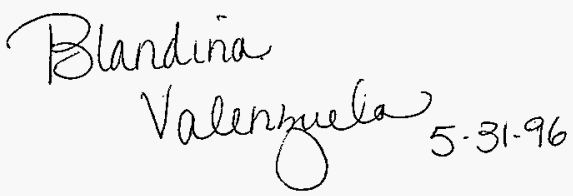

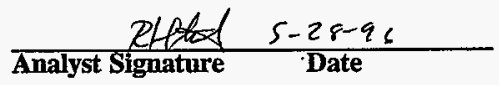

Data Entry Comments:

Units shown for $Q C$ (SPK \& STD) may not reflect the actual units. $D L=$ Detection Limit, $S=$ Worklist Slot Number, $R=$ Replicate Number, $A=$ Aliquot Code. 


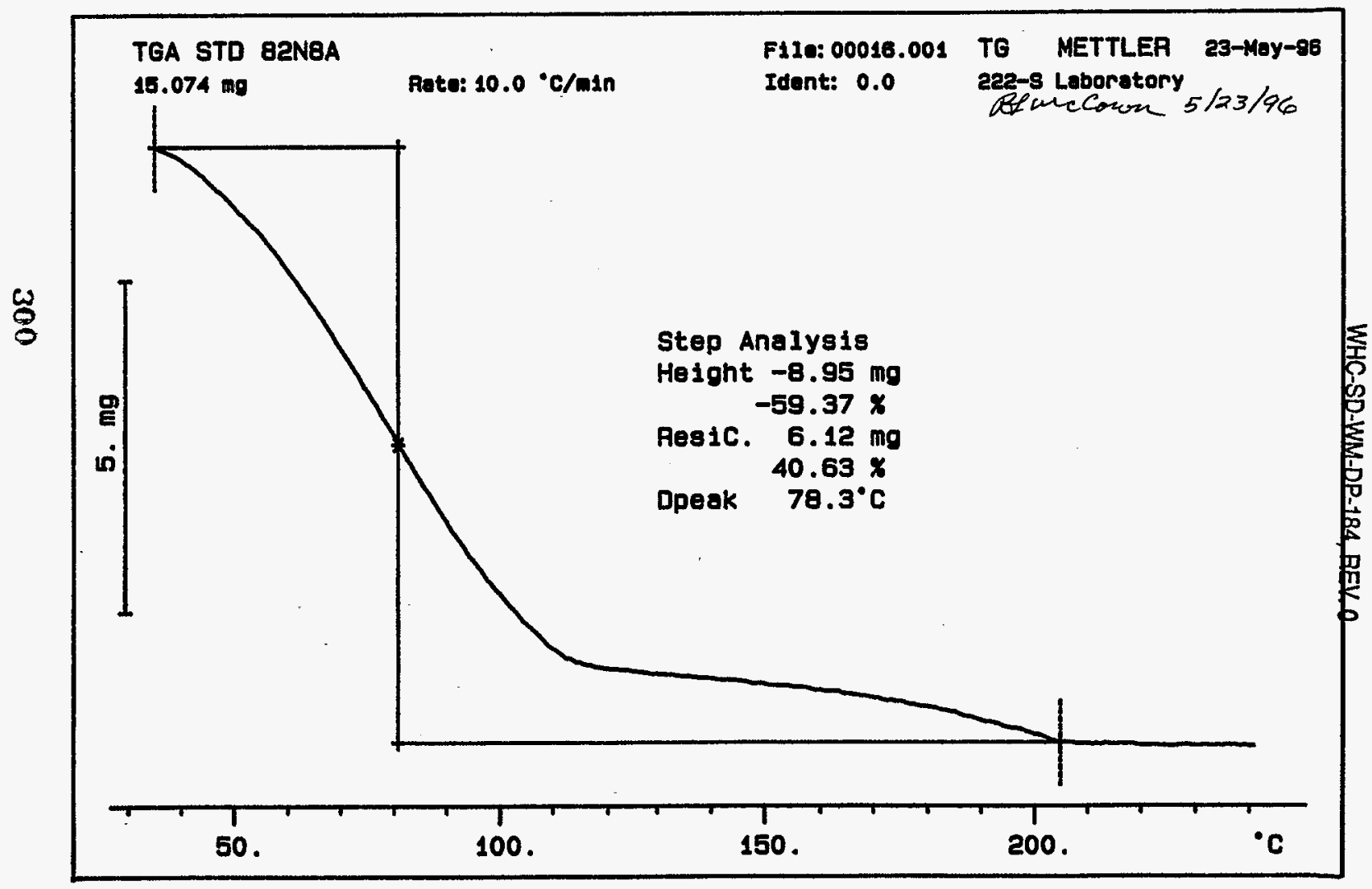




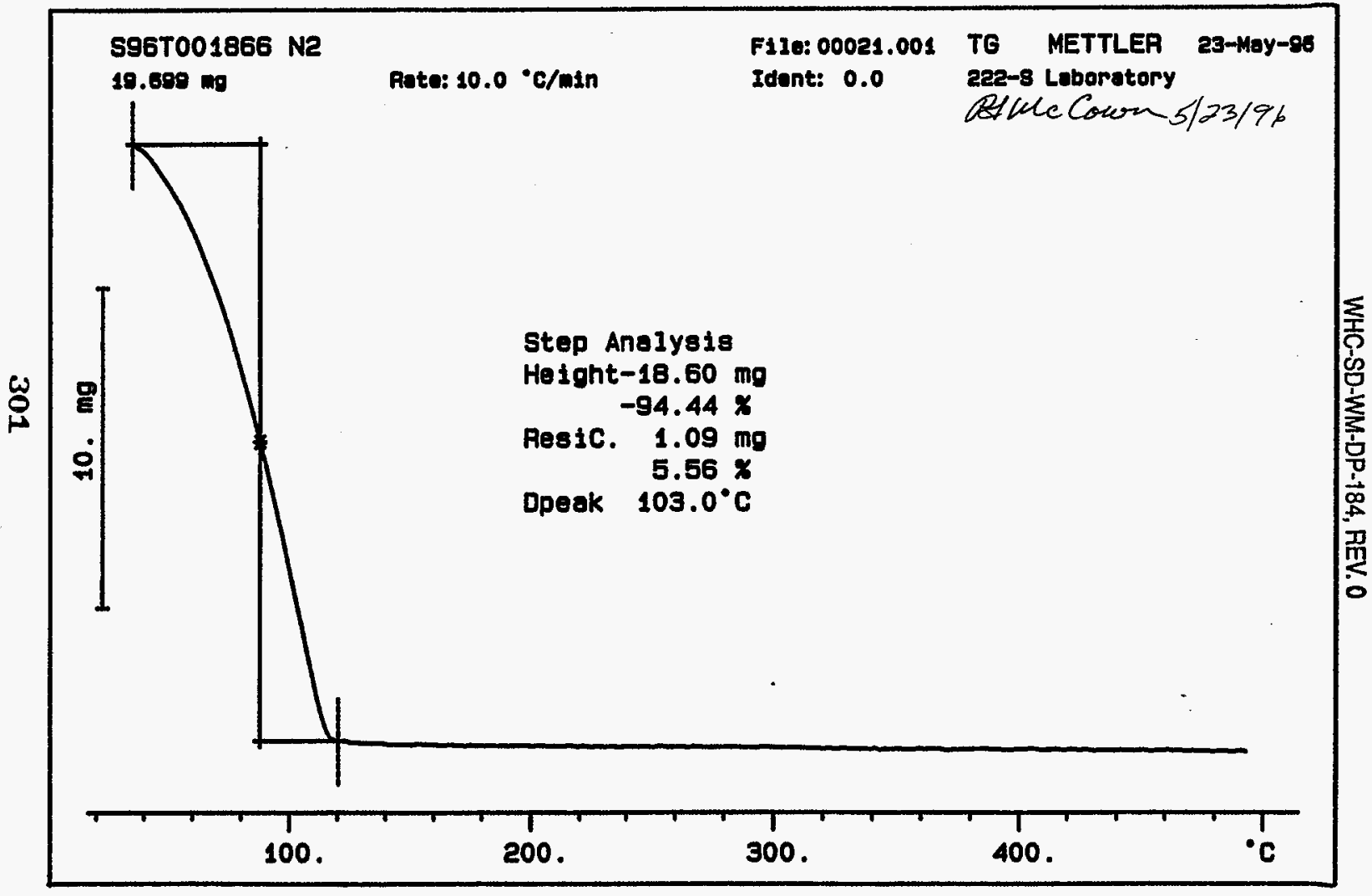




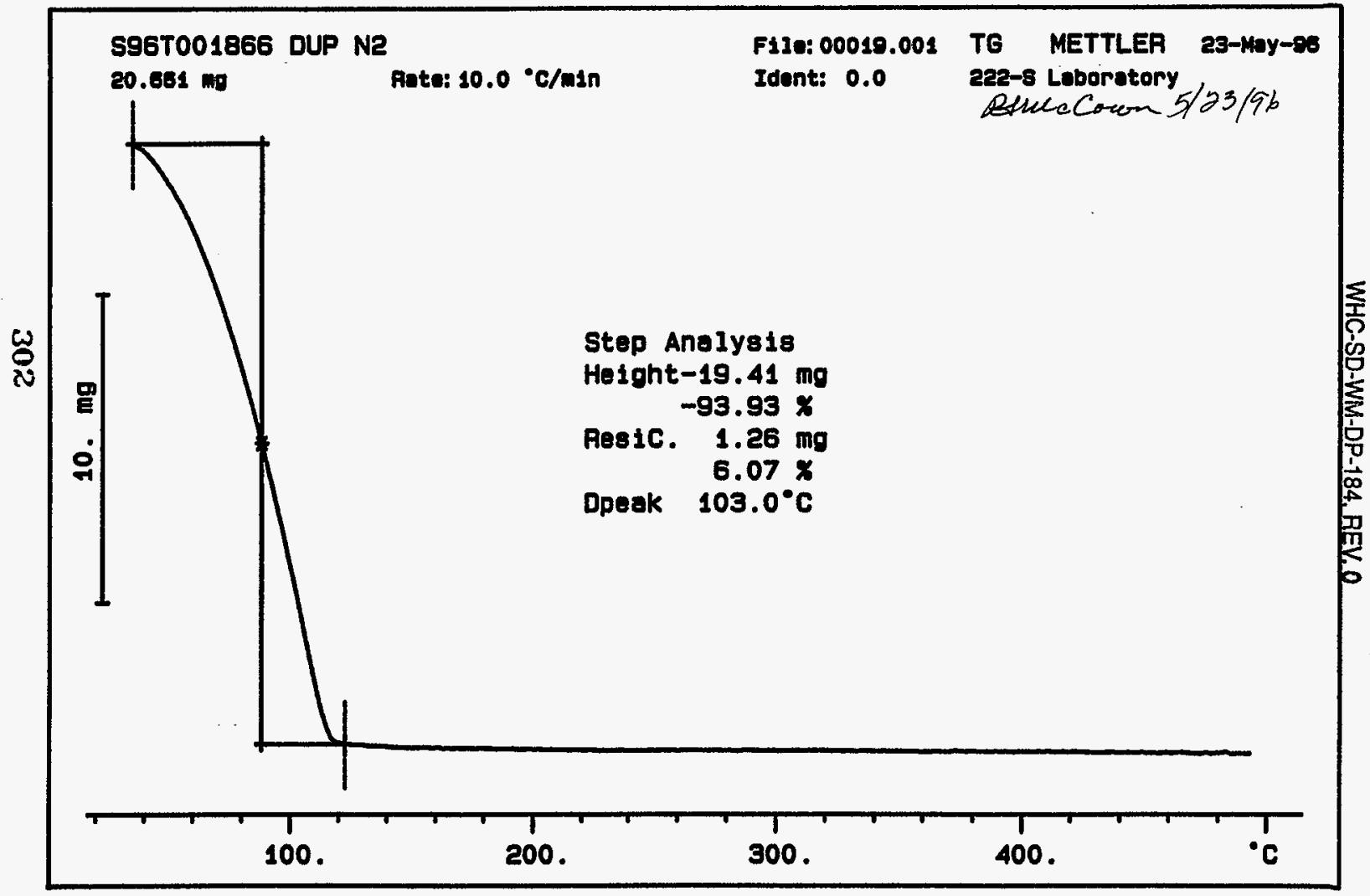




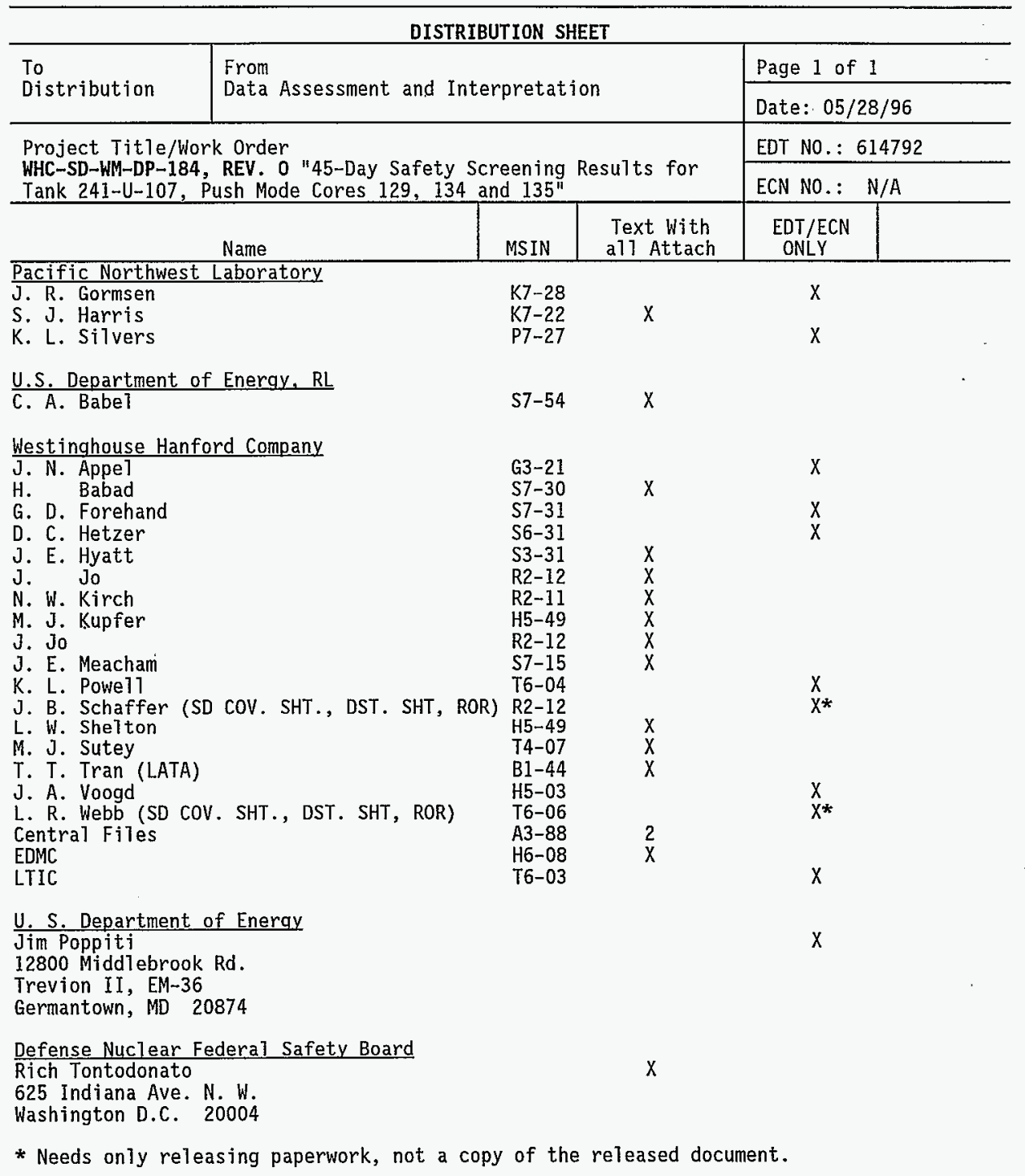

